\begin{tabular}{|c|c|c|c|}
\hline \multicolumn{2}{|c|}{$\begin{array}{c}\text { 2. To: (Receiving organization) } \\
\text { Distribution }\end{array}$} & $\begin{array}{l}\text { 3. From: (originating organization) } \\
\text { Data Assessment and } \\
\text { Interpretation }\end{array}$ & $\begin{array}{r}\text { 4. Related EDT No.: } \\
\text { N/A }\end{array}$ \\
\hline \multicolumn{2}{|c|}{$\begin{array}{l}\text { 5. Proj./Prog./Dept./Div.: } \\
\text { Tank 241-AN-105/Waste } \\
\text { Management/DAI/Process } \\
\text { Engineering }\end{array}$} & $\begin{array}{l}\text { 6. Design Authority/ Design Agent/Cog. } \\
\text { Engr.: } \quad \text { Jaiduk Jo }\end{array}$ & $\begin{array}{l}\text { 7. Purchase order No.: } \\
\qquad N / A\end{array}$ \\
\hline \multirow{2}{*}{\multicolumn{3}{|c|}{$\begin{array}{l}\text { 8. originator Remarks: } \\
\text { This document is being released into the supporting document } \\
\text { system for retrievability purposes. }\end{array}$}} & $\begin{array}{c}\text { 9. Equip./Component No.: } \\
N / A\end{array}$ \\
\hline & & & $\begin{array}{l}\text { 10. System/Bldg./Facility: } \\
241-A N-105\end{array}$ \\
\hline \multirow{3}{*}{\multicolumn{3}{|c|}{$\begin{array}{l}\text { 11. Receiver Remarks: } \\
\text { For release. }\end{array}$}} & $\begin{array}{r}\text { 12. Major Assm. Dwg. No.: } \\
\text { N/A }\end{array}$ \\
\hline & & & $\begin{array}{l}\text { 13. Permit/Permit Application No.: } \\
\text { N/A }\end{array}$ \\
\hline & & & $\begin{array}{c}\text { 14. Requi red Response Date: } \\
04 / 30 / 97\end{array}$ \\
\hline
\end{tabular}

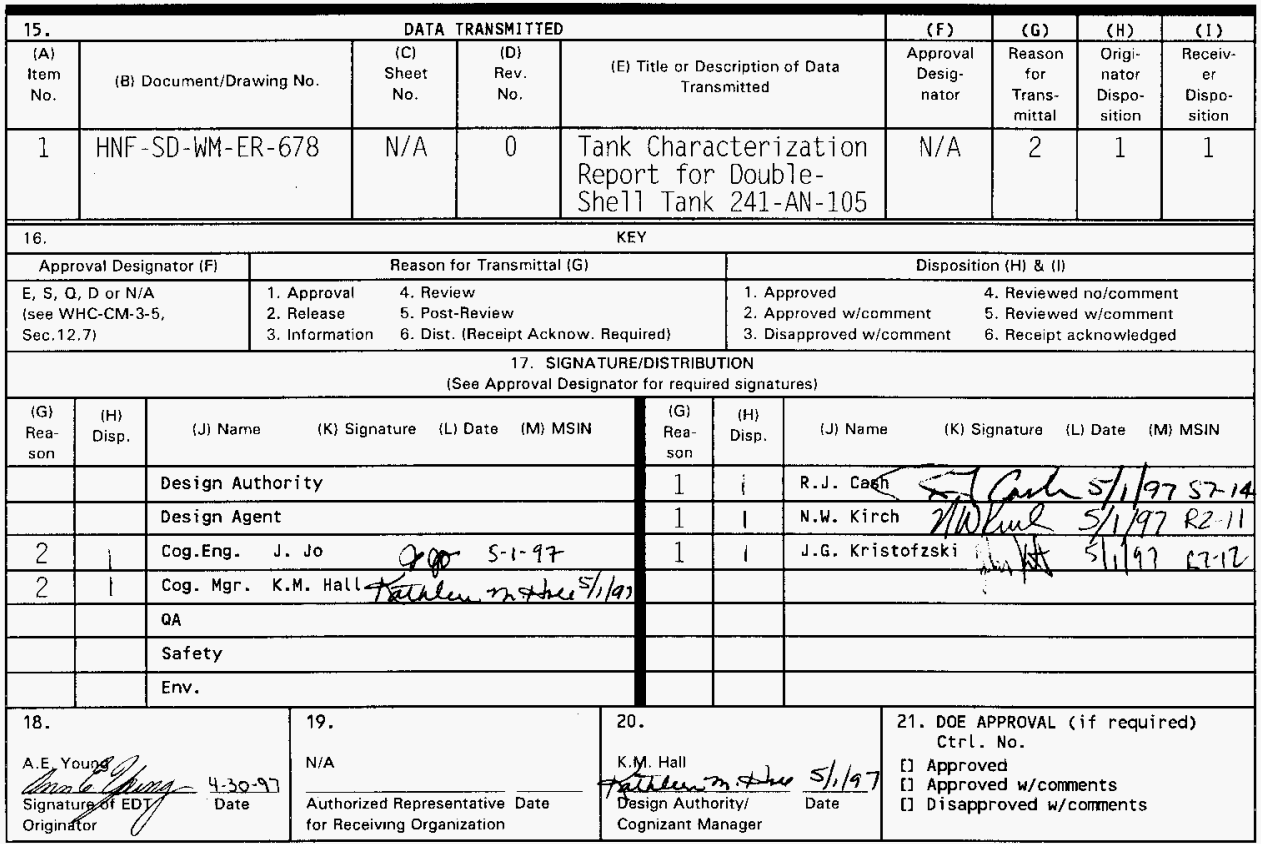




\section{Tank Characterization Report for Double-Shell Tank 241-AN-105}

Jaiduk Jo

Lockheed Martin Hanford Corp., Richland. WA 99352

U.S. Department of Energy Contract DE-AC06-87RL10930

EDT/ECN: EDT-617654 UC: 2070

Org Code: 74620

B\&R Code: EW 3120074 Total Pages: $335^{\circ}$

Key Words: Waste Characterization, Double-Shel1 Tank. DST, Tank 241-AN105. Tank AN-105, AN-105. AN Farm. Tank Characterization Report. TCR, Waste Inventory, TPA Mi lestone M-44

Abstract: This document summarizes the information on the historical uses. present status, and the sampling and analysis results of waste stored in Tank 241-AN-105. This report supports the requirements of the Tri-Party Agreement Milestone M-44-10.

TRADEMARK DISCLAIMER. Reference herein to any specific commercial product, process, or service by trade name, trademark, manufacturer, or otherwise, does not necessarily constitute or imply its endorsement, recommendation, or favoring by the United States Government or any agency thereof or $i$ ts contractors or subcontractors.

Printed in the United States of America. To obtain copies of this document, contact: WHC/BCS Document Control Services, P.O. Box 1970, Mailstop H6-08, Richland WA 99352, Phone (509) 372-2420; Fax (509) 376-4989.
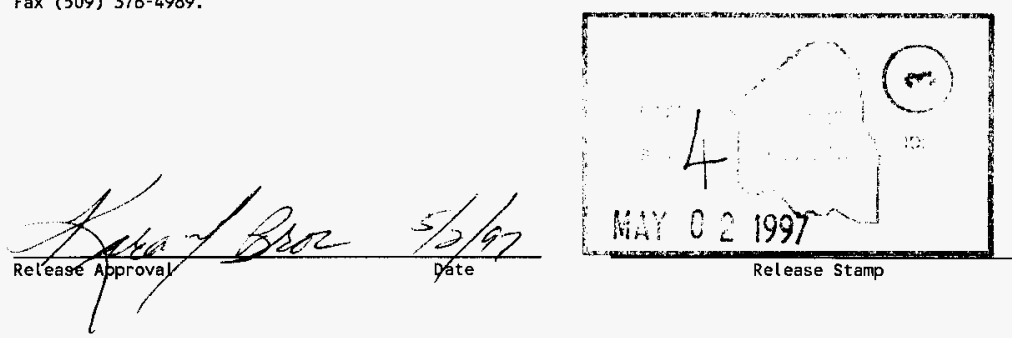

Approved for Public Release 


\title{
Tank Characterization Report for Double-Shell Tank 241-AN-105
}

\author{
J. Jo \\ L. W. Shelton Jr. \\ Lockheed Martin Hanford Corporation \\ T. L. Weish \\ B\&W Protec, Inc. \\ J. Stroup \\ Fluor Daniel Northwest, Inc
}

Date Published

May 1997

Prepared for the U.S. Department of Energy

Assistant Secretary for Environmental Management

Project Hanford Management Contractor for the

U.S. Department of Energy under Contract DE-ACO6-96RL13200

Approved for public release; distribution is unlimited 


\section{CONTENTS}

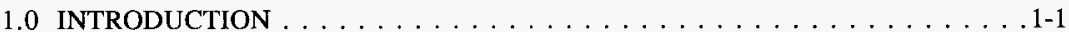

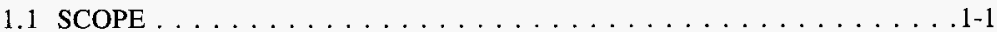

1.2 TANK BACKGROUND $\ldots \ldots \ldots \ldots \ldots \ldots \ldots \ldots \ldots \ldots \ldots$

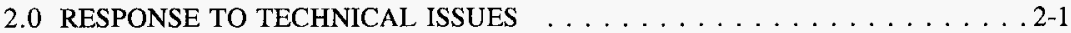

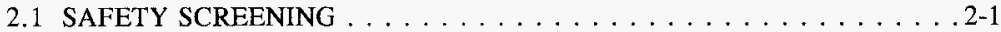

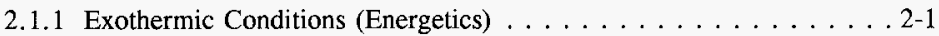

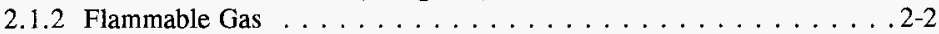

2.1 .3 Criticality . . . . . . . . . . . . . . 2-2

2.2 FLAMMABLE GAS TANK SAFETY EVALUATION $\ldots \ldots \ldots \ldots .2-2$

2.3 OTHER TECHNICAL ISSUES . . . . . . . . . . . . . . . 2-4

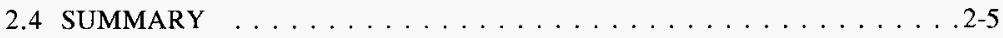

3.0 BEST-BASIS STANDARD INVENTORY ESTIMATE $\ldots \ldots \ldots \ldots \ldots$. $\ldots \ldots$

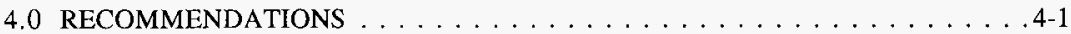

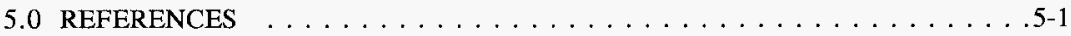

APPENDIXES

APPENDIX A HISTORICAL TANK INFORMATION $\ldots \ldots \ldots \ldots \ldots \ldots$ A-1

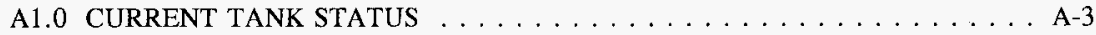

A2.0 TANK DESIGN AND BACKGROUND $\ldots \ldots \ldots \ldots \ldots \ldots \ldots \ldots$ A-4

A3.0 PROCESS KNOWLEDGE $\ldots \ldots \ldots \ldots \ldots \ldots \ldots \ldots \ldots \ldots \ldots \ldots$

A3.1 WASTE TRANSFER HISTORY $\ldots \ldots \ldots \ldots \ldots \ldots \ldots \ldots \ldots$ A-8

A3.2 HISTORICAL ESTIMATION OF TANK CONTENTS $\ldots \ldots \ldots \ldots$ A .9

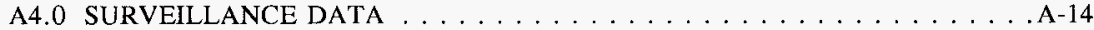

A4.1 SURFACE LEVEL READINGS $\ldots \ldots \ldots \ldots \ldots \ldots \ldots \ldots \ldots$ A-14

A4.2 INTERNAL TANK TEMPERATURES $\ldots \ldots \ldots \ldots \ldots \ldots \ldots$. . . . . . .

A4.3 TANK 241 -AN-105 PHOTOGRAPHS . . . . . . . . . . . A-14

A4.4 STANDARD HYDROGEN MONITORING SYSTEMS . . . . . . . A-15

A5.0 APPENDIX A REFERENCES $\ldots \ldots \ldots \ldots \ldots \ldots \ldots \ldots \ldots$ A-18 


\section{CONTENTS (cont)}

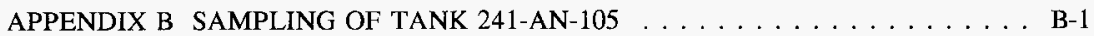

B1.0 TANK SAMPLING OVERVIEW $\ldots \ldots \ldots \ldots \ldots \ldots \ldots \ldots \ldots$

B1.1 DESCRIPTION OF SAMPLING EVENT $\ldots \ldots \ldots \ldots \ldots \ldots \ldots$

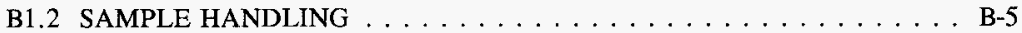

B1.3 SAMPLE ANALYSIS $\ldots \ldots \ldots \ldots \ldots \ldots \ldots \ldots \ldots \ldots$

B1.4 DESCRIPTION OF HISTORICAL SAMPLING EVENT . . . . . . . . B-20

B2.0 ANALYTICAL RESULTS . . . . . . . . . . . . . . . . . . B-29

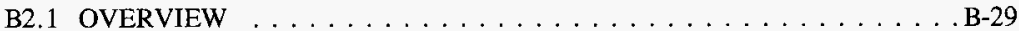

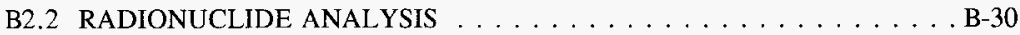

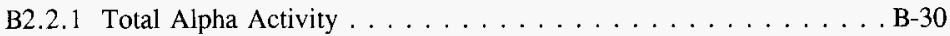

B2.2.2 Total Beta . . . . . . . . . . . . . . . . . B-30

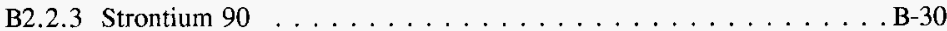

B2.2.4 Gamma Energy Analysis . . . . . . . . . . . B-30

B2.2.5 Total Uranium . . . . . . . . . . . . . . . . . B-31

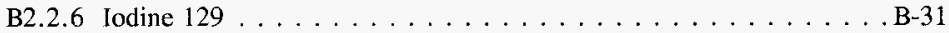

B2.2.7 Technecium $99 \ldots \ldots \ldots \ldots \ldots \ldots \ldots \ldots \ldots \ldots . . . \ldots \ldots$

B2.2.8 Alpha Energy Analyses . . . . . . . . . . . . . . . B-31

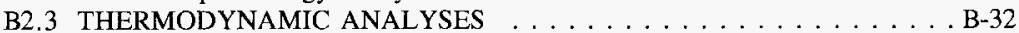

B2.3.1 Thermogravimetric Analysis . . . . . . . . . . B-32

B2.3.2 Differential Scanning Calorimetry $\ldots \ldots \ldots \ldots \ldots \ldots \ldots$ B-33

B2.4 DENSITY AND SPECIFIC GRAVITY $\ldots \ldots \ldots \ldots \ldots \ldots \ldots$ B-33

B2.5 INORGANIC ANALYSES . . . . . . . . . . . . . . . B-33

B2.5.1 Inductively Coupled Plasma . . . . . . . . . . . . . . B-34

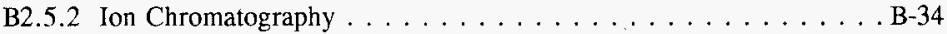

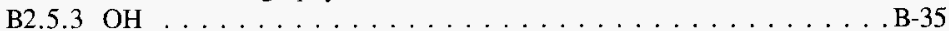

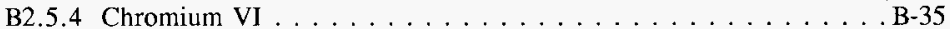

B2.6 TOTAL INORGANIC CARBON/TOTAL ORGANIC CARBON

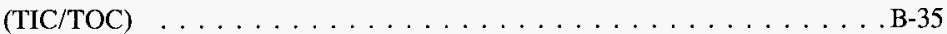

B2.7 VAPOR PHASE MEASUREMENT $\ldots \ldots \ldots \ldots \ldots \ldots \ldots \ldots \ldots$ B-35

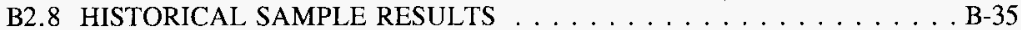

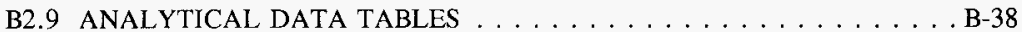

B3.0 ASSESSMENT OF CHARACTERIZATION RESULTS $\ldots \ldots \ldots \ldots \ldots$. . . . . . .

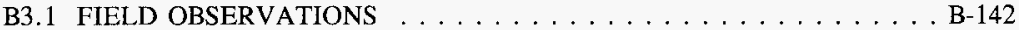

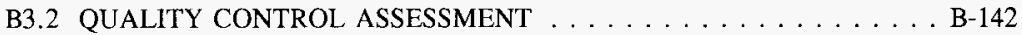

B3.3 DATA CONSISTENCY CHECKS . . . . . . . . . . . . . B B-143

B3.3.1 Comparison of Results from Different Analytical Methods . . . B-143

B3.3.2 Mass and Charge Balance . . . . . . . . . . . . . B-144 


\section{CONTENTS (cont)}

B3.4 MEAN CONCENTRATIONS AND CONFIDENCE INTERVALS . . . B-148 B3.4.1 Mean Concentrations . . . . . . . . . . . . . . B-149

B3.4.2 Analysis of Variance Model . . . . . . . . . . B-183

B3.4.3 Sampling Based Tank Inventory . . . . . . . . . B-186

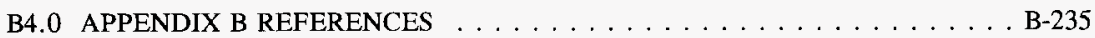

APPENDIX C STATISTICAL ANALYSIS FOR ISSUE RESOLUTION . . . . . . C C-1

C1.0 STATISTICS FOR SAFETY SCREENING DATA QUALITY OBJECTIVE . . . C-3

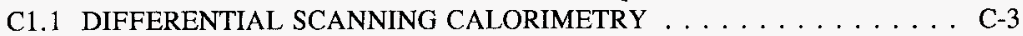

C1.2 TOTAL ALPHA $\ldots \ldots \ldots \ldots \ldots \ldots \ldots \ldots \ldots \ldots \ldots$

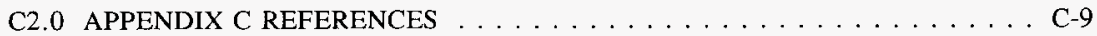

APPENDIX D EVALUATION TO ESTABLISH BEST-BASIS INVENTORY FOR

TANK $241-$ AN-105 . . . . . . . . . . . . . . . . D-1

D1.O CHEMICAL INFORMATION SOURCES $\ldots \ldots \ldots \ldots$ D-3

D2.0 COMPARISON OF COMPONENT INVENTORY VALUES $\ldots \ldots \ldots \ldots$ D-3

D3.0 COMPONENT INVENTORY EVALUATION $\ldots \ldots \ldots \ldots \ldots$ D-7

D4.0 DEFINE THE BEST-BASIS AND ESTABLISH COMPONENT

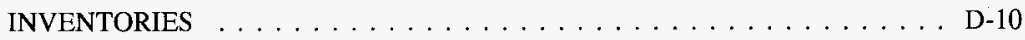

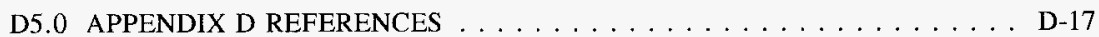

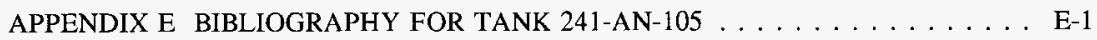


HNF-SD-WM-ER-678 Rev. 0

\section{LIST OF FIGURES}

A2-1 Riser Configuration for Tank AN-105 . . . . . . . . . . . . . A-6

A2-2 Tank 241-AN-105 Cross Section and Schematic . . . . . . . . . . . A-7

A3-1 Tank Layer Model $\ldots \ldots \ldots \ldots \ldots \ldots \ldots \ldots \ldots \ldots$. . . . . . . . . . . . .

A4-1 Tank 241-AN-105 Level History . . . . . . . . . . . . . . . . A-16

A4-2 Tank 241-AN-105 Weekly High Temperature Plot . . . . . . . . . . . . . A-17 


\section{LIST OF TABLES}

1-1 Summary of Recent Sampling $\ldots \ldots \ldots \ldots \ldots \ldots \ldots \ldots \ldots \ldots \ldots \ldots \ldots$

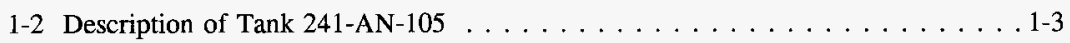

2-1 Heat Load for Tank 241-AN-105 Based on Radionuclide Content . . . . . . 2-5

2-2 Summary of Safety Screening and Historical Flammable Gas . . . . . . 2-5

3-1 Best-Basis Inventory Estimates for Nonradioactive Components in Tank 241-AN-105 Supernatant as of May 31, $1996 \ldots \ldots \ldots \ldots$. . . . . . .

3-2 Best-Basis Inventory Estimates for Radioactive Components in Tank 241-AN-105 Supernatant as of May 31, 1996 . . . . . . . . . 3-3

3-3 Best-Basis Inventory Estimates for Nonradioactive Components in Tank 241-AN-105 Salt Slurry as of May 31, $1996 \ldots \ldots \ldots \ldots$. . . . . . . .

3-4 Best-Basis Inventory Estimates for Radioactive Components in Tank 241-AN-105 Salt Slurry as of May 31, $1996 \ldots \ldots \ldots$. . . . . . . . .

3-5 Best-Basis Total Inventory Estimates for Nonradioactive Components

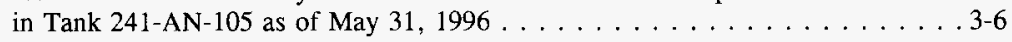

3-6 Best-Basis Total Inventory Estimates for Radioactive Components in

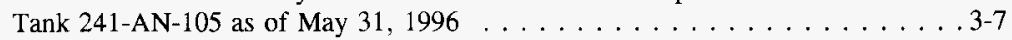

4-1 Acceptance of Tank 241-AN-105 Sampling and Analysis . . . . . . . . . 4-1

4-2 Acceptance of Evaluation of Characterization Data and Information for Tank $241-\mathrm{AN}-105 \ldots \ldots \ldots \ldots \ldots . . \ldots \ldots$. . . . . . . . . . .

A1-1 Tank Contents Status Summary $\ldots \ldots \ldots \ldots \ldots \ldots \ldots$ A-4

A2-1 Tank 241-AN-105 Risers . . . . . . . . . . . . A-5

A3-1 Tank 241-AN-105 Major Transfers . . . . . . . . . . . . . . A-9

A3-2 Historical Tank Inventory Estimate $\ldots \ldots \ldots \ldots \ldots \ldots \ldots \ldots \ldots$ A-12 


\section{LIST OF TABLES (cont)}

B1-1 Integrated Data Quality Objective Requirements for Tank 241-AN-105 . . . . B-5

B1-2a Sample Receipt and Extrusion Information for 241-AN-105, Core $152 \ldots$. . . B-6

B1-2b Sample Receipt and Extrusion Information for $241-A N-105$, Core $153 \ldots$. . B-13

B1-3 Tank 241-AN-105 Sample Analysis Summary . . . . . . . . . . . . . . B-21

B1-4 Analytical Procedures $\ldots \ldots \ldots \ldots \ldots \ldots \ldots \ldots \ldots \ldots \ldots$

B2-1 Analytical Presentation Tables . . . . . . . . . . . . . . . . . . B-29

B2-2 Supernatant Sample of May $1984 \ldots \ldots \ldots \ldots \ldots \ldots \ldots \ldots \ldots$

B2-3 Supernatant Sample of November $1984 \ldots \ldots \ldots \ldots \ldots \ldots \ldots$. . . . . . . . . . . . . .

B2-4 Tank 241-AN-105 Analytical Results: Total Alpha (Alpha Rad) . . . . . . . . B-39

B2-5 Tank 241-AN-105 Analytical Results: Total Alpha (Alpha) . . . . . . . . . . . B-40

B2-6 Tank 241-AN-105 Analytical Results: Total Beta. . . . . . . . . . . . . . . B-40

B2-7 Tank 241-AN-105 Analytical Results: Total Uranium (Uranium Gross Kinetic Phos.) . . . . . . . . . . . . . . . . . B-41

B2-8 Tank 241-AN-105 Analytical Results: Americium-241 (AEA) . . . . . . . . B-41

B2-9 Tank 241-AN-105 Analytical Results: Cm-243/244 (AEA) . . . . . . . . . . B-41

B2-10 Tank 241-AN-105 Analytical Results: Americium-241 . . . . . . . . . . . . B-41

B2-11 Tank 241-AN-105 Analytical Results: Cm-243/244 . . . . . . . . . . . B-42

B2-12 Tank 241-AN-105 Analytical Results: Americium-241 (GEA) . . . . . . . . . B-42

B2-13 Tank 241-AN-105 Analytical Results: Cesium-137 (GEA) . . . . . . . . . . B-42

B2-14 Tank 241-AN-105 Analytical Results: Cobalt-60 (GEA) . . . . . . . . . . . . B-43

B2-15 Tank 241-AN-105 Analytical Results: Europium-154 (GEA) . . . . . . . . . . B-43

B2-16 Tank 241-AN-105 Analytical Results: Europium-155 (GEA) . . . . . . . . . B-43 


\section{LIST OF TABLES (cont)}

B2-17 Tank 241-AN-105 Analytical Results: Iodine-129 . . . . . . . . . . . . B-44

B2-18 Tank 241-AN-105 Analytical Results: H-3 (Liquid

Scintillation) $\ldots \ldots \ldots \ldots \ldots \ldots \ldots \ldots \ldots \ldots \ldots \ldots$. . . . . . . . . .

B2-19 Tank 241-AN-105 Analytical Results: Neptunium-237 . . . . . . . . . . . . B-44

B2-20 Tank 241-AN-105 Analytical Results: Plutonium-239/40 . . . . . . . . . B B-45

B2-21 Tank 241-AN-105 Analytical Results: Strontium-89/90 . . . . . . . . . . B-45

B2-22 Tank 241-AN-105 Analytical Results: Technetium-99 . . . . . . . . . . B-45

B2-23 Tank 241-AN-105 Analytical Results: Exotherm - (DSC) . . . . . . . . . . B-46

B2-24 Tank 241-AN-105 Analytical Results: Percent Water (TGA) . . . . . . . . . B-48

B2-25 Tank 241-AN-105 Analytical Results: Bulk Density . . . . . . . . . . . B-50

B2-26 Tank 241-AN-105 Analytical Results: Specific Gravity . . . . . . . . . . B B-51

B2-27 Tank 241-AN-105 Analytical Results: Aluminum (ICP) . . . . . . . . . . B-52

B2-28 Tank 241-AN-105 Analytical Results: Bismuth (ICP) $\ldots \ldots \ldots \ldots \ldots \ldots$ B-55

B2-29 Tank 241-AN-105 Analytical Results: Boron (ICP) $\ldots \ldots \ldots \ldots \ldots$. . . . .58

B2-30 Tank 241-AN-105 Analytical Results: Cadmium (ICP) . . . . . . . . . . B-61

B2-31 Tank 241-AN-105 Analytical Results: Calcium (ICP) $\ldots \ldots \ldots \ldots \ldots \ldots$ B-64

B2-32 Tank 241-AN-105 Analytical Results: Chromium (ICP) . . . . . . . . . . B-67

B2-33 Tank 241-AN-105 Analytical Results: Copper (ICP) . . . . . . . . . . . B-70

B2-34 Tank 241-AN-105 Analytical Results: Iron (ICP) $\ldots \ldots \ldots \ldots \ldots \ldots$ B-73

B2-35 Tank 241-AN-105 Analytical Results: Lead (ICP) $\ldots \ldots \ldots \ldots \ldots \ldots$. . . . . . .

B2-36 Tank 241-AN-105 Analytical Results: Lithium (ICP) $\ldots \ldots \ldots \ldots$. . . . B-79

B2-37 Tank 241-AN-105 Analytical Results: Magnesium (ICP) $\ldots \ldots \ldots \ldots \ldots$ B-82 


\section{LIST OF TABLES (cont)}

B2-38 Tank 241-AN-105 Analytical Results: Manganese (ICP) $\ldots \ldots \ldots \ldots \ldots$. . . . . .

B2-39 Tank 241-AN-105 Analytical Results: Molybdenum (ICP) $\ldots \ldots \ldots \ldots$. . B-88

B2-40 Tank 241-AN-105 Analytical Results: Nickel (ICP) $\ldots \ldots \ldots \ldots \ldots$. . . . B-91

B2-41 Tank 241-AN-105 Analytical Results: Phosphorus (ICP) $\ldots \ldots \ldots \ldots \ldots$ B-93

B2-42 Tank 241-AN-105 Analytical Results: Potassium (ICP) $\ldots \ldots \ldots \ldots$. . . . . B-96

B2-43 Tank 241-AN-105 Analytical Results: Silicon (ICP) $\ldots \ldots \ldots \ldots \ldots$. . . . . . .

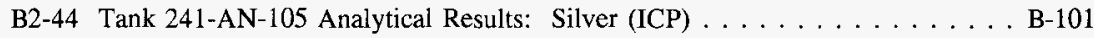

B2-45 Tank 241-AN-105 Analytical Results: Sodium (ICP) . . . . . . . . . . B B-104

B2-46 Tank 241-AN-105 Analytical Results: Strontium (ICP) $\ldots \ldots \ldots \ldots \ldots$ B-107

B2-47 Tank 241-AN-105 Analytical Results: Sulfur (ICP) $\ldots \ldots \ldots \ldots \ldots$ B-110

B2-48 Tank 241-AN-105 Analytical Results: Zinc (ICP) $\ldots \ldots \ldots \ldots \ldots \ldots$ B-113

B2-49 Tank 241-AN-105 Analytical Results: Zirconium (ICP) . . . . . . . . . B B-116

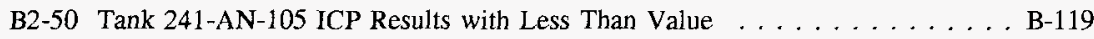

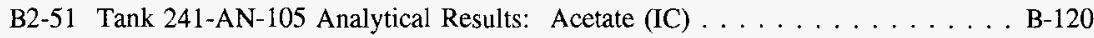

B2-52 Tank 241-AN-105 Analytical Results: Bromide (IC) . . . . . . . . . . B-122

B2-53 Tank 241-AN-105 Analytical Results: Chloride (IC) . . . . . . . . . . . B-124

B2-54 Tank 241-AN-105 Analytical Results: Fluoride (IC) $\ldots \ldots \ldots \ldots \ldots$. . . . . . . .

B2-55 Tank 241-AN-105 Analytical Results: Formate (IC) $\ldots \ldots \ldots \ldots \ldots$. . . . . . .

B2-56 Tank 241-AN-105 Analytical Results: Nitrate (IC) . . . . . . . . . . . . B-130

B2-57 Tank 241-AN-105 Analytical Results: Nitrite (IC) $\ldots \ldots \ldots \ldots \ldots \ldots$. . . . . . . . .

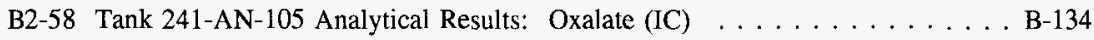




\section{LIST OF TABLES (cont)}

B2-59 Tank 241-AN-105 Analytical Results: Phosphate (IC) . . . . . . . . . . . B-136

B2-60 Tank 241-AN-105 Analytical Results: Sulfate (IC) $\ldots \ldots \ldots \ldots \ldots$. . . . 138

B2-61 Tank 241-AN-105 Analytical Results: Hydroxide . . . . . . . . . . . . . B-140

B2-62 Tank 241-AN-105 Analytical Results: Hydroxide . . . . . . . . . . B-140

B2-63 Tank 241-AN-105 Analytical Results: Hexavalent Chromium-VI . . . . . . B B-140

B2-64 Tank 241-AN-105 Analytical Results: Total

Organic Carbon by Coulometry $\ldots \ldots \ldots \ldots \ldots \ldots \ldots \ldots$. . . . . . . . . .

B2-65 Tank 241-AN-105 Analytical Results: Total

Organic Carbon by Persulfate Coulometry . . . . . . . . . . . B-141

B2-66 Tank 241-AN-105 Analytical Results: Total Inorganic Carbon (TIC) . . . . . . . . . . . . . . . . . . . . B-141

B2-67 Tank 241-AN-105 Vapor Sampling Results . . . . . . . . . . . . . B-141

B3-1 Phosphate vs Phosphorous $\ldots \ldots \ldots \ldots \ldots \ldots \ldots \ldots \ldots$ B-144

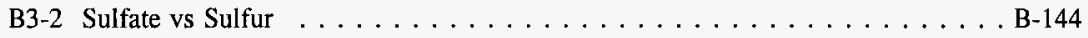

B3-3 Supernatant Cation Mass and Charge Data $\ldots \ldots \ldots \ldots \ldots \ldots$ B-145

B3-4 Supernatant Anion Mass and Charge Data . . . . . . . . . . . . . B-145

B3-5 Mass Balance Totals . . . . . . . . . . . . . . . . . . . . . . . B-146

B3-6 Salt Slurry Cation Mass and Charge Data . . . . . . . . . . . . B-147

B3-7 Salt Slurry Anion Mass and Charge Data . . . . . . . . . . . . . . B-147

B3-8 Mass Balance Totals $\ldots \ldots \ldots \ldots \ldots \ldots \ldots \ldots \ldots \ldots \ldots \ldots$ B-148

B3-9 Summary Statistics - Supernatant (Nested ANOVA) $\ldots \ldots \ldots \ldots$. . . . B-150

B3-10 Summary Statistics - Supernatant (One-way ANOVA) $\ldots \ldots \ldots \ldots \ldots$ B-152

B3-11 Analytes (Supernatant) with $>50$ Percent "Less Than" Values . . . . . . . B-154 


\section{LIST OF TABLES (cont)}

B3-12 Summary Statistics - Supernatant $\ldots \ldots \ldots \ldots \ldots \ldots$ B-155

B3-13 Supernatant Composite (Core 152, Segments 1-14) Analytes with $>50$ Percent "Less Than" Values . . . . . . . . . . . . . . . . B-157

B3-14 Summary Statistics (per g basis) - Sludge (Nested ANOVA with Riser Term) $\ldots \ldots \ldots \ldots \ldots \ldots \ldots \ldots \ldots \ldots$. . . . . . . . . . . . . .

B3-15 Summary Statistics (per g basis) - Sludge (Nested ANOVA without Riser) $\ldots \ldots \ldots \ldots \ldots \ldots \ldots \ldots \ldots \ldots \ldots$. . . . . . . . . . . . .

B3-16 Analytes (Sludge - per $\mathrm{g}$ basis) with $>50$ Percent "Less Than" Values . . . . . . . . . . . . . . . . . . . . . . . . . B-164

B3-17 Summary Statistics - Sludge Composite (Core 152, Segments 14-20) $\ldots \ldots$. B-166

B3-18 Sludge Composite (Core 152, Segments 14-20) Analytes with > 50 Percent "Less Than" Values . . . . . . . . . . . . . . . . . . . . B-168

B3-19 Summary Statistics - Sludge Composite (Core 152, Segment 22) . . . . . . B-169

B3-20 Sludge Composite (Core 152, Segment 22) Analytes with $>50$ Percent "Less Than" Values . . . . . . . . . . . . . . . . . . . B-171

B3-21 Tank 241-AN-105 Sludge Bulk Density Values $\ldots \ldots \ldots \ldots \ldots \ldots$. . . . . . . . .

B3-22 Summary Statistics (per $\mathrm{mL}$ basis) - Sludge (Nested ANOVA with Riser Term)

B3-23 Summary Statistics (per $\mathrm{mL}$ basis) - Sludge (Nested ANOVA without Riser) $\ldots \ldots \ldots \ldots \ldots \ldots$. . . . . . . B-175

B3-24 Sludge (per L basis) - Analytes with $>50$ Percent "Less Than" Values . . . . . . . . . . . . . . . . . . . . . . . . . . . B-177

B3-25 Summary Statistics (per g basis) - Crust $\ldots \ldots \ldots \ldots \ldots$. . . . . . . . . . .

B3-26 Analytes (Crust - per g basis) with $>50$ Percent "Less Than" Values . . . . . . . . . . . . . . . . . . . . . . . B-181

B3-27 Analytical-Based Supernate Inventory for Tank 241-AN-105 (Nested ANOVA - Segment Data) . . . . . . . . . . . . . . . . . B-187 


\section{LIST OF TABLES (cont)}

B3-28 Analytical-Based Supernate Inventory for Tank 241-AN-105

(One-way ANOVA - Segment Data) . . . . . . . . . . . . . . . B-189

B3-29 Analytical-Based Supernatant Inventory for Tank 241-AN-105

("Less-thans" - Segment Data)

B3-30 Analytical-Based Supernatant Inventory for Tank 241-AN-105 - (Supernatant

Composite Sample - Core 152, Segments 1-14) . . . . . . . . . . . . B-192

B3-31 Analytical-Based Salt Slurry Inventory for Tank 241-AN-105

(Nested ANOVA with the Riser Term - Segment Data) . . . . . . . . . . B-195

B3-32 Analytical-Based Salt Slurry Inventory for Tank 241-AN-105

(Nested ANOVA without the Riser Term - Segment Data) . . . . . . . . . B-197

B3-33 Analytes (Sludge - Segment Data) with $>50$ Percent

"Less Than" Values . . . . . . . . . . . . . . . . . . . . . . . . . . . B-199

B3-34 Analytical-Based Salt Slurry Tank Inventory - Composite

(Core 152, Segments $14-20$ ) . . . . . . . . . . . . . . B-201

B3-35 Analytical-Based Salt Slurry Tank Inventory - Composite

(Core 152 , Segment 22) . . . . . . . . . . . . . . . B-204

B3-36 Tank Inventory - Based on Segment Data . . . . . . . . . . . . . . B-207

B3-37 Tank Inventory - Based on Composite Data . . . . . . . . . . . . . . B-211

B3-38 Tank Mean Concentration - Based on Segment Data

(Per Volume Basis) . . . . . . . . . . . . . . . . . B-218

B3-39 Tank Mean Concentration - Based on Segment Data

(Per Weight Basis) . . . . . . . . . . . . . . . . . . . . . B-223

B3-40 Tank Mean Concentration - Based on Composite Sample Data

(Per Volume Basis) . . . . . . . . . . . . . . . . . . . B-227

B3-41 Tank Mean Concentration - Based on Composite Sample Data

(Per Weight Basis) . . . . . . . . . . . . . . . . . . . . B-231

C1-1 Tank 241-AN-105 Differential Scanning Calorimetry

Results $(\mathrm{J} / \mathrm{g}$ dry $) \ldots \ldots \ldots \ldots \ldots \ldots \ldots \ldots . \ldots \ldots$ 


\section{LIST OF TABLES (cont)}

C1-2 Tank 241-AN-105 Total Alpha Results . . . . . . . . . . . C-7

D2-1 Sampling and Hanford Defined Waste Model Inventory Estimates for Nonradioactive Components in Double-Shell Tank 241-AN $105 \ldots \ldots \ldots$. . .

D2-2 Sampling and Hanford Defined Waste Model Inventory Estimates for Radioactive Components in Double-Shell Tank 241-AN-105.

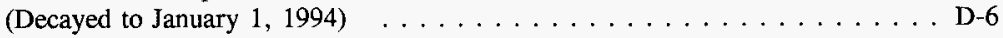

D3-1 Comparison of June 1996 Core Sample-based Inventories $\ldots \ldots \ldots \ldots$ D-8

D4-1 Best-Basis Inventory Estimates for Nonradioactive Components in Tank 241-AN-105 Supernatant as of October $31,1996 \ldots \ldots \ldots \ldots$ D-10

D4-2 Best-Basis Inventory Estimates for Radioactive Components in Tank 241-AN-105 Supernatant as of October $31,1996 \ldots \ldots \ldots \ldots$ D-11

D4-3 Best-Basis Inventory Estimates for Nonradioactive Components in Tank 241-AN-105 Salt Slurry as of October 31, $1996 \ldots \ldots \ldots \ldots \ldots$. . . . . . .

D4-4 Best-Basis Inventory Estimates for Radioactive Components in Tank 241-AN-105 Salt Slurry as of October 31, $1996 \ldots \ldots \ldots \ldots$. . . . . . .

D4-5 Best-Basis Total Inventory Estimates for Nonradioactive Components in Tank 241-AN-105 as of October 31, $1996 \ldots \ldots \ldots \ldots \ldots$ D-14

D4-6 Best-Basis Total Inventory Estimates for Radioactive Components in Tank 241-AN-105 as of October $31,1996 \ldots \ldots \ldots \ldots \ldots \ldots$ D-16 


\section{LIST OF TERMS}

$1 \mathrm{C}$

AEA

ANOVA

AT

BD

Btu/hr

Ci

$\mathrm{Ci} / \mathrm{L}$

CI

$\mathrm{cm}$

$\mathrm{cP}$

df

DL

DOE

DQO

DSC

DSSF

DSS

DST

EB

Ecology

FIC

$\mathrm{ft}$

FTIR

GC

GCS

GEA

$\mathrm{g}$

$\mathrm{g} / \mathrm{cc}$

$\mathrm{g} / \mathrm{L}$

$\mathrm{g} / \mathrm{mL}$

HDW

HTCE

IC

ICP

in.

$\mathrm{J} / \mathrm{g}$

$\mathrm{kg}$

$\mathrm{kgal}$

$\mathrm{kL}$

$\mathrm{kW}$ first cycle decontamination waste

alpha energy analysis

analysis of variance

alpha total

below detection limit

British thermal units per hour

curies

curies per liter

confidence interval

centimeter

centipoise

degrees of freedom

drainable liquid

U.S. Department of Energy

data quality objectives

differential scanning calorimetry

double-shell slurry feed

double-shell slurry

double-shell tank

evaporator bottoms

Washington State Department of Ecology

Food Instrument Corporation

feet

Fourier transform infrared spectrometer

gas chromatographs

gas characterization system

gravimetric energy analysis

gram

grams per cubic centimeter

grams per liter

grams per milliliter

Hanford defined waste

historical tank content estimate

ion chromatography

inductively coupled plasma spectroscopy

inch

joules per gram

kilogram

kilogallon

kiloliter

kilowatt 


\section{LIST OF TERMS (Continued)}

LANL

LEL

LFL

LH

LL

$\mathrm{m}$

$M$

MDA

$\mathrm{mL}$

$\mathrm{mg}$

$\mathrm{mm}$

NA

n/a

NCPLX

NR

ppm

QC

REML

RGS

RPD

SACS

SAP

SHMS

SMM

STP

T1SltCk

TB

TBP

TCP

TCR

TGA

TIC

TLM

TOC

TSAP

TWRS

UH

UL

VFI

VSS

W

$\mathrm{W} / \mathrm{Ci}$
Los Alamos National Laboratory

lower explosive limit

lower flammability limit

lower half

lower limit

meter

moles per liter

minimum detectable activity

milliliter

milligram

millimeter

not available

not applicable

non-complexed

not reported

parts per million

quality control

restricted maximum likelihood estimation methods

retained gas sampler

relative percent difference

Surveillance Analysis Computer System

sampling and analysis plan

standard hydrogen monitoring systems

supernatant mixing model

standard temperature and pressure

242-T Evaporator saltcake waste, 1951-55

total beta

tributyl phosphate

tank characterization plan

tank characterization report

thermogravimetric analysis

total inorganic carbon

tank layer model

total organic carbon

Tank Sampling and Analysis Plan

Tank Waste Remediation System

upper half

upper limit

void fraction instrument

vapor sampling system

watts

watts per curies 


\section{LIST OF TERMS (Continued)}

WSTRS

wt \%

${ }^{\circ} \mathrm{C}$

${ }^{\circ} \mathrm{F}$

$\mu \mathrm{Ci} / \mathrm{g}$

$\mu \mathrm{Ci} / \mathrm{L}$

$\mu \mathrm{Ci} / \mathrm{mL}$

$\mu \mathrm{eq} / \mathrm{g}$

$\mu \mathrm{g}$

$\mu \mathrm{g} / \mathrm{mL}$

$\mu \mathrm{gC} / \mathrm{g}$

$\mu \mathrm{g} / \mathrm{g}$ waste status and transaction record summary weight percent degrees Centigrade degrees Fahrenheit microcuries per gram microcuries per liter microcuries per milliliter microequivalents per gram microgram micrograms per milliliter micrograms of carbon per gram micrograms per gram 
HNF-SD-WM-ER-678 Rev. 0

This page intentionally left blank. 


\subsection{INTRODUCTION}

A major function of the Tank Waste Remediation System (TWRS) is to characterize wastes in support of waste management and disposal activities at the Hanford Site. Analytical data from sampling and analysis, along with other available information about a tank, are compiled and maintained in a tank characterization report (TCR). This report and its appendixes serve as the TCR for double-shell tank 241-AN-105.

The objectives of this report are: 1) to use characterization data in response to technical issues associated with tank 241-AN-105 waste; and 2) to provide a standard characterization of this waste in terms of a best-basis inventory estimate. The response to technical issues is summarized in Section 2.0, and the best-basis inventory estimate is presented in Section 3.0. Recommendations regarding safety status and additional sampling needs are provided in Section 4.0. Supporting data and information are contained in the appendices. This report also supports the requirements of the Hanford Federal Facility Agreement and Consent Order (Ecology et al. 1996) milestone M-44-10.

\subsection{SCOPE}

Characterization information presented in this report originated from sample analyses and known historical sources. While only the results of recent sample events will be used to fulfill the requirements of the data quality objectives (DQOs), other information can be used to support (or question) conclusions derived from these results. Historical information for tank 241-AN-105, provided in Appendix A, includes surveillance information, records pertaining to waste transfers and tank operations, and expected tank contents derived from a process knowledge model.

The recent sampling events listed in Table 1-1, as well as sample data obtained before 1989, are summarized in Appendix B along with the sampling results. The results of the 1996 sampling events, also reported in the laboratory data package (Steen 1997), satisfied the data requirements specified in the tank characterization plan (TCP) for this tank (Winkleman 1996). The statistical analysis and numerical manipulation of data used in issue resolution are reported in Appendixes B and C. Appendix D contains the evaluation to establish the best basis for the inventory estimate and the statistical analysis performed for this evaluation. A bibliography that resulted from an in-depth literature search of all known information sources applicable to tank 241-AN-105 and its respective waste types is contained in Appendix E. The reports listed in Appendix E may be found in the Tank Characterization Resource Center. 
Table 1-1. Summary of Recent Sampling.

\begin{tabular}{|l|l|l|l|l|l|}
\hline $\begin{array}{l}\text { Sampleidule } \\
\text { Cores } 152,153 \\
(6 / 10 / 96-6 / 28 / 96)\end{array}$ & $\begin{array}{l}\text { Solid and } \\
\text { Liquid }\end{array}$ & Risers 12A and 7B & half segment & $>90$ percent \\
\hline $\begin{array}{l}\text { Vapor sample } \\
(6 / 25 / 96-6 / 28 / 96)\end{array}$ & Gas & $\begin{array}{l}\text { Tank headspace, Risers } \\
12 \mathrm{~A} \text { and } 7 \mathrm{~B}, 6 \mathrm{~m} \\
(20 \mathrm{ft}) \text { below top of } \\
\text { risers }\end{array}$ & $\mathrm{n} / \mathrm{a}$ & $\mathrm{n} / \mathrm{a}$ \\
\hline
\end{tabular}

Notes:

$\mathrm{n} / \mathrm{a} \quad=$ not applicable

Dates are provided in the $\mathrm{mm} / \mathrm{dd} / \mathrm{yy}$ format.

\subsection{TANK BACKGROUND}

Tank 241-AN-105 is located in the 200 East Area AN Tank Farm on the Hanford Site. The tank went into service in 1982, receiving water. The tank received double-shell slurry feed waste from tank 241-AW-102 from December 1982 until the first quarter of 1983. The tank received non-complexed waste from tank $241-\mathrm{AN}-104$ in the first quarter of 1984 . A small amount of unknown waste was received in the third quarter of 1984 . Most of the waste was removed during the first quarter of 1985 for an evaporator campaign. From the first quarter to the second quarter of 1985 , the tank received double-shell slurry feed waste from tank 241-AW-102 via the evaporator (Agnew et al. 1997a). The last transfer for this tank consist of small flush water in 1995 and 1996.

A description of tank 241-AN-105 is summarized in Table 1-2. The tank has an operating capacity of $4,390 \mathrm{~kL}(1,160 \mathrm{kgal})$, and presently contains an estimated 4,270 kL (1,129 kgal) of double-shell slurry feed waste (Hanlon 1996). The tank is on the Hydrogen Watch List (Public Law 101-510). 
Table 1-2. Description of Tank 241-AN-105.

\begin{tabular}{|c|c|}
\hline 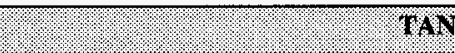 & 110\% \\
\hline Type & Double-Shell \\
\hline Constructed & 1980-1981 \\
\hline In-service & 1982 \\
\hline Diameter & $22.9 \mathrm{~m}(75.0 \mathrm{ft})$ \\
\hline Operating depth & $10.7 \mathrm{~m}(35 \mathrm{ft})$ \\
\hline Capacity & $4,390 \mathrm{~kL}(1,160 \mathrm{kgal})$ \\
\hline Bottom Shape & Flat \\
\hline Ventilation & Active \\
\hline (19.1. & \\
\hline Waste classification & Double-shell slurry feed \\
\hline Total waste volume ${ }^{1}$ & $4,270 \mathrm{~kL}(1,129 \mathrm{kgal})$ \\
\hline Supernatant volume $^{2}$ & $4,270 \mathrm{~kL}(1,129 \mathrm{kgal})$ \\
\hline Saltcake volume $^{2}$ & $0 \mathrm{~kL}(0 \mathrm{kgal})$ \\
\hline Sludge volume & $0 \mathrm{~kL}(0 \mathrm{kgal})$ \\
\hline Drainable interstitial liquid volume & $0 \mathrm{~kL}(0 \mathrm{kgal})$ \\
\hline Waste surface level (June 30, 1996) & $10.4 \mathrm{~m}(34.2 \mathrm{ft})$ \\
\hline Temperature (Jan. 1990 to Feb. 1997) & $19.2^{\circ} \mathrm{C}\left(66.6^{\circ} \mathrm{F}\right)$ to $46.2^{\circ} \mathrm{C}\left(116^{\circ} \mathrm{F}\right)$ \\
\hline Integrity & Sound \\
\hline Watch List & Flammable Gas \\
\hline SA & MIE \\
\hline $\begin{array}{l}\text { Core samples and tank headspace } \\
\text { flammability }\end{array}$ & June 1996 \\
\hline $\mathrm{SE}$ & IUs \\
\hline Active & esent \\
\hline
\end{tabular}

Notes:

'Waste volume is estimated from surface level measurements (May 1996).

${ }^{2}$ Although Hanlon (1996) reports that the tank contains all supernate waste, it is evident from the core samples (Steen 1997) that the bottom $40 \%$ of the waste is probably salt slurry. 
HNF-SD-WM-ER-678 Rev. 0

This page intentionally left blank. 


\subsection{RESPONSE TO TECHNICAL ISSUES}

Two technical issues have been identified for tank 241-AN-105 (Brown et al. 1996). They are:

- Safety Screening: Does the waste pose or contribute to any recognized potential safety problems?

- Flammable Gas: Data from the core samples are needed to provide an understanding of the tank contents so that: (1) insight may be obtained on the mechanisms for gas generation, retention, and release, (2) models of the waste behavior can be developed to support safety analysis and development of mitigation methods, (3) stimulants for waste studies can be developed, and (4) modeling of the release of gases, and subsequent potential for ignition in the dome space, can be done to support hazard analyses.

The Tank Safety and Analysis Plan (SAP) (Eggers 1996) provides the types of sampling and analysis used to address the above issues. Data from the recent analysis of two core samples and tank vapor space flammability measurements, as well as available historical information, provided the means to respond to these two issues. This response is detailed in the following sections. See Appendix B for sample and analysis data for tank 241-AN-105.

\subsection{SAFETY SCREENING}

The data needed to screen the waste in tank 241-AN-105 for potential safety problems is documented in Tank Safety Screening Data Quality Objective (Dukelow et al. 1995). These potential safety problems are exothermic conditions in the waste; flammable gases in the waste and/or tank headspace; and criticality conditions in the waste. Each of these conditions is addressed separately below.

\subsubsection{Exothermic Conditions (Energetics)}

The first requirement outlined in the safety screening DQO (Dukelow et al. 1995) is to ensure that there are not sufficient exothermic constituents in tank 241-AN-105 to cause a safety hazard. Because of this requirement, energetics in the tank 241-AN-105 waste were evaluated. The safety screening $\mathrm{DQO}$ required that solid samples be tested for energetics every $24 \mathrm{~cm}(9.5 \mathrm{in}$.) to determine if the energetics exceed the safety threshold limit. Note that the first 14 segments were mostly composed of drainable liquid and therefore, were not subdivided into upper and lower samples.

Results obtained using differential scanning calorimetry (DSC) indicated that exotherms were well below notification limits of $480 \mathrm{~J} / \mathrm{g}$ on a dry weight basis with the maximum value of $174 \mathrm{~J} / \mathrm{g}$ (Steen 1996). Furthermore, the upper limits of the one-sided 95 percent confidence 
interval for the DSC results were below notification limit with the maximum value of 355 $\mathrm{J} / \mathrm{g}$. These results suggest that there is no energetic concern in this tank.

Historically, there is evidence of the waste showing exothermic behavior. Waste transfer records indicate the major waste type expected to be found in the tank is double-shell slurry (Agnew et al. 1997a). These waste types are expected to have organic constituents (Sasaki 1984). Analysis of a sample taken in 1984 also showed evidence of exotherms (Mauss 1984).

\subsubsection{Flammable Gas}

Vapor phase measurements, taken in the tank headspace prior to the core samples in June 1996 , indicated that no flammable gas was detected $(0.3$ percent of the lower flammability limit [LFL]). Data from these vapor phase measurements are presented in Appendix B. Prior to this sampling event there have been two gas release events that resulted in concentrations greater than 25 percent of the LFL (Wilkins et al. 1997).

\subsubsection{Criticality}

The safety threshold limit is $1 \mathrm{~g}{ }^{239} \mathrm{Pu}$ per liter of waste. Assuming all alpha is from ${ }^{239} \mathrm{Pu}$, with maximum density of $1.66 \mathrm{~g} / \mathrm{mL}$ for salt slurry the safety threshold limit is equivalent to $37 \mu \mathrm{Ci} / \mathrm{g}$ (conversion used the maximum density of $1.66 \mathrm{~g} / \mathrm{mL}$ observed for the sludge subsamples) of salt slurry and $61.5 \mu \mathrm{Ci} / \mathrm{g}$ of liquid of alpha activity. The core samples were analyzed in accordance with the safety screening DQO (Dukelow et al. 1995).

Waste samples were tested for total alpha activity for each core sample. Concentrations in all samples were well below this limit with the maximum value of $0.133 \mu \mathrm{Ci} / \mathrm{g}$.

Additionally, as required by the DQO, the upper limit of the one-sided 95 percent confidence interval for these results was less than $37 \mu \mathrm{Ci} / \mathrm{g}$ with the maximum value of $0.18 \mu \mathrm{Ci} / \mathrm{g}$. The method used to calculate confidence limits is contained in Appendix C. The data show that there is no criticality concern with this tank.

\subsection{FLAMMABLE GAS TANK SAFETY EVALUATION}

The purpose of the flammable gas tank safety evaluation is to obtain data to develop mitigation methods, to support tank behavior models needed for making safety analyses, and to support evaluations, of chemical mechanisms for gas production and release. These data needs are met by the sampling event and retained gas sampler (RGS) in June 1996, and by void fraction instrument (VFI) deployed in December 1995.

Tank 241-AN-105 was the second double-shell tank sampled for retained gases. The RGS was used in two risers within this tank to obtain eight segments. The current waste level is 
about $1040 \mathrm{~cm}$ (410 in.) and consists of a nonconvective layer 400 to $460 \mathrm{~cm}$ (160 to 180 in.) in depth, 580 to $640 \mathrm{~cm}$ (230 to $250 \mathrm{in}$.) of convective liquid, and a noncontinuous floating crust layer that may be up to $30 \mathrm{~cm}$ (12 in.) thick. The head space volume in tank 241-AN-105 is about $1,047 \mathrm{~m}^{3}\left(37,000 \mathrm{ft}^{3}\right)$.

Retained gas measurements and estimated solubilities show three major constituents in the gas/vapor phase (free gas) of the nonconvective and convective layers: $27 \mathrm{~mol} \%$ nitrogen, 60 mol\% hydrogen, and $11 \mathrm{~mol} \%$ nitrous oxide. The remainder is ammonia, methane, and other hydrocarbons. The measured local ammonia concentrations in tank 241-AN-105 ranged from 890 to $2100 \mu$ mole/liter of waste, more than 99.9 percent of the ammonia is dissolved in the liquid. Integrating the local concentrations leads to a total amount, if it were vapor (which is improbable), of approximately $99 \mathrm{~m}^{3}\left(3,500 \mathrm{ft}^{3}\right)$ of ammonia at standard temperature and pressure (STP) (Shekarriz et al, 1997).

The extraction results show that the insoluble gases were primarily retained in the lower, nonconvective layer. Based on the estimated solubilities and RGS measurements of gas concentrations, about 4.5 percent by volume (in-situ) of the nonconvective layer was filled with free gas, while 0.3 percent by volume (in-situ) of the convective (upper) layer was filled with free gas. Local calculated void fractions based on RGS data were in close agreement with the VFI results for the segments above $200 \mathrm{~cm}(80$ in.) elevation. While the lower two segments did not have VFI measurements to compare, the trends pointed to reasonable estimates of the void fraction. The maximum void fraction measured with RGS was approximately 11.1 percent in this tank. Although the void fractions appear to be consistent for both risers, some variations in the gas composition were observed between the two, indicating some lateral variation. The calculated hydrogen inventory in both phases of the nonconvective and convective layers of tank $241-\mathrm{AN}-105$ is $103 \mathrm{~m}^{3}\left(3,600 \mathrm{ft}^{3}\right)$, based on integrated RGS measurements (Shekarriz et al. 1997).

The x-ray results are significantly different from those of the previous two tanks in severa] ways: 1) large gas pockets were observed in tank 241-AN-105 that account for a large portion of the measured void fraction. This contrasts with the observations made for tank 241-AW-101 waste, where the major portion of the gas was observed to be smaller than the detection threshold of the x-ray imaging system $(<0.5 \mathrm{~mm}) ; 2)$ no fractures or irregularly shaped bubbles were observed in this tank, unlike what was observed in tank 241-A-101 waste. Densities measured in this tank, based on $x$-ray images, ranged from 1.65 to $1.83 \mathrm{~g} / \mathrm{cc}$ (Shekarriz et al. 1997).

The ball rheometer and VFI were run in tank 241-AN-105 in riser $1 \mathrm{~B}$ on December 15 and 18,1995 , and in $16 \mathrm{~B}$ on December 20 and 22,1995 , respectively. Two traverses were accomplished with the VFI in riser $1 \mathrm{~B}$ and three in 16B. The ball sank to within about 110 $\mathrm{cm}$ (43 in.) of the tank bottom in riser $1 \mathrm{~B}$ and to within about $20 \mathrm{~cm}$ ( $7.9 \mathrm{in}$.) of the bottom in $16 \mathrm{~B}$. No significant gas releases were observed on the waste surface or on the gas monitoring equipment during VFI operation. 
No measurements could be taken below about $150 \mathrm{~cm}$ (59 in.) because of interference by a plastic sleeve required for contamination control. The sleeve used in tank 241-AN-105 was made of thicker material than those encountered in previous tests. To estimate the average void fraction and total gas volume, the assumption was made that void fraction in the unsampled lower $150 \mathrm{~cm}$ (59 in.) of the waste was represented by the available measurements below $215 \mathrm{~cm}(85 \mathrm{in}$.), which is consistent with trends observed in tank 241-AW-101 and 241-SY-103 (Stewart et al. 1996).

The nonconvective layer was split into three sublayers with boundaries of 0 to $215 \mathrm{~cm}(85$ in.) and $215 \mathrm{~cm}(85$ in.) to $330 \mathrm{~cm}$ (130 in.) and $330 \mathrm{~cm}(130$ in.) to $450 \mathrm{~cm}$ (177 in.), by a visual interpretation of the void profile. The overall average void is $3.8 \pm 0.6$ percent. The unsampled lower $150 \mathrm{~cm}(59 \mathrm{~cm})$ is assumed to be represented by the void measurements below $215 \mathrm{~cm}(85 \mathrm{in}$.). If the void below $150 \mathrm{~cm}(59 \mathrm{in}$.) is actually higher by 25 percent (6.5 percent void instead of 5.2 percent), the total standard gas volume in the nonconvective layer increases by $18 \mathrm{~m}^{3}\left(635 \mathrm{ft}^{3}\right)$, well within the standard deviation of $44 \mathrm{~m}^{3}\left(1550 \mathrm{ft}^{3}\right)$.

The viscosity of the convective layer was calculated to be $36 \mathrm{cP}$, bounded above by $55 \mathrm{cP}$ and below by $15 \mathrm{cP}$, from the ball drag force data at speeds of 30 and $50 \mathrm{~cm} / \mathrm{s}$ in risers $1 \mathrm{~B}$ and $16 \mathrm{~B}$. The apparent viscosity at strain rates are $0.01,0.1$ and $1.0 \mathrm{sec}^{-1}$ on the first and last passes. After the ball has traversed through the layer four times, the apparent viscosity decreases considerably (Stewart et al. 1996).

\subsection{OTHER TECHNICAL ISSUES}

A factor in assessing tank safety is the heat generation and temperature of the waste. Heat is generated in the tanks from radioactive decay. An estimate of the tank heat load can be calculated based on the results from the 1996 sample event. The heat load value calculated from the 1996 sample events was $2.4 \mathrm{~kW}(8,100 \mathrm{Btu} / \mathrm{hr})$, as shown in Table $2-1$. The heat load estimate based on the tank process history was $11.4 \mathrm{~kW}(39,000 \mathrm{Btu} / \mathrm{hr}$ ) (Agnew et al. 1997b). The heat load estimate based on the tank headspace temperature was $7.42 \mathrm{~kW}$ $(25,000 \mathrm{Btu} / \mathrm{hr})($ Kummerer 1994). These estimates are well below the limit of the maximum heat load, $20.5 \mathrm{~kW}(70,000 \mathrm{Btu} / \mathrm{hr})$, allowed for double-shell tanks (Haubach 1996). 
Table 2-1. Heat Load for Tank 241-AN-105 Based on Radionuclide Content.

\begin{tabular}{|c|c|c|c|c|}
\hline & 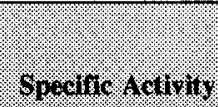 & 1rojertors & 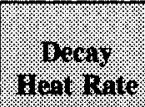 & 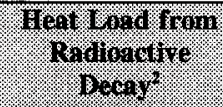 \\
\hline radivindrat & 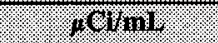 & \% & W & w \\
\hline${ }^{241} \mathrm{Am}$ & 1.01 & 4310 & 0.0328 & 140 \\
\hline${ }^{137} \mathrm{Cs}$ & 470 & $2,000,000$ & 0.00101 & 2,020 \\
\hline${ }^{89 / 90} \mathrm{Sr}$ & 7.42 & 31,680 & 0.00669 & 210 \\
\hline Total & & & & 2,370 \\
\hline
\end{tabular}

Notes:

${ }^{1}$ Include daughter radionuclides.

${ }^{2}$ Only analytes contributing to the heat generation rate above $100 \mathrm{~W}$ are included.

\subsection{SUMMARY}

The results from all analyses performed to address potential safety issues showed that no primary analyte exceeded safety decision threshold limits. The analyses for the flammable gas was completed to obtain data to develop mitigation methods, to support tank behavior models needed for making safety analyses, and to support evaluations of chemical mechanisms for gas production and release. The analyses results are summarized in Table 2-2.

Table 2-2. Summary of Safety Screening and Historical Flammable Gas. (2 sheets)

\begin{tabular}{|c|c|c|}
\hline Lssie & Sub issue & Resull \\
\hline \multirow[t]{3}{*}{$\begin{array}{l}\text { Safety } \\
\text { screening }\end{array}$} & Energetics & $\begin{array}{l}\text { All exothermic reactions below } 480 \mathrm{~J} / \mathrm{g} \text {. Highest } 95 \\
\text { percent confidence interval upper limit }=384 \mathrm{~J} / \mathrm{g} \text {. }\end{array}$ \\
\hline & Flammable gas & $\begin{array}{l}\text { Vapor measurement reported } 0.3 \text { percent of lower } \\
\text { flammability limit. (Combustible gas meter). }\end{array}$ \\
\hline & Criticality & $\begin{array}{l}\text { All analyses well below } 37 \mu \mathrm{Ci} / \mathrm{g} \text { total alpha (within } \\
95 \text { percent confidence limit on each sample). }\end{array}$ \\
\hline
\end{tabular}


Table 2-2. Summary of Safety Screening and Historical Flammable Gas. (2 sheets)

\begin{tabular}{|l|l|l|l|l|}
\hline \multirow{2}{*}{$\begin{array}{l}\text { Flammable } \\
\text { gas }\end{array}$} & $\begin{array}{l}\text { Mechanism of gas } \\
\text { generation, } \\
\text { retention, and } \\
\text { released }\end{array}$ & $\mathrm{N} / \mathrm{R}$ \\
\cline { 2 - 4 } & $\begin{array}{l}\text { Waste behavior } \\
\text { model }\end{array}$ & $\mathrm{N} / \mathrm{R}$ \\
\cline { 2 - 3 } & $\begin{array}{l}\text { Simulent } \\
\text { development }\end{array}$ & $\mathrm{N} / \mathrm{R}$ \\
\cline { 2 - 3 } & $\begin{array}{l}\text { Model to support } \\
\text { hazard analysis }\end{array}$ & $\mathrm{N} / \mathrm{R}$ \\
\hline
\end{tabular}

Note:

$$
\mathrm{N} / \mathrm{R}=\text { not reviewed }
$$




\subsection{BEST-BASIS STANDARD INVENTORY ESTIMATE}

Information about the chemical and/or physical properties of tank wastes is used to perform safety analyses, engineering evaluations, and risk assessments associated with waste management activities, as well as to address regulatory issues. Waste management activities include overseeing tank farm operations and identifying, monitoring, and resolving safety issues associated with these operations and with the tank wastes. Disposal activities involve designing equipment, processes, and facilities for retrieving wastes and processing the wastes into a form that is suitable for long-term storage.

Chemical inventory information generally is derived using two approaches: 1) component inventories are estimated using the results of sample analyses; and 2) component inventories are predicted using a model based on process knowledge and historical information. The most recent model was developed by Los Alamos National Laboratory (LANL) (Agnew et al. 1997b). Information derived from these two different approaches is often inconsistent.

An effort is underway to provide waste inventory estimates that will serve as standard characterization information for the various waste management activities (Hodgson and LeClair 1996). As part of this effort, an evaluation of available chemical information for tank 241-AN-105 was performed that included:

- Steen (1997) and Appendix B of this report, which provides characterization results from the June 1996 core sampling event.

- A feed projection document that supported grout treatment facility studies provides estimates of the waste in tank 241 - AN-105 based on 242-A Evaporator slurry product data (Hendrickson 1994).

- The Hanford defined waste (HDW) model document (Agnew et al. 1997b) provides tank content estimates, in terms of component concentrations and inventories.

Results from this evaluation, detailed in Appendix D, support using the sampling data as the basis for the best estimate inventory for tank $241-\mathrm{AN}-105$ for the following reasons:

1. The June 1996 sampling event provides the most recent data for the waste. 
2. Estimates based on 242-A Evaporator product sample data agrees with the June 1996 data.

3. The HDW model estimate overestimates slurry and supernatant inventories due to solubility assumptions that are biased high.

Best-basis inventory estimates for tank 241-AN-105 are presented in Tables 3-1 through 3-6. Radionuclide values are decayed to January 1, 1994.

Table 3-1. Best-Basis Inventory Estimates for Nonradioactive Components in Tank 241-AN-105 Supernatant as of May 31, 1996. (2 sheets)

\begin{tabular}{|c|c|c|c|}
\hline 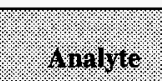 & 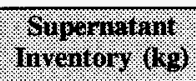 & (S. Masis: or $\mathrm{A}$ ? & 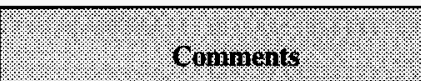 \\
\hline $\mathrm{Al}$ & 99,200 & $S$ & \\
\hline$\overline{\mathrm{Bi}}$ & $<134$ & $S$ & \\
\hline $\mathrm{Ca}$ & $<154$ & $S$ & \\
\hline $\mathrm{Cl}$ & 26,100 & $S$ & \\
\hline $\mathrm{TIC}$ as $\mathrm{CO}_{3}$ & 26,200 & $S$ & Core composite data \\
\hline $\mathrm{Cr}$ & 573 & $\mathrm{~S}$ & \\
\hline $\mathbf{F}$ & 445 & $S$ & RPD $>20 \%$ Composite $=<138$ \\
\hline $\mathrm{Fe}$ & 120 & $S$ & Segment-based $<77.1$ \\
\hline $\mathbf{K}$ & 16,700 & S & \\
\hline $\mathrm{La}$ & $<77.1$ & $S$ & \\
\hline $\mathrm{Mn}$ & $<15.2$ & $S$ & \\
\hline $\mathrm{Na}$ & 644,000 & $S$ & \\
\hline $\mathrm{Ni}$ & $<30.7$ & $S$ & \\
\hline $\mathrm{NO}_{2}$ & 323,000 & $S$ & \\
\hline $\mathrm{NO}_{3}$ & 420,500 & $\mathrm{~S}$ & \\
\hline $\mathrm{OH}$ & 150,000 & $\mathrm{~S}$ & Core composite data \\
\hline $\mathrm{Pb}$ & 146 & $\mathrm{~S}$ & Composite $<154$ \\
\hline $\mathrm{PO}_{4}$ & 2,850 & S & \\
\hline$\overline{\mathrm{Si}}$ & 492 & $S$ & \\
\hline $\mathrm{SO}_{4}$ & 4,840 & $S$ & \\
\hline $\mathrm{Sr}$ & $<13.4$ & $S$ & \\
\hline TOC & 15,600 & $\mathrm{~S}$ & Core composite data \\
\hline
\end{tabular}


Table 3-1. Best-Basis Inventory Estimates for Nonradioactive Components in Tank 241-AN-105 Supernatant as of May 31, 1996. (2 sheets)

\begin{tabular}{|c|c|c|c|}
\hline 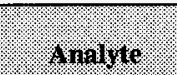 & 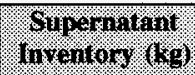 & $(6.1 \%, 0.9)$ & (20minitis \\
\hline $\mathrm{U}_{\text {TOTAL }}$ & 9.13 & $S$ & Core composite data \\
\hline $\mathrm{Zr}$ & $<15.5$ & $S$ & \\
\hline$\%$ Water & 51.9 & $S$ & \\
\hline Density, $\mathrm{g} / \mathrm{mL}$ & 1.42 & $S$ & \\
\hline
\end{tabular}

Notes:

${ }^{\text {'S }}$ = sample-based, $\mathrm{M}$ = Hanford defined waste model-based, $\mathrm{E}$ = engineering assessment-based $\mathrm{NR}=$ not reported

$\mathrm{RPD}$ = relative percent difference.

Table 3-2. Best-Basis Inventory Estimates for Radioactive Components in Tank 241-AN-105 Supernatant as of May 31, 1996. (Decayed to January 1, 1994)

\begin{tabular}{|c|c|c|}
\hline (1. & 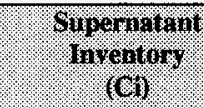 & (3. \\
\hline${ }^{60} \mathrm{Co}$ & $<0.050$ & $S$ \\
\hline${ }^{90} \mathrm{Sr}$ & 112 & $S$ \\
\hline${ }^{90} \mathrm{Y}$ & 112 & $S$ \\
\hline${ }^{99} \mathrm{Tc}$ & 701 & $S$ \\
\hline${ }^{129} I$ & 0.685 & $S$ \\
\hline${ }^{137} \mathrm{Cs}$ & 1,290 & $S$ \\
\hline${ }^{137 m} \mathrm{Ba}$ & 1,230 & $S$ \\
\hline${ }^{154} \mathrm{Eu}$ & $<250$ & $S$ \\
\hline${ }^{155} \mathrm{Eu}$ & $<835$ & $S$ \\
\hline${ }^{237} \mathrm{~Np}$ & $<0.0566$ & $S$ \\
\hline${ }^{239 / 240} \mathrm{Pu}$ & 0.0953 & $S$ \\
\hline${ }^{241} \mathrm{Am}$ & 0.100 & $S$ \\
\hline${ }^{243 / 244} \mathrm{Cm}$ & 0.110 & $S$ \\
\hline
\end{tabular}

Notes:

'S = sample-based, $M=$ Hanford defined waste model-based, $\mathrm{E}=$ engineering assessment-based. 
Table 3-3. Best-Basis Inventory Estimates for Nonradioactive Components in Tank 241-AN-105 Salt Slurry as of May 31, 1996.

\begin{tabular}{|c|c|c|c|}
\hline Analve & 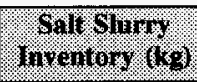 & (5., Mas, of (i)? & Comment \\
\hline $\mathrm{Al}$ & 56,500 & $S$ & \\
\hline $\mathrm{Bi}$ & 147 & $S$ & \\
\hline $\mathrm{Ca}$ & 942 & $S$ & \\
\hline $\mathrm{Cl}$ & 14,620 & $S$ & \\
\hline TIC as $\mathrm{CO}_{3}$ & 169,000 & $S$ & Core composite data \\
\hline $\mathrm{Cr}$ & 4,470 & $S$ & \\
\hline $\mathbf{F}$ & 3,400 & $S$ & \\
\hline $\mathrm{Fe}$ & 167 & $\mathrm{~S}$ & \\
\hline $\bar{K}$ & 8,670 & $\mathrm{~S}$ & \\
\hline $\mathrm{La}$ & $<504$ & $\mathrm{~S}$ & \\
\hline $\mathrm{Mn}$ & 44.2 & $S$ & \\
\hline $\mathrm{Na}$ & 456,000 & $\mathrm{~S}$ & \\
\hline $\mathrm{Ni}$ & 97.8 & $\mathrm{~s}$ & \\
\hline $\mathrm{NO}_{2}$ & 181,000 & $S$ & \\
\hline $\mathrm{NO}_{3}$ & 308,000 & $\mathrm{~S}$ & \\
\hline$\overline{\mathrm{OH}}$ & 64,000 & $S$ & Core composite data \\
\hline $\mathrm{Pb}$ & 119 & $\mathrm{~S}$ & \\
\hline $\mathrm{PO}_{4}$ & 17,300 & $S$ & \\
\hline $\mathrm{Si}$ & 458 & $S$ & \\
\hline $\mathrm{SO}_{4}$ & 18,000 & $\mathrm{~s}$ & \\
\hline $\mathrm{Sr}$ & $<18.7$ & $\mathrm{~S}$ & Core composite data \\
\hline$\overline{\text { TOC }}$ & 14,300 & $\mathrm{~S}$ & Core composite data \\
\hline$\overline{U_{\text {TOTAL }}}$ & 233 & $\mathrm{~S}$ & Core composite data \\
\hline$\%$ Water & 44.7 & $\mathrm{~S}$ & \\
\hline Density, $\mathrm{g} / \mathrm{mL}$ & 1.59 & $\mathrm{~S}$ & \\
\hline $\mathrm{Zr}$ & 20.0 & S & \\
\hline
\end{tabular}

Notes:

' $\mathrm{S}=$ sample-based, $\mathrm{M}=$ Hanford Defined Waste model-based, $\mathrm{E}$ = engineering assessment-based. 
Table 3-4. Best-Basis Inventory Estimates for Radioactive Components in Tank 241-AN-105 Salt Slurry as of May 31, 1996.

(Decayed to January 1, 1994)

\begin{tabular}{|c|c|c|}
\hline t. Triagle & 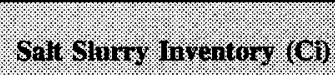 & 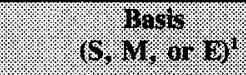 \\
\hline${ }^{60} \mathrm{Co}$ & 6.41 & $S$ \\
\hline${ }^{90} \mathrm{Sr}$ & 33,500 & $S$ \\
\hline${ }^{90} \mathrm{Y}$ & 33,500 & $S$ \\
\hline${ }^{99} \mathrm{Tc}$ & 473 & $\mathrm{~S}$ \\
\hline${ }^{129} \mathrm{I}$ & 10.3 & $S$ \\
\hline${ }^{137} \mathrm{Cs}$ & 83,500 & $S$ \\
\hline${ }^{137 m} \mathrm{Ba}$ & 79,300 & $S$ \\
\hline${ }^{154} \mathrm{Eu}$ & $<315$ & $S$ \\
\hline${ }^{155} \mathrm{Eu}$ & $<1,420$ & $S$ \\
\hline${ }^{237} \mathrm{~Np}$ & $<25.7$ & $\mathrm{~S}$ \\
\hline${ }^{239 / 240} \mathrm{Pu}$ & 6.33 & $S$ \\
\hline${ }^{241} \mathrm{Am}$ & 9.97 & $S$ \\
\hline${ }^{243 / 244} \mathrm{Cm}$ & $<4.16$ & $S$ \\
\hline
\end{tabular}

Notes:
'S = sample-based, $\mathrm{M}=$ Hanford defined waste model-based,
$\mathrm{E}=$ engineering assessment-based. 
Table 3-5. Best-Basis Total Inventory Estimates for Nonradioactive Components in Tank 241-AN-105 as of May 31, 1996.

\begin{tabular}{|c|c|c|}
\hline W.1. & 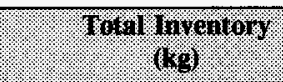 & 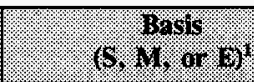 \\
\hline $\mathrm{Al}$ & 156,000 & S \\
\hline $\mathrm{Bi}$ & 214 & $\mathrm{~S}$ \\
\hline $\mathrm{Ca}$ & 2,140 & $S$ \\
\hline $\mathrm{Cl}$ & 40,800 & $\mathrm{~S}$ \\
\hline TIC as $\mathrm{CO}_{3}$ & 197,000 & $\mathrm{~S}$ \\
\hline $\mathrm{Cr}$ & 5,040 & $\mathrm{~s}$ \\
\hline $\mathbf{F}$ & 3,850 & $S$ \\
\hline $\mathrm{Fe}$ & 287 & $S$ \\
\hline $\mathrm{K}$ & 25,400 & $S$ \\
\hline $\mathrm{La}$ & $<581$ & $S$ \\
\hline Mn & $<59.4$ & $\mathrm{~S}$ \\
\hline $\mathrm{Na}$ & $1.10 \mathrm{E}+06$ & $S$ \\
\hline $\mathrm{Ni}$ & $<129$ & $\mathrm{~S}$ \\
\hline $\mathrm{NO}_{2}$ & 504,000 & $S$ \\
\hline $\mathrm{NO}_{3}$ & 728,000 & $S$ \\
\hline$\overline{\mathrm{OH}}$ & 214,000 & $S$ \\
\hline $\mathrm{Pb}$ & 265 & $S$ \\
\hline $\mathrm{PO}_{4}$ & 20,200 & $S$ \\
\hline $\mathrm{Si}$ & 950 & $\mathrm{~s}$ \\
\hline $\mathrm{SO}_{4}$ & 22,900 & $S$ \\
\hline $\mathrm{Sr}$ & $<32.1$ & $S$ \\
\hline TOC & 29,900 & $S$ \\
\hline $\mathrm{U}_{\text {TOTAL }}$ & 242 & $\mathrm{~s}$ \\
\hline $\mathrm{Zr}$ & $<43.5$ & $S$ \\
\hline$\%$ Water & 46.6 & $S$ \\
\hline Density, $\mathrm{g} / \mathrm{mL}$ & 1.56 & $S$ \\
\hline
\end{tabular}

Notes:

${ }^{1} \mathrm{~S}=$ sample-based, $\mathrm{M}=$ Hanford Defined Waste model-based, $\mathrm{E}=$ engineering assessment-based. 
Table 3-6. Best-Basis Total Inventory Estimates for Radioactive Components in Tank 241-AN-105 as of May 31, 1996.

(Decayed to January 1, 1994)

\begin{tabular}{|c|c|c|}
\hline 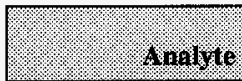 & $\begin{array}{l}\text { Tothl Gremony } \\
\text { (c) }\end{array}$ & (5, M, o, $\mathrm{Gl}$ \\
\hline${ }^{60} \mathrm{Co}$ & $<6.46$ & $S$ \\
\hline${ }^{90} \mathrm{Sr}$ & 33,600 & $S$ \\
\hline${ }^{90} \mathrm{Y}$ & 33,600 & $S$ \\
\hline${ }^{99} \mathrm{Tc}$ & 1,170 & $S$ \\
\hline${ }^{129} \mathrm{I}$ & 11.0 & $\bar{S}$ \\
\hline${ }^{137} \mathrm{Cs}$ & 84,800 & $S$ \\
\hline \begin{tabular}{|l|}
$137 \mathrm{~m}$ \\
$\mathrm{Ba}$
\end{tabular} & 80,600 & $S$ \\
\hline${ }^{154} \mathrm{Eu}$ & $<565$ & $s$ \\
\hline${ }^{1{ }^{155} \mathrm{Eu}}$ & $<2,260$ & $S$ \\
\hline${ }^{237} \mathrm{~Np}$ & 25.8 & $S$ \\
\hline${ }^{239 / 240} \mathrm{Pu}$ & 6.43 & $S$ \\
\hline${ }^{241} \mathrm{Am}$ & 10.1 & $S$ \\
\hline \begin{tabular}{|l|}
$243 / 244$ \\
$\mathrm{Cm}$
\end{tabular} & $<4.27$ & $\mathrm{~S}$ \\
\hline
\end{tabular}

Notes:

${ }^{1} \mathrm{~S}=$ sample-based, $\mathrm{M}=$ Hanford defined waste model-based, $\mathrm{E}$ = engineering assessment-based. 
HNF-SD-WM-ER-678 Rev. 0

This page intentionally left blank. 


\subsection{RECOMMENDATIONS}

All analytical results for the safety screening DQO were well within the safety notification limits. The sampling and analysis activities performed for tank 241-AN-105 have met all requirements for all of the applicable DQO documents. Furthermore, a characterization best-basis inventory was developed for the tank contents.

Table 4-1 summarizes the status of the Project Hanford Management Contractor (PHMC) TWRS Program review and acceptance of the sampling and analysis results reported in this tank characterization report. All DQO issues required to be addressed by sampling and analysis are listed in column one of Table 4-1. The second column indicates whether the requirements of the DQO were met by the sampling and analysis activities performed and is answered with a "yes" or a "no." The third column indicates concurrence and acceptance by the program in TWRS that is responsible for the DQO that the sampling and analysis activities performed adequately meet the needs of the DQO. A "yes" or "no" in column three indicates acceptance or disapproval of the sampling and analysis information presented in the TCR. If the results/information have not yet been reviewed, "N/R" is shown in the column.

Table 4-1. Acceptance of Tank 241-AN-105 Sampling and Analysis.

\begin{tabular}{|c|c|c|}
\hline Issure & $\begin{array}{l}\text { Sampling and } \\
\text { remalysis }\end{array}$ & $\begin{array}{l}\text { WRS Progran } \\
\text { Acceutance }\end{array}$ \\
\hline Safety Screening DQO & Yes & Yes \\
\hline Flammable Gas Tank Safety Program & Yes & Yes \\
\hline Vapor Screening & Yes & Yes \\
\hline
\end{tabular}

Table 4-2 summarizes the status of the PHMC TWRS Program review and acceptance of the evaluations and other characterization information contained in this report. The evaluations specifically outlined in this report are the best-basis inventory evaluation and the evaluation to determine whether the tank is safe, conditionally safe, or unsafe. Column one lists the different evaluations performed in this report. Columns two and three are in the same format as Table 4-1. The manner in which concurrence and acceptance are summarized is also the same as that in Table $4-1$. 
Table 4-2. Acceptance of Evaluation of Characterization Data and Information for Tank 241-AN-105.

\begin{tabular}{|c|c|c|}
\hline Kssie & grarinarion & 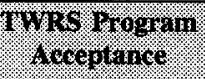 \\
\hline $\begin{array}{l}\text { Safety categorization } \\
\text { (tank is safe) }\end{array}$ & Yes & Yes \\
\hline Flammable Gas Tank Safety Program & $\mathrm{N} / \mathrm{R}$ & N/R \\
\hline
\end{tabular}

The waste currently in tank 241-AN-105 should be monitored continuously because of gas release events. In addition, no additional liquid and solid characterization efforts are needed at this time. Finally, there were no unexpected findings that would affect the ability to retrieve and dispose of the waste safely.

One final comment regarding the safety screening DQO needs to be made. The one-sided confidence intervals that were used to determine whether or not ${ }^{239} \mathrm{Pu}$ and DSC are below the DQO stated threshold limits were performed on each individual sample as required by the DQO. 


\subsection{REFERENCES}

Agnew, S. F., R. A. Corbin, T. B. Duran, K. A. Jurgensen, T. P. Ortiz, and B. L. Young, 1997a, Waste Status and Transaction Record Summary WSTRS Revision 4, LA-UR-97-311, Rev. 0, Los Alamos National Laboratory, New Mexico.

Agnew, S. F., J. Boyer, R. A. Corbin, T. B. Duran, J. R. Fitzpatrick, K. A. Jurgensen, T. P. Ortiz, and B. L. Young, 1997b, Hanford Tank Chemical and Radionuclide Inventories: HDW Model Revision 4, LA-UR-96-3680, Los Alamos National Laboratory, Los Alamos, New Mexico.

Brown, T. M., S. J. Eberlein, J. W. Hunt, and T. J. Kunthara, 1996, Tank Waste Characterization Basis, WHC-SD-WM-TA-164, Rev. 2, Westinghouse Hanford Company, Richland, Washington.

Dukelow, G. T., J. W. Hunt, H. Babad, and J. E. Meacham, 1995, Tank Safety Screening Data Quality Objective, WHC-SD-WM-SP-004, Rev. 2, Westinghouse Hanford Company, Richland, Washington.

Ecology, EPA and DOE, 1996, Hanford Federal Facility Agreement and Consent Order, as amended, Washington State Department of Ecology, U.S. Environmental Protection Agency, and U.S. Department of Energy, Olympia, Washington.

Eggers, R. F., 1996,Tank 241-AN-105 Push Mode Core Sampling and Analysis Plan, WHC-SD-WM-TSAP-027, Rev. 1, Westinghouse Hanford Company, Richland, Washington.

Hanlon, B. M., 1996, Waste Tank Summary Report for Month Ending May 31, 1996, WHC-EP-0182-98, Westinghouse Hanford Company, Richland, Washington.

Haubach, E. C., 1996, Double Shell Tank Interim Operational Safety Requirements, WHC-SD-WM-OSR-016, Rev. 0E, Westinghouse Hanford Company, Richland Washington

Hendrickson, D. W., 1994, Grout Treatment Facility Waste Feed Projections, WHC-SD-WM-TI-528, Westinghouse Hanford Company, Richland, Washington.

Hodgson, K. M. and M. D. LeClair, 1996, Work Plan for Defining a Standard Inventory Estimate for Wastes Stored in Hanford Site Underground Tanks, WHC-SD-WM-WP-311, Rev. 1, Lockheed Martin Hanford Corporation, Richland, Washington. 
Kummerer, M., 1994, Topical Report on Heat Removal Characteristics of Waste Storage

Tanks, WHC-SD-WM-SARR-010, Rev. 0, Westinghouse Hanford Company, Richland, Washington.

Mauss, B. M., 1984, Chemical Compositions of 102-AY, 101-AW, 105-AN, and 104-AW, (letter \# 65453-84-348 to E. G. Gratny, November 9), Rockwell Hanford Operation, Richland, Washington.

Public Law 101-510, 1990, "Safety Measures for Waste Tanks at Hanford Nuclear Reservation," Section 3137 of National Defense Authorization Act for Fiscal Year 1991.

Sasaki L. M., 1984, Results of Organic Analysis of Double Shell Slurry, (letter \# 65611-84-053 to D. J. Fisher, April 5, 1984), Rockwell Hanford Operation, Richland, Washington.

Shekarriz, A., R. E. Bauer, D. R. Rector, N. S. Cannon, L. A. Mahoney, B. E. Hey, M. A. Chieda, C. G. Linshooten, J. M. Bates, F. J. Reitz, E. R. Siciliano, 1997, Retained Gas Sampler Measurement Results for Hanford Waste Tanks 241-AW-101, 241-A-101, 241-AN-105, 241-AN-104, and 241-AN-103, PNNL-11450, Rev. 1, Pacific Northwest National Laboratory, Richland, Washington.

Steen, F. H., 1997, Tank 241-AN-105, Cores 152 and 153 Analytical Results for the Final Report, HNF-SD-WM-DP-199, Rev. 1, Rust Federal Service of Hanford, Inc., Richland, Washington.

Steen, F. H., 1996, Tank 241-AN-105, Cores 152 and 153, Analytical Results for The 45 Day Report, HNF-SD-WM-DP-199, Rev. 0, Westinghouse Hanford Company, Richland, Washington.

Stewart, C. W., J. M. Alzheimer, M. E. Brewster, G. Chen, R. E. Mendoza, H. C. Reid, C. L. Shepard, and G. Terrones, 1996, In Situ Rheology and Gas Volume in Hanford Double-shell Waste Tanks, PNNL-11296, Pacific Northwest National Laboratory, Richland, Washington.

Wilkins, N. E., R. E. Bauer, and D. M. Ogden, 1997, Results of Vapor Space Monitoring of Flammable Gas Watch List Tanks, HNF-SD-WM-TI-797, Rev. 1, Lockheed Martin Hanford Corporation, Richland, Washington.

Winkleman, W. D., 1996, Tank 24I-AN-105 Tank Characterization Plan, WHC-SD-WM-TP-385, Rev. 3, Westinghouse Hanford Company, Richland, Washington. 
HNF-SD-WM-ER-678 Rev. 0

APPENDIX A

HISTORICAL TANK INFORMATION

A-1 
HNF-SD-WM-ER-678 Rev. 0

This page intentionally left blank.

A-2 


\section{APPENDIX A}

\section{HISTORICAL TANK INFORMATION}

Appendix A describes tank 241-AN-105 based on historical information. For this report, historical information includes any information about the fill history, waste types, surveillance, or modeling data about the tank. This information is necessary for providing a balanced assessment of the sampling and analytical results.

This appendix contains the following information:

- Section A1: Current status of the tank, including the current waste levels as well as the stabilization and isolation status of the tank.

- Section A2: Information about the design of the tank.

- Section A3: Process knowledge of the tank; that is, the waste transfer history and the estimated contents of the tank based on modeling data.

- Section A4: Surveillance data for tank 241-AN-105, including surface-level readings, temperatures, and a description of the waste surface based on photographs.

- Section A5: References for Appendix A.

Historical sampling results (results from samples obtained prior to 1989) are included in Appendix B.

\section{A1.0 CURRENT TANK STATUS}

As of May 31, 1996, tank 241-AN-105 contained an estimated 4,270 kL $(1,129 \mathrm{kgal})$ of double-shell slurry feed waste (Hanlon 1996). The waste volumes were estimated using a ENRAF $^{1}$ surface-level gauge. The volumes of the waste phases found in the tank are shown in Table A1-1. The tank has an operating capacity of 4,390 $\mathrm{kL}(1,160 \mathrm{kgal})$.

Tank 241-AN-105 is on the Hydrogen Watch List (Public Law 101-510), and, therefore, is prohibited from receiving waste. The tank is actively ventilated and categorized as sound.

\footnotetext{
${ }^{1}$ ENRAF is a trademark of the ENRAF Corporation, Houston, Texas.
} 
Table A1-1. Tank Contents Status Summary'.

\begin{tabular}{|c|c|}
\hline Waste t ope & . $11 .(\mathrm{ogal}$ \\
\hline Total waste & $4,270(1,129)$ \\
\hline Supernatant $^{2}$ & $4,270(1,129)$ \\
\hline Sludge $^{2}$ & $0(0)$ \\
\hline Saltcake $^{2}$ & $0 \quad(0)$ \\
\hline Drainable interstitial liquid & $0(0)$ \\
\hline Drainable liquid remaining & $4,270(1,129)$ \\
\hline Pumpable liquid remaining & $4,270(1,129)$ \\
\hline
\end{tabular}

Notes:

\begin{abstract}
${ }^{1}$ Hanlon 1996
${ }^{2}$ Although the Waste Tank Summary Report (Hanlon 1996) reports that the tank contents are all supernatant waste, it is evident from the appearance of the core samples that approximately 40 percent of the bottom of the waste is salt slurry.
\end{abstract}

\title{
A2.0 TANK DESIGN AND BACKGROUND
}

The AN Tank Farm is a double-shell tank farm built between 1980 and 1981. This tank farm consists of seven, type 100 series, $4,390 \mathrm{~kL}(1,160 \mathrm{kgal})$ tanks. These tanks were designed for boiling waste with a maximum fluid temperature of $177^{\circ} \mathrm{C}\left(350^{\circ} \mathrm{F}\right)$. The 241-AN Tank Farm does not use a cascade system between tanks.

Tank 241-AN-105 was constructed of $460 \mathrm{~mm}(1.5 \mathrm{ft})$ thick concrete walls and a $380 \mathrm{~mm}$ $(1.25 \mathrm{ft})$ thick concrete dome. The mild carbon steel liner on the bottom is $13 \mathrm{~mm}(1 / 2 \mathrm{in}$.) thick while the lower portion of the sides are $19 \mathrm{~mm}(3 / 4 \mathrm{in}$.). The upper portion of the sides are $13 \mathrm{~mm}$ ( $1 / 2$ in.) thick and the dome liner is $9.5 \mathrm{~mm}(3 / 8 \mathrm{in}$.) thick steel. The inner liner has been heat-treated and stress-relieved. The secondary liner is made of $9.5 \mathrm{~mm}$ (3/8 in.) mild carbon steel. The outer liner has not been heat-treated. The tank has a flat bottom and a maximum operating depth of $10.7 \mathrm{~m}(35 \mathrm{ft})$. The tank is set on an insulated, reinforced concrete foundation. This foundation has a grid of drain slots designed to collect any tank leakage and divert it to a leak detection well. The grid also served as an escape route for free water released from the concrete grout during initial heating of the tank. Various coatings and sealants were used to ensure that no leaks and intrusions occurred.

Tank 241-AN-105 has 22 risers, ranging in diameter from $102 \mathrm{~mm}$ (4 in.) to $1.07 \mathrm{~m}$ (42 in.), that provide access to the tank and 37 risers that provide access to the annulus. Table A2-1 shows numbers, diameters, and descriptions of the risers (annular risers not included). Figure A2-1 shows the riser configuration. 
Table A2-1. Tank 241-AN-105 Risers. ${ }^{1,2,3}$

\begin{tabular}{|c|c|c|c|}
\hline Wertore & (1010 & Whores & 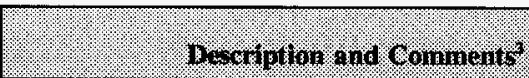 \\
\hline 102 & $1 \mathrm{~A}$ & 4 & Sludge measurement port \\
\hline 103 & $1 B^{4}$ & 4 & Sludge measurement port, (12 in. cover) \\
\hline 101 & $1 \mathrm{C}$ & 4 & Sludge measurement port \\
\hline 104 & $2 \mathrm{~A}$ & 4 & ENRAF $^{(8)}$ level gauge \\
\hline 105 & $3 \mathrm{~A}$ & 12 & Supernatant pump, Central pump pit \\
\hline 106 & $4 \mathrm{~A}$ & 4 & Thermocouple tree \\
\hline 108 & $5 \mathrm{~A}$ & 42 & Manhole, below grade \\
\hline 107 & $5 B$ & 42 & Manhole, below grade \\
\hline 112 & $7 \mathrm{~A}$ & 12 & Tank ventilation \\
\hline 111 & $7 \mathrm{~B}^{4}$ & 12 & Spare \\
\hline 125 & $10 \mathrm{~A}^{4}$ & 4 & Tank pressure \\
\hline 126 & $11 \mathrm{~A}$ & 42 & Slurry distributor, Central pump pit \\
\hline 127 & $12 \mathrm{~A}^{4}$ & 12 & Observation port, spare \\
\hline 128 & $13 \mathrm{~A}$ & 4 & Tank pressure \\
\hline 129 & $14 \mathrm{~A}$ & 4 & Supernatant return, Central pump pit \\
\hline 131 & $15 A^{4}$ & 4 & Spare, (12 in. cover) \\
\hline 130 & $15 \mathrm{~B}$ & 4 & High liquid level sensor \\
\hline 134 & $16 \mathrm{~A}$ & 4 & Sludge measurement port, (12 in. cover) \\
\hline 132 & $16 \mathrm{~B}^{4}$ & 4 & Sludge measurement port \\
\hline 133 & $16 \mathrm{C}$ & 4 & Sludge measurement port \\
\hline 155 & $21 \mathrm{~A}$ & 4 & Spare \\
\hline 156 & $22 \mathrm{~A}^{4}$ & 4 & Sludge measurement port \\
\hline
\end{tabular}

Notes:

${ }^{1}$ Salazar (1994)
${ }^{2}$ Hanford Drawings: H-14-01050, H-2-71995

${ }^{3}$ Tank risers only, annulus risers excluded.

${ }^{4}$ Denotes risers tentatively available for sampling (Lipnicki 1996).

Risers 1B, 10A, 15A, 16B, and 22A (each $102 \mathrm{~mm}$ [4 in.] in diameter) and risers $7 \mathrm{~B}$ and 12A (305 mm [12-in.] in diameter) are available for sampling (Lipnicki 1996). A tank cross-section showing the approximate waste level along with a schematic of the tank equipment is in Figure A2-2. 
Figure A2-1. Riser Configuration for Tank AN-105.

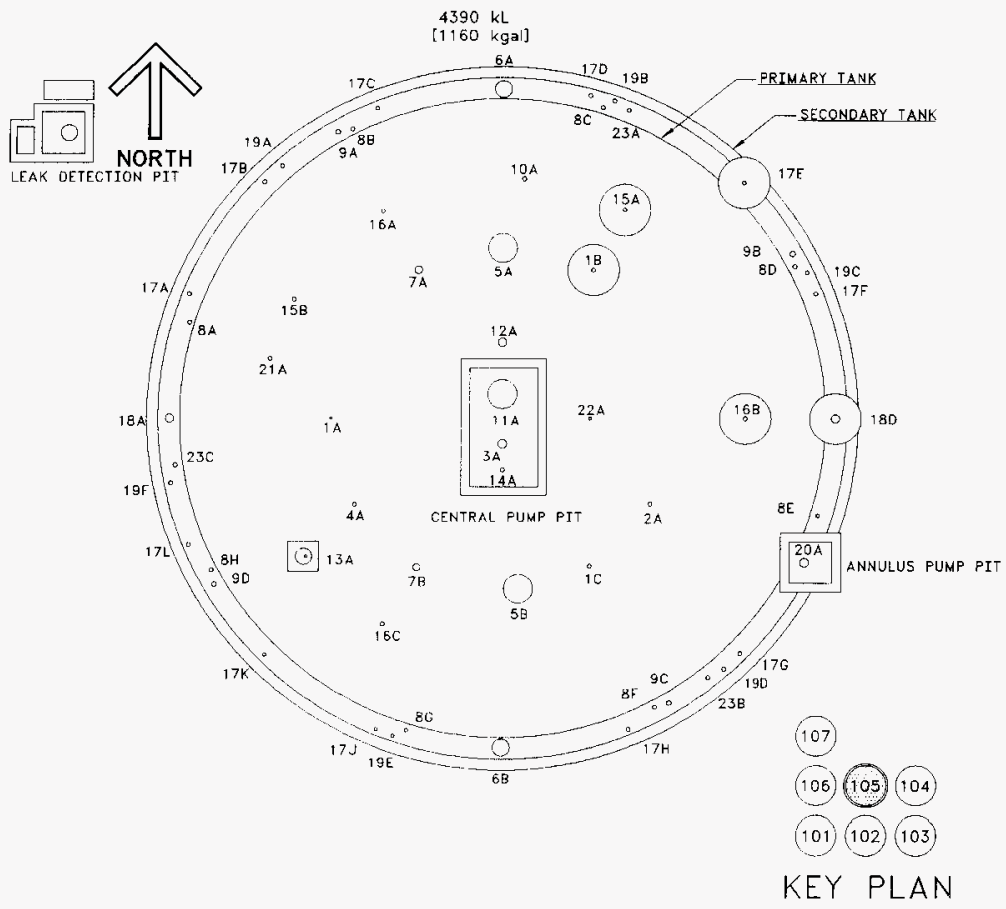


Figure A2-2. Tank 241-AN-105 Cross Section and Schematic.

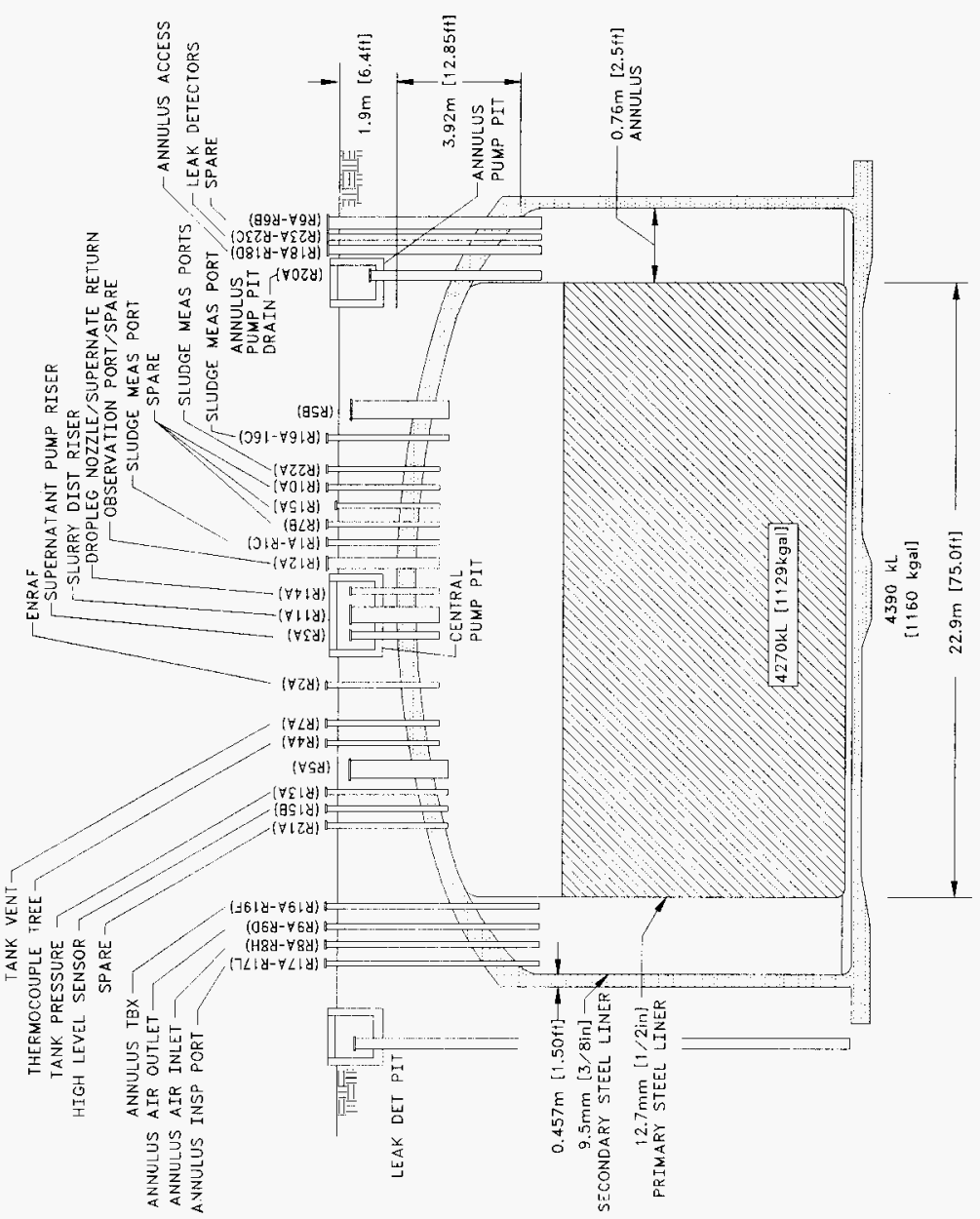




\section{A3.0 PROCESS KNOWLEDGE}

The sections below: 1) provide information about the transfer history of tank 241-AN-105; 2) describe the process wastes that made up the transfers; and 3) give an estimate of the current tank contents based on transfer history.

\section{A3.1 WASTE TRANSFER HISTORY}

Table A3-1 summarizes the waste transfer history of tank 241-AN-105 using (Agnew et al. 1997a) and the Hanford Site tank transfer database. Water was initially added to tank 241-AN-105 in the second quarter of 1982. During the fourth quarter of 1982 through the first quarter of 1983, the tank received double-shell slurry feed waste from tank 241-AW-102. A small amount of waste from an unknown source was added in the third quarter of 1984. The tank received non-complexed waste from tank 241-AN-104 in the first quarter of 1984. Most of the waste was removed to tank 241-AW-102 during the first quarter of 1985 for the evaporator campaign, leaving approximately $583 \mathrm{~kL}$ (154 kgal) waste in tank 241-AN-105. During the first quarter of 1985 to the second quarter of 1985 , the tank received double-shell slurry feed waste from tank $241-\mathrm{AW}-102$ via the evaporator, then waste reception ceased. The only transfer since that time has been an addition of flush water from miscellaneous sources from the fourth quarter of 1995 to the first quarter of 1996. 
Table A3-1. Tank 241-AN-105 Major Transfers. ${ }^{1,2}$

\begin{tabular}{|c|c|c|c|c|c|}
\hline \multirow{2}{*}{ 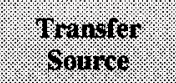 } & \multirow{2}{*}{ 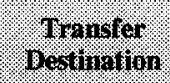 } & \multirow[b]{2}{*}{ Mrote Ifoe } & \multirow{2}{*}{ Ferog } & \multicolumn{2}{|c|}{ Estiniated Waste Yolame } \\
\hline & & & & 14 & (Ka) \\
\hline $\begin{array}{l}\text { Miscellaneous } \\
\text { source }\end{array}$ & & Flush water & 1982 & 42 & 11 \\
\hline 241-AW-102 & & DSSF & $1982-1983$ & 2377 & 628 \\
\hline 241-AN-104 & & NCPLX & 1984 & 1890 & 499 \\
\hline Unknown & & Unknown & 1984 & 26 & 7 \\
\hline & 241-AW-102 & DSSF/NCPLX & 1985 & -3610 & -954 \\
\hline 241-AW-102 & & DSSF & 1985 & 3676 & 971 \\
\hline $\begin{array}{l}\text { Miscellaneous } \\
\text { source }\end{array}$ & & Flush water & $1995-1996$ & 11 & 3 \\
\hline
\end{tabular}

Notes:

DSSF $=$ Double-Shell Slurry Feed - Waste concentrated just before reaching the sodium aluminate saturation boundary in the evaporator without exceeding receiver tank composition limits. This form is not as concentrated as double shell slurry (DSS).

NCPLX $=\quad$ Non-complexed - General waste term applied to all Hanford Site liquors not identified as complexed.

${ }^{1}$ Agnew et al. 1997a

${ }^{2}$ Because only major transfers are listed, the sum of these transfers will not equal the current tank waste volume.

\section{A3.2 HISTORICAL ESTIMATION OF TANK CONTENTS}

The historical transfer data used for this estimate are from the following sources:

- Waste Status and Transaction Record Summary for the Southwest Quadrant of the Hanford 200 East Area (WSTRS) (Agnew et al. 1997a). WSTRS is a tank-by-tank quarterly summary spreadsheet of waste transactions.

- Hanford Tank Chemical and Radionuclide Inventories: HDW Model Rev. 4 (Agnew et al. 1997b). This document contains the Hanford Defined Waste (HDW) list, the Supernatant Mixing Model (SMM), and the Tank Layer Model (TLM), and the historical tank inventory estimate.

- The HDW list is a list of approximately 50 waste types with nominal analyte composition for both sludge and supernatant layer. The TLM defines the 
sludge and saltcake layers in each tank using waste composition and waste transfer information. Supernatant Mixing Model (SMM) is a subroutine within the HDW model that calculates the volume and composition of certain supernatant blends and concentrates.

Using these records, the TLM defines the sludge and saltcake layers in each tank. The HDW list provides typical composition expected for approximately 50 waste types. The SMM uses information from WSTRS, the HDW list, and the TLM to describe the supernatants and concentrates in each tank. Together the WSTRS, HDW list, TLM, and SMM determine each tank's inventory estimate. These model predictions are considered estimates that require further evaluation using analytical data.

Based on Agnew et al. (1997b), tank 241-AN-105 contains only a layer of 4,270 kL (1,129 kgal) of DSSF waste. Figure A3-1 shows a graphical representation of the estimated waste type and volume for the tank layer. The historical tank content estimate model predicts tank 241-AN-105 to contain very large (greater than one weight percent) amounts of sodium, aluminum, nitrite, nitrate, and hydroxide. Table A3-2 shows the historical estimate of the expected waste constituents and their concentrations. 
HNF-SD-WM-ER-678 Rev. 0

Figure A3-1. Tank Layer Model.

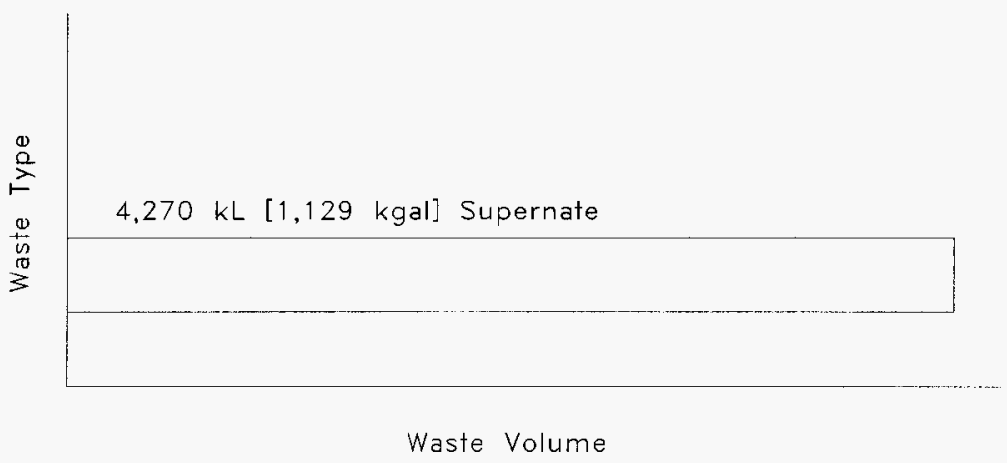


Table A3-2. Historical Tank Inventory Estimate. ${ }^{1,2}$ (2 sheets)

\begin{tabular}{|c|c|c|c|}
\hline Total inveriony Estinate & 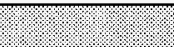 & : & \\
\hline Puysieal I roperties & & & \\
\hline Total Waste & $8.04 \mathrm{E}+06 \mathrm{~kg}$ & $3 \mathrm{E}+03 \mathrm{kgal})$ & \\
\hline Heat Load & $11.4 \mathrm{~kW}(3.90$ & 04 BTU/hr) & \\
\hline Bulk Density & $1.88(\mathrm{~g} / \mathrm{mL})$ & & \\
\hline Water weight percent & 17.2 & & \\
\hline $\begin{array}{l}\text { Total Organic Carbon } \\
\text { weight percent Carbon (wet) }\end{array}$ & 1.36 & & \\
\hline Ghernical Gonstifiuents & 1. & 1. $10 / g$ & 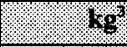 \\
\hline $\mathrm{Na}^{+}$ & 20.5 & $2.51 \mathrm{E}+05$ & $2.02 \mathrm{E}+06$ \\
\hline $\mathrm{Al}^{3+}$ & 2.35 & $3.38 \mathrm{E}+04$ & $2.71 \mathrm{E}+05$ \\
\hline $\mathrm{Fe}^{3+}$ (total $\left.\mathrm{Fe}\right)$ & $1.56 \mathrm{E}-02$ & 464 & $3.73 \mathrm{E}+03$ \\
\hline $\mathrm{Cr}^{3+}$ & 0.160 & $4.42 \mathrm{E}+03$ & $3.56 \mathrm{E}+04$ \\
\hline $\mathrm{Bi}^{3+}$ & $1.87 \mathrm{E}-03$ & 208 & $1.67 \mathrm{E}+03$ \\
\hline $\mathrm{La}^{3+}$ & $1.99 \mathrm{E}-05$ & 1.47 & 11.8 \\
\hline $\mathrm{Hg}^{2+}$ & $1.38 \mathrm{E}-05$ & 1.47 & 11.8 \\
\hline $\mathrm{Zr}\left(\right.$ as $\left.\mathrm{ZrO}(\mathrm{OH})_{2}\right)$ & $3.82 \mathrm{E}-04$ & 18.5 & 149 \\
\hline $\mathrm{Pb}^{2+}$ & $1.74 \mathrm{E}-03$ & 191 & $1.54 \mathrm{E}+03$ \\
\hline $\mathrm{Ni}^{2+}$ & $1.06 \mathrm{E}-02$ & 331 & $2.66 \mathrm{E}+03$ \\
\hline $\mathrm{Sr}^{2+}$ & 0 & 0 & 0 \\
\hline $\mathrm{Mn}^{4+}$ & $9.35 \mathrm{E}-03$ & 273 & $2.20 \mathrm{E}+03$ \\
\hline $\mathrm{Ca}^{2+}$ & $5.57 \mathrm{E}-02$ & $1.19 \mathrm{E}+03$ & $9.56 \mathrm{E}+03$ \\
\hline $\mathrm{K}^{+}$ & 0.106 & $2.22 \mathrm{E}+03$ & $1.78 \mathrm{E}+04$ \\
\hline $\mathrm{OH}^{-}$ & 13.7 & $1.24 \mathrm{E}+05$ & $9.97 \mathrm{E}+05$ \\
\hline $\mathrm{NO}_{3}{ }^{-}$ & 7.08 & $2.34 \mathrm{E}+05$ & $1.88 \mathrm{E}+06$ \\
\hline$\widehat{\mathrm{NO}_{2}}$ & 3.10 & $7.58 \mathrm{E}+04$ & $6.09 \mathrm{E}+05$ \\
\hline $\mathrm{CO}_{3}{ }^{2-}$ & 0.910 & $2.91 \mathrm{E}+04$ & $2.34 \mathrm{E}+05$ \\
\hline $\mathrm{PO}_{4}^{3-}$ & 0.184 & $9.29 \mathrm{E}+03$ & $7.47 \mathrm{E}+04$ \\
\hline $\mathrm{SO}_{4}^{2-}$ & 0.386 & $1.97 \mathrm{E}+04$ & $1.59 \mathrm{E}+05$ \\
\hline $\mathrm{Si}\left(\right.$ as $\mathrm{SiO}_{3}{ }^{2}$ ) & 0.113 & $1.69 \mathrm{E}+03$ & $1.36 \mathrm{E}+04$ \\
\hline
\end{tabular}


Table A3-2. Historical Tank Inventory Estimate. ${ }^{1,2}$ (2 sheets)

\begin{tabular}{|c|c|c|c|}
\hline Total linerolor Estinite & & & \\
\hline Giemeal Goustivents (Gorr d) & 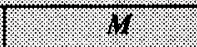 & $1.6 \mathrm{c}$ & 148 \\
\hline $\mathrm{F}^{-}$ & 0.107 & $1.09 \mathrm{E}+03$ & $8.72 \mathrm{E}+03$ \\
\hline$\overline{\mathrm{Cl}^{-}}$ & 0.361 & $6.81 \mathrm{E}+03$ & $5.48 \mathrm{E}+04$ \\
\hline $\mathrm{C} 4 \mathrm{H} 507^{3-}$ & $4.80 \mathrm{E}-02$ & $4.83 \mathrm{E}+03$ & $3.88 \mathrm{E}+04$ \\
\hline EDTA $^{4-}$ & $3.14 \mathrm{E}-02$ & $4.82 \mathrm{E}+03$ & $3.88 \mathrm{E}+04$ \\
\hline HEDTA $^{3-}$ & $5.48 \mathrm{E}-02$ & $7.99 \mathrm{E}+03$ & $6.42 \mathrm{E}+04$ \\
\hline glycolate & 0.261 & $1.04 \mathrm{E}+04$ & $8.38 \mathrm{E}+04$ \\
\hline acetate & $2.59 \mathrm{E}-02$ & 815 & $6.55 \mathrm{E}+03$ \\
\hline oxalate & 2.06 & 1.22 & 9.8 \\
\hline DBP & $3.40 \mathrm{E}-02$ & $3.80 \mathrm{E}+03$ & $2.06 \mathrm{E}+04$ \\
\hline butanol & $3.40 \mathrm{E}-02$ & $1.34 \mathrm{E}+03$ & $1.08 \mathrm{E}+04$ \\
\hline $\mathrm{NH}_{3}$ & 0.114 & $1.03 \mathrm{E}+03$ & $8.27 \mathrm{E}+03$ \\
\hline $\mathrm{Fe}(\mathrm{CN})_{6}{ }^{4-}$ & 0 & 0 & 0 \\
\hline Ratiologieal constituents. & & & \\
\hline $\mathrm{Pu}$ & $9.21 \mathrm{E}-04(\mathrm{~g} / \mathrm{L})$ & & $3.94(\mathrm{~kg})$ \\
\hline $\mathrm{U}$ & $1.35 \mathrm{E}-02(M)$ & $1.71 \mathrm{E}+03(\mu \mathrm{g} / \mathrm{g})$ & $1.38 \mathrm{E}+04(\mathrm{~kg})$ \\
\hline $\mathrm{Cs}$ & $0.346(\mathrm{Ci} / \mathrm{L})$ & $184(\mu \mathrm{Ci} / \mathrm{g})$ & $1.48 \mathrm{E}+06(\mathrm{Ci})$ \\
\hline Sr-90 & 0.155 & $82.7(\mu \mathrm{Ci} / \mathrm{g})$ & $6.65 \mathrm{E}+05(\mathrm{Ci})$ \\
\hline
\end{tabular}

Notes:

'Agnew et al. (1997b)

${ }^{2}$ The historical tank inventory predictions have not been validated and should be used with caution.

${ }^{3}$ Differences exist among the inventories in this column and the inventories calculated from the two sets of concentrations. 


\section{A4.0 SURVEILLANCE DATA}

Tank 241-AN-105 surveillance data consists of surface-level measurements (liquid and solid), temperature monitoring inside the tank (waste and headspace). The annulus is ventilated and continually monitored by radiation and leak detectors for evidence of primary tank leakage. These data provide the basis for determining tank integrity.

\section{A4.1 SURFACE LEVEL READINGS}

A Food Instrument Corporation (FIC) gauge was used to monitor the waste surface level in tank 241-AN-105 through riser 2A in the automatic mode until July 15, 1995, and in the manual mode until July 31,1995 . Currently, surface levels are currently taken via a manual and automatic ENRAF ${ }^{\oplus}$ gauge. This tank is on the Hydrogen/Flammable Gas Watch List and is prohibited from receiving waste. A graphical representation of the volume measurements is presented as a level history graph in Figure A4-1.

\section{A4.2 INTERNAL TANK TEMPERATURES}

Tank 241-AN-105 has a single thermocouple tree with 18 thermocouples to monitor the waste temperature through riser $4 \mathrm{~A}$. Elevations are available for all thermocouples. Plots of the individual thermocouple readings can be found in the AN Tank Farm supporting document for the HTCE (Brevick et al. 1995).

Temperature data, obtained from the Surveillance Analysis Computer System (SACS) (LMHC 1997), were recorded from January 1990 to February 1997. Data were available for all 18 thermocouples. The mean temperature of the SACS data is $39.1{ }^{\circ} \mathrm{C}\left(102.3^{\circ} \mathrm{F}\right)$ with a minimum of $19.2^{\circ} \mathrm{C}\left(66.6^{\circ} \mathrm{F}\right)$ and a maximum of $46.7^{\circ} \mathrm{C}\left(116^{\circ} \mathrm{F}\right)$. The mean temperature of the SACS data over the last year (February 1996 through February 1997) is $38.7^{\circ} \mathrm{C}\left(100.5^{\circ} \mathrm{F}\right)$ with a minimum of $19.2^{\circ} \mathrm{C}\left(66.6^{\circ} \mathrm{F}\right)$ and a maximum of $45.2^{\circ} \mathrm{C}$ $\left(113.4^{\circ} \mathrm{F}\right)$. On February 3,1997 , the lowest temperature recorded was $20^{\circ} \mathrm{C}\left(68^{\circ} \mathrm{F}\right)$ on thermocouple 17 (located near the surface of the waste). The highest temperature recorded was $41.9^{\circ} \mathrm{C}\left(107.4^{\circ} \mathrm{F}\right)$ on thermocouple 5 (located in the waste). A graph of the weekly high temperature can be found in Figure A4-2.

\section{A4.3 TANK 241-AN-105 PHOTOGRAPHS}

The January 1988 photographic montage of the tank 241-AN-105 interior shows a typical saltcake waste surface that is produced by the Hanford Site evaporators. The colors are off-white with patches of yellow. The tank walls and dome show signs of rust. Equipment visible in the photographs are a level probe, a thermocouple tree, a saltwell screen, an FIC probe, and a slurry distributor. Since the photographs were taken, the level of the waste has 
only changed slightly because of slurry growth from gas generation. Tank 241-AN-105 has a video camera that has been operational since 1995 . The photographic montage should be a fairly accurate representation of the current tank waste appearance (Brevick et al 1995).

\section{A4.4 STANDARD HYDROGEN MONITORING SYSTEMS (SHMS)}

An standard hydrogen monitoring systems (SHMS) was installed in this tank in October 1994 that monitors hydrogen with two Whittaker cells. A gas characterization system (GCS) was installed in April 1996. A GCS comprises three instruments: two gas chromatographs (GC) and a Fourier transform infrared spectrometer (FTIR). One GC measures hydrogen, the other measures nitrous oxide and methane.

The FTIR measures ammonia concentrations. The maximum hydrogen concentration measured since the SHMS was installed was 1.6 percent on August 21, 1995. Concentration reached 1.5 percent on May 30, 1996. Background hydrogen concentration in tank 241-

AN-105 is typically below $100 \mathrm{ppm}$ nitrous oxide, about $20 \mathrm{ppm}$, ammonia at about $10 \mathrm{ppm}$ and methane registers 0 ppm (Stewart et al. 1996). 


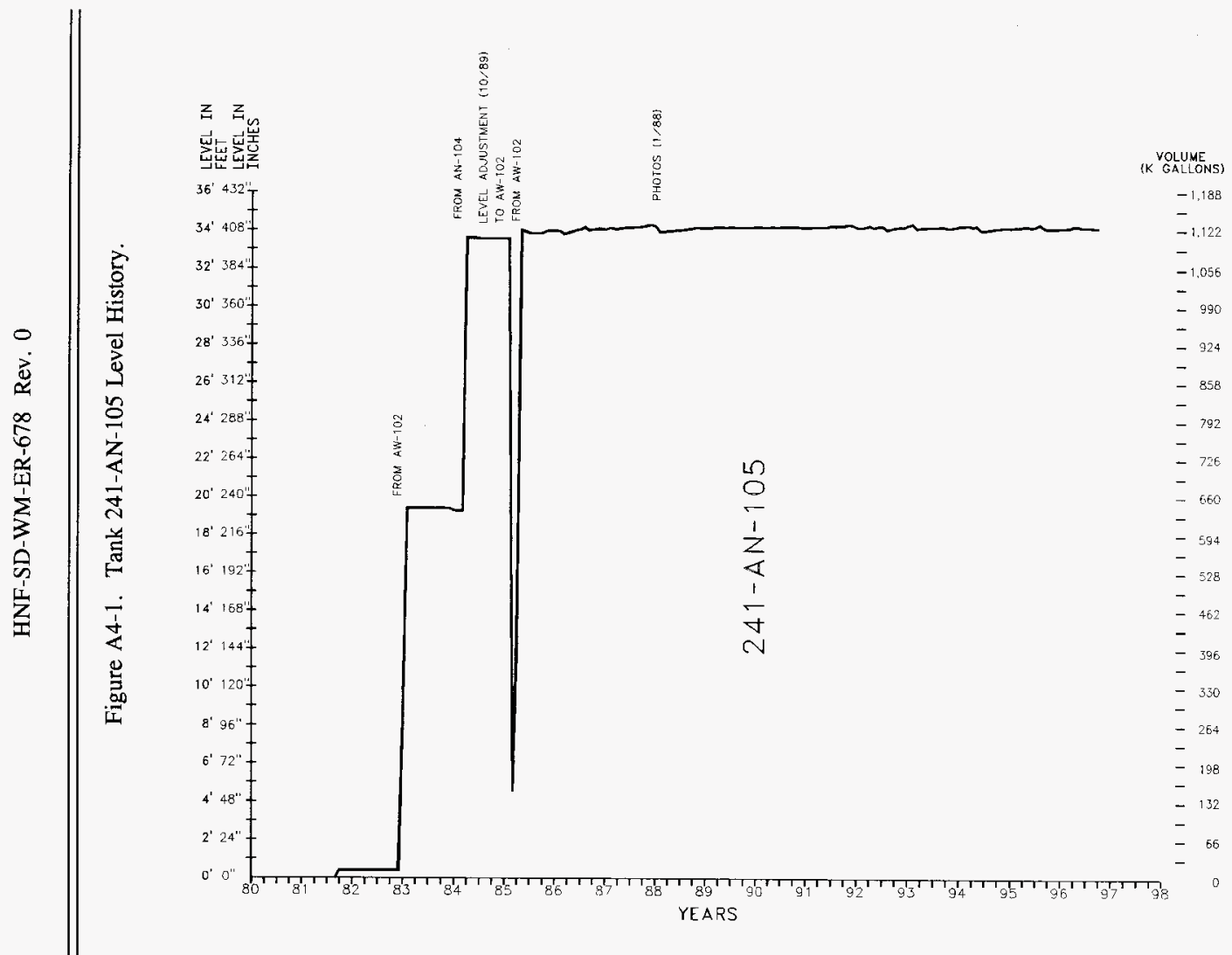

$\stackrel{0}{2}$ 
Figure A4-2. Tank 241-AN-105 Weekly High Temperature Plot.

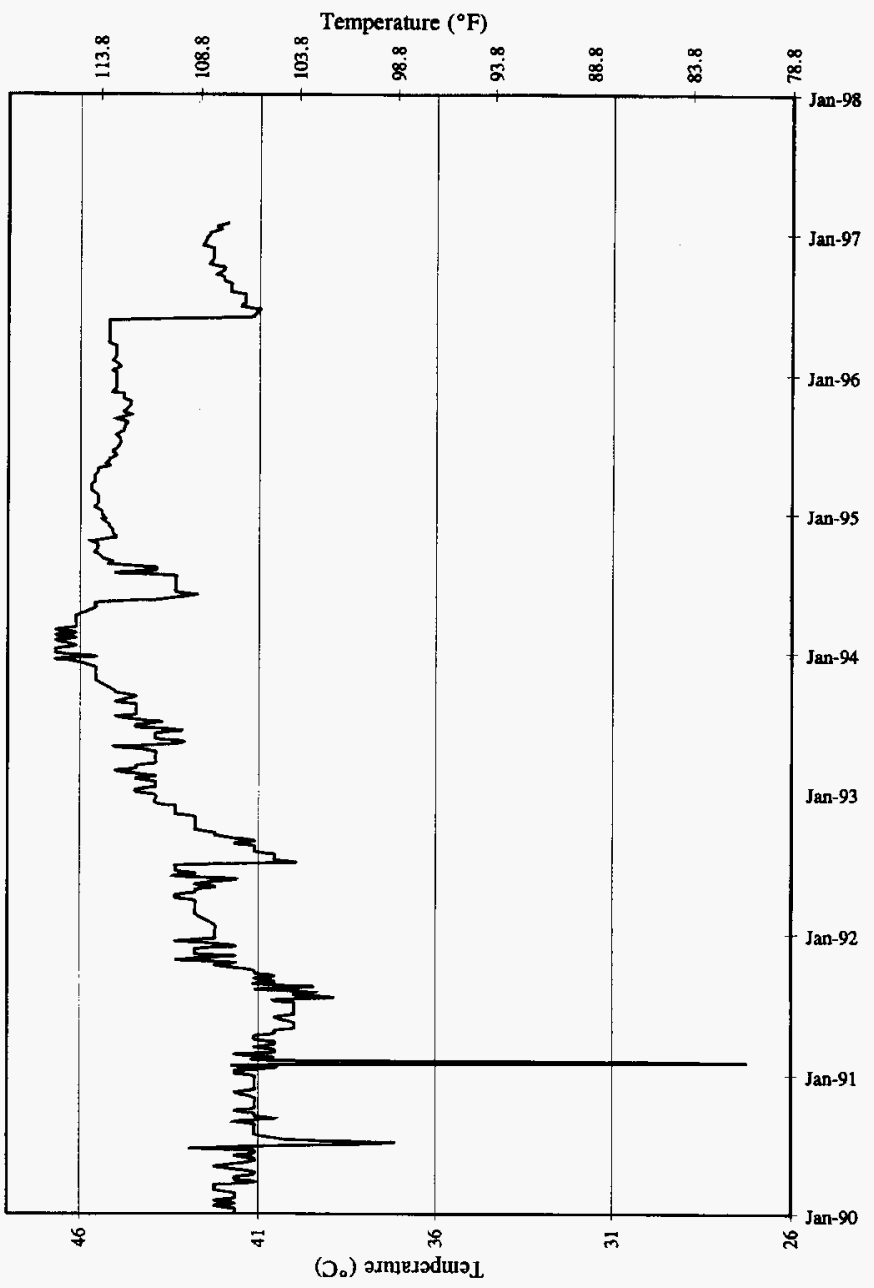




\section{A5.0 APPENDIX A REFERENCES}

Agnew, S. F., R. A. Corbin, T. B. Duran, K. A. Jurgensen, T. P. Ortiz, and B. L. Young, 1997a, Waste Status and Transaction Record Summary WSTRS, Rev 4, LA-UR-97-311, Rev. 0, Los Alamos National Laboratory, New Mexico.

Agnew, S. F., J. Boyer, R. A. Corbin, T. B. Duran, J. R. Fitzpatrick, K. A. Jurgensen, T. P. Ortiz, and B. L. Young, 1997b, Hanford Tank Chemical and Radionuclide Inventories: HDW Model Rev. 4, LA-UR-96-3680, Los Alamos National Laboratory, Los Alamos, New Mexico.

Brevick, C. H., L. A. Gaddis, and S. D. Consort, 1995, Supporting Document for the Southeast Quadrant Historical Tank Content Estimate for AN Tank Farm - Volume I and 2, WHC-SD-WM-ER-314, Rev. 0, Westinghouse Hanford Company, Richland, Washington.

Hanlon, B. M., 1996, Waste Tank Summary Report for Month Ending May 31, 1996, WHC-EP-0182-98, Westinghouse Hanford Company, Richland, Washington.

Lipnicki, J., 1996, Waste Tank Risers Available for Sampling, WHC-SD-WM-TI-710, Rev. 3, Westinghouse Hanford Company, Richland, Washington.

LMHC, 1997, Surveillance Analysis Computer System, In: SYBASE/Visual Basic (mainframe), Lockheed Martin Hanford Corporation, Richland, Washington.

Public Law 101-510, 1990, "Safety Measures for Waste Tanks at Hanford Nuclear Reservation," Section 3137 of National Defense Authorization Act for Fiscal Year 1991.

Salazar, B. E., 1994, Double-Shell Underground Waste Storage Tank Riser Survey, WHC-SD-RE-TI-093, Rev. 4, Westinghouse Hanford Company, Richland, Washington. 
Stewart, C. W., J. M. Alzheimer, C. L. Shepard, G. Terrones, G. Chen, N. E. Wilkins, 1996, In Situ Determination of Rheological Properties and Void Fraction: Hanford Waste Tank 241-AN-105, PNNLMIT:021696, Pacific Northwest National Laboratory, Richland, Washington.

Wilkins, N. E., R. E. Bauer, D. M. Ogden, Results of Vapor Space Monitoring of Flammable Gas Watch List Tanks, HNF-SD-WM-TI-797, Rev. 1, Lockheed Martin Hanford Corporation, Richland, Washington.

WHC, 1995, Done Penetration Schedules Tanks 101-107, H-14-010501, Sheet 3, Rev. 0, Westinghouse Hanford Company, Richland, Washington.

WHC, 1996, Piping Plan Tank 105, H2-71995, Rev. 12, Westinghouse Hanford Company, Richland, Washington. 
HNF-SD-WM-ER-678 Rev. 0

This page intentionally left blank. 
HNF-SD-WM-ER-678 Rev. 0

APPENDIX B

SAMPLING OF TANK 241-AN-105

B-1 
HNF-SD-WM-ER-678 Rev. 0

This page intentionally left blank. 


\section{APPENDIX B}

\section{SAMPLING OF TANK 241-AN-105}

Appendix B provides sampling and analysis information for each known sampling event for tank 241-AN-105 and provides an assessment of the core sample results.

- Section B1: Tank Sampling Overview

- Section B2: Analytical Results

- Section B3: Assessment of Characterization Results

- Section B4: References for Appendix B.

Future sampling of tank 241-AN-105 will be appended to the above list.

\section{B1.0 TANK SAMPLING OVERVIEW}

This section describes the June 1996 sampling and analysis events for tank 241-AN-105. Core samples were taken to satisfy the requirements of the Tank Safety Screening Data Quality Objective (Dukelow et al. 1995), and the Flammable Gas Safety Program: Data Requirements for Core Sample Analysis Developed through the Data Quality Objectives (DQO) Process (McDuffie and Johnson 1995). The sampling and analyses were performed in accordance with the Tank 241-AN-105 Push Mode Core Sampling and Analysis Plan (Eggers 1996). The sampling and analysis plan (SAP) also instructed that 4.0 liters of supernatant from the tank were to be obtained as part of the scheduled push mode core sampling event. The sample will be used to support the Privatization initiative. Two supernatant samples were also taken from this tank in 1984 (Jansky 1984) to support evaporator Campaign Run 84-5. One supernatant sample was taken from this tank in August 1984 to support evaporator Campaign Run 85-3. These sample events are discussed in Section B1.4. 


\section{B1.1 DESCRIPTION OF SAMPLING EVENT}

Twenty-two push mode core segments were removed from tank 241-AN-105 riser 12A (Core 152) between June 10,1996, and June 18, 1996. Segments were received by the 222-S Laboratory between June 11, 1996, and June 24, 1996. Selected segments $(15,17,19$ and 21) were sampled using the Retained Gas Sampler (RGS) and extruded by the Process Chemistry and Statistical Analysis Group.

The second twenty-two push mode core segments were removed from tank 241-AN-105 riser 7B (Core 153) between June 25, 1996, and June 28, 1996. Segments were received by the 222-S Laboratory between June 28, 1996, and August 9, 1996. Selected segments (4, 16, 18 and 20) were sampled using the Retained Gas Sampler and extruded by the Process Chemistry and Statistical Analysis Group.

A field blank was provided to the 222-S Laboratory with core 153 . It underwent the same analysis as the drainable liquid as instructed by the TSAP (Eggers 1996).

Lithium bromide solution used as hydrostatic head fluid during sampling, was provided to the 222-S Laboratory with core 153. It underwent Inductively Coupled Plasma Spectroscopy (ICP) and Ion Chromatography (IC) analyses as instructed by the TSAP (Eggers 1996).

A vertical profile is used to satisfy the safety screening data quality objective (DQO). Safety screening analyses include: total alpha to determine criticality, differential scanning calorimetry (DSC) to ascertain the fuel energy value, and thermogravimetric analysis (TGA) to obtain the total moisture content. In addition, combustible gas meter readings in the tank headspace were performed to measure flammability. The current revision of the safety screening DQO (Dukelow et al. 1995) also requires bulk density measurements.

Tank 241-AN-105 also was evaluated for the Flammable Gas Tank Safety Program. The specified analyses include: DSC, TGA, bulk density, viscosity, void fraction, total inorganic carbon (TIC) total organic carbon (TOC), formate, oxalate, ICP, IC, and radiochemistry.

Sampling and analytical requirements from the safety screening and Flammable Gas DQOs are summarized in Table $\mathrm{B} 1-1$. 
Table B1-1. Integrated Data Quality Objective Requirements for Tank 241-AN-105. ${ }^{1}$

\begin{tabular}{|c|c|c|c|}
\hline Sampling & 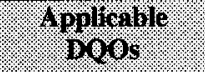 & 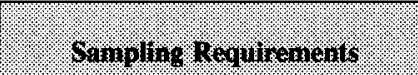 & Wharom \\
\hline \multirow[t]{2}{*}{ Core sampling } & Safety screening & $\begin{array}{l}\text { Core samples from a minimum of } \\
\text { two risers separated radially to the } \\
\text { maximum extent possible. }\end{array}$ & $\begin{array}{l}\text { - Energetics } \\
\text { - Moisture content } \\
\text { - Total alpha }\end{array}$ \\
\hline & Flammable gas & At least one complete core & $\begin{array}{l}\text { - Energetics } \\
\text { - Moisture content } \\
\text { - Bulk density } \\
\text { - Viscosity } \\
\text { - Void fraction } \\
\text { - TIC/TOC } \\
\text { - Formate } \\
\text { - Oxalate } \\
\text { - OH } \\
\text { - ICP } \\
\text { - IC } \\
\text { - Radiochemistry }\end{array}$ \\
\hline $\begin{array}{l}\text { Combustible } \\
\text { gas meter } \\
\text { reading }\end{array}$ & Safety screening & $\begin{array}{l}\text { Measurement in a minimum of one } \\
\text { location within tank vapor space. }\end{array}$ & $\begin{array}{l}\text { - Flammable gas } \\
\text { concentration }\end{array}$ \\
\hline Vapor & $\begin{array}{l}\text { Organic solvent } \\
\text { screening }\end{array}$ & Steel canisters & -Organic vapor \\
\hline
\end{tabular}

Notes:

\author{
'Eggers 1996 \\ ${ }^{2}$ Sampling for organic solvent screening has not been performed.
}

\title{
B1.2 SAMPLE HANDLING
}

The core samples were shipped to the 222-S Laboratory for subsampling and analysis. Samples were assigned LABCORE numbers and were subjected to visual inspection for color, clarity, and solids content. The radiation dose rate on contact was also measured. The salt slurry samples were divided into upper and lower half-segments. The material was homogenized and subsampled for laboratory analyses and archiving. Subsamples of each half-segment were then recombined and subsampled for core composite analyses. Tables B1-2a and B1-2b gives the subsampling scheme and sample description for core 152 and 153 , respectively. 
Table B1-2a. Sample Receipt and Extrusion Information for 241-AN-105, Core 152. (7 sheets)

\begin{tabular}{|c|c|c|c|c|c|c|c|c|}
\hline $\begin{array}{l}\text { Castomer } \\
\text {. } 10 \text {. }\end{array}$ & Se: & $\begin{array}{l}\text { Date } \\
\text { Sannpled! }\end{array}$ & $\begin{array}{l}\text { Bate } \\
\text { Received }\end{array}$ & Extruded & $\begin{array}{l}\text { Inches } \\
\text { Exiruded? }\end{array}$ & $\begin{array}{l}\text {. Liquid: } \\
\text { Recovered (g) }\end{array}$ & 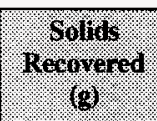 & Sample Desorifilion \\
\hline $96-253$ & 1 & 6/10/96 & 6/11/96 & 6/17/96 & 2.5 & 256.8-Drainable & $\begin{array}{l}\text { 37.6-upper } \\
\text { half }\end{array}$ & $\begin{array}{l}\text { The solids were light gray in } \\
\text { color and resembled a salt } \\
\text { slurry. The liquid was green } \\
\text { in color and opaque. } \\
\text { Collected } 190 \mathrm{~mL} \text { of liquid. } \\
\text { No organic layer was } \\
\text { observed. }\end{array}$ \\
\hline $96-254$ & 2 & $6 / 10 / 96$ & $6 / 11 / 96$ & $6 / 17 / 96$ & 0.0 & 426.3-Drainable & 0.0 & $\begin{array}{l}\text { Extruded light gray solids and } \\
\text { resembled a wet salt. The } \\
\text { liquid was green in color and } \\
\text { opaque. Collected } 305 \mathrm{~mL} \text { of } \\
\text { liquid. No organic layer was } \\
\text { observed. }\end{array}$ \\
\hline $96-255$ & 3 & $6 / 11 / 96$ & $6 / 11 / 96$ & $6 / 18 / 96$ & 0.0 & 406.2-Drainable & 0.0 & $\begin{array}{l}\text { Extruded an unmeasurable } \\
\text { amount of light gray solids } \\
\text { and resembled a wet salt. The } \\
\text { liquid was green in color and } \\
\text { opaque. Collected } 280 \mathrm{~mL} \text { of } \\
\text { liquid. No organic layer was } \\
\text { observed. }\end{array}$ \\
\hline
\end{tabular}


Table B1-2a. Sample Receipt and Extrusion Information for 241-AN-105, Core 152. (7 sheets)

\begin{tabular}{|c|c|c|c|c|c|c|c|c|}
\hline $\begin{array}{l}\text { Customer } \\
\text {. }\end{array}$ & Seg. & Sampled & $\begin{array}{l}\text { Date } \\
\text { Received }\end{array}$ & Extruded & $\begin{array}{l}\text { Thinges: } \\
\text { Extrualed? }\end{array}$ & $\begin{array}{l}\text { Uiquid } \\
\text { Recotered (g) }\end{array}$ & 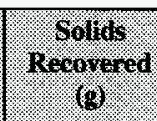 & Sample resciplon \\
\hline $96-256$ & 4 & 6/11/96 & $6 / 12 / 96$ & 6/18/96 & 0.0 & 429.3-Drainable & 0.0 & $\begin{array}{l}\text { Extruded an unmeasurable } \\
\text { amount of light gray solids } \\
\text { and resembled a wet salt. The } \\
\text { liquid was green in color and } \\
\text { opaque. Collected } 300 \mathrm{~mL} \text { of } \\
\text { liquid. No organic layer was } \\
\text { observed. }\end{array}$ \\
\hline $96-257$ & 5 & $6 / 11 / 96$ & $6 / 12 / 96$ & $6 / 18 / 96$ & 0.0 & 435.8-Drainable & 0.0 & $\begin{array}{l}\text { Extruded an unmeasurable } \\
\text { amount of light gray solids } \\
\text { and resembled a wet salt. The } \\
\text { liquid was green in color and } \\
\text { opaque. Collected } 310 \mathrm{~mL} \text { of } \\
\text { liquid. No organic layer was } \\
\text { observed. }\end{array}$ \\
\hline $96-258$ & 6 & $6 / 11 / 96$ & $6 / 12 / 96$ & $6 / 19 / 96$ & 0.0 & 429.5-Drainable & 0.0 & $\begin{array}{l}\text { Extruded an unmeasurable } \\
\text { amount of light gray solids } \\
\text { and resembled a wet salt. The } \\
\text { liquid was green in color and } \\
\text { opaque. Collected } 290 \mathrm{~mL} \text { of } \\
\text { liquid. No organic layer was } \\
\text { observed. }\end{array}$ \\
\hline
\end{tabular}


Table B1-2a. Sample Receipt and Extrusion Information for 241-AN-105, Core 152. (7 sheets)

\begin{tabular}{|c|c|c|c|c|c|c|c|c|}
\hline Cistoner & $8 \times 8$ & Sampled: & Received & Bxiruded & IErinuded? & $\begin{array}{l}\text { Iiquid } \\
\text { Recovered (g) }\end{array}$ & $\begin{array}{l}\text { Solitis } \\
\text { Recovered } \\
\mathrm{g} \text { ) }\end{array}$ & Sample Dicuription \\
\hline $96-259$ & 7 & 6/11/96 & $6 / 12 / 96$ & $6 / 27 / 96$ & 0.0 & 433.4-Drainable & 0.0 & $\begin{array}{l}\text { Extruded an unmeasurable } \\
\text { amount of light gray solids } \\
\text { and resembled a wet salt. The } \\
\text { liquid was green in color and } \\
\text { opaque. Collected } 290 \mathrm{~mL} \text { of } \\
\text { liquid. No organic layer was } \\
\text { observed. }\end{array}$ \\
\hline $96-260$ & 8 & $6 / 12 / 96$ & $6 / 12 / 96$ & $6 / 27 / 96$ & 0.0 & 429.3-Drainable & 0.0 & $\begin{array}{l}\text { Extruded an unmeasurable } \\
\text { amount of light gray solids } \\
\text { and resembled a wet salt. The } \\
\text { liquid was green in color and } \\
\text { opaque. Collected } 300 \mathrm{mLs} \text { of } \\
\text { liquid. }\end{array}$ \\
\hline $96-261$ & 9 & $6 / 12 / 96$ & $6 / 12 / 96$ & $6 / 27 / 96$ & 0.0 & 378.7-Drainable & 0.0 & $\begin{array}{l}\text { Extruded an unmeasurable } \\
\text { amount of light gray solids } \\
\text { and resembled a wet salt. The } \\
\text { liquid was green in color and } \\
\text { opaque. Collected } 250 \mathrm{~mL} \text { of } \\
\text { liquid. No organic layer was } \\
\text { observed. }\end{array}$ \\
\hline
\end{tabular}


Table B1-2a. Sample Receipt and Extrusion Information for 241-AN-105, Core 152. (7 sheets)

\begin{tabular}{|c|c|c|c|c|c|c|c|c|}
\hline gristomer & 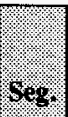 & Garer & gereived & Exirader & Gxiraded? & 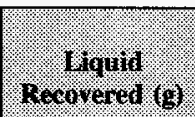 & $\begin{array}{l}\text { Solitis } \\
\text { Recovered! } \\
(\mathbf{8})\end{array}$ & (1. \\
\hline $96-262$ & 10 & $6 / 12 / 96$ & $6 / 13 / 96$ & $6 / 27 / 96$ & 0.0 & 392.2-Drainable & 0.0 & $\begin{array}{l}\text { Extruded an unmeasurable } \\
\text { amount of light gray solids } \\
\text { and resembled a wet salt. The } \\
\text { liquid was green in color and } \\
\text { opaque. Collected } 250 \mathrm{~mL} \text { of } \\
\text { liquid. No organic layer was } \\
\text { observed. }\end{array}$ \\
\hline $96-263$ & 11 & $6 / 12 / 96$ & $6 / 13 / 96$ & $6 / 27 / 96$ & 0.0 & 431.4-Drainable & 0.0 & $\begin{array}{l}\text { Extruded an unmeasurable } \\
\text { amount of light gray solids } \\
\text { and resembled a wet salt. The } \\
\text { liquid was green in color and } \\
\text { opaque. Collected } 300 \mathrm{~mL} \text { of } \\
\text { liquid. No organic layer was } \\
\text { observed. }\end{array}$ \\
\hline
\end{tabular}




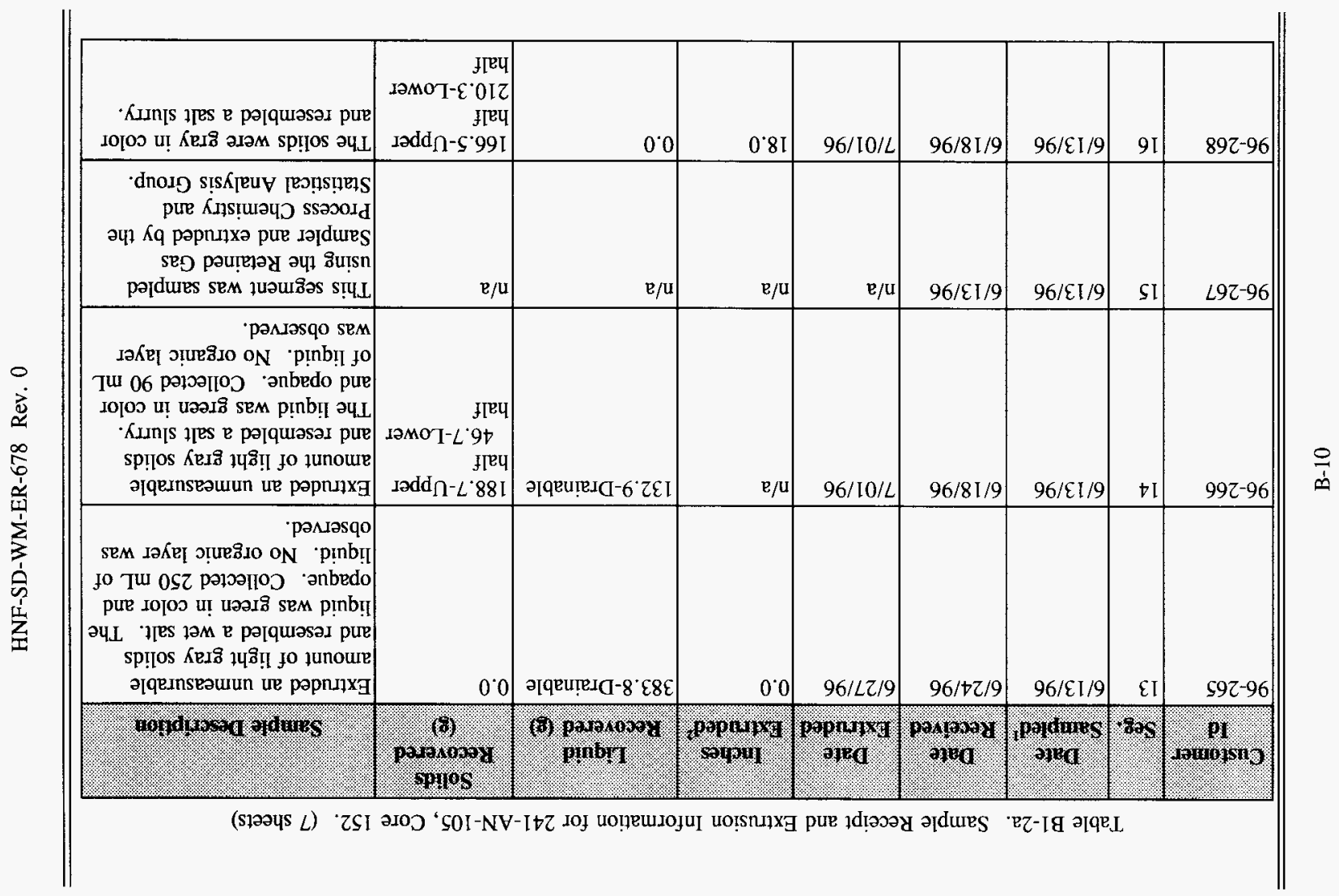


Table B1-2a. Sample Receipt and Extrusion Information for 241-AN-105, Core 152. (7 sheets)

\begin{tabular}{|c|c|c|c|c|c|c|c|c|}
\hline (ristomer. & (5eg. & Sorrested & 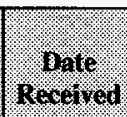 & 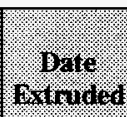 & Inchics: & 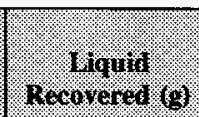 & 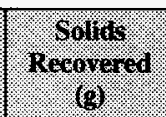 & (1. \\
\hline $96-269$ & 17 & $6 / 14 / 96$ & $6 / 18 / 96$ & $n / a$ & $\mathrm{n} / \mathrm{a}$ & $n / a$ & $\mathrm{n} / \mathrm{a}$ & $\begin{array}{l}\text { This segment was sampled } \\
\text { using the Retained Gas } \\
\text { Sampler and extruded by the } \\
\text { Process Chemistry and } \\
\text { Statistical Analysis Group. }\end{array}$ \\
\hline $96-270$ & 18 & $6 / 14 / 96$ & $6 / 18 / 96$ & $7 / 08 / 96$ & 19.0 & 0.0 & $\begin{array}{l}\text { 188.0-Upper } \\
\text { half } \\
\text { 195.5-Lower } \\
\text { half }\end{array}$ & $\begin{array}{l}\text { The solids were gray in color } \\
\text { and resembled a wet salt. }\end{array}$ \\
\hline $96-271$ & 19 & $6 / 14 / 96$ & $6 / 18 / 96$ & $n / a$ & $n / a$ & $\mathrm{n} / \mathrm{a}$ & $n / a$ & $\begin{array}{l}\text { This segment was sampled } \\
\text { using the Retained Gas } \\
\text { Sampler and extruded by the } \\
\text { Process Chemistry and } \\
\text { Statistical Analysis Group. }\end{array}$ \\
\hline $96-272$ & 20 & $6 / 14 / 96$ & $6 / 18 / 96$ & $7 / 08 / 96$ & 17.0 & 0.0 & $\begin{array}{l}\text { 198.9-Upper } \\
\text { half } \\
\text { 198.0-Lower } \\
\text { half }\end{array}$ & $\begin{array}{l}\text { The solids were light to dark } \\
\text { gray in color and resembled a } \\
\text { wet salt and a salt slurry. }\end{array}$ \\
\hline
\end{tabular}


Table B1-2a. Sample Receipt and Extrusion Information for 241-AN-105, Core 152. (7 sheets)

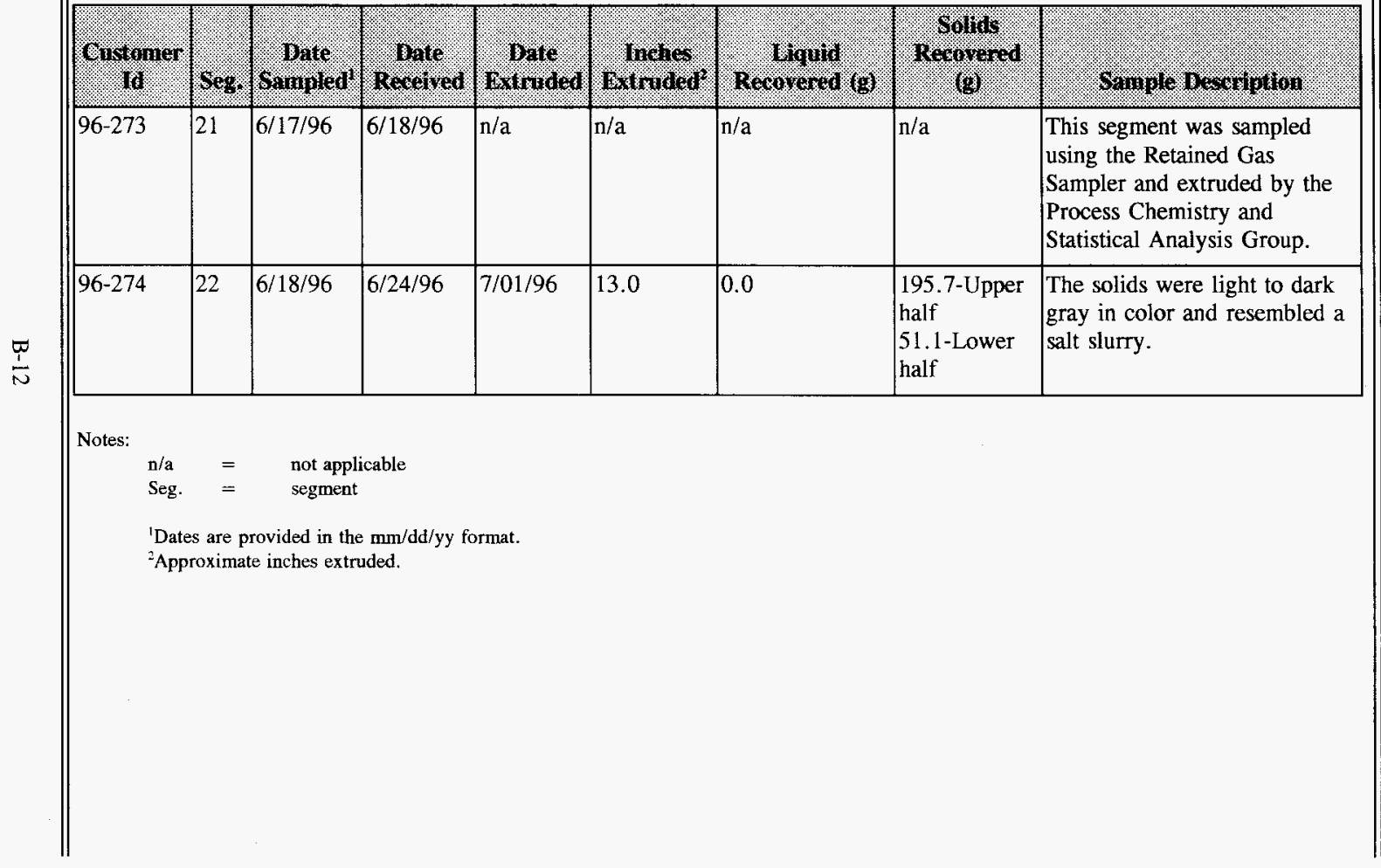


Table B1-2b. Sample Receipt and Extrusion Information for 241-AN-105, Core 153. (7 sheets)

\begin{tabular}{|c|c|c|c|c|c|c|c|c|}
\hline Costomer: & seg. & Sarioled & herorivo & Wriruded & Indingled & Revoricied $(0)$ & 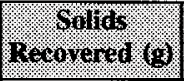 & 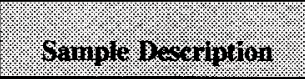 \\
\hline $96-275$ & 1 & $6 / 25 / 96$ & $8 / 09 / 96$ & $8 / 14 / 96$ & 0.0 & 256.8-Drainable & 0.0 & $\begin{array}{l}\text { Extruded an unmeasurable } \\
\text { amount of light gray } \\
\text { solids that resembled a } \\
\text { salt slurry. The liquid } \\
\text { was green in color and } \\
\text { opaque. Collected } 165 \\
\text { mL of liquid. No organic } \\
\text { layer was observed. }\end{array}$ \\
\hline $96-276$ & 2 & $6 / 25 / 96$ & $7 / 30 / 96$ & $8 / 05 / 96$ & 0.0 & 422.8-Drainable & 0.0 & $\begin{array}{l}\text { Extruded an unmeasurable } \\
\text { amount of light gray } \\
\text { solids that resembled a } \\
\text { salt slurry. The liquid } \\
\text { was green in color and } \\
\text { opaque. Collected } 320 \\
\text { mL of liquid. No organic } \\
\text { layer was observed. }\end{array}$ \\
\hline $96-277$ & 3 & $6 / 26 / 96$ & $8 / 09 / 96$ & $8 / 14 / 96$ & 0.0 & 367.0-Drainable & 0.0 & $\begin{array}{l}\text { Extruded an unmeasurable } \\
\text { amount of light gray } \\
\text { solids that resembled a } \\
\text { wet salt. The liquid was } \\
\text { green in color and } \\
\text { opaque. Collected } 250 \\
\text { mL of liquid. No organic } \\
\text { layer was observed. }\end{array}$ \\
\hline
\end{tabular}


Table B1-2b. Sample Receipt and Extrusion Information for 241-AN-105, Core 153. (7 sheets)

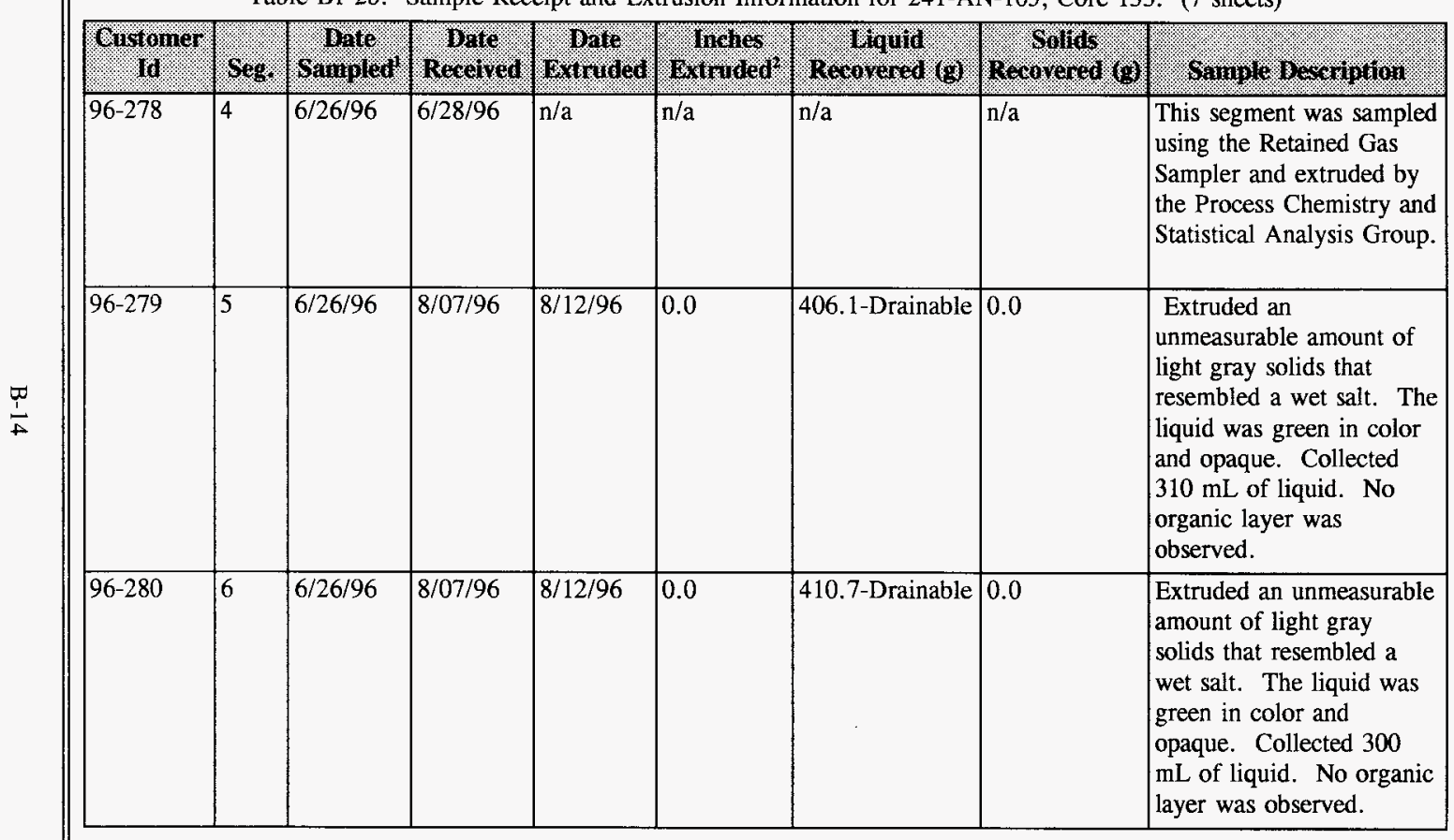


Table B1-2b. Sample Receipt and Extrusion Information for 241-AN-105, Core 153. (7 sheets)

\begin{tabular}{|c|c|c|c|c|c|c|c|c|}
\hline (4astomer & Seg. & Sampled & Mare & Gariog & Grinebes: & 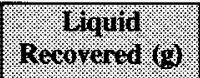 & Recovergor $(\mathrm{g})$ & Siriple Dereriplion. \\
\hline $96-281$ & 7 & $6 / 26 / 96$ & $8 / 06 / 96$ & $8 / 12 / 96$ & 0.0 & 402.6-Drainable & 0.0 & $\begin{array}{l}\text { Extruded an unmeasurable } \\
\text { amount of light gray } \\
\text { solids that resembled a } \\
\text { wet salt. The liquid was } \\
\text { green in color and } \\
\text { opaque. Collected } 295 \\
\text { mL of liquid. No organic } \\
\text { layer was observed. }\end{array}$ \\
\hline $96-282$ & 8 & $6 / 26 / 96$ & $7 / 03 / 96$ & $7 / 09 / 96$ & 0.0 & 426.7-Drainable & 0.0 & $\begin{array}{l}\text { Extruded an unmeasurable } \\
\text { amount of light gray } \\
\text { solids that resembled a } \\
\text { wet salt. The liquid was } \\
\text { green in color and } \\
\text { opaque. Collected } 290 \\
\text { mL of liquid. No organic } \\
\text { layer was observed. }\end{array}$ \\
\hline $96-283$ & 9 & $6 / 26 / 96$ & $7 / 02 / 96$ & $7 / 08 / 96$ & 0.0 & 428.9-Drainable & 0.0 & $\begin{array}{l}\text { Extruded an unmeasurable } \\
\text { amount of light gray } \\
\text { solids that resembled a } \\
\text { wet salt. The liquid was } \\
\text { green in color and } \\
\text { opaque. Collected } 300 \\
\text { mL of liquid. No organic } \\
\text { layer was observed. }\end{array}$ \\
\hline
\end{tabular}


Table B1-2b. Sample Receipt and Extrusion Information for 241-AN-105, Core 153. (7 sheets)

\begin{tabular}{|c|c|c|c|c|c|c|c|c|}
\hline Gistomer & Segs. & Gamioled & Worterero & Wriviodit & Intrided & Reromerad & Recoverer $(9)$ & 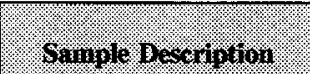 \\
\hline $96-284$ & 10 & $6 / 27 / 96$ & $7 / 30 / 96$ & $8 / 05 / 96$ & 0.0 & 386.3-Drainable & 0.0 & $\begin{array}{l}\text { Extruded an unmeasurable } \\
\text { amount of light gray } \\
\text { solids that resembled a } \\
\text { wet salt. The liquid was } \\
\text { green in color and } \\
\text { opaque. Collected } 290 \\
\text { mL of liquid. No organic } \\
\text { layer was observed. }\end{array}$ \\
\hline $96-285$ & 11 & $6 / 27 / 96$ & $7 / 02 / 96$ & $7 / 08 / 96$ & 0.0 & 420.0-Drainable & 0.0 & $\begin{array}{l}\text { Extruded an unmeasurable } \\
\text { amount of light gray } \\
\text { solids that resembled a } \\
\text { wet salt. The liquid was } \\
\text { green in color and } \\
\text { opaque. Collected } 290 \\
\text { mL of liquid. No organic } \\
\text { layer was observed. }\end{array}$ \\
\hline $96-286$ & 12 & $6 / 27 / 96$ & $7 / 02 / 96$ & $7 / 08 / 96$ & 0.0 & 407.2-Drainable & 0.0 & $\begin{array}{l}\text { Extruded an unmeasurable } \\
\text { amount of light gray } \\
\text { solids that resembled a } \\
\text { wet salt. The liquid was } \\
\text { green in color and } \\
\text { opaque. Collected } 290 \\
\text { mL of liquid. No organic } \\
\text { layer was observed. }\end{array}$ \\
\hline
\end{tabular}


Table B1-2b. Sample Receipt and Extrusion Information for 241-AN-105, Core 153. (7 sheets)

\begin{tabular}{|c|c|c|c|c|c|c|c|c|}
\hline Sistomer & 808 & Sampled & Weoryed & Sorided & Goradrates & Recorord $(8)$ & Frooverod & 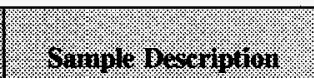 \\
\hline $96-287$ & 13 & $6 / 27 / 96$ & $7 / 03 / 96$ & $7 / 09 / 96$ & 0.0 & 431.4-Drainable & 0.0 & $\begin{array}{l}\text { Extruded an unmeasurable } \\
\text { amount of light gray } \\
\text { solids that resembled a } \\
\text { wet salt. The liquid was } \\
\text { green in color and } \\
\text { opaque. Collected } 300 \\
\text { mL of liquid. }\end{array}$ \\
\hline $96-288$ & 14 & $6 / 27 / 96$ & $6 / 28 / 96$ & $8 / 07 / 96$ & 13.0 & 130.1-Drainable & $\begin{array}{l}248.6 \text {-Lower } \\
\text { half }\end{array}$ & $\begin{array}{l}\text { The solids were medium } \\
\text { to dark gray in color and } \\
\text { resembled a salt slurry. } \\
\text { The liquid was green in } \\
\text { color and opaque. } \\
\text { Collected } 100 \mathrm{~mL} \text { of } \\
\text { liquid. No organic layer } \\
\text { was observed. }\end{array}$ \\
\hline $96-290$ & 16 & $6 / 27 / 96$ & $6 / 28 / 96$ & $\mathrm{n} / \mathrm{a}$ & $\mathrm{n} / \mathrm{a}$ & $\mathrm{n} / \mathrm{a}$ & $n / a$ & $\begin{array}{l}\text { This segment was sampled } \\
\text { using the Retained Gas } \\
\text { Sampler and extruded by } \\
\text { the Process Chemistry and } \\
\text { Statistical Analysis Group. }\end{array}$ \\
\hline
\end{tabular}


Table B1-2b. Sample Receipt and Extrusion Information for 241-AN-105, Core 153. (7 sheets)

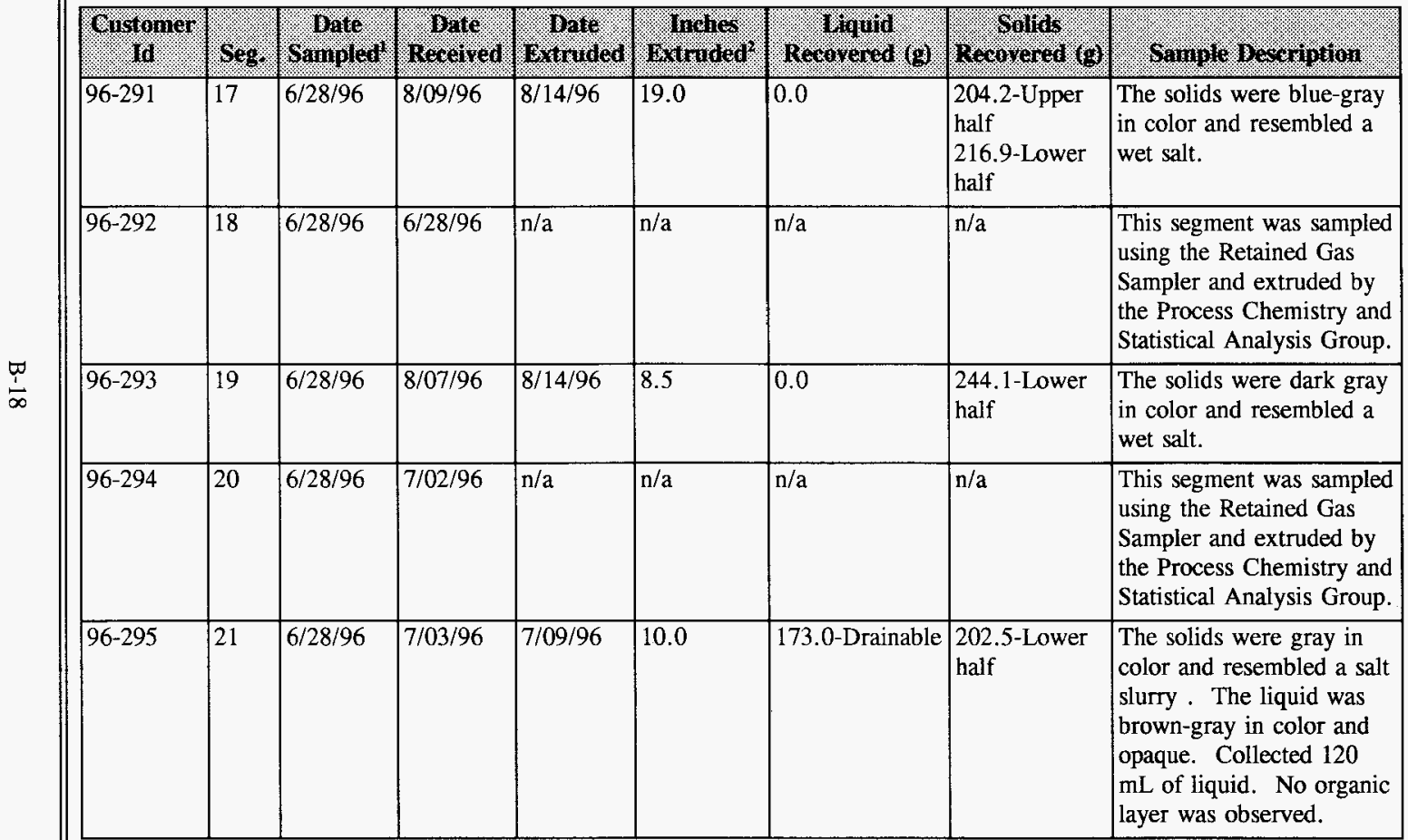


Table B1-2b. Sample Receipt and Extrusion Information for 241-AN-105, Core 153. (7 sheets)

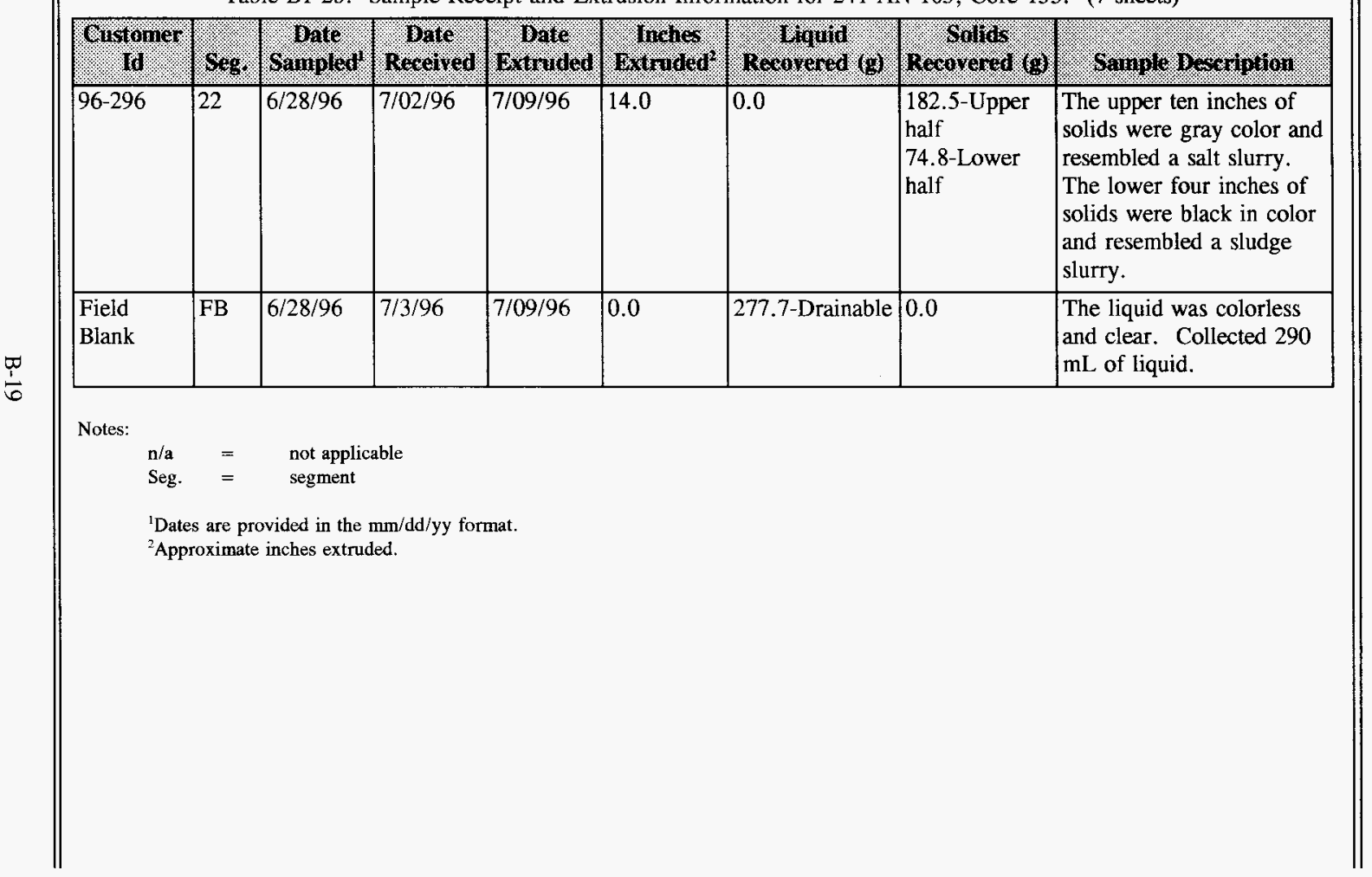




\section{B1.3 SAMPLE ANALYSIS}

The analyses performed on the core samples were limited to those required by the safety screening DQO and the flammable gas DQO. The analyses required by the safety screening DQO included analyses for thermal properties by DSC, moisture content by TGA, and content of fissile material by total alpha activity analysis. The flammable gas DQO required a full set of analytes to be analyzed by IC and ICP as well as TOC, TIC and various radionuclides.

Differential scanning calorimetry and TGA were performed on $8.665-\mathrm{mg}$ to $45.550-\mathrm{mg}$ samples. Quality control tests included performing the analyses in duplicate, and the use of standards. Moisture content was also measured by a gravimetric method.

Total alpha activity measurements were performed on samples that had been fused in a matrix of potassium hydroxide and then dissolved in acid. The resulting solution was then dried on a counting planchet and counted in an alpha proportional counter. Quality control tests included standards, spikes, blanks, and duplicate analyses.

Ion chromatography was performed on samples that had been prepared by water digestion. Quality control tests included standards, spikes, blanks, and duplicate analyses. The SAP required that the full suite of IC analytes be measured.

Inductively coupled plasma spectrometry was performed on samples that had been prepared by a fusion procedure, followed by dissolution in acid. Quality control tests included standards, blanks, spikes, and duplicate analyses. The SAP required that the full suite of ICP elements be analyzed.

All reported analyses were performed in accordance with approved laboratory procedures. A list of the sample numbers and applicable analyses is presented in Table B1-3. The sample preparation procedure numbers and analysis procedure numbers are presented in Table B1-4.

\section{B1.4 DESCRIPTION OF HISTORICAL SAMPLING EVENT}

Sampling data for tank 241-AN-105 have been obtained to support evaporator Campaign Runs 84-5 and 85-3 in 1984 and reported on May 10, 1984 (Jansky 1984) and November 9, 1984 (Mauss 1984), respectively. The data are presented in Section B2.8. Pre-1989 analytical data have not been validated and should be used with caution.

No information was available regarding sample handling for this tank. The reason for the sampling was given as "242-A Evaporator Campaign." 
Table B1-3. Tank 241-AN-105 Sample Analysis Summary. (7 Sheets)

\begin{tabular}{|c|c|c|c|c|}
\hline Mest & Iforitinglion & Sanule portinn & Whingle & Muntrses \\
\hline \multirow[t]{18}{*}{$12 \mathrm{~A}$} & \multirow[t]{18}{*}{ Core 152} & \multirow[t]{15}{*}{ Core composite } & S96T004039 & Alpha rad, TGA, DSC, IC \\
\hline & & & S96T004049 & $\begin{array}{l}\text { Furnace oxidation (TOC), } \\
\text { Pu239/240, alpha rad, TIC/TOC, } \\
\mathrm{Np} 237, \mathrm{Am} 241, \mathrm{I} 129, \mathrm{GEA}, \mathrm{Tc} \text {, } \\
\mathrm{Sr}\end{array}$ \\
\hline & & & S96T004144 & ICP \\
\hline & & & S96T004207 & Bulk density \\
\hline & & & S96T004208 & Bulk density \\
\hline & & & S96T004254 & TIC/TOC, TGA, DSC \\
\hline & & & S96T004256 & $\begin{array}{l}\text { Pu239/240, Np237, alpha, GEA, } \\
\text { AEA, Tc, Sr, U }\end{array}$ \\
\hline & & & S96T004257 & ICP \\
\hline & & & S96T004258 & Liq. Scin., I129, Cr+6, OH, IC \\
\hline & & & \$96T004259 & TIC/TOC, TGA, DSC \\
\hline & & & S96T004261 & $\begin{array}{l}\text { Pu239/240, Np237, alpha, GEA, } \\
\text { AEA, Tc, Sr, U }\end{array}$ \\
\hline & & & S96T004262 & ICP \\
\hline & & & S96T004263 & Liq. Scin., I129, Cr+6, OH, IC \\
\hline & & & S96T004831 & $\begin{array}{l}\text { Scintillation, } \mathrm{OH} \text { Direct, } \mathrm{Cr}+6 \text {, } \\
\mathrm{ICP}, \mathrm{IC}\end{array}$ \\
\hline & & & S96T005546 & Uranium gross (kinetic phos.), \\
\hline & & \multirow[t]{3}{*}{ Whole } & S96T003732 & $\begin{array}{l}\text { Alpha rad, TGA, SpG, ICP, } \\
\text { DSC, IC }\end{array}$ \\
\hline & & & \$96T003739 & $\begin{array}{l}\text { Alpha rad, TGA, SpG, ICP, } \\
\text { DSC, IC }\end{array}$ \\
\hline & & & S96T003740 & $\begin{array}{l}\text { Alpha rad, TGA, SpG, ICP, } \\
\text { DSC, IC }\end{array}$ \\
\hline
\end{tabular}


Table B1-3. Tank 241-AN-105 Sample Analysis Summary. (7 Sheets)

\begin{tabular}{|c|c|c|c|c|}
\hline Rrer & 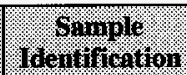 & shinjole pointon & Minion & thalyses \\
\hline \multirow[t]{19}{*}{$12 \mathrm{~A}$} & \multirow[t]{19}{*}{ Core 152} & \multirow[t]{11}{*}{ Whole } & S96T003741 & $\begin{array}{l}\text { Alpha rad, TGA, SpG, ICP, } \\
\text { DSC, IC }\end{array}$ \\
\hline & & & S96T003742 & $\begin{array}{l}\text { Alpha rad, TGA, SpG, ICP, } \\
\text { DSC, IC }\end{array}$ \\
\hline & & & S96T003743 & $\begin{array}{l}\text { Alpha rad, TGA, SpG, ICP, } \\
\text { DSC, IC }\end{array}$ \\
\hline & & & S96T003744 & $\begin{array}{l}\text { Alpha rad, TGA, SpG, ICP, } \\
\text { DSC, IC }\end{array}$ \\
\hline & & & S96T003745 & $\begin{array}{l}\text { Alpha rad, TGA, SpG, ICP, } \\
\text { DSC, IC }\end{array}$ \\
\hline & & & S96T003746 & $\begin{array}{l}\text { Alpha rad, TGA, SpG, ICP, } \\
\text { DSC, IC }\end{array}$ \\
\hline & & & S96T003747 & $\begin{array}{l}\text { Alpha rad, TGA, SpG, ICP, } \\
\text { DSC, IC }\end{array}$ \\
\hline & & & S96T003748 & $\begin{array}{l}\text { Alpha rad, TGA, SpG, ICP, } \\
\text { DSC, IC }\end{array}$ \\
\hline & & & S96T003749 & $\begin{array}{l}\text { Alpha rad, DSC/TGA, TGA, } \\
\text { SpG, ICP, IC }\end{array}$ \\
\hline & & & S96T003750 & $\begin{array}{l}\text { Alpha rad, TGA, SpG, ICP, } \\
\text { DSC, IC }\end{array}$ \\
\hline & & & S96T003751 & $\begin{array}{l}\text { Alpha rad, TGA, SpG, ICP, } \\
\text { DSC, IC }\end{array}$ \\
\hline & & \multirow[t]{8}{*}{ Upper sample } & S96T003704 & Bulk density \\
\hline & & & S96T003706 & Bulk density \\
\hline & & & S96T003707 & Bulk density \\
\hline & & & S96T003710 & Bulk density \\
\hline & & & S96T003712 & Bulk density \\
\hline & & & S96T003714 & Bulk density \\
\hline & & & S96T003731 & TGA, DSC \\
\hline & & & S96T003792 & TGA, DSC \\
\hline
\end{tabular}


Table B1-3. Tank 241-AN-105 Sample Analysis Summary. (7 Sheets)

\begin{tabular}{|c|c|c|c|c|}
\hline titser. & 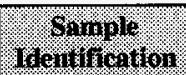 & 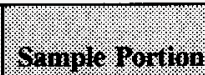 & Whinge & 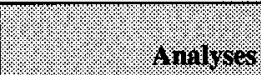 \\
\hline \multirow[t]{26}{*}{$12 \mathrm{~A}$} & \multirow[t]{26}{*}{ Core 152} & \multirow[t]{22}{*}{ Upper sample } & S96T003794 & TGA, DSC \\
\hline & & & S96T003796 & TGA, DSC \\
\hline & & & S96T003798 & TGA, DSC \\
\hline & & & S96T003816 & TGA, DSC \\
\hline & & & S96T003892 & Alpha, ICP \\
\hline & & & S96T003893 & Alpha, ICP \\
\hline & & & S96T003894 & ICP \\
\hline & & & \$96T003895 & ICP \\
\hline & & & \$96T003896 & ICP \\
\hline & & & S96T003897 & ICP \\
\hline & & & S96T003898 & ICP \\
\hline & & & S96T003899 & ICP \\
\hline & & & S96T003901 & ICP \\
\hline & & & S96T003903 & ICP \\
\hline & & & S96T003905 & ICP \\
\hline & & & S96T003906 & ICP \\
\hline & & & S96T003907 & IC \\
\hline & & & S96T003908 & IC \\
\hline & & & S96T003910 & IC \\
\hline & & & S96T003912 & IC \\
\hline & & & S96T003914 & IC \\
\hline & & & S96T003915 & IC \\
\hline & & \multirow[t]{4}{*}{ Lower sample } & S96T003705 & Bulk density \\
\hline & & & S96T003708 & Bulk density \\
\hline & & & S96T003711 & Bulk density \\
\hline & & & S96T003793 & TGA, DSC \\
\hline
\end{tabular}


Table B1-3. Tank 241-AN-105 Sample Analysis Summary. (7 Sheets)

\begin{tabular}{|c|c|c|c|c|}
\hline riser & 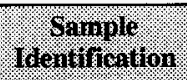 & Shmple soyinn & 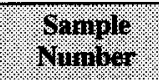 & Miniges? \\
\hline \multirow[t]{15}{*}{$12 \mathrm{~A}$} & \multirow[t]{15}{*}{ Core 152} & \multirow[t]{15}{*}{ Lower sample } & S96T003795 & TGA, DSC \\
\hline & & & S96T003797 & TGA, DSC \\
\hline & & & S96T003817 & TGA, DSC \\
\hline & & & S96T003827 & Alpha, ICP \\
\hline & & & S96T003828 & ICP \\
\hline & & & S96T003829 & IC \\
\hline & & & S96T003889 & Alpha, ICP \\
\hline & & & S96T003890 & Alpha, ICP \\
\hline & & & S96T003891 & Alpha, ICP \\
\hline & & & S96T003900 & ICP \\
\hline & & & S96T003902 & ICP \\
\hline & & & S96T003904 & ICP \\
\hline & & & \$96T003909 & IC \\
\hline & & & S96T003911 & IC \\
\hline & & & S96T003913 & IC \\
\hline \multirow[t]{7}{*}{$7 \mathrm{~B}$} & \multirow[t]{7}{*}{ Core 153} & \multirow[t]{7}{*}{ Whole } & S96T003631 & ICP, IC \\
\hline & & & S96T003974 & $\begin{array}{l}\text { OH direct, alpha rad, TGA, SpG, } \\
\text { ICP, DSC, IC }\end{array}$ \\
\hline & & & S96T003975 & $\begin{array}{l}\text { Alpha rad, TGA, SpG, ICP, } \\
\text { DSC, IC }\end{array}$ \\
\hline & & & S96T003976 & $\begin{array}{l}\text { Alpha rad, TGA, SpG, ICP, } \\
\text { DSC, IC }\end{array}$ \\
\hline & & & S96T003977 & $\begin{array}{l}\text { Alpha rad, TGA, SpG, ICP, } \\
\text { DSC, IC }\end{array}$ \\
\hline & & & S96T003978 & $\begin{array}{l}\text { Alpha rad, DSC/TGA, SpG, ICP, } \\
\text { IC }\end{array}$ \\
\hline & & & S96T003979 & $\begin{array}{l}\text { Alpha rad, DSC/TGA, SpG, ICP, } \\
\text { IC }\end{array}$ \\
\hline
\end{tabular}


Table B1-3. Tank 241-AN-105 Sample Analysis Summary. (7 Sheets)

\begin{tabular}{|c|c|c|c|c|}
\hline Reser & irenimitcaton & Sampilo $301010 \mathrm{a}$ & Mambior & 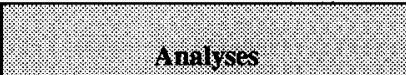 \\
\hline \multirow[t]{18}{*}{$7 \mathrm{~B}$} & \multirow[t]{18}{*}{ Core 153} & \multirow[t]{10}{*}{ Whole } & S96T003980 & $\begin{array}{l}\text { Alpha rad, DSC/TGA, SpG, ICP, } \\
\text { IC }\end{array}$ \\
\hline & & & S96T004003 & $\begin{array}{l}\text { Uranium gross (kinetic phos.), } \\
\mathrm{H}_{3}, \mathrm{PU} 239 / 240, \mathrm{~Np} 237, \mathrm{Am} 241, \\
\mathrm{I} 129, \mathrm{Cr}+6, \mathrm{GEA}, \mathrm{Tc}, \mathrm{Sr}\end{array}$ \\
\hline & & & S96T004308 & $\begin{array}{l}\text { Alpha rad, TGA, SpG, ICP, } \\
\text { DSC, IC }\end{array}$ \\
\hline & & & S96T004309 & $\begin{array}{l}\text { Alpha rad, DSC/TGA, SpG, ICP, } \\
\text { IC }\end{array}$ \\
\hline & & & S96T004333 & $\begin{array}{l}\text { Alpha rad, DSC/TGA, SpG, ICP, } \\
\text { IC }\end{array}$ \\
\hline & & & S96T004419 & $\begin{array}{l}\text { Alpha rad, TGA, SpG, ICP, } \\
\text { DSC, IC }\end{array}$ \\
\hline & & & S96T004420 & $\begin{array}{l}\text { Alpha rad, TGA, SpG, ICP, } \\
\text { DSC, IC }\end{array}$ \\
\hline & & & \begin{tabular}{|l|} 
S96T004421 \\
\end{tabular} & $\begin{array}{l}\text { Alpha rad, DSC/TGA, SpG, ICP, } \\
\text { IC }\end{array}$ \\
\hline & & & S96T004433 & $\begin{array}{l}\text { Alpha rad, DSC/TGA, SpG, ICP, } \\
\text { IC }\end{array}$ \\
\hline & & & S96T004434 & $\begin{array}{l}\text { Alpha rad, TGA, SpG, ICP, } \\
\text { DSC, IC }\end{array}$ \\
\hline & & \multirow[t]{8}{*}{ Upper sample } & S96T003973 & Bulk density \\
\hline & & & S96T003983 & TGA, DSC \\
\hline & & & S96T003986 & ICP \\
\hline & & & S96T003989 & ICP \\
\hline & & & S96T003992 & IC \\
\hline & & & S96T004302 & Bulk density \\
\hline & & & S96T004310 & TGA, DSC \\
\hline & & & S96T004314 & ICP \\
\hline
\end{tabular}


Table B1-3. Tank 241-AN-105 Sample Analysis Summary. (7 Sheets)

\begin{tabular}{|c|c|c|c|c|}
\hline $\mathrm{riser}$ & Therinimation & 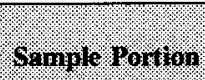 & Whaple & 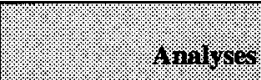 \\
\hline \multirow[t]{26}{*}{$7 \mathrm{~B}$} & \multirow[t]{26}{*}{ Core 153} & \multirow[t]{7}{*}{ Upper sample } & S96T004315 & ICP \\
\hline & & & S96T004316 & $\mathrm{IC}$ \\
\hline & & & \$96T004428 & Bulk density \\
\hline & & & S96T004435 & TGA, DSC \\
\hline & & & S96T004441 & $\mathrm{ICP}$ \\
\hline & & & S96T004444 & ICP \\
\hline & & & S96T004447 & IC \\
\hline & & \multirow[t]{19}{*}{ Lower sample } & S96T003971 & Bulk density \\
\hline & & & S96T003972 & Bulk density \\
\hline & & & \$96T003981 & TGA, DSC \\
\hline & & & S96T003982 & TGA, DSC \\
\hline & & & S96T003984 & Alpha, ICP \\
\hline & & & S96T003985 & Alpha, ICP \\
\hline & & & S96T003987 & ICP \\
\hline & & & S96T003988 & ICP \\
\hline & & & S96T003990 & IC \\
\hline & & & S96T003991 & IC \\
\hline & & & S96T004303 & Bulk density \\
\hline & & & S96T004311 & TGA, DSC \\
\hline & & & S96T004317 & Alpha, ICP \\
\hline & & & S96T004318 & ICP \\
\hline & & & S96T004319 & IC \\
\hline & & & S96T004334 & Bulk density \\
\hline & & & S96T004335 & TGA, DSC \\
\hline & & & S96T004337 & Alpha, ICP \\
\hline & & & S96T004338 & ICP \\
\hline
\end{tabular}


Table B1-3. Tank 241-AN-105 Sample Analysis Summary. (7 Sheets)

\begin{tabular}{|c|c|c|c|c|}
\hline Reser & 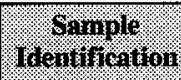 & Gample roxitin & \%ofmole & 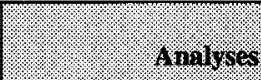 \\
\hline \multirow[t]{13}{*}{$7 B$} & \multirow[t]{13}{*}{ Core 153} & \multirow[t]{13}{*}{ Lower sample } & S96T004339 & IC \\
\hline & & & S96T004427 & Bulk density \\
\hline & & & S96T004429 & Bulk density \\
\hline & & & S96T004436 & TGA, DSC \\
\hline & & & S96T004437 & TGA, DSC \\
\hline & & & S96T004442 & Alpha, ICP \\
\hline & & & S96T004443 & Alpha, ICP \\
\hline & & & S96T004445 & ICP \\
\hline & & & S96T004446 & ICP \\
\hline & & & S96T004448 & IC \\
\hline & & & S96T004449 & IC \\
\hline & & & S96T005325 & IC \\
\hline & & & \$96T005326 & IC \\
\hline
\end{tabular}


Table B1-4. Analytical Procedures. (2 sheets)

\begin{tabular}{|c|c|c|c|}
\hline Wings & 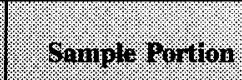 & Wroprom & 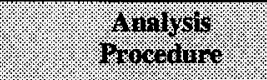 \\
\hline DSC & Solid/liquid & $n / a$ & $\begin{array}{l}\text { LA-514-114 Rev. C-1 } \\
\text { LA-514-113 Rev. C-1 }\end{array}$ \\
\hline TGA & Solid/liquid & $\mathrm{n} / \mathrm{a}$ & $\begin{array}{l}\text { LA-514-114 Rev. C-1 } \\
\text { LA-560-112 Rev. B-1 }\end{array}$ \\
\hline Bulk Density & Solid & $n / a$ & LO-160-103 Rev. B-0 \\
\hline Sp.G. & Liquid & $n / a$ & LA-510-112 Rev. C-3 \\
\hline $\mathrm{U}$ & $\begin{array}{l}\text { Solid } \\
\text { Liquid }\end{array}$ & $\begin{array}{l}\text { LA-549-141 Rev. F-0 } \\
\text { n/a }\end{array}$ & LA-925-009 Rev. A-1 \\
\hline IC & $\begin{array}{l}\text { Solid } \\
\text { Liquid }\end{array}$ & $\begin{array}{l}\text { LA-504-101 Rev. E-0 } \\
\text { n/a }\end{array}$ & LA-533-105 Rev. D-1 \\
\hline ICP & $\begin{array}{l}\text { Solid } \\
\text { Liquid }\end{array}$ & $\begin{array}{l}\text { LA-505-159 Rev. D-0 } \\
\text { LA-549-141 Rev. F-0 } \\
\text { n/a }\end{array}$ & $\begin{array}{l}\text { LA-505-151 Rev. D-3 } \\
\text { LA-505-161 Rev. B-1 }\end{array}$ \\
\hline $\mathrm{OH}-$ & $\begin{array}{l}\text { Solid } \\
\text { Liquid }\end{array}$ & $\begin{array}{l}\text { LA-504-101 Rev, E-0 } \\
\mathrm{n} / \mathrm{a}\end{array}$ & LA-211-102 Rev. C-0 \\
\hline TIC/TOC & Solid/liquid & $\mathrm{n} / \mathrm{a}$ & LA-342-100 Rev. D-0 \\
\hline $\mathrm{Cr}(\mathrm{VI})$ & $\begin{array}{l}\text { Solid } \\
\text { Liquid }\end{array}$ & $\begin{array}{l}\text { LA-504-101 Rev. E-0 } \\
\mathrm{n} / \mathrm{a}\end{array}$ & LA-265-101 Rev. B-0 \\
\hline TOC & Liquid & $\mathrm{n} / \mathrm{a}$ & LA-344-105 Rev. D-1 \\
\hline${ }^{3} \mathrm{H}$ & $\begin{array}{l}\text { Solid } \\
\text { Liquid }\end{array}$ & $\begin{array}{l}\text { LA-504-101 Rev. E-0 } \\
\mathrm{n} / \mathrm{a}\end{array}$ & LA-218-114 Rev. B-0 \\
\hline${ }^{129} \mathrm{I}$ & $\begin{array}{l}\text { Solid } \\
\text { Liquid }\end{array}$ & $\begin{array}{l}\text { LA-504-101 Rev. E-0 } \\
\text { n/a }\end{array}$ & LA-378-103 Rev. C-0 \\
\hline${ }^{99} \mathrm{Tc}$ & $\begin{array}{l}\text { Solid } \\
\text { Liquid }\end{array}$ & $\begin{array}{l}\text { LA-549-141 Rev. F-0 } \\
\mathrm{n} / \mathrm{a}\end{array}$ & LA-438-101 Rev. D-2 \\
\hline${ }^{237} \mathrm{~Np}$ & $\begin{array}{l}\text { Solid } \\
\text { Liquid }\end{array}$ & $\begin{array}{l}\text { LA-549-141 Rev. F-0 } \\
\mathrm{n} / \mathrm{a}\end{array}$ & LA-933-141 Rev. H-1 \\
\hline $\mathrm{AT} / \mathrm{TB}$ & $\begin{array}{l}\text { Solid } \\
\text { Liquid }\end{array}$ & $\begin{array}{l}\text { LA-549-101 Rev. F-0 } \\
\text { n/a }\end{array}$ & LA-508-101 Rev. D-2 \\
\hline GEA & $\begin{array}{l}\text { Solid } \\
\text { Liquid }\end{array}$ & $\begin{array}{l}\text { LA-549-101 Rev. F-0 } \\
\text { n/a }\end{array}$ & LA-548-121 Rev. E-0 \\
\hline
\end{tabular}


Table B1-4. Analytical Procedures. (2 sheets)

\begin{tabular}{|c|c|c|c|}
\hline Analyisis: & Sample Fortion & 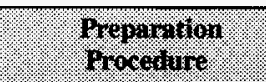 & 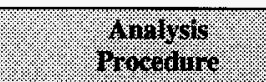 \\
\hline${ }^{241} \mathrm{Am} /{ }^{244} \mathrm{Cm}$ & $\begin{array}{l}\text { Solid } \\
\text { Liquid }\end{array}$ & $\begin{array}{l}\text { LA-549-101 Rev. F-0 } \\
\text { n/a }\end{array}$ & LA-953-103 Rev. B-0 \\
\hline${ }^{90} \mathrm{Sr}$ & $\begin{array}{l}\text { Solid } \\
\text { Liquid }\end{array}$ & $\begin{array}{l}\text { LA-549-141 Rev. F-0 } \\
\text { n/a }\end{array}$ & LA-220-101 Rev. D-1 \\
\hline${ }^{239} \mathrm{Pu}$ & $\begin{array}{l}\text { Solid } \\
\text { Liquid }\end{array}$ & $\begin{array}{l}\text { LA-549-101 Rev. F-0 } \\
\mathrm{n} / \mathrm{a}\end{array}$ & LA-943-128 Rev. B-0 \\
\hline
\end{tabular}

Note:

$\mathbf{n} / \mathbf{a}=$ not applicable (these are direct samples).

\section{B2.0 ANALYTICAL RESULTS}

\section{B2.1 OVERVIEW}

This section summarizes the sampling and analytical results associated with the June 1996 sampling and analysis of tank 241-AN-105. The total alpha activity, percent water, energetics, IC, and ICP analytical results associated with this tank are presented in Table B2-1. These results are documented in Steen (1997).

Table B2-1. Analytical Presentation Tables.

\begin{tabular}{|c|c|}
\hline Anavis & Table nunber \\
\hline Radionuclides & B2-4 through B2-22 \\
\hline Thermodynamic analyses & $\mathrm{B} 2-23$ and $\mathrm{B} 2-24$ \\
\hline Density and specific gravity & $\mathrm{B} 2-25$ and $\mathrm{B} 2-26$ \\
\hline Inorganic analysis & B2-27 through B2-63 \\
\hline TIC/TOC & B2-64 through B2-66 \\
\hline Vapor sampling results & B2-67 \\
\hline
\end{tabular}




\section{B2.2 RADIONUCLIDE ANALYSIS}

\section{B2.2.1 Total Alpha Activity}

Total alpha activity analyses were performed on direct liquid core composite subsamples and the field blank. The solid core composite subsamples were prepared for analysis by performing a fusion digest in duplicate. The highest result returned was $0.133 \mu \mathrm{Ci} / \mathrm{g}$. The sample results for total alpha are given in Tables B2-4 and B2-5. High relative percent differences (RPDs) ( $>20$ percent) were reported for the solid core composite subsamples and can be attributed to sample inhomogeneity. No reruns were requested because of the low alpha activity in the samples. The standard recoveries and spike recoveries for this analysis were within the required limits (Steen 1997).

\section{B2.2.2 Total Beta}

Total beta (TB) activity analyses were performed on direct liquid core composite subsamples and the field blank. The solid core composite subsamples were prepared for analysis by performing a fusion digest in duplicate. Low levels of beta activity were noted in some preparation blanks, however, the levels of contamination are inconsequential when compared to the result of the sample and do not impact sample data quality. The standard recoveries, spike recoveries and RPDs for this analysis were within the required limits. The sample results for total beta are given in Table B2-6.

\section{B2.2.3 Strontium 90}

Strontium 90 analyses were performed on direct liquid core composite subsamples and the field blank. The solid core composite subsamples were prepared for analysis by performing a fusion digest in duplicate. The standard recoveries and RPDs for this analysis were within the required limits. Strontium 90 analytical results are given in Table B2-21.

\section{B2.2.4 Gamma Energy Analysis}

Gamma energy analysis (GEA), procedure number LA-548-121, was used to measure the activity of ${ }^{134} \mathrm{Cs},{ }^{137} \mathrm{Cs},{ }^{94} \mathrm{Nb},{ }^{106} \mathrm{Rh} / \mathrm{Ru},{ }^{125} \mathrm{Sb},{ }^{144} \mathrm{Ce}$, and ${ }^{60} \mathrm{Co}$. GEA analyses were performed on direct liquid core composite subsamples and the field blank. The solid core composite subsamples were prepared for analysis by performing a fusion digest in duplicate. Gamma energy analytical results are listed in Tables B2-12 through B2-16.

Actual detection limits for GEA analytes are not currently available. The latest GEA software does not currently report a minimum detectable activity (MDA). If an analyte is reported as "less than," the value reported is the detection limit. 
Low levels of cesium activity were noted in some preparation blanks, however, the levels of contamination are inconsequential when compared to the result of the sample and do not impact sample data quality. The standard recoveries and RPDs for this analysis were within the required limits.

\section{B2.2.5 Total Uranium}

Uranium (U) analyses were performed on direct liquid core composite subsamples and the field blank. The solid core composite subsamples were prepared for analysis by performing a fusion digest in duplicate. The standard recoveries, spike recoveries and RPDs for this analysis were within the required limits. Total Uranium analytical results are listed in Table B2-7.

\section{B2.2.6 Iodine 129}

${ }^{129}$ I analyses were performed on direct liquid core composite subsamples and the field blank. The solid core composite subsamples were prepared for analysis by performing a water digest. The standard recoveries and RPDs for this analysis were within the required limits. Iodine analytical results are listed in Table B2-17.

\section{B2.2.7 Technecium 99}

${ }^{99} \mathrm{Tc}$ analyses were performed on direct liquid core composite subsamples and the field blank. The solid core composite subsamples were prepared for analysis by performing a fusion digest in duplicate. The standard recoveries and RPDs for this analysis were within the required limits. Technecium analytical results are listed in Table B2-22.

\section{B2.2.8 Alpha Energy Analyses}

B2.2.8.1 Americium $\left({ }^{241} \mathbf{A m}\right) .{ }^{241} \mathrm{Am}$ analyses were performed on direct liquid core composite subsamples and the field blank. The solid core composite subsamples were prepared for analysis by performing a fusion digest in duplicate. High RPDs were reported for the solid core composite samples. This can be attributed to the heterogenous nature of the samples. No rerun was requested due to the low americium activity in the samples. The standard recoveries for this analysis were within the required limits. Americium analytical results are listed in Tables B2-8, B2-10, and B2-12.

B2.2.8.2 Curium $\left({ }^{243 / 244} \mathrm{Cm}\right)$. ${ }^{243 / 244} \mathrm{Cm}$ analyses were performed on direct liquid core composite subsamples and the field blank. The solid core composite subsamples were 
prepared for analysis by performing a fusion digest in duplicate. The RPDs for this analysis were within the required limits. Curium analytical results are listed in Tables B2-9 and $\mathrm{B} 2-11$.

B2.2.8.3 Plutonium ( $\left.{ }^{239 / 240} \mathrm{Pu}\right)$. ${ }^{239 / 240} \mathrm{Pu}$ analyses were performed on direct liquid core composite subsamples and the field blank. The solid core composite subsamples were prepared for analysis by performing a fusion digest in duplicate. The standard recoveries and RPDs for this analysis were within the required limits. Plutonium analytical results are listed in Table B2-20.

\section{B2.3 THERMODYNAMIC ANALYSES}

As required by the safety screening and flammable gas DQOs, TGA and DSC were performed on the solids and liquids.

\section{B2.3.1 Thermogravimetric Analysis}

Thermogravimetric analysis measures the mass of a sample while its temperature is increased at a constant rate. Nitrogen is passed over the sample during heating to remove any released gases. Any decrease in the weight of a sample during TGA represents a loss of gaseous matter from the sample, either through evaporation or through a reaction that forms gas phase products. The moisture content is estimated by assuming that all TGA sample weight loss up to a certain temperature (typically 150 to $200^{\circ} \mathrm{C}$ ) is due to water evaporation. The temperature limit for moisture loss is chosen by the operator at an inflection point on the TGA plot. Other volatile matter fractions can often be differentiated by inflection points as well.

Tank 241-AN-105 samples were analyzed by TGA using either procedure LA-514-114, Rev. C- 0 on a Perkin-Elmer ${ }^{1}$ TGA 7 instrument, or procedure LA-560-112, Rev. B-0 on a Mettler $^{2}$ TG 50 instrument. The TGA results for tank $241-\mathrm{AN}-105$ are presented in Table B2-25. All samples exhibited a large weight loss between the ambient temperature and $200^{\circ} \mathrm{C}\left(392^{\circ} \mathrm{F}\right)$. The weight percent water values for the tank supernatant and salt slurry were between 36.14 and 54.2. The standard recoveries and RPDs for this analysis were within the required limits. TGA results are presented in Table B2-24.

\footnotetext{
${ }^{1}$ Perkin-Elmer is a trademark of Perkins Research \& Manufacturing Company, Incorporated, Canoga Park, California.

${ }^{2}$ Mettler is a trademark of Mettler Instrument Corporation, Anaheim, California.
} 


\section{B2.3.2 Differential Scanning Calorimetry}

In a DSC analysis, heat absorbed or emitted by a substance is measured while the temperature of the sample is heated at a constant rate. Nitrogen is passed over the sample material to remove any gases being released. The onset temperature for an endothermic or exothermic event is determined graphically. The DSC analyses for tank 241-AN-105 were performed using either procedure LA-514-113, Rev. C-0 on a Mettler ${ }^{\text {TM }}$ DSC 20 instrument or procedure LA-514-114, Rev. C-0 on a Perkin-Elmer ${ }^{\mathrm{rm}}$ DSC 7 instrument. No sample exceeded the safety screening DQO decision criteria threshold of $480 \mathrm{~J} / \mathrm{g}$. The highest individual sample and duplicate exothermic results for liquid were 36.4 and $414 \mathrm{~J} / \mathrm{g}$, respectively. Because of the high RPD, a rerun was requested. The DSC results were 0.0 and $29.3 \mathrm{~J} / \mathrm{g}$ for the sample and duplicate, respectively. The highest individualsample for solid was $174 \mathrm{~J} / \mathrm{g}$. The upper limit of a 95 percent confidence interval on the mean for each sample was calculated.

Transitions 1 and 2 represent endothermic events, both of which are caused by water evaporation. High RPDs ( $>20$ percent) were reported for the solid core composite subsamples. The RPDs can be attributed to the heterogenous nature of the samples and no reruns were requested. The standard recoveries for this analysis were within the required limits. DSC results are presented in Table B2-23.

\section{B2.4 DENSITY AND SPECIFIC GRAVITY}

Density/specific gravity measurements were performed on all subsegments. The subsegment-level results for salt slurry densities ranged from 1.52 to $1.66 \mathrm{~g} / \mathrm{mL}$. The specific gravity measurements for the liquid samples ranged from 1.35 to 1.44. The bulk density and specific gravity are presented in Tables B2-25 and B2-26.

\section{B2.5 INORGANIC ANALYSES}

The ICP and IC analyses were performed for the anions and cations respectively. In the sections below, a table is provided for each analyte.

In each table, the "Mean" column is the average of the result and duplicate values. All values, including those below the detection level (denoted by the less-than symbol, "<"), were averaged. If both sample and duplicate values were non-detected, the mean is expressed as a non-detected value. If one value were detected while the other were not, the mean is expressed as a detected value. If both values were detected, the mean is expressed as a detected value. 


\section{B2.5.1 Inductively Coupled Plasma}

The ICP analyses were performed per procedures LA-505-161, Rev. B-1, or LA-505-151, Rev. D-3, depending on the ICP instrument used. A full suite of analytes were reported. Phosphorus was analyzed as a cross-check for the phosphate results reported from IC analyses. The liquid subsamples were prepared for analysis by an acid adjustment of the direct subsample. Solid subsamples were prepared for analysis by performing both an acid digest and a fusion.

Only the quality control (QC) for requested analytes ( $\mathrm{Li}, \mathrm{Na}, \mathrm{Al}, \mathrm{Cr}, \mathrm{Ca}, \mathrm{Fe}, \mathrm{K}, \mathrm{U}, \mathrm{Zn}, \mathrm{Zr}$, $\mathrm{Ba}, \mathrm{Si}, \mathrm{B}, \mathrm{Bi}, \mathrm{Mn}, \mathrm{Ni}$ ) were reviewed for this report. Other "opportunistic" analyte results are included in this appendix. These analytes do not have customer defined QC parameters and are not discussed. High RPDs ( $>20$ percent) were reported for several analytes ( $\mathrm{Li}, \mathrm{Al}$, $\mathrm{Cr}, \mathrm{Ca}, \mathrm{Fe}, \mathrm{Zn}, \mathrm{Zr}, \mathrm{Si}, \mathrm{B}, \mathrm{Mn}, \mathrm{Ni}$ ) and were the result of sample inhomogeneity. Reruns were not requested. Spike recoveries outside of the required range were reported for $\mathrm{Al}, \mathrm{Ca}$, $\mathrm{Cr}, \mathrm{Fe}, \mathrm{Li}, \mathrm{K}, \mathrm{Si}, \mathrm{Na}$ and $\mathrm{Zr}$. Spike recoveries for major analytes are not valid because the spike is too small compared to the sample concentration. Serial dilutions are performed by diluting a sample by five-fold. The results from the serial dilution may be found in the raw data and are signified by an "_L" at the end of the sample number. For acceptable performance the percent difference between the serial dilution and the undiluted results must be $\leq 10$ percent, one sample had a serial dilution greater than ten percent. This inaccuracy can be attributed to the low concentration of the analyte with respect to the detection limit. There did not appear to be any analytical anomalies or difficulties during the analyses of Ba or $\mathrm{U}$.

The concentrations of metals in the samples are shown in Tables B2-27 through B2-50. The results from two preparation methods, fusion and acid, are presented for the metals.

\section{B2.5.2 Ion Chromatography}

The IC analyses were performed on direct subsamples of liquid samples. The solid subsamples were prepared for analysis by performing a water digest. Samples for ion chromatography were performed in duplicate per procedure LA-533-105, Rev. D-1. All analytes reported by the IC instrument were requested.

High RPDs ( $>20$ percent) were reported for several analytes and can be attributed to sample inhomogeneity. Spike recoveries outside of the required range ( 75 percent to 125 percent) were reported for acetate, $\mathrm{Cl}^{-}, \mathrm{F}^{-}, \mathrm{NO}_{2}^{-}$and $\mathrm{NO}_{3}^{-}$ions. The chemist noted that the spike failures were due to matrix interferences from organic acids. Bromide was detected in 34 of the 52 subsamples submitted for analysis and was an indication of hydrostatic head fluid intrusion into the samples. The standard recoveries for IC analysis were within the required limits. IC analyses results are given in Tables B2-51 through B2-60. 


\section{B2.5.3 OH}

The $\mathrm{OH}$ analyses were performed on direct liquid core composite subsamples and the field blank. The solid core composite subsamples were prepared for analysis by performing a water digest. The standard recoveries and RPDs for this analysis were within the required limits. $\mathrm{OH}$ results are presented in Tables B2-61 and B2-62.

\section{B2.5.4 Chromium VI (CrVI)}

The $\mathrm{Cr}$ (VI) analyses were performed on direct liquid core composite subsamples and the field blank. The solid core composite subsamples were prepared for analysis by performing a water digest. The standard recoveries, spike recoveries and RPDs for this analysis were within the required limits. $\mathrm{Cr}(\mathrm{VI})$ results are presented in Table B2-63.

\section{B2.6 TOTAL INORGANIC CARBON/TOTAL ORGANIC CARBON (TIC/TOC)}

TIC/TOC by persulfate/coulometry analyses were performed on the core composite samples. None of the results exceeded the TOC notification limit of $30,000 \mu \mathrm{gC} / \mathrm{g}$. Low concentrations of TIC and TOC were noted in some preparation blanks, however, the levels of contamination are inconsequential when compared to the result of the sample and do not impact sample data quality. The standard recoveries, spike recoveries and RPDs for these analyses were within the required limits. TIC/TOC results are presented in Tables B2-64 through B2-66.

\section{B2.7 VAPOR PHASE MEASUREMENT}

During June 1996, tank dome space gas samples were taken from tank 241-AN-105. These measurements supported the safety screening DQO (Dukelow et al. 1995). The vapor phase screening was taken for flammability issues. The vapor phase measurements were taken $20 \mathrm{ft}$ below risers $7 \mathrm{~B}$ and $12 \mathrm{~A}$ in the dome space of the tank and results were obtained in the field (that is, no gas sample was sent to the laboratory for analysis). The average results of the vapor phase measurements are provided in Table B2-67.

\section{B2.8 HISTORICAL SAMPLE RESULTS}

The results of the first sampling event in 1984 results are shown in Table B2-2 (Jansky 1984). The results of second sampling event performed in 1984 are shown in Table B2-3 (Mauss 1984). This tank was used as a receiver tank for the 242-A Evaporator. Because evaporated waste has been added to the tank since these sampling events, the sample probably does not represent present tank contents. The sample was analyzed for specific 
constituents and the results showed high concentrations of sodium, hydroxide, TOC, nitrate, and nitrite. These data have not been validated and should be used with caution.

Table B2-2. Supernatant Sample of May 1984. ${ }^{1,2}$

\begin{tabular}{|c|c|c|c|}
\hline T": & C. That 241 & & \\
\hline (2. :. & 19639 & 18900 & Wrivinin \\
\hline l. & Tryoricrit D & & 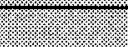 \\
\hline Specific gravity & 1.39 & NA & \\
\hline $\mathrm{pH}$ & 13.30 & NA & \\
\hline 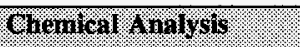 & 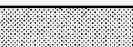 & 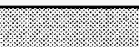 & . \\
\hline $\mathrm{OH}^{+}$ & 2.43 & 2.33 & $M$ \\
\hline$\overline{\mathrm{Al}}$ & 1.10 & 1.08 & $M$ \\
\hline $\mathrm{Na}$ & 8.71 & 7.08 & $M$ \\
\hline $\mathrm{NO}_{2}^{-}$ & 1.85 & 1.83 & $M$ \\
\hline $\mathrm{NO}_{3}^{-}$ & 1.99 & 2.86 & $M$ \\
\hline $\mathrm{CO}_{3}{ }^{2-}$ & 0.445 & 0.434 & $\bar{M}$ \\
\hline $\mathrm{SO}_{4}^{2-}$ & 0.0413 & $\mathrm{BD}$ & $M$ \\
\hline $\mathrm{PO}_{4}{ }^{3-}$ & 0.0152 & 0.0147 & $\bar{M}$ \\
\hline TOC & 4.05 & 3.94 & $\mathrm{~g} / \mathrm{L}$ \\
\hline $\mathrm{Cl}^{-}$ & 0.252 & 0.215 & $\bar{M}$ \\
\hline K & 0.105 & 0.0789 & $M$ \\
\hline Radiological a nelysis: & & & 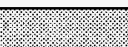 \\
\hline${ }^{137} \mathrm{Cs}$ & $3.82 \mathrm{E}+05$ & $3.61 \mathrm{E}+05$ & $\mu \mathrm{Ci} / \mathrm{L}$ \\
\hline
\end{tabular}

Notes:

$\mathrm{NA}=$ not available
$\mathrm{BD}=$ below detection limit

'Pre-1989 analytical data have not been validated and should be used with caution.

${ }^{2}$ Jansky (1984). 
Table B2-3. Supernatant Sample of November 1984., ${ }^{1,2}$

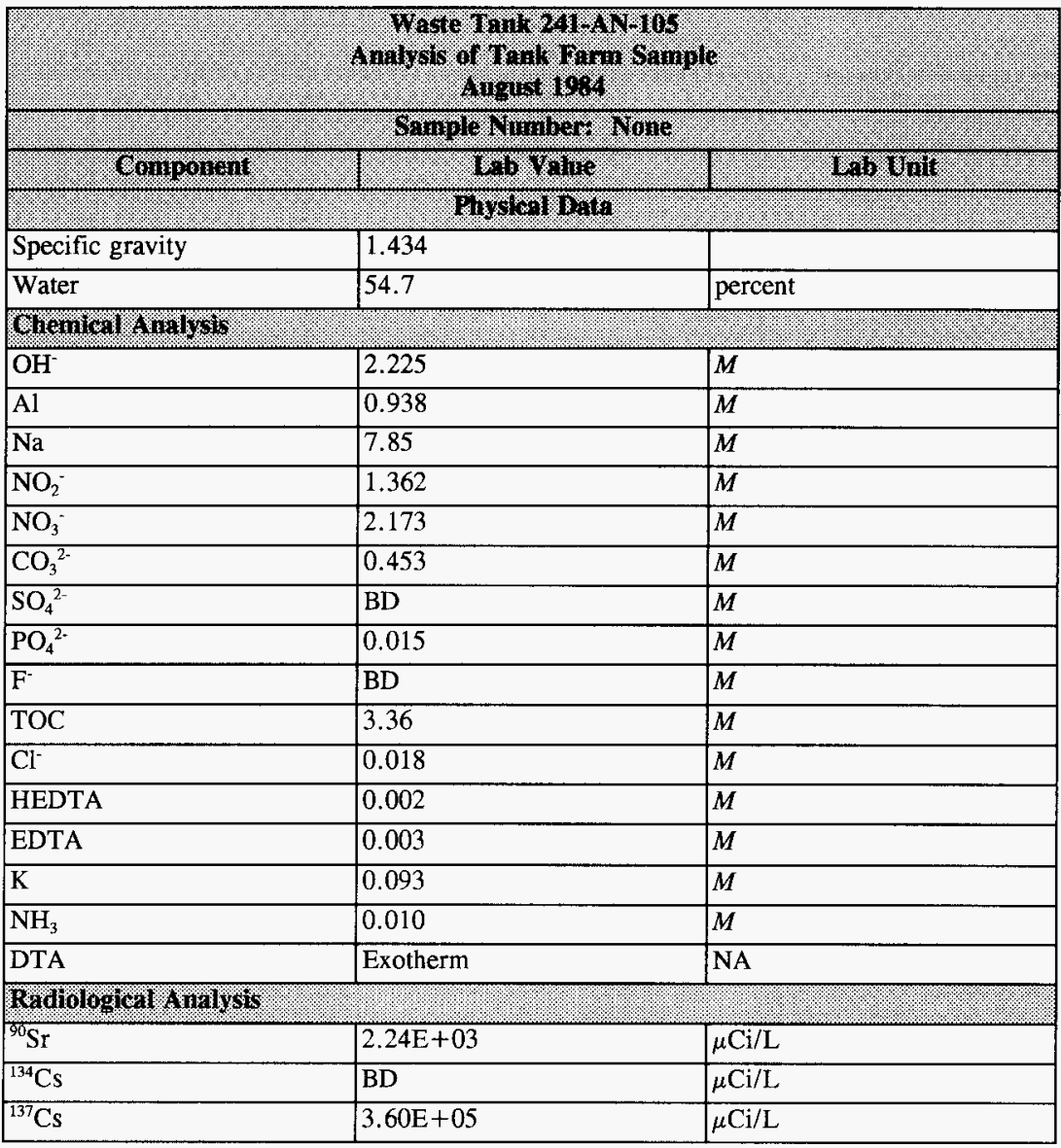

Notes:

$\mathrm{BD}=$ below detection limit

$\mathrm{NA}=$ not available

'Pre-1989 analytical data have not been validated and should be used with caution.

${ }^{2}$ Mauss (1984). 


\section{B2.9 ANALYTICAL DATA TABLES}

For most analytes (except for some physical and rheological measurements), the data tables consist of six columns. The first column lists the sample number. Note that for each primary/duplicate pair, the sample number is for the primary result. The second column lists the core from which the samples were derived. The third column lists the sample portion from which the aliquots were taken. The final three columns display the primary and duplicate analytical values and a mean for each sample/duplicate pair.

The four QC parameters assessed in conjunction with the tank 241-AN-105 samples were standard recoveries, spike recoveries, duplicate analyses (RPDs), and blanks. The QC criteria specified in the SAP (Eggers 1996) for safety screening DQO were 90 to 110 percent recovery for standards and spikes and $\leq 10$ percent for RPDs. The only QC parameter for which limits are not specified in the SAP is blank contamination. The limits for blanks are set forth in guidelines followed by the laboratory, and all data results presented in this report have met those guidelines. Sample and duplicate pairs in which any of the QC parameters were outside of these limits are footnoted in the sample mean column of the following data summary tables with an a, b, c, d, e, or $\mathrm{f}$ as follows:

- $\quad$ " $\mathrm{a}$ " indicates that the standard recovery was below the QC range.

- $\quad \mathrm{b} "$ indicates that the standard recovery was above the QC range.

- $\quad \mathrm{c} "$ indicates that the spike recovery was below the QC range.

- $\quad \mathrm{d}$ " indicates that the spike recovery was above the QC range.

- $\quad \mathrm{e} "$ indicates that the RPD was greater than the QC limit range.

- $\quad \mathrm{f}$ " indicates that there was blank contamination. 
Table B2-4. Tank 241-AN-105 Analytical Results: Total Alpha (Alpha Rad).

\begin{tabular}{|c|c|c|c|c|c|}
\hline Hampor & ogmple & 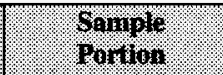 & $\mathrm{R} e+411$ & Dipliogte & ranin \\
\hline \multicolumn{2}{|c|}{ Wuings } & & 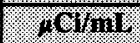 & 101112 & W $617 \%$ \\
\hline S96T003732 & 152: 1 & Drainable Liquid & $<0.02$ & 0.02 & $<0.02^{\mathrm{QC:b}}$ \\
\hline S96T003739 & 152: 2 & Drainable Liquid & $<0.02$ & $<0.02$ & $<0.02^{\mathrm{QC}: \mathrm{b}}$ \\
\hline S96T003740 & 152: 3 & Drainable Liquid & $<0.01$ & 0.04 & $<0.02^{\mathrm{QC:b}}$ \\
\hline \$96T003741 & 152: 4 & Drainable Liquid & $<0.02$ & $<0.027$ & $<0.02^{\mathrm{QC:b}}$ \\
\hline S96T003742 & 152: 5 & Drainable Liquid & $<0.01$ & $<0.02$ & $<0.019^{Q \mathrm{QC}: \mathrm{b}}$ \\
\hline S96T003743 & 152: 6 & Drainable Liquid & $<0.02$ & $<0.04$ & $<0.03$ \\
\hline S96T003744 & 152: 7 & Drainable Liquid & 0.01 & $<0.02$ & $<0.01$ \\
\hline S96T003745 & 152: 8 & Drainable Liquid & $<0.02$ & $<0.02$ & $<0.02$ \\
\hline S96T003746 & 152: 9 & Drainable Liquid & $<0.02$ & $<0.02$ & $<0.02$ \\
\hline S96T003747 & $152: 10$ & Drainable Liquid & $<0.03$ & $<0.01$ & $<0.02$ \\
\hline S96T003748 & $152: 11$ & Drainable Liquid & $<0.05$ & $<0.08$ & $<0.06$ \\
\hline S96T003749 & $152: 12$ & Drainable Liquid & $<0.05$ & $<0.05$ & $<0.05$ \\
\hline S96T003750 & $152: 13$ & Drainable Liquid & $<0.03$ & $<0.04$ & $<0.04$ \\
\hline S96T003751 & $152: 14$ & Drainable Liquid & $<0.07$ & $<0.06$ & $<0.07$ \\
\hline S96T004433 & 153: 1 & Drainable Liquid & $<0.02$ & $<0.02$ & $<0.02$ \\
\hline S96T004308 & 153: 2 & Drainable Liquid & $<0.01$ & $<0.00$ & $<0.01^{\mathrm{QC:f}}$ \\
\hline S96T004434 & 153: 3 & Drainable Liquid & $<0.01$ & $<0.01$ & $<0.01$ \\
\hline S96T004419 & 153: 5 & Drainable Liquid & $<0.01$ & $<0.01$ & $<0.01$ \\
\hline S96T004420 & 153: 6 & Drainable Liquid & $<0.01$ & $<0.01$ & $<0.01$ \\
\hline \$96T004421 & 153: 7 & Drainable Liquid & $<0.01$ & $<0.01$ & $<0.01^{\mathrm{QC}: \mathrm{a}}$ \\
\hline S96T003975 & 153: 8 & Drainable Liquid & $<0.02$ & $<0.14$ & $<0.08$ \\
\hline S96T003976 & 153: 9 & Drainable Liquid & 0.04 & $<0.02$ & $<0.03$ \\
\hline S96T004309 & $153: 10$ & Drainable Liquid & $<0.0071$ & $<0.0071$ & $<0.0071^{\text {QC:f }}$ \\
\hline S96T003979 & $153: 11$ & Drainable Liquid & $<0.02$ & $<0.03$ & $<0.02$ \\
\hline S96T003977 & $153: 12$ & Drainable Liquid & $<0.02$ & $<0.03$ & $<0.02$ \\
\hline S96T003978 & $153: 13$ & Drainable Liquid & $<0.02$ & $<0.01$ & $<0.02$ \\
\hline S96T004333 & $153: 14$ & Drainable Liquid & $<0.00$ & 0.01 & $<0.01^{\mathrm{QC}: \mathrm{f}}$ \\
\hline S96T003980 & $153: 21$ & Drainable Liquid & $<0.01$ & $<0.02$ & $<0.02$ \\
\hline S96T004039 & Core 152 & Liquid Composite & $<0.01$ & $<0.01$ & $<0.01$ \\
\hline
\end{tabular}


Table B2-5. Tank 241-AN-105 Analytical Results: Total Alpha (Alpha).

\begin{tabular}{|c|c|c|c|c|c|}
\hline Wample. & saminger & Samplo & gresing & Biplicate & Men \\
\hline oddas & 115010 & & $1001 \%$ & 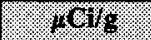 & 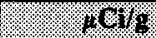 \\
\hline S96T003893 & 152: 1 & Upper half & $<0.01$ & $<0.02$ & $<0.01^{\mathrm{QC: \textrm {a }}}$ \\
\hline S96T003827 & $152: 14$ & Lower half & $<0.02$ & $<0.01$ & $<0.02^{\mathrm{QC:a}}$ \\
\hline S96T003889 & $152: 16$ & Lower half & $<0.03$ & $<0.02$ & $<0.02$ \\
\hline S96T003890 & $152: 18$ & Lower half & $<0.01$ & $<0.01$ & $<0.01$ \\
\hline S96T003891 & $152: 20$ & Lower half & 0.00 & 0.00 & $0.00^{\mathrm{QC} ; \mathrm{b}}$ \\
\hline S96T003892 & $152: 22$ & Upper half & 0.01 & 0.01 & $0.01^{\mathrm{QC}: \mathrm{b}, \mathrm{e}}$ \\
\hline S96T004337 & $153: 14$ & Lower half & $<0.00$ & 0.00 & $<0.00$ \\
\hline S96T004317 & $153: 15$ & Lower half & $<0.00$ & 0.00 & $<0.00$ \\
\hline S96T004442 & $153: 17$ & Lower half & 0.00 & 0.00 & 0.00 \\
\hline S96T004443 & $153: 19$ & Lower half & 0.01 & 0.01 & $0.01^{\mathrm{QC}: c}$ \\
\hline S96T003984 & $153: 21$ & Lower half & 0.00 & 0.00 & $0.00^{\mathrm{QC}: \mathrm{e}}$ \\
\hline S96T003985 & $153: 22$ & Lower half & 0.13 & 0.11 & $0.12^{\mathrm{QC}: \mathrm{e}}$ \\
\hline S96T004256 & \multirow[t]{2}{*}{ Core 152} & Solid Composite & 0.01 & 0.01 & $0.01^{\mathrm{QC}: b, e, f}$ \\
\hline S96T004261 & & Solid Composite & 0.06 & 0.05 & $0.05^{\mathrm{QC}: b, e, f}$ \\
\hline
\end{tabular}

Table B2-6. Tank 241-AN-105 Analytical Results: Total Beta.

\begin{tabular}{|c|c|c|c|c|c|}
\hline $\begin{array}{l}\text { gample } \\
\text { Mumpor }\end{array}$ & 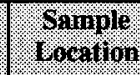 & Pomplo & resall & Buplicale & Woan \\
\hline \multicolumn{2}{|c|}{ YHoum } & & $101 / 11$ & 10 HN & $101 \mathrm{~mL}$ \\
\hline S96T004049 & Core 152 & Liquid Composite & 542 & 534 & 538 \\
\hline Sollds: & ruston & & $101 \%$ & 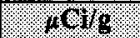 & $16 \%$ \\
\hline S96T004256 & Core 152 & Solid Composite & 328 & 325 & 326.5 \\
\hline S96T004261 & & Solid Composite & 268 & 281 & 274.5 \\
\hline
\end{tabular}


Table B2-7. Tank 241-AN-105 Analytical Results: Total Uranium (Uranium Gross Kinetic Phos.)

\begin{tabular}{|c|c|c|c|c|c|}
\hline Sample & 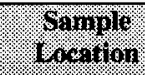 & $\begin{array}{l}\text { Sourow } \\
\text { 801010m }\end{array}$ & $\mathrm{P}-414$ & Dublicalo & Monu \\
\hline Wols & ? & & Hos & 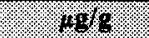 & $18 \%$ \\
\hline S96T005546 & Core 152 & $\begin{array}{l}\text { Liquid } \\
\text { Composite }\end{array}$ & 2.64789 & 2.37324 & $2.51056^{\mathrm{QC:f}}$ \\
\hline
\end{tabular}

Table B2-8. Tank 241-AN-105 Analytical Results: Americium-241 (AEA).

\begin{tabular}{|c|c|c|c|c|c|}
\hline Sumpor & Somplor & Portion & resull & Duplicale & Mean \\
\hline Solids & Mision & & 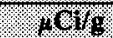 & aris & $100 \mathrm{~s}$ \\
\hline S96T004256 & \multirow[t]{2}{*}{ Core 152} & Solid Composite & 0.005 & 0.003 & $0.41^{\mathrm{QC}: e}$ \\
\hline S96T004261 & & Solid Composite & 0.04 & 0.03 & $0.03^{\mathrm{QC}: \mathrm{C}}$ \\
\hline
\end{tabular}

Table B2-9. Tank 241-AN-105 Analytical Results: Cm-243/244 (AEA).

\begin{tabular}{|c|c|c|c|c|c|}
\hline Samplo & ogople & Samblo & resull & Duplicate & Menn \\
\hline Wouns & Triaion & & 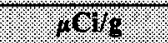 & \% $1 / 6$ & 4018 \\
\hline S96T004256 & \multirow[t]{2}{*}{ Core 152} & Solid Composite & $<9.670 \mathrm{E}-04$ & $<8.240 \mathrm{E}-04$ & $<8.955 \mathrm{E}-04$ \\
\hline S96T004261 & & Solid Composite & $<0.006$ & $<0.005$ & $<0.05$ \\
\hline
\end{tabular}

Table B2-10. Tank 241-AN-105 Analytical Results: Americium-241 (Am-241).

\begin{tabular}{|c|c|c|c|c|c|}
\hline Somplo & Gocolisn & Sample & Resilit & Duplicite & Wean \\
\hline \multicolumn{3}{|c|}{ Wriugs } & Wrot & 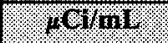 & H $/ \mathrm{m}$ \\
\hline S96T004049 & Core 152 & Liquid Composite & $<3.910 \mathrm{E}-05$ & $<3.520 \mathrm{E}-05$ & $<3.715 \mathrm{E}-05$ \\
\hline
\end{tabular}


Table B2-11. Tank 241-AN-105 Analytical Results: Cm-243/244 (Am-241).

\begin{tabular}{|c|c|c|c|c|c|}
\hline $\begin{array}{l}\text { Sample } \\
\text { Nirmber: }\end{array}$ & & $\begin{array}{l}\text { Sample } \\
\text { Fortion }\end{array}$ & Resull & guplorate & riegn \\
\hline \multicolumn{2}{|c|}{ Trouts: } & & 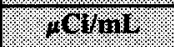 & (chisil & If COMI \\
\hline $96 \mathrm{~T} 00404$ & ore 152 & Liquid Composite & $<3.910 \mathrm{E}-05$ & $<3.520 \mathrm{E}-05$ & $<3.715 \mathrm{E}-05$ \\
\hline
\end{tabular}

Table B2-12. Tank 241-AN-105 Analytical Results: Americium-241 (GEA).

\begin{tabular}{|c|c|c|c|c|c|}
\hline $\begin{array}{l}\text { Sample: } \\
\text { Number. }\end{array}$ & $\begin{array}{l}\text { Sarmple: } \\
\text { Eocalton }\end{array}$ & $\begin{array}{l}\text { Samples. } \\
\text { Fontion. }\end{array}$ & Resint & Bupl & Mrean \\
\hline \multicolumn{2}{|c|}{ Elquías } & 1.9 & IOTini: & \#CHIII & INAm \\
\hline S96T004049 & Core 152 & Liquid Composite & $<0.85$ & $<0.85$ & $<0.85$ \\
\hline Sollids: & fusion & 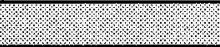 & rês & $1901 \%$ & m.1. \\
\hline S96T004256 & \multirow[t]{2}{*}{ Core 152} & Solid Composite & $<0.82$ & $<0.82$ & $<0.82$ \\
\hline S96T004261 & & Solid Composite & $<0.47$ & $<0.49$ & $<0.48$ \\
\hline
\end{tabular}

Table B2-13. Tank 241-AN-105 Analytical Results: Cesium-137 (GEA).

\begin{tabular}{|c|c|c|c|c|c|}
\hline $\begin{array}{l}\text { Sample: } \\
\text { Namber. }\end{array}$ & $\begin{array}{l}\text { Sample: } \\
\text { socation }\end{array}$ & $\begin{array}{l}\text { Sample: } \\
\text { Portion }\end{array}$ & Resull & Duplicate & Mean. \\
\hline \multicolumn{2}{|c|}{ Tiquitis: } & 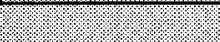 & OON111 & HOHIII & WCrini: \\
\hline S96T004049 & Core 152 & Liquid Composite & 473 & 476 & 474.5 \\
\hline Sollots: & Hivion & & 1018. & $10 \% \mathrm{~g}$ & 1.0.9. \\
\hline S96T004256 & \multirow[t]{2}{*}{ Core 152} & Solid Composite & 305.2 & 303 & 304.1 \\
\hline S96T004261 & & Solid Composite & 205 & 207 & 206 \\
\hline
\end{tabular}


Table B2-14. Tank 241-AN-105 Analytical Results: Cobalt-60 (GEA).

\begin{tabular}{|c|c|c|c|c|c|}
\hline Shmplo & 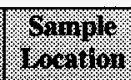 & 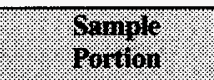 & $\mathrm{P}=911 \%$ & Buplicato & Mener \\
\hline \multicolumn{2}{|c|}{ Woupros } & & $\% \propto 111$ & $16 \% 11$ & W 111 \\
\hline S96T004049 & Core 152 & Liquid Composite & $<0.01$ & $<0.01$ & $<0.01$ \\
\hline Soling & $19 \%$ & & 1016. & $10 \%$ & 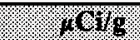 \\
\hline S96T004256 & \multirow[t]{2}{*}{ Core 152} & Solid Composite & $<0.03$ & $<0.02$ & $<0.034$ \\
\hline S96T004261 & & Solid Composite & $<0.01$ & $<0.01$ & $<0.01$ \\
\hline
\end{tabular}

Table B2-15. Tank 241-AN-105 Analytical Results: Europium-154 (GEA).

\begin{tabular}{|c|c|c|c|c|c|}
\hline Wanglo & Souplou & 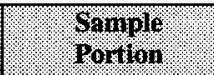 & gesilit. & Duplicate & Mean \\
\hline \multicolumn{2}{|c|}{ Wus } & & 4Q Wring & Kornu & Morinit \\
\hline S96T004049 & Core 152 & Liquid Composite & $<0.09$ & $<0.09$ & $<0.09$ \\
\hline solvas & fuston & & 1018 & 10/g & 1019 \\
\hline S96T004256 & \multirow[t]{2}{*}{ Core 152} & Solid Composite & $<0.08$ & $<0.09$ & $<0.09$ \\
\hline S96T004261 & & Solid Composite & $<0.06$ & $<0.07$ & $<0.07$ \\
\hline
\end{tabular}

Table B2-16. Tank 241-AN-105 Analytical Results: Europium-155 (GEA).

\begin{tabular}{|c|c|c|c|c|c|}
\hline Wamoloc. & 609100 & Samper & Resull & Biplicate & Mean \\
\hline \multicolumn{3}{|c|}{ Houirs } & W & (101110 & \% 1111 \\
\hline S96T004049 & Core 152 & Liquid Composite & $<0.32$ & $<0.32$ & $<0.32$ \\
\hline Whits & frision & & (x) $1 / 9$ & $1 \% 1 / g$ & $10 \%$ \\
\hline S96T004256 & \multirow[t]{2}{*}{ Core 152} & Solid Composite & $<0.38$ & $<0.38$ & $<0.38$ \\
\hline S96T004261 & & Solid Composite & $<0.22$ & $<0.23$ & $<0.22$ \\
\hline
\end{tabular}


Table B2-17. Tank 241-AN-105 Analytical Results: Iodine-129 (I129).

\begin{tabular}{|c|c|c|c|c|c|}
\hline $\begin{array}{l}\text { Sample } \\
\text { Voringer }\end{array}$ & oponole & 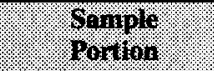 & 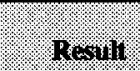 & Ouplions: & Mean \\
\hline \multicolumn{2}{|c|}{ Sour } & & 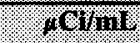 & $1 \% 11$ & Wogu \\
\hline S96T004049 & Core 152 & Liquid Composite & $2.460 \mathrm{E}-04$ & $2.890 \mathrm{E}-04$ & $2.675 \mathrm{E}-04$ \\
\hline \multicolumn{2}{|c|}{ Sollidsr yater oluget } & & 4018 & $101 / 9$ & 181/\% \\
\hline S96T004258 & \multirow[t]{2}{*}{ Core 152} & Solid Composite & $<0.0033$ & $<0.0039$ & $<0.0036$ \\
\hline S96T004263 & & Solid Composite & $<0.0030$ & $<0.0031$ & $<0.0030$ \\
\hline
\end{tabular}

Table B2-18. Tank 241-AN-105 Analytical Results: H-3 (Liq. Scin.).

\begin{tabular}{|c|c|c|c|c|c|}
\hline Namber & 6ramplo & 8019010 & rivesult & Diplleate & Wran \\
\hline \multicolumn{3}{|c|}{ Wquas } & 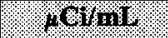 & $4 \mathrm{~W}_{17}$ & Winin \\
\hline S96T004831 & $\begin{array}{l}\text { Core } \\
152\end{array}$ & $\begin{array}{l}\text { Liquid } \\
\text { Composite }\end{array}$ & $<3.960 \mathrm{E}-05$ & $<3.900 \mathrm{E}-05$ & $<3.930 \mathrm{E}-05^{\mathrm{QC}: \mathrm{f}}$ \\
\hline \multicolumn{3}{|c|}{ Solids yaler digest } & MO & $\% 1 \%$ & moras \\
\hline S96T004258 & \multirow[t]{2}{*}{$\begin{array}{l}\text { Core } \\
152\end{array}$} & Solid Composite & 0.0024 & 0.0019 & $0.0022^{\mathrm{QC:f}}$ \\
\hline S96T004263 & & Solid Composite & $<6.330 \mathrm{E}-04$ & $<6.280 \mathrm{E}-04$ & $<6.305 \mathrm{E}-04$ \\
\hline
\end{tabular}

Table B2-19. Tank 241-AN-105 Analytical Results: Neptunium-237 (Np-237).

\begin{tabular}{|c|c|c|c|c|c|}
\hline Sampro & Whamp & Shmple & reanill & Wuplisate & Milan \\
\hline \multicolumn{2}{|c|}{ Wiquias } & & Hovim & NQWin & Rorin \\
\hline S96T004049 & Core 152 & Liquid Composite & $<2.060 \mathrm{E}-05$ & $<2.210 \mathrm{E}-05$ & $<2.135 \mathrm{E}-05^{\mathrm{QC}: \mathrm{A}}$ \\
\hline \multicolumn{2}{|c|}{ Sollas . fosiol. } & & 140 & $140 \%$ & \%119 \\
\hline S96T004256 & \multirow[t]{2}{*}{ Core 152} & Solid Composite & 0.0103 & 0.0109 & $0.0106^{\mathrm{QC:a}}$ \\
\hline S96T004261 & & Solid Composite & $<0.0153$ & $<0.0162$ & $<0.0157^{\mathrm{QC:a}}$ \\
\hline
\end{tabular}


Table B2-20. Tank 241-AN-105 Analytical Results: Plutonium-239/40 (Pu-239/240).

\begin{tabular}{|c|c|c|c|c|c|}
\hline 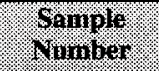 & 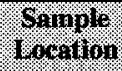 & somosos & iesuil: & Buplicate & Moan \\
\hline \multicolumn{2}{|c|}{ Wiving } & & $4 \times 110$ & M & 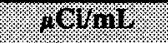 \\
\hline S96T004049 & Core 152 & Liquid Composite & $<3.470 \mathrm{E}-05$ & $<3.720 \mathrm{E}-05$ & $<3.595 \mathrm{E}-05$ \\
\hline \multicolumn{2}{|c|}{ Soldis. } & & 16\% & 19: & 1\%8 \\
\hline S96T004256 & \multirow[t]{2}{*}{ Core 152} & Solid Composite & $<0.0018$ & $<0.0017$ & $<0.0017$ \\
\hline S96T004261 & & Solid Composite & 0.0075 & 0.0075 & 0.0075 \\
\hline
\end{tabular}

Table B2-21. Tank 241-AN-105 Analytical Results: Strontium-89/90 (Sr).

\begin{tabular}{|c|c|c|c|c|c|}
\hline Wample & 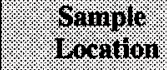 & 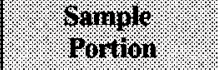 & nesillt & Bupliste & Mean \\
\hline \multicolumn{3}{|c|}{ Liquids } & $\mu \mathrm{Ci} / \mathrm{mL}$ & $\mu \mathrm{Ci} / \mathrm{mL}$ & $\mu \mathrm{Ci} / \mathrm{mL}$ \\
\hline S96T004049 & Core 152 & Liquid Composite & 0.042 & 0.0408 & 0.0414 \\
\hline \multicolumn{2}{|c|}{ Solfids fiston } & & Wros & (1) & $10 \%$ \\
\hline S96T004256 & Core 152 & Solid Composite & 9.41 & 8.91 & 9.16 \\
\hline S96T004261 & & Solid Composite & 35.8 & 37.3 & 36.55 \\
\hline
\end{tabular}

Table B2-22. Tank 241-AN-105 Analytical Results: Technetium-99 (Tc).

\begin{tabular}{|c|c|c|c|c|c|}
\hline Wanger & Somplo & Dovibon & $\mathrm{hesilt}$ & Ouplicate & 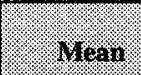 \\
\hline \multicolumn{2}{|c|}{ Wiguris } & 1. & Hornil & Wim & $1 \% 1 \mathrm{~m}$ \\
\hline S96T004049 & Core 152 & Liquid Composite & 0.27 & 0.26 & 0.27 \\
\hline 101100 & 119ion. & & $n \% 1 \%$ & 4019. & \% \\
\hline S96T004256 & \multirow[t]{2}{*}{ Core 152} & Solid Composite & 0.18 & 0.18 & 0.18 \\
\hline S96T004261 & & Solid Composite & 0.14 & 0.12 & 0.13 \\
\hline
\end{tabular}


Table B2-23. Tank 241-AN-105 Analytical Results: Exotherm - (DSC). (2 sheets)

\begin{tabular}{|c|c|c|c|c|c|}
\hline Mamplo & $4.806110 \%$ & Whomple & R=vil & Bullidate & mern \\
\hline \multicolumn{2}{|c|}{ Wrory } & 3.1 .2 .21$. & Wy & 196 & Wy \\
\hline S96T004434 & 153:3 & Drainable liquid & 16.8 & 19.9 & 18.35 \\
\hline S96T004419 & 153: 5 & Drainable liquid & 18 & 205.2 & $111.6^{\mathrm{QC:e}}$ \\
\hline S96T003976 & 153:9 & Drainable liquid & 2.2 & 0 & $1.1^{\mathrm{QC}: \mathrm{e}}$ \\
\hline S96T003977 & $153: 12$ & Drainable liquid & 0 & 52.4 & $26.2^{\mathrm{QC}: \mathrm{e}}$ \\
\hline S96T003749 & $152: 12$ & Drainable liquid & 42.6 & 64.9 & $53.75^{\text {QC:e }}$ \\
\hline S96T004433 & 153: 1 & Drainable liquid & 12.7 & 15.9 & $14.3^{\mathrm{QC}: e}$ \\
\hline \$96T004419 & 153: 5 & Drainable liquid & & 14.53 & 14.53 \\
\hline S96T004421 & 153: 7 & Drainable liquid & & 34.54 & 34.54 \\
\hline S96T004309 & $153: 10$ & Drainable liquid & 13.55 & 19.54 & $16.54^{\mathrm{QC}: \mathrm{e}}$ \\
\hline S96T004333 & $153: 14$ & Drainable liquid & 34.8 & 57.7 & $46.25^{\mathrm{QC:e}}$ \\
\hline \multicolumn{2}{|c|}{ Solvos. } & & $1 / 19$ & $1.16 \mathrm{~g}$ & (w. \\
\hline S96T003731 & 152: 1 & Upper half & 5 & 7.3 & $6.15^{\mathrm{QC}: \mathrm{c}}$ \\
\hline S96T003816 & $152: 14$ & Upper half & 28.4 & 24.2 & 26.3 \\
\hline S96T003817 & & Lower half & 16.1 & 7.6 & $11.85^{\mathrm{QC}: \mathrm{e}}$ \\
\hline S96T003792 & $152: 16$ & Upper half & 24.3 & 37.7 & $31^{\mathrm{QC:e}}$ \\
\hline S96T003793 & & Lower half & 27.2 & 28 & 27.6 \\
\hline S96T003794 & $152: 18$ & Upper half & 26.5 & 24.3 & 25.4 \\
\hline \$96T003795 & & Lower half & 30.7 & 32.7 & 31.7 \\
\hline S96T003796 & $152: 20$ & Upper half & 38.5 & 39.7 & 39.1 \\
\hline S96T003798 & $152: 22$ & Upper half & 59 & 73 & $66^{\mathrm{QC:c}}$ \\
\hline S96T004335 & $153: 14$ & Lower half & 31 & 39 & $35^{\text {QC:e }}$ \\
\hline S96T004310 & $153: 15$ & Upper half & 40.4 & 23.9 & $32.15^{\mathrm{QC}: e}$ \\
\hline S96T004311 & & Lower half & 34.7 & 57.1 & $45.9^{\mathrm{QC}: \mathrm{e}}$ \\
\hline
\end{tabular}


HNF-SD-WM-ER-678 Rev. 0

Table B2-23. Tank 241-AN-105 Analytical Results: Exotherm - (DSC). (2 sheets)

\begin{tabular}{|c|c|c|c|c|c|}
\hline $\begin{array}{l}\text { Sample: } \\
\text { Number: }\end{array}$ & $\begin{array}{l}\text { Sample } \\
\text { I rogatlon }\end{array}$ & $\begin{array}{l}\text { Simple: } \\
\text { rotion }\end{array}$ & Resill, & Buplisale & 1.thin \\
\hline \multicolumn{2}{|c|}{ Solitis } & V: & $\sqrt{19}$ & $1 / 18$ & 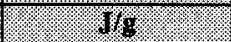 \\
\hline S96T004435 & $153: 17$ & Upper half & 48.7 & 94.1 & $71.4^{\mathrm{QC}: \mathrm{e}}$ \\
\hline S96T004436 & & Lower half & 15.8 & $\overline{19.6}$ & $17.7^{\mathrm{QC}: \mathrm{e}}$ \\
\hline S96T004437 & $153: 19$ & Lower half & 22.9 & 44.4 & $33.65^{\mathrm{QC}: \mathrm{e}}$ \\
\hline S96T003981 & $153: 21$ & Lower half & 21 & 18.2 & 19.6 \\
\hline S96T003983 & $153: 22$ & Upper half & 39.1 & 48.6 & $43.85^{\text {QC:e }}$ \\
\hline S96T003982 & & Lower half & 57.5 & 94.8 & $76.15^{\mathrm{QC}: c}$ \\
\hline S96T004254 & Core 152 & Solid composite & 43.7 & 31.7 & $37.7^{\mathrm{QC:e}}$ \\
\hline S96T004259 & & Solid composite & 60.4 & 54.1 & 57.25 \\
\hline
\end{tabular}


Table B2-24. Tank 241-AN-105 Analytical Results: Percent Water (TGA). (2 sheets)

\begin{tabular}{|c|c|c|c|c|c|}
\hline Whimolo & 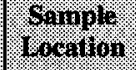 & $\operatorname{sor} 801$ & Result & Butiligato & Mon \\
\hline \multicolumn{2}{|c|}{ Wouke } & & $\%$ & $\%$ & 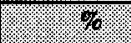 \\
\hline S96T003732 & $152: 1$ & Drainable liquid & 49.57 & 51.74 & 50.65 \\
\hline S96T003739 & $152: 2$ & Drainable liquid & 50.2 & 50.57 & 50.38 \\
\hline S96T003742 & 152: 5 & Drainable liquid & 50.5 & 50.85 & 50.67 \\
\hline S96T003743 & 152: 6 & Drainable liquid & 50.07 & 50.4 & 50.23 \\
\hline S96T003744 & $152: 7$ & Drainable liquid & 50.91 & 50.37 & 50.64 \\
\hline$\$ 96 T 003745$ & 152: 8 & Drainable liquid & 50.42 & 52.16 & 51.29 \\
\hline S96T003748 & \multirow[t]{2}{*}{$152: 11$} & Drainable liquid & 50.51 & 50.66 & 50.58 \\
\hline S96T003748 & & Drainable liquid & 49.75 & 30.12 & $39.93^{\mathrm{QC}: \mathrm{c}}$ \\
\hline S96T003749 & $152: 12$ & Drainable liquid & 50.07 & 50.46 & 50.26 \\
\hline \$96T004308 & 153: 2 & Drainable liquid & 50.38 & 50.23 & 50.30 \\
\hline S96T004434 & 153: 3 & Drainable liquid & 51.07 & 51.41 & 51.24 \\
\hline S96T004419 & 153: 5 & Drainable liquid & 50.41 & 50.54 & 50.47 \\
\hline S96T004420 & 153: 6 & Drainable liquid & 50.1 & 51 & 50.55 \\
\hline$\$ 96 \mathrm{T003975}$ & 153: 8 & Drainable liquid & 49.99 & 50.16 & 50.07 \\
\hline S96T003976 & 153: 9 & Drainable liquid & 50.51 & 50.33 & 50.42 \\
\hline S96T003977 & $153: 12$ & Drainable liquid & 49.68 & 50.25 & 49.96 \\
\hline$\$ 96 \mathrm{~T} 004039$ & Core 152 & Liquid composite & 53.57 & 46.67 & 50.12 \\
\hline \multicolumn{2}{|c|}{ Qollds. } & & \% & $18 \%$ & \%). \\
\hline$\$ 96 T 003731$ & 152: 1 & Upper half & 46.68 & 40.81 & 43.74 \\
\hline S96T003816 & \multirow[t]{2}{*}{$152: 14$} & Upper half & 47.82 & 46.02 & 46.92 \\
\hline \$96T003817 & & Lower half & 44.82 & 43.89 & 44.35 \\
\hline S96T003792 & \multirow[t]{2}{*}{$152: 16$} & Upper half & 44.31 & 43.63 & 43.97 \\
\hline S96T003793 & & Lower half & 42.04 & 44.58 & 43.31 \\
\hline S96T003794 & \multirow[t]{2}{*}{$152: 18$} & Upper half & 42.46 & 44.41 & 43.43 \\
\hline S96T003795 & & Lower half & 43.23 & 45.83 & 44.53 \\
\hline S96T003796 & \multirow[t]{2}{*}{$152: 20$} & Upper half & 45.15 & 46.04 & 45.59 \\
\hline S96T003797 & & Lower half & 36.14 & 39.33 & 37.73 \\
\hline S96T003798 & $152: 22$ & Upper half & 38.88 & 40.86 & 39.87 \\
\hline S96T004335 & $153: 14$ & Lower half & 46.87 & 45.88 & 46.37 \\
\hline
\end{tabular}


Table B2-24. Tank 241-AN-105 Analytical Results: Percent Water (TGA). (2 sheets)

\begin{tabular}{|c|c|c|c|c|c|}
\hline Shingly & \%ognole & 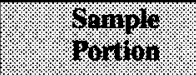 & Resulit & Bullicalo & 184n \\
\hline \multicolumn{2}{|c|}{ 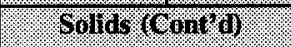 } & & 86. & 10. & $\%$ \\
\hline S96T004310 & \multirow[t]{2}{*}{$153: 15$} & Upper half & 47.55 & 48.04 & 47.79 \\
\hline S96T004311 & & Lower half & 52.82 & 50.12 & 51.47 \\
\hline S96T004435 & \multirow[t]{2}{*}{$153: 17$} & Upper half & 44.7 & 38.2 & 41.45 \\
\hline S96T004436 & & Lower half & 44.54 & 42.57 & 43.55 \\
\hline S96T004437 & $153: 19$ & Lower half & 46.16 & 44.59 & 45.37 \\
\hline S96T003981 & $153: 21$ & Lower half & 44.99 & 45.6 & 45.29 \\
\hline S96T003983 & \multirow[t]{2}{*}{$153: 22$} & Upper half & 44.17 & 43.15 & 43.66 \\
\hline S96T003982 & & Lower half & 45.87 & 45.21 & 45.54 \\
\hline S96T004254 & \multirow[t]{2}{*}{ Core 152} & Solid composite & 46.19 & 45.29 & 45.74 \\
\hline S96T004259 & & Solid composite & 42.61 & 42.87 & 42.74 \\
\hline S96T003740 & 152: 3 & Drainable liquid & 50.43 & 49.69 & 50.06 \\
\hline S96T003741 & $152: 4$ & Drainable liquid & 50.12 & 50.83 & 50.47 \\
\hline S96T003746 & 152:9 & Drainable liquid & 50.8 & 50.03 & 50.41 \\
\hline S96T003747 & $152: 10$ & Drainable liquid & 50.12 & 49.66 & 49.89 \\
\hline S96T003750 & $152: 13$ & Drainable liquid & 48.76 & 50.22 & 49.49 \\
\hline S96T003751 & $152: 14$ & Drainable liquid & 51.31 & 50.95 & 51.13 \\
\hline S96T004433 & 153: 1 & Drainable liquid & 49.71 & 49.3 & 49.50 \\
\hline
\end{tabular}


Table B2-25. Tank 241-AN-105 Analytical Results: Bulk Density (bulk density).

\begin{tabular}{|c|c|c|c|c|}
\hline Whoph & 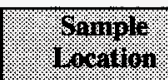 & (1. & Ressur & 110 \\
\hline \multicolumn{3}{|c|}{ Wolvit } & $8 / 111$ & $1 / 11 \%$ \\
\hline S96T003714 & 152: 1 & Upper half & 1.52 & 1.52 \\
\hline S96T003704 & $152: 14$ & Upper half & 1.53 & 1.53 \\
\hline S96T003706 & \multirow[t]{2}{*}{$152: 16$} & Upper half & 1.59 & 1.59 \\
\hline S96T003705 & & Lower half & 1.58 & 1.58 \\
\hline S96T003707 & \multirow[t]{2}{*}{$152: 18$} & Upper half & 1.66 & 1.66 \\
\hline S96T003708 & & Lower half & 1.64 & 1.64 \\
\hline S96T003710 & \multirow[t]{2}{*}{$152: 20$} & Upper half & 1.58 & 1.58 \\
\hline S96T003711 & & Lower half & 1.6 & 1.6 \\
\hline S96T003712 & $152: 22$ & Upper half & 1.64 & 1.64 \\
\hline S96T004334 & $153: 14$ & Lower half & 1.56 & 1.56 \\
\hline S96T004302 & \multirow[t]{2}{*}{$153: 15$} & Upper half & 1.52 & 1.52 \\
\hline S96T004303 & & \begin{tabular}{|l} 
Lower half \\
\end{tabular} & 1.56 & 1.56 \\
\hline S96T004428 & \multirow[t]{2}{*}{$153: 17$} & Upper half & 1.52 & 1.52 \\
\hline S96T004427 & & Lower half & 1.53 & 1.53 \\
\hline S96T004429 & $153: 19$ & Lower half & 1.56 & 1.56 \\
\hline S96T003971 & $153: 21$ & Lower half & 1.57 & 1.57 \\
\hline S96T003973 & \multirow[t]{2}{*}{$153: 22$} & Upper half & 1.58 & 1.58 \\
\hline S96T003972 & & Lower half & 1.6 & 1.6 \\
\hline S96T004207 & \multirow[t]{2}{*}{ Core 152} & Solid composite & 1.56 & 1.56 \\
\hline S96T004208 & & Solid composite & 1.67 & 1.67 \\
\hline
\end{tabular}


Table B2-26. Tank 241-AN-105 Analytical Results: Specific Gravity (SpG).

\begin{tabular}{|c|c|c|c|c|c|}
\hline $\begin{array}{l}\text { Samplo } \\
\text { Muniloc }\end{array}$ & \%orangion & . & 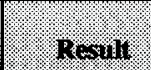 & Buploute & 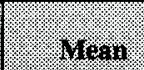 \\
\hline \multicolumn{3}{|c|}{ Hrivitu } & . intules. & inifss & linings \\
\hline S96T003732 & 152: 1 & Drainable liquid & 1.41 & 1.40 & 1.41 \\
\hline S96T003739 & $152: 2$ & Drainable liquid & 1.40 & 1.41 & 1.40 \\
\hline S96T003740 & 152: 3 & Drainable liquid & 1.40 & 1.41 & 1.40 \\
\hline S96T003741 & 152: 4 & Drainable liquid & 1.39 & 1.40 & 1.39 \\
\hline S96T003742 & 152: 5 & Drainable liquid & 1.41 & 1.40 & 1.41 \\
\hline$\$ 96 \mathrm{~T} 003743$ & 152: 6 & Drainable liquid & 1.39 & 1.40 & 1.40 \\
\hline S96T003744 & 152: 7 & Drainable liquid & 1.43 & 1.43 & 1.43 \\
\hline S96T003745 & 152: 8 & Drainable liquid & 1.34 & 1.35 & 1.35 \\
\hline S96T003746 & 152: 9 & Drainable liquid & 1.40 & 1.4 & 1.40 \\
\hline S96T003747 & $152: 10$ & Drainable liquid & 1.37 & 1.37 & 1.37 \\
\hline S96T003748 & $152: 11$ & Drainable liquid & 1.38 & 1.38 & 1.38 \\
\hline S96T003749 & $152: 12$ & Drainable liquid & 1.39 & 1.38 & 1.38 \\
\hline \$96T003750 & $152: 13$ & Drainable liquid & 1.37 & 1.38 & 1.38 \\
\hline S96T003751 & $152: 14$ & Drainable liquid & 1.45 & 1.39 & 1.39 \\
\hline S96T004433 & 153: 1 & Drainable liquid & 1.43 & 1.44 & 1.44 \\
\hline S96T004308 & 153: 2 & Drainable liquid & 1.53 & 1.45 & 1.49 \\
\hline S96T004434 & 153: 3 & Drainable liquid & 1.46 & 1.46 & 1.46 \\
\hline S96T004419 & 153: 5 & Drainable liquid & 1.44 & 1.43 & 1.43 \\
\hline S96T004420 & 153: 6 & Drainable liquid & 1.38 & 1.34 & 1.36 \\
\hline S96T004421 & 153: 7 & Drainable liquid & 1.51 & 1.50 & 1.50 \\
\hline S96T003975 & 153: 8 & Drainable liquid & 1.43 & 1.42 & 1.43 \\
\hline S96T003976 & 153: 9 & Drainable liquid & 1.47 & 1.44 & 1.46 \\
\hline S96T004309 & $153: 10$ & Drainable liquid & 1.36 & 1.46 & 1.41 \\
\hline S96T003979 & 153:11 & Drainable liquid & 1.46 & 1.44 & 1.45 \\
\hline S96T003977 & 153:12 & Drainable liquid & 1.46 & 1.42 & 1.44 \\
\hline S96T003978 & $153: 13$ & Drainable liquid & 1.47 & 1.47 & 1.47 \\
\hline S96T004333 & 153:14 & Drainable liquid & 1.45 & 1.44 & 1.44 \\
\hline S96T003980 & 153:21 & Drainable liquid & 1.41 & 1.41 & 1.41 \\
\hline S96T005546 & Core 152 & Liquid composite & 1.42 & 1.41 & 1.42 \\
\hline
\end{tabular}


Table B2-27. Tank 241-AN-105 Analytical Results: Aluminum (ICP). (3 sheets)

\begin{tabular}{|c|c|c|c|c|c|}
\hline 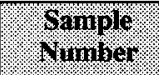 & Sample & 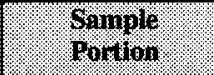 & misuli & Wullicatio & Menil \\
\hline \multicolumn{2}{|c|}{ Womas } & & 14918 & $40 \%$ & 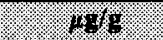 \\
\hline S96T003732 & $152: 1$ & Drainable liquid & $30,845.1$ & $30,140.8$ & $30,493^{\mathrm{QC}: \mathrm{c}}$ \\
\hline S96T003739 & 152: 2 & Drainable liquid & $28,802.8$ & $27,535.2$ & $28,169^{\mathrm{QC}: c}$ \\
\hline S96T003740 & 152: 3 & Drainable liquid & $28,802.8$ & $26,619.7$ & $27,711.3^{\mathrm{QC}: \mathrm{c}}$ \\
\hline S96T003741 & 152: 4 & Drainable liquid & $28,802.8$ & $27,746.5$ & $28,274.6^{\mathrm{QC}: \mathrm{d}}$ \\
\hline S96T003742 & 152: 5 & Drainable liquid & $29,859.2$ & $27,957.7$ & $28,908.5^{\mathrm{QC}: \mathrm{c}}$ \\
\hline S96T003743 & 152: 6 & Drainable liquid & $32,042.3$ & $33,732.4$ & $32,887.3^{\mathrm{QC} \mathrm{c}}$ \\
\hline S96T003744 & 152: 7 & Drainable liquid & $25,352.1$ & $28,028.2$ & $26,690.1^{\mathrm{QC}: \mathrm{d}}$ \\
\hline S96T003745 & 152: 8 & Drainable liquid & $26,408.5$ & $32,887.3$ & $29,647.9^{\text {QC d } \mathrm{e}}$ \\
\hline S96T003746 & 152: 9 & Drainable liquid & $29,084.5$ & $29,929.6$ & $29,507^{\text {QC:d }}$ \\
\hline S96T003747 & $152: 10$ & Drainable liquid & $29,295.8$ & $29,859.2$ & $29,577.5^{\mathrm{QC}: \mathrm{c}}$ \\
\hline S96T003748 & $152: 11$ & Drainable liquid & $29,225.4$ & $29,577.5$ & $29,401.4$ \\
\hline S96T003749 & $152: 12$ & Drainable liquid & $29,154.9$ & $28,380.3$ & $28,767.6^{\mathrm{QC}: \mathrm{c}}$ \\
\hline S96T003750 & $152: 13$ & Drainable liquid & $28,239.4$ & $28,028.2$ & $28,133.8^{\mathrm{QC}: \mathrm{d}}$ \\
\hline S96T003751 & $152: 14$ & Drainable liquid & $27,253.5$ & $27,535.2$ & $27,394.4^{\mathrm{QC}: \mathrm{e}}$ \\
\hline S96T004433 & 153: 1 & Drainable liquid & $32,887.3$ & $30,563.4$ & $31,725.4$ \\
\hline \$96T004308 & 153: 2 & Drainable liquid & $30,915.5$ & $29,929.6$ & $30,422.5$ \\
\hline S96T004434 & 153: 3 & Drainable liquid & $27,746.5$ & $29,718.3$ & $28,732.4^{\mathrm{QC}: \mathrm{d}}$ \\
\hline S96T004419 & $153: 5$ & Drainable liquid & $31,126.8$ & $30,774.6$ & $30,950.7^{\mathrm{eC} d \mathrm{~d}}$ \\
\hline S96T004420 & 153: 6 & Drainable liquid & $29,084.5$ & $31,408.5$ & $30,246.5^{\mathrm{QC}: \mathrm{d}}$ \\
\hline S96T004421 & 153: 7 & Drainable liquid & $29,929.6$ & $32,323.9$ & $31,126.8^{\mathrm{QC}: \mathrm{d}}$ \\
\hline S96T003975 & 153: 8 & Drainable liquid & $25,140.8$ & $24,225.4$ & $24,683.1^{\mathrm{QC}: \mathrm{d}}$ \\
\hline S96T003976 & 153: 9 & Drainable liquid & $26,267.6$ & $31,267.6$ & $28,767.6^{\mathrm{QC}: \mathrm{d}}$ \\
\hline S96T004309 & $153: 10$ & Drainable liquid & $30,422.5$ & $31,690.1$ & $31,056.3^{\mathrm{QC}: \mathrm{c}}$ \\
\hline S96T003979 & $153: 11$ & Drainable liquid & $30,211.3$ & $30,070.4$ & $30,140,8^{Q C: c}$ \\
\hline S96T003977 & $153: 12$ & Drainable liquid & $29,788.7$ & $30,563.4$ & $30,176,1^{\mathrm{QC}: \mathrm{d}}$ \\
\hline S96T003978 & $153: 13$ & Drainable liquid & $28,239.4$ & $26,056.3$ & $27,147.9$ \\
\hline S96T004333 & $153: 14$ & Drainable liquid & $31,197.2$ & $31,267.6$ & $31,232.4^{Q C: d}$ \\
\hline S96T003980 & $153: 21$ & Drainable liquid & $19,295.8$ & $19,014.1$ & $19,154.9^{\mathrm{QC}: c}$ \\
\hline S96T004831 & Core 152 & Liquid composite & $30,563.4$ & $30,845.1$ & $30,704.2^{d}$ \\
\hline
\end{tabular}


Table B2-27. Tank 241-AN-105 Analytical Results: Aluminum (ICP). (3 sheets)

\begin{tabular}{|c|c|c|c|c|c|}
\hline Wample & 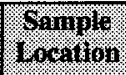 & 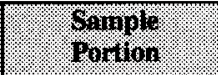 & nesill & Burileque & Mrenin \\
\hline \multicolumn{2}{|c|}{ 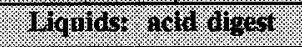 } & & $\sqrt{1898}$ & 40 & 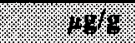 \\
\hline S96T004144 & Core 152 & Liquid Composite & $25,070.4$ & $25,211.3$ & $25,140.8$ \\
\hline \multicolumn{2}{|c|}{ 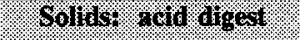 } & & 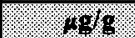 & 1018 & 1418 \\
\hline S96T003906 & $152: 1$ & Upper half & 25,100 & 24,800 & $24,950^{\mathrm{QC}: \mathrm{c}}$ \\
\hline S96T003898 & $152: 14$ & Upper half & 25,100 & 25,900 & 25,500 \\
\hline \$96T003828 & & Lower half & 25,500 & 25,800 & 25,650 \\
\hline S96T003899 & $152: 16$ & Upper half & 23,300 & 23,900 & 23,600 \\
\hline S96T003900 & & Lower half & 24,100 & 24,700 & 24,400 \\
\hline S96T003901 & $152: 18$ & Upper half & 23,100 & 21,900 & 22,500 \\
\hline S96T003902 & & Lower half & 23,100 & 24,100 & 23,600 \\
\hline S96T003903 & $152: 20$ & Upper half & 24,800 & 24,400 & 24,600 \\
\hline S96T003904 & & Lower half & 20,700 & 21,000 & 20,850 \\
\hline S96T003905 & $152: 22$ & Upper half & 19,400 & 19,600 & 19,500 \\
\hline S96T004338 & $153: 14$ & Lower half & 23,700 & 23,900 & 23,800 \\
\hline S96T004315 & $153: 15$ & Upper half & 23,100 & 22,300 & 22,700 \\
\hline S96T004318 & & Lower half & 23,300 & 23,200 & 23,250 \\
\hline S96T004444 & $153: 17$ & Upper half & 22,400 & 22,900 & 22,650 \\
\hline S96T004445 & & Lower half & 21,600 & 21,400 & 21,500 \\
\hline S96T004446 & $153: 19$ & Lower half & 20,900 & 21,200 & 21,050 \\
\hline S96T003988 & $153: 21$ & Lower half & 19,200 & 19,000 & 19,100 \\
\hline \$96T003989 & $153: 22$ & Upper half & 19,300 & 20,800 & 20,050 \\
\hline S96T003987 & & Lower half & 15,200 & 16,500 & $15,850^{\mathrm{QC}: \mathrm{c}}$ \\
\hline S96T004257 & Core 152 & Solid composite & 20,200 & 20,700 & 20,450 \\
\hline S96T004262 & & Solid composite & 15,200 & 13,700 & 14,450 \\
\hline
\end{tabular}


Table B2-27. Tank 241-AN-105 Analytical Results: Aluminum (ICP). (3 sheets)

\begin{tabular}{|c|c|c|c|c|c|}
\hline $\begin{array}{l}\text { Sample } \\
\text { Nomer }\end{array}$ & 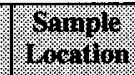 & 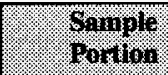 & 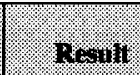 & Bupligate & $1,1.411$ \\
\hline Sollist & Now1. & 6. & $40 / 9$ & $18 \%$ & 1019 \\
\hline S96T003893 & 152: 1 & Upper half & 25,600 & 24,400 & 25,000 \\
\hline S96T003894 & $152: 14$ & Upper half & 25,900 & 25,300 & 25,600 \\
\hline S96T003827 & & Lower half & 25,400 & 26,300 & 25,850 \\
\hline S96T003895 & $152: 16$ & Upper half & 24,300 & 24,000 & 24,150 \\
\hline S96T003889 & & Lower half & 24,800 & 24,200 & 24,500 \\
\hline S96T003896 & $152: 18$ & Upper half & 22,100 & 22,700 & 22,400 \\
\hline S96T003890 & & Lower half & 23,800 & 24,000 & 23,900 \\
\hline S96T003897 & $152: 20$ & Upper half & 23,600 & 23,200 & 23,400 \\
\hline S96T003891 & & Lower half & 19,100 & 18,400 & 18,750 \\
\hline S96T003892 & $152: 22$ & Upper half & 21,800 & 21,900 & 21,850 \\
\hline S96T004337 & $153: 14$ & Lower half & 27,100 & 26,000 & 26,550 \\
\hline S96T004314 & $153: 15$ & Upper half & 24,900 & 24,500 & 24,700 \\
\hline S96T004317 & & Lower half & 25,100 & 22,700 & 23,900 \\
\hline S96T004441 & $153: 17$ & Upper half & 24,200 & 24,500 & 24,350 \\
\hline S96T004442 & & Lower half & 24,600 & 24,300 & 24,450 \\
\hline S96T004443 & $153: 19$ & Lower half & 23,600 & 24,700 & 24,150 \\
\hline S96T003984 & $153: 21$ & Lower half & 22,200 & 22,900 & 22,550 \\
\hline S96T003986 & $153: 22$ & Upper half & 19,000 & 20,300 & 19,650 \\
\hline S96T003985 & & Lower half & 13,800 & 14,300 & 14,050 \\
\hline
\end{tabular}


Table B2-28. Tank 241-AN-105 Analytical Results: Bismuth (ICP). (3 sheets)

\begin{tabular}{|c|c|c|c|c|c|}
\hline $\begin{array}{l}\text { Sominl } \\
\text { Mrinot }\end{array}$ & Somple & 6.62010 & 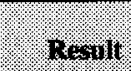 & Thollonte & 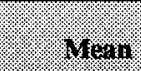 \\
\hline \multicolumn{2}{|c|}{ Mruirs } & & 48 & 1868 & $4 y_{6}$ \\
\hline S96T003732 & 152: 1 & Drainable liquid & $<28.24$ & $<28.24$ & $<28.24$ \\
\hline S96T003739 & 152: 2 & Drainable liquid & $<28.24$ & $<28.24$ & $<28.24$ \\
\hline S96T003740 & $152: 3$ & Drainable liquid & $<28.24$ & $<28.24$ & $<28.24$ \\
\hline S96T003741 & 152: 4 & Drainable liquid & $<28.24$ & $<28.24$ & $<28.24$ \\
\hline S96T003742 & $152: 5$ & Drainable liquid & $<28.24$ & $<28.24$ & $<28.24$ \\
\hline S96T003743 & 152: 6 & Drainable liquid & $<28.24$ & $<28.24$ & $<28.24$ \\
\hline S96T003744 & $152: 7$ & Drainable liquid & $<28.24$ & $<28.24$ & $<28.24$ \\
\hline S96T003745 & $152: 8$ & Drainable liquid & $<28.24$ & $<28.24$ & $<28.24$ \\
\hline S96T003746 & 152: 9 & Drainable liquid & $<28.24$ & $<28.24$ & $<28.24$ \\
\hline S96T003747 & $152: 10$ & Drainable liquid & $<42.32$ & $<42.32$ & $<42.32$ \\
\hline S96T003748 & $152: 11$ & Drainable liquid & $<42.32$ & $<42.32$ & $<42.32$ \\
\hline S96T003749 & $152: 12$ & Drainable liquid & $<42.32$ & $<42.32$ & $<42.32$ \\
\hline S96T003750 & $152: 13$ & Drainable liquid & $<42.32$ & $<42.32$ & $<42.32$ \\
\hline S96T003751 & $152: 14$ & Drainable liquid & $<28.24$ & $<28.24$ & $<28.24$ \\
\hline S96T004433 & 153: 1 & Drainable liquid & $<28.24$ & $<28.24$ & $<28.24$ \\
\hline S96T004308 & 153: 2 & Drainable liquid & $<42.32$ & $<42.32$ & $<42.32$ \\
\hline S96T004434 & 153: 3 & Drainable liquid & $<28.24$ & $<28.24$ & $<28.24$ \\
\hline S96T004419 & 153: 5 & Drainable liquid & $<42.32$ & $<42.32$ & $<42.32$ \\
\hline S96T004420 & 153: 6 & Drainable liquid & $<42.32$ & $<42.32$ & $<42.32$ \\
\hline S96T004421 & 153: 7 & Drainable liquid & $<28.24$ & $<28.24$ & $<28.24$ \\
\hline S96T003975 & 153: 8 & Drainable liquid & $<28.24$ & $<28.24$ & $<28.24$ \\
\hline S96T003976 & 153: 9 & Drainable liquid & $<28.24$ & $<28.24$ & $<28.24$ \\
\hline S96T004309 & $153: 10$ & Drainable liquid & $<42.32$ & $<42.32$ & $<42.32$ \\
\hline S96T003979 & 153:11 & Drainable liquid & $<42.32$ & $<42.32$ & $<42.32$ \\
\hline S96T003977 & $153: 12$ & Drainable liquid & $<42.32$ & $<42.32$ & $<42.32$ \\
\hline S96T003978 & $153: 13$ & Drainable liquid & $<42.32$ & $<42.32$ & $<42.32$ \\
\hline S96T004333 & $153: 14$ & Drainable liquid & $<42.32$ & $<42.32$ & $<42.32$ \\
\hline S96T003980 & $153: 21$ & Drainable liquid & $<42.32$ & $<42.32$ & $<42.32$ \\
\hline S96T004831 & Core 152 & Liquid composite & $<42.32$ & $<42.32$ & $<42.32$ \\
\hline
\end{tabular}


Table B2-28. Tank 241-AN-105 Analytical Results: Bismuth (ICP). (3 sheets)

\begin{tabular}{|c|c|c|c|c|c|}
\hline Sampio & Wample & Sample & $\mathrm{R}=\mathrm{nil}$ & Diplicate & ncan \\
\hline \multicolumn{2}{|c|}{ IFgmar ard digesi } & & $121 \%$ & $10 \%$ & 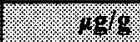 \\
\hline S96T004144 & Core 152 & Liquid composite & $<36.90$ & $<35.21$ & $<36.05$ \\
\hline \multicolumn{2}{|c|}{ Sollifo rqug digast } & & 1098 & 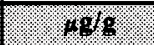 & $6 \%$ \\
\hline S96T003906 & 152: 1 & Upper half & $<38.6$ & $<40.3$ & $<39.45$ \\
\hline S96T003898 & \multirow[t]{2}{*}{$152: 14$} & Upper half & $<39.3$ & $<38.8$ & $<39.05$ \\
\hline S96T003828 & & Lower half & $<40.4$ & $<39.3$ & $<39.85$ \\
\hline \$96T003899 & \multirow[t]{2}{*}{$152: 16$} & Upper half & $<39.6$ & $<39.8$ & $<39.7$ \\
\hline S96T003900 & & Lower half & $<39.3$ & $<39.3$ & $<39.3$ \\
\hline \$96T003901 & \multirow[t]{2}{*}{$152: 18$} & Upper half & $<38.9$ & $<39.6$ & $<39.25$ \\
\hline S96T003902 & & Lower half & $<40$ & $<40.4$ & $<40.2$ \\
\hline S96T003903 & \multirow[t]{2}{*}{$152: 20$} & Upper half & $<38.3$ & $<37.6$ & $<37.95$ \\
\hline S96T003904 & & Lower half & $<37.5$ & $<37.1$ & $<37.3$ \\
\hline \$96T003905 & $152: 22$ & Upper half & $<39.6$ & $<41.4$ & $<40.5$ \\
\hline S96T004338 & $153: 14$ & Lower half & $<46.7$ & $<47.8$ & $<47.25$ \\
\hline S96T004315 & \multirow[t]{2}{*}{$153: 15$} & Upper half & $<47.6$ & $<47.8$ & $<47.7$ \\
\hline S96T004318 & & Lower half & $<47.9$ & $<45.1$ & $<46.5$ \\
\hline S96T004444 & \multirow[t]{2}{*}{$153: 17$} & Upper half & $<48.3$ & $<47.5$ & $<47.9$ \\
\hline S96T004445 & & Lower half & $<37.1$ & $<36.6$ & $<36.85$ \\
\hline S96T004446 & $153: 19$ & Lower half & $<38.2$ & $<38.2$ & $<38.2$ \\
\hline S96T003988 & $153: 21$ & Lower half & $<35.6$ & $<35.6$ & $<35.6$ \\
\hline S96T003989 & \multirow[t]{2}{*}{$153: 22$} & Upper half & $<46.5$ & $<46.2$ & $<46.35$ \\
\hline S96T003987 & & Lower half & 62.6 & 71.8 & 67.2 \\
\hline S96T004257 & \multirow[t]{2}{*}{ Core 152} & Solid composite & $<38.3$ & $<40.3$ & $<39.3^{\mathrm{QC}: \mathrm{a}}$ \\
\hline S96T004262 & & Solid composite & $<38.9$ & $<37$ & $<37.95$ \\
\hline
\end{tabular}


Table B2-28. Tank 241-AN-105 Analytical Results: Bismuth (ICP). (3 sheets)

\begin{tabular}{|c|c|c|c|c|c|}
\hline Sample & sample & omomis & ntwill & Buflistro & Herin \\
\hline Wolds & frow & & $10 \%$ & 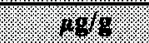 & 40 \\
\hline S96T003893 & 152: 1 & Upper half & $<1,980$ & $<2,010$ & $<1,995$ \\
\hline S96T003894 & \multirow[t]{2}{*}{$152: 14$} & Upper half & $<2,040$ & $<2,000$ & $<2,020$ \\
\hline S96T003827 & & Lower half & $<2,010$ & $<2,060$ & $<2,035$ \\
\hline S96T003895 & \multirow[t]{2}{*}{$152: 16$} & Upper half & $<2,040$ & $<2,010$ & $<2,025$ \\
\hline S96T003889 & & Lower half & $<2,030$ & $<2,000$ & $<2,015$ \\
\hline S96T003896 & \multirow[t]{2}{*}{$152: 18$} & Upper half & $<2,070$ & $<2,010$ & $<2,040$ \\
\hline \$96T003890 & & Lower half & $<2,060$ & $<2,070$ & $<2,065$ \\
\hline S96T003897 & \multirow[t]{2}{*}{$152: 20$} & Upper half & $<2,120$ & $<2,070$ & $<2,095$ \\
\hline S96T003891 & & Lower half & $<2,040$ & $<2,060$ & $<2,050$ \\
\hline S96T003892 & $152: 22$ & Upper half & $<2,090$ & $<2,120$ & $<2,105$ \\
\hline S96T004337 & $153: 14$ & Lower half & $<2,030$ & $<2,030$ & $<2,030$ \\
\hline S96T004314 & \multirow[t]{2}{*}{$153: 15$} & Upper half & $<1,910$ & $<1,920$ & $<1,915$ \\
\hline S96T004317 & & Lower half & $<2,000$ & $<2,030$ & $<2,015$ \\
\hline \$96Т004441 & \multirow[t]{2}{*}{$153: 17$} & Upper half & $<1,960$ & $<1,960$ & $<1,960$ \\
\hline S96T004442 & & Lower half & $<2,010$ & $<2,000$ & $<2,005$ \\
\hline S96T004443 & $153: 19$ & Lower half & $<2,020$ & $<2,000$ & $<2,010$ \\
\hline S96T003984 & $153: 21$ & Lower half & $<2,010$ & $<2,010$ & $<2,010$ \\
\hline S96T003986 & \multirow[t]{2}{*}{$153: 22$} & Upper half & $<2,000$ & $<2,030$ & $<2,015$ \\
\hline S96T003985 & & Lower half & $<2,010$ & $<2,010$ & $<2,010$ \\
\hline
\end{tabular}


Table B2-29. Tank 241-AN-105 Analytical Results: Boron (ICP). (3 sheets)

\begin{tabular}{|c|c|c|c|c|c|}
\hline Kumpor & Y.s.mple & $6011181 \%$ & 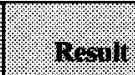 & Hoplisare & Hen \\
\hline \multicolumn{2}{|c|}{ 19pinis } & & 1018 & 12818 & 1.818 \\
\hline S96T003732 & 152: 1 & Drainable liquid & 50.98 & 49.57 & 50.28 \\
\hline S96T003739 & 152: 2 & Drainable liquid & 52.04 & 51.12 & 51.58 \\
\hline S96T003740 & 152: 3 & Drainable liquid & 55.21 & 52.60 & 53.90 \\
\hline S96T003741 & 152: 4 & Drainable liquid & 54.22 & 50.14 & 52.18 \\
\hline S96T003742 & $152: 5$ & Drainable liquid & 51.47 & 47.95 & 49.71 \\
\hline S96T003743 & 152: 6 & Drainable liquid & 54.64 & 59.01 & 56.83 \\
\hline S96T003744 & 152: 7 & Drainable liquid & 44.78 & 50.42 & 47.60 \\
\hline S96T003745 & 152: 8 & Drainable liquid & 52.67 & 65.07 & $58.87^{\mathrm{QC:e}}$ \\
\hline S96T003746 & 152: 9 & Drainable liquid & 53.38 & 53.38 & 53.38 \\
\hline S96T003747 & $152: 10$ & Drainable liquid & 54.29 & 56.40 & 55.35 \\
\hline S96T003748 & $152: 11$ & Drainable liquid & 53.66 & 55.56 & 54.61 \\
\hline S96T003749 & $152: 12$ & Drainable liquid & 54.29 & 52.81 & 53.55 \\
\hline S96T003750 & $152: 13$ & Drainable liquid & 48.45 & 50.49 & 49.47 \\
\hline S96T003751 & $152: 14$ & Drainable liquid & 53.23 & 52.81 & 53.02 \\
\hline S96T004433 & 153: 1 & Drainable liquid & 53.16 & 51.90 & 52.53 \\
\hline S96T004308 & 153: 2 & Drainable liquid & 52.81 & 52.39 & 52.60 \\
\hline S96T004434 & 153: 3 & Drainable liquid & 46.05 & 53.80 & 49.92 \\
\hline \begin{tabular}{|l|} 
S96T004419 \\
\end{tabular} & 153: 5 & Drainable liquid & 54.36 & 51.90 & 53.13 \\
\hline S96T004420 & 153: 6 & Drainable liquid & 48.66 & 53.94 & 51.30 \\
\hline \begin{tabular}{|l|} 
S96T004421 \\
\end{tabular} & 153: 7 & Drainable liquid & 51.33 & 56.19 & 53.76 \\
\hline S96T003975 & 153: 8 & Drainable liquid & 47.46 & 46.61 & 47.04 \\
\hline S96T003976 & 153:9 & Drainable liquid & 46.54 & 56.83 & 51.69 \\
\hline$\$ 967004309$ & $153: 10$ & Drainable liquid & 51.54 & 54.71 & 53.13 \\
\hline S96T003979 & $153: 11$ & Drainable liquid & 52.46 & 54.71 & 53.59 \\
\hline \$96T003977 & $153: 12$ & Drainable liquid & 53.23 & 53.66 & 53.45 \\
\hline S96T003978 & $153: 13$ & Drainable liquid & 50.14 & 45.35 & 47.74 \\
\hline S96T004333 & $153: 14$ & Drainable liquid & 54.78 & 52.53 & 53.66 \\
\hline S96T003980 & 153:21 & Drainable liquid & 44.08 & 42.18 & 43.13 \\
\hline S96T004831 & Core 152 & Liquid composite & 52.25 & 50.21 & 51.23 \\
\hline
\end{tabular}


Table B2-29. Tank 241-AN-105 Analytical Results: Boron (ICP). (3 sheets)

\begin{tabular}{|c|c|c|c|c|c|}
\hline Whinglo & Wamplo & 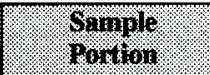 & $24+111$ & bujlicale & 10011 \\
\hline \multicolumn{2}{|c|}{ Irquirs act digest? } & & $\% 18$ & 496 & 68 \\
\hline \$96T004144 & Core 152 & Liquid composite & 109.85 & 128.87 & $119.36^{\mathrm{QC:f}}$ \\
\hline \multicolumn{2}{|c|}{ 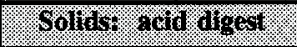 } & & $\%$ & $4 t_{5}$ & 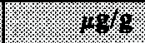 \\
\hline S96T003906 & 152: 1 & Upper half & 97.4 & 111 & 104.2 \\
\hline S96T003898 & \multirow[t]{2}{*}{$152: 14$} & Upper half & 84.6 & 92.7 & 88.65 \\
\hline S96T003828 & & Lower half & 88.7 & 90 & 89.35 \\
\hline S96T003899 & \multirow[t]{2}{*}{$152: 16$} & Upper half & 59.3 & 67.7 & 63.5 \\
\hline S96T003900 & & Lower half & 58.6 & 76.7 & $67.65^{\mathrm{QC:e}}$ \\
\hline S96T003901 & \multirow[t]{2}{*}{$152: 18$} & Upper half & 72.5 & 83.8 & 78.15 \\
\hline S96T003902 & & Lower half & 108 & 69.6 & $88.8^{\mathrm{QC:b}, c}$ \\
\hline S96T003903 & \multirow[t]{2}{*}{$152: 20$} & Upper half & 89.6 & 77.3 & $83.45^{\mathrm{QC}: b}$ \\
\hline S96T003904 & & Lower half & 66.1 & 66 & $66.05^{\mathrm{QC}: \mathrm{b}}$ \\
\hline S96T003905 & $152: 22$ & Upper half & 60.7 & 63 & $61.85^{\mathrm{QC}: \mathrm{b}}$ \\
\hline S96T004338 & $153: 14$ & Lower half & 115 & 77.2 & $96.1^{\mathrm{QCe} e}$ \\
\hline S96T004315 & \multirow[t]{2}{*}{$153: 15$} & Upper half & 103 & 84.5 & 93.75 \\
\hline S96T004318 & & Lower half & 104 & 91.2 & 97.6 \\
\hline S96T004444 & \multirow[t]{2}{*}{$153: 17$} & Upper half & 103 & 147 & $125^{\mathrm{QC}: \mathrm{e}}$ \\
\hline S96T004445 & & Lower half & 75.7 & 115 & $95.35^{\mathrm{QC}: \mathrm{e}}$ \\
\hline S96T004446 & 153:19 & Lower half & 119 & 108 & 113.5 \\
\hline S96T003988 & $153: 21$ & Lower half & 81.9 & 102 & $91.95^{\text {QC:e }}$ \\
\hline S96T003989 & \multirow[t]{2}{*}{$153: 22$} & Upper half & 82.3 & 97.5 & 89.9 \\
\hline S96T003987 & & Lower half & 144 & 109 & $126.5^{\mathrm{QC}: \mathrm{e}}$ \\
\hline S96T004257 & \multirow[t]{2}{*}{ Core 152} & Solid composite & 105 & 128 & 116.5 \\
\hline S96T004262 & & Solid composite & 136 & 91.2 & $113.6^{\mathrm{QC}: \mathrm{e}}$ \\
\hline
\end{tabular}


Table B2-29. Tank 241-AN-105 Analytical Results: Boron (ICP). (3 sheets)

\begin{tabular}{|c|c|c|c|c|c|}
\hline $\begin{array}{l}\text { Sample } \\
\text { Niminer. }\end{array}$ & Siriple & $\begin{array}{l}\text { Sranple } \\
\text { romtlon }\end{array}$ & Re-1i1) & Doplicute. & Mran \\
\hline Solids: & Wron & 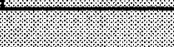 & $1.498 \%$ & mors & 1908 \\
\hline S96T003893 & $152: 1$ & Upper half & $<988$ & $<1,010$ & $<999$ \\
\hline S96T003894 & \multirow[t]{2}{*}{$152: 14$} & Upper half & $<1,020$ & $<998$ & $<1,009$ \\
\hline S96T003827 & & Lower half & $<1,010$ & $<1,030$ & $<1,020$ \\
\hline S96T003895 & \multirow[t]{2}{*}{$152: 16$} & Upper half & $<1,020$ & $<1,000$ & $<1,010$ \\
\hline S96T003889 & & Lower half & $<1,010$ & $<1,000$ & $<1,005$ \\
\hline S96T003896 & \multirow[t]{2}{*}{$152: 18$} & Upper half & $<1,030$ & $<1,000$ & $<1,015$ \\
\hline S96T003890 & & Lower half & $<1,030$ & $<1,040$ & $<1,035$ \\
\hline S96T003897 & \multirow[t]{2}{*}{$152: 20$} & Upper half & $<1,060$ & $<1,040$ & $<1,050$ \\
\hline S96T003891 & & Lower half & $<1,020$ & $<1,030$ & $<1,025$ \\
\hline S96T003892 & $152: 22$ & Upper half & $<1,040$ & $<1,060$ & $<1,050$ \\
\hline S96T004337 & $153: 14$ & Lower half & $<1,020$ & $<1,010$ & $<1,015$ \\
\hline S96T004314 & \multirow[t]{2}{*}{$153: 15$} & Upper half & $<953$ & $<958$ & $<955.5$ \\
\hline S96T004317 & & Lower half & $<998$ & $<1,020$ & $<1,009$ \\
\hline S96T004441 & \multirow[t]{2}{*}{$153: 17$} & Upper half & $<980$ & $<982$ & $<981$ \\
\hline S96T004442 & & Lower half & $<1,010$ & $<1,000$ & $<1,005$ \\
\hline S96T004443 & $153: 19$ & Lower half & $<1,010$ & $<1,000$ & $<1,005$ \\
\hline S96T003984 & $153: 21$ & Lower half & $<1,010$ & $<1,000$ & $<1,005$ \\
\hline S96T003986 & \multirow[t]{2}{*}{$153: 22$} & Upper half & $<999$ & $<1,020$ & $<1,009.5$ \\
\hline S96T003985 & & Lower half & $<1,010$ & $<1,010$ & $<1,010$ \\
\hline
\end{tabular}


Table B2-30. Tank 241-AN-105 Analytical Results: Cadmium (ICP). (3 sheets)

\begin{tabular}{|c|c|c|c|c|c|}
\hline Mample & Marpolo & Whapros & Resilin & Borbllarie & 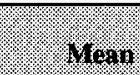 \\
\hline \multicolumn{2}{|c|}{ Wring } & & 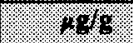 & $\%$ & 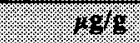 \\
\hline S96T003732 & 152: 1 & Drainable liquid & $<1.41$ & $<1.41$ & $<1.40$ \\
\hline S96T003739 & 152: 2 & Drainable liquid & $<1.41$ & $<1.41$ & $<1.40$ \\
\hline S96T003740 & 152: 3 & Drainable liquid & $<1.41$ & $<1.41$ & $<1.40$ \\
\hline S96T003741 & 152: 4 & Drainable liquid & $<1.41$ & $<1.41$ & $<1.40$ \\
\hline S96T003742 & 152: 5 & Drainable liquid & $<1.41$ & $<1.41$ & $<1.40$ \\
\hline S96T003743 & 152: 6 & Drainable liquid & $<1.41$ & $<1.41$ & $<1.40$ \\
\hline S96T003744 & 152: 7 & Drainable liquid & $<1.41$ & $<1.41$ & $<1.40$ \\
\hline S96T003745 & 152: 8 & Drainable liquid & $<1.41$ & $<1.41$ & $<1.40$ \\
\hline S96T003746 & 152: 9 & Drainable liquid & $<1.41$ & $<1.41$ & $<1.40$ \\
\hline S96T003747 & $152: 10$ & Drainable liquid & $<2.11$ & $<2.11$ & $<2.11$ \\
\hline S96T003748 & 152:11 & Drainable liquid & $<2.11$ & $<2.11$ & $<2.11$ \\
\hline \$96T003749 & $152: 12$ & Drainable liquid & $<2.11$ & $<2.11$ & $<2.11$ \\
\hline S96T003750 & $152: 13$ & Drainable liquid & $<2.11$ & $<2.11$ & $<2.11$ \\
\hline S96T003751 & $152: 14$ & Drainable liquid & $<1.40$ & $<1.40$ & $<1.40$ \\
\hline S96T004433 & 153: 1 & Drainable liquid & $<1.40$ & $<1.40$ & $<1.40$ \\
\hline \$96T004308 & 153: 2 & Drainable liquid & $<2.11$ & $<2.11$ & $<2.11$ \\
\hline S96T004434 & 153: 3 & Drainable liquid & $<1.40$ & $<1.40$ & $<1.40$ \\
\hline S96T004419 & 153: 5 & Drainable liquid & $<2.11$ & $<2.11$ & $<2.11$ \\
\hline S96T004420 & 153: 6 & Drainable liquid & $<2.11$ & $<2.11$ & $<2.11$ \\
\hline S96T004421 & 153: 7 & Drainable liquid & $<1.40$ & $<1.40$ & $<1.40$ \\
\hline S96T003975 & 153: 8 & Drainable liquid & $<1.40$ & $<1.40$ & $<1.40$ \\
\hline$\$ 967003976$ & 153:9 & Drainable liquid & $<1.40$ & $<1.40$ & $<1.40$ \\
\hline S96T004309 & $153: 10$ & Drainable liquid & $<2.11$ & $<2.11$ & $<2.11$ \\
\hline S96T003979 & 153:11 & Drainable liquid & $<2.11$ & $<2.11$ & $<2.11$ \\
\hline S96T003977 & $153: 12$ & Drainable liquid & $<2.11$ & $<2.11$ & $<2.11$ \\
\hline S96T003978 & $153: 13$ & Drainable liquid & $<2.11$ & $<2.11$ & $<2.11$ \\
\hline \$96T004333 & 153:14 & Drainable liquid & $<2.11$ & $<2.11$ & $<2.11$ \\
\hline S96T003980 & $153: 21$ & Drainable liquid & $<2.11$ & $<2.11$ & $<2.11$ \\
\hline
\end{tabular}


Table B2-30. Tank 241-AN-105 Analytical Results: Cadmium (ICP). (3 sheets)

\begin{tabular}{|c|c|c|c|c|c|}
\hline $\begin{array}{l}\text { Wamelo } \\
\text { WHinos }\end{array}$ & opaple & 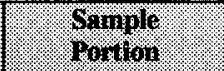 & $1 \times 0111$ & Dupllatis. & 444 \\
\hline S96T004831 & Core 152 & Liquid composite & $<2.11$ & $<2.11$ & $<2.11$ \\
\hline \multicolumn{2}{|c|}{ 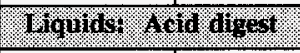 } & 6 & 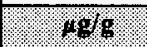 & $\sqrt{1}+496$ & 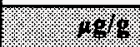 \\
\hline S96t004144 & Core 152 & Liquid Composite & $<1.84507$ & $<1.76056$ & $<1.80282$ \\
\hline \multicolumn{2}{|c|}{ 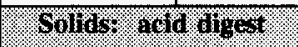 } & 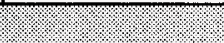 & 1.6 .18 & 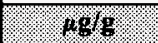 & 48 \\
\hline \$96T003906 & 152: 1 & Upper half & 3.31 & 3.76 & 3.53 \\
\hline S96T003898 & \multirow{2}{*}{$152: 14$} & Upper half & 5.73 & 5.39 & 5.56 \\
\hline S96T003828 & & Lower half & 4.82 & 5.9 & $5.36^{\mathrm{QC}: \mathrm{e}}$ \\
\hline S96T003899 & \multirow[t]{2}{*}{$152: 16$} & Upper half & 9.5 & 9.61 & 9.55 \\
\hline S96T003900 & & Lower half & 10.8 & 11.6 & 11.2 \\
\hline S96T003901 & \multirow[t]{2}{*}{$152: 18$} & Upper half & 20.9 & 19.4 & 20.15 \\
\hline S96T003902 & & Lower half & 12.9 & 13 & 12.95 \\
\hline S96T003903 & \multirow[t]{2}{*}{$152: 20$} & Upper half & 13.6 & 13.2 & 13.4 \\
\hline S96T003904 & & Lower half & 7 & 7.01 & 7.00 \\
\hline S96T003905 & $152: 22$ & Upper half & 8.52 & 8.41 & 8.46 \\
\hline S96T004338 & $153: 14$ & Lower half & 7.57 & 8.81 & 8.19 \\
\hline S96T004315 & \multirow[t]{2}{*}{$153: 15$} & Upper haif & 5.49 & 5.24 & 5.36 \\
\hline S96T004318 & & Lower half & 5.04 & 5.06 & 5.05 \\
\hline S96T004444 & \multirow[t]{2}{*}{$153: 17$} & Upper half & 10.8 & 12.5 & 11.65 \\
\hline$S 96 \mathrm{~T} 004445$ & & Lower half & 18.4 & 21.6 & 20 \\
\hline S96T004446 & $153: 19$ & Lower half & 13.3 & 13.7 & 13.5 \\
\hline S96T003988 & $153: 21$ & Lower half & 4.44 & 4.64 & 4.54 \\
\hline S96T003989 & \multirow[t]{2}{*}{$153: 22$} & Upper half & 11.4 & 9.85 & 10.62 \\
\hline S96T003987 & & Lower half & 36.9 & 40.1 & 38.5 \\
\hline S96T004257 & \multirow[t]{2}{*}{ Core 152} & Solid composite & 8.13 & 6.46 & 7.29 \\
\hline S96T004262 & & Solid composite & 14.4 & 13.3 & 13.85 \\
\hline
\end{tabular}


Table B2-30. Tank 241-AN-105 Analytical Results: Cadmium (ICP). (3 sheets)

\begin{tabular}{|c|c|c|c|c|c|}
\hline Gavple & Gample & Somple & nt,nlt & Mollenic & $404 \mathrm{n}$ \\
\hline Bollas & 14013 & . & $10 \%$ & 40 & $40 \%$ \\
\hline \$96T003893 & 152: 1 & Upper half & $<98.8$ & $<101$ & $<99.9$ \\
\hline S96T003894 & \multirow[t]{2}{*}{$152: 14$} & Upper half & $<102$ & $<99.8$ & $<100.9$ \\
\hline S96T003827 & & Lower half & $<101$ & $<103$ & $<102$ \\
\hline S96T003895 & \multirow[t]{2}{*}{$152: 16$} & Upper half & $<102$ & $<100$ & $<101$ \\
\hline S96T003889 & & Lower half & $<101$ & $<100$ & $<100.5$ \\
\hline S96T003896 & \multirow[t]{2}{*}{$152: 18$} & Upper half & $<103$ & $<100$ & $<101.5$ \\
\hline S96T003890 & & Lower half & $<103$ & $<104$ & $<103.5$ \\
\hline S96T003897 & \multirow[t]{2}{*}{$152: 20$} & Upper half & $<106$ & $<104$ & $<105$ \\
\hline S96T003891 & & Lower half & $<102$ & $<103$ & $<102.5$ \\
\hline S96T003892 & $152: 22$ & Upper half & $<104$ & $<106$ & $<105$ \\
\hline S96T004337 & 153:14 & Lower half & $<102$ & $<101$ & $<101.5$ \\
\hline S96T004314 & \multirow[t]{2}{*}{$153: 15$} & Upper half & $<95.3$ & $<95.8$ & $<95.55$ \\
\hline S96T004317 & & Lower half & $<99.8$ & $<102$ & $<100.9$ \\
\hline S96T004441 & \multirow[t]{2}{*}{$153: 17$} & Upper half & $<98$ & $<98.2$ & $<98.1$ \\
\hline \$96T004442 & & Lower half & $<101$ & $<100$ & $<100.5$ \\
\hline S96T004443 & $153: 19$ & Lower half & $<101$ & $<100$ & $<100.5$ \\
\hline S96T003984 & $153: 21$ & Lower half & $<101$ & $<100$ & $<100.5$ \\
\hline S96T003986 & \multirow[t]{2}{*}{$153: 22$} & Upper half & $<99.9$ & $<102$ & $<100.95$ \\
\hline S96T003985 & & Lower half & $<101$ & $<101$ & $<101$ \\
\hline
\end{tabular}


Table B2-31. Tank 241-AN-105 Analytical Results: Calcium (ICP). (3 sheets)

\begin{tabular}{|c|c|c|c|c|c|}
\hline Gampor & Wanple & $80 \%$ worle & (r. $=11 \%$ & Diglicats & $\sqrt{169 n}$ \\
\hline \multicolumn{2}{|c|}{ Wignids } & & 6458 & $128 \%$ & 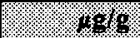 \\
\hline S96T003732 & 152: 1 & Drainable liquid & $<28.23$ & $<28.23$ & $<28.23$ \\
\hline S96T003739 & $152: 2$ & Drainable liquid & $<28.23$ & $<28.23$ & $<28.23$ \\
\hline S96T003740 & 152: 3 & Drainable liquid & $<28.23$ & $<28.23$ & $<28.23$ \\
\hline S96T003741 & 152: 4 & Drainable liquid & $<28.23$ & $<28.23$ & $<28.23$ \\
\hline S96T003742 & 152: 5 & Drainable liquid & $<28.23$ & $<28.23$ & $<28.23$ \\
\hline S96T003743 & $152: 6$ & Drainable liquid & $<28.23$ & $<28.23$ & $<28.23$ \\
\hline S96T003744 & $152: 7$ & Drainable liquid & $<28.23$ & $<28.23$ & $<28.23$ \\
\hline S96T003745 & $152: 8$ & Drainable liquid & $<28.23$ & $<28.23$ & $<28.23$ \\
\hline S96T003746 & 152: 9 & Drainable liquid & $<28.23$ & $<28.23$ & $<28.23$ \\
\hline \$96T003747 & $152: 10$ & Drainable liquid & $<42.32$ & $<42.32$ & $<42.32$ \\
\hline S96T003748 & $152: 11$ & Drainable liquid & $<42.32$ & $<42.32$ & $<42.32$ \\
\hline \$96T003749 & $152: 12$ & Drainable liquid & $<42.32$ & $<42.32$ & $<42.32$ \\
\hline S96T003750 & $152: 13$ & Drainable liquid & $<42.32$ & $<42.32$ & $<42.32$ \\
\hline S96T003751 & $152: 14$ & Drainable liquid & $<28.23$ & $<28.23$ & $<28.23$ \\
\hline S96T004433 & 153: 1 & Drainable liquid & $<28.23$ & $<28.23$ & $<28.23$ \\
\hline S96T004308 & 153: 2 & Drainable liquid & $<42.32$ & $<42.32$ & $<42.32$ \\
\hline S96T004434 & 153: 3 & Drainable liquid & $<28.23$ & $<28.23$ & $<28.23$ \\
\hline \$96T004419 & 153: 5 & Drainable liquid & $<42.32$ & $<42.32$ & $<42.32$ \\
\hline S96T004420 & 153: 6 & Drainable liquid & $<42.32$ & $<42.32$ & $<42.32$ \\
\hline S96T004421 & 153: 7 & Drainable liquid & $<28.23$ & $<28.23$ & $<28.23$ \\
\hline S96T003975 & 153: 8 & Drainable liquid & $<28.23$ & $<28.23$ & $<28.23$ \\
\hline S96T003976 & 153: 9 & Drainable liquid & $<28.23$ & $<28.23$ & $<28.23$ \\
\hline S96T004309 & $153: 10$ & Drainable liquid & $<42.32$ & $<42.32$ & $<42.32$ \\
\hline S96T003979 & $153: 11$ & Drainable liquid & $<42.32$ & $<42.32$ & $<42.32$ \\
\hline \$96T003977 & $153: 12$ & Drainable liquid & $<42.32$ & $<42.32$ & $<42.32$ \\
\hline S96T003978 & $153: 13$ & Drainable liquid & $<42.32$ & $<42.32$ & $<42.32$ \\
\hline S96T004333 & $153: 14$ & Drainable liquid & $<42.32$ & $<42.32$ & $<42.32$ \\
\hline S96T003980 & $153: 21$ & Drainable liquid & $<42.32$ & $<42.32$ & $<42.32$ \\
\hline S96T004831 & Core 152 & Liquid composite & $<42.32$ & $<42.32$ & $<42.32$ \\
\hline
\end{tabular}


Table B2-31. Tank 241-AN-105 Analytical Results: Calcium (ICP). (3 sheets)

\begin{tabular}{|c|c|c|c|c|c|}
\hline Wample & Sample & 46 onmple & $\mathrm{n} x \rightarrow 11$ & Diplicate & Men \\
\hline \multicolumn{2}{|c|}{ 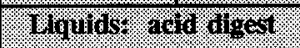 } & . & 1018 & 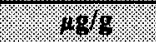 & 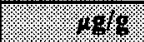 \\
\hline S96T004144 & Core 152 & Liquid composite & 635.91 & 600.70 & $618.31^{\mathrm{QC}: b, f}$ \\
\hline \multicolumn{2}{|c|}{ 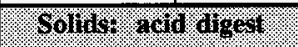 } & & 148 & 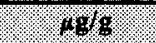 & $168 \mathrm{~g}$ \\
\hline S96T003906 & 152: 1 & Upper half & 108 & 182 & $145^{\mathrm{QC}: \mathrm{e}}$ \\
\hline S96T003898 & \multirow[t]{2}{*}{$152: 14$} & Upper half & 214 & 158 & $186^{\mathrm{QC}: \mathrm{e}}$ \\
\hline S96T003828 & & Lower half & 130 & 161 & $145.5^{\mathrm{QC}: e}$ \\
\hline S96T003899 & \multirow[t]{2}{*}{$152: 16$} & Upper half & 323 & 280 & 301.5 \\
\hline S96T003900 & & Lower half & 279 & 536 & $407.5^{\mathrm{QC:e}}$ \\
\hline S96T003901 & \multirow[t]{2}{*}{$152: 18$} & Upper half & 420 & 328 & $374^{\mathrm{QC}: \mathrm{e}}$ \\
\hline S96T003902 & & Lower half & 240 & 237 & 238.5 \\
\hline S96T003903 & \multirow[t]{2}{*}{$152: 20$} & Upper half & 253 & 258 & 255.5 \\
\hline S96T003904 & & Lower half & 408 & 400 & 404 \\
\hline S96T003905 & $152: 22$ & Upper half & 310 & $\overline{532}$ & $421^{\text {QC:e }}$ \\
\hline S96T004338 & $153: 14$ & Lower half & 1,050 & 788 & $919^{\text {QC:b,e }}$ \\
\hline S96T004315 & \multirow[t]{2}{*}{$153: 15$} & Upper half & 708 & 856 & $782^{\mathrm{QC:b}}$ \\
\hline S96T004318 & & Lower half & 589 & 540 & $564.5^{\mathrm{QC}: \mathrm{b}}$ \\
\hline S96T004444 & \multirow[t]{2}{*}{$153: 17$} & Upper half & 624 & 624 & $624^{\mathrm{QC}: b}$ \\
\hline S96T004445 & & Lower half & 820 & 611 & $715.5^{\mathrm{QC}: \mathrm{b}, \mathrm{e}}$ \\
\hline S96T004446 & $153: 19$ & Lower half & 1,470 & 1,120 & $1,295^{\mathrm{QC}: \mathrm{b}, \mathrm{e}}$ \\
\hline S96T003988 & $153: 21$ & Lower half & 632 & 641 & $636.5^{\mathrm{QC}: b}$ \\
\hline S96T003989 & \multirow[t]{2}{*}{$153: 22$} & Upper half & 1,000 & 1,200 & $1,100^{\mathrm{QC}: \mathrm{b}}$ \\
\hline S96T003987 & & Lower half & 2,010 & 2,270 & $2,140^{\mathrm{QC}: \mathrm{b}}$ \\
\hline S96T004257 & \multirow[t]{2}{*}{ Core 152} & Solid composite & 209 & 222 & $215.5^{\mathrm{QC}: \mathrm{c}}$ \\
\hline S96T004262 & & Solid composite & 355 & 302 & 328.5 \\
\hline
\end{tabular}


Table B2-31. Tank 241-AN-105 Analytical Results: Calcium (ICP). (3 sheets)

\begin{tabular}{|c|c|c|c|c|c|}
\hline $\begin{array}{l}\text { Samply } \\
\text { Number }\end{array}$ & $\begin{array}{l}\text { Sumple } \\
\text { Eocation }\end{array}$ & $\begin{array}{l}\text { Sample. } \\
\text { romtlon }\end{array}$ & nesint & Buplicate & Mein \\
\hline Solids: & Wrion & (2) & 1018 & $1.08 \%$ & . 198 \\
\hline S96T003893 & 152: 1 & Upper half & $<1,980$ & $<2,010$ & $<1,995$ \\
\hline S96T003894 & \multirow[t]{2}{*}{$152: 14$} & Upper half & $<2,040$ & $<2,000$ & $<2,020$ \\
\hline S96T003827 & & Lower half & $<2,010$ & $<2,060$ & $<2,035$ \\
\hline S96T003895 & \multirow[t]{2}{*}{$152: 16$} & Upper half & $<2,040$ & $<2,010$ & $<2,025$ \\
\hline S96T003889 & & Lower half & $<2,030$ & $<2,000$ & $<2,015$ \\
\hline S96T003896 & \multirow[t]{2}{*}{$152: 18$} & Upper half & $<2,070$ & $<2,010$ & $<2,040$ \\
\hline S96T003890 & & Lower half & $<2,060$ & $<2,070$ & $<2,065$ \\
\hline S96T003897 & \multirow[t]{2}{*}{$152: 20$} & Upper half & $<2,120$ & $<2,070$ & $<2,095$ \\
\hline S96T003891 & & Lower half & $<2,040$ & $<2,060$ & $<2,050$ \\
\hline S96T003892 & $152: 22$ & Upper half & $<2,090$ & $<2,120$ & $<2,105$ \\
\hline S96T004337 & $153: 14$ & Lower half & $<2,030$ & $<2,030$ & $<2,030$ \\
\hline S96T004314 & \multirow[t]{2}{*}{$153: 15$} & Upper half & $<1,910$ & $<1,920$ & $<1,915$ \\
\hline S96T004317 & & Lower half & $<2,000$ & $<2,030$ & $<2,015$ \\
\hline S96T004441 & \multirow[t]{2}{*}{$153: 17$} & Upper half & $<1,960$ & $<1,960$ & $<1,960$ \\
\hline S96T004442 & & Lower half & $<2,010$ & $<2,000$ & $<2,005$ \\
\hline S96T004443 & $153: 19$ & Lower half & $<2,020$ & $<2,000$ & $<2,010$ \\
\hline S96T003984 & $153: 21$ & Lower half & $<2,010$ & $<2,010$ & $<2,010$ \\
\hline S96T003986 & \multirow[t]{2}{*}{$153: 22$} & Upper half & $<2,000$ & $<2,030$ & $<2,015$ \\
\hline S96T003985 & & Lower half & $<2,010$ & $<2,010$ & $<2,010$ \\
\hline
\end{tabular}


Table B2-32. Tank 241-AN-105 Analytical Results: Chromium (ICP). (3 sheets)

\begin{tabular}{|c|c|c|c|c|c|}
\hline 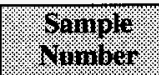 & Gramplo & Sornir & Pesill & Miglicate & Whan \\
\hline \multicolumn{2}{|c|}{ Wours } & & $1 \% 7_{6}$ & $4 \mathrm{~g} g$ & 14918 \\
\hline S96T003732 & 152: 1 & Drainable liquid & 164.78 & 158.45 & 161.62 \\
\hline S96T003739 & 152: 2 & Drainable liquid & 141.54 & 139.43 & 140.49 \\
\hline S96T003740 & 152: 3 & Drainable liquid & 142.25 & 137.32 & 139.78 \\
\hline S96T003741 & 152: 4 & Drainable liquid & 150.70 & 143.66 & 147.18 \\
\hline S96T003742 & 152: 5 & Drainable liquid & 148.59 & 142.25 & 145.42 \\
\hline S96T003743 & 152: 6 & Drainable liquid & 166.19 & 178.87 & 172.53 \\
\hline S96T003744 & 152: 7 & Drainable liquid & 127.46 & 140.14 & 133.80 \\
\hline S96T003745 & 152: 8 & Drainable liquid & 137.32 & 167.60 & 152.46 \\
\hline S96T003746 & 152:9 & Drainable liquid & 149.29 & 154.22 & 151.76 \\
\hline S96T003747 & $152: 10$ & Drainable liquid & 150 & 153.52 & 151.76 \\
\hline S96T003748 & $152: 11$ & Drainable liquid & 149.29 & 150.70 & 150 \\
\hline S96T003749 & $152: 12$ & Drainable liquid & 150.70 & 146.47 & 148.59 \\
\hline S96T003750 & $152: 13$ & Drainable liquid & 145.07 & 142.25 & 143.66 \\
\hline S96T003751 & $152: 14$ & Drainable liquid & 138.02 & 137.32 & 137.67 \\
\hline S96T004433 & 153: 1 & Drainable liquid & 278.16 & 261.97 & 270.07 \\
\hline S96T004308 & 153: 2 & Drainable liquid & 156.33 & 151.40 & 153.87 \\
\hline S96T004434 & 153: 3 & Drainable liquid & 195.77 & 194.36 & 195.07 \\
\hline S96T004419 & 153: 5 & Drainable liquid & 157.74 & 154.22 & 155.98 \\
\hline S96T004420 & 153: 6 & Drainable liquid & 147.18 & 158.45 & 152.81 \\
\hline S96T004421 & 153: 7 & Drainable liquid & 271.83 & 183.09 & $227.46^{\mathrm{QC}: \mathrm{e}}$ \\
\hline S96T003975 & 153: 8 & Drainable liquid & 145.77 & 143.66 & 144.71 \\
\hline S96T003976 & 153: 9 & Drainable liquid & 133.80 & 158.45 & 146.12 \\
\hline S96T004309 & $153: 10$ & Drainable liquid & 152.81 & 161.26 & 157.04 \\
\hline S96T003979 & 153:11 & Drainable liquid & 151.40 & 148.59 & 150 \\
\hline \$96T003977 & $153: 12$ & Drainable liquid & 147.88 & 153.52 & 150.70 \\
\hline S96T003978 & $153: 13$ & Drainable liquid & 140.14 & 128.87 & 134.50 \\
\hline S96T004333 & $153: 14$ & Drainable liquid & 152.11 & 154.93 & 153.52 \\
\hline S96T003980 & $153: 21$ & Drainable liquid & 110.56 & 109.15 & 109.85 \\
\hline S96T004831 & Core 152 & Liquid composite & 155.63 & 157.74 & 156.69 \\
\hline
\end{tabular}


Table B2-32. Tank 241-AN-105 Analytical Results: Chromium (ICP). (3 sheets)

\begin{tabular}{|c|c|c|c|c|c|}
\hline Gample & Samplor & . & i. resill & Pyplicale & Mren \\
\hline \multicolumn{2}{|c|}{ Finjivist afd digest } & (l) & 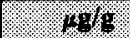 & $49 / 8$ & 1898 \\
\hline S96T004144 & Core 152 & Liquid composite & 131.69 & 131.69 & 131.69 \\
\hline \multicolumn{2}{|c|}{ Solds, aro dingost } & & 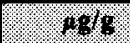 & 10 & 1848 \\
\hline S96T003906 & 152: 1 & Upper half & 758 & 733 & 745.5 \\
\hline S96T003898 & \multirow[t]{2}{*}{$152: 14$} & Upper half & 1,230 & 1,270 & 1,250 \\
\hline S96T003828 & & Lower half & 1,210 & 1,220 & 1,215 \\
\hline \$96T003899 & \multirow[t]{2}{*}{$152: 16$} & Upper half & 2,020 & 2,070 & 2,045 \\
\hline S96T003900 & & Lower half & 1,710 & 1,750 & 1,730 \\
\hline S96T003901 & \multirow[t]{2}{*}{$152: 18$} & Upper half & 2,510 & 2,380 & 2,445 \\
\hline S96T003902 & & Lower half & 2,250 & 2,310 & 2,280 \\
\hline S96T003903 & \multirow[t]{2}{*}{$152: 20$} & Upper half & 3,470 & 3,380 & 3,425 \\
\hline S96T003904 & & Lower half & 1,560 & 1,590 & 1,575 \\
\hline$\$ 96 \mathrm{~T} 003905$ & $152: 22$ & Upper half & 1,990 & 2,080 & 2,035 \\
\hline$\$ 96 \mathrm{~T} 004338$ & $153: 14$ & Lower half & 1,840 & 2,010 & 1,925 \\
\hline \$96T004315 & \multirow[t]{2}{*}{$153: 15$} & Upper half & 1,350 & 1,400 & 1,375 \\
\hline S96T004318 & & Lower half & 1,210 & 1,330 & 1,270 \\
\hline S96T004444 & \multirow[t]{2}{*}{$153: 17$} & Upper half & 2,690 & 2,650 & 2,670 \\
\hline S96T004445 & & Lower half & 4,240 & 4,370 & 4,305 \\
\hline S96T004446 & $153: 19$ & Lower half & 2,980 & 3,160 & 3,070 \\
\hline S96T003988 & $153: 21$ & Lower half & 1,170 & 1,120 & 1,145 \\
\hline S96T003989 & \multirow[t]{2}{*}{$153: 22$} & Upper half & 2,360 & 2,030 & 2,195 \\
\hline S96T003987 & & Lower half & 6,100 & 6,510 & 6,305 \\
\hline S96T004257 & \multirow[t]{2}{*}{ Core 152} & Solid composite & 1,600 & 1,370 & $1,485^{\mathrm{QC}: c, \mathrm{e}}$ \\
\hline S96T004262 & & Solid composite & 2,900 & 2,580 & 2,740 \\
\hline
\end{tabular}


Table B2-32. Tank 241-AN-105 Analytical Results: Chromium (ICP). (3 sheets)

\begin{tabular}{|c|c|c|c|c|c|}
\hline ofimple & 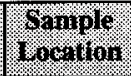 & ( ormple & . & Goolutar & 48 \\
\hline \multicolumn{3}{|c|}{ 6oling } & 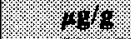 & 1896 & $14 / 8$ \\
\hline \$96T003893 & 152: 1 & Upper half & 721 & 712 & 716.5 \\
\hline S96T003894 & \multirow[t]{2}{*}{$152: 14$} & Upper half & 1,230 & 1,230 & 1,230 \\
\hline S96T003827 & & Lower half & 1,150 & 1,220 & 1,185 \\
\hline S96T003895 & \multirow[t]{2}{*}{$152: 16$} & Upper half & 2,050 & 2,130 & 2,090 \\
\hline S96T003889 & & Lower half & 1,700 & 1,750 & 1,725 \\
\hline S96T003896 & \multirow[t]{2}{*}{$152: 18$} & Upper half & 2,420 & 2,440 & 2,430 \\
\hline S96T003890 & & Lower half & 2,260 & 2,320 & 2,290 \\
\hline S96T003897 & \multirow[t]{2}{*}{$152: 20$} & Upper half & 3,950 & 3,810 & 3,880 \\
\hline S96T003891 & & Lower half & 1,980 & 1,910 & 1,945 \\
\hline \$96T003892 & $152: 22$ & Upper half & 1,580 & 1,620 & 1,600 \\
\hline S96T004337 & $153: 14$ & Lower half & 1,930 & 1,980 & 1,955 \\
\hline S96T004314 & \multirow[t]{2}{*}{$153: 15$} & Upper half & 1,330 & 1,520 & 1,425 \\
\hline S96T004317 & & Lower half & 1,510 & 1,790 & 1,650 \\
\hline \$96T004441 & \multirow[t]{2}{*}{$153: 17$} & Upper half & 3,710 & 3,410 & 3,560 \\
\hline S96T004442 & & Lower half & 4,800 & 5,090 & 4,945 \\
\hline S96T004443 & $153: 19$ & Lower half & 3,890 & 3,330 & 3,610 \\
\hline S96T003984 & $153: 21$ & Lower half & 1,480 & 1,430 & 1,455 \\
\hline S96T003986 & \multirow[t]{2}{*}{$153: 22$} & Upper half & 2,730 & 2,600 & 2,665 \\
\hline S96T003985 & & Lower half & 5,680 & 5,490 & 5,585 \\
\hline
\end{tabular}


HNF-SD-WM-ER-678 Rev. 0

Table B2-33. Tank 241-AN-105 Analytical Results: Copper (ICP). (3 sheets)

\begin{tabular}{|c|c|c|c|c|c|}
\hline Wamplo & Saraple & Shmple & Pansul & Guplatale & 196a) \\
\hline \multicolumn{2}{|c|}{ Whirio } & & $18 \%$ & 49 & 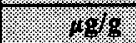 \\
\hline S96T003732 & 152: 1 & Drainable liquid & $<2.82$ & $<2.82$ & $<2.82$ \\
\hline S96T003739 & 152: 2 & Drainable liquid & $<2.82$ & $<2.82$ & $<2.82$ \\
\hline S96T003740 & $152: 3$ & Drainable liquid & $<2.82$ & $<2.82$ & $<2.82$ \\
\hline S96T003741 & 152: 4 & Drainable liquid & $<2.82$ & $<2.82$ & $<2.82$ \\
\hline S96T003742 & 152: 5 & Drainable liquid & $<2.82$ & $<2.82$ & $<2.82$ \\
\hline S96T003743 & 152: 6 & Drainable liquid & 3.55 & 3.67 & 3.61 \\
\hline S96T003744 & 152: 7 & Drainable liquid & $<2.82$ & $<2.82$ & $<2.82$ \\
\hline S96T003745 & 152: 8 & Drainable liquid & 3.30 & $<2.82$ & $<3.06$ \\
\hline S96T003746 & 152:9 & Drainable liquid & $<2.82$ & $<2.82$ & $<2.82$ \\
\hline S96T003747 & $152: 10$ & Drainable liquid & $<4.23$ & $<4.23$ & $<4.23$ \\
\hline S96T003748 & $152: 11$ & Drainable liquid & $<4.23$ & $<4.23$ & $<4.23$ \\
\hline S96T003749 & $152: 12$ & Drainable liquid & $<4.23$ & $<4.23$ & $<4.23$ \\
\hline S96T003750 & $152: 13$ & Drainable liquid & $<4.23$ & $<4.23$ & $<4.23$ \\
\hline S96T003751 & $152: 14$ & Drainable liquid & $<2.82$ & $<2.82$ & $<2.82$ \\
\hline S96T004433 & 153: 1 & Drainable liquid & 5.97 & 4.99 & 5.48 \\
\hline S96T004308 & 153: 2 & Drainable liquid & $<4.23$ & $<4.23$ & $<4.23$ \\
\hline S96T004434 & 153: 3 & Drainable liquid & 7.18 & 4.74 & $5.96^{\mathrm{QC}: c}$ \\
\hline S96T004419 & 153: 5 & Drainable liquid & $<4.23$ & $<4.23$ & $<4.23$ \\
\hline S96T004420 & 153: 6 & Drainable liquid & $<4.23$ & $<4.23$ & $<4.23$ \\
\hline S96T004421 & 153: 7 & Drainable liquid & $<2.82$ & $<2.82$ & $<2.82$ \\
\hline S96T003975 & 153: 8 & Drainable liquid & $<2.82$ & $<2.82$ & $<2.82$ \\
\hline S96T003976 & 153: 9 & Drainable liquid & $<2.82$ & $<2.82$ & $<2.82$ \\
\hline S96T004309 & $153: 10$ & Drainable liquid & $<4.23$ & $<4.23$ & $<4.23$ \\
\hline S96T003979 & 153:11 & Drainable liquid & $<4.23$ & $<4.23$ & $<4.23$ \\
\hline S96T003977 & $153: 12$ & Drainable liquid & $<4.23$ & $<4.23$ & $<4.23$ \\
\hline S96T003978 & $153: 13$ & Drainable liquid & $<4.23$ & $<4.23$ & $<4.23$ \\
\hline S96T004333 & $153: 14$ & Drainable liquid & $<4.23$ & $<4.23$ & $<4.23$ \\
\hline S96T003980 & $153: 21$ & Drainable liquid & $<4.23$ & $<4.23$ & $<4.23$ \\
\hline S96T004831 & Core 152 & Liquid composite & $<4.23$ & $<4.23$ & $<4.23$ \\
\hline
\end{tabular}


Table B2-33. Tank 241-AN-105 Analytical Results: Copper (ICP). (3 sheets)

\begin{tabular}{|c|c|c|c|c|c|}
\hline Wample & 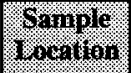 & . & (. & Bupliging. & Mran \\
\hline \multicolumn{2}{|c|}{ 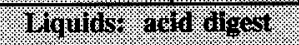 } & 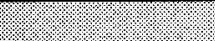 & $1.6 \%$ & 498 & $48 \%$ \\
\hline S96T004144 & Core 152 & Liquid composite & $<3.69$ & 4.42 & $<4.05$ \\
\hline \multicolumn{2}{|c|}{ Sollot atd tigest } & & 498 & 69 & $8 \%$ \\
\hline S96T003906 & $152: 1$ & Upper half & 4.71 & 4.32 & 4.515 \\
\hline \$96T003898 & \multirow[t]{2}{*}{$152: 14$} & Upper half & 7.29 & 5.88 & $6.58^{\mathrm{QC}: \mathrm{e}}$ \\
\hline S96T003828 & & Lower half & 7.15 & 6.24 & 6.69 \\
\hline S96T003899 & \multirow[t]{2}{*}{$152: 16$} & Upper half & 6.28 & 6.48 & 6.38 \\
\hline S96T003900 & & Lower half & 5.35 & 6.03 & 5.69 \\
\hline \$96T003901 & \multirow[t]{2}{*}{$152: 18$} & Upper half & 6 & 6.17 & 6.08 \\
\hline S96T003902 & & Lower half & 5.29 & 10.5 & $7.89^{Q \mathrm{Ce}}$ \\
\hline \$96T003903 & \multirow[t]{2}{*}{$152: 20$} & Upper half & 6.9 & 5.91 & 6.40 \\
\hline \$96T003904 & & Lower half & 7.81 & 9.05 & 8.43 \\
\hline S96T003905 & $152: 22$ & Upper half & 3.98 & $<4.14$ & $<4.06$ \\
\hline S96T004338 & $153: 14$ & Lower half & 5.7 & 6.91 & 6.30 \\
\hline S96T004315 & \multirow[t]{2}{*}{$153: 15$} & Upper half & 8.1 & 5.85 & $6.97^{\mathrm{QC:e}}$ \\
\hline S96T004318 & & Lower half & 5.71 & 6.05 & 5.88 \\
\hline S96T004444 & \multirow[t]{2}{*}{$153: 17$} & Upper half & 7.37 & 11.4 & $9.38^{\mathrm{QC}: e}$ \\
\hline S96T004445 & & Lower half & 8.15 & 13.7 & $10.92^{\mathrm{QC}: e}$ \\
\hline S96T004446 & $153: 19$ & Lower half & 7.11 & 6.87 & 6.99 \\
\hline S96T003988 & $153: 21$ & Lower half & 4.34 & 4.43 & 4.38 \\
\hline S96T003989 & \multirow[t]{2}{*}{$153: 22$} & Upper half & 6.28 & 4.79 & $5.53^{\mathrm{QC:c}}$ \\
\hline S96T003987 & & Lower half & 6.82 & 18.1 & $12.46^{\mathrm{QC}: \mathrm{e}}$ \\
\hline S96T004257 & \multirow[t]{2}{*}{ Core 152} & Solid composite & 4.88 & 5.34 & $5.11^{\mathrm{QC}: \mathrm{c}}$ \\
\hline S96T004262 & & Solid composite & 7.04 & 5.02 & $6.03^{\mathrm{QC}: \mathrm{c}}$ \\
\hline
\end{tabular}


HNF-SD-WM-ER-678 Rev. 0

Table B2-33. Tank 241-AN-105 Analytical Results: Copper (ICP). (3 sheets)

\begin{tabular}{|c|c|c|c|c|c|}
\hline Samples & Sample & 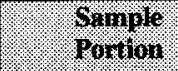 & Resill. & Muplicale & yegro \\
\hline \multicolumn{3}{|c|}{ Solths Yulon } & $18 \%$ & 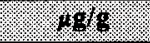 & $48 \%$ \\
\hline S96T003893 & 152: 1 & Upper half & $<198$ & $<201$ & $<199.5$ \\
\hline S96T003894 & \multirow[t]{2}{*}{$152: 14$} & Upper half & $<204$ & $<200$ & $<202$ \\
\hline S96T003827 & & Lower half & $<201$ & $<206$ & $<203.5$ \\
\hline S96T003895 & \multirow[t]{2}{*}{$152: 16$} & Upper half & $<204$ & $<201$ & $<202.5$ \\
\hline S96T003889 & & Lower half & $<203$ & $<200$ & $<201.5$ \\
\hline S96T003896 & \multirow[t]{2}{*}{$152: 18$} & Upper half & $<207$ & $<201$ & $<204$ \\
\hline S96T003890 & & Lower half & $<206$ & $<207$ & $<206.5$ \\
\hline S96T003897 & \multirow[t]{2}{*}{$152: 20$} & Upper half & $<212$ & $<207$ & $<209.5$ \\
\hline S96T003891 & & Lower half & $<204$ & $<206$ & $<205$ \\
\hline S96T003892 & $152: 22$ & Upper half & $<209$ & $<212$ & $<210.5$ \\
\hline S96T004337 & $153: 14$ & Lower half & $<203$ & $<203$ & $<203$ \\
\hline S96T004314 & \multirow[t]{2}{*}{$153: 15$} & Upper half & $<191$ & $<192$ & $<191.5$ \\
\hline$\$ 96 T 004317$ & & Lower half & $<200$ & $<203$ & $<201.5$ \\
\hline S96T004441 & \multirow[t]{2}{*}{$153: 17$} & Upper half & $<196$ & $<196$ & $<196$ \\
\hline S96T004442 & & Lower half & $<201$ & $<200$ & $<200.5$ \\
\hline S96T004443 & $153: 19$ & Lower half & $<202$ & $<200$ & $<201$ \\
\hline$\$ 96 \mathrm{T003984}$ & $153: 21$ & Lower half & $<201$ & $<201$ & $<201$ \\
\hline S96T003986 & \multirow[t]{2}{*}{$153: 22$} & Upper half & $<200$ & $<203$ & $<201.5$ \\
\hline S96T003985 & & Lower half & $<201$ & $<201$ & $<201$ \\
\hline
\end{tabular}


Table B2-34. Tank 241-AN-105 Analytical Results: Iron (ICP). (3 sheets)

\begin{tabular}{|c|c|c|c|c|c|}
\hline Saniolo & $\begin{array}{l}\text { ofunople } \\
\text { Hocalion }\end{array}$ & 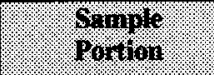 & $\ln (4)$ & Buplicalo & Yoto \\
\hline \multicolumn{2}{|c|}{ Wrouls } & & 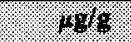 & 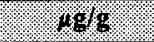 & $40 \%$ \\
\hline S96T003732 & 152: 1 & Drainable liquid & $<14.15$ & $<14.15$ & $<14.15$ \\
\hline S96T003739 & 152: 2 & Drainable liquid & $<14.15$ & $<14.15$ & $<14.15$ \\
\hline S96T003740 & 152: 3 & Drainable liquid & $<14.15$ & $<14.15$ & $<14.15$ \\
\hline S96T003741 & $152: 4$ & Drainable liquid & $<14.15$ & $<14.15$ & $<14.15$ \\
\hline S96T003742 & 152: 5 & Drainable liquid & $<14.15$ & $<14.15$ & $<14.15$ \\
\hline S96T003743 & 152: 6 & Drainable liquid & $<14.15$ & $<14.15$ & $<14.15$ \\
\hline S96T003744 & 152: 7 & Drainable liquid & $<14.15$ & $<14.15$ & $<14.15$ \\
\hline S96T003745 & $152: 8$ & Drainable liquid & $<14.15$ & $<14.15$ & $<14.15$ \\
\hline S96T003746 & $152: 9$ & Drainable liquid & $<14.15$ & $<14.15$ & $<14.15$ \\
\hline S96T003747 & $152: 10$ & Drainable liquid & $<21.19$ & $<21.19$ & $<21.19$ \\
\hline S96T003748 & $152: 11$ & Drainable liquid & $<21.19$ & $<21.19$ & $<21.19$ \\
\hline S96T003749 & $152: 12$ & Drainable liquid & $<21.19$ & $<21.19$ & $<21.19$ \\
\hline S96T003750 & $152: 13$ & Drainable liquid & $<21.19$ & $<21.19$ & $<21.19$ \\
\hline S96T003751 & $152: 14$ & Drainable liquid & $<14.15$ & $<14.15$ & $<14.15$ \\
\hline S96T004433 & 153: 1 & Drainable liquid & $<14.15$ & $<14.15$ & $<14.15$ \\
\hline S96T004308 & 153: 2 & Drainable liquid & $<21.19$ & $<21.19$ & $<21.19$ \\
\hline S96T004434 & $153: 3$ & Drainable liquid & $<14.15$ & $<14.15$ & $<14.15$ \\
\hline S96T004419 & 153: 5 & Drainable liquid & $<21.19$ & $<21.19$ & $<21.19$ \\
\hline S96T004420 & 153: 6 & Drainable liquid & $<21.19$ & $<21.19$ & $<21.19$ \\
\hline S96T004421 & 153: 7 & Drainable liquid & $<14.15$ & $<14.15$ & $<14.15$ \\
\hline S96T003975 & 153: 8 & Drainable liquid & $<14.15$ & $<14.15$ & $<14.15$ \\
\hline S96T003976 & 153: 9 & Drainable liquid & $<14.15$ & $<14.15$ & $<14.15$ \\
\hline S96T004309 & $153: 10$ & Drainable liquid & $<21.19$ & $<21.19$ & $<21.19$ \\
\hline S96T003979 & 153:11 & Drainable liquid & $<21.19$ & $<21.19$ & $<21.19$ \\
\hline S96T003977 & $153: 12$ & Drainable liquid & $<21.19$ & $<21.19$ & $<21.19$ \\
\hline S96T003978 & $153: 13$ & Drainable liquid & $<21.19$ & $<21.19$ & $<21.19$ \\
\hline S96T004333 & $153: 14$ & Drainable liquid & $<21.19$ & $<21.19$ & $<21.19$ \\
\hline S96T003980 & $153: 21$ & Drainable liquid & $<21.19$ & $<21.19$ & $<21.19$ \\
\hline S96T004831 & Core 152 & Liquid composite & $<21.19$ & $<21.19$ & $<21.19$ \\
\hline
\end{tabular}


Table B2-34. Tank 241-AN-105 Analytical Results: Iron (ICP). (3 sheets)

\begin{tabular}{|c|c|c|c|c|c|}
\hline Shinply & Waroplo & 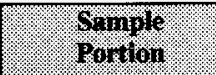 & Resill & Buplorato & 19an \\
\hline \multicolumn{2}{|c|}{ 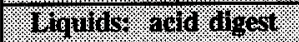 } & : & 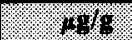 & $69 / 9$ & 1698 \\
\hline S96T004144 & Core 152 & Liquid composite & 51.05 & 38.80 & $44.92^{\mathrm{QC}: \mathrm{e}}$ \\
\hline \multicolumn{2}{|c|}{ Solkst vid digest } & & 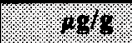 & 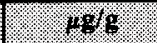 & $19 \%$ \\
\hline S96T003906 & 152: 1 & Upper half & 44.8 & 32.8 & $38.8^{\mathrm{QC}: \mathrm{e}}$ \\
\hline S96T003898 & \multirow[t]{2}{*}{$152: 14$} & Upper half & 68.8 & 64.9 & 66.85 \\
\hline S96T003828 & & Lower half & 46.1 & 47.1 & 46.6 \\
\hline S96T003899 & \multirow[t]{2}{*}{$152: 16$} & Upper half & 72.6 & 327 & $199.8^{\mathrm{QC}: \mathrm{e}}$ \\
\hline S96T003900 & & Lower half & 90.4 & 80 & 85.2 \\
\hline S96T003901 & \multirow[t]{2}{*}{$152: 18$} & Upper half & 179 & 171 & 175 \\
\hline S96T003902 & & Lower half & 78.7 & 74.8 & 76.75 \\
\hline S96T003903 & \multirow[t]{2}{*}{$152: 20$} & Upper half & 82.1 & 90.7 & 86.4 \\
\hline S96T003904 & & Lower half & 61.2 & 59 & 60.1 \\
\hline S96T003905 & $152: 22$ & Upper half & 157 & 123 & $140^{\mathrm{QC:e}}$ \\
\hline \$96T004338 & 153:14 & Lower half & 54.6 & 61.4 & 58 \\
\hline S96T004315 & \multirow[t]{2}{*}{$153: 15$} & Upper half & 83.6 & 62.5 & $73.05^{\mathrm{QC}: \mathrm{e}}$ \\
\hline S96T004318 & & Lower half & 35.8 & 36.6 & 36.2 \\
\hline S96T004444 & \multirow[t]{2}{*}{$153: 17$} & Upper half & 60.2 & 62.3 & 61.25 \\
\hline S96T004445 & & Lower half & 91.6 & 86.9 & 89.25 \\
\hline S96T004446 & $153: 19$ & Lower half & 101 & 70.9 & $85.95^{\mathrm{QC}: \mathrm{e}}$ \\
\hline S96T003988 & $153: 21$ & Lower half & 183 & 175 & 179 \\
\hline S96T003989 & \multirow[t]{2}{*}{$153: 22$} & Upper half & 142 & 124 & 133 \\
\hline S96T003987 & & Lower half & 538 & 451 & 494.5 \\
\hline S96T004257 & \multirow[t]{2}{*}{ Core 152} & Solid composite & 71.7 & 50.6 & $61.15^{\mathrm{QC:e,c}}$ \\
\hline S96T004262 & & Solid composite & 154 & 139 & 146.5 \\
\hline
\end{tabular}


Table B2-34. Tank 241-AN-105 Analytical Results: Iron (ICP). (3 sheets)

\begin{tabular}{|c|c|c|c|c|c|}
\hline Waninglo. & Wariple & . & Resin! & Diplonte & (1) \\
\hline \multicolumn{2}{|c|}{ 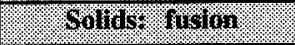 } & & $18 \%$ & $\% 4 \%$ & $8 \%$ \\
\hline \$96T003893 & 152: 1 & Upper half & $<988$ & $<1,010$ & $<999$ \\
\hline S96T003894 & \multirow[t]{2}{*}{$152: 14$} & Upper half & $<1,020$ & $<998$ & $<1,009$ \\
\hline S96T003827 & & Lower half & $<1,010$ & $<1,030$ & $<1,020$ \\
\hline S96T003895 & \multirow[t]{2}{*}{$152: 16$} & Upper half & $<1,020$ & $<1,000$ & $<1,010$ \\
\hline S96T003889 & & Lower half & $<1,010$ & $<1,000$ & $<1,005$ \\
\hline S96T003896 & \multirow[t]{2}{*}{$152: 18$} & Upper half & $<1,030$ & $<1,000$ & $<1,015$ \\
\hline S96T003890 & & Lower half & $<1,030$ & $<1,040$ & $<1,035$ \\
\hline S96T003897 & \multirow[t]{2}{*}{$152: 20$} & Upper half & $<1,060$ & $<1,040$ & $<1,050$ \\
\hline S96T003891 & & Lower half & $<1,020$ & $<1,030$ & $<1,025$ \\
\hline S96T003892 & $152: 22$ & Upper half & $<1,040$ & $<1,060$ & $<1,050$ \\
\hline S96'T004337 & $153: 14$ & Lower half & $<1,020$ & $<1,010$ & $<1,015$ \\
\hline S96T004314 & \multirow[t]{2}{*}{$153: 15$} & Upper half & $<953$ & $<958$ & $<955.5$ \\
\hline S96T004317 & & Lower half & $<998$ & $<1,020$ & $<1,009$ \\
\hline S96T004441 & \multirow[t]{2}{*}{$153: 17$} & Upper half & $<980$ & $<982$ & $<981$ \\
\hline S96T004442 & & Lower half & $<1,010$ & $<1,000$ & $<1,005$ \\
\hline S96T004443 & $153: 19$ & Lower half & $<1,010$ & $<1,000$ & $<1,005$ \\
\hline S96T003984 & $153: 21$ & Lower half & $<1,010$ & $<1,000$ & $<1,005$ \\
\hline S96T003986 & \multirow[t]{2}{*}{$153: 22$} & Upper half & $<999$ & $<1,020$ & $<1,009.5$ \\
\hline S96T003985 & & Lower half & $<1,010$ & $<1,010$ & $<1,010$ \\
\hline
\end{tabular}


HNF-SD-WM-ER-678 Rev. 0

Table B2-35. Tank 241-AN-105 Analytical Results: Lead (ICP). (3 sheets)

\begin{tabular}{|c|c|c|c|c|c|}
\hline 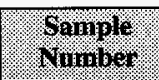 & Gample & $\begin{array}{l}\text { Shmple } \\
2 \%+6101\end{array}$ & $6 \times 111$ & Miplicrir & 18011 \\
\hline \multicolumn{2}{|c|}{ 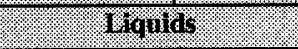 } & & 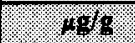 & (19) & 489 \\
\hline S96T003732 & 152: 1 & Drainable liquid & 36.26 & 38.02 & 37.14 \\
\hline S96T003739 & 152: 2 & Drainable liquid & 30.98 & 34.22 & 32.60 \\
\hline S96T003740 & 152: 3 & Drainable liquid & 33.80 & 30.84 & 32.32 \\
\hline S96T003741 & 152: 4 & Drainable liquid & 36.47 & 33.02 & 34.75 \\
\hline S96T003742 & 152: 5 & Drainable liquid & 36.33 & 34.71 & 35.52 \\
\hline S96T003743 & $152: 6$ & Drainable liquid & 37.46 & 42.95 & 40.21 \\
\hline S96T003744 & 152: 7 & Drainable liquid & 30.42 & 31.76 & 31.09 \\
\hline S96T003745 & 152: 8 & Drainable liquid & 33.16 & 35 & 34.08 \\
\hline S96T003746 & 152:9 & Drainable liquid & 36.90 & 34.85 & 35.88 \\
\hline \$96T003747 & $152: 10$ & Drainable liquid & $<42.32$ & 42.95 & $<42.64$ \\
\hline S96T003748 & $152: 11$ & Drainable liquid & $<42.32$ & $<42.32$ & $<42.32$ \\
\hline S96T003749 & $152: 12$ & Drainable liquid & $<42.32$ & $<42.32$ & $<42.32$ \\
\hline S96T003750 & $152: 13$ & Drainable liquid & 43.02 & $<42.32$ & $<42.67$ \\
\hline S96T003751 & $152: 14$ & Drainable liquid & 35.84 & 30.70 & 33.27 \\
\hline S96T004433 & 153: 1 & Drainable liquid & 35.63 & 32.67 & 34.15 \\
\hline S96T004308 & 153: 2 & Drainable liquid & $<42.32$ & $<42.32$ & $<42.32$ \\
\hline S96T004434 & 153: 3 & Drainable liquid & 31.54 & 35.77 & 33.66 \\
\hline S96T004419 & 153: 5 & Drainable liquid & $<42.32$ & $<42.32$ & $<42.32$ \\
\hline S96T004420 & 153: 6 & Drainable liquid & $<42.32$ & $<42.32$ & $<42.32$ \\
\hline S96T004421 & 153: 7 & Drainable liquid & 30.70 & 34.01 & 32.35 \\
\hline S96T003975 & 153: 8 & Drainable Liquid & 34.2254 & 30.7746 & 32.5 \\
\hline S96T003976 & 153: 9 & Drainable liquid & 34.43 & 35.21 & 34.82 \\
\hline \$96T004309 & $153: 10$ & Drainable liquid & $<42.32$ & $<42.32$ & $<42.32$ \\
\hline S96T003979 & 153:11 & Drainable liquid & $<42.32$ & $<42.32$ & $<42.32$ \\
\hline S96T003977 & $153: 12$ & Drainable liquid & $<42.32$ & $<42.32$ & $<42.32$ \\
\hline S96T003978 & $153: 13$ & Drainabje liquid & $<42.32$ & $<42.32$ & $<42.32$ \\
\hline S96T004333 & $153: 14$ & Drainable liquid & $<42.32$ & $<42.32$ & $<42.32$ \\
\hline S96T003980 & $153: 21$ & Drainable liquid & $<42.32$ & $<42.32$ & $<42.32$ \\
\hline
\end{tabular}


Table B2-35. Tank 241-AN-105 Analytical Results: Lead (ICP). (3 sheets)

\begin{tabular}{|c|c|c|c|c|c|}
\hline Samiple & gomplo & Sarpole & $\operatorname{Ros} \times 111$ & Buplente & viran \\
\hline \multicolumn{2}{|c|}{ Gquits a ard figest } & X: & 1485 & $10 \%$ & 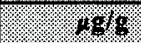 \\
\hline S96T004144 & Core 152 & Liquid composite & $<36.90$ & $<35.21$ & $<36.05$ \\
\hline S96T004831 & Core 152 & Liquid composite & $<42.32$ & $<42.32$ & $<42.32$ \\
\hline \multicolumn{2}{|c|}{ Solingt rag ofgedt } & 6 & 48 & 196 & $1+y_{0}$ \\
\hline S96T003906 & 152: 1 & Upper half & $<38.6$ & 41.4 & $<40$ \\
\hline S96T003898 & \multirow[t]{2}{*}{$152: 14$} & Upper half & $<39.3$ & $<38.8$ & $<39.05$ \\
\hline S96 T003828 & & Lower half & $<40.4$ & $<39.3$ & $<39.85$ \\
\hline S96T003899 & \multirow[t]{2}{*}{$152: 16$} & Upper half & 41 & $<39.8$ & $<40.4$ \\
\hline S96T003900 & & Lower half & 42.8 & 41.9 & 42.35 \\
\hline S96T003901 & \multirow[t]{2}{*}{$152: 18$} & Upper half & 47.3 & $<39.6$ & $<43.45$ \\
\hline S96T003902 & & Lower half & 44.3 & $<40.4$ & $<42.35$ \\
\hline \$96T003903 & \multirow[t]{2}{*}{$152: 20$} & Upper half & 43.2 & 42.9 & 43.05 \\
\hline S96T003904 & & Lower half & $<37.5$ & 38.5 & $<38$ \\
\hline S96T003905 & $152: 22$ & Upper half & 45.5 & 41.7 & 43.6 \\
\hline S96T004338 & $153: 14$ & Lower half & $<46.7$ & $<47.8$ & $<47.25$ \\
\hline S96T004315 & \multirow[t]{2}{*}{$153: 15$} & Upper half & $<47.6$ & $<47.8$ & $<47.7$ \\
\hline S96T004318 & & Lower half & $<47.9$ & $<45.1$ & $<46.5$ \\
\hline \$96T004444 & \multirow[t]{2}{*}{$153: 17$} & Upper half & $<48.3$ & $<47.5$ & $<47.9$ \\
\hline S96T004445 & & Lower half & 45.4 & 56.1 & $50.75^{\text {QCe }}$ \\
\hline S96T004446 & $153: 19$ & Lower half & 43.2 & 41.7 & 42.45 \\
\hline S96T003989 & \multirow[t]{2}{*}{$153: 22$} & Upper half & 47.9 & 51.9 & 49.9 \\
\hline \$96T003987 & & Lower half & 115 & 118 & 116.5 \\
\hline S96T004257 & \multirow[t]{2}{*}{ Core 152} & Solid composite & $<38.3$ & $<40.3$ & $<39.3$ \\
\hline \$96T004262 & & Solid composite & 51.8 & 47.6 & 49.7 \\
\hline
\end{tabular}


Table B2-35. Tank 241-AN-105 Analytical Results: Lead (ICP). (3 sheets)

\begin{tabular}{|c|c|c|c|c|c|}
\hline Sample & $\% 0 \% 1010$ & . & $4+111$ & Buplicate & Hern. \\
\hline 6010 & $1610 \%$ & & $6 \%$ & 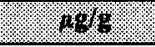 & $\%$ \\
\hline S96T003893 & 152: 1 & Upper half & $<1,980$ & $<2,010$ & $<1,995$ \\
\hline S96T003894 & \multirow[t]{2}{*}{$152: 14$} & Upper half & $<2,040$ & $<2,000$ & $<2,020$ \\
\hline S96T003827 & & Lower half & $<2,010$ & $<2,060$ & $<2,035$ \\
\hline S96T003895 & \multirow[t]{2}{*}{$152: 16$} & Upper half & $<2,040$ & $<2,010$ & $<2,025$ \\
\hline S96T003889 & & Lower half & $<2,030$ & $<2,000$ & $<2,015$ \\
\hline S96T003896 & \multirow[t]{2}{*}{$152: 18$} & Upper half & $<2,070$ & $<2,010$ & $<2,040$ \\
\hline S96T003890 & & Lower half & $<2,060$ & $<2,070$ & $<2,065$ \\
\hline \$96T003897 & \multirow[t]{2}{*}{$152: 20$} & Upper half & $<2,120$ & $<2,070$ & $<2,095$ \\
\hline S96T003891 & & Lower half & $<2,040$ & $<2,060$ & $<2,050$ \\
\hline S96T003892 & $152: 22$ & Upper half & $<2,090$ & $<2,120$ & $<2,105$ \\
\hline S96T004337 & $153: 14$ & Lower half & $<2,030$ & $<2,030$ & $<2,030$ \\
\hline S96T004314 & \multirow[t]{2}{*}{$153: 15$} & Upper half & $<1,910$ & $<1,920$ & $<1,915$ \\
\hline S96T004317 & & Lower half & $<2,000$ & $<2,030$ & $<2,015$ \\
\hline S96T004441 & \multirow[t]{2}{*}{$153: 17$} & Upper half & $<1,960$ & $<1,960$ & $<1,960$ \\
\hline S96T004442 & & Lower half & $<2,010$ & $<2,000$ & $<2,005$ \\
\hline S96T004443 & $153: 19$ & Lower half & $<2,020$ & $<2,000$ & $<2,010$ \\
\hline S96T003984 & $153: 21$ & Lower half & $<2,010$ & $<2,010$ & $<2,010$ \\
\hline S96T003986 & \multirow[t]{2}{*}{$153: 22$} & Upper half & $<2,000$ & $<2,030$ & $<2,015$ \\
\hline S96T003985 & & Lower half & $<2,010$ & $<2,010$ & $<2,010$ \\
\hline
\end{tabular}


Table B2-36. Tank 241-AN-105 Analytical Results: Lithium (ICP) ${ }^{1}$. (3 sheets)

\begin{tabular}{|c|c|c|c|c|c|}
\hline $\begin{array}{l}\text { Sanple } \\
\text { Yunilor }\end{array}$ & Trampres & Q9inole & postil & Bupleate & $110 \%$ \\
\hline \multicolumn{2}{|c|}{ Wopras } & & 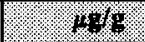 & $16 \%$ & 48 \\
\hline S96T003732 & 152: 1 & Drainable liquid & $<2.82$ & $<2.82$ & $<2.82$ \\
\hline S96T003739 & 152: 2 & Drainable liquid & 3.61 & 3.64 & 3.63 \\
\hline S96T003740 & 152: 3 & Drainable liquid & $<2.82$ & $<2.82$ & $<2.82$ \\
\hline S96T003741 & 152: 4 & Drainable liquid & 3.68 & 3.68 & 3.68 \\
\hline S96T003742 & 152: 5 & Drainable liquid & 3.23 & $<2.82$ & $<3.02$ \\
\hline S96T003743 & 152: 6 & Drainable liquid & 3.78 & 4.61 & 4.20 \\
\hline S96T003744 & $152: 7$ & Drainable liquid & 7.74 & $<2.82$ & $<5.28^{\mathrm{QC:e}}$ \\
\hline S96T003745 & 152: 8 & Drainable liquid & 19.22 & 4.07 & $11.64^{\mathrm{QC:e}}$ \\
\hline S96T003746 & 152: 9 & Drainable liquid & 4.18 & 4.25 & 4.21 \\
\hline S96T003747 & $152: 10$ & Drainable liquid & $<4.23$ & $<4.23$ & $<4.23$ \\
\hline S96T003748 & $152: 11$ & Drainable liquid & $<4.23$ & $<4.23$ & $<4.23$ \\
\hline \$96T003749 & $152: 12$ & Drainable liquid & 4.71 & 4.66 & 4.69 \\
\hline S96T003750 & $152: 13$ & Drainable liquid & $<4.23$ & $<4.23$ & $<4.23$ \\
\hline S96T003751 & $152: 14$ & Drainable liquid & 5.32 & $<2.82$ & $<4.07^{\mathrm{QC} \cdot \mathrm{C}}$ \\
\hline S96T004433 & 153: 1 & Drainable liquid & $<2.82$ & $<2.82$ & $<2.82$ \\
\hline \$96T004308 & 153: 2 & Drainable liquid & $<4.23$ & $<4.23$ & $<4.23$ \\
\hline \$96T004434 & 153: 3 & Drainable liquid & 9.71 & 7.74 & $8.73^{\mathrm{QC}: \mathrm{e}}$ \\
\hline S96T004419 & 153: 5 & Drainable liquid & $<4.23$ & $<4.23$ & $<4.23$ \\
\hline S96T004420 & 153: 6 & Drainable liquid & $<4.23$ & $<4.23$ & $<4.23$ \\
\hline S96T004421 & 153: 7 & Drainable liquid & 14.78 & 4.94 & $9.86^{\text {QC:e }}$ \\
\hline S96T003975 & 153: 8 & Drainable liquid & 5.56 & 8.80 & $7.18^{\mathrm{QC} e c}$ \\
\hline S96T003976 & 153: 9 & Drainable liquid & $<2.82$ & 2.92 & $<2.87$ \\
\hline S96T004309 & $153: 10$ & Drainable liquid & $<4.23$ & $<4.23$ & $<4.23$ \\
\hline S96T003979 & 153:11 & Drainable liquid & $<4.23$ & $<4.23$ & $<4.23$ \\
\hline S96T003977 & 153:12 & Drainable liquid & $<4.23$ & $<4.23$ & $<4.23$ \\
\hline S96T003978 & $153: 13$ & Drainable liquid & $<4.23$ & $<4.23$ & $<4.23$ \\
\hline S96T004333 & $153: 14$ & Drainable liquid & $<4.23$ & $<4.23$ & $<4.23$ \\
\hline S96T003980 & $153: 21$ & Drainable liquid & $<4.23$ & $<4.23$ & $<4.23$ \\
\hline S96T004831 & Core 152 & Liquid composite & $<4.23$ & $<4.23$ & $<4.23$ \\
\hline
\end{tabular}


Table B2-36. Tank 241-AN-105 Analytical Results: Lithium (ICP) ${ }^{1}$. (3 sheets)

\begin{tabular}{|c|c|c|c|c|c|}
\hline Sample & Tomple & 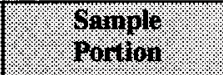 & Resail & Auplicate & Hegin \\
\hline \multicolumn{2}{|c|}{ 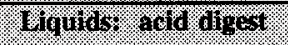 } & 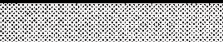 & 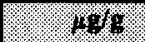 & 1018 & 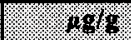 \\
\hline S96T004144 & Core 152 & Liquid Composite & 4.09859 & 4.30282 & 4.2007 \\
\hline \multicolumn{2}{|c|}{ Sollas wad higest } & & 1019 & $29 \%$ & 1969 \\
\hline S96T003906 & $152: 1$ & Upper half & $<3.86$ & $<4.03$ & $<3.94$ \\
\hline S96T003898 & \multirow[t]{2}{*}{$152: 14$} & Upper half & 30.1 & 30.3 & 30.2 \\
\hline S96T003828 & & Lower half & 12.8 & 13.5 & 13.15 \\
\hline \begin{tabular}{|l|} 
S96T003899 \\
\end{tabular} & \multirow[t]{2}{*}{$152: 16$} & Upper half & 8.94 & 7.13 & $8.03^{\mathrm{QC} c \mathrm{c}}$ \\
\hline \$96T003900 & & Lower half & $<3.93$ & $<3.93$ & $<3.93$ \\
\hline S96T003901 & \multirow[t]{2}{*}{$152: 18$} & Upper half & 7.25 & 4.05 & $5.65^{Q C: e}$ \\
\hline S96T003902 & & Lower half & 12 & 12.8 & 12.4 \\
\hline S96T003903 & \multirow[t]{2}{*}{$152: 20$} & Upper half & 12.2 & 12 & 12.1 \\
\hline S96T003904 & & Lower half & $<3.75$ & $<3.71$ & $<3.73$ \\
\hline S96T003905 & $152: 22$ & Upper half & 82.5 & 6.75 & $44.62^{\mathrm{QC}: \mathrm{e}}$ \\
\hline S96T004338 & $153: 14$ & Lower half & 7.91 & 8.34 & 8.12 \\
\hline S96T004315 & \multirow[t]{2}{*}{$153: 15$} & Upper half & 29 & 30.2 & 29.6 \\
\hline S96T004318 & & Lower half & 8.11 & 8.81 & 8.46 \\
\hline S96T004444 & \multirow[t]{2}{*}{$153: 17$} & Upper half & 9.25 & 11.3 & 10.27 \\
\hline \$96T004445 & & Lower half & 9.55 & 13.2 & $11.37^{\mathrm{QC:e}}$ \\
\hline S96T004446 & 153:19 & Lower half & 14.6 & 15.4 & 15 \\
\hline S96T003988 & $153: 21$ & Lower half & 64.3 & 62.8 & 63.55 \\
\hline S96T003989 & \multirow[t]{2}{*}{$153: 22$} & Upper half & 8.85 & 12.2 & $10.52^{\mathrm{QC}: \mathrm{e}}$ \\
\hline S96T003987 & & Lower half & 26.7 & 28.3 & 27.5 \\
\hline S96T004257 & \multirow[t]{2}{*}{ Core 152} & Solid composite & 8.6 & 8.8 & 8.7 \\
\hline S96T004262 & & Solid composite & 7.32 & 22.1 & $14.71^{\mathrm{QC}: \mathrm{e}}$ \\
\hline
\end{tabular}


Table B2-36. Tank 241-AN-105 Analytical Results: Lithium (ICP) ${ }^{1}$. (3 sheets)

\begin{tabular}{|c|c|c|c|c|c|}
\hline \%1110\% & 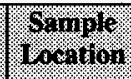 & 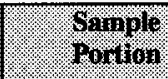 & Romil & Diplicate. & Menin \\
\hline Solido & Tision & & 48 & 1488 & 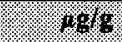 \\
\hline S96'T003893 & 152: 1 & Upper half & $<198$ & $<201$ & $<199.5$ \\
\hline S96T003894 & $152: 14$ & Upper half & $<204$ & $<200$ & $<202$ \\
\hline S96T003827 & & Lower half & $<201$ & $<206$ & $<203.5$ \\
\hline S96T003895 & $152: 16$ & Upper half & $<204$ & $<201$ & $<202.5$ \\
\hline S96T003889 & & Lower half & $<203$ & $<200$ & $<201.5$ \\
\hline S96T003896 & $152: 18$ & Upper half & $<207$ & $<201$ & $<204$ \\
\hline S96T003890 & & Lower half & $<206$ & $<207$ & $<206.5$ \\
\hline S96T003897 & $152: 20$ & Upper half & $<212$ & $<207$ & $<209.5$ \\
\hline S96T003891 & & Lower half & $<204$ & $<206$ & $<205$ \\
\hline S96T003892 & $152: 22$ & Upper half & $<209$ & $<212$ & $<210.5$ \\
\hline S96T004337 & $153: 14$ & Lower half & $<203$ & $<203$ & $<203$ \\
\hline S96T004314 & $153: 15$ & Upper half & $<191$ & $<192$ & $<191.5$ \\
\hline S96T004317 & & Lower half & $<200$ & $<203$ & $<201.5$ \\
\hline S96T004441 & $153: 17$ & Upper half & $<196$ & $<196$ & $<196$ \\
\hline S96T004442 & & Lower half & $<201$ & $<200$ & $<200.5$ \\
\hline S96T004443 & $153: 19$ & Lower half & $<202$ & $<200$ & $<201$ \\
\hline S96T003984 & $153: 21$ & Lower half & $<201$ & $<201$ & $<201$ \\
\hline S96T003986 & $153: 22$ & Upper half & $<200$ & $<203$ & $<201.5$ \\
\hline S96T003985 & & Lower half & $<201$ & $<201$ & $<201$ \\
\hline
\end{tabular}

Note:

${ }^{1}$ Lithium is from hydrostatic head fluid. 
Table B2-37. Tank 241-AN-105 Analytical Results: Magnesium (ICP). (3 sheets)

\begin{tabular}{|c|c|c|c|c|c|}
\hline Sample & ormple & 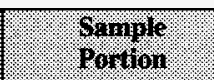 & Resili & Suplicale & Mean \\
\hline \multicolumn{2}{|c|}{ Wourds } & & $8 y^{2}$ & 1989 & 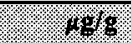 \\
\hline S96T003732 & $152: 1$ & Drainable liquid & $<28.23$ & $<28.23$ & $<28.23$ \\
\hline S96T003739 & 152: 2 & Drainable liquid & $<28.23$ & $<28.23$ & $<28.23$ \\
\hline S96T003740 & 152: 3 & Drainable liquid & $<28.23$ & $<28.23$ & $<28.23$ \\
\hline S96'T003741 & 152: 4 & Drainable liquid & $<28.23$ & $<28.23$ & $<28.23$ \\
\hline S96T003742 & $152: 5$ & Drainable liquid & $<28.23$ & $<28.23$ & $<28.23$ \\
\hline S96T003743 & 152: 6 & Drainable liquid & $<28.23$ & $<28.23$ & $<28.23$ \\
\hline S96T003744 & 152: 7 & Drainable liquid & $<28.23$ & $<28.23$ & $<28.23$ \\
\hline S96T003745 & 152: 8 & Drainable liquid & $<28.23$ & $<28.23$ & $<28.23$ \\
\hline S96T003746 & 152: 9 & Drainable liquid & $<28.23$ & $<28.23$ & $<28.23$ \\
\hline S96T003747 & $152: 10$ & Drainable liquid & $<42.32$ & $<42.32$ & $<42.32$ \\
\hline S96T003748 & $152: 11$ & Drainable liquid & $<42.32$ & $<42.32$ & $<42.32$ \\
\hline S96T003749 & $152: 12$ & Drainable liquid & $<42.32$ & $<42.32$ & $<42.32$ \\
\hline S96T003750 & $152: 13$ & Drainable liquid & $<42.32$ & $<42.32$ & $<42.32$ \\
\hline S96T003751 & $152: 14$ & Drainable liquid & $<28.23$ & $<28.23$ & $<28.23$ \\
\hline S96T004433 & $153: 1$ & Drainable liquid & $<28.23$ & $<28.23$ & $<28.23$ \\
\hline \$96T004308 & 153: 2 & Drainable liquid & $<42.32$ & $<42.32$ & $<42.32$ \\
\hline S96T004434 & 153: 3 & Drainable liquid & $<28.23$ & $<28.23$ & $<28.23$ \\
\hline S96T004419 & 153: 5 & Drainable liquid & $<42.32$ & $<42.32$ & $<42.32$ \\
\hline S96T004420 & 153: 6 & Drainable liquid & $<42.32$ & $<42.32$ & $<42.32$ \\
\hline S96T004421 & 153: 7 & Drainable liquid & $<28.23$ & $<28.23$ & $<28.23$ \\
\hline S96T003975 & 153: 8 & Drainable liquid & $<28.23$ & $<28.23$ & $<28.23$ \\
\hline S96T003976 & 153: 9 & Drainable liquid & $<28.23$ & $<28.23$ & $<28.23$ \\
\hline S96T004309 & $153: 10$ & Drainable liquid & $<42.32$ & $<42.32$ & $<42.32$ \\
\hline S96T003979 & $153: 11$ & Drainable liquid & $<42.32$ & $<42.32$ & $<42.32$ \\
\hline S96T003977 & $153: 12$ & Drainable liquid & $<42.32$ & $<42.32$ & $<42.32$ \\
\hline S96T003978 & $153: 13$ & Drainable liquid & $<42.32$ & $<42.32$ & $<42.32$ \\
\hline \begin{tabular}{|l|} 
S96T004333 \\
\end{tabular} & 153:14 & Drainable liquid & $<42.32$ & $<42.32$ & $<42.32$ \\
\hline S96T003980 & $153: 21$ & Drainable liquid & $<42.32$ & $<42.32$ & $<42.32$ \\
\hline S96T004831 & Core 152 & Liquid composite & $<42.32$ & $<42.32$ & $<42.32$ \\
\hline
\end{tabular}


Table B2-37. Tank 241-AN-105 Analytical Results: Magnesium (ICP). (3 sheets)

\begin{tabular}{|c|c|c|c|c|c|}
\hline Wainolit & Sample & . Trmple & Pasull & 3rpiloule & Mean \\
\hline \multicolumn{2}{|c|}{ 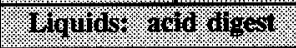 } & & $10 \%$ & $20 \%$ & 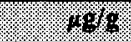 \\
\hline \$96T004144 & Core 152 & Liquid composite & $<36.90$ & $<35.21$ & $<36.05$ \\
\hline \multicolumn{2}{|c|}{ Solmos agroghert } & & 1980 & $4 \mathrm{~g} \%$ & 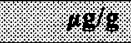 \\
\hline S96T003906 & 152: 1 & Upper half & $<38.6$ & $<40.3$ & $<39.45$ \\
\hline S96T003898 & \multirow[t]{2}{*}{$152: 14$} & Upper half & $<39.3$ & $<38.8$ & $<39.05$ \\
\hline S96T003828 & & Lower half & $<40.4$ & $<39.3$ & $<39.85$ \\
\hline S96T003899 & \multirow[t]{2}{*}{$152: 16$} & Upper half & $<39.6$ & $<39.8$ & $<39.7$ \\
\hline S96T003900 & & Lower half & $<39.3$ & $<39.3$ & $<39.3$ \\
\hline S96T003901 & \multirow[t]{2}{*}{$152: 18$} & Upper half & $<38.9$ & $<39.6$ & $<39.25$ \\
\hline S96T003902 & & Lower half & $<40$ & $<40.4$ & $<40.2$ \\
\hline S96T003903 & \multirow[t]{2}{*}{$152: 20$} & Upper half & $<38.3$ & $<37.6$ & $<37.95$ \\
\hline S96T003904 & & Lower half & $<37.5$ & $<37.1$ & $<37.3$ \\
\hline S96T003905 & $152: 22$ & Upper half & $<39.6$ & $<41.4$ & $<40.5$ \\
\hline S96T004338 & 153:14 & Lower half & $<46.7$ & $<47.8$ & $<47.25$ \\
\hline S96T004315 & \multirow[t]{2}{*}{$153: 15$} & Upper half & $<47.6$ & $<47.8$ & $<47.7$ \\
\hline S96T004318 & & Lower half & $<47.9$ & $<45.1$ & $<46.5$ \\
\hline S96T004444 & \multirow[t]{2}{*}{$153: 17$} & Upper half & $<48.3$ & $<47.5$ & $<47.9$ \\
\hline S96T004445 & & Lower half & $<37.1$ & $<36.6$ & $<36.85$ \\
\hline S96T004446 & $153: 19$ & Lower half & $<38.2$ & $<38.2$ & $<38.2$ \\
\hline S96T003988 & $153: 21$ & Lower half & $<35.6$ & $<35.6$ & $<35.6$ \\
\hline S96T003989 & \multirow[t]{2}{*}{$153: 22$} & Upper half & $<46.5$ & $<46.2$ & $<46.35$ \\
\hline S96T003987 & & Lower half & 58.3 & 60.9 & 59.6 \\
\hline S96T004257 & \multirow[t]{2}{*}{ Core 152} & Solid composite & $<38.3$ & $<40.3$ & $<39.3$ \\
\hline S96T004262 & & Solid composite & $<38.9$ & $<37$ & $<37.95$ \\
\hline
\end{tabular}


Table B2-37. Tank 241-AN-105 Analytical Results: Magnesium (ICP). (3 sheets)

\begin{tabular}{|c|c|c|c|c|c|}
\hline 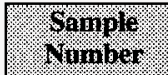 & Sorinple & Srmple & Aesull & Buplicile & . \\
\hline Soligs, & Giviris & ? & $1 / 8 / 8$ & 1098 & $49 \%$ \\
\hline \$96T003893 & 152: 1 & Upper half & $<1,980$ & $<2,010$ & $<1,995$ \\
\hline S96T003894 & \multirow[t]{2}{*}{$152: 14$} & Upper half & $<2,040$ & $<2,000$ & $<2,020$ \\
\hline S96T003827 & & Lower half & $<2,010$ & $<2,060$ & $<2,035$ \\
\hline S96T003895 & \multirow[t]{2}{*}{$152: 16$} & Upper half & $<2,040$ & $<2,010$ & $<2,025$ \\
\hline \$96T003889 & & Lower half & $<2,030$ & $<2,000$ & $<2,015$ \\
\hline S96T003896 & \multirow[t]{2}{*}{$152: 18$} & Upper half & $<2,070$ & $<2,010$ & $<2,040$ \\
\hline S96T003890 & & Lower half & $<2,060$ & $<2,070$ & $<2,065$ \\
\hline S96T003897 & \multirow[t]{2}{*}{$152: 20$} & Upper half & $<2,120$ & $<2,070$ & $<2,095$ \\
\hline S96T003891 & & Lower half & $<2,040$ & $<2,060$ & $<2,050$ \\
\hline S96T003892 & $152: 22$ & Upper half & $<2,090$ & $<2,120$ & $<2,105$ \\
\hline S96T004337 & $153: 14$ & Lower half & $<2,030$ & $<2,030$ & $<2,030$ \\
\hline S96T004314 & \multirow[t]{2}{*}{$153: 15$} & Upper half & $<1,910$ & $<1,920$ & $<1,915$ \\
\hline S96T004317 & & Lower half & $<2,000$ & $<2,030$ & $<2,015$ \\
\hline S96T004441 & \multirow[t]{2}{*}{$153: 17$} & Upper half & $<1,960$ & $<1,960$ & $<1,960$ \\
\hline S96T004442 & & Lower half & $<2,010$ & $<2,000$ & $<2,005$ \\
\hline S96T004443 & $153: 19$ & Lower half & $<2,020$ & $<2,000$ & $<2,010$ \\
\hline S96T003984 & $153: 21$ & Lower half & $<2,010$ & $<2,010$ & $<2,010$ \\
\hline S96T003986 & \multirow[t]{2}{*}{$153: 22$} & Upper half & $<2,000$ & $<2,030$ & $<2,015$ \\
\hline S96T003985 & & Lower half & $<2,010$ & $<2,010$ & $<2,010$ \\
\hline
\end{tabular}


Table B2-38. Tank 241-AN-105 Analytical Results: Manganese (ICP). (3 sheets)

\begin{tabular}{|c|c|c|c|c|c|}
\hline Wamplo & Ganple & 3.3nure & arsill & Biplicito & Meran \\
\hline \multicolumn{2}{|c|}{ Yough } & & 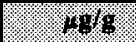 & 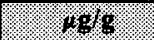 & 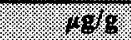 \\
\hline S96T003732 & 152: 1 & Drainable liquid & $<2.82$ & $<2.82$ & $<2.82$ \\
\hline S96T003739 & 152: 2 & Drainable liquid & $<2.82$ & $<2.82$ & $<2.82$ \\
\hline S96T003740 & 152: 3 & Drainable liquid & $<2.82$ & $<2.82$ & $<2.82$ \\
\hline S96T003741 & 152: 4 & Drainable liquid & $<2.82$ & $<2.82$ & $<2.82$ \\
\hline S96T003742 & 152: 5 & Drainable liquid & $<2.82$ & $<2.82$ & $<2.82$ \\
\hline S96T003743 & 152: 6 & Drainable liquid & $<2.82$ & $<2.82$ & $<2.82$ \\
\hline S96T003744 & 152:7 & Drainable liquid & $<2.82$ & $<2.82$ & $<2.82$ \\
\hline S96T003745 & 152: 8 & Drainable liquid & $<2.82$ & $<2.82$ & $<2.82$ \\
\hline S96T003746 & 152: 9 & Drainable liquid & $<2.82$ & $<2.82$ & $<2.82$ \\
\hline \$96T003747 & $152: 10$ & Drainable liquid & $<4.23$ & $<4.23$ & $<4.23$ \\
\hline S96T003748 & $152: 11$ & Drainable liquid & $<4.23$ & $<4.23$ & $<4.23$ \\
\hline S96T003749 & $152: 12$ & Drainable liquid & $<4.23$ & $<4.23$ & $<4.23$ \\
\hline S96T003750 & $152: 13$ & Drainable liquid & $<4.23$ & $<4.23$ & $<4.23$ \\
\hline S96T003751 & $152: 14$ & Drainable liquid & $<2.82$ & $<2.82$ & $<2.82$ \\
\hline S96T004433 & 153: 1 & Drainable liquid & $<2.82$ & $<2.82$ & $<2.82$ \\
\hline S96T004308 & 153: 2 & Drainable liquid & $<4.23$ & $<4.23$ & $<4.23$ \\
\hline S96T004434 & 153: 3 & Drainable liquid & $<2.82$ & $<2.82$ & $<2.82$ \\
\hline \$96T004419 & 153: 5 & Drainable liquid & $<4.23$ & $<4.23$ & $<4.23$ \\
\hline S96T004420 & 153: 6 & Drainable liquid & $<4.23$ & $<4.23$ & $<4.23$ \\
\hline S96T004421 & 153: 7 & Drainable liquid & $<2.82$ & $<2.82$ & $<2.82$ \\
\hline S96T003975 & 153: 8 & Drainable liquid & $<2.82$ & $<2.82$ & $<2.82$ \\
\hline \$96T003976 & 153: 9 & Drainable liquid & $<2.82$ & $<2.82$ & $<2.82$ \\
\hline S96T004309 & $153: 10$ & Drainable liquid & $<4.23$ & $<4.23$ & $<4.23$ \\
\hline S96T003979 & $153: 11$ & Drainable liquid & $<4.23$ & $<4.23$ & $<4.23$ \\
\hline S96T003977 & $153: 12$ & Drainable liquid & $<4.23$ & $<4.23$ & $<4.23$ \\
\hline S96T003978 & $153: 13$ & Drainable liquid & $<4.23$ & $<4.23$ & $<4.23$ \\
\hline S96T004333 & $153: 14$ & Drainable liquid & $<4.23$ & $<4.23$ & $<4.23$ \\
\hline S96T003980 & $153: 21$ & Drainable liquid & $<4.23$ & $<4.23$ & $<4.23$ \\
\hline S96T004831 & Core 152 & Liquid composite & $<4.23$ & $<4.23$ & $<4.23$ \\
\hline
\end{tabular}


Table B2-38. Tank 241-AN-105 Analytical Results: Manganese (ICP). (3 sheets)

\begin{tabular}{|c|c|c|c|c|c|}
\hline Saminge & Sample: & S Soritongle & 2) & Biplicate & MYat \\
\hline \multicolumn{2}{|c|}{ 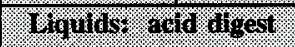 } & & $14 \mathrm{~g}$ & f. & 8910 \\
\hline S96T004144 & Core 152 & Liquid composite & $<3.69$ & $<3.52$ & $<3.60$ \\
\hline \multicolumn{2}{|c|}{ 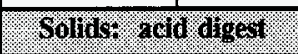 } & & 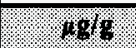 & 1018 & $\log$ \\
\hline S96T003906 & 152: 1 & Upper half & $<3.86$ & $<4.03$ & $<3.945$ \\
\hline S96T003898 & \multirow[t]{2}{*}{$152: 14$} & Upper half & 4.06 & 4.14 & 4.1 \\
\hline S96T003828 & & Lower half & $<4.04$ & $<3.93$ & $<3.985$ \\
\hline \$96T003899 & \multirow[t]{2}{*}{$152: 16$} & Upper half & 8.4 & 10.3 & $9.35^{\mathrm{QC}: \mathrm{e}}$ \\
\hline S96T003900 & & Lower half & 12.8 & 13.1 & 12.95 \\
\hline S96T003901 & \multirow[t]{2}{*}{$152: 18$} & Upper half & 30 & 28.6 & 29.3 \\
\hline S96T003902 & & Lower half & 11.1 & 11.1 & 11.1 \\
\hline S96T003903 & \multirow[t]{2}{*}{$152: 20$} & Upper half & 9.47 & 9.45 & 9.46 \\
\hline S96T003904 & & Lower half & 10.1 & 10.3 & 10.2 \\
\hline S96T003905 & $152: 22$ & Upper half & 33.2 & 34.3 & 33.75 \\
\hline S96T004338 & $153: 14$ & Lower half & 5.3 & 5.77 & 5.53 \\
\hline S96T004315 & \multirow[t]{2}{*}{$153: 15$} & Upper half & 5.15 & 4.86 & 5.00 \\
\hline S96T004318 & & Lower half & $<4.79$ & 5.03 & $<4.91$ \\
\hline S96T004444 & \multirow[t]{2}{*}{$153: 17$} & Upper half & 7.52 & 8.62 & 8.07 \\
\hline S96T004445 & & Lower half & 10.3 & 13.2 & 11.75 \\
\hline S96T004446 & $153: 19$ & Lower half & 10.5 & 10.4 & 10.45 \\
\hline S96T003988 & $153: 21$ & Lower half & 9.4 & 8.89 & 9.14 \\
\hline S96T003989 & \multirow[t]{2}{*}{$153: 22$} & Upper half & 48.8 & 42.3 & 45.55 \\
\hline S96T003987 & & Lower half & 151 & 165 & 158 \\
\hline S96T004257 & \multirow[t]{2}{*}{ Core 152} & Solid composite & 8.46 & 7.18 & $7.82^{Q C: a, c, c}$ \\
\hline S96T004262 & & Solid composite & 52.2 & 46.6 & 49.4 \\
\hline
\end{tabular}


Table B2-38. Tank 241-AN-105 Analytical Results: Manganese (ICP). (3 sheets)

\begin{tabular}{|c|c|c|c|c|c|}
\hline $\begin{array}{l}\text { Sample } \\
\text { Number }\end{array}$ & $\begin{array}{l}\text { Sample } \\
\text { Uocation }\end{array}$ & $\begin{array}{l}\text { Grimple } \\
\text { Portion }\end{array}$ & Resilu & Duplicate & Mean \\
\hline Sollas & Woilon & & 1980 & 1.196 & 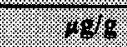 \\
\hline S96T003893 & 152: 1 & Upper half & $<198$ & $<201$ & $<199.5$ \\
\hline S96T003894 & \multirow[t]{2}{*}{$152: 14$} & Upper half & $<204$ & $<200$ & $<202$ \\
\hline S96T003827 & & Lower half & $<201$ & $<206$ & $<203.5$ \\
\hline S96T003895 & \multirow[t]{2}{*}{$152: 16$} & Upper half & $<204$ & $<201$ & $<202.5$ \\
\hline S96T003889 & & Lower half & $<203$ & $<200$ & $<201.5$ \\
\hline S96T003896 & \multirow[t]{2}{*}{$152: 18$} & Upper half & $<207$ & $<201$ & $<204$ \\
\hline S96T003890 & & Lower half & $<206$ & $<207$ & $<206.5$ \\
\hline S96T003897 & \multirow[t]{2}{*}{$152: 20$} & Upper half & $<212$ & $<207$ & $<209.5$ \\
\hline S96T003891 & & Lower half & $<204$ & $<206$ & $<205$ \\
\hline S96T003892 & $152: 22$ & Upper half & $<209$ & $<212$ & $<210.5$ \\
\hline S96T004337 & $153: 14$ & Lower half & $<203$ & $<203$ & $<203$ \\
\hline S96T004314 & \multirow[t]{2}{*}{$153: 15$} & Upper half & $<191$ & $<192$ & $<191.5$ \\
\hline S96T004317 & & Lower half & $<200$ & $<203$ & $<201.5$ \\
\hline S96T004441 & \multirow[t]{2}{*}{$153: 17$} & Upper half & $<196$ & $<196$ & $<196$ \\
\hline \$96T004442 & & Lower half & $<201$ & $<200$ & $<200.5$ \\
\hline S96T004443 & $153: 19$ & Lower half & $<202$ & $<200$ & $<201$ \\
\hline S96T003984 & $153: 21$ & Lower half & $<201$ & $<201$ & $<201$ \\
\hline S96T003986 & \multirow[t]{2}{*}{$153: 22$} & Upper half & $<200$ & $<203$ & $<201.5$ \\
\hline S96T003985 & & Lower half & $<201$ & $<201$ & $<201$ \\
\hline
\end{tabular}


Table B2-39. Tank 241-AN-105 Analytical Results: Molybdenum (ICP). (3 sheets)

\begin{tabular}{|c|c|c|c|c|c|}
\hline ramplor & Saruple & $60 \% 1019$ & recsin & Miplicale & Hentin \\
\hline \multicolumn{2}{|c|}{ Gigids } & & 1,40 & 1018 & 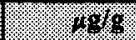 \\
\hline S96T003732 & 152: 1 & Drainable liquid & 71.12 & 68.66 & 69.89 \\
\hline S96T003739 & 152: 2 & Drainable liquid & 66.69 & 66.90 & 66.79 \\
\hline S96T003740 & $152: 3$ & Drainable liquid & 67.25 & 65.35 & 66.30 \\
\hline S96T003741 & 152: 4 & Drainable liquid & 69.43 & 65.98 & 67.71 \\
\hline S96T003742 & 152: 5 & Drainable liquid & 70.42 & 68.02 & 69.22 \\
\hline S96T003743 & 152: 6 & Drainable liquid & 72.53 & 76.76 & 74.64 \\
\hline \begin{tabular}{|l|} 
S96'T003744 \\
\end{tabular} & 152: 7 & Drainable liquid & 58.09 & 64.78 & 61.44 \\
\hline S96T003745 & 152: 8 & Drainable liquid & 60.07 & 76.05 & 68.06 \\
\hline S96T003746 & 152: 9 & Drainable liquid & 66.54 & 68.23 & 67.39 \\
\hline S96T003747 & $152: 10$ & Drainable liquid & 69.57 & 69.15 & 69.36 \\
\hline S96T003748 & $152: 11$ & Drainable liquid & 66.83 & 69.29 & 68.06 \\
\hline S96T003749 & $152: 12$ & Drainable liquid & 69.08 & 66.54 & 67.81 \\
\hline S96T003750 & $152: 13$ & Drainable liquid & 65.77 & 64.15 & 64.96 \\
\hline S96T003751 & $152: 14$ & Drainable liquid & 61.69 & 62.32 & 62.00 \\
\hline S96T004433 & 153: 1 & Drainable liquid & 73.94 & 70.42 & 72.18 \\
\hline S96T004308 & 153: 2 & Drainable liquid & 71.12 & 68.45 & 69.78 \\
\hline S96T004434 & 153: 3 & Drainable liquid & 65 & 72.53 & 68.76 \\
\hline S96T004419 & 153: 5 & Drainable liquid & 71.83 & 72.53 & 72.18 \\
\hline S96T004420 & 153: 6 & Drainable liquid & 68.09 & 73.94 & 71.02 \\
\hline S96T004421 & 153: 7 & Drainable liquid & 69.08 & 72.53 & 70.80 \\
\hline S96T003975 & $153: 8$ & Drainable liquid & 64.08 & 59.92 & 62.00 \\
\hline S96T003976 & 153: 9 & Drainable liquid & 58.45 & 70.07 & 64.26 \\
\hline S96T004309 & $153: 10$ & Drainable liquid & 71.12 & 73.94 & 72.53 \\
\hline \$96T003979 & 153:11 & Drainable liquid & 68.02 & 67.46 & 67.74 \\
\hline S96T003977 & $153: 12$ & Drainable liquid & 67.60 & 71.12 & 69.36 \\
\hline S96T003978 & $153: 13$ & Drainable liquid & 63.80 & 58.59 & 61.19 \\
\hline S96T004333 & $153: 14$ & Drainable liquid & 72.53 & 72.53 & 72.53 \\
\hline S96T003980 & $153: 21$ & Drainable liquid & 51.26 & 50 & 50.63 \\
\hline 6S96T004831 & Core 152 & Liquid composite & 70.42 & 71.83 & 71.12 \\
\hline
\end{tabular}


Table B2-39. Tank 241-AN-105 Analytical Results: Molybdenum (ICP). (3 sheets)

\begin{tabular}{|c|c|c|c|c|c|}
\hline Samilo & Wamplo & $\begin{array}{l}\text { Whinge } \\
201101\end{array}$ & $8<-111$ & Divilicale & 4011 \\
\hline \multicolumn{2}{|c|}{ 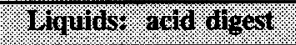 } & & $16 \%$ & $48 \%$ & 618 \\
\hline S96T004144 & Core 152 & Liquid composite & 57.95 & 58.30 & 58.13 \\
\hline \multicolumn{2}{|c|}{ Solds 2 cogdigert } & & $48 \%$ & $108 \mathrm{~s}$ & ofy \\
\hline S96T003906 & 152: 1 & Upper half & 57.8 & 56.6 & 57.2 \\
\hline S96T003898 & \multirow[t]{2}{*}{$152: 14$} & Upper half & 58.9 & 60 & 59.45 \\
\hline S96T003828 & & Lower half & 59.5 & 59.4 & 59.45 \\
\hline S96T003899 & \multirow[t]{2}{*}{$152: 16$} & Upper half & 55.8 & 55.4 & 55.6 \\
\hline S96T003900 & & Lower half & 56.8 & 57.6 & 57.2 \\
\hline S96T003901 & \multirow[t]{2}{*}{$152: 18$} & Upper half & 54.3 & 50.6 & 52.45 \\
\hline S96T003902 & & Lower half & 53.4 & 54.5 & 53.95 \\
\hline S96T003903 & \multirow[t]{2}{*}{$152: 20$} & Upper half & 55.6 & 57.4 & 56.5 \\
\hline S96T003904 & & Lower half & 48.8 & 49.2 & 49 \\
\hline S96T003905 & $152: 22$ & Upper half & 48.4 & 49.8 & 49.1 \\
\hline S96T004338 & 153:14 & Lower half & 53.5 & 51.8 & 52.65 \\
\hline S96T004315 & \multirow[t]{2}{*}{ 153:15 } & Upper half & 53.3 & 49.3 & 51.3 \\
\hline S96T004318 & & Lower half & 50.2 & 51.4 & 50.8 \\
\hline S96T004444 & \multirow[t]{2}{*}{$153: 17$} & Upper half & 50.9 & 51.9 & 51.4 \\
\hline S96T004445 & & Lower half & 50.9 & 52.1 & 51.5 \\
\hline \$96T004446 & $153: 19$ & Lower half & 49.1 & 47.4 & 48.25 \\
\hline S96T003988 & $153: 21$ & Lower half & 43.8 & 43.7 & 43.75 \\
\hline S96T003989 & \multirow[t]{2}{*}{$153: 22$} & Upper half & 46.8 & 51.9 & 49.35 \\
\hline S96T003987 & & Lower half & 36.7 & 37.5 & 37.1 \\
\hline S96T004257 & \multirow[t]{2}{*}{ Core 152} & Solid composite & 45.8 & 45.9 & 45.85 \\
\hline S96T004262 & & Solid composite & 37.4 & 33.4 & 35.4 \\
\hline
\end{tabular}


Table B2-39. Tank 241-AN-105 Analytical Results: Molybdenum (ICP). (3 sheets)

\begin{tabular}{|c|c|c|c|c|c|}
\hline $\begin{array}{l}\text { Sampl } \\
6117 \% \text { of }\end{array}$ & Ganple & 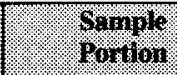 & Pranull & Buplicate & Henar \\
\hline \multicolumn{2}{|c|}{ Soritisy rigion } & & $62 / 5$ & $48 / 8$ & 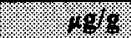 \\
\hline \$96T003893 & 152: 1 & Upper half & $<988$ & $<1,010$ & $<999$ \\
\hline \$96T003894 & \multirow[t]{2}{*}{$152: 14$} & Upper half & $<1,020$ & $<998$ & $<1,009$ \\
\hline S96T003827 & & Lower half & $<1,010$ & $<1,030$ & $<1,020$ \\
\hline S96T003895 & \multirow[t]{2}{*}{$152: 16$} & Upper half & $<1,020$ & $<1,000$ & $<1,010$ \\
\hline S96T003889 & & Lower half & $<1,010$ & $<1,000$ & $<1,005$ \\
\hline \$96T003896 & \multirow[t]{2}{*}{$152: 18$} & Upper half & $<1,030$ & $<1,000$ & $<1,015$ \\
\hline S96T003890 & & Lower half & $<1,030$ & $<1,040$ & $<1,035$ \\
\hline \$96T003897 & \multirow[t]{2}{*}{$152: 20$} & Upper half & $<1,060$ & $<1,040$ & $<1,050$ \\
\hline S96T003891 & & Lower half & $<1,020$ & $<1,030$ & $<1,025$ \\
\hline \$96T003892 & $152: 22$ & Upper half & $<1,040$ & $<1,060$ & $<1,050$ \\
\hline S96T004337 & $153: 14$ & Lower half & $<1,020$ & $<1,010$ & $<1,015$ \\
\hline$\$ 96 \mathrm{~T} 004314$ & \multirow[t]{2}{*}{$153: 15$} & Upper half & $<953$ & $<958$ & $<955.5$ \\
\hline S96T004317 & & Lower half & $<998$ & $<1,020$ & $<1,009$ \\
\hline \$96Т004441 & \multirow[t]{2}{*}{$153: 17$} & Upper half & $<980$ & $<982$ & $<981$ \\
\hline S96T004442 & & Lower half & $<1,010$ & $<1,000$ & $<1,005$ \\
\hline S96T004443 & $153: 19$ & Lower half & $<1,010$ & $<1,000$ & $<1,005$ \\
\hline S96T003984 & $153: 21$ & Lower half & $<1,010$ & $<1,000$ & $<1,005$ \\
\hline S96T003986 & \multirow[t]{2}{*}{$153: 22$} & Upper half & $<999$ & $<1,020$ & $<1,009.5$ \\
\hline S96T003985 & & Lower half & $<1,010$ & $<1,010$ & $<1,010$ \\
\hline
\end{tabular}


Table B2-40. Tank 241-AN-105 Analytical Results: Nickel (ICP). (2 sheets)

\begin{tabular}{|c|c|c|c|c|c|}
\hline Oampic & Gingole & 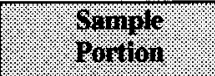 & pesil: & Buplowie. & $\log (\operatorname{leg}$ \\
\hline \multicolumn{2}{|c|}{ Wrind } & & 180 & $1 \%$ & $45 \%$ \\
\hline S96T003732 & $152: 1$ & Drainable liquid & $<5.64$ & $<5.64$ & $<5.64$ \\
\hline S96T003739 & 152: 2 & Drainable liquid & $<5.64$ & $<5.64$ & $<5.64$ \\
\hline S96T003740 & 152: 3 & Drainable liquid & $<5.64$ & $<5.64$ & $<5.64$ \\
\hline S96T003741 & 152: 4 & Drainable liquid & $<5.64$ & $<5.64$ & $<5.64$ \\
\hline S96T003742 & 152: 5 & Drainable liquid & $<5.64$ & $<5.64$ & $<5.64$ \\
\hline S96T003743 & 152: 6 & Drainable liquid & 7.46 & 7.25 & 7.35 \\
\hline S96T003744 & $152: 7$ & Drainable liquid & $<5.64$ & $<5.64$ & $<5.64$ \\
\hline S96T003745 & $152: 8$ & Drainable liquid & $<5.64$ & $<5.64$ & $<5.64$ \\
\hline S96T003746 & 152: 9 & Drainable liquid & $<5.64$ & $<5.64$ & $<5.64$ \\
\hline S96T003747 & $152: 10$ & Drainable liquid & $<8.45$ & $<8.45$ & $<8.45$ \\
\hline S96T003748 & $152: 11$ & Drainable liquid & $<8.45$ & $<8.45$ & $<8.45$ \\
\hline S96T003749 & $152: 12$ & Drainable liquid & $<8.45$ & $<8.45$ & $<8.45$ \\
\hline S96T003750 & $152: 13$ & Drainable liquid & $<8.45$ & $<8.45$ & $<8.45$ \\
\hline S96T003751 & $152: 14$ & Drainable liquid & $<5.64$ & $<5.64$ & $<5.64$ \\
\hline \$96T004433 & $153: 1$ & Drainable liquid & $<5.64$ & $<5.64$ & $<5.64$ \\
\hline S96T004308 & $153: 2$ & Drainable liquid & $<8.45$ & $<8.45$ & $<8.45$ \\
\hline S96T004434 & $153: 3$ & Drainable liquid & $<5.64$ & $<5.64$ & $<5.64$ \\
\hline S96T004419 & 153: 5 & Drainable liquid & $<8.45$ & $<8.45$ & $<8.45$ \\
\hline S96T004420 & 153: 6 & Drainable liquid & $<8.45$ & $<8.45$ & $<8.45$ \\
\hline \$96T004421 & $153: 7$ & Drainable liquid & $<5.64$ & $<5.64$ & $<5.64$ \\
\hline S96T003975 & 153: 8 & Drainable liquid & $<5.64$ & $<5.64$ & $<5.64$ \\
\hline S96T003976 & 153: 9 & Drainable liquid & $<5.64$ & $<5.64$ & $<5.64$ \\
\hline S96T004309 & $153: 10$ & Drainable liquid & $<8.45$ & $<8.45$ & $<8.45$ \\
\hline S96T003979 & $153: 11$ & Drainable liquid & $<8.45$ & $<8.45$ & $<8.45$ \\
\hline S96T003977 & $153: 12$ & Drainable liquid & $<8.45$ & $<8.45$ & $<8.45$ \\
\hline S96T003978 & $153: 13$ & Drainable liquid & $<8.45$ & $<8.45$ & $<8.45$ \\
\hline S96T004333 & 153:14 & Drainable liquid & $<8.45$ & $<8.45$ & $<8.45$ \\
\hline S96T003980 & $153: 21$ & Drainable liquid & $<8.45$ & $<8.45$ & $<8.45$ \\
\hline S96T004831 & Core 152 & Liquid composite & $<8.45$ & $<8.45$ & $<8.45$ \\
\hline
\end{tabular}


Table B2-40. Tank 241-AN-105 Analytical Results: Nickel (ICP). (2 sheets)

\begin{tabular}{|c|c|c|c|c|c|}
\hline 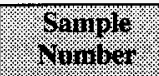 & Sornole. & . & mosilit & Muplicule? & Heari \\
\hline \multicolumn{2}{|c|}{ 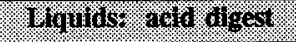 } & & $18 \%$ & $\%$ & 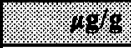 \\
\hline \$96T004144 & Core 152 & Liquid composite & $<7.39$ & $<7.03$ & $<7.21$ \\
\hline \multicolumn{2}{|c|}{ Sollos ad aigest } & . & $8 \mathrm{~g}$ & 49 & 1089 \\
\hline$\$ 96 T 003906$ & 152: 1 & Upper half & 12.6 & 16.3 & $14.45^{\mathrm{QC}: e}$ \\
\hline S96T003898 & $152: 14$ & Upper half & 20.5 & 20.3 & 20.4 \\
\hline S96T003828 & & Lower half & 20.5 & 19.9 & 20.2 \\
\hline S96T003899 & $152: 16$ & Upper half & 34.3 & 37.9 & 36.1 \\
\hline \$96T003900 & & Lower half & 36.5 & 43.7 & 40.1 \\
\hline S96T003901 & $152: 18$ & Upper half & 72.5 & 71.1 & 71.8 \\
\hline S96T003902 & & Lower half & 55.7 & 52 & 53.85 \\
\hline S96T003903 & $152: 20$ & Upper half & 59.1 & 54.3 & 56.7 \\
\hline S96T003904 & & Lower half & 28.2 & 25.8 & 27 \\
\hline \$96T003905 & $152: 22$ & Upper half & 29.8 & 27.8 & 28.8 \\
\hline \$96T004338 & $153: 14$ & Lower half & 28.7 & 34 & 31.35 \\
\hline S96T004315 & $153: 15$ & Upper half & 23.7 & 24.3 & 24 \\
\hline S96T004318 & & Lower half & 21.6 & 19.5 & 20.55 \\
\hline S96T004444 & $153: 17$ & Upper half & 46.6 & 46.6 & 46.6 \\
\hline S96T004445 & & Lower half & 78.4 & 83 & 80.7 \\
\hline S96T004446 & $153: 19$ & Lower half & 58.7 & 60.7 & 59.7 \\
\hline \$96T003988 & $153: 21$ & Lower half & 24.6 & 22.5 & 23.55 \\
\hline S96T003989 & $153: 22$ & Upper half & 35.5 & 28.6 & $32.05^{\mathrm{QC}: \mathrm{e}}$ \\
\hline S96T003987 & & Lower half & 65.2 & 67.2 & 66.2 \\
\hline S96T004257 & Core 152 & Solid composite & 34.9 & 28.6 & $31.75^{\mathrm{QC}: \mathrm{e}}$ \\
\hline S96T004262 & & Solid composite & 36.5 & 30.8 & 33.65 \\
\hline
\end{tabular}


Table B2-41. Tank 241-AN-105 Analytical Results: Phosphorus (ICP). (3 sheets)

\begin{tabular}{|c|c|c|c|c|c|}
\hline Samplo & ogmple & H. & Pes114 & Duplieres & $19 \operatorname{lig} 1$ \\
\hline \multicolumn{2}{|c|}{ Worids } & & 1696 & 6.108 & 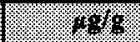 \\
\hline S96T003732 & 152: 1 & Drainable liquid & 287.32 & 248.59 & 267.95 \\
\hline S96T003739 & 152: 2 & Drainable liquid & 243.66 & 237.32 & 240.49 \\
\hline \$96T003740 & 152: 3 & Drainable liquid & 240.84 & 235.21 & 238.02 \\
\hline S96T003741 & $152: 4$ & Drainable liquid & 242.25 & 228.16 & 235.21 \\
\hline S96T003742 & $152: 5$ & Drainable liquid & 253.52 & 232.39 & 242.95 \\
\hline S96T003743 & 152: 6 & Drainable liquid & 285.91 & 329.57 & 307.74 \\
\hline \$96T003744 & 152: 7 & Drainable liquid & 269.71 & 261.26 & 265.49 \\
\hline S96T003745 & $152: 8$ & Drainable liquid & 873.23 & 291.54 & $582.39^{\mathrm{QC}: c, e}$ \\
\hline S96T003746 & 152: 9 & Drainable liquid & 293.66 & 290.84 & 292.25 \\
\hline S96T003747 & $152: 10$ & Drainable liquid & 230.28 & 233.09 & 231.69 \\
\hline S96T003748 & $152: 11$ & Drainable liquid & 231.69 & 219.01 & 225.35 \\
\hline S96T003749 & $152: 12$ & Drainable liquid & 230.98 & 227.46 & 229.22 \\
\hline S96T003750 & $152: 13$ & Drainable liquid & 226.05 & 222.53 & 224.29 \\
\hline S96T003751 & $152: 14$ & Drainable liquid & 371.83 & 270.42 & $321.12^{\mathrm{QC}: \mathrm{c}, \mathrm{e}}$ \\
\hline S96T004433 & 153: 1 & Drainable liquid & 461.97 & 461.26 & 461.62 \\
\hline S96T004308 & 153: 2 & Drainable liquid & 277.46 & 259.15 & 268.31 \\
\hline S96T004434 & 153: 3 & Drainable liquid & 302.81 & 306.33 & 304.57 \\
\hline S96T004419 & 153: 5 & Drainable liquid & 271.83 & 252.81 & 262.32 \\
\hline S96T004420 & 153: 6 & Drainable liquid & 256.33 & 260.56 & 258.45 \\
\hline S96T004421 & 153: 7 & Drainable liquid & 347.88 & 310.56 & 329.22 \\
\hline S96T003975 & 153: 8 & Drainable liquid & 249.29 & 247.18 & 248.23 \\
\hline S96T003976 & 153: 9 & Drainable liquid & 264.08 & 306.33 & $285.21^{\text {QC:d }}$ \\
\hline S96T004309 & $153: 10$ & Drainable liquid & 257.74 & 327.46 & $292.60^{\mathrm{QC}: \mathrm{e}}$ \\
\hline S96T003979 & $153: 11$ & Drainable liquid & 245.77 & 241.54 & 243.66 \\
\hline S96T003977 & $153: 12$ & Drainable liquid & 253.52 & 252.81 & 253.16 \\
\hline S96T003978 & $153: 13$ & Drainable liquid & 249.29 & 213.38 & 231.33 \\
\hline \$96T004333 & $153: 14$ & Drainable liquid & 263.38 & 261.26 & 262.32 \\
\hline S96T003980 & $153: 21$ & Drainable liquid & 272.53 & 266.90 & 269.71 \\
\hline S96T004831 & Core 152 & Liquid composite & 274.64 & 278.16 & 276.40 \\
\hline
\end{tabular}


Table B2-41. Tank 241-AN-105 Analytical Results: Phosphorus (ICP). (3 sheets)

\begin{tabular}{|c|c|c|c|c|c|}
\hline Sariplot & Sompros & 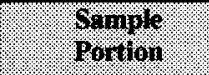 & Presill & Muplieate & Mean \\
\hline \multicolumn{2}{|c|}{ 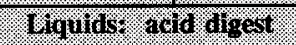 } & & 68 & 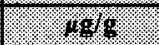 & 48 \\
\hline S96T004144 & Core 152 & Liquid composite & 236.62 & 241.54 & 239.08 \\
\hline \multicolumn{2}{|c|}{ solkst acis dig ou } & & 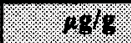 & os & 48 \\
\hline S96T003906 & 152: 1 & Upper half & 1,330 & 939 & $1,134.5^{\mathrm{QC}: \mathrm{e}}$ \\
\hline S96T003898 & \multirow[t]{2}{*}{$152: 14$} & Upper half & 358 & 297 & 327.5 \\
\hline S96T003828 & & Lower half & 389 & 382 & 385.5 \\
\hline S96T003899 & \multirow[t]{2}{*}{$152: 16$} & Upper half & 653 & 510 & $581.5^{\mathrm{QC}: \mathrm{e}}$ \\
\hline S96T003900 & & Lower half & 642 & 684 & 663 \\
\hline S96T003901 & \multirow[t]{2}{*}{$152: 18$} & Upper half & 789 & 762 & 775.5 \\
\hline S96T003902 & & Lower half & 849 & 925 & 887 \\
\hline S96T003903 & \multirow[t]{2}{*}{$152: 20$} & Upper half & 694 & 578 & 636 \\
\hline S96T003904 & & Lower half & 1,670 & 581 & $1,125.5^{\mathrm{QC}: \mathrm{e}}$ \\
\hline S96T003905 & $152: 22$ & Upper half & 2,520 & 2,580 & 2,550 \\
\hline S96T004338 & $153: 14$ & Lower half & 492 & 485 & 488.5 \\
\hline S96T004315 & \multirow[t]{2}{*}{$153: 15$} & Upper half & 360 & 380 & 370 \\
\hline S96T004318 & & Lower half & 399 & 372 & 385.5 \\
\hline S96T004444 & \multirow[t]{2}{*}{$153: 17$} & Upper half & 577 & 518 & 547.5 \\
\hline S96T004445 & & Lower half & 799 & 785 & 792 \\
\hline S96T004446 & 153:19 & Lower half & 731 & 2,020 & $1,375.5^{\text {QC:e }}$ \\
\hline S96T003988 & $153: 21$ & Lower half & 455 & 455 & 455 \\
\hline S96T003989 & \multirow[t]{2}{*}{$153: 22$} & Upper half & 5,300 & 3,850 & $4,575^{\mathrm{QC}: \mathrm{e}}$ \\
\hline S96T003987 & & Lower half & 29,700 & 27,000 & 28,350 \\
\hline S96T004257 & \multirow[t]{2}{*}{ Core 152} & Solid composite & 418 & 386 & 402 \\
\hline S96T004262 & & Solid composite & 15,000 & 13,200 & $14,100^{\mathrm{QC}: \mathrm{c}}$ \\
\hline
\end{tabular}


Table B2-41. Tank 241-AN-105 Analytical Results: Phosphorus (ICP). (3 sheets)

\begin{tabular}{|c|c|c|c|c|c|}
\hline Shangor & 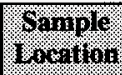 & 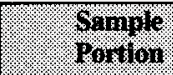 & incillu & Huplicile & rogn \\
\hline Solloss & 14\% & & 198 & $49 / 8$ & 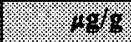 \\
\hline S96T003893 & 152: 1 & Upper half & $<3,950$ & $<4,020$ & $<3,985$ \\
\hline S96T003894 & \multirow[t]{2}{*}{$152: 14$} & Upper half & $<4,070$ & $<3,990$ & $<4,030$ \\
\hline S96T003827 & & Lower half & $<4,030$ & $<4,120$ & $<4,075$ \\
\hline S96T003895 & \multirow[t]{2}{*}{$152: 16$} & Upper half & $<4,090$ & $<4,020$ & $<4,055$ \\
\hline S96T003889 & & Lower half & $<4,060$ & $<4,010$ & $<4,035$ \\
\hline S96T003896 & \multirow[t]{2}{*}{$152: 18$} & Upper half & $<4,130$ & $<4,020$ & $<4,075$ \\
\hline S96T003890 & & Lower half & $<4,120$ & $<4,140$ & $<4,130$ \\
\hline S96T003897 & \multirow[t]{2}{*}{$152: 20$} & Upper half & $<4,240$ & $<4,140$ & $<4,190$ \\
\hline S96T003891 & & Lower half & $<4,080$ & $<4,110$ & $<4,095$ \\
\hline S96T003892 & $152: 22$ & Upper half & $<4,180$ & $<4,250$ & $<4,215$ \\
\hline S96T004337 & $153: 14$ & Lower half & $<4,060$ & $<4,060$ & $<4,060$ \\
\hline \$96T004314 & \multirow[t]{2}{*}{$153: 15$} & Upper half & $<3,810$ & $<3,830$ & $<3,820$ \\
\hline S96T004317 & & Lower half & $<3,990$ & $<4,060$ & $<4,025$ \\
\hline S96T004441 & \multirow[t]{2}{*}{$153: 17$} & Upper half & $<3,920$ & $<3,930$ & $<3,925$ \\
\hline S96T004442 & & Lower half & $<4,030$ & $<4,000$ & $<4,015$ \\
\hline S96 T004443 & $153: 19$ & Lower half & $<4,040$ & $<4,000$ & $<4,020$ \\
\hline S96T003984 & $153: 21$ & Lower half & $<4,030$ & $<4,010$ & $<4,020$ \\
\hline S96T003986 & \multirow[t]{2}{*}{$153: 22$} & Upper half & 5,140 & 4,570 & 4,855 \\
\hline S96T003985 & & Lower half & 35,700 & 33,100 & 34,400 \\
\hline
\end{tabular}


Table B2-42. Tank 241-AN-105 Analytical Results: Potassium (ICP). (2 sheets)

\begin{tabular}{|c|c|c|c|c|c|}
\hline $\begin{array}{l}\text { Oampil } \\
\text { Mumpor }\end{array}$ & 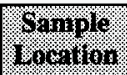 & 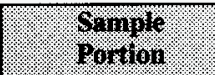 & Resill & Bupliculo & Mean \\
\hline \multicolumn{2}{|c|}{ Wquids } & & 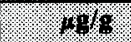 & 408 & 1696 \\
\hline S96T003732 & $152: 1$ & Drainable liquid & $4,852.11$ & $4,690.14$ & $4,771.13$ \\
\hline S96T003739 & 152: 2 & Drainable liquid & $4,521.13$ & $4,577.46$ & $4,549.3$ \\
\hline S96T003740 & 152: 3 & Drainable liquid & $4,521.13$ & $4,507.04$ & $4,514.08$ \\
\hline S96T003741 & 152: 4 & Drainable liquid & $4,753.52$ & $4,514.08$ & $4,633.8$ \\
\hline S96T003742 & $152: 5$ & Drainable liquid & $4,704.23$ & $4,612.68$ & $4,658.45^{\mathrm{QC}: c}$ \\
\hline S96T003743 & $152: 6$ & Drainable liquid & 5,000 & $5,295.77$ & $5,147.89^{Q C: c}$ \\
\hline S96T003744 & 152: 7 & Drainable liquid & $3,880.28$ & $4,260.56$ & $4,070.42^{Q C d d}$ \\
\hline S96T003745 & 152: 8 & Drainable liquid & $4,267.61$ & $4,809.86$ & $4,538.73^{Q C \cdot d}$ \\
\hline S96T003746 & $152: 9$ & Drainable liquid & $4,359.15$ & $4,570.42$ & $4,464.79^{Q C: d}$ \\
\hline S96T003747 & $152: 10$ & Drainable liquid & $4,570.42$ & $4,697.18$ & $4,633.8$ \\
\hline \$96T003748 & $152: 11$ & Drainable liquid & $4,570.42$ & $4,669.01$ & $4,619.72^{\mathrm{QC}: \mathrm{d}}$ \\
\hline \$96T003749 & $152: 12$ & Drainable liquid & $4,647.89$ & $4,521.13$ & $4,584.51$ \\
\hline S96T003750 & $152: 13$ & Drainable liquid & $4,514.08$ & $4,443.66$ & $4,478.87^{\mathrm{QC:d}}$ \\
\hline S96T003751 & $152: 14$ & Drainable liquid & $4,274.65$ & $4,359.15$ & $4,316.9^{\mathrm{QC}: \mathrm{c}}$ \\
\hline S96T004433 & 153: 1 & Drainable liquid & $4,873.24$ & $4,507.04$ & $4,690.14$ \\
\hline S96T004308 & 153: 2 & Drainable liquid & $4,739.44$ & $4,626.76$ & $4,683.1$ \\
\hline S96T004434 & 153: 3 & Drainable liquid & $4,274.65$ & $4,647.89$ & $4,461.27^{\mathrm{QC:d}}$ \\
\hline \$96T004419 & 153: 5 & Drainable liquid & $4,901.41$ & $4,950.7$ & $4,926.06$ \\
\hline S96T004420 & 153: 6 & Drainable liquid & $4,676.06$ & 5,000 & $4,838.03^{\mathrm{QC} \cdot \mathrm{d}}$ \\
\hline S96T004421 & 153: 7 & Drainable liquid & $4,528.17$ & $4,922.54$ & $4,725.35^{\mathrm{QC:d}}$ \\
\hline S96T003975 & 153: 8 & Drainable liquid & $4,457.75$ & $4,154.93$ & $4,306.34$ \\
\hline S96T003976 & 153: 9 & Drainable liquid & $4,091.55$ & $4,859.15$ & $4,475.35^{\mathrm{QC:d}}$ \\
\hline \$96T004309 & $153: 10$ & Drainable liquid & $4,676.06$ & $4,880.28$ & $4,778.17^{\mathrm{QC:c}}$ \\
\hline \$96T003979 & $153: 11$ & Drainable liquid & $4,873.24$ & $4,697.18$ & $4,785.21^{\mathrm{QC}: c}$ \\
\hline S96T003977 & $153: 12$ & Drainable liquid & $4,704.23$ & $4,873.24$ & $4,788.73^{\mathrm{QC:d}}$ \\
\hline S96T003978 & $153: 13$ & Drainable liquid & $4,429.58$ & $4,112.68$ & $4,271.13^{\mathrm{QC}: \mathrm{d}}$ \\
\hline S96T004333 & $153: 14$ & Drainable liquid & $5,028.17$ & $4,964.79$ & $4,996.48$ \\
\hline \$96T003980 & $153: 21$ & Drainable liquid & $3,577.46$ & 3,500 & $3,538.73$ \\
\hline S96T004831 & Core 152 & Liquid composite & $4,570.42$ & $4,612.68$ & $4,591.55^{\mathrm{QC:d}}$ \\
\hline
\end{tabular}


Table B2-42. Tank 241-AN-105 Analytical Results: Potassium (ICP). (2 sheets)

\begin{tabular}{|c|c|c|c|c|c|}
\hline Sample & Wample & $\begin{array}{l}\text { Wmale } \\
6 \% 10 \%\end{array}$ & $\mathrm{p}=\mathrm{sull}$ & 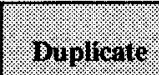 & 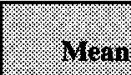 \\
\hline \multicolumn{2}{|c|}{ 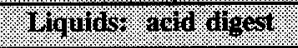 } & 位 & $14 \%$ & 180 & $10 \%$ \\
\hline S96T004144 & Core 152 & Liquid composite & $3,760.56$ & $3,711.27$ & $3,735.92$ \\
\hline \multicolumn{2}{|c|}{ 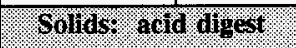 } & $\sqrt{1.92}$ & 40 & $1.4 .8 \%$ & $19 \%$ \\
\hline S96T003906 & 152: 1 & Upper half & 3,960 & 3,790 & 3,875 \\
\hline S96T003898 & $152: 14$ & Upper half & 3,900 & 3,980 & 3,940 \\
\hline S96T003828 & & Lower half & 3,950 & 4,020 & 3,985 \\
\hline S96T003899 & $152: 16$ & Upper half & 3,610 & 3,760 & 3,685 \\
\hline S96T003900 & & Lower half & 3,770 & 3,770 & 3,770 \\
\hline S96T003901 & $152: 18$ & Upper half & 3,610 & 3,420 & 3,515 \\
\hline S96T003902 & & Lower half & 3,700 & 3,720 & 3,710 \\
\hline$\$ 96 T 003903$ & $152: 20$ & Upper half & 3,890 & 3,730 & 3,810 \\
\hline S96T003904 & & Lower half & 3,260 & 3,290 & 3,275 \\
\hline S96T003905 & $152: 22$ & Upper half & 2,910 & 3,080 & 2,995 \\
\hline S96T004338 & $153: 14$ & Lower half & 3,780 & 3,850 & 3,815 \\
\hline S96T004315 & $153: 15$ & Upper half & 3,560 & 3,500 & 3,530 \\
\hline S96T004318 & & Lower half & 3,720 & 3,760 & 3,740 \\
\hline S96T004444 & $153: 17$ & Upper half & 3,650 & 3,630 & 3,640 \\
\hline S96T004445 & & Lower half & 3,390 & 3,320 & 3,355 \\
\hline S96T004446 & $153: 19$ & Lower half & 3,200 & 3,270 & 3,235 \\
\hline S96T003988 & $153: 21$ & Lower half & 2,940 & 2,900 & 2,920 \\
\hline S96T003989 & $153: 22$ & Upper half & 3,030 & 3,350 & 3,190 \\
\hline S96T003987 & & Lower half & 2,190 & 2,500 & 2,345 \\
\hline S96T004257 & Core 152 & Solid composite & 3,070 & 3,110 & $3,090^{\mathrm{QC:a}}$ \\
\hline \$96T004262 & & Solid composite & 2,240 & 2,050 & 2,145 \\
\hline
\end{tabular}


Table B2-43. Tank 241-AN-105 Analytical Results: Silicon (ICP). (3 sheets)

\begin{tabular}{|c|c|c|c|c|c|}
\hline Wamplo & \%oringle & $.6 \%$ monle & resill & Onplicate & (rean \\
\hline \multicolumn{2}{|c|}{ Worys } & & $4 \%$ & $8 \%$ & $18 \%$ \\
\hline S96T003732 & 152: 1 & Drainable liquid & 157.74 & 154.93 & 156.33 \\
\hline S96T003739 & 152: 2 & Drainable liquid & 133.09 & 130.28 & 131.69 \\
\hline S96T003740 & 152: 3 & Drainable liquid & 153.52 & 144.36 & 148.94 \\
\hline S96T003741 & 152: 4 & Drainable liquid & 145.07 & 139.43 & 142.25 \\
\hline S96T003742 & 152: 5 & Drainable liquid & 191.54 & 160.56 & 176.05 \\
\hline S96T003743 & $152: 6$ & Drainable liquid & 178.16 & 190.84 & 184.50 \\
\hline S96T003744 & 152: 7 & Drainable liquid & 137.32 & 151.40 & 144.36 \\
\hline S96T003745 & 152: 8 & Drainable liquid & 186.62 & 205.63 & 196.12 \\
\hline S96T003746 & 152: 9 & Drainable liquid & 142.25 & 145.77 & 144.01 \\
\hline S96T003747 & $152: 10$ & Drainable liquid & 135.91 & 138.73 & 137.32 \\
\hline \$96T003748 & $152: 11$ & Drainable liquid & 161.97 & 162.67 & 162.32 \\
\hline S96T003749 & $152: 12$ & Drainable liquid & 145.77 & 141.54 & 143.66 \\
\hline S96T003750 & $152: 13$ & Drainable liquid & 143.66 & 144.36 & 144.01 \\
\hline S96T003751 & $152: 14$ & Drainable liquid & 170.42 & 162.67 & 166.54 \\
\hline S96T004433 & 153: 1 & Drainable liquid & 214.78 & 196.47 & 205.63 \\
\hline S96T004308 & 153: 2 & Drainable liquid & 131.69 & 127.46 & 129.57 \\
\hline S96T004434 & 153: 3 & Drainable liquid & 126.76 & 138.02 & 132.39 \\
\hline S96T004419 & 153: 5 & Drainable liquid & 163.38 & 161.26 & 162.32 \\
\hline S96T004420 & $153: 6$ & Drainable liquid & 135.21 & 145.07 & 140.14 \\
\hline S96T004421 & 153: 7 & Drainable liquid & 158.45 & 153.52 & 155.98 \\
\hline S96T003975 & 153: 8 & Drainable liquid & 145.77 & 142.25 & 144.01 \\
\hline \$96T003976 & 153: 9 & Drainable liquid & 141.54 & 171.83 & 156.69 \\
\hline \$96T004309 & $153: 10$ & Drainable liquid & 137.32 & 147.18 & 142.25 \\
\hline \$96T003979 & 153:11 & Drainable liquid & 166.90 & 164.08 & 165.49 \\
\hline S96T003977 & $153: 12$ & Drainable liquid & 162.67 & 174.64 & 168.66 \\
\hline S96T003978 & $153: 13$ & Drainable liquid & 160.56 & 147.88 & 154.22 \\
\hline S96T004333 & $153: 14$ & Drainable liquid & 116.19 & 112.67 & 114.43 \\
\hline S96T003980 & $153: 21$ & Drainable liquid & 138.02 & 134.50 & 136.26 \\
\hline S96T004831 & Core 152 & Liquid composite & 116.90 & 116.90 & 116.90 \\
\hline
\end{tabular}


Table B2-43. Tank 241-AN-105 Analytical Results: Silicon (ICP). (3 sheets)

\begin{tabular}{|c|c|c|c|c|c|}
\hline Gample & 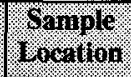 & Prongle & Besili: & Buplicate & 164n \\
\hline \multicolumn{2}{|c|}{ 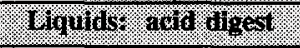 } & $\sqrt[3]{3}$ & 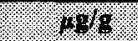 & $1.9 / 8$ & 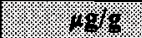 \\
\hline S96T004144 & Core 152 & Liquid composite & 146.47 & 162.67 & $154.57^{\text {QC:b,f }}$ \\
\hline \multicolumn{2}{|c|}{ 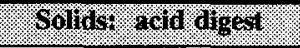 } & & $0 \% 18$ & 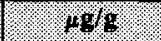 & 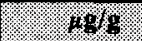 \\
\hline S96T003906 & 152: 1 & Upper half & 219 & 201 & $210^{\mathrm{QC}: \mathrm{b}}$ \\
\hline S96T003898 & \multirow[t]{2}{*}{$152: 14$} & Upper half & 138 & 145 & $141.5^{\mathrm{QC} b \mathrm{~b}}$ \\
\hline S96T003828 & & Lower half & 165 & 181 & $173^{\mathrm{QC}: \mathrm{b}}$ \\
\hline S96T003899 & \multirow[t]{2}{*}{$152: 16$} & Upper half & 134 & 142 & $138^{\mathrm{QC}: \mathrm{b}}$ \\
\hline S96T003900 & & Lower half & 91.2 & 116 & $103.6^{\mathrm{QC}: \mathrm{b}, \mathrm{e}}$ \\
\hline S96T003901 & \multirow[t]{2}{*}{$152: 18$} & Upper half & 103 & 145 & $124^{\mathrm{QC:b,e}}$ \\
\hline S96T003902 & & Lower half & 141 & 86.6 & $113.8^{\mathrm{QC}: \mathrm{b}, \mathrm{e}}$ \\
\hline S96T003903 & \multirow[t]{2}{*}{$152: 20$} & Upper half & 149 & 147 & $148^{\mathrm{QC}: \mathrm{b}}$ \\
\hline S96T003904 & & Lower half & 117 & 110 & $113.5^{\mathrm{QC}: \mathrm{b}}$ \\
\hline S96T003905 & $152: 22$ & Upper half & 115 & 102 & $108.5^{\mathrm{QC}: \mathrm{b}}$ \\
\hline S96'T004338 & $153: 14$ & Lower half & 142 & $<23.9$ & $<82.95$ \\
\hline \$96T004315 & \multirow[t]{2}{*}{$153: 15$} & Upper half & 175 & 180 & 177.5 \\
\hline S96T004318 & & Lower half & 61.7 & 170 & $115.85^{Q \mathrm{QC:} e}$ \\
\hline S96T004444 & \multirow[t]{2}{*}{$153: 17$} & Upper half & 159 & 153 & 156 \\
\hline S96T004445 & & Lower half & 190 & 176 & 183 \\
\hline S96T004446 & $153: 19$ & Lower half & 126 & 164 & $145^{\mathrm{QC}: e}$ \\
\hline S96T003988 & $153: 21$ & Lower half & 136 & 135 & 135.5 \\
\hline \$96T003989 & \multirow[t]{2}{*}{$153: 22$} & Upper half & 167 & 136 & $151.5^{\mathrm{QC:b,d,e}}$ \\
\hline S96T003987 & & Lower half & 113 & 109 & $111^{\mathrm{QC}: \mathrm{b}}$ \\
\hline S96T004257 & \multirow[t]{2}{*}{ Core 152} & Solid composite & 139 & 218 & $178.5^{\mathrm{QC}, \mathrm{b}, \mathrm{d}, \mathrm{e}}$ \\
\hline S96T004262 & & Solid composite & 621 & 328 & $474.5^{\text {QC:b,e }}$ \\
\hline
\end{tabular}


Table B2-43. Tank 241-AN-105 Analytical Results: Silicon (ICP). (3 sheets)

\begin{tabular}{|c|c|c|c|c|c|}
\hline $\begin{array}{l}\text { Sample: } \\
\text { Nomber. }\end{array}$ & $\begin{array}{l}\text { Sample } \\
\text { Yocation }\end{array}$ & $\begin{array}{l}\text { Sample } \\
\text { Tortion }\end{array}$ & Resilit & Dipolicate & Mean \\
\hline Solligs & Tusion & 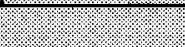 & 1480 & 1018 & 1.498. \\
\hline S96T003893 & 152: 1 & Upper half & $<988$ & $<1,010$ & $<999$ \\
\hline S96T003894 & \multirow[t]{2}{*}{$152: 14$} & Upper half & $<1,020$ & $<998$ & $<1,009$ \\
\hline S96T003827 & & Lower half & $<1,010$ & $<1,030$ & $<1,020$ \\
\hline S96T003895 & \multirow[t]{2}{*}{$152: 16$} & Upper half & $<1,020$ & $<1,000$ & $<1,010$ \\
\hline S96T003889 & & Lower half & $<1,010$ & $<1,000$ & $<1,005$ \\
\hline S96T003896 & \multirow[t]{2}{*}{$152: 18$} & Upper half & $<1,030$ & $<1,000$ & $<1,015$ \\
\hline S96T003890 & & Lower half & $<1,030$ & $<1,040$ & $<1,035$ \\
\hline S96T003897 & \multirow[t]{2}{*}{$152: 20$} & Upper half & $<1,060$ & $<1,040$ & $<1,050$ \\
\hline S96T003891 & & Lower half & $<1,020$ & $<1,030$ & $<1,025$ \\
\hline S96T003892 & $152: 22$ & Upper half & $<1,040$ & $<1,060$ & $<1,050$ \\
\hline S96T004337 & 153:14 & Lower half & $<1,020$ & 2,450 & $<1,735$ \\
\hline S96T004314 & \multirow[t]{2}{*}{$153: 15$} & Upper half & $<953$ & $<958$ & $<955.5$ \\
\hline S96T004317 & & Lower half & $<998$ & $<1,020$ & $<1,009$ \\
\hline S96T004441 & \multirow[t]{2}{*}{$153: 17$} & Upper half & $<980$ & $<<982$ & $<981$ \\
\hline S96T004442 & & Lower half & 1,830 & $<1,000$ & $<1,415$ \\
\hline S96T004443 & $153: 19$ & Lower half & $<1,010$ & $<1,000$ & $<1,005$ \\
\hline S96T003984 & $153: 21$ & Lower half & $<1,010$ & $<1,000$ & $<1,005$ \\
\hline S96T003986 & \multirow[t]{2}{*}{$153: 22$} & Upper half & $<999$ & $<1,020$ & $<1,009.5$ \\
\hline S96T003985 & & Lower half & $<1,010$ & $<1,010$ & $<1,010$ \\
\hline
\end{tabular}


Table B2-44. Tank 241-AN-105 Analytical Results: Silver (ICP). (3 sheets)

\begin{tabular}{|c|c|c|c|c|c|}
\hline $\begin{array}{l}\text { Samplc } \\
\text { Numbro }\end{array}$ & Whomple & ( & $\mathrm{P}(\mathrm{m}, \mathrm{in}$ & OHol10.10 & 164n \\
\hline \multicolumn{2}{|c|}{ Kolving } & & 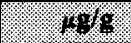 & 4.19 & 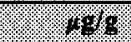 \\
\hline S96T003732 & 152: 1 & Drainable liquid & 12.67 & 12.11 & 12.39 \\
\hline S96T003739 & 152: 2 & Drainable liquid & 11.90 & 11.97 & 11.93 \\
\hline S96T003740 & 152: 3 & Drainable liquid & 11.90 & 11.69 & 11.79 \\
\hline S96T003741 & 152: 4 & Drainable liquid & 12.60 & 11.76 & 12.18 \\
\hline S96T003742 & 152: 5 & Drainable liquid & 12.74 & 12.25 & 12.5 \\
\hline S96T003743 & 152: 6 & Drainable liquid & 13.87 & 14.01 & 13.94 \\
\hline S96T003744 & 152: 7 & Drainable liquid & 10.91 & 11.97 & 11.44 \\
\hline S96T003745 & 152: 8 & Drainable liquid & 12.25 & 14.01 & 13.13 \\
\hline S96T003746 & 152: 9 & Drainable liquid & 12.67 & 12.67 & 12.67 \\
\hline S96T003747 & $152: 10$ & Drainable liquid & 12.11 & 11.90 & 12.00 \\
\hline S96T003748 & $152: 11$ & Drainable liquid & 12.11 & 12.46 & 12.28 \\
\hline S96T003749 & $152: 12$ & Drainable liquid & 12.60 & 11.83 & 12.21 \\
\hline S96T003750 & $152: 13$ & Drainable liquid & 11.76 & 11.83 & 11.79 \\
\hline S96T003751 & $152: 14$ & Drainable liquid & 12.04 & 11.61 & 11.83 \\
\hline S96T004433 & 153: 1 & Drainable liquid & 14.08 & 12.95 & 13.52 \\
\hline S96T004308 & 153: 2 & Drainable liquid & 12.81 & 11.97 & 12.39 \\
\hline S96T004434 & 153: 3 & Drainable liquid & 12.53 & 13.16 & 12.85 \\
\hline \$96T004419 & 153: 5 & Drainable liquid & 13.38 & 13.30 & 13.34 \\
\hline S96T004420 & 153: 6 & Drainable liquid & 12.60 & 14.15 & 13.38 \\
\hline S96T004421 & 153: 7 & Drainable liquid & 12.81 & 13.80 & 13.30 \\
\hline S96T003975 & 153: 8 & Drainable liquid & 12.18 & 11.12 & 11.65 \\
\hline S96T003976 & 153: 9 & Drainable liquid & 11.33 & 13.09 & 12.21 \\
\hline \$96T004309 & $153: 10$ & Drainable liquid & 12.04 & 13.23 & 12.64 \\
\hline S96T003979 & $153: 11$ & Drainable liquid & 13.38 & 12.81 & 13.09 \\
\hline S96T003977 & $153: 12$ & Drainable liquid & 12.25 & 13.09 & 12.67 \\
\hline S96T003978 & $153: 13$ & Drainable liquid & 11.90 & 11.12 & 11.51 \\
\hline S96T004333 & 153:14 & Drainable liquid & 13.52 & 13.66 & 13.59 \\
\hline S96T003980 & $153: 21$ & Drainable liquid & 11.05 & 10.91 & 10.98 \\
\hline S96T004831 & Core 152 & Liquid composite & 13.02 & 12.32 & 12.67 \\
\hline
\end{tabular}


Table B2-44. Tank 241-AN-105 Analytical Results: Silver (ICP). (3 sheets)

\begin{tabular}{|c|c|c|c|c|c|}
\hline Marimilo & gontion & Shmole & Resilt & Puplicate & 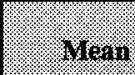 \\
\hline \multicolumn{2}{|c|}{ Wiquitiss ard digest } & & $\%$ & 4848 & 696 \\
\hline S96T004144 & Core 152 & Liquid composite & 10.84 & 10.49 & 10.66 \\
\hline \multicolumn{2}{|c|}{ 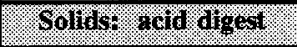 } & & pas & 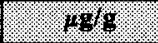 & 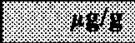 \\
\hline S96T003906 & 152: 1 & Upper half & 13.7 & 13.4 & $13.55^{\mathrm{QC:a,c}}$ \\
\hline S96T003898 & \multirow[t]{2}{*}{$152: 14$} & Upper half & 13.8 & 13.6 & $13.7^{\mathrm{QC}: \mathrm{a}}$ \\
\hline S96T003828 & & Lower half & 13.5 & 14.2 & $13.85^{\mathrm{QC}: \mathrm{a}}$ \\
\hline S96T003899 & \multirow[t]{2}{*}{$152: 16$} & Upper half & 14.8 & 15.1 & $14.95^{\mathrm{QC} ; \mathrm{a}}$ \\
\hline S96T003900 & & Lower half & 14.7 & 14.7 & $14.7^{\mathrm{QC}: \mathrm{a}}$ \\
\hline S96T003901 & \multirow[t]{2}{*}{$152: 18$} & Upper half & 16.1 & 15.6 & $15.85^{\mathrm{QC:a}}$ \\
\hline S96T003902 & & Lower half & 16 & 15.8 & $15.9^{\mathrm{QC}: \mathrm{a}}$ \\
\hline S96T003903 & \multirow[t]{2}{*}{$152: 20$} & Upper half & 15.1 & 14.9 & $15^{\mathrm{QC:a}}$ \\
\hline S96T003904 & & Lower half & 16.2 & 16 & $16.1^{\mathrm{QC} \cdot \mathrm{a}}$ \\
\hline S96T003905 & $152: 22$ & Upper half & 17.2 & 17.7 & $17.45^{\mathrm{QC}: \mathrm{a}}$ \\
\hline S96T004338 & $153: 14$ & Lower half & 13.8 & 13.4 & $13.6^{\mathrm{QC} \cdot \mathrm{a}}$ \\
\hline S96T004315 & \multirow[t]{2}{*}{$153: 15$} & Upper half & 12.2 & 12.1 & $12.15^{\mathrm{QC}: \mathrm{a}}$ \\
\hline S96T004318 & & Lower half & 12.5 & 12.7 & $12.6^{\mathrm{QC}: \mathrm{a}}$ \\
\hline S96T004444 & \multirow[t]{2}{*}{$153: 17$} & Upper half & 12.6 & 13.2 & $12.9^{\mathrm{QC}: \mathrm{a}}$ \\
\hline S96T004445 & & Lower half & 13.6 & 14.5 & $14.05^{\mathrm{QC} ; \mathrm{a}}$ \\
\hline S96T004446 & $153: 19$ & Lower half & 13.3 & 14 & $13.65^{\mathrm{QC}: \mathrm{a}}$ \\
\hline S96T003988 & $153: 21$ & Lower half & 13.7 & 13.4 & $13.55^{\mathrm{QC}: \mathrm{a}}$ \\
\hline S96T003989 & \multirow[t]{2}{*}{$153: 22$} & Upper half & 19.1 & 16.2 & 17.65 \\
\hline S96T003987 & & Lower half & 98.7 & 107 & 102.85 \\
\hline S96T004257 & \multirow[t]{2}{*}{ Core 152} & Solid composite & 12.3 & 11.5 & 11.9 \\
\hline S96T004262 & & Solid composite & 44.8 & 40.1 & 42.45 \\
\hline
\end{tabular}


Table B2-44. Tank 241-AN-105 Analytical Results: Silver (ICP). (3 sheets)

\begin{tabular}{|c|c|c|c|c|c|}
\hline Hamolo & Srapolo & 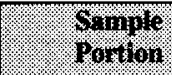 & Resuil & Bubitarte & Worn \\
\hline Nollos & $11 \% 109$ & & 48 & 4s & $4 \%$ \\
\hline S96T003893 & 152: 1 & Upper half & $<198$ & $<201$ & $<199.5$ \\
\hline S96T003894 & \multirow[t]{2}{*}{$152: 14$} & Upper half & $<204$ & $<200$ & $<202$ \\
\hline S96T003827 & & Lower half & $<201$ & $<206$ & $<203.5$ \\
\hline S96T003895 & \multirow[t]{2}{*}{$152: 16$} & Upper half & $<204$ & $<201$ & $<202.5$ \\
\hline S96T003889 & & Lower half & $<203$ & $<200$ & $<201.5$ \\
\hline S96T003896 & \multirow[t]{2}{*}{$152: 18$} & Upper half & $<207$ & $<201$ & $<204$ \\
\hline S96T003890 & & Lower half & $<206$ & $<207$ & $<206.5$ \\
\hline S96T003897 & \multirow[t]{2}{*}{$152: 20$} & Upper half & $<212$ & $<207$ & $<209.5$ \\
\hline S96T003891 & & Lower half & $<204$ & $<206$ & $<205$ \\
\hline S96T003892 & $152: 22$ & Upper half & $<209$ & $<212$ & $<210.5$ \\
\hline S96T004337 & 153:14 & Lower half & $<203$ & $<203$ & $<203$ \\
\hline S96T004314 & \multirow[t]{2}{*}{$153: 15$} & Upper half & $<191$ & $<192$ & $<191.5$ \\
\hline S96T004317 & & Lower half & $<200$ & $<203$ & $<201.5$ \\
\hline S96T004441 & \multirow[t]{2}{*}{$153: 17$} & Upper half & $<196$ & $<196$ & $<196$ \\
\hline S96T004442 & & Lower half & $<201$ & $<200$ & $<200.5$ \\
\hline S96T004443 & $153: 19$ & Lower half & $<202$ & $<200$ & $<201$ \\
\hline S96T003984 & $153: 21$ & Lower half & $<201$ & $<201$ & $<201$ \\
\hline S96T003986 & \multirow[t]{2}{*}{$153: 22$} & Upper half & $<200$ & $<203$ & $<201.5$ \\
\hline S96T003985 & & Lower half & $<201$ & $<201$ & $<201$ \\
\hline
\end{tabular}


Table B2-45. Tank 241-AN-105 Analytical Results: Sodium (ICP). (3 sheets)

\begin{tabular}{|c|c|c|c|c|c|}
\hline $\begin{array}{l}\text { Smiple } \\
\text { Thinge }\end{array}$ & $\begin{array}{l}\text { samplo. } \\
\text { varition }\end{array}$ & 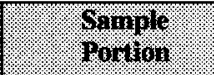 & Resull: & Muplatere. & $16 \sin$ \\
\hline \multicolumn{3}{|c|}{ Maugs } & $10 \%$ & 1098 & $18 \%$ \\
\hline S96T003732 & 152: 1 & Drainable liquid & $1.782 \mathrm{E}+05$ & $1.732 \mathrm{E}+05$ & $1.757 \mathrm{E}+05^{\mathrm{QC}: \mathrm{c}}$ \\
\hline S96T003739 & 152: 2 & Drainable liquid & $1.683 \mathrm{E}+05$ & $1.690 \mathrm{E}+05$ & $1.687 \mathrm{E}+05^{\mathrm{QC:c}}$ \\
\hline S96T003740 & 152: 3 & Drainable liquid & $1.683 \mathrm{E}+05$ & $1.648 \mathrm{E}+05$ & $1.666 \mathrm{E}+05^{\mathrm{QC}: \mathrm{c}}$ \\
\hline S96T003741 & 152: 4 & Drainable liquid & $1.768 \mathrm{E}+05$ & $1.648 \mathrm{E}+05$ & $1.708 \mathrm{E}+05^{\mathrm{QC}: \mathrm{c}}$ \\
\hline S96T003742 & 152: 5 & Drainable liquid & $1.768 \mathrm{E}+05$ & $1.683 \mathrm{E}+05$ & $1.725 \mathrm{E}+05^{\mathrm{QC}: \mathrm{c}}$ \\
\hline S96T003743 & 152: 6 & Drainable liquid & $1.873 \mathrm{E}+05$ & $1.951 \mathrm{E}+05$ & $1.912 \mathrm{E}+05^{\mathrm{QC}: \mathrm{c}}$ \\
\hline S96T003744 & $152: 7$ & Drainable liquid & $1.507 \mathrm{E}+05$ & $1.655 \mathrm{E}+05$ & $1.581 \mathrm{E}+05^{\mathrm{QC}: \mathrm{d}}$ \\
\hline S96T003745 & 152: 8 & Drainable liquid & $1.662 \mathrm{E}+05$ & $1.909 \mathrm{E}+05$ & $1.785 \mathrm{E}+05^{\mathrm{QC}: \mathrm{d}}$ \\
\hline S96T003746 & 152: 9 & Drainable liquid & $1.690 \mathrm{E}+05$ & $1.732 \mathrm{E}+05$ & $1.711 \mathrm{E}+05^{\mathrm{QC:d}}$ \\
\hline S96T003747 & $152: 10$ & Drainable liquid & $1.718 \mathrm{E}+05$ & $1.747 \mathrm{E}+05$ & $1.732 \mathrm{E}+05^{\mathrm{QC:c}}$ \\
\hline S96T003748 & $152: 11$ & Drainable liquid & $1.704 \mathrm{E}+05$ & $1.718 \mathrm{E}+05$ & $1.711 \mathrm{E}+05^{\mathrm{QC}: \mathrm{d}}$ \\
\hline S96T003749 & $152: 12$ & Drainable liquid & $1.690 \mathrm{E}+05$ & $1.648 \mathrm{E}+05$ & $1.669 \mathrm{E}+05^{\mathrm{QC}: \mathrm{c}}$ \\
\hline S96T003750 & $152: 13$ & Drainable liquid & $1.676 \mathrm{E}+05$ & $1.662 \mathrm{E}+05$ & $1.669 \mathrm{E}+05^{\mathrm{QC:d}}$ \\
\hline S96T003751 & $152: 14$ & Drainable liquid & $1.648 \mathrm{E}+05$ & $1.655 \mathrm{E}+05$ & $1.651 \mathrm{E}+05^{\mathrm{QC}: c}$ \\
\hline S96T004433 & 153: 1 & Drainable liquid & $1.894 \mathrm{E}+05$ & $1.768 \mathrm{E}+05$ & $1.831 \mathrm{E}+05^{\mathrm{QC}: \mathrm{d}}$ \\
\hline S96T004308 & 153: 2 & Drainable liquid & $1.817 \mathrm{E}+05$ & $1.747 \mathrm{E}+05$ & $1.782 \mathrm{E}+05^{\mathrm{QC}: \mathrm{c}}$ \\
\hline S96T004434 & 153: 3 & Drainable liquid & $1.641 \mathrm{E}+05$ & $1.803 \mathrm{E}+05$ & $1.722 \mathrm{E}+05^{\mathrm{QC:d}}$ \\
\hline S96T004419 & 153: 5 & Drainable liquid & $1.845 \mathrm{E}+05$ & $1.817 \mathrm{E}+05$ & $1.831 \mathrm{E}+05^{\mathrm{QC:d}}$ \\
\hline S96T004420 & 153: 6 & Drainable liquid & $1.732 \mathrm{E}+05$ & $1.866 \mathrm{E}+05$ & $1.799 \mathrm{E}+05^{\mathrm{QC:d}}$ \\
\hline S96T004421 & 153: 7 & Drainable liquid & $1.796 \mathrm{E}+05$ & $1.930 \mathrm{E}+05$ & $1.863 \mathrm{E}+05^{\mathrm{QC:d}}$ \\
\hline S96T003975 & 153: 8 & Drainable liquid & $1.683 \mathrm{E}+05$ & $1.570 \mathrm{E}+05$ & $1.627 \mathrm{E}+05^{\mathrm{QC}: c}$ \\
\hline S96T003976 & 153: 9 & Drainable liquid & $1.535 \mathrm{E}+05$ & $1.817 \mathrm{E}+05$ & $1.676 \mathrm{E}+05^{\mathrm{QC:d}}$ \\
\hline S96T004309 & $153: 10$ & Drainable liquid & $1.789 \mathrm{E}+05$ & $1.873 E+05$ & $1.831 \mathrm{E}+05^{\mathrm{QC:c}}$ \\
\hline S96T003979 & 153:11 & Drainable liquid & $1.775 \mathrm{E}+05$ & $1.761 \mathrm{E}+05$ & $1.768 \mathrm{E}+05^{Q \mathrm{QC:c}}$ \\
\hline S96T003977 & $153: 12$ & Drainable liquid & $1.775 \mathrm{E}+05$ & $1.817 \mathrm{E}+05$ & $1.796 \mathrm{E}+05^{Q \mathrm{C}: \mathrm{d}}$ \\
\hline S96T003978 & $153: 13$ & Drainable liquid & $1.669 E+05$ & $1.535 \mathrm{E}+05$ & $1.602 \mathrm{E}+05^{\mathrm{QC:c}}$ \\
\hline S96T004333 & $153: 14$ & Drainable liquid & $1.873 \mathrm{E}+05$ & $1.852 \mathrm{E}+05$ & $1.863 \mathrm{E}+05^{\mathrm{QC}: c}$ \\
\hline S96T003980 & $153: 21$ & Drainable liquid & $1.570 \mathrm{E}+05$ & $1.542 \mathrm{E}+05$ & $1.556 \mathrm{E}+05^{\mathrm{QC:c}}$ \\
\hline S96T004831 & Core 152 & Liquid composite & $1.789 \mathrm{E}+05$ & $1.810 \mathrm{E}+05$ & $1.799 \mathrm{E}+05^{\mathrm{QC:d}}$ \\
\hline
\end{tabular}


Table B2-45. Tank 241-AN-105 Analytical Results: Sodium (ICP). (3 sheets)

\begin{tabular}{|c|c|c|c|c|c|}
\hline Sanole & 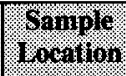 & 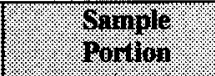 & Result & miplicale & 16 \\
\hline \multicolumn{2}{|c|}{ 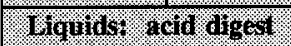 } & & 1018 & 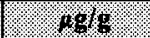 & $12 \%$ \\
\hline S96T004144 & Core 152 & Liquid composite & $1.486 \mathrm{E}+05$ & $1.486 \mathrm{E}+05$ & $1.486 \mathrm{E}+05$ \\
\hline \multicolumn{2}{|c|}{ Golidst acto dyst } & & 18.8 & 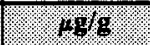 & 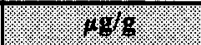 \\
\hline S96T003906 & $152: 1$ & Upper half & $1.870 \mathrm{E}+05$ & $1.780 \mathrm{E}+05$ & $1.825 \mathrm{E}+05^{\mathrm{QC}: \mathrm{b}, \mathrm{c}}$ \\
\hline S96T003898 & \multirow[t]{2}{*}{$152: 14$} & Upper half & $1.790 \mathrm{E}+05$ & $1.810 \mathrm{E}+05$ & $1.800 \mathrm{E}+05^{Q C: b}$ \\
\hline S96T003828 & & Lower half & $1.830 \mathrm{E}+05$ & $1.850 \mathrm{E}+05$ & $1.840 \mathrm{E}+05^{\mathrm{QC}: \mathrm{b}}$ \\
\hline S96T003899 & \multirow[t]{2}{*}{$152: 16$} & Upper half & $1.870 \mathrm{E}+05$ & $1.920 \mathrm{E}+05$ & $1.895 \mathrm{E}+05^{\mathrm{QC}: \mathrm{b}}$ \\
\hline S96T003900 & & Lower half & $1.880 \mathrm{E}+05$ & $1.920 \mathrm{E}+05$ & $1.900 \mathrm{E}+05^{\mathrm{QC} \cdot \mathrm{b}}$ \\
\hline \$96T003901 & \multirow[t]{2}{*}{$152: 18$} & Upper half & $1.980 \mathrm{E}+05$ & $1.950 \mathrm{E}+05$ & $1.965 \mathrm{E}+05^{\mathrm{QC}: \mathrm{b}}$ \\
\hline S96T003902 & & Lower half & $1.970 \mathrm{E}+05$ & $1.950 \mathrm{E}+05$ & $1.960 \mathrm{E}+05^{\mathrm{QC:b,c}}$ \\
\hline S96T003903 & \multirow[t]{2}{*}{$152: 20$} & Upper half & $1.910 \mathrm{E}+05$ & $1.860 \mathrm{E}+05$ & $1.885 \mathrm{E}+05^{\mathrm{QC}: \mathrm{b}}$ \\
\hline S96T003904 & & Lower half & $2.030 \mathrm{E}+05$ & $1.990 \mathrm{E}+05$ & $2.010 \mathrm{E}+05^{\mathrm{QC}: \mathrm{b}}$ \\
\hline \$96T003905 & $152: 22$ & Upper half & $1.930 \mathrm{E}+05$ & $1.970 \mathrm{E}+05$ & $1.950 \mathrm{E}+05^{\mathrm{QC}: \mathrm{b}}$ \\
\hline S96T004338 & $153: 14$ & Lower half & $1.720 \mathrm{E}+05$ & $1.740 \mathrm{E}+05$ & $1.730 \mathrm{E}+05^{\mathrm{QC}: \mathrm{b}, \mathrm{c}}$ \\
\hline S96T004315 & \multirow[t]{2}{*}{$153: 15$} & Upper half & $1.690 \mathrm{E}+05$ & $1.680 \mathrm{E}+05$ & $1.685 \mathrm{E}+05^{\mathrm{QC}: \mathrm{b}}$ \\
\hline S96T004318 & & Lower half & $1.690 \mathrm{E}+05$ & $1.700 \mathrm{E}+05$ & $1.695 \mathrm{E}+05^{\mathrm{QC}: \mathrm{b}, \mathrm{d}}$ \\
\hline S96T004444 & \multirow[t]{2}{*}{$153: 17$} & Upper half & $1.730 \mathrm{E}+05$ & $1.730 \mathrm{E}+05$ & $1.730 \mathrm{E}+05^{\mathrm{QC}: \mathrm{b}}$ \\
\hline S96T004445 & & Lower half & $1.670 \mathrm{E}+05$ & $1.660 \mathrm{E}+05$ & $1.665 \mathrm{E}+05$ \\
\hline S96T004446 & $153: 19$ & Lower half & $1.710 \mathrm{E}+05$ & $1.790 E+05$ & $1.750 \mathrm{E}+05$ \\
\hline \$96T003988 & $153: 21$ & Lower half & $1.800 \mathrm{E}+05$ & $1.710 \mathrm{E}+05$ & $1.755 \mathrm{E}+05^{\mathrm{QC}: \mathrm{c}}$ \\
\hline S96T003989 & \multirow[t]{2}{*}{$153: 22$} & Upper half & $1.880 \mathrm{E}+05$ & $1.780 \mathrm{E}+05$ & $1.830 \mathrm{E}+05^{\mathrm{QC}: \mathrm{b}}$ \\
\hline S96T003987 & & Lower half & $1.800 \mathrm{E}+05$ & $1.810 \mathrm{E}+05$ & $1.805 \mathrm{E}+05^{\mathrm{QC:b, \varepsilon}}$ \\
\hline S96T004257 & \multirow[t]{2}{*}{ Core 152} & Solid composite & $1.570 \mathrm{E}+05$ & $1.530 \mathrm{E}+05$ & $1.550 \mathrm{E}+05^{\mathrm{QC}: \mathrm{b}}$ \\
\hline S96T004262 & & Solid composite & $1.750 \mathrm{E}+05$ & $1.540 \mathrm{E}+05$ & $1.645 \mathrm{E}+05$ \\
\hline
\end{tabular}


Table B2-45. Tank 241-AN-105 Analytical Results: Sodium (ICP). (3 sheets)

\begin{tabular}{|c|c|c|c|c|c|}
\hline Samplo & Samplo & $\frac{6911810}{901401}$ & Terilli: & Duplicite & 14an. \\
\hline Soliofs & Tision: & & $10 \%$ & 1818 & 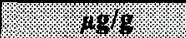 \\
\hline S96T003893 & 152: 1 & Upper half & $2.380 \mathrm{E}+05$ & $2.540 \mathrm{E}+05$ & $2.460 \mathrm{E}+05^{\mathrm{QC}: c}$ \\
\hline S96T003894 & \multirow[t]{2}{*}{$152: 14$} & Upper half & $2.380 \mathrm{E}+05$ & $2.160 \mathrm{E}+05$ & $2.270 \mathrm{E}+05$ \\
\hline \$96T003827 & & Lower half & $2.350 \mathrm{E}+05$ & $2.220 \mathrm{E}+05$ & $2.285 \mathrm{E}+05^{\mathrm{QC}: \mathrm{d}}$ \\
\hline \$96T003895 & \multirow[t]{2}{*}{$152: 16$} & Upper half & $2.500 \mathrm{E}+05$ & $2.220 \mathrm{E}+05$ & $2.360 \mathrm{E}+05$ \\
\hline S96T003889 & & Lower half & $2.250 \mathrm{E}+05$ & $2.350 \mathrm{E}+05$ & $2.300 \mathrm{E}+05$ \\
\hline S96T003896 & \multirow[t]{2}{*}{$152: 18$} & Upper half & $2.410 \mathrm{E}+05$ & $2.380 \mathrm{E}+05$ & $2.395 \mathrm{E}+05^{\mathrm{QC:d}}$ \\
\hline S96T003890 & & Lower half & $2.580 \mathrm{E}+05$ & $2.450 \mathrm{E}+05$ & $2.515 \mathrm{E}+05$ \\
\hline S96T003897 & \multirow[t]{2}{*}{$152: 20$} & Upper half & $2.280 \mathrm{E}+05$ & $2.270 \mathrm{E}+05$ & $2.275 \mathrm{E}+05$ \\
\hline S96T003891 & & Lower half & $2.320 \mathrm{E}+05$ & $2.250 \mathrm{E}+05$ & $2.285 \mathrm{E}+05$ \\
\hline S96T003892 & $152: 22$ & Upper half & $2.390 \mathrm{E}+05$ & $2.320 \mathrm{E}+05$ & $2.355 \mathrm{E}+05$ \\
\hline S96T004337 & $153: 14$ & Lower half & $2.220 \mathrm{E}+05$ & $2.150 \mathrm{E}+05$ & $2.185 \mathrm{E}+05^{\mathrm{QC}: \mathrm{C}}$ \\
\hline S96T004314 & \multirow[t]{2}{*}{$153: 15$} & Upper half & $2.150 \mathrm{E}+05$ & $2.170 \mathrm{E}+05$ & $2.160 \mathrm{E}+05^{\mathrm{QC}: \mathrm{c}}$ \\
\hline S96T004317 & & Lower half & $2.230 \mathrm{E}+05$ & $2.300 \mathrm{E}+05$ & $2.265 \mathrm{E}+05^{\mathrm{QC}: c}$ \\
\hline S96T004441 & \multirow[t]{2}{*}{$153: 17$} & Upper half & $2.230 \mathrm{E}+05$ & $2.250 \mathrm{E}+05$ & $2.240 \mathrm{E}+05^{\mathrm{QC}: \mathrm{c}}$ \\
\hline S96T004442 & & Lower half & $2.150 \mathrm{E}+05$ & $2.200 \mathrm{E}+05$ & $2.175 \mathrm{E}+05$ \\
\hline S96T004443 & $153: 19$ & Lower half & $2.190 \mathrm{E}+05$ & $2.210 \mathrm{E}+05$ & $2.200 \mathrm{E}+05$ \\
\hline S96T003984 & $153: 21$ & Lower half & $2.280 \mathrm{E}+05$ & $2.240 \mathrm{E}+05$ & $2.260 \mathrm{E}+05^{\mathrm{QC}: \mathrm{c}}$ \\
\hline S96T003986 & \multirow[t]{2}{*}{$153: 22$} & Upper half & $2.380 \mathrm{E}+05$ & $2.350 \mathrm{E}+05$ & $2.365 \mathrm{E}+05$ \\
\hline S96T003985 & & Lower half & $2.130 \mathrm{E}+05$ & $2.140 E+05$ & $2.135 \mathrm{E}+05$ \\
\hline
\end{tabular}


Table B2-46. Tank 241-AN-105 Analytical Results: Strontium (ICP). (3 sheets)

\begin{tabular}{|c|c|c|c|c|c|}
\hline Wamping & Sornople & 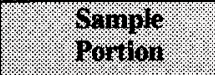 & icsur. & Bupltaras & 18can \\
\hline \multicolumn{2}{|c|}{ Wofins } & & 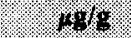 & 4818 & 498 \\
\hline S96T003732 & $152: 1$ & Drainable liquid & $<2.82$ & $<2.82$ & $<2.82$ \\
\hline S96T003739 & 152: 2 & Drainable liquid & $<2.82$ & $<2.82$ & $<2.82$ \\
\hline S96T003740 & $152: 3$ & Drainable liquid & $<2.82$ & $<2.82$ & $<2.82$ \\
\hline S96T003741 & $152: 4$ & Drainable liquid & $<2.82$ & $<2.82$ & $<2.82$ \\
\hline S96T003742 & 152: 5 & Drainable liquid & $<2.82$ & $<2.82$ & $<2.82$ \\
\hline \$96T003743 & 152: 6 & Drainable liquid & $<2.82$ & $<2.82$ & $<2.82$ \\
\hline S96T003744 & 152: 7 & Drainable liquid & $<2.82$ & $<2.82$ & $<2.82$ \\
\hline S96T003745 & $152: 8$ & Drainable liquid & $<2.82$ & $<2.82$ & $<2.82$ \\
\hline S96T003746 & 152: 9 & Drainable liquid & $<2.82$ & $<2.82$ & $<2.82$ \\
\hline S96T003747 & $152: 10$ & Drainable liquid & $<4.23$ & $<4.23$ & $<4.23$ \\
\hline S96T003748 & $152: 11$ & Drainable liquid & $<4.23$ & $<4.23$ & $<4.23$ \\
\hline \$96T003749 & $152: 12$ & Drainable liquid & $<4.23$ & $<4.23$ & $<4.23$ \\
\hline S96T003750 & $152: 13$ & Drainable liquid & $<4.23$ & $<4.23$ & $<4.23$ \\
\hline S96T003751 & $152: 14$ & Drainable liquid & $<2.82$ & $<2.82$ & $<2.82$ \\
\hline S96T004433 & 153: 1 & Drainable liquid & $<2.82$ & $<2.82$ & $<2.82$ \\
\hline S96T004308 & 153: 2 & Drainable liquid & $<4.23$ & $<4.23$ & $<4.23$ \\
\hline S96T004434 & 153: 3 & Drainable liquid & $<2.82$ & $<2.82$ & $<2.82$ \\
\hline S96T004419 & $153: 5$ & Drainable liquid & $<4.23$ & $<4.23$ & $<4.23$ \\
\hline S96T004420 & 153: 6 & Drainable liquid & $<4.23$ & $<4.23$ & $<4.23$ \\
\hline S96T004421 & 153: 7 & Drainable liquid & $<2.82$ & $<2.82$ & $<2.82$ \\
\hline S96T003975 & 153: 8 & Drainable jiquid & $<2.82$ & $<2.82$ & $<2.82$ \\
\hline S96T003976 & $153: 9$ & Drainable liquid & $<2.82$ & $<2.82$ & $<2.82$ \\
\hline S96T004309 & $153: 10$ & Drainable liquid & $<4.23$ & $<4.23$ & $<4.23$ \\
\hline \$96T003979 & $153: 11$ & Drainable liquid & $<4.23$ & $<4.23$ & $<4.23$ \\
\hline S96T003977 & $153: 12$ & Drainable liquid & $<4.23$ & $<4.23$ & $<4.23$ \\
\hline S96T003978 & $153: 13$ & Drainable liquid & $<4.23$ & $<4.23$ & $<4.23$ \\
\hline S96T004333 & $153: 14$ & Drainable liquid & $<4.23$ & $<4.23$ & $<4.23$ \\
\hline S96T003980 & $153: 21$ & Drainable liquid & $<4.23$ & $<4.23$ & $<4.23$ \\
\hline S96T004831 & Core 152 & Liquid composite & $<4.23$ & $<4.23$ & $<4.23$ \\
\hline
\end{tabular}


Table B2-46. Tank 241-AN-105 Analytical Results: Strontium (ICP). (3 sheets)

\begin{tabular}{|c|c|c|c|c|c|}
\hline 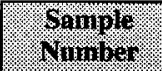 & Sanplo & 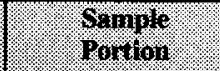 & (2) & Brolizito & 1.6 .14817 \\
\hline \multicolumn{2}{|c|}{ 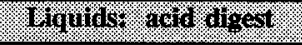 } & & 1649 & 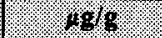 & $19 \%$ \\
\hline S96T004144 & Core 152 & Liquid composite & $<3.69$ & $<3.52$ & $<3.60$ \\
\hline \multicolumn{2}{|c|}{ 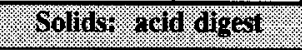 } & (1. & 898 & 160 & 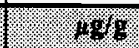 \\
\hline S96T003906 & 152: 1 & Upper half & $<3.86$ & $<4.03$ & $<3.94$ \\
\hline S96T003898 & \multirow[t]{2}{*}{$152: 14$} & Upper half & $<3.93$ & $<3.88$ & $<3.90$ \\
\hline \$96T003828 & & Lower half & $<4.04$ & $<3.93$ & $<3.98$ \\
\hline S96T003899 & \multirow[t]{2}{*}{$152: 16$} & Upper half & $<3.96$ & $<3.98$ & $<3.97$ \\
\hline S96T003900 & & Lower half & $<3.93$ & $<3.93$ & $<3.93$ \\
\hline S96T003901 & \multirow[t]{2}{*}{$152: 18$} & Upper half & $<3.89$ & $<3.96$ & $<3.92$ \\
\hline S96T003902 & & Lower half & $<4$ & $<4.04$ & $<4.02$ \\
\hline S96T003903 & \multirow[t]{2}{*}{$152: 20$} & Upper half & $<3.83$ & $<3.76$ & $<3.79$ \\
\hline S96T003904 & & Lower half & $<3.75$ & $<3.71$ & $<3.73$ \\
\hline S96T003905 & $152: 22$ & Upper half & $<3.96$ & $<4.14$ & $<4.05$ \\
\hline S96T004338 & 153:14 & Lower half & $<4.67$ & $<4.78$ & $<4.72$ \\
\hline \$96T004315 & \multirow[t]{2}{*}{$153: 15$} & Upper half & $<4.76$ & $<4.78$ & $<4.77$ \\
\hline S96T004318 & & Lower half & $<4.79$ & $<4.51$ & $<4.65$ \\
\hline S96T004444 & \multirow[t]{2}{*}{$153: 17$} & Upper half & $<4.83$ & $<4.75$ & $<4.79$ \\
\hline S96T004445 & & Lower half & $<3.71$ & 3.75 & $<3.73$ \\
\hline S96T004446 & $153: 19$ & Lower half & $<3.82$ & $<3.82$ & $<3.82$ \\
\hline \$96T003988 & $153: 21$ & Lower half & $<3.56$ & $<3.56$ & $<3.56$ \\
\hline S96T003989 & \multirow[t]{2}{*}{$153: \overline{22}$} & Upper half & $<4.65$ & $<4.62$ & $<4.63$ \\
\hline S96T003987 & & Lower half & 9.06 & 9.73 & 9.39 \\
\hline S96T004257 & \multirow[t]{2}{*}{ Core 152} & Solid composite & $<3.83$ & $<4.03$ & $<3.93$ \\
\hline S96T004262 & & Solid composite & $<3.89$ & $<3.7$ & $<3.79$ \\
\hline
\end{tabular}


Table B2-46. Tank 241-AN-105 Analytical Results: Strontium (ICP). (3 sheets)

\begin{tabular}{|c|c|c|c|c|c|}
\hline $\begin{array}{l}\text { Sample } \\
\text { Nimber }\end{array}$ & $\begin{array}{l}\text { Sample } \\
\text { liocation }\end{array}$ & $\begin{array}{l}\text { Sampie } \\
\text { Torion }\end{array}$ & Presill & Buplionte & Mean \\
\hline Sollos: & Gision & & 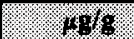 & $\pi .18$ & 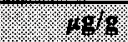 \\
\hline S96T003893 & 152: 1 & Upper half & $<198$ & $<201$ & $<199.5$ \\
\hline S96T003894 & \multirow[t]{2}{*}{$152: 14$} & Upper half & $<204$ & $<200$ & $<202$ \\
\hline S96T003827 & & Lower half & $<201$ & $<206$ & $<203.5$ \\
\hline S96T003895 & \multirow[t]{2}{*}{$152: 16$} & Upper half & $<204$ & $<201$ & $<202.5$ \\
\hline S96T003889 & & Lower half & $<203$ & $<200$ & $<201.5$ \\
\hline S96T003896 & \multirow[t]{2}{*}{$152: 18$} & Upper half & $<207$ & $<201$ & $<204$ \\
\hline S96T003890 & & Lower half & $<206$ & $\begin{array}{l}<207 \\
\end{array}$ & $<206.5$ \\
\hline S96T003897 & \multirow[t]{2}{*}{$152: 20$} & Upper half & $<212$ & $<207$ & $<209.5$ \\
\hline S96T003891 & & Lower half & $<204$ & $<206$ & $<205$ \\
\hline S96T003892 & $152: 22$ & Upper half & $<209$ & $<212$ & $<210.5$ \\
\hline S96T004337 & $153: 14$ & Lower half & $<203$ & $<203$ & $<203$ \\
\hline S96T004314 & \multirow[t]{2}{*}{$153: 15$} & Upper half & $<191$ & $<192$ & $<191.5$ \\
\hline S96T004317 & & Lower half & $<200$ & $<203$ & $<201.5$ \\
\hline S96T004441 & \multirow[t]{2}{*}{$153: 17$} & Upper half & $<196$ & $<196$ & $<196$ \\
\hline S96T004442 & & Lower half & $<201$ & $<200$ & $<200.5$ \\
\hline S96T004443 & $153: 19$ & Lower half & $<202$ & $<200$ & $<201$ \\
\hline S96T003984 & $153: 21$ & Lower half & $<201$ & $<201$ & $<201$ \\
\hline S96T003986 & \multirow[t]{2}{*}{$153: 22$} & Upper half & $<200$ & $<203$ & $<201.5$ \\
\hline S96T003985 & & Lower half & $<201$ & $<201$ & $<201$ \\
\hline
\end{tabular}


Table B2-47. Tank 241-AN-105 Analytical Results: Sulfur (ICP). (3 sheets)

\begin{tabular}{|c|c|c|c|c|c|}
\hline Wham & Wompie & 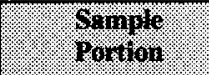 & 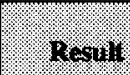 & Duplicats & Menn \\
\hline \multicolumn{2}{|c|}{ Wrofirs } & & 4.48 & 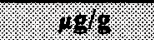 & 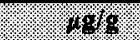 \\
\hline S96T003732 & $152: 1$ & Drainable liquid & 711.26 & 690.14 & 700.70 \\
\hline S96T003739 & 152: 2 & Drainable liquid & 654.93 & 500.70 & $577.81^{\text {QC:e,e }}$ \\
\hline S96T003740 & $152: 3$ & Drainable liquid & 669.71 & 490.84 & $580.28^{Q C: c}$ \\
\hline S96T003741 & 152: 4 & Drainable liquid & 570.42 & 533.80 & $552.11^{\text {QC:d }}$ \\
\hline S96T003742 & $152: 5$ & Drainable liquid & 640.84 & 541.54 & $591.19^{\mathrm{QC}: c}$ \\
\hline S96T003743 & $152: 6$ & Drainable liquid & 732.39 & 795.77 & 764.08 \\
\hline S96T003744 & 152: 7 & Drainable liquid & 598.59 & 667.60 & $633.09^{\mathrm{QC:d}}$ \\
\hline S96T003745 & 152: 8 & Drainable liquid & 574.64 & 682.39 & $628.52^{\mathrm{QC}: \mathrm{d}}$ \\
\hline S96T003746 & 152:9 & Drainable liquid & 686.62 & 694.36 & 690.49 \\
\hline S96T003747 & $152: 10$ & Drainable liquid & 668.31 & 685.21 & 676.76 \\
\hline S96T003748 & $152: 11$ & Drainable liquid & 677.46 & 686.62 & 682.04 \\
\hline S96T003749 & $152: 12$ & Drainable liquid & 672.53 & 650 & 661.26 \\
\hline S96T003750 & $152: 13$ & Drainable liquid & 657.04 & 653.52 & 655.28 \\
\hline S96T003751 & $152: 14$ & Drainable liquid & 678.87 & 688.02 & 683.45 \\
\hline S96T004433 & 153: 1 & Drainable liquid & 781.69 & 732.39 & 757.04 \\
\hline S96T004308 & 153: 2 & Drainable liquid & 725.35 & 718.31 & 721.83 \\
\hline S96T004434 & 153: 3 & Drainable liquid & 550.70 & 583.80 & $567.25^{\mathrm{QC}: \mathrm{d}}$ \\
\hline S96T004419 & 153: 5 & Drainable liquid & 718.31 & 718.31 & 718.31 \\
\hline S96T004420 & 153: 6 & Drainable liquid & 661.26 & 718.31 & 689.78 \\
\hline S96T004421 & 153: 7 & Drainable liquid & 676.76 & 753.52 & 715.14 \\
\hline S96T003975 & 153: 8 & Drainable liquid & 462.67 & 434.50 & 448.59 \\
\hline S96T003976 & 153: 9 & Drainable liquid & 615.49 & 732.39 & 673.94 \\
\hline S96T004309 & $153: 10$ & Drainable liquid & 718.31 & 739.43 & 728.87 \\
\hline S96T003979 & $153: 11$ & Drainable liquid & 697.18 & 683.80 & 690.49 \\
\hline S96T003977 & $153: 12$ & Drainable liquid & 688.73 & 704.22 & 696.47 \\
\hline S96T003978 & $153: 13$ & Drainable liquid & 645.77 & 600 & 622.88 \\
\hline S96T004333 & $153: 14$ & Drainable liquid & 688.02 & 718.31 & 703.16 \\
\hline S96T003980 & $153: 21$ & Drainable liquid & 936.62 & 922.53 & 929.57 \\
\hline S96T004831 & Core 152 & Liquid composite & 725.35 & 699.29 & 712.32 \\
\hline
\end{tabular}


Table B2-47. Tank 241-AN-105 Analytical Results: Sulfur (ICP). (3 sheets)

\begin{tabular}{|c|c|c|c|c|c|}
\hline Samplo & $4 \%$ & 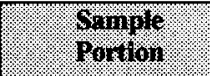 & Pesial & Buplicate & Me日i: \\
\hline \multicolumn{2}{|c|}{ Wquidst ard alges/ } & & 148 & $\%$ & $148 \%$ \\
\hline S96T004144 & Core 152 & Liquid composite & 615.49 & 619.71 & 617.60 \\
\hline \multicolumn{2}{|c|}{ 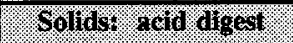 } & & 1018 & 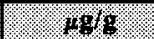 & $1 \%$ \\
\hline S96T003906 & 152: 1 & Upper half & 1,580 & 1,500 & 1,540 \\
\hline S96T003898 & \multirow[t]{2}{*}{$152: 14$} & Upper half & 2,030 & 2,080 & 2,055 \\
\hline S96T003828 & & Lower half & 2,180 & 2,230 & 2,205 \\
\hline S96T003899 & \multirow[t]{2}{*}{$152: 16$} & Upper half & 2,940 & 2,990 & 2,965 \\
\hline \$96T003900 & & Lower half & 3,100 & 3,150 & 3,125 \\
\hline S96T003901 & \multirow[t]{2}{*}{$152: 18$} & Upper half & 3,300 & 3,230 & 3,265 \\
\hline S96T003902 & & Lower half & 3,170 & 3,260 & 3,215 \\
\hline S96T003903 & \multirow[t]{2}{*}{$152: 20$} & Upper half & 2,740 & 2,720 & 2,730 \\
\hline \$96T003904 & & Lower half & 3,390 & 3,430 & 3,410 \\
\hline S96T003905 & $152: 22$ & Upper half & 2,610 & 2,700 & 2,655 \\
\hline S96T004338 & $153: 14$ & Lower half & 2,050 & 2,210 & 2,130 \\
\hline \$96T004315 & \multirow[t]{2}{*}{$153: 15$} & Upper half & 2,190 & 2,270 & 2,230 \\
\hline S96T004318 & & Lower half & 2,000 & 2,150 & 2,075 \\
\hline S96T004444 & \multirow[t]{2}{*}{$153: 17$} & Upper half & 2,230 & 2,220 & 2,225 \\
\hline \$96T004445 & & Lower half & 2,310 & 2,360 & 2,335 \\
\hline S96T004446 & $153: 19$ & Lower half & 2,470 & 2,570 & 2,520 \\
\hline S96T003988 & $153: 21$ & Lower half & 2,820 & 2,710 & 2,765 \\
\hline S96T003989 & \multirow[t]{2}{*}{$153: 22$} & Upper half & 2,370 & 2,190 & 2,280 \\
\hline S96T003987 & & Lower half & 711 & 754 & 732.5 \\
\hline S96T004257 & \multirow[t]{2}{*}{ Core 152} & Solid composite & 2,320 & 2,030 & 2,175 \\
\hline S96T004262 & & Solid composite & 1,530 & 1,360 & 1,445 \\
\hline
\end{tabular}


Table B2-47. Tank 241-AN-105 Analytical Results: Sulfur (ICP). (3 sheets)

\begin{tabular}{|c|c|c|c|c|c|}
\hline Warnols & Gample & 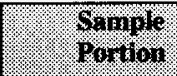 & 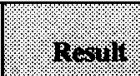 & Buplicale & (8) \\
\hline $6010 \%$ & 40 & & 45 & 40 & $48 \%$ \\
\hline S96T003893 & 152: 1 & Upper half & $<1,980$ & $<2,010$ & $<1,995$ \\
\hline S96T003894 & \multirow[t]{2}{*}{$152: 14$} & Upper half & $<2,040$ & $<2,000$ & $<2,020$ \\
\hline \$96T003827 & & Lower half & $<2,010$ & 2,080 & $<2,045$ \\
\hline S96T003895 & \multirow[t]{2}{*}{$152: 16$} & Upper half & 2,890 & 2,880 & 2,885 \\
\hline S96T003889 & & Lower half & 3,000 & 3,140 & 3,070 \\
\hline S96T003896 & \multirow[t]{2}{*}{$152: 18$} & Upper half & 3,250 & 3,350 & 3,300 \\
\hline S96T003890 & & Lower half & 3,270 & 3,290 & 3,280 \\
\hline S96T003897 & \multirow[t]{2}{*}{$152: 20$} & Upper half & 3,100 & 3,030 & 3,065 \\
\hline S96T003891 & & Lower half & 2,480 & 2,490 & 2,485 \\
\hline S96T003892 & $152: 22$ & Upper half & 3,420 & 3,690 & 3,555 \\
\hline S96T004337 & 153:14 & Lower half & 2,050 & 2,120 & 2,085 \\
\hline S96T004314 & \multirow[t]{2}{*}{$153: 15$} & Upper half & 2,060 & 2,550 & $2,305^{\mathrm{QC}: \mathrm{e}}$ \\
\hline S96T004317 & & Lower half & 2,390 & 2,640 & 2,515 \\
\hline \$96T004441 & \multirow[t]{2}{*}{$153: 17$} & Upper half & 3,040 & 2,640 & 2,840 \\
\hline S96T004442 & & Lower half & 2,650 & 2,660 & 2,655 \\
\hline S96T004443 & 153:19 & Lower half & 3,210 & 2,720 & 2,965 \\
\hline S96T003984 & $153: 21$ & Lower half & 3,500 & 3,490 & 3,495 \\
\hline S96T003986 & \multirow[t]{2}{*}{$153: 22$} & Upper half & 2,520 & 2,530 & 2,525 \\
\hline \$96T003985 & & Lower half & $<2,010$ & $<2,010$ & $<2,010$ \\
\hline
\end{tabular}


Table B2-48. Tank 241-AN-105 Analytical Results: Zinc (ICP). (3 sheets)

\begin{tabular}{|c|c|c|c|c|c|}
\hline Sumber & Wample & 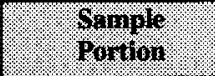 & resilit & Bupligato & Hean \\
\hline \multicolumn{2}{|c|}{ W } & & 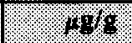 & ( $.6 \%$ & $401 \%$ \\
\hline S96T003732 & 152: 1 & Drainable liquid & 16.54 & 16.54 & 16.54 \\
\hline S96T003739 & 152: 2 & Drainable liquid & 13.09 & 15.49 & 14.29 \\
\hline S96T003740 & 152: 3 & Drainable liquid & 15.63 & 17.60 & 16.61 \\
\hline S96T003741 & 152: 4 & Drainable liquid & 13.09 & 11.69 & 12.39 \\
\hline S96T003742 & 152: 5 & Drainable liquid & 18.23 & 14.15 & $16.19^{\mathrm{QC}: \mathrm{e}}$ \\
\hline S96T003743 & 152: 6 & Drainable liquid & 20.63 & 21.33 & 20.98 \\
\hline S96T003744 & 152: 7 & Drainable liquid & 22.88 & 25.77 & 24.33 \\
\hline S96T003745 & 152: 8 & Drainable liquid & 18.73 & 24.64 & $21.69^{\text {QC:e }}$ \\
\hline S96T003746 & 152: 9 & Drainable liquid & 23.38 & 21.40 & 22.39 \\
\hline S96T003747 & $152: 10$ & Drainable liquid & 11.69 & 12.46 & 12.07 \\
\hline S96T003748 & 152:11 & Drainable liquid & 11.33 & 11.40 & 11.37 \\
\hline S96T003749 & $152: 12$ & Drainable liquid & 11.47 & 11.26 & 11.37 \\
\hline S96T003750 & $152: 13$ & Drainable liquid & 10.35 & 10.35 & 10.35 \\
\hline S96T003751 & $152: 14$ & Drainable liquid & 31.97 & 28.80 & 30.38 \\
\hline S96T004433 & 153: 1 & Drainable liquid & 57.11 & 38.09 & $47.60^{\mathrm{QC}: \mathrm{e}}$ \\
\hline S96T004308 & 153: 2 & Drainable liquid & 16.54 & 14.85 & 15.70 \\
\hline S96T004434 & 153: 3 & Drainable liquid & 39.01 & 33.73 & 36.37 \\
\hline S96T004419 & 153: 5 & Drainable liquid & 29.43 & 29.36 & 29.40 \\
\hline S96T004420 & 153: 6 & Drainable liquid & 25.84 & 27.60 & 26.72 \\
\hline S96T004421 & 153: 7 & Drainable liquid & 46.33 & 42.25 & 44.29 \\
\hline S96 T003975 & 153: 8 & Drainable liquid & 40.49 & 28.94 & $34.71^{\mathrm{QC:e}}$ \\
\hline S96T003976 & 153: 9 & Drainable liquid & 38.30 & 34.08 & 36.19 \\
\hline S96T004309 & $153: 10$ & Drainable liquid & 17.18 & 17.32 & 17.25 \\
\hline S96T003979 & $153: 11$ & Drainable liquid & 10.21 & 9.78 & 10 \\
\hline S96T003977 & $153: 12$ & Drainable liquid & 11.12 & 11.47 & 11.30 \\
\hline S96T003978 & $153: 13$ & Drainable liquid & 9.43 & 8.80 & 9.11 \\
\hline S96T004333 & 153:14 & Drainable liquid & 30.56 & 29.71 & 30.14 \\
\hline S96T003980 & $153: 21$ & Drainable liquid & 27.67 & 26.97 & 27.32 \\
\hline S96T004831 & Core 152 & Liquid composite & 12.04 & 12.74 & 12.39 \\
\hline
\end{tabular}


Table B2-48. Tank 241-AN-105 Analytical Results: Zinc (ICP). (3 sheets)

\begin{tabular}{|c|c|c|c|c|c|}
\hline Yamber & aranple & $\begin{array}{l}\text { Sranple } \\
\text { Porthor }\end{array}$ & $27=311$ & Oyplicale & 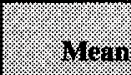 \\
\hline \multicolumn{2}{|c|}{ Windor add disert } & & 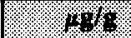 & 1988 & 45 \\
\hline S96T004144 & Core 152 & Liquid composite & 54.71 & 52.46 & $53.59^{\mathrm{QC}: \mathrm{f}}$ \\
\hline \multicolumn{2}{|c|}{ 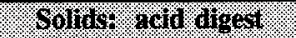 } & & 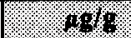 & 1489 & 109 \\
\hline S96T003906 & 152: 1 & Upper half & 32.2 & 31.2 & 31.7 \\
\hline S96T003898 & \multirow[t]{2}{*}{$152: 14$} & Upper half & 36.1 & 35.7 & 35.9 \\
\hline S96T003828 & & Lower half & 35.9 & 37.4 & 36.65 \\
\hline S96T003899 & \multirow[t]{2}{*}{$152: 16$} & Upper half & 35.9 & 35.4 & 35.65 \\
\hline S96T003900 & & Lower half & 37.2 & 41.9 & 39.55 \\
\hline S96T003901 & \multirow[t]{2}{*}{$152: 18$} & Upper half & 40 & 38.9 & 39.45 \\
\hline \$96T003902 & & Lower half & 34.8 & 38 & 36.4 \\
\hline S96T003903 & \multirow[t]{2}{*}{$152: 20$} & Upper half & 37 & 38.3 & 37.65 \\
\hline S96T003904 & & Lower half & 38.2 & 35.6 & 36.9 \\
\hline S96T003905 & $152: 22$ & Upper half & 36.5 & 37.9 & 37.2 \\
\hline S96T004338 & $153: 14$ & Lower half & 57.9 & 54 & 55.95 \\
\hline S96T004315 & \multirow[t]{2}{*}{$153: 15$} & Upper half & 57.2 & 42.1 & $49.65^{\mathrm{QC}: \mathrm{e}}$ \\
\hline S96T004318 & & Lower half & 43.2 & 40.1 & 41.65 \\
\hline S96T004444 & \multirow[t]{2}{*}{$153: 17$} & Upper half & 42.3 & 41.8 & 42.05 \\
\hline S96T004445 & & Lower half & 30.5 & 42.1 & $36.3^{\mathrm{QC}: \mathrm{e}}$ \\
\hline S96T004446 & $153: 19$ & Lower half & 66.8 & 43.9 & $55.35^{\mathrm{QC}: \mathrm{e}}$ \\
\hline S96T003988 & $153: 21$ & Lower half & 38 & 47.4 & $42.7^{Q C: e}$ \\
\hline S96T003989 & \multirow[t]{2}{*}{$153: 22$} & Upper half & 49.5 & 51.5 & 50.5 \\
\hline S96T003987 & & Lower half & 63.2 & 75.3 & 69.25 \\
\hline S96T004257 & \multirow[t]{2}{*}{ Core 152} & Solid composite & 7.78 & 8.58 & $8.18^{\mathrm{QC}: \mathrm{a}, \mathrm{c}}$ \\
\hline S96T004262 & & Solid composite & 13.4 & 9.7 & $11.55^{\mathrm{QC:e}}$ \\
\hline
\end{tabular}


Table B2-48. Tank 241-AN-105 Analytical Results: Zinc (ICP). (3 sheets)

\begin{tabular}{|c|c|c|c|c|c|}
\hline $\begin{array}{l}\text { Sample } \\
\text { Nomber }\end{array}$ & $\begin{array}{l}\text { Sample } \\
\text { U Gcartion }\end{array}$ & $\begin{array}{l}\text { Somple } \\
\text { Porilon }\end{array}$ & Resull & Buplicite & Mean \\
\hline \multicolumn{2}{|c|}{ Solltiss Iirion } & 2: & $1 \% 8 / 8$ & $10 \mathrm{~g} / 8$ & . \\
\hline S96T003893 & $152: 1$ & Upper half & 704 & 586 & 645 \\
\hline S96T003894 & \multirow[t]{2}{*}{$152: 14$} & Upper half & 397 & 616 & $506.5^{\mathrm{QC:c}}$ \\
\hline S96T003827 & & Lower half & 432 & 810 & $621^{\mathrm{QC}: \mathrm{e}}$ \\
\hline S96T003895 & \multirow[t]{2}{*}{$152: 16$} & Upper half & 780 & 350 & $565^{\mathrm{QC}: e}$ \\
\hline S96T003889 & & Lower half & $<203$ & $<200$ & $<201.5$ \\
\hline S96T003896 & \multirow[t]{2}{*}{$152: 18$} & Upper half & $<207$ & $<201$ & $<204$ \\
\hline S96T003890 & & Lower half & $<206$ & $<207$ & $<206.5$ \\
\hline S96T003897 & \multirow[t]{2}{*}{$152: 20$} & Upper half & $<212$ & $<207$ & $<209.5$ \\
\hline S96T003891 & & Lower half & 375 & 375 & 375 \\
\hline S96T003892 & $152: 22$ & Upper half & 657 & 770 & 713.5 \\
\hline S96T004337 & $153: 14$ & Lower half & $<203$ & $<203$ & $<203$ \\
\hline S96T004314 & \multirow[t]{2}{*}{$153: 15$} & Upper half & $<191$ & $<192$ & $<191.5$ \\
\hline S96T004317 & & Lower half & $<200$ & $<203$ & $<201.5$ \\
\hline S96T004441 & \multirow[t]{2}{*}{$153: 17$} & Upper half & $<196$ & $<196$ & $<196$ \\
\hline S96T004442 & & Lower half & $<201$ & $<200$ & $<200.5$ \\
\hline S96T004443 & $153: 19$ & Lower half & $<202$ & $<200$ & $<201$ \\
\hline S96T003984 & $153: 21$ & Lower half & $<201$ & $<201$ & $<201$ \\
\hline S96T003986 & \multirow[t]{2}{*}{$153: 22$} & Upper half & $<200$ & $<203$ & $<201.5$ \\
\hline S96T003985 & & Lower half & $<201$ & $<201$ & $<201$ \\
\hline
\end{tabular}


Table B2-49. Tank 241-AN-105 Analytical Results: Zirconium (ICP). (3 sheets)

\begin{tabular}{|c|c|c|c|c|c|}
\hline $\begin{array}{l}\text { Sample } \\
\text { Number }\end{array}$ & $\begin{array}{l}\text { Sarmple } \\
\text { Tication }\end{array}$ & $\begin{array}{l}\text { Sample } \\
\text { Portion }\end{array}$ & Resilu & Duplitarte & Mrean \\
\hline \multicolumn{2}{|c|}{ Trapión: } & & 1968 & 4968 & 19818 \\
\hline S96T003732 & 152: 1 & Drainable liquid & 3.31 & 3.35 & 3.33 \\
\hline \begin{tabular}{|l|} 
S96T003739 \\
\end{tabular} & 152: 2 & Drainable liquid & 3 & $<2.82$ & $<2.91$ \\
\hline \begin{tabular}{|l|} 
S96T003740 \\
\end{tabular} & $152: 3$ & Drainable liquid & 3.19 & 2.95 & 3.07 \\
\hline S96T003741 & 152: 4 & Drainable liquid & 3.21 & 3.18 & 3.19 \\
\hline \begin{tabular}{|l} 
S96T003742 \\
\end{tabular} & $152: 5$ & Drainable liquid & 3.59 & 3.46 & 3.52 \\
\hline S96T003743 & $152: 6$ & Drainable liquid & 2.86 & 3.61 & $3.23^{\mathrm{QC}: \mathrm{e}}$ \\
\hline S96T003744 & 152: 7 & Drainable liquid & $<2.82$ & $<2.82$ & $<2.82$ \\
\hline S96T003745 & 152: 8 & Drainable liquid & $<2.82$ & 2.82 & $<2.82$ \\
\hline S96T003746 & 152: 9 & Drainable liquid & 2.85 & $<2.82$ & $<2.83$ \\
\hline S96T003747 & $152: 10$ & Drainable liquid & $<4.23$ & $<4.23$ & $<4.23$ \\
\hline \begin{tabular}{|l|} 
S96T003748 \\
\end{tabular} & $152: 11$ & Drainable liquid & 4.28 & $<4.23$ & $<4.26$ \\
\hline S96T003749 & $152: 12$ & Drainable liquid & $<4.23$ & $<4.23$ & $<4.23$ \\
\hline S96T003750 & $152: 13$ & Drainable liquid & $<4.23$ & $<4.23$ & $<4.23$ \\
\hline S96T003751 & $152: 14$ & Drainable liquid & $<2.82$ & $<2.82$ & $<2.82$ \\
\hline \$96T004433 & 153: 1 & Drainable liquid & 2.86 & $<2.82$ & $<2.84$ \\
\hline S96T004308 & 153: 2 & Drainable liquid & $<4.23$ & $<4.23$ & $<4.23$ \\
\hline S96T004434 & 153: 3 & Drainable liquid & $<2.82$ & 2.92 & $<2.87$ \\
\hline S96T004419 & 153: 5 & Drainable liquid & $<4.23$ & $<4.23$ & $<4.23$ \\
\hline S96T004420 & 153: 6 & Drainable liquid & $<4.23$ & $<4.23$ & $<4.23$ \\
\hline \begin{tabular}{|l|} 
S96T004421 \\
\end{tabular} & 153: 7 & Drainable liquid & $<2.82$ & $<2.82$ & $<2.82$ \\
\hline S96T003975 & 153: 8 & Drainable liquid & 3.05 & $<2.82$ & $<2.94$ \\
\hline S96T003976 & 153: 9 & Drainable liquid & $<2.82$ & 2.95 & $<2.89$ \\
\hline S96T004309 & $153: 10$ & Drainable liquid & $<4.23$ & $<4.23$ & $<4.23$ \\
\hline S96T003979 & $153: 11$ & Drainable liquid & $<4.23$ & $<4.23$ & $<4.23$ \\
\hline S96T003977 & $153: 12$ & Drainable liquid & $<4.23$ & $<4.23$ & $<4.23$ \\
\hline S96T003978 & $153: 13$ & Drainable liquid & $<4.23$ & $<4.23$ & $<4.23$ \\
\hline S96T004333 & $153: 14$ & Drainable liquid & $<4.23$ & $<4.23$ & $<4.23$ \\
\hline S96T003980 & $153: 21$ & Drainable liquid & $<4.23$ & $<4.23$ & $<4.23$ \\
\hline S96T004831 & Core 152 & Liquid composite & $<4.23$ & $<4.23$ & $<4.23$ \\
\hline
\end{tabular}


Table B2-49. Tank 241-AN-105 Analytical Results: Zirconium (ICP). (3 sheets)

\begin{tabular}{|c|c|c|c|c|c|}
\hline $\begin{array}{l}\text { Sample: } \\
\text { Number. }\end{array}$ & $\begin{array}{l}\text { Sample } \\
\text { Laration }\end{array}$ & $\begin{array}{l}\text { Sample: } \\
\text { Potion }\end{array}$ & Resill & Dipliarte. & Mran \\
\hline \multicolumn{2}{|c|}{ Tiquids arerd diges } & 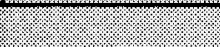 & 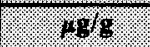 & $1=48 \mathrm{~g}$ & 1298. \\
\hline S96T004144 & Core 152 & Liquid composite & $<3.69014$ & 3.52113 & $<3.60563$ \\
\hline \multicolumn{2}{|c|}{ Sollds; actid digest } & & 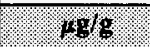 & $109 / 8$ & $1097 \mathrm{~s}$ \\
\hline S96T003906 & $152: 1$ & Upper half & $<3.86$ & $<4.03$ & $<3.94$ \\
\hline S96T003898 & \multirow[t]{2}{*}{$152: 14$} & Upper half & 4.44 & 4.7 & 4.57 \\
\hline S96T003828 & & Lower half & 4.44 & 4.15 & 4.29 \\
\hline S96T003899 & \multirow[t]{2}{*}{$152: 16$} & Upper half & 6.23 & 6.37 & 6.3 \\
\hline S96T003900 & & Lower half & 6.64 & 7.5 & 7.07 \\
\hline S96T003901 & \multirow[t]{2}{*}{$152: 18$} & Upper half & 8.92 & 8.77 & 8.84 \\
\hline S96T003902 & & Lower half & 7.8 & 7.43 & 7.61 \\
\hline S96T003903 & \multirow[t]{2}{*}{$152: 20$} & Upper half & 7.5 & 7.56 & 7.53 \\
\hline S96T003904 & & Lower half & 7.45 & 7.72 & 7.58 \\
\hline S96T003905 & $152: 22$ & Upper half & 25.2 & 25.3 & 25.25 \\
\hline S96T004338 & $153: 14$ & Lower half & 4.92 & 4.85 & 4.88 \\
\hline S96T004315 & \multirow[t]{2}{*}{$153: 15$} & Upper half & 5.32 & 5.66 & 5.49 \\
\hline S96T004318 & & Lower half & $<4.79$ & $<4.51$ & $<4.65$ \\
\hline S96T004444 & \multirow[t]{2}{*}{ 153:17 } & Upper half & 5.58 & 6.53 & 6.05 \\
\hline S96T004445 & & Lower half & 8.09 & 12.1 & $10.09^{\mathrm{QC:e}}$ \\
\hline S96T004446 & $153: 19$ & Lower half & 6.61 & 7.08 & 6.84 \\
\hline S96T003988 & $153: 21$ & Lower half & 5.93 & 5.55 & 5.74 \\
\hline S96T003989 & \multirow[t]{2}{*}{$153: 22$} & Upper half & 6.95 & $<4.62$ & $<5.78$ \\
\hline S96T003987 & & Lower half & 33.1 & 118 & $75.55^{\mathrm{QC}: \mathrm{e}}$ \\
\hline S96T004257 & \multirow[t]{2}{*}{ Core 152} & Solid composite & 4.51 & $<4.03$ & $<4.27$ \\
\hline S96T004262 & & Solid composite & 52.7 & 59 & 55.85 \\
\hline
\end{tabular}


Table B2-49. Tank 241-AN-105 Analytical Results: Zirconium (ICP). (3 sheets)

\begin{tabular}{|c|c|c|c|c|c|}
\hline $\begin{array}{l}\text { Sample } \\
\text { Number }\end{array}$ & $\begin{array}{l}\text { Coripoli. } \\
\text { Socation }\end{array}$ & $\begin{array}{l}\text { Sample } \\
\text { Yorthor }\end{array}$ & Rosing & Buplicate & Mran \\
\hline Sollor: & fivion. & & 1.495 & $.18 / 8$ & 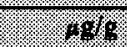 \\
\hline S96T003893 & $152: 1$ & Upper half & $<198$ & $<201$ & $<199.5$ \\
\hline S96T003894 & \multirow[t]{2}{*}{$152: 14$} & Upper half & $<204$ & $<200$ & $<202$ \\
\hline S96T003827 & & Lower half & $<201$ & $<206$ & $<203.5$ \\
\hline S96T003895 & \multirow[t]{2}{*}{$152: 16$} & Upper half & $<204$ & $<201$ & $<202.5$ \\
\hline S96T003889 & & Lower half & $<203$ & $<200$ & $<201.5$ \\
\hline S96T003896 & \multirow[t]{2}{*}{$152: 18$} & Upper half & $<207$ & $<201$ & $<204$ \\
\hline S96T003890 & & Lower half & $<206$ & $<207$ & $<206.5$ \\
\hline S96T003897 & \multirow[t]{2}{*}{$152: 20$} & Upper half & $<212$ & $<207$ & $<209.5$ \\
\hline S96T003891 & & Lower half & $<204$ & $<206$ & $<205$ \\
\hline S96T003892 & $152: 22$ & Upper half & $<209$ & $<212$ & $<210.5$ \\
\hline S96T004337 & 153:14 & Lower half & $<203$ & $<203$ & $<203$ \\
\hline S96T004314 & \multirow[t]{2}{*}{$153: 15$} & Upper half & $<191$ & $<192$ & $<191.5$ \\
\hline S96T004317 & & Lower half & $<200$ & $<203$ & $<201.5$ \\
\hline S96T004441 & \multirow[t]{2}{*}{$153: 17$} & Upper half & $<196$ & $<196$ & $<196$ \\
\hline S96T004442 & & Lower half & $<201$ & $<200$ & $<200.5$ \\
\hline S96T004443 & $153: 19$ & Lower half & $<202$ & $<200$ & $<201$ \\
\hline S96T003984 & $153: 21$ & Lower half & $<201$ & $<201$ & $<201$ \\
\hline S96T003986 & \multirow[t]{2}{*}{$153: 22$} & Upper half & $<200$ & $<203$ & $<201.5$ \\
\hline $25-1643985$ & & Lower half & 373 & 480 & $426.5^{\mathrm{QC}: \mathrm{e}}$ \\
\hline
\end{tabular}


HNF-SD-WM-ER-678 Rev. 0

Table B2-50. Tank 241-AN-105 ICP Results with Less Than Value.

\begin{tabular}{|c|c|}
\hline 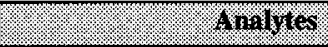 & 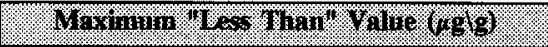 \\
\hline Antimony & $<1270$ \\
\hline Arsenic & $<2120$ \\
\hline Barium & $<1060$ \\
\hline Beryllium & $<106$ \\
\hline Cerium & $<2120$ \\
\hline Cobalt & $<424$ \\
\hline Lanthanum & $<1060$ \\
\hline Neodymium & $<2120$ \\
\hline Samarium & $<2120$ \\
\hline Selenium & $<2120$ \\
\hline Thallium & $<4250$ \\
\hline Titanium & $<2120$ \\
\hline Total Uranium & $<10,600$ \\
\hline Vanadium & $<30$ \\
\hline
\end{tabular}


Table B2-51. Tank 241-AN-105 Analytical Results: Acetate (IC). (2 sheets)

\begin{tabular}{|c|c|c|c|c|c|}
\hline 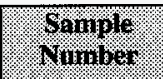 & Somplem & . & . & 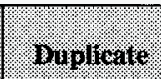 & Mrain \\
\hline \multicolumn{2}{|c|}{ Wrapos } & & 6048 & 1818 & $141 \%$ \\
\hline S96T003732 & 152: 1 & Drainable liquid & 541.12 & 478.87 & 510 \\
\hline S96T003739 & 152: 2 & Drainable liquid & 526.40 & 511.97 & 519.19 \\
\hline S96T003740 & $152: 3$ & Drainable liquid & 483.80 & 504.22 & 494.01 \\
\hline S96T003741 & 152: 4 & Drainable liquid & 511.54 & $<298.59$ & $<405.07$ \\
\hline S96T003742 & $152: 5$ & Drainable liquid & 343.94 & $<298.59$ & $<321.26$ \\
\hline S96T003743 & 152: 6 & Drainable liquid & $<298.59$ & $<298.59$ & $<298.59$ \\
\hline S96T003744 & $152: 7$ & Drainable liquid & 472.95 & 401.40 & 437.18 \\
\hline S96T003745 & 152: 8 & Drainable liquid & 314.50 & 323.23 & 318.87 \\
\hline S96T003746 & 152: 9 & Drainable liquid & 23.35 & 22.95 & 23.15 \\
\hline \$96T003747 & $152: 10$ & Drainable liquid & 374.50 & 378.87 & 376.69 \\
\hline S96T003748 & $152: 11$ & Drainable liquid & 309.78 & 286.62 & 298.20 \\
\hline S96T003749 & $152: 12$ & Drainable liquid & 291.12 & 319.01 & 305.07 \\
\hline S96T003750 & $152: 13$ & Drainable liquid & 424.01 & 421.83 & 422.92 \\
\hline S96T003751 & $152: 14$ & Drainable liquid & 493.23 & 533.09 & 513.16 \\
\hline S96T004433 & 153: 1 & Drainable liquid & $1,068.31$ & 488.73 & $778.52^{\mathrm{QC}: \mathrm{e}}$ \\
\hline S96T004308 & 153: 2 & Drainable liquid & 408.45 & 457.74 & 433.09 \\
\hline S96T004434 & 153: 3 & Drainable liquid & 318.52 & 321.12 & 319.82 \\
\hline S96T004419 & 153: 5 & Drainable liquid & 424.29 & 375.35 & 399.82 \\
\hline S96T004420 & 153: 6 & Drainable liquid & 567.46 & 669.71 & 618.59 \\
\hline S96T004421 & 153: 7 & Drainable liquid & 302.81 & 535.91 & $419.36^{\text {QC:e }}$ \\
\hline S96T003975 & 153: 8 & Drainable liquid & 166.69 & 173.23 & 169.96 \\
\hline S96T003976 & 153: 9 & Drainable liquid & 474.50 & 490.14 & 482.32 \\
\hline S96T004309 & $153: 10$ & Drainable liquid & 572.11 & 604.22 & 588.16 \\
\hline S96T003979 & $153: 11$ & Drainable liquid & 471.05 & 461.26 & 466.16 \\
\hline S96T003977 & $153: 12$ & Drainable liquid & 435.49 & 486.62 & 461.05 \\
\hline S96T003978 & $153: 13$ & Drainable liquid & 419.64 & 456.33 & 437.99 \\
\hline S96T004333 & $153: 14$ & Drainable liquid & 543.80 & 556.33 & 550.07 \\
\hline S96T003980 & $153: 21$ & Drainable liquid & 923.94 & 866.19 & 895.07 \\
\hline S96T004831 & Core 152 & Liquid composite & 451.33 & 452.11 & 451.72 \\
\hline
\end{tabular}


Table B2-51. Tank 241-AN-105 Analytical Results: Acetate (IC). (2 sheets)

\begin{tabular}{|c|c|c|c|c|c|}
\hline 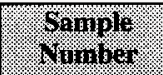 & Q & 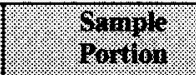 & 329.111 & 90 picate. & Mean \\
\hline \multicolumn{2}{|c|}{ 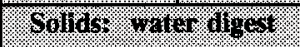 } & & 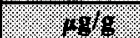 & 49 & (1) \\
\hline S96T003915 & 152: 1 & Upper half & 757.2 & $<159$ & $<458.1$ \\
\hline \$96T003907 & \multirow[t]{2}{*}{$152: 14$} & Upper half & 855.9 & 782 & 818.95 \\
\hline \$96T003829 & & Lower half & 655.4 & 764 & 709.7 \\
\hline S96T003908 & \multirow[t]{2}{*}{$152: 16$} & Upper half & $<166.5$ & 736 & $<451.25$ \\
\hline S96T003909 & & Lower half & 667.7 & 571 & 619.35 \\
\hline S96T003910 & \multirow[t]{2}{*}{$152: 18$} & Upper half & 622.6 & 599 & 610.8 \\
\hline S96T003911 & & Lower half & 784.6 & 437 & $610.8^{\mathrm{QC}: c}$ \\
\hline S96T003912 & \multirow[t]{2}{*}{$152: 20$} & Upper half & 798.5 & 450 & $624.25^{\mathrm{QC}: c}$ \\
\hline S96T003913 & & Lower half & 860.8 & 756 & 808.4 \\
\hline S96T003914 & $152: 22$ & Upper half & 655 & 645 & 650 \\
\hline S96T004339 & $153: 14$ & Lower half & 827.1 & 808 & 817.55 \\
\hline S96T004316 & \multirow[t]{2}{*}{$153: 15$} & Upper half & 557.3 & 560 & 558.65 \\
\hline S96T004319 & & Lower half & $<82.06$ & $<83.4$ & $<82.73$ \\
\hline S96T004447 & \multirow[t]{3}{*}{$153: 17$} & Upper half & 389.3 & 394 & 391.65 \\
\hline S96T004448 & & Lower half & 765.6 & 805 & 785.3 \\
\hline S96T005325 & & Lower half & 536.9 & 501 & 518.95 \\
\hline S96T004449 & \multirow[t]{2}{*}{$153: 19$} & Lower half & 914.4 & 897 & 905.7 \\
\hline S96T005326 & & Lower half & 536.4 & 533 & 534.7 \\
\hline S96T003990 & $153: 21$ & Lower half & 823.4 & 729 & 776.2 \\
\hline S96T003992 & \multirow[t]{2}{*}{$153: 22$} & Upper half & 719.6 & 591 & 655.3 \\
\hline S96T003991 & & Lower half & $<82.5$ & $<83$ & $<82.75$ \\
\hline S96T004258 & \multirow[t]{2}{*}{ Core 152} & Solid composite & 470.1 & 474 & $472.05^{\mathrm{QC:}}$ \\
\hline S96T004263 & & Solid composite & $<79.01$ & $<78.3$ & $<78.65$ \\
\hline
\end{tabular}


Table B2-52. Tank 241-AN-105 Analytical Results: Bromide (IC) ${ }^{1}$. (2 sheets)

\begin{tabular}{|c|c|c|c|c|c|}
\hline Wamplo & $\begin{array}{l}\text { Ganople } \\
\text { sacation }\end{array}$ & Somile & mesill. & Mupligite & Mean \\
\hline \multicolumn{2}{|c|}{ Fids } & & $18 \%$ & $69 \%$ & 1496 \\
\hline S96T003732 & 152: 1 & Drainable liquid & $<367.46$ & 461.97 & $<414.71$ \\
\hline S96T003739 & 152: 2 & Drainable liquid & 724.64 & 746.47 & 735.56 \\
\hline S96T003740 & 152: 3 & Drainable liquid & 864.08 & 816.90 & 840.49 \\
\hline S96T003741 & 152: 4 & Drainable liquid & 601.26 & $<457.04$ & $<529.15$ \\
\hline S96T003742 & 152: 5 & Drainable liquid & $<904.93$ & $<908.45$ & $<906.69$ \\
\hline S96T003743 & 152: 6 & Drainable liquid & $1,173.24$ & $1,169.01$ & $1,171.13$ \\
\hline S96T003744 & 152: 7 & Drainable liquid & 745.77 & 760.56 & 753.16 \\
\hline S96T003745 & 152: 8 & Drainable liquid & 544.36 & 564.08 & 554.22 \\
\hline S96T003746 & 152: 9 & Drainable liquid & 505.35 & 523.94 & 514.64 \\
\hline S96T003747 & $152: 10$ & Drainable liquid & 760.56 & 732.39 & 746.47 \\
\hline S96T003748 & $152: 11$ & Drainable liquid & 450.28 & 460.56 & 455.42 \\
\hline S96T003749 & $152: 12$ & Drainable liquid & 716.19 & 732.39 & 724.29 \\
\hline S96T003750 & $152: 13$ & Drainable liquid & 811.97 & 802.81 & 807.39 \\
\hline S96T003751 & $152: 14$ & Drainable liquid & $1,168.31$ & $1,169.01$ & $1,168.66$ \\
\hline S96T004433 & 153: 1 & Drainable liquid & $<364.50$ & $<364.78$ & $<364.64$ \\
\hline S96T004308 & 153: 2 & Drainable liquid & 594.64 & 603.52 & 599.08 \\
\hline S96T004434 & 153: 3 & Drainable liquid & 670.56 & 670.42 & 670.49 \\
\hline S96T004419 & 153: 5 & Drainable liquid & 734.50 & 760.56 & 747.53 \\
\hline S96T004420 & 153: 6 & Drainable liquid & 816.90 & 774.64 & 795.77 \\
\hline S96T004421 & 153: 7 & Drainable liquid & 723.23 & 711.26 & 717.25 \\
\hline S96T003975 & 153: 8 & Drainable liquid & 821.83 & 823.94 & 822.88 \\
\hline S96T003976 & 153:9 & Drainable liquid & 459.15 & 473.94 & 466.54 \\
\hline S96T004309 & $153: 10$ & Drainable liquid & 699.43 & 672.53 & 685.98 \\
\hline S96T003979 & $153: 11$ & Drainable liquid & 471.76 & 477.46 & 474.61 \\
\hline S96T003977 & $153: 12$ & Drainable liquid & 558.59 & 588.73 & 573.66 \\
\hline S96T003978 & $153: 13$ & Drainable liquid & 540.84 & 552.81 & 546.83 \\
\hline S96T004333 & $153: 14$ & Drainable liquid & 583.80 & 583.80 & 583.80 \\
\hline S96T003980 & $153: 21$ & Drainable liquid & $4,152.82$ & $4,190.14$ & $4,171.48$ \\
\hline S96T004039 & Core 152 & Liquid composite & $<367.46$ & $<367.60$ & $<367.53$ \\
\hline
\end{tabular}


Table B2-52. Tank 241-AN-105 Analytical Results: Bromide (IC) ${ }^{1}$. (2 sheets)

\begin{tabular}{|c|c|c|c|c|c|}
\hline Sampor & $\begin{array}{l}\text { gample } \\
\text { gomiloi }\end{array}$ & Manplo & Psoul & Monilicale. & 101n \\
\hline \multicolumn{2}{|c|}{ Soldis r w ror frost } & & $6 \%$ : & 48 & $18 \%$ \\
\hline S96T003915 & 152: 1 & Upper half & $<514.4$ & $<512$ & $<513.2$ \\
\hline S96T003907 & \multirow[t]{2}{*}{$152: 14$} & Upper half & 657.8 & 724 & 690.9 \\
\hline S96T003829 & & Lower half & $<274.8$ & 340 & $<307.4$ \\
\hline S96T003908 & \multirow[t]{2}{*}{$152: 16$} & Upper half & $<281.4$ & $<270$ & $<275.7$ \\
\hline S96T003909 & & Lower half & $<1,030$ & $<1,030$ & $<1,030$ \\
\hline S96T003910 & \multirow[t]{2}{*}{$152: 18$} & Upper half & $<1,052$ & $<1,020$ & $<1,036$ \\
\hline S96T003911 & & Lower half & $<1,020$ & $<1,000$ & $<1,010$ \\
\hline S96T003912 & \multirow[t]{2}{*}{$152: 20$} & Upper half & $<1,045$ & $<1,070$ & $<1,057.5$ \\
\hline S96T003913 & & Lower half & $<527$ & $<572$ & $<549.5$ \\
\hline S96T003914 & $152: 22$ & Upper half & $<578.5$ & $<539$ & $<558.75$ \\
\hline S96T004339 & $153: 14$ & Lower half & 506.2 & $<503$ & $<504.6$ \\
\hline S96T004316 & \multirow[t]{2}{*}{$153: 15$} & Upper half & 758.2 & 730 & 744.1 \\
\hline S96T004319 & & Lower half & 560.8 & 576 & 568.4 \\
\hline S96T004447 & \multirow[t]{2}{*}{$153: 17$} & Upper half & 515.7 & 537 & 526.35 \\
\hline S96T005325 & & Lower half & $<484$ & $<478$ & $<481$ \\
\hline S96T005326 & $153: 19$ & Lower half & $<485.6$ & $<491$ & $<488.3$ \\
\hline S96T003990 & 153:21 & Lower half & 575.4 & 625 & 600.2 \\
\hline S96T003992 & \multirow[t]{2}{*}{$153: 22$} & Upper half & $<616$ & $<630$ & $<623$ \\
\hline S96T003991 & & Lower half & 835 & 793 & 814 \\
\hline S96T004258 & Core 152 & Solid composite & $<558.2$ & $<538$ & $<548.1$ \\
\hline S96T004263 & & Solid composite & $<493.8$ & $<489$ & $<491.4$ \\
\hline
\end{tabular}

Note:

\footnotetext{
${ }^{1}$ Bromide is in hydrostatic head fluid.
} 
Table B2-53. Tank 241-AN-105 Analytical Results: Chloride (IC). (2 sheets)

\begin{tabular}{|c|c|c|c|c|c|}
\hline hamplo & Ganple & $\frac{6}{601 m p 10}$ & Resisit & Dublilerite & Mean \\
\hline \multicolumn{2}{|c|}{ Wogras } & & $18 \mathrm{~g}$ & 498 & $\% 5$ \\
\hline S96T003732 & 152: 1 & Drainable liquid & $6,946.48$ & $6,605.63$ & $6,776.06$ \\
\hline S96T003739 & 152: 2 & Drainable liquid & $6,510.56$ & $6,542.25$ & $6,526.41$ \\
\hline S96T003740 & 152: 3 & Drainable liquid & $7,042.25$ & $7,183.1$ & $7,112.68$ \\
\hline S96T003741 & 152: 4 & Drainable liquid & $6,986.62$ & $7,183.1$ & $7,084.86$ \\
\hline S96T003742 & 152: 5 & Drainable liquid & $2,021.83$ & $2,042.25$ & $2,032.04$ \\
\hline S96T003743 & 152: 6 & Drainable liquid & $17,852.1$ & 18,169 & $18,010.6^{\mathrm{QC:d}}$ \\
\hline S96T003744 & 152: 7 & Drainable liquid & $7,098.59$ & $7,042.25$ & $7,070.42$ \\
\hline S96T003745 & 152: 8 & Drainable liquid & $6,850.7$ & $6,845.07$ & $6,847.89$ \\
\hline S96T003746 & 152: 9 & Drainable liquid & $6,764.79$ & $6,626.76$ & $6,695.77$ \\
\hline S96T003747 & $152: 10$ & Drainable liquid & $6,801.41$ & $6,697.18$ & $6,749.3$ \\
\hline S96T003748 & $152: 11$ & Drainable liquid & $7,084.51$ & $7,112.68$ & $7,098.59$ \\
\hline S96T003749 & $152: 12$ & Drainable liquid & $6,921.13$ & $6,964.79$ & $6,942.96$ \\
\hline S96T003750 & $152: 13$ & Drainable liquid & $7,084.51$ & $6,950.7$ & $7,017.61$ \\
\hline S96T003751 & $152: 14$ & Drainable liquid & $7,471.83$ & $7,394.37$ & $7,433.1^{\mathrm{QC}: \mathrm{c}}$ \\
\hline S96T004433 & 153: 1 & Drainable liquid & $7,492.96$ & $7,323.94$ & $7,408.45$ \\
\hline S96T004308 & 153: 2 & Drainable liquid & $7,154.93$ & $7,042.25$ & $7,098.59$ \\
\hline S96T004434 & 153: 3 & Drainable liquid & $7,147.89$ & $6,964.79$ & $7,056.34$ \\
\hline S96T004419 & 153: 5 & Drainable liquid & $7,246.48$ & $7,042.25$ & $7,144.37$ \\
\hline S96T004420 & 153: 6 & Drainable liquid & $7,232.39$ & $7,394.37$ & $7,313.38$ \\
\hline S96T004421 & 153: 7 & Drainable liquid & $7,647.89$ & $7,746.48$ & $7,697.18$ \\
\hline S96T003975 & 153: 8 & Drainable liquid & $6,748.59$ & $6,711.27$ & $6,729.93$ \\
\hline S96T003976 & 153: 9 & Drainable liquid & $6,652.82$ & $6,774.65$ & $6,713.73$ \\
\hline S96T004309 & $153: 10$ & Drainable liquid & $6,561.27$ & $6,309.86$ & $6,435.56$ \\
\hline S96T003979 & $153: 11$ & Drainable liquid & $6,786.62$ & $6,746.48$ & $6,766.55$ \\
\hline S96T003977 & $153: 12$ & Drainable liquid & $6,952.11$ & $7,183.1$ & $7,067.61$ \\
\hline S96T003978 & 153:13 & Drainable liquid & $6,658.45$ & $6,669.01$ & $6,663.73$ \\
\hline S96T004333 & $153: 14$ & Drainable liquid & $7,176.06$ & $7,112.68$ & $7,144.37^{\mathrm{QC:d}}$ \\
\hline S96T003980 & $153: 21$ & Drainable liquid & $6,559.15$ & $6,436.62$ & $6,497.89$ \\
\hline S96T004039 & Core 152 & Liquid composite & $7,147.89$ & $7,183.1$ & $7,165.49$ \\
\hline
\end{tabular}


Table B2-53. Tank 241-AN-105 Analytical Results: Chloride (IC). (2 sheets)

\begin{tabular}{|c|c|c|c|c|c|}
\hline Sumplo & Ganation & 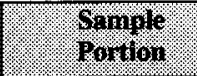 & 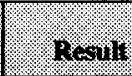 & Buplicale & Herr. \\
\hline \multicolumn{2}{|c|}{ 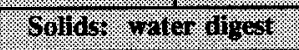 } & & 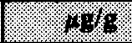 & 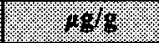 & 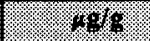 \\
\hline S96T003915 & 152: 1 & Upper half & 5,620 & 5,350 & 5,485 \\
\hline S96T003907 & \multirow[t]{2}{*}{$152: 14$} & Upper half & 5,550 & 5,710 & 5,630 \\
\hline S96T003829 & & Lower half & 3,764 & 4,050 & 3,907 \\
\hline S96T003908 & \multirow[t]{2}{*}{$152: 16$} & Upper half & 4,155 & 4,080 & $4,117.5$ \\
\hline S96T003909 & & Lower half & 5,891 & 5,800 & $5,845.5$ \\
\hline S96T003910 & \multirow[t]{2}{*}{$152: 18$} & Upper half & 5,317 & 5,130 & $5,223.5$ \\
\hline S96T003911 & & Lower half & 5,181 & 4,980 & $5,080.5$ \\
\hline S96T003912 & \multirow[t]{2}{*}{$152: 20$} & Upper half & 5,236 & 5,180 & 5,208 \\
\hline \$96T003913 & & Lower half & 5,280 & 4,630 & $4,955^{\mathrm{QC:c}}$ \\
\hline S96T003914 & $152: 22$ & Upper half & 4,439 & 4,430 & $4,434.5$ \\
\hline S96T004339 & $153: 14$ & Lower half & 7,253 & 6,050 & $6,651.5^{\mathrm{QC:c}}$ \\
\hline S96T004316 & \multirow[t]{2}{*}{$153: 15$} & Upper half & 5,846 & 5,530 & 5,688 \\
\hline S96T004319 & & Lower half & 4,538 & 5,490 & $5,014^{\mathrm{QC}: \mathrm{d}}$ \\
\hline S96T004447 & \multirow[t]{2}{*}{$153: 17$} & Upper half & 4,924 & 6,090 & $5,507^{\mathrm{QC:c}}$ \\
\hline S96T005325 & & Lower half & 6,715 & 6,260 & $6,487.5$ \\
\hline \$96T005326 & 153:19 & Lower half & 6,529 & 6,170 & $6,349.5$ \\
\hline S96T003990 & $153: 21$ & Lower half & 3,943 & 5,280 & $4,611.5^{\mathrm{QC}: \mathrm{d}, \mathrm{c}}$ \\
\hline S96T003992 & \multirow[t]{2}{*}{$153: 22$} & Upper half & 4,904 & 4,780 & 4,842 \\
\hline S96T003991 & & Lower half & 3,753 & 3,480 & $3,616.5$ \\
\hline S96T004258 & \multirow[t]{2}{*}{ Core 152} & Solid composite & 5,851 & 5,910 & $5,880.5$ \\
\hline S96T004263 & & Solid composite & 4,345 & 3,800 & $4,072.5$ \\
\hline
\end{tabular}


Table B2-54. Tank 241-AN-105 Analytical Results: Fluoride (IC). (2 sheets)

\begin{tabular}{|c|c|c|c|c|c|}
\hline Whinge & ogniple: & 6onmle & nesili: & Byplicato. & 1) \\
\hline \multicolumn{2}{|c|}{ Yyoriars } & & 49 & Wor & $8 \mathrm{~s}$ \\
\hline S96T003732 & 152: 1 & Drainable liquid & $<37.90$ & $<37.88$ & $<37.89$ \\
\hline S96T003739 & 152: 2 & Drainable liquid & 348.45 & 328.16 & 338.31 \\
\hline S96T003740 & 152: 3 & Drainable liquid & 356.90 & 354.22 & $355.56^{\mathrm{QC}: c}$ \\
\hline S96T003741 & 152: 4 & Drainable liquid & 358.09 & 335.91 & 347.00 \\
\hline \$96T003742 & 152: 5 & Drainable liquid & $<93.38$ & $<93.66$ & $<93.52$ \\
\hline S96T003743 & 152: 6 & Drainable liquid & $<93.38$ & $<93.66$ & $<93.52^{\mathrm{QC} ; \mathrm{d}}$ \\
\hline S96T003744 & $152: 7$ & Drainable liquid & 308.16 & 302.11 & $305.14^{\mathrm{QC}: \mathrm{c}}$ \\
\hline S96T003745 & 152: 8 & Drainable liquid & $<37.90$ & $<37.88$ & $<37.89^{\mathrm{QC:d}}$ \\
\hline S96T003746 & 152:9 & Drainable liquid & $<37.90$ & $<37.88$ & $<37.89$ \\
\hline \$96T003747 & $152: 10$ & Drainable liquid & 699.71 & $<37.88$ & $<368.80$ \\
\hline S96T003748 & $152: 11$ & Drainable liquid & 314.78 & 329.57 & 322.18 \\
\hline S96r003749 & $152: 12$ & Drainable liquid & 329.64 & 325.35 & 327.5 \\
\hline S96T003750 & $152: 13$ & Drainable liquid & $<37.90$ & $<37.88$ & $<37.89$ \\
\hline S96T003751 & $152: 14$ & Drainable liquid & $<37.90$ & $<37.88$ & $<37.89^{\mathrm{QC}: \mathrm{c}}$ \\
\hline \$96T004433 & 153: 1 & Drainable liquid & 324.577 & 321.12 & $322.85^{Q C: e}$ \\
\hline S96T004308 & 153: 2 & Drainable liquid & 316.549 & 303.52 & 310.03 \\
\hline S96T004434 & 153: 3 & Drainable liquid & 291.901 & 304.93 & 298.41 \\
\hline S96T004419 & 153: 5 & Drainable liquid & 308.944 & 314.78 & 311.86 \\
\hline S96T004420 & 153: 6 & Drainable liquid & 279.718 & 292.25 & $285.98^{\mathrm{QC}: c}$ \\
\hline S96T004421 & 153: 7 & Drainable liquid & 294.93 & 286.62 & 290.77 \\
\hline S96T003975 & 153: 8 & Drainable liquid & 310.07 & 290.84 & 300.45 \\
\hline S96T003976 & 153: 9 & Drainable liquid & 323.87 & 302.11 & 312.99 \\
\hline S96T004309 & $153: 10$ & Drainable liquid & 304.93 & 287.32 & 296.12 \\
\hline S96T003979 & $153: 11$ & Drainable liquid & $<37.90$ & $<37.88$ & $<37.89^{\mathrm{QC}: \mathrm{d}}$ \\
\hline S96T003977 & $153: 12$ & Drainable liquid & $<37.90$ & $<37.88$ & $<37.89^{\mathrm{QC}: \mathrm{d}}$ \\
\hline S96T003978 & $153: 13$ & Drainable liquid & $<37.90$ & $<37.88$ & $<37.89$ \\
\hline S96T004333 & $153: 14$ & Drainable liquid & 318.66 & 309.15 & $313.90^{\mathrm{QC:c}}$ \\
\hline S96T003980 & $153: 21$ & Drainable liquid & $<37.90$ & $<37.88$ & $<37.89$ \\
\hline S96T004039 & Core 152 & Liquid composite & $<37.90$ & $<37.88$ & $<37.89^{\text {QC:d }}$ \\
\hline
\end{tabular}


Table B2-54. Tank 241-AN-105 Analytical Results: Fluoride (IC). (2 sheets)

\begin{tabular}{|c|c|c|c|c|c|}
\hline Wonpola & Graple & \%amp & $(x+1) 1$ & Wuplisate & Mori \\
\hline \multicolumn{2}{|c|}{ Sollass roter bigert } & & 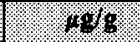 & $18 \%$ & 48 \\
\hline S96T003915 & 152: 1 & Upper half & 269.1 & 527 & $398.05^{\text {QC:e }}$ \\
\hline S96T003907 & \multirow[t]{2}{*}{$152: 14$} & Upper half & 777.2 & 239 & $508.1^{\mathrm{QC}: \mathrm{e}}$ \\
\hline S96T003829 & & Lower half & 411.8 & 455 & 433.4 \\
\hline S96T003908 & \multirow[t]{2}{*}{$152: 16$} & Upper half & 708.3 & 728 & 718.15 \\
\hline S96T003909 & & Lower half & 1,778 & 1,430 & $1,604^{\mathrm{QC} \cdot \mathrm{e}}$ \\
\hline S96T003910 & \multirow[t]{2}{*}{$152: 18$} & Upper half & 1,841 & 1,840 & $1,840.5$ \\
\hline S96T003911 & & Lower half & 2,654 & 2,640 & 2,647 \\
\hline S96T003912 & \multirow[t]{2}{*}{$152: 20$} & Upper half & 2,800 & 2,510 & 2,655 \\
\hline S96T003913 & & Lower half & 1,712 & 1,890 & $1,801^{\mathrm{QC}: \mathrm{c}}$ \\
\hline S96T003914 & $152: 22$ & Upper half & 1,214 & 1,120 & 1,167 \\
\hline S96T004339 & $153: 14$ & Lower half & 1,411 & 1,150 & $1,280.5^{\mathrm{QC}: \mathrm{e}}$ \\
\hline$\$ 96 \mathrm{~T} 004316$ & \multirow[t]{2}{*}{$153: 15$} & Upper half & 1,126 & 1,170 & 1,148 \\
\hline S96T004319 & & Lower half & 1,093 & 1,090 & $1,091.5$ \\
\hline S96T004447 & \multirow[t]{2}{*}{$153: 17$} & Upper half & 1,206 & 1,360 & 1,283 \\
\hline S96T005325 & & Lower half & 1,221 & 1,260 & $1,240.5^{\mathrm{QC}: \mathrm{d}}$ \\
\hline S96T005326 & 153:19 & Lower half & 1,093 & 1,320 & $1,206.5$ \\
\hline S96T003990 & $153: 21$ & Lower half & 1,150 & 1,380 & $1,265^{\mathrm{QC:c}}$ \\
\hline S96T003992 & \multirow[t]{2}{*}{$153: 22$} & Upper half & 2,680 & 2,500 & 2,590 \\
\hline S96T003991 & & Lower half & 10,140 & 8,650 & 9,395 \\
\hline S96T004258 & \multirow[t]{2}{*}{ Core 152} & Solid composite & 1,353 & 929 & $1,141^{\mathrm{QC}: \mathfrak{c}, \mathrm{e}}$ \\
\hline S96T004263 & & Solid composite & 5,180 & 4,870 & 5,025 \\
\hline
\end{tabular}


Table B2-55. Tank 241-AN-105 Analytical Results: Formate (IC). (2 sheets)

\begin{tabular}{|c|c|c|c|c|c|}
\hline Samplo & Wonple & 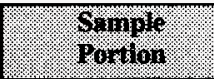 & 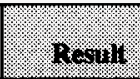 & 9upligate & Wern \\
\hline \multicolumn{2}{|c|}{ Wrolso } & & 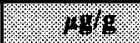 & 498 & $\%$ \\
\hline S96T003732 & 152: 1 & Drainable liquid & $1,226.76$ & $1,169.01$ & $1,197.89$ \\
\hline S96T003739 & 152: 2 & Drainable liquid & $1,164.79$ & $1,204.23$ & $1,184.51$ \\
\hline S96T003740 & $152: 3$ & Drainable liquid & $1,085.21$ & $1,077.46$ & $1,081.34$ \\
\hline S96T003741 & 152: 4 & Drainable liquid & $1,080.99$ & $1,084.51$ & $1,082.75$ \\
\hline S96T003742 & 152: 5 & Drainable liquid & $1,084.51$ & $1,112.68$ & $1,098.59$ \\
\hline S96T003743 & 152: 6 & Drainable liquid & $1,220.42$ & $1,176.06$ & $1,198.24$ \\
\hline S96T003744 & 152: 7 & Drainable liquid & $1,129.58$ & $1,021.13$ & $1,075.35$ \\
\hline \$96T003745 & 152: 8 & Drainable liquid & $1,044.37$ & $1,042.25$ & $1,043.31$ \\
\hline S96T003746 & 152:9 & Drainable liquid & 36.831 & 36.338 & 36.5845 \\
\hline S96T003747 & $152: 10$ & Drainable liquid & 961.268 & 964.789 & 963.028 \\
\hline S96T003748 & $152: 11$ & Drainable liquid & $1,054.93$ & $1,077.46$ & $1,066.2$ \\
\hline S96T003749 & $152: 12$ & Drainable liquid & 1,200 & $1,232.39$ & $1,216.2$ \\
\hline S96T003750 & $152: 13$ & Drainable liquid & $1,133.8$ & $1,133.8$ & $1,133.8$ \\
\hline S96T003751 & $152: 14$ & Drainable liquid & $1,198.59$ & $1,232.39$ & $1,215.49$ \\
\hline S96T004433 & 153: 1 & Drainable liquid & $1,296.48$ & $1,309.86$ & $1,303.17$ \\
\hline S96T004308 & $153: 2$ & Drainable liquid & $1,191.55$ & $1,274.65$ & $1,233.1$ \\
\hline S96T004434 & 153: 3 & Drainable liquid & 735.211 & 718.31 & 726.761 \\
\hline S96T004419 & 153: 5 & Drainable liquid & $1,147.89$ & $1,098.59$ & $1,123.24$ \\
\hline S96T004420 & 153: 6 & Drainable liquid & $1,207.04$ & $1,436.62$ & $1,321.83$ \\
\hline S96T004421 & 153: 7 & Drainable liquid & $1,332.39$ & $1,267.61$ & 1,300 \\
\hline S96T003975 & 153: 8 & Drainable liquid & 417.817 & 379.577 & 398.697 \\
\hline \$96T003976 & 153: 9 & Drainable liquid & $1,239.44$ & $1,218.31$ & $1,228.87$ \\
\hline S96T004309 & $153: 10$ & Drainable liquid & $1,436.62$ & $1,394.37$ & $1,415.49$ \\
\hline \$96T003979 & $153: 11$ & Drainable liquid & $1,193.66$ & $1,246.48$ & $1,220.07$ \\
\hline S96T003977 & $153: 12$ & Drainable liquid & $1,307.04$ & $1,246.48$ & $1,276.76$ \\
\hline \$96T003978 & $153: 13$ & Drainable liquid & $1,116.9$ & $1,183.1$ & 1,150 \\
\hline S96T004333 & 153:14 & Drainable liquid & $1,367.61$ & $1,359.15$ & $1,363.38$ \\
\hline \$96T003980 & $153: 21$ & Drainable liquid & $1,051.41$ & 964.789 & $1,008.1$ \\
\hline S96T004831 & Core 152 & Liquid composite & $1,140.85$ & $1,140.85$ & $1,140.85$ \\
\hline
\end{tabular}


Table B2-55. Tank 241-AN-105 Analytical Results: Formate (IC). (2 sheets)

\begin{tabular}{|c|c|c|c|c|c|}
\hline Wamplo & Gaviple & (3) & Pesintin & Buplicate & Yeail \\
\hline \multicolumn{2}{|c|}{ Sollow w wer ilgest } & & 1018 & 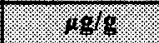 & 898 \\
\hline S96T003915 & 152: 1 & Upper half & 1,355 & 1,240 & $1,297.5$ \\
\hline S96T003907 & \multirow[t]{2}{*}{$152: 14$} & Upper half & 1,356 & 1,250 & 1,303 \\
\hline S96T003829 & & Lower half & 1,021 & 1,170 & $1,095.5$ \\
\hline S96T003908 & \multirow[t]{2}{*}{$152: 16$} & Upper half & 1,290 & 1,250 & 1,270 \\
\hline S96T003909 & & Lower half & 1,213 & 1,110 & $1,161.5$ \\
\hline S96T003910 & \multirow[t]{2}{*}{$152: 18$} & Upper half & 1,179 & 1,120 & $1,149.5$ \\
\hline S96T003911 & & Lower half & 772.8 & 888 & 830.4 \\
\hline S96T003912 & \multirow[t]{2}{*}{$152: 20$} & Upper half & 889.3 & 968 & 928.65 \\
\hline S96T003913 & & Lower half & 1,016 & 988 & 1,002 \\
\hline S96T003914 & $152: 22$ & Upper half & 1,099 & 1,020 & $1,059.5$ \\
\hline S96T004339 & 153:14 & Lower half & 1,172 & 1,180 & 1,176 \\
\hline S96T004316 & \multirow[t]{2}{*}{$153: 15$} & Upper half & 1,194 & 1,140 & 1,167 \\
\hline S96T004319 & & Lower half & 664.5 & 715 & $689.75^{\mathrm{QC} c}$ \\
\hline S96T004447 & \multirow[t]{3}{*}{$153: 17$} & Upper half & 801.5 & 826 & 813.75 \\
\hline S96T004448 & & Lower half & 1,018 & 1,130 & 1,074 \\
\hline S96T005325 & & Lower half & 1,186 & 1,060 & 1,123 \\
\hline \$96T004449 & \multirow[t]{2}{*}{$153: 19$} & Lower half & 1,114 & 1,090 & 1,102 \\
\hline S96T005326 & & Lower half & 1,136 & 1,140 & 1,138 \\
\hline S96T003990 & $153: 21$ & Lower half & 1,265 & 1,170 & $1,217.5$ \\
\hline S96T003992 & \multirow[t]{2}{*}{$153: 22$} & Upper half & 1,071 & 1,030 & $1,050.5$ \\
\hline S96T003991 & & Lower half & 985.1 & 894 & 939.55 \\
\hline S96T004258 & \multirow[t]{2}{*}{ Core 152} & Solid composite & 1,217 & 1,230 & $1,223.5$ \\
\hline S96'T004263 & & Solid composite & 1,122 & 1,110 & 1,116 \\
\hline
\end{tabular}


Table B2-56. Tank 241-AN-105 Analytical Results: Nitrate (IC). (2 sheets)

\begin{tabular}{|c|c|c|c|c|c|}
\hline Mamplo & oample & 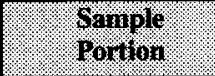 & $10+11$ & Bifolicals & Meario \\
\hline \multicolumn{3}{|c|}{ \%6IIt } & $1 \% \%$ & 18189 & 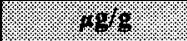 \\
\hline S96T003732 & 152: 1 & Drainable liquid & $1.120 \mathrm{E}+05$ & $1.113 \mathrm{E}+05$ & $1.116 \mathrm{E}+05$ \\
\hline S96T003739 & 152: 2 & Drainable liquid & $1.128 \mathrm{E}+05$ & $1.134 \mathrm{E}+05$ & $1.131 \mathrm{E}+05$ \\
\hline S96T003740 & $152: 3$ & Drainable liquid & $1.211 \mathrm{E}+05$ & $1.225 \mathrm{E}+05$ & $1.218 \mathrm{E}+05$ \\
\hline S96T003741 & 152: 4 & Drainable liquid & $1.151 \mathrm{E}+05$ & $1.162 \mathrm{E}+05$ & $1.157 \mathrm{E}+05$ \\
\hline S96T003742 & 152: 5 & Drainable liquid & $26,845.1$ & $26,126.8$ & $26,485.9$ \\
\hline S96T003743 & 152: 6 & Drainable liquid & $2.952 \mathrm{E}+05$ & $2.951 \mathrm{E}+05$ & $2.951 \mathrm{E}+05^{\mathrm{QC} d \mathrm{~d}}$ \\
\hline S96T003744 & 152: 7 & Drainable liquid & $1.189 \mathrm{E}+05$ & $1.190 \mathrm{E}+05$ & $1.189 \mathrm{E}+05$ \\
\hline S96T003745 & 152: 8 & Drainable liquid & $1.146 \mathrm{E}+05$ & $1.134 \mathrm{E}+05$ & $1.140 \mathrm{E}+05$ \\
\hline S96T003746 & 152: 9 & Drainable liquid & $92,112.7$ & $91,549.3$ & 91,831 \\
\hline S96T003747 & $152: 10$ & Drainable liquid & $1.111 \mathrm{E}+05$ & $1.099 \mathrm{E}+05$ & $1.105 \mathrm{E}+05$ \\
\hline \$96T003748 & $152: 11$ & Drainable liquid & $1.154 \mathrm{E}+05$ & $1.155 \mathrm{E}+05$ & $1.155 \mathrm{E}+05$ \\
\hline S96T003749 & $152: 12$ & Drainable liquid & $1.072 \mathrm{E}+05$ & $1.092 \mathrm{E}+05$ & $1.082 \mathrm{E}+05$ \\
\hline S96T003750 & $152: 13$ & Drainable liquid & $1.168 \mathrm{E}+05$ & $1.169 \mathrm{E}+05$ & $1.168 \mathrm{E}+05$ \\
\hline S96T003751 & $152: 14$ & Drainable liquid & $1.123 \mathrm{E}+05$ & $1.120 \mathrm{E}+05$ & $1.121 \mathrm{E}+05$ \\
\hline S96T004433 & 153: 1 & Drainable liquid & $1.166 \mathrm{E}+05$ & $1.155 \mathrm{E}+05$ & $1.160 \mathrm{E}+05$ \\
\hline S96T004308 & 153: 2 & Drainable liquid & $1.142 \mathrm{E}+05$ & $1.141 E+05$ & $1.141 \mathrm{E}+05$ \\
\hline S96T004434 & $153: 3$ & Drainable liquid & $1.131 \mathrm{E}+05$ & $1.134 \mathrm{E}+05$ & $1.132 \mathrm{E}+05$ \\
\hline S96T004419 & 153: 5 & Drainable liquid & $1.194 \mathrm{E}+05$ & $1.197 \mathrm{E}+05$ & $1.195 \mathrm{E}+05$ \\
\hline S96T004420 & 153: 6 & Drainable liquid & $1.116 \mathrm{E}+05$ & $1.113 \mathrm{E}+05$ & $1.114 \mathrm{E}+05$ \\
\hline S96T004421 & 153: 7 & Drainable liquid & $1.172 \mathrm{E}+05$ & $1.183 \mathrm{E}+05$ & $1.178 \mathrm{E}+05$ \\
\hline \$96T003975 & 153: 8 & Drainable liquid & $1.125 \mathrm{E}+05$ & $1.120 \mathrm{E}+05$ & $1.122 \mathrm{E}+05$ \\
\hline S96T003976 & 153: 9 & Drainable liquid & $1.092 \mathrm{E}+05$ & $1.078 \mathrm{E}+05$ & $1.085 \mathrm{E}+05$ \\
\hline S96T004309 & $153: 10$ & Drainable liquid & $1.088 \mathrm{E}+05$ & $1.049 E+05$ & $1.069 E+05$ \\
\hline S96T003979 & $153: 11$ & Drainable liquid & $1.080 \mathrm{E}+05$ & $1.078 \mathrm{E}+05$ & $1.079 \mathrm{E}+05$ \\
\hline S96T003977 & $153: 12$ & Drainable liquid & $1.186 \mathrm{E}+05$ & $1.211 \mathrm{E}+05$ & $1.199 \mathrm{E}+05$ \\
\hline S96T003978 & $153: 13$ & Drainable liquid & $1.125 \mathrm{E}+05$ & $1.127 \mathrm{E}+05$ & $1.126 \mathrm{E}+05$ \\
\hline S96T004333 & $153: 14$ & Drainable liquid & $1.217 \mathrm{E}+05$ & $1.218 \mathrm{E}+05$ & $1.218 \mathrm{E}+05$ \\
\hline \$96T003980 & $153: 21$ & Drainable liquid & $1.227 \mathrm{E}+05$ & $1.218 \mathrm{E}+05$ & $1.223 E+05$ \\
\hline \$96T004039 & Core 152 & Liquid composite & $1.182 \mathrm{E}+05$ & $1.176 \mathrm{E}+05$ & $1.179 \mathrm{E}+05$ \\
\hline
\end{tabular}


Table B2-56. Tank 241-AN-105 Analytical Results: Nitrate (IC). (2 sheets)

\begin{tabular}{|c|c|c|c|c|c|}
\hline 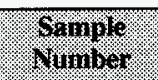 & Grampils & Sornove & Resill & Buplicate & (rean \\
\hline \multicolumn{2}{|c|}{ Wollast later digat. } & & $18 \%$ & $18 \%$ & $48 / 8$ \\
\hline S96T003915 & 152: 1 & Upper half & $1.489 \mathrm{E}+05$ & $1.580 \mathrm{E}+05$ & $1.535 \mathrm{E}+05$ \\
\hline S96T003907 & \multirow[t]{2}{*}{$152: 14$} & Upper half & $1.059 \mathrm{E}+05$ & $1.060 \mathrm{E}+05$ & $1.060 \mathrm{E}+05$ \\
\hline S96T003829 & & Lower half & 60,510 & 65,600 & 63,055 \\
\hline S96T003908 & \multirow[t]{2}{*}{$152: 16$} & Upper half & 69,420 & 80,700 & 75,060 \\
\hline S96T003909 & & Lower half & 95,370 & 96,900 & 96,135 \\
\hline S96T003910 & \multirow[t]{2}{*}{$152: 18$} & Upper half & $1.121 \mathrm{E}+05$ & $1.120 \mathrm{E}+05$ & $1.121 \mathrm{E}+05$ \\
\hline S96T003911 & & Lower half & $1.181 \mathrm{E}+05$ & $1.140 E+05$ & $1.161 \mathrm{E}+05$ \\
\hline S96T003912 & \multirow[t]{2}{*}{$152: 20$} & Upper half & 90,680 & 99,200 & 94,940 \\
\hline S96T003913 & & Lower half & $1.553 \mathrm{E}+05$ & $1.560 \mathrm{E}+05$ & $1.557 \mathrm{E}+05^{\mathrm{QC}: c}$ \\
\hline S96T003914 & $152: 22$ & Upper half & $1.651 \mathrm{E}+05$ & $1.690 \mathrm{E}+05$ & $1.671 \mathrm{E}+05$ \\
\hline S96T004339 & $153: 14$ & Lower half & $1.177 \mathrm{E}+05$ & $1.020 \mathrm{E}+05$ & $1.099 \mathrm{E}+05^{\mathrm{QC}: c}$ \\
\hline \$96T004316 & \multirow[t]{2}{*}{$153: 15$} & Upper half & $1.022 \mathrm{E}+05$ & $1.030 \mathrm{E}+05$ & $1.026 \mathrm{E}+05$ \\
\hline S96T004319 & & Lower half & 94,440 & 99,300 & $96,870^{\mathrm{QC}: \mathrm{d}}$ \\
\hline S96T004447 & \multirow[t]{2}{*}{$153: 17$} & Upper half & 77,020 & & $88,510^{\mathrm{QC:d,e}}$ \\
\hline S96T005325 & & Lower half & $1.048 \mathrm{E}+05$ & 97,700 & $1.013 \mathrm{E}+05$ \\
\hline S96T005326 & $153: 19$ & Lower half & $1.140 \mathrm{E}+05$ & $1.270 \mathrm{E}+05$ & $1.205 \mathrm{E}+05$ \\
\hline S96T003990 & $153: 21$ & Lower half & $1.162 \mathrm{E}+05$ & $1.860 E+05$ & $1.511 \mathrm{E}+05^{\mathrm{QCd}, \mathrm{e}}$ \\
\hline S96T003992 & \multirow[t]{2}{*}{$153: 22$} & Upper half & $1.585 \mathrm{E}+05$ & $1.690 \mathrm{E}+05$ & $1.638 \mathrm{E}+05$ \\
\hline \$96T003991 & & Lower half & 88,760 & 75,600 & 82,180 \\
\hline S96T004258 & \multirow[t]{2}{*}{ Core 152} & Solid composite & $1.169 \mathrm{E}+05$ & $1.090 \mathrm{E}+05$ & $1.130 \mathrm{E}+05$ \\
\hline S96T004263 & & Solid composite & $1.749 \mathrm{E}+05$ & $1.470 \mathrm{E}+05$ & $1.610 \mathrm{E}+05$ \\
\hline
\end{tabular}


Table B2-57. Tank 241-AN-105 Analytical Results: Nitrite (IC). (2 sheets)

\begin{tabular}{|c|c|c|c|c|c|}
\hline Q & Wample & 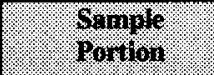 & $\mathrm{R}=\mathbf{1 1 1}$ & Bupliante & Kerin \\
\hline \multicolumn{2}{|c|}{ Woris: } & & 1018 & 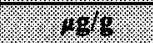 & 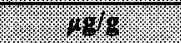 \\
\hline S96T003732 & 152: 1 & Drainable liquid & $90,422.5$ & $88,028.2$ & $89,225.4$ \\
\hline S96T003739 & 152: 2 & Drainable liquid & 84,507 & $85,211.3$ & $84,859.2$ \\
\hline S96T003740 & 152: 3 & Drainable liquid & $91,126.8$ & $90,140.8$ & $90,633.8$ \\
\hline S96T003741 & 152: 4 & Drainable liquid & $88,450.7$ & $89,436.6$ & $88,943.7$ \\
\hline S96T003742 & 152: 5 & Drainable liquid & $22,647.9$ & $21,760.6$ & $22,204.2$ \\
\hline S96T003743 & 152: 6 & Drainable liquid & $2.317 \mathrm{E}+05$ & $2.317 \mathrm{E}+05$ & $2.317 \mathrm{E}+05^{\mathrm{QC:d}}$ \\
\hline S96T003744 & 152: 7 & Drainable liquid & $84,929.6$ & $85,915.5$ & $85,422.5$ \\
\hline S96T003745 & $152: 8$ & Drainable liquid & $89,084.5$ & $88,028.2$ & $88,556.3$ \\
\hline S96T003746 & 152: 9 & Drainable liquid & $72,676.1$ & $72,535.2$ & $72,605.6$ \\
\hline S96T003747 & $152: 10$ & Drainable liquid & $86,901.4$ & $86,619.7$ & $86,760.6$ \\
\hline S96T003748 & $152: 11$ & Drainable liquid & 88,662 & $88,028.2$ & $88,345.1$ \\
\hline S96T003749 & $152: 12$ & Drainable liquid & 85,493 & $88,028.2$ & $86,760.6$ \\
\hline S96T003750 & $152: 13$ & Drainable liquid & $88,943.7$ & $88,732.4$ & 88,838 \\
\hline S96T003751 & $152: 14$ & Drainable liquid & $84,014.1$ & 84,507 & $84,260.6$ \\
\hline S96T004433 & 153: 1 & Drainable liquid & $90,563.4$ & $89,436.6$ & 90,000 \\
\hline S96T004308 & 153: 2 & Drainable liquid & $87,957.7$ & $86,619.7$ & $87,288.7$ \\
\hline S96T004434 & 153: 3 & Drainable liquid & $86,549.3$ & $85,915.5$ & $86,232.4$ \\
\hline S96T004419 & 153: 5 & Drainable liquid & $87,464.8$ & $87,323.9$ & $87,394.4$ \\
\hline S96T004420 & 153: 6 & Drainable liquid & $81,760.6$ & $81,690.1$ & $81,725.4$ \\
\hline S96T004421 & 153: 7 & Drainable liquid & $84,154.9$ & $85,211.3$ & $84,683.1$ \\
\hline S96T003975 & 153: 8 & Drainable liquid & $85,140.8$ & $85,211.3$ & $85,176.1$ \\
\hline S96T003976 & 153: 9 & Drainable liquid & $82,676.1$ & $83,098.6$ & $82,887.3$ \\
\hline S96T004309 & $153: 10$ & Drainable liquid & $80,985.9$ & 78,169 & $79,577.5$ \\
\hline S96T003979 & $153: 11$ & Drainable liquid & $85,915.5$ & $87,323.9$ & $86,619.7$ \\
\hline S96T003977 & $153: 12$ & Drainable liquid & $88,732.4$ & $92,253.5$ & 90,493 \\
\hline S96T003978 & $153: 13$ & Drainable liquid & $86,056.3$ & $85,915.5$ & $85,985.9$ \\
\hline S96T004333 & $153: 14$ & Drainable liquid & $89,154.9$ & $88,732.4$ & $88,943.7$ \\
\hline S96T003980 & $153: 21$ & Drainable liquid & $66,725.4$ & $66,126.8$ & $66,426.1$ \\
\hline S96T004039 & Core 152 & Liquid composite & $92,042.3$ & $91,549.3$ & $91,795.8$ \\
\hline
\end{tabular}


Table B2-57. Tank 241-AN-105 Analytical Results: Nitrite (IC). (2 sheets)

\begin{tabular}{|c|c|c|c|c|c|}
\hline Yamprs & Somple & $\begin{array}{l}\text { 6rimole } \\
\text { Porolor }\end{array}$ & $\mathrm{P}=111 \mathrm{x}$ & Buplicalo & Mean. \\
\hline \multicolumn{2}{|c|}{ 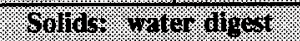 } & & 40 & 1016 & $48 \%$ \\
\hline S96T003915 & 152: 1 & Upper half & 74,500 & 71,300 & 72,900 \\
\hline \$96T003907 & \multirow[t]{2}{*}{$152: 14$} & Upper half & 74,600 & 77,100 & 75,850 \\
\hline \$96T003829 & & Lower half & 47,360 & 52,300 & 49,830 \\
\hline S96T003908 & \multirow[t]{2}{*}{$152: 16$} & Upper half & 52,530 & 52,900 & 52,715 \\
\hline \$96T003909 & & Lower half & 72,380 & 72,900 & 72,640 \\
\hline$\$ 96 T 003910$ & \multirow[t]{2}{*}{$152: 18$} & Upper half & 66,210 & 65,600 & 65,905 \\
\hline S96T003911 & & Lower half & 65,210 & 63,700 & 64,455 \\
\hline S96T003912 & \multirow[t]{2}{*}{$152: 20$} & Upper half & 64,900 & 64,400 & 64,650 \\
\hline S96T003913 & & Lower half & 66,290 & 57,600 & $61,945^{\mathrm{QC}: \mathrm{c}}$ \\
\hline \$96T003914 & $152: 22$ & Upper half & 53,110 & 52,800 & 52,955 \\
\hline S96T004339 & $153: 14$ & Lower half & 87,220 & 77,500 & $82,360^{Q C \cdot c}$ \\
\hline S96T004316 & \multirow[t]{2}{*}{$153: 15$} & Upper half & 72,840 & 70,000 & 71,420 \\
\hline S96T004319 & & Lower half & 56,450 & 68,200 & $62,325^{\mathrm{QC}: \mathrm{d}}$ \\
\hline \$96T004447 & \multirow[t]{2}{*}{$153: 17$} & Upper half & 58,930 & 72,700 & $65,815^{\mathrm{QC}: \mathrm{e}}$ \\
\hline \$96T005325 & & Lower half & 78,630 & 73,800 & 76,215 \\
\hline S96T005326 & $153: 19$ & Lower half & 75,230 & 72,600 & 73,915 \\
\hline S96T003990 & $153: 21$ & Lower half & 48,110 & 65,300 & $56,705^{\mathrm{QC}: \mathrm{d}, \mathrm{e}}$ \\
\hline S96T003992 & \multirow[t]{2}{*}{$153: 22$} & Upper half & 60,530 & 57,000 & 58,765 \\
\hline S96T003991 & & Lower half & 43,370 & 39,300 & 41,335 \\
\hline S96T004258 & \multirow[t]{2}{*}{ Core 152} & Solid composite & 72,900 & 73,200 & 73,050 \\
\hline S96T004263 & & Solid composite & 51,100 & 44,300 & 47,700 \\
\hline
\end{tabular}


Table B2-58. Tank 241-AN-105 Analytical Results: Oxalate (IC). (2 sheets)

\begin{tabular}{|c|c|c|c|c|c|}
\hline Wamplich & Whiplo & 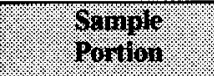 & 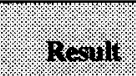 & Bupliciver. & 404 \\
\hline \multicolumn{2}{|c|}{ Wing } & & $14 \%$ & 498 & $48 \%$ \\
\hline S96T003732 & 152: 1 & Drainable liquid & $<306.19$ & $<306.33$ & $<306.26$ \\
\hline S96T003739 & 152: 2 & Drainable liquid & $<380.91$ & $<380.98$ & $<380.95$ \\
\hline S96T003740 & 152: 3 & Drainable liquid & $<380.91$ & $<380.98$ & $<380.95$ \\
\hline S96T003741 & 152: 4 & Drainable liquid & $<380.91$ & $<380.98$ & $<380.95$ \\
\hline S96T003742 & $152: 5$ & Drainable liquid & $<754.22$ & $<753.52$ & $<753.87$ \\
\hline S96T003743 & $152: 6$ & Drainable liquid & $<754.22$ & $<753.52$ & $<753.87$ \\
\hline \$96T003744 & 152: 7 & Drainable liquid & $<306.19$ & 347.88 & $<327.04$ \\
\hline S96T003745 & 152: 8 & Drainable liquid & $<306.19$ & $<306.33$ & $<306.26$ \\
\hline \$96T003746 & 152: 9 & Drainable liquid & $<306.19$ & $<306.33$ & $<306.26$ \\
\hline S96T003747 & $152: 10$ & Drainable liquid & 11,331 & $<306.33$ & $<5,818.66$ \\
\hline S96T003748 & $152: 11$ & Drainable liquid & 181.26 & 190.14 & 185.70 \\
\hline S96T003749 & $152: 12$ & Drainable liquid & $<380.91$ & $<380.98$ & $<380.95$ \\
\hline S96r003750 & $152: 13$ & Drainable liquid & $<306.19$ & $<306.33$ & $<306.26$ \\
\hline S96T003751 & $152: 14$ & Drainable liquid & $<306.19$ & $<306.33$ & $<306.26$ \\
\hline S96T004433 & 153: 1 & Drainable liquid & $<306.19$ & 333.80 & $<320$ \\
\hline S96T004308 & 153: 2 & Drainable liquid & $<306.19$ & $<306.33$ & $<306.26$ \\
\hline S96T004434 & $153: 3$ & Drainable liquid & $<306.19$ & $<306.33$ & $<306.26$ \\
\hline S96T004419 & 153: 5 & Drainable liquid & $<306.19$ & $<306.33$ & $<306.26$ \\
\hline S96T004420 & 153: 6 & Drainable liquid & 196.69 & 209.85 & 203.27 \\
\hline S96T004421 & 153: 7 & Drainable liquid & $<194.15$ & 220.42 & $<207.28$ \\
\hline S96T003975 & 153: 8 & Drainable liquid & $<380.91$ & 409.85 & $<395.38$ \\
\hline S96T003976 & 153: 9 & Drainable liquid & 189.93 & 192.95 & 191.44 \\
\hline S96T004309 & $153: 10$ & Drainable liquid & $<306.19$ & $<306.33$ & $<306.26$ \\
\hline \$96T003979 & $153: 11$ & Drainable liquid & $<306.19$ & $<306.33$ & $<306.26$ \\
\hline S96T003977 & $153: 12$ & Drainable liquid & $<306.19$ & $<306.33$ & $<306.26$ \\
\hline S96T003978 & $153: 13$ & Drainable liquid & $<306.19$ & $<306.33$ & $<306.26$ \\
\hline S96T004333 & $153: 14$ & Drainable liquid & 400.28 & 480.28 & 440.28 \\
\hline S96T003980 & $153: 21$ & Drainable liquid & $<306.19$ & $<306.33$ & $<306.26$ \\
\hline S96T004039 & Core 152 & Liquid composite & $<306.19$ & $<306.33$ & $<306.26$ \\
\hline
\end{tabular}


Table B2-58. Tank 241-AN-105 Analytical Results: Oxalate (IC). (2 sheets)

\begin{tabular}{|c|c|c|c|c|c|}
\hline F Sample & $\begin{array}{l}\text { Sample } \\
\text { Locition }\end{array}$ & $\begin{array}{l}\text { Sample } \\
\text { Pontiona }\end{array}$ & resuil & Buplicate: & Mean \\
\hline \multicolumn{2}{|c|}{ Solids varter aigest } & & 18\% & (x) $6 / 8$ & 1148 \\
\hline S96T003915 & 152: 1 & Upper half & 4,558 & 4,390 & 4,474 \\
\hline S96T003907 & \multirow[t]{2}{*}{$152: 14$} & Upper half & 8,005 & 7,920 & $7,962.5$ \\
\hline S96T003829 & & Lower half & 4,784 & 5,400 & 5,092 \\
\hline S96T003908 & \multirow[t]{2}{*}{$152: 16$} & Upper half & 5,474 & 5,660 & 5,567 \\
\hline S96T003909 & & Lower half & 4,641 & 4,860 & $4,750.5$ \\
\hline S96T003910 & \multirow[t]{2}{*}{$152: 18$} & Upper half & 5,005 & 5,010 & $5,007.5$ \\
\hline S96T003911 & & Lower half & 5,208 & 5,900 & 5,554 \\
\hline S96T003912 & \multirow[t]{2}{*}{$152: 20$} & Upper half & 10,090 & 10,100 & 10,095 \\
\hline S96T003913 & & Lower half & 4,950 & 4,490 & 4,720 \\
\hline S96T003914 & $152: 22$ & Upper half & 4,422 & 4,350 & 4,386 \\
\hline S96T004339 & $153: 14$ & Lower half & 11,670 & 8,580 & $10,125^{\mathrm{QC:e}}$ \\
\hline S96T004316 & \multirow[t]{2}{*}{$153: 15$} & Upper half & 8,707 & 8,480 & $8,593.5$ \\
\hline S96T004319 & & Lower half & 7,645 & 8,020 & $7,832.5$ \\
\hline S96T004447 & \multirow[t]{2}{*}{$153: 17$} & Upper half & 9,762 & 10,000 & 9,881 \\
\hline S96T005325 & & Lower half & 11,820 & 12,200 & 12,010 \\
\hline S96T005326 & $153: 19$ & Lower half & 5,632 & 6,640 & 6,136 \\
\hline S96T003990 & $153: 21$ & Lower half & 2,886 & 3,900 & $3,393^{\mathrm{QC}: c}$ \\
\hline S96T003992 & \multirow[t]{2}{*}{$153: 22$} & Upper half & 6,058 & 5,580 & 5,819 \\
\hline S96T003991 & & Lower half & 9,900 & 8,730 & 9,315 \\
\hline S96T004258 & \multirow[t]{2}{*}{ Core 152} & Solid composite & 6,294 & 6,690 & 6,492 \\
\hline S96T004263 & & Solid composite & 6,302 & 5,460 & 5,881 \\
\hline
\end{tabular}


Table B2-59. Tank 241-AN-105 Analytical Results: Phosphate (IC). (2 sheets)

\begin{tabular}{|c|c|c|c|c|c|}
\hline $\begin{array}{l}\text { Samplo } \\
\text { Thinodr }\end{array}$ & Somple & 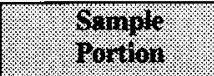 & Hesil: & ouplitare & 148rin \\
\hline \multicolumn{2}{|c|}{ Wogias } & & 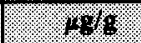 & 49 & $6 \%$ \\
\hline S96T003732 & 152: 1 & Drainable liquid & 871.12 & 347.88 & $609.50^{\mathrm{QC:e}}$ \\
\hline S96T003739 & 152: 2 & Drainable liquid & $<431.33$ & 711.26 & $<571.30$ \\
\hline \$96T003740 & 152: 3 & Drainable liquid & $<431.33$ & 774.64 & $<602.99$ \\
\hline S96T003741 & 152: 4 & Drainable liquid & 854.22 & $<430.98$ & $<642.60$ \\
\hline S96T003742 & 152: 5 & Drainable liquid & $<854.22$ & $<852.11$ & $<853.16$ \\
\hline S96T003743 & 152: 6 & Drainable liquid & $1,375.35$ & $1,274.65$ & 1,325 \\
\hline S96T003744 & 152: 7 & Drainable liquid & 526.47 & 470.42 & 498.45 \\
\hline S96T003745 & 152: 8 & Drainable liquid & 452.11 & 463.38 & 457.74 \\
\hline$\$ 96 T 003746$ & 152: 9 & Drainable liquid & $<346.76$ & $<346.47$ & $<346.62$ \\
\hline \$96T003747 & $152: 10$ & Drainable liquid & $12,176.1$ & $<346.47$ & $<6,261.27$ \\
\hline S96T003748 & $152: 11$ & Drainable liquid & 499.22 & 433.09 & 466.16 \\
\hline S96T003749 & $152: 12$ & Drainable liquid & 483.66 & $<430.98$ & $<457.32$ \\
\hline \$96T003750 & $152: 13$ & Drainable liquid & $<346.76$ & $<346.47$ & $<346.62$ \\
\hline \$96T003751 & $152: 14$ & Drainable liquid & 934.50 & $<346.47$ & $<640.49$ \\
\hline S96T004433 & 153: 1 & Drainable liquid & 461.47 & 509.85 & 485.66 \\
\hline S96T004308 & 153: 2 & Drainable liquid & 446.47 & 426.76 & 436.62 \\
\hline S96T004434 & 153: 3 & Drainable liquid & 423.31 & 524.64 & $473.97^{\mathrm{QC:e}}$ \\
\hline S96T004419 & 153: 5 & Drainable liquid & 551.12 & 447.88 & $499.50^{\text {oC:e }}$ \\
\hline S96T004420 & 153: 6 & Drainable liquid & 476.47 & 469.01 & 472.74 \\
\hline \$96T004421 & 153: 7 & Drainable liquid & 479.71 & 451.40 & 465.56 \\
\hline S96T003975 & 153: 8 & Drainable liquid & $<431.33$ & $<430.98$ & $<431.16$ \\
\hline S96T003976 & 153: 9 & Drainable liquid & 529.08 & 551.40 & 540.24 \\
\hline$\$ 96 \mathrm{T004309}$ & $153: 10$ & Drainable liquid & 462.74 & 397.88 & 430.31 \\
\hline S96T003979 & $153: 11$ & Drainable liquid & 572.04 & 435.91 & $503.97^{\text {QC:e }}$ \\
\hline \$96T003977 & 153:12 & Drainable liquid & 372.25 & 544.36 & $458.31^{Q C: c}$ \\
\hline S96T003978 & $153: 13$ & Drainable liquid & 589.85 & 424.64 & $507.25^{\mathrm{QC}: \mathrm{c}}$ \\
\hline S96T004333 & 153:14 & Drainable liquid & 464.78 & 428.87 & 446.83 \\
\hline S96T003980 & 153:21 & Drainable liquid & 524.64 & 374.64 & $449.64^{\mathrm{QC}: \mathrm{e}}$ \\
\hline S96T004039 & Core 152 & Liquid composite & 447.04 & 421.12 & 434.08 \\
\hline
\end{tabular}


Table B2-59. Tank 241-AN-105 Analytical Results: Phosphate (IC). (2 sheets)

\begin{tabular}{|c|c|c|c|c|c|}
\hline Shinpis. & Q quinple & Gomple & 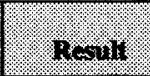 & Finilicale & Worn \\
\hline \multicolumn{2}{|c|}{ Sollows wat alost } & & $1.6 \%$ & 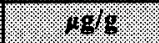 & 49 \\
\hline S96T003915 & 152: 1 & Upper half & 3,618 & 3,550 & 3,584 \\
\hline S96T003907 & \multirow[t]{2}{*}{$152: 14$} & Upper half & 1,424 & 1,550 & 1,487 \\
\hline S96T003829 & & Lower half & $<259.3$ & 524 & $<391.65$ \\
\hline S96T003908 & \multirow[t]{2}{*}{$152: 16$} & Upper half & 1,041 & 1,680 & $1,360.5^{\mathrm{QC}: \mathrm{e}}$ \\
\hline S96T003909 & & Lower half & 1,913 & 1,760 & $1,836.5$ \\
\hline S96T003910 & \multirow[t]{2}{*}{$152: 18$} & Upper half & 2,260 & 2,690 & 2,475 \\
\hline S96T003911 & & Lower half & 1,697 & 1,420 & $1,558.5$ \\
\hline S96T003912 & \multirow[t]{2}{*}{$152: 20$} & Upper half & 2,607 & 1,910 & $2,258.5^{\mathrm{QC}: \varepsilon}$ \\
\hline S96T003913 & & Lower half & 1,360 & 3,860 & $2,610^{\mathrm{QC}: \mathrm{e}}$ \\
\hline S96T003914 & $152: 22$ & Upper half & 6,698 & 5,850 & 6,274 \\
\hline S96T004339 & $153: 14$ & Lower half & 1,204 & 1,340 & 1,272 \\
\hline S96T004316 & \multirow[t]{2}{*}{$153: 15$} & Upper half & 675.3 & 1,050 & $862.65^{\mathrm{QC}: e}$ \\
\hline S96T004319 & & Lower half & 739.2 & 573 & $656.1^{\mathrm{QC}: \mathrm{e}}$ \\
\hline S96T004447 & \multirow[t]{2}{*}{$153: 17$} & Upper half & 986.8 & 1,510 & $1,248.4^{\mathrm{QC:c}}$ \\
\hline S96T005325 & & Lower half & 2,001 & 2,680 & $2,340.5^{\mathrm{QC}: \mathrm{e}}$ \\
\hline S96T005326 & $153: 19$ & Lower half & 3,315 & 5,500 & $4,407.5^{\mathrm{QC}: \mathrm{e}}$ \\
\hline S96T003990 & $153: 21$ & Lower half & 1,634 & 996 & $1,315^{\mathrm{QC}: \mathrm{e}}$ \\
\hline S96T003992 & \multirow[t]{2}{*}{$153: 22$} & Upper half & 12,090 & 10,400 & 11,245 \\
\hline S96T003991 & & Lower half & $1.041 \mathrm{E}+05$ & $1.020 \mathrm{E}+05$ & $1.031 \mathrm{E}+05$ \\
\hline S96T004258 & \multirow[t]{2}{*}{ Core 152} & Solid composite & 1,281 & 1,130 & $1,205.5$ \\
\hline S96T004263 & & Solid composite & 47,950 & 43,400 & 45,675 \\
\hline
\end{tabular}


Table B2-60. Tank 241-AN-105 Analytical Results: Sulfate (IC). (2 sheets)

\begin{tabular}{|c|c|c|c|c|c|}
\hline Whinoly & 6 mole & . $640 \%$ & Perail. & Buplichis & . \\
\hline \multicolumn{3}{|c|}{ Wring } & $1 \%$ & (18) & 489 \\
\hline S96T003732 & 152: 1 & Drainable liquid & 733.09 & 830.98 & 782.04 \\
\hline S96T003739 & 152: 2 & Drainable liquid & 688.87 & 929.57 & $809.22^{\mathrm{QC} c \mathrm{c}}$ \\
\hline S96T003740 & $152: 3$ & Drainable liquid & 895.07 & 704.22 & $799.64^{\text {QC:0 }}$ \\
\hline S96T003741 & 152: 4 & Drainable liquid & 759.85 & 795.77 & 777.81 \\
\hline S96T003742 & 152: 5 & Drainable liquid & $<976.05$ & $<978.87$ & $<977.46$ \\
\hline S96T003743 & $152: 6$ & Drainable liquid & $2,041.55$ & $2,647.89$ & $2,344.72^{\mathrm{QC}: \mathrm{c}}$ \\
\hline S96T003744 & 152: 7 & Drainable liquid & 766.19 & 816.90 & 791.54 \\
\hline S96T003745 & 152: 8 & Drainable liquid & 669.50 & 659.85 & 664.68 \\
\hline S96T003746 & 152: 9 & Drainable liquid & 816.19 & 677.46 & 746.83 \\
\hline S96T003747 & $152: 10$ & Drainable liquid & 802.81 & 781.69 & 792.25 \\
\hline S96T003748 & $152: 11$ & Drainable liquid & 603.02 & 664.78 & 633.90 \\
\hline \$96T003749 & $152: 12$ & Drainable liquid & 750.70 & 802.81 & 776.76 \\
\hline S96T003750 & $152: 13$ & Drainable liquid & 804.22 & 760.56 & 782.39 \\
\hline S96T003751 & $152: 14$ & Drainable liquid & 971.12 & 992.95 & 982.04 \\
\hline S96T004433 & 153: 1 & Drainable liquid & 778.16 & 753.52 & 765.84 \\
\hline S96T004308 & 153: 2 & Drainable liquid & 740.84 & 823.94 & 782.39 \\
\hline S96T004434 & $153: 3$ & Drainable liquid & 773.94 & 760.56 & 767.25 \\
\hline S96T004419 & 153: 5 & Drainable liquid & 757.04 & 823.94 & 790.49 \\
\hline S96T004420 & 153: 6 & Drainable liquid & 698.02 & 638.02 & 668.02 \\
\hline S96T004421 & 153: 7 & Drainable liquid & 694.01 & 670.42 & 682.21 \\
\hline S96T003975 & 153: 8 & Drainable liquid & 800 & 894.36 & 847.18 \\
\hline S96T003976 & 153: 9 & Drainable liquid & 592.74 & 584.50 & 588.62 \\
\hline S96T004309 & $153: 10$ & Drainable liquid & 766.90 & 732.39 & 749.64 \\
\hline S96T003979 & $153: 11$ & Drainable liquid & 741.54 & 725.35 & 733.45 \\
\hline \$96T003977 & 153:12 & Drainable liquid & 704.93 & 795.77 & 750.35 \\
\hline S96T003978 & $153: 13$ & Drainable liquid & 834.50 & 760.56 & 797.53 \\
\hline S96T004333 & $153: 14$ & Drainable liquid & 854.93 & 964.78 & 909.85 \\
\hline S96T003980 & $153: 21$ & Drainable liquid & $1,996.48$ & $2,014.08$ & $2,005.28$ \\
\hline S96T004039 & Core 152 & Liquid composite & 709.85 & 654.22 & 682.04 \\
\hline
\end{tabular}


Table B2-60. Tank 241-AN-105 Analytical Results: Sulfate (IC). (2 sheets)

\begin{tabular}{|c|c|c|c|c|c|}
\hline Mininer & \%ample & Tample & restil & 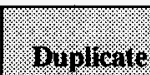 & Ifan \\
\hline \multicolumn{2}{|c|}{ Solds - warer algest? } & 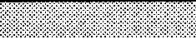 & 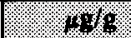 & $10 \%$ & U. \\
\hline S96T003915 & 152: 1 & Upper half & 3,453 & 3,430 & $3,441.5$ \\
\hline S96T003907 & \multirow[t]{2}{*}{$152: 14$} & Upper half & 4,510 & 4,680 & 4,595 \\
\hline S96T003829 & & Lower half & 3,329 & 3,510 & $3,419.5$ \\
\hline S96T003908 & \multirow[t]{2}{*}{$152: 16$} & Upper half & 5,725 & 5,820 & $5,772.5$ \\
\hline S96T003909 & & Lower half & 8,548 & 8,740 & 8,644 \\
\hline S96T003910 & \multirow[t]{2}{*}{$152: 18$} & Upper half & 8,990 & 8,720 & 8,855 \\
\hline S96T003911 & & Lower half & 7,789 & 8,030 & $7,909.5$ \\
\hline S96T003912 & \multirow[t]{2}{*}{$152: 20$} & Upper half & 8,341 & 7,350 & $7,845.5$ \\
\hline S96T003913 & & Lower half & 9,465 & 9,060 & $9,262.5$ \\
\hline S96T003914 & $152: 22$ & Upper half & 6,435 & 6,110 & $6,272.5$ \\
\hline S96T004339 & $153: 14$ & Lower half & 6,943 & 5,360 & $6,151.5^{\mathrm{QC}: c}$ \\
\hline S96T004316 & \multirow[t]{2}{*}{$153: 15$} & Upper half & 5,887 & 5,750 & $5,818.5$ \\
\hline S96T004319 & & Lower half & 5,130 & 5,280 & 5,205 \\
\hline S96T004447 & \multirow[t]{2}{*}{$153: 17$} & Upper half & 5,922 & 6,460 & 6,191 \\
\hline S96T005325 & & Lower half & 6,637 & 6,390 & $6,513.5$ \\
\hline S96T005326 & $153: 19$ & Lower half & 5,459 & 5,910 & $5,684.5$ \\
\hline S96T003990 & $153: 21$ & Lower half & 6,037 & 8,560 & $7,298.5^{\mathrm{QC}: \mathrm{o}}$ \\
\hline S96T003992 & \multirow[t]{2}{*}{$153: 22$} & Upper half & 5,974 & 5,690 & 5,832 \\
\hline \$96T003991 & & Lower half & 1,339 & 1,260 & $1,299.5$ \\
\hline S96T004258 & \multirow[t]{2}{*}{ Core 152} & Solid composite & 7,215 & 7,730 & $7,472.5$ \\
\hline S96T004263 & & Solid composite & 4,397 & 3,960 & $4,178.5$ \\
\hline
\end{tabular}


HNF-SD-WM-ER-678 Rev. 0

Table B2-61. Tank 241-AN-105 Analytical Results: Hydroxide (OH Direct).

\begin{tabular}{|c|c|c|c|c|c|}
\hline Somplo & 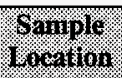 & $\begin{array}{l}\text { ogmple } \\
.00101\end{array}$ & Resill & Bupliople & Mein \\
\hline \multicolumn{2}{|c|}{ Wor } & & (tgis & 48 & 14\% \\
\hline S96T004831 & Core 152 & Liquid composite & $39,859.2$ & $42,605.6$ & $41,232.4^{\mathrm{QC}: \mathrm{f}}$ \\
\hline
\end{tabular}

Table B2-62. Tank 241-AN-105 Analytical Results: Hydroxide (OH).

\begin{tabular}{|c|c|c|c|c|c|}
\hline $\begin{array}{l}\text { Sample } \\
\text { Gumbo }\end{array}$ & $\begin{array}{l}\text { Saniple } \\
\text { Koverofon }\end{array}$ & Somple & resulth & Buplionite & 14an \\
\hline \multicolumn{2}{|c|}{ 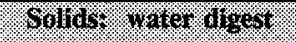 } & & 4848 & 108 & 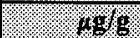 \\
\hline S96T004258 & \multirow[t]{2}{*}{ Core 152} & Solid composite & 23,600 & 24,500 & 24,050 \\
\hline S96T004263 & & Solid composite & 22,400 & 21,600 & 22,000 \\
\hline
\end{tabular}

Table B2-63. Tank 241-AN-105 Analytical Results: Hexavalent Chromium-VI $(\mathrm{Cr}+6)$.

\begin{tabular}{|c|c|c|c|c|c|}
\hline Wannole & Womple & Wample & Resull & Buplicte & Mean \\
\hline \multicolumn{3}{|c|}{ Wivings: } & 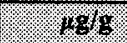 & $6,6 / 9$ & $18 \mathrm{~g}$ \\
\hline S96T004831 & Core 152 & Liquid composite & 108.45 & 107.74 & 108.09 \\
\hline \multicolumn{3}{|c|}{ ovilds. water digest } & 1.8\% & \%g/g & 498 \\
\hline S96T004258 & \multirow[t]{2}{*}{ Core 152} & Solid composite & 118 & 120 & 119 \\
\hline S96T004263 & & Solid composite & 107 & 101 & 104 \\
\hline
\end{tabular}

Table B2-64. Tank 241-AN-105 Analytical Results: Total Organic Carbon by Coulometry.

\begin{tabular}{|c|c|c|c|c|c|}
\hline Yaminglo & Wornol & $1046 \%$ & resilit & Huplicate & Migan \\
\hline Wig & & & 1818 & $148 \% 8$ & 1888 \\
\hline S96T004049 & Core 152 & Liquid composite & $4,316.9$ & $4,274.65$ & $4,295.77^{\text {QC:f }}$ \\
\hline
\end{tabular}


Table B2-65. Tank 241-AN-105 Analytical Results: Total Organic Carbon by Persulfate Coulometry.

\begin{tabular}{|c|c|c|c|c|c|}
\hline $\begin{array}{l}\text { Sample: } \\
\text { Number. }\end{array}$ & $\begin{array}{l}\text { Sample } \\
\text { Location }\end{array}$ & $\begin{array}{l}\text { Sample. } \\
\text { Foitiono. }\end{array}$ & Resull: & Duplizate. & Mean \\
\hline \multicolumn{2}{|c|}{ Travius } & (2:-3: & 1968 & $19 \mathrm{~g} g$ & 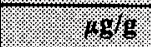 \\
\hline S96T004049 & Core 152 & Liquid composite & $1,929.58$ & $1,718.31$ & $1,823.94^{\mathrm{QC}: \mathrm{f}}$ \\
\hline \multicolumn{2}{|c|}{ Solids } & 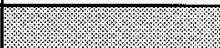 & 4g/s. & $19 \mathrm{~g} / \mathrm{g}$ & 1496 \\
\hline S96T004254 & Core 152 & Solid composite & 4,900 & 5,950 & $5,425^{\mathrm{QC}: \mathrm{e}}$ \\
\hline S96T004259 & & Solid composite & 4,330 & 4,630 & 4,480 \\
\hline
\end{tabular}

Table B2-66. Tank 241-AN-105 Analytical Results: Total Inorganic Carbon (TIC).

\begin{tabular}{|c|c|c|c|c|c|}
\hline $\begin{array}{l}\text { Siminile. } \\
\text { Niminber. }\end{array}$ & $\begin{array}{c}\text { Sample } \\
\text { licarion }\end{array}$ & $\begin{array}{l}\text { Sample: } \\
\text { rortion. }\end{array}$ & Result & Duplicate & Menon. \\
\hline \multicolumn{2}{|c|}{ Tirquids } & 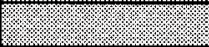 & mg 6 & $4 \mathrm{~g} 8$ & 1898 \\
\hline S96T004049 & Core 152 & Liquid composite & $1,563.38$ & $1,528.17$ & $1,545.77^{\mathrm{QC}: \mathrm{f}}$ \\
\hline \multicolumn{2}{|c|}{ Solins } & & $16 \mathrm{~g} / \mathrm{g}$ & 1.098 & 498 \\
\hline S96T004254 & Core 152 & Solid composite & 12,500 & 13,600 & $13,050^{\mathrm{QC:c}}$ \\
\hline S96T004259 & & Solid composite & 8,510 & 7,990 & 8,250 \\
\hline
\end{tabular}

Table B2-67. Tank 241-AN-105 Vapor Sampling Results.

\begin{tabular}{|l|l|}
\hline \multicolumn{1}{|c|}{ Measurenent } \\
\hline Total Organic Carbon (TOC) & \multicolumn{1}{|c|}{ Sample } \\
\hline Lower Explosive Limit (LEL) & 4.6 parts per million (ppm) \\
\hline Oxygen & 0.3 percent of LEL \\
\hline Ammonia & 20.9 percent \\
\hline
\end{tabular}




\section{B3.0 ASSESSMENT OF CHARACTERIZATION RESULTS}

The purpose of this chapter is to discuss the overall quality and consistency of the current sampling results for tank $241-\mathrm{AN}-105$, and to present the results of the calculation of an analytical-based inventory.

This section also evaluates sampling and analysis factors that may impact interpretation of the data. These factors are used to assess the overall quality and consistency of the data and to identify any limitations in the use of the data.

\section{B3.1 FIELD OBSERVATIONS}

The safety screening DQO (Dukelow et al. 1995) requires all vertical waste profiles be from two widely spaced risers. The flammable gas DQO (McDuffie 1995) requires at least one complete core will be taken. These requirements were fulfilled. Contamination by hydrostatic head fluid resulted in adjusting the weight percent water results for one subsegment. The waste recovered in segments with less than full recovery was assumed to be representative of the whole segment.

\section{B3.2 QUALITY CON'TROL ASSESSMENT}

The usual quality control assessment includes an evaluation of the appropriate standard recoveries, spike recoveries, duplicate analyses, and blanks that are performed in conjunction with the chemical analyses. All the pertinent quality control tests were conducted on the 1996 core samples, allowing a full assessment regarding the accuracy and precision of the data. The SAP (Eggers 1996) established the specific criteria for all analytes. Sample and duplicate pairs that had one or more quality control $(\mathrm{QC})$ results outside the specified criteria were identified by footnotes in the data summary tables.

The standard and spike recovery results provide an estimate of the accuracy of the analysis. If a standard or spike recovery is above or below the given criterion, the analytical results may be biased high or low, respectively. The precision is estimated by the relative percent difference (RPD), which is defined as the absolute value of the difference between the primary and duplicate samples, divided by their mean, times one hundred.

A high RPD was reported for two of the forty subsamples submitted for total alpha activity. This may have been caused by subsequent self-shielding. Reruns were deemed unnecessary as the sample results were far below the action limit. 
Some of the high RPDs for the IC analytes may be attributable to sample homogeneity problems. The high RPD and poor spike recovery for fluoride can be attributed to the fact that the fluoride peak is very near the baseline and suffers interference from the slightly larger chloride peak.

Many of the ICP analytes also had one or more QC parameters outside the specified limits. The poor spike recoveries for sodium may be due to the high concentration of sodium in the samples (samples cannot be spiked to levels much greater than already present). The high concentrations of sodium required high dilutions for all ICP samples. These high dilutions in turn can cause poor or meaningless spike recoveries and RPDs for those ICP elements that had either very high concentrations or were close to the detection limit. Finally, none of the samples exceeded the criterion for preparation blanks; thus, contamination was not a problem.

In summary, the vast majority of the $\mathrm{QC}$ results were within the boundaries specified in the SAPs. The discrepancies mentioned here and footnoted in the data summary tables should not impact either the validity or the use of the data.

\section{B3.3 DATA CONSISTENCY CHECKS}

Comparisons of different analytical methods can help to assess the consistency and quality of the data. Several comparisons were possible with the data set provided by the two core samples, including a comparison of phosphorous as analyzed by ICP with phosphate as analyzed by IC, and a comparison of weight percent water by TGA with the weight percent water by gravimetry. In addition, mass and charge balances were calculated to help assess the overall data consistency.

\section{B3.3.1 Comparison of Results from Different Analytical Methods}

The following data consistency checks compare the results from two different analytical methods. A close comparison between the two methods strengthens the credibility of both results, whereas a poor comparison brings the reliability of the data into question. All analytical mean results were calculated from tables in Section B2.0.

The analytical phosphorous mean result as determined by ICP was $1,219 \mu \mathrm{g} / \mathrm{g}$, which converts to $3,734 \mu \mathrm{g} / \mathrm{g}$ of phosphate. This compared well with the IC phosphate mean result of $3,870 / \mathrm{g}$ (Table B3-1). The RPD between these two phosphate results was 4.8 percent. 
Table B3-1. Phosphate vs Phosphorous.

\begin{tabular}{|c|c|c|c|c|c|}
\hline \multirow[b]{2}{*}{ inalyes. } & \multicolumn{2}{|c|}{ Supernate } & Salt Slim & $\begin{array}{l}\text { Weighited } \\
\text { Mens? }\end{array}$ & Ciculited Men \\
\hline & $0.9 / 11$ & 148/8 & 1086 & 498 & $1=0918$ \\
\hline $\mathrm{P}$ & 400 & 282 & 2,460 & 1,219 & 3,734 \\
\hline$\overline{\mathrm{PO}_{4}}$ & 1,060 & 746 & 8,010 & 3,870 & 3,870 \\
\hline
\end{tabular}

Note:

${ }^{1}$ Weighted Mean was calculated using the following formula: $0.57 *$ [supernatant] $+0.43 *$ [salt slurry]

The ICP sulfur value of $1,567 \mu \mathrm{g} / \mathrm{g}$, which represents total sulfur, is equivalent to 4,701 $\mu \mathrm{g} / \mathrm{g}$ of sulfate. The IC result for sulfate was $3,179 \mu \mathrm{g} / \mathrm{g}$ (Table B3-2), with an RPD between the two values of 39 percent. These results are expected. Because ICP measures total sulfur and its result is usually larger than or equal to the IC sulfate value, which is a measurement of the soluble sulfur.

Table B3-2. Sulfate vs Sulfur.

\begin{tabular}{|c|c|c|c|c|c|}
\hline \multirow[b]{2}{*}{ Analyter. } & \multicolumn{2}{|c|}{ Supernate } & Sall SHirg & $\begin{array}{l}\text { Weighted } \\
\text { Mean' }\end{array}$ & $\begin{array}{l}\text { Calculated } \\
\text { Miean. }\end{array}$ \\
\hline & $18 \mathrm{gnu}$ & 1018 & 1988 & ( & $1808 \mathrm{~s}$ \\
\hline S & 937 & 660 & 2,770 & 1,567 & 4,701 \\
\hline $\mathrm{SO}_{4}$ & 1,180 & 831 & 6,270 & 3,170 & 3,179 \\
\hline
\end{tabular}

Note:

'Weighted Mean was calculated using the following formula: $0.57 *$ [supernatant] $+0.43 *$ [salt slurry].

\section{B3.3.2 Mass and Charge Balance}

The principle objective in performing mass and charge balances is to determine if the measurements are self-consistent. In calculating the balances, only analytes listed in Section B2.0 detected at a concentration of $1,000 \mu \mathrm{g} / \mathrm{g}$ or greater were considered. Separate mass and charge balances were calculated for the supernatant and salt slurry layers because these waste phases were analyzed separately. The results of these comparisons are presented in the following sections. 
B3.3.2.1 Mass and Charge Balances for the Supernatant. All analytical results used in calculating the supernatant mass and charge balances were first converted from $\mu \mathrm{g} / \mathrm{mL}$ to $\mu \mathrm{g} / \mathrm{g}$ (using the supernatant specific gravity mean of $1.42 \mathrm{~g} / \mathrm{mL}$ ) before use in the tables. The anions listed in Table B3-4 were assumed to be present as sodium salts and were expected to balance the positive charge exhibited by the cations. Phosphate, as determined by IC, is assumed to be completely water soluble and appears only in the anion mass and charge calculations. The concentrations of cationic species in Table B3-3, the anionic species in Table B3-4, and the percent water were ultimately used to calculate the mass balance.

Table B3-3. Supernatant Cation Mass and Charge Data.

\begin{tabular}{|c|c|c|c|c|}
\hline Anvilyte & $\begin{array}{l}\text { Conqentration } \\
(\mu \mathrm{g} / \mathrm{g}) \text {. }\end{array}$ & Asponed & $\begin{array}{l}\text { Concentration of } \\
\text { A wamed Species } \\
(\mu \mathrm{g} / \mathrm{g})\end{array}$ & gharge \\
\hline Potassium & 4,620 & $\mathrm{~K}^{+}$ & 4,620 & 88.00 \\
\hline Sodium & 174,000 & $\mathrm{Na}^{+}$ & 174,000 & $7,956.00$ \\
\hline Total & & & 178,600 & 8,040 \\
\hline
\end{tabular}

Table B3-4. Supernatant Anion Mass and Charge Data.

\begin{tabular}{|c|c|c|c|c|}
\hline Analyte & $\begin{array}{l}\text { Eoncentrition } \\
\text { ( } / \mathrm{g}) \text {. }\end{array}$ & $\begin{array}{l}\text { A srumed } \\
\text { Species }\end{array}$ & 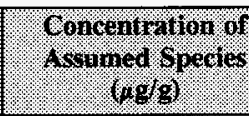 & $\begin{array}{l}\text { gharge } \\
\text { (meoge) }\end{array}$ \\
\hline Aluminum & 29,400 & $\mathrm{Al}(\mathrm{OH})_{4}$ & 103,400 & 1088 \\
\hline Chloride & 7,200 & $\mathrm{Cl}^{-}$ & 7,200 & 203 \\
\hline Formate & 1,100 & $\mathrm{COO}^{-}$ & 1,080 & 25 \\
\hline Hydroxide & 41,300 & $\widehat{\mathrm{OH}^{-}}$ & 41,300 & 2,430 \\
\hline Nitrate & 117,000 & $\mathrm{NO}_{3}^{-}$ & 117,000 & 1,890 \\
\hline Nitrite & 89,000 & $\mathrm{NO}_{2}^{-}$ & 89,000 & 1,935 \\
\hline Phosphate & 750 & $\mathrm{PO}_{4}{ }^{3-}$ & 750 & 24 \\
\hline \multicolumn{3}{|l|}{ Total } & 359,700 & 7,600 \\
\hline
\end{tabular}


The mass balance was calculated from the formula below. The factor 0.0001 is the conversion factor from $\mu \mathrm{g} / \mathrm{g}$ to weight percent.

$$
\begin{aligned}
& \text { Mass balance }=\quad \% \text { Water }+0.0001 \times\{\text { Total Analyte Concentration }\} \\
& =\quad \% \text { Water }+0.0001 \times \mathrm{Na}^{+}+\mathrm{K}^{+}+\mathrm{Al}(\mathrm{OH})_{4}^{-}+\mathrm{Cl}^{-} \\
& \left.+\mathrm{COO}^{-}+\mathrm{OH}^{-}+\mathrm{NO}_{2}^{-}+\mathrm{NO}_{3}^{-}+\mathrm{PO}_{4}^{-3}\right\}
\end{aligned}
$$

The total analyte concentration calculated from the above equation is $538,000 \mu \mathrm{g} / \mathrm{g}$. The mean weight percent water is 49.9 percent, or $499,000 \mu \mathrm{g} / \mathrm{g}$. The mass balance resulting

\begin{tabular}{|c|c|c|}
\hline ( & 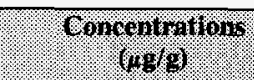 & (10) \\
\hline Total from Table B3-3 & 178,600 & 8,040 \\
\hline Total from Table B3-4 & 359,700 & 7,600 \\
\hline Water percent & 499,000 & 0 \\
\hline Grand Total & $1,037,000$ & 440 \\
\hline
\end{tabular}
from adding the percent water to the total analyte concentration is 104 percent (Table B3-5).

Table B3-5. Mass Balance Totals.

The following equations demonstrate the derivation of total cations and total anions; the charge balance is the ratio of these two values.

$$
\begin{aligned}
\text { Total cations }(\mu \mathrm{eq} / \mathrm{g})= & {\left[\mathrm{K}^{+}\right] / 39+\left[\mathrm{Na}^{+}\right] / 23.0=8,040 \mu \mathrm{eq} / \mathrm{g} } \\
\text { Total anions }(\mu \mathrm{eq} / \mathrm{g})= & {\left[\mathrm{Al}(\mathrm{OH})_{4} / 95\right]+\left[\mathrm{Cl}^{-}\right] / 35.5+\left[\mathrm{COO}^{-}\right] / 44.0+\left[\mathrm{OH}^{-}\right] / 17.0+} \\
& {\left[\mathrm{NO}_{2}^{-}\right] / 46.0+\left[\mathrm{NO}_{3}^{-}\right] / 62.0+\left[\mathrm{PO}_{4}^{-3}\right] / 31.7=7,598 \mu \mathrm{eq} / \mathrm{g} }
\end{aligned}
$$

The charge balance obtained by dividing the sum of the positive charge by the sum of the negative charge was 1.06 .

In summary, the above calculations yield reasonable mass and charge balance values (close to 1.00 for charge balance and 100 percent for mass balance), indicating that the analytical results for the supernatant are generally self-consistent.

B3.3.3.1 Mass and Charge Balances for the Salt Slurry. The positive charges attributed to sodium and potassium were expected to balance the negative charges exhibited by the anions. Sulfur was assumed to be present as sulfate. Phosphate, as determined by IC, is assumed to be completely water soluble and appears only in the anion mass and charge calculations. The concentrations of cationic species in Table B3-6, the anionic species in 
Table B3-7, and the weight percent water were ultimately used to calculate the mass balance.

The mass balance was calculated from the formula below. The factor 0.0001 is the conversion factor from $\mu \mathrm{g} / \mathrm{g}$ to weight percent.

$$
\begin{gathered}
\text { Mass balance }=\% \text { Water }+0.0001 \times\{\text { Total Analyte Concentration }\} \\
=\% \text { Water }+0.0001 \times\left\{\mathrm{Al}(\mathrm{OH})_{3}+\mathrm{Cr}(\mathrm{OH})_{3}+\mathrm{Na}^{+}+\mathrm{K}^{+}+\mathrm{Cl}^{-}\right. \\
\quad \begin{array}{c}
+\mathrm{COO}^{-}+(\mathrm{COO})_{2}{ }^{2-}+\mathrm{F}^{-}+\mathrm{NO}_{2}^{-}+\mathrm{NO}_{3}^{-}+\mathrm{OH}^{-}+\mathrm{PO}_{4}^{-3}+ \\
\left.\mathrm{SO}_{4}^{-2}\right\}
\end{array}
\end{gathered}
$$

\begin{tabular}{|c|c|c|c|c|}
\hline Analyse & 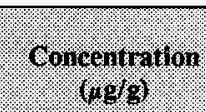 & a vsuined & 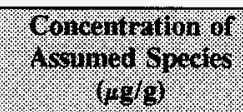 & (2.). \\
\hline Chromium & 2,480 & $\mathrm{Cr}(\mathrm{OH})_{3}$ & 4,900 & 0.00 \\
\hline Potassium & 3,450 & $\mathrm{~K}^{+}$ & 3,450 & 88.00 \\
\hline Sodium & 183,000 & $\mathrm{Na}^{+}$ & 183,000 & $7,956.00$ \\
\hline \multicolumn{3}{|l|}{ Total } & 191,300 & 8,040 \\
\hline
\end{tabular}

\begin{tabular}{|c|c|c|c|c|}
\hline . & 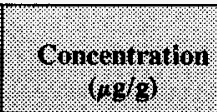 & $6.40 \mathrm{sumed}$ & 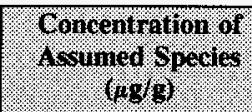 & (6.). \\
\hline Aluminum & 23,100 & $\mathrm{~A} 1(\mathrm{OH})_{4}$ & 81,300 & 856 \\
\hline Chloride & 5,180 & $\mathrm{Cl}^{-}$ & 5,180 & 146 \\
\hline Fluoride & 1,850 & $\mathrm{~F}$ & 1,850 & 97 \\
\hline Formate & 1,060 & $\mathrm{COO}^{-}$ & 1,060 & 23 \\
\hline Hydroxide & 24,100 & $\mathrm{OH}^{-}$ & 24,100 & 1,420 \\
\hline Nitrate & 111,000 & $\mathrm{NO}_{3}^{-}$ & 111,000 & 1,790 \\
\hline Nitrite & 63,900 & $\mathrm{NO}_{2}^{-}$ & 63,900 & 1,389 \\
\hline Oxalate & 6,970 & $(\mathrm{COO})_{2}{ }^{2-}$ & 6,970 & 158 \\
\hline Phosphate & 8,010 & $\mathrm{PO}_{4}{ }^{3-}$ & 8,010 & 252 \\
\hline Sulfate & 62,700 & $\mathrm{SO}_{4}{ }^{2-}$ & 62,700 & 1,306 \\
\hline \multicolumn{3}{|l|}{ Total } & 366,100 & 7,440 \\
\hline
\end{tabular}

Table B3-6. Salt Slurry Cation Mass and Charge Data.

Table B3-7. Salt Slurry Anion Mass and Charge Data. 
The total analyte concentrations calculated from the above equation is $557,400 \mu \mathrm{g} / \mathrm{g}$. The mean weight percent water in salt slurry is 44.4 percent, or $444,000 \mu \mathrm{g} / \mathrm{g}$. The mass balance resulting from adding the percent water to the total analyte concentration is 100 percent (Table B3-8).

The following equations demonstrate the derivation of total cations and total anions in the salt slurry; the charge balance is the ratio of these two values.

$$
\begin{aligned}
\text { Total cations }(\mu \mathrm{eq} / \mathrm{g})= & {\left[\mathrm{K}^{+}\right] / 39+\left[\mathrm{Na}^{+}\right] / 23.0=8,040 \mu \mathrm{eq} / \mathrm{g} } \\
\text { Total anions }(\mu \mathrm{eq} / \mathrm{g})=\quad & {\left[\mathrm{A} 1(\mathrm{OH})_{4}{ }^{-} / 95\right]+\left[\mathrm{F}^{-}\right] / 19.0+\left[\mathrm{Cl}^{-}\right] / 35.5+\left[\mathrm{COO}^{-}\right] / 44.0+} \\
& {\left[\left(\mathrm{COO}_{2}{ }^{2-}\right] / 44.0+\left[\mathrm{NO}_{2}^{-}\right] / 46.0+\left[\mathrm{NO}_{3}^{-}\right] / 62.0+\left[\mathrm{OH}^{-}\right] / 17.0+\right.} \\
& {\left[\mathrm{PO}_{4}^{-3}\right] / 31.7+\left[\mathrm{SO}_{4}^{-2}\right] / 48=7,440 \mu \mathrm{eq} / \mathrm{g} }
\end{aligned}
$$

The charge balance obtained by dividing the sum of the positive charge by the sum of the negative charge was 1.08 .

In summary, the above calculations yield reasonable mass and charge balance values (close to

\begin{tabular}{|c|c|c|}
\hline 10.10 & $\begin{array}{l}\text { Concentrifions } \\
\text { ig/g) }\end{array}$ & Charge (uequg) \\
\hline Total from Table B3-6 & 191,300 & 8040 \\
\hline Total from Table B3-7 & 366,100 & 7440 \\
\hline Water percent & 444,000 & 0 \\
\hline Total & $1,001,400$ & 600 \\
\hline
\end{tabular}
1.00 for charge balance and 100 percent for mass balance), indicating that the analytical results are consistent.

Table B3-8. Mass Balance Totals.

\section{B3.4 MEAN CONCENTRATIONS AND CONFIDENCE INTERVALS}

The following statistical evaluation was performed using the analytical data generated from tank 241-AN-105 core samples. The core samples were obtained June 1996 from two risers (core 152 and core 153) the full length of the waste in the tank (22 segments). The crust was a portion of segment 1 . The remainder of segment 1 through segment 13 and a portion of segment 14 were supernatant samples. The remainder of segment 14 through segment 22 were salt slurry (sludge) samples. The three sets of data (crust, supernatant, and sludge) were analyzed separately. 
For each data set, a mean concentration and the associated variability were calculated for each analyte. A two-sided 95 percent confidence interval for the mean concentration was also calculated for each analyte. The confidence interval takes into account the sampling and analytical uncertainties. The upper and lower limits (UL and LL) of a two-sided 95 percent confidence interval for the mean are:

$$
\hat{\boldsymbol{\mu}} \pm \mathrm{t}_{(\mathrm{d}, 0.025)} \times \hat{\boldsymbol{\sigma}}_{\hat{\mu}} .
$$

In these equations, $\hat{\mu}$ is the estimate of the mean concentration, $\hat{\sigma}_{\hat{\mu}}$ is the estimate of the standard deviation of the mean concentration, and $t_{d f, 0.025)}$ is the quantile from Student's $t$ distribution with $d f$ degrees of freedom for a two-sided 95 percent confidence interval. The mean, $\hat{\mu}$, and the standard deviation of the mean, $\hat{\sigma}_{\hat{\mu}}$, were estimated using restricted maximum likelihood estimation (REML) methods.

\section{B3.4.1 Mean Concentrations}

B3.4.1.1 Supernatant Data. The statistics in this section were based on analytical data from the 1996 sampling event of tank 24l-AN-105. The 1996 data were statistically evaluated using two different models. The first model used a nested analysis of variance (ANOVA): the data are identified by segment within riser. The second model used one-way ANOVA: the data are identified by one variable (the segment). Analysis of variance techniques were used to estimate the mean and its associated variability for all analytes that had at least 50 percent of the reported data as quantitative values.

For those analytes that had a mixture of both quantitative values and "less than" values, the ANOVA was computed using two different methodologies.

The upper value of the "less than" (for example, 3.5 for $<3.5$ ) was used to represent all "less than" analytical values in the first computation. This produces a bias of unknown magnitude in both the mean analyte concentration and the variance associated with the mean; the mean analyte concentration is biased high. The extension ".w" was added to the analyte name in the tables to distinguish which analyte was statistically analyzed using "less than" values.

The "less than" values were deleted in the second computation. Deleting data produces unbalanced data sets which complicates the statistical analysis. Deleting data decreases the number of degrees of freedom. Deleting data also produces a bias of unknown magnitude in both the mean analyte concentration and the variance associated with the mean. The extension ".wo" was added to the analyte name in the tables to distinguish which analyte was statistically analyzed with the "less than" values deleted. The extension ".adl" represents acid dilution. 
No ANOVA estimates were computed for analytes that had less than 50 percent of the reported data as quantitative values. The data for some of the analytes consisted of "outliers." The statistical analysis was performed with the outliers in the data set and with the outliers deleted from the data set. The extension ".ed" was used in the tables to indicate which analytes had outliers deleted in the statistical analysis.

The mean concentration estimates for the supernatant layer, along with the two-sided 95 percent confidence interval for the mean concentration, are given in Table B3-9 (nested ANOVA) and Table B3-10 (one-way ANOVA) for those analytes with at least 50 percent of the reported data as quantitative values. For some of the analytes, the lower limit of the 95 percent confidence interval was a negative value due to the magnitude of the variability. Because the actual concentration of a tank sample cannot be less than zero, the lower limit is reported as zero. The analytes in Table B3-9 where $\hat{\sigma}_{\text {riser }}$ is significantly different from zero are marked with a "*". The riser variable is an indicator of spatial variability.

For those analytes where $\hat{\sigma}_{\text {riser }}$ is not significantly different from zero, the mean concentrations and the variances of the mean concentrations calculated using the two statistical models (one-way ANOVA and nested ANOVA) are not significantly different. In these cases, the one-way ANOVA model is more appropriate.

The analytes (supernatant layer) which had less than 50 percent of the reported data as quantitative values are listed in Table B3-11. Table B3-11 cites the largest value observed from the analytical results.

Table B3-9. Summary Statistics - Supernatant (Nested ANOVA). (2 sheets)

\begin{tabular}{|c|c|c|c|c|c|c|}
\hline Arayte & Wuins & . & 40 & $d y$ & W. & (1) \\
\hline \%water.tga & wt\% & $4.99 \mathrm{E}+01$ & $3.70 \mathrm{E}-01$ & 1 & $4.52 \mathrm{E}+01$ & $5.46 \mathrm{E}+01$ \\
\hline \%water.tga.ed & wt\% & $5.02 \mathrm{E}+01$ & $1.75 \mathrm{E}-01$ & 1 & $4.80 \mathrm{E}+01$ & $5.25 \mathrm{E}+01$ \\
\hline Acetate.ic.w & $\mu \mathrm{g} / \mathrm{mL}$ & $6.00 \mathrm{E}+02$ & $6.86 \mathrm{E}+01$ & 1 & $0.00 \mathrm{E}+00$ & $1.47 \mathrm{E}+03$ \\
\hline Acetate.ic.wo & $\mu \mathrm{g} / \mathrm{mL}$ & $6.11 E+02$ & $5.82 \mathrm{E}+01$ & 1 & $0.00 \mathrm{E}+00$ & $1.35 \mathrm{E}+03$ \\
\hline Ag.icp.adl & $\mu \mathrm{g} / \mathrm{mL}$ & $1.78 \mathrm{E}+01$ & $3.47 \mathrm{E}-01$ & 1 & $1.34 \mathrm{E}+01$ & $2.22 \mathrm{E}+01$ \\
\hline Al.icp.adl & $\mu \mathrm{g} / \mathrm{mL}$ & $4.17 \mathrm{E}+04$ & $5.36 \mathrm{E}+02$ & 1 & $3.48 \mathrm{E}+04$ & $4.85 \mathrm{E}+04$ \\
\hline B.icp.adl & $\mu \mathrm{g} / \mathrm{mL}$ & $7.44 \mathrm{E}+01$ & $7.59 \mathrm{E}-01$ & 1 & $6.47 \mathrm{E}+01$ & $8.40 \mathrm{E}+01$ \\
\hline${ }^{1} \mathrm{Br}^{-}$.ic.w & $\mu \mathrm{g} / \mathrm{mL}$ & $9.64 \mathrm{E}+02$ & $8.39 \mathrm{E}+01$ & 1 & $0.00 \mathrm{E}+00$ & $2.03 E+03$ \\
\hline${ }^{1} \mathrm{Br}^{-}$.ic.wo & $\mu \mathrm{g} / \mathrm{mL}$ & $9.77 \mathrm{E}+02$ & $6.63 \mathrm{E}+01$ & 1 & $1.35 \mathrm{E}+02$ & $1.82 \mathrm{E}+03$ \\
\hline $\mathrm{Cl}^{-}$.ic & $\mu \mathrm{g} / \mathrm{mL}$ & $1.02 \mathrm{E}+04$ & $6.50 \mathrm{E}+02$ & 1 & $1.98 \mathrm{E}+03$ & $1.85 \mathrm{E}+04$ \\
\hline Cl.ic.ed & $\mu \mathrm{g} / \mathrm{mL}$ & $9.92 \mathrm{E}+03$ & $8.50 \mathrm{E}+01$ & 1 & $8.84 \mathrm{E}+03$ & $1.10 \mathrm{E}+04$ \\
\hline Cr.icp.adl & $\mu \mathrm{g} / \mathrm{mL}$ & $2.25 \mathrm{E}+02$ & $1.44 \mathrm{E}+01$ & 1 & $4.20 \mathrm{E}+01$ & $4.08 \mathrm{E}+02$ \\
\hline DSC.dry & $\mathrm{J} / \mathrm{g}$ dry & $2.21 \mathrm{E}+01$ & $1.44 \mathrm{E}+01$ & 1 & $0.00 \mathrm{E}+00$ & $2.05 \mathrm{E}+02$ \\
\hline DSC.wet & $\mathrm{J} / \mathrm{g}$ wet & $1.10 \mathrm{E}+01$ & $7.18 \mathrm{E}+00$ & 1 & $0.00 \mathrm{E}+00$ & $1.02 \mathrm{E}+02$ \\
\hline
\end{tabular}


Table B3-9. Summary Statistics - Supernatant (Nested ANOVA). (2 sheets)

\begin{tabular}{|c|c|c|c|c|c|c|}
\hline Wiriot & Wilits & Xy & 6 & of & 14 & \%) \\
\hline $\mathrm{F}^{-}$.ic.w & $\mu \mathrm{g} / \mathrm{mL}$ & $3.10 \mathrm{E}+02$ & $3.67 \mathrm{E}+01$ & 1 & $0.00 \mathrm{E}+00$ & $7.77 \mathrm{E}+02$ \\
\hline F-.ic.w.ed & $\mu \mathrm{g} / \mathrm{mL}$ & $2.94 \mathrm{E}+02$ & $5.02 \mathrm{E}+01$ & 1 & $0.00 \mathrm{E}+00$ & $9.31 \mathrm{E}+02$ \\
\hline $\mathrm{F}^{-}$,ic.wo ${ }^{*}$ & $\mu \mathrm{g} / \mathrm{mL}$ & $4.86 \mathrm{E}+02$ & $5.71 \mathrm{E}+01$ & 1 & $0.00 \mathrm{E}+00$ & $1.21 \mathrm{E}+03$ \\
\hline $\mathrm{F}^{-}$.ic.wo.ed * & $\mu \mathrm{g} / \mathrm{mL}$ & $4.52 \mathrm{E}+02$ & $2.01 \mathrm{E}+01$ & 1 & $1.97 E+02$ & $7.07 \mathrm{E}+02$ \\
\hline Formate.ic & $\mu \mathrm{g} / \mathrm{mL}$ & $1.56 \mathrm{E}+03$ & $8.28 \mathrm{E}+01$ & 1 & $5.08 \mathrm{E}+02$ & $2.61 E+03$ \\
\hline K.icp.adl & $\mu \mathrm{g} / \mathrm{mL}$ & $6.56 \mathrm{E}+03$ & $7.17 \mathrm{E}+01$ & 1 & $5.65 \mathrm{E}+03$ & $7.47 \mathrm{E}+03$ \\
\hline Mo.icp.adl & $\mu \mathrm{g} / \mathrm{mL}$ & $9.67 \mathrm{E}+01$ & $9.88 \mathrm{E}-01$ & 1 & $8.41 \mathrm{E}+01$ & $1.09 \mathrm{E}+02$ \\
\hline $\mathrm{NO}_{2}^{-}$.ic & $\mu \mathrm{g} / \mathrm{mL}$ & $1.27 \mathrm{E}+05$ & $8.54 \mathrm{E}+03$ & 1 & $1.80 \mathrm{E}+04$ & $2.35 \mathrm{E}+05$ \\
\hline $\mathrm{NO}_{2}^{-}$.ic.ed & $\mu \mathrm{g} / \mathrm{mL}$ & $1.22 \mathrm{E}+05$ & $1.11 \mathrm{E}+03$ & 1 & $1.08 \mathrm{E}+05$ & $1.36 \mathrm{E}+05$ \\
\hline $\mathrm{NO}_{3}^{-} \cdot \mathrm{ic}$ & $\mu \mathrm{g} / \mathrm{mL}$ & $1.66 \mathrm{E}+05$ & $1.09 \mathrm{E}+04$ & 1 & $2.77 \mathrm{E}+04$ & $3.04 \mathrm{E}+05$ \\
\hline $\mathrm{NO}_{3}^{-}$.ic.ed & $\mu \mathrm{g} / \mathrm{mL}$ & $1.61 \mathrm{E}+05$ & $1.75 \mathrm{E}+03$ & 1 & $1.39 \mathrm{E}+05$ & $1.83 \mathrm{E}+05$ \\
\hline Na.icp.adl & $\mu \mathrm{g} / \mathrm{mL}$ & $2.47 \mathrm{E}+05$ & $4.02 \mathrm{E}+03$ & 1 & $1.96 \mathrm{E}+05$ & $2.98 \mathrm{E}+05$ \\
\hline P.icp.adl & $\mu \mathrm{g} / \mathrm{mL}$ & $4.00 \mathrm{E}+02$ & $2.11 \mathrm{E}+01$ & 1 & $1.32 \mathrm{E}+02$ & $6.68 \mathrm{E}+02$ \\
\hline P.icp.adl.ed & $\mu \mathrm{g} / \mathrm{mL}$ & $3.85 \mathrm{E}+02$ & $1.90 \mathrm{E}+01$ & 1 & $1.43 \mathrm{E}+02$ & $6.27 \mathrm{E}+02$ \\
\hline $\mathrm{PO}_{4}{ }^{3-}$.ic. $\mathrm{W}$ & $\mu \mathrm{g} / \mathrm{mL}$ & $1.06 \mathrm{E}+03$ & $3.78 \mathrm{E}+02$ & 1 & $0.00 \mathrm{E}+00$ & $5.86 \mathrm{E}+03$ \\
\hline $\mathrm{PO}_{4}{ }^{3-}$.ic.w.ed & $\mu \mathrm{g} / \mathrm{mL}$ & $7.54 \mathrm{E}+02$ & $8.09 \mathrm{E}+01$ & 1 & $0.00 \mathrm{E}+00$ & $1.78 \mathrm{E}+03$ \\
\hline $\mathrm{PO}_{4}{ }^{3-}$.ic.wo & $\mu \mathrm{g} / \mathrm{mL}$ & $1.56 \mathrm{E}+03$ & $9.06 \mathrm{E}+02$ & 1 & $0.00 \mathrm{E}+00$ & $1.31 \mathrm{E}+04$ \\
\hline $\mathrm{PO}_{4}{ }^{3-}$.ic.wo.ed * & $\mu \mathrm{g} / \mathrm{mL}$ & $8.39 \mathrm{E}+02$ & $1.64 \mathrm{E}+02$ & 1 & $0.00 \mathrm{E}+00$ & $2.93 \mathrm{E}+03$ \\
\hline Pb.icp.adl.w & $\mu \mathrm{g} / \mathrm{mL}$ & $5.38 \mathrm{E}+01$ & $1.43 \mathrm{E}+00$ & 1 & $3.57 \mathrm{E}+01$ & $7.20 \mathrm{E}+01$ \\
\hline Pb.icp.adl.wo & $\mu \mathrm{g} / \mathrm{mL}$ & $4.95 \mathrm{E}+01$ & $1.60 \mathrm{E}+00$ & 1 & $2.92 \mathrm{E}+01$ & $6.98 \mathrm{E}+01$ \\
\hline S.icp.adl & $\mu \mathrm{g} / \mathrm{mL}$ & $9.37 \mathrm{E}+02$ & $1.93 E+01$ & 1 & $6.91 E+02$ & $1.18 \mathrm{E}+03$ \\
\hline $\mathrm{SO}_{4}^{2-}$.ic. W & $\mu \mathrm{g} / \mathrm{mL}$ & $1.18 \mathrm{E}+03$ & $1.05 \mathrm{E}+02$ & 1 & $0.00 \mathrm{E}+00$ & $2.52 \mathrm{E}+03$ \\
\hline $\mathrm{SO}_{4}^{2-}$ ic.w.ed & $\mu \mathrm{g} / \mathrm{mL}$ & $1.10 \mathrm{E}+03$ & $2.68 \mathrm{E}+01$ & 1 & $7.61 \mathrm{E}+02$ & $1.44 \mathrm{E}+03$ \\
\hline $\mathrm{SO}_{4}^{2-}$.ic.wo & $\mu \mathrm{g} / \mathrm{mL}$ & $1.18 \mathrm{E}+03$ & $1.01 \mathrm{E}+02$ & 1 & $0.00 \mathrm{E}+00$ & $2.46 \mathrm{E}+03$ \\
\hline $\mathrm{SO}_{4}{ }^{2-}$.ic.wo.ed & $\mu \mathrm{g} / \mathrm{mL}$ & $1.09 \mathrm{E}+03$ & $2.31 \mathrm{E}+01$ & 1 & $7.96 \mathrm{E}+02$ & $1.38 \mathrm{E}+03$ \\
\hline Si.icp.adl & $\mu \mathrm{g} / \mathrm{mL}$ & $2.18 \mathrm{E}+02$ & $5.60 \mathrm{E}+00$ & 1 & $1.47 \mathrm{E}+02$ & $2.89 \mathrm{E}+02$ \\
\hline SpG * & $\ldots$ & $1.42 \mathrm{E}+00$ & $2.59 \mathrm{E}-02$ & 1 & $1.09 \mathrm{E}+00$ & $1.75 \mathrm{E}+00$ \\
\hline Zn.icp.adl * & $\mu \mathrm{g} / \mathrm{mL}$ & $3.12 \mathrm{E}+01$ & $6.83 \mathrm{E}+00$ & 1 & $0.00 \mathrm{E}+00$ & $1.18 \mathrm{E}+02$ \\
\hline
\end{tabular}

Notes:

* $=\hat{\sigma}_{\text {riser }}$ is significantly different from zero (evidence of horizontal heterogeneity).

${ }^{1} \mathrm{Br}$ is one of the tracers for hydrostatic head fluid. 
Table B3-10. Summary Statistics - Supernatant (One-way ANOVA). (2 sheets)

\begin{tabular}{|c|c|c|c|c|c|c|}
\hline Halo & Grits & 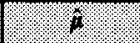 & 8 & or & Wy & Y) \\
\hline$\%$ water.tga & wt \% & $4.99 \mathrm{E}+01$ & $3.70 \mathrm{E}-01$ & 26 & $4.91 \mathrm{E}+01$ & $5.07 \mathrm{E}+01$ \\
\hline \%water.tga.ed & wt\% & $5.03 \mathrm{E}+01$ & $1.04 \mathrm{E}-01$ & 26 & $5.00 \mathrm{E}+01$ & $5.05 \mathrm{E}+01$ \\
\hline Acetate.ic.w & $\mu \mathrm{g} / \mathrm{mL}$ & $5.98 \mathrm{E}+02$ & $3.97 \mathrm{E}+01$ & 26 & $5.16 \mathrm{E}+02$ & $6.80 \mathrm{E}+02$ \\
\hline Acetate.ic.wo & $\mu \mathrm{g} / \mathrm{mL}$ & $6.12 E+02$ & $4.12 \mathrm{E}+01$ & 25 & $5.27 \mathrm{E}+02$ & $6.96 \mathrm{E}+02$ \\
\hline Ag.icp.adl & $\mu \mathrm{g} / \mathrm{mL}$ & $1.78 \mathrm{E}+01$ & $1.90 \mathrm{E}-01$ & 26 & $1.74 \mathrm{E}+01$ & $1.82 \mathrm{E}+01$ \\
\hline Al.icp.adl & $\mu \mathrm{g} / \mathrm{mL}$ & $4.17 E+04$ & $4.81 E+02$ & 26 & $4.07 \mathrm{E}+04$ & $4.26 \mathrm{E}+04$ \\
\hline B.icp.adl & $\mu \mathrm{g} / \mathrm{mL}$ & $7.44 \mathrm{E}+01$ & $7.37 \mathrm{E}-01$ & 26 & $7.28 \mathrm{E}+01$ & $7.59 \mathrm{E}+01$ \\
\hline${ }^{1} \mathrm{Br}$.ic.w & $\mu \mathrm{g} / \mathrm{mL}$ & $9.66 \mathrm{E}+02$ & $5.49 \mathrm{E}+01$ & 26 & $8.54 \mathrm{E}+02$ & $1.08 \mathrm{E}+03$ \\
\hline${ }^{1} \mathrm{Br}$.ic.wo & $\mu \mathrm{g} / \mathrm{mL}$ & $9.78 \mathrm{E}+02$ & $5.37 \mathrm{E}+01$ & 24 & $8.68 \mathrm{E}+02$ & $1.09 \mathrm{E}+03$ \\
\hline $\mathrm{Cl}^{-} . \mathrm{ic}$ & $\mu \mathrm{g} / \mathrm{mL}$ & $1.02 \mathrm{E}+04$ & $6.50 \mathrm{E}+02$ & 26 & $8.91 \mathrm{E}+03$ & $1.16 \mathrm{E}+04$ \\
\hline Cl.ic.ed & $\mu \mathrm{g} / \mathrm{mL}$ & $9.92 \mathrm{E}+03$ & $8.50 \mathrm{E}+01$ & 24 & $9.75 \mathrm{E}+03$ & $1.01 \mathrm{E}+04$ \\
\hline Cr.icp.adl & $\mu \mathrm{g} / \mathrm{mL}$ & $2.25 E+02$ & $8.02 \mathrm{E}+00$ & 26 & $2.08 \mathrm{E}+02$ & $2.41 \mathrm{E}+02$ \\
\hline DSC.dry & $\mathrm{J} / \mathrm{g}$ dry & $2.17 \mathrm{E}+01$ & $8.53 \mathrm{E}+00$ & 26 & $4.19 \mathrm{E}+00$ & $3.92 \mathrm{E}+01$ \\
\hline DSC.wet & $\mathrm{J} / \mathrm{g}$ wet & $1.08 \mathrm{E}+01$ & $4.23 E+00$ & 26 & $2.11 E+00$ & $1.95 \mathrm{E}+01$ \\
\hline F-ic.w & $\mu \mathrm{g} / \mathrm{mL}$ & $3.10 \mathrm{E}+02$ & $3.67 \mathrm{E}+01$ & 26 & $2.35 \mathrm{E}+02$ & $3.86 \mathrm{E}+02$ \\
\hline F-ic.w.ed & $\mu \mathrm{g} / \mathrm{mL}$ & $2.93 \mathrm{E}+02$ & $3.70 \mathrm{E}+01$ & 26 & $2.17 \mathrm{E}+02$ & $3.69 \mathrm{E}+02$ \\
\hline Formate.ic & $\mu \mathrm{g} / \mathrm{mL}$ & $1.56 \mathrm{E}+03$ & $7.99 \mathrm{E}+01$ & 26 & $1.39 \mathrm{E}+03$ & $1.72 \mathrm{E}+03$ \\
\hline K.icp.adl & $\mu \mathrm{g} / \mathrm{mL}$ & $6.56 E+03$ & $6.38 \mathrm{E}+01$ & 26 & $6.43 E+03$ & $6.69 E+03$ \\
\hline Mo.icp.adl & $\mu \mathrm{g} / \mathrm{mL}$ & $9.67 \mathrm{E}+01$ & $9.87 \mathrm{E}-01$ & 26 & $9.46 \mathrm{E}+01$ & $9.87 \mathrm{E}+01$ \\
\hline $\mathrm{NO}_{2}^{-}$.ic & $\mu \mathrm{g} / \mathrm{mL}$ & $1.27 \mathrm{E}+05$ & $8.54 \mathrm{E}+03$ & 26 & $1.09 \mathrm{E}+05$ & $1.44 \mathrm{E}+05$ \\
\hline $\mathrm{NO}_{2}^{-}$.ic.ed & $\mu \mathrm{g} / \mathrm{mL}$ & $1.22 \mathrm{E}+05$ & $1.11 \mathrm{E}+03$ & 24 & $1.20 \mathrm{E}+05$ & $1.25 \mathrm{E}+05$ \\
\hline $\mathrm{NO}_{3}^{-} \cdot \mathrm{ic}$ & $\mu \mathrm{g} / \mathrm{mL}$ & $1.66 E+05$ & $1.09 \mathrm{E}+04$ & 26 & $1.44 \mathrm{E}+05$ & $1.88 \mathrm{E}+05$ \\
\hline $\mathrm{NO}_{3}^{-}$.ic.ed & $\mu \mathrm{g} / \mathrm{mL}$ & $1.61 E+05$ & $1.75 \mathrm{E}+03$ & 24 & $1.57 \mathrm{E}+05$ & $1.64 \mathrm{E}+05$ \\
\hline Na.icp.adl & $\mu \mathrm{g} / \mathrm{mL}$ & $2.47 \mathrm{E}+05$ & $2.32 \mathrm{E}+03$ & 26 & $2.42 \mathrm{E}+05$ & $2.52 \mathrm{E}+05$ \\
\hline P.icp.adl & $\mu \mathrm{g} / \mathrm{mL}$ & $4.00 \mathrm{E}+02$ & $2.11 \mathrm{E}+01$ & 26 & $3.57 \mathrm{E}+02$ & $4.43 \mathrm{E}+02$ \\
\hline P.icp.adl.ed & $\mu \mathrm{g} / \mathrm{mL}$ & $3.85 \mathrm{E}+02$ & $1.33 \mathrm{E}+01$ & 26 & $3.57 \mathrm{E}+02$ & $4.12 E+02$ \\
\hline $\mathrm{PO}_{4}{ }^{3-}$.ic.w & $\mu \mathrm{g} / \mathrm{mL}$ & $1.06 \mathrm{E}+03$ & $3.09 \mathrm{E}+02$ & 26 & $4.29 E+02$ & $1.70 \mathrm{E}+03$ \\
\hline $\mathrm{PO}_{4}{ }^{3}$.ic.w.ed & $\mu \mathrm{g} / \mathrm{mL}$ & $7.55 \mathrm{E}+02$ & $5.21 \mathrm{E}+01$ & 26 & $6.48 \mathrm{E}+02$ & $8.62 \mathrm{E}+02$ \\
\hline $\mathrm{PO}_{4}{ }^{3 \cdot}$.ic.wo & $\mu \mathrm{g} / \mathrm{mL}$ & $1.54 \mathrm{E}+03$ & $7.18 \mathrm{E}+02$ & 22 & $5.42 \mathrm{E}+01$ & $3.03 E+03$ \\
\hline Pb.icp.adl.w & $\mu \mathrm{g} / \mathrm{mL}$ & $5.38 \mathrm{E}+01$ & $1.21 \mathrm{E}+00$ & 26 & $5.13 \mathrm{E}+01$ & $5.63 \mathrm{E}+01$ \\
\hline Pb.icp.adl.wo & $\mu \mathrm{g} / \mathrm{mL}$ & $4.99 \mathrm{E}+01$ & $1.11 \mathrm{E}+00$ & 16 & $4.75 \mathrm{E}+01$ & $5.22 \mathrm{E}+01$ \\
\hline
\end{tabular}


Table B3-10. Summary Statistics - Supernatant (One-way ANOVA). (2 sheets)

\begin{tabular}{|c|c|c|c|c|c|c|}
\hline Wnalye & Miniss. & \% & \% & all & Why & W. \\
\hline S.icp.adl & $\mu \mathrm{g} / \mathrm{mL}$ & $9.37 \mathrm{E}+02$ & $1.93 \mathrm{E}+01$ & 26 & $8.97 \mathrm{E}+02$ & $9.76 \mathrm{E}+02$ \\
\hline $\mathrm{SO}_{4}{ }^{2-}$.ic.w & $\mu \mathrm{g} / \mathrm{mL}$ & $1.18 \mathrm{E}+03$ & $8.60 \mathrm{E}+01$ & 26 & $1.01 \mathrm{E}+03$ & $1.36 \mathrm{E}+03$ \\
\hline $\mathrm{SO}_{4}^{2-}$.ic.w.ed & $\mu \mathrm{g} / \mathrm{mL}$ & $1.10 \mathrm{E}+03$ & $2.50 \mathrm{E}+01$ & 25 & $1.05 \mathrm{E}+03$ & $1.15 \mathrm{E}+03$ \\
\hline $\mathrm{SO}_{4}{ }^{2-}$.ic.wo & $\mu \mathrm{g} / \mathrm{mL}$ & $1.18 \mathrm{E}+03$ & $8.90 \mathrm{E}+01$ & 25 & $9.92 \mathrm{E}+02$ & $1.36 \mathrm{E}+03$ \\
\hline $\mathrm{SO}_{4}{ }^{2-}$.ic.wo.ed & $\mu \mathrm{g} / \mathrm{mL}$ & $1.09 \mathrm{E}+03$ & $2.31 \mathrm{E}+01$ & 24 & $1.04 \mathrm{E}+03$ & $1.14 \mathrm{E}+03$ \\
\hline Si.icp.adlb & $\mu \mathrm{g} / \mathrm{mLbm}$ & $2.18 \mathrm{E}+02$ & $5.60 \mathrm{E}+00$ & 26 & $2.07 \mathrm{E}+02$ & $2.30 \mathrm{E}+02$ \\
\hline
\end{tabular}

Note:

${ }^{1} \mathrm{Br}$ is one of the tracers for hydrostatic head fluid. 
Table B3-11. Analytes (Supernatant) with $>50$ Percent "Less Than" Values.

\begin{tabular}{|l|l|l|}
\hline \multicolumn{1}{|c|}{ Analyte } & \multicolumn{1}{|c|}{ Unil } & \\
\hline As.icp.adl & $\mu \mathrm{g} / \mathrm{mL}$ & $<60.1$ \\
\hline Ba.icp.adl & $\mu \mathrm{g} / \mathrm{mL}$ & $<30.1$ \\
\hline Be.icp.adl & $\mu \mathrm{g} / \mathrm{mL}$ & $<3$ \\
\hline Bi.icp.adl & $\mu \mathrm{g} / \mathrm{mL}$ & $<60.1$ \\
\hline Ca.icp.adl & $\mu \mathrm{g} / \mathrm{mL}$ & $<60.1$ \\
\hline Cd.icp.adl & $\mu \mathrm{g} / \mathrm{mL}$ & $<3$ \\
\hline Ce.icp.adl & $\mu \mathrm{g} / \mathrm{mL}$ & $<60.1$ \\
\hline Co.icp.adl & $\mu \mathrm{g} / \mathrm{mL}$ & $<12$ \\
\hline Cu.icp.adl & $\mu \mathrm{g} / \mathrm{mL}$ & 10.2 \\
\hline Fe.icp.adl & $\mu \mathrm{g} / \mathrm{mL}$ & $<30.1$ \\
\hline La.icp.adl & $\mu \mathrm{g} / \mathrm{mL}$ & $<30.1$ \\
\hline Li.icp.adl & $\mu \mathrm{g} / \mathrm{mL}$ & 27.3 \\
\hline Mg.icp.adl & $\mu \mathrm{g} / \mathrm{mL}$ & $<60.1$ \\
\hline Mn.icp.adl & $\mu \mathrm{g} / \mathrm{mL}$ & $<6.01$ \\
\hline Nd.icp.adl & $\mu \mathrm{g} / \mathrm{mL}$ & $<60.1$ \\
\hline Ni.icp.adl & $\mu \mathrm{g} / \mathrm{mL}$ & $<12$ \\
\hline Oxalate.ic & $\mu \mathrm{g} / \mathrm{mL}$ & 16100 \\
\hline Sb.icp.adl & $\mu \mathrm{g} / \mathrm{mL}$ & $<36.1$ \\
\hline Se.icp.adl & $\mu \mathrm{g} / \mathrm{mL}$ & $<60.1$ \\
\hline Sm.icp.adl & $\mu \mathrm{g} / \mathrm{mL}$ & $<60.1$ \\
\hline Sr.icp.adl & $\mu \mathrm{g} / \mathrm{mL}$ & $<6.01$ \\
\hline Ti.icp.adl & $\mu \mathrm{g} / \mathrm{mL}$ & $<6.01$ \\
\hline Tl.icp.adl & $\mu \mathrm{g} / \mathrm{mL}$ & $<120$ \\
\hline Total Alpha & $\mu \mathrm{Ci} / \mathrm{mL}$ & $<0.145$ \\
\hline U.icp.adl & $\mu \mathrm{g} / \mathrm{mL}$ & $<300$ \\
\hline V.icp.adl & $\mu \mathrm{g} / \mathrm{mL}$ & 6.09 \\
\hline Zr.icp.adl & $\mu \mathrm{g} / \mathrm{mL}$ & \\
\hline & & $<30.1$ \\
\hline
\end{tabular}

Note:

${ }^{1} \mathrm{Li}$ is one of the tracers for hydrostatic head fluid. 
A supernatant composite sample was formed from supernatant subsamples from Core 152 segments 1-14. The arithmetic mean, the associated variability, and a two-sided 95 percent confidence interval for the mean concentration were calculated for each analyte with at least 50 percent of the reported data as quantitative values. The confidence interval takes into account only the analytical uncertainty. The summary statistics are listed in Table B3-12 and Table B3-13 contains the analytes which had less than 50 percent of the reported data as quantative values.

Table B3-12. Summary Statistics - Supernatant Composite (Core 152, Segments 1-14). (2 sheets)

\begin{tabular}{|c|c|c|c|c|c|c|}
\hline 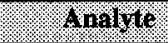 & Wints & 12 & 6 & ar & W. & 11. \\
\hline$\%$ water.tga & wt\% & $5.01 \mathrm{E}+01$ & $3.45 \mathrm{E}+00$ & 1 & $6.28 \mathrm{E}+00$ & $9.40 \mathrm{E}+01$ \\
\hline Acetate.ic & $\mu \mathrm{g} / \mathrm{mL}$ & $6.42 \mathrm{E}+02$ & $5.00 \mathrm{E}-01$ & 1 & $6.35 \mathrm{E}+02$ & $6.48 \mathrm{E}+02$ \\
\hline Ag.icp.a & $\mu \mathrm{g} / \mathrm{mL}$ & $1.52 \mathrm{E}+01$ & $2.50 \mathrm{E}-01$ & 1 & $1.20 \mathrm{E}+01$ & $1.83 \mathrm{E}+01$ \\
\hline Ag.icp.adl & $\mu \mathrm{g} / \mathrm{mL}$ & $1.80 \mathrm{E}+01$ & $5.00 \mathrm{E}-01$ & 1 & $1.16 \mathrm{E}+01$ & $2.44 \mathrm{E}+01$ \\
\hline Al.icp.a & $\mu \mathrm{g} / \mathrm{mL}$ & $3.57 \mathrm{E}+04$ & $1.00 \mathrm{E}+02$ & 1 & $3.44 \mathrm{E}+04$ & $3.70 \mathrm{E}+04$ \\
\hline Al.icp.adl & $\mu \mathrm{g} / \mathrm{mL}$ & $4.36 \mathrm{E}+04$ & $2.00 \mathrm{E}+02$ & 1 & $4.11 \mathrm{E}+04$ & $4.61 E+04$ \\
\hline B.icp.a & $\mu \mathrm{g} / \mathrm{mL}$ & $1.70 E+02$ & $1.35 \mathrm{E}+01$ & 1 & $0.00 \mathrm{E}+00$ & $3.41 \mathrm{E}+02$ \\
\hline B.icp.adl & $\mu \mathrm{g} / \mathrm{mL}$ & $7.28 \mathrm{E}+01$ & $1.45 \mathrm{E}+00$ & 1 & $5.43 E+01$ & $9.12 \mathrm{E}+01$ \\
\hline Ca.icp.a & $\mu \mathrm{g} / \mathrm{mL}$ & $8.78 \mathrm{E}+02$ & $2.50 \mathrm{E}+01$ & 1 & $5.60 E+02$ & $1.20 \mathrm{E}+03$ \\
\hline $\mathrm{Cl}^{\text {. ic }}$ & $\mu \mathrm{g} / \mathrm{mL}$ & $1.02 \mathrm{E}+04$ & $0.00 \mathrm{E}+00$ & 1 & $1.02 \mathrm{E}+04$ & $1.02 \mathrm{E}+04$ \\
\hline Cr.icp.a & $\mu \mathrm{g} / \mathrm{mL}$ & $1.87 \mathrm{E}+02$ & $0.00 \mathrm{E}+00$ & 1 & $1.87 \mathrm{E}+02$ & $1.87 \mathrm{E}+02$ \\
\hline Cr.icp.adl & $\mu \mathrm{g} / \mathrm{mL}$ & $2.23 \mathrm{E}+02$ & $1.50 \mathrm{E}+00$ & 1 & $2.03 \mathrm{E}+02$ & $2.42 \mathrm{E}+02$ \\
\hline Cr(VI).spec & $\mu \mathrm{g} / \mathrm{mL}$ & $1.54 \mathrm{E}+02$ & $5.00 \mathrm{E}-01$ & 1 & $1.47 \mathrm{E}+02$ & $1.60 \mathrm{E}+02$ \\
\hline${ }^{137}$ Cs.gea & $\mu \mathrm{Ci} / \mathrm{mL}$ & $4.75 \mathrm{E}+02$ & $1.50 \mathrm{E}+00$ & 1 & $4.55 \mathrm{E}+02$ & $4.94 \mathrm{E}+02$ \\
\hline DSC.dry & $\mathrm{J} / \mathrm{g}$ dry & $0.00 \mathrm{E}+00$ & $0.00 \mathrm{E}+00$ & 1 & $0.00 \mathrm{E}+00$ & $0.00 \mathrm{E}+00$ \\
\hline DSC.wet & $\mathrm{J} / \mathrm{g}$ wet & $0.00 \mathrm{E}+00$ & $0.00 \mathrm{E}+00$ & 1 & $0.00 \mathrm{E}+00$ & $0.00 \mathrm{E}+00$ \\
\hline Fe.icp.a & $\mu \mathrm{g} / \mathrm{mL}$ & $6.38 \mathrm{E}+01$ & $8.70 \mathrm{E}+00$ & 1 & $0.00 \mathrm{E}+00$ & $1.74 \mathrm{E}+02$ \\
\hline Formate.ic & $\mu \mathrm{g} / \mathrm{mL}$ & $1.62 \mathrm{E}+03$ & $0.00 \mathrm{E}+00$ & 1 & $1.62 \mathrm{E}+03$ & $1.62 \mathrm{E}+03$ \\
\hline${ }^{129} \mathrm{I}$ & $\mu \mathrm{Ci} / \mathrm{mL}$ & $2.68 \mathrm{E}-04$ & $2.15 \mathrm{E}-05$ & 1 & $0.00 \mathrm{E}+00$ & $5.41 \mathrm{E}-04$ \\
\hline K.icp.a & $\mu \mathrm{g} / \mathrm{mL}$ & $5.31 \mathrm{E}+03$ & $3.50 \mathrm{E}+01$ & 1 & $4.86 \mathrm{E}+03$ & $5.75 \mathrm{E}+03$ \\
\hline K.icp.adl & $\mu \mathrm{g} / \mathrm{mL}$ & $6.52 \mathrm{E}+03$ & $3.00 \mathrm{E}+01$ & 1 & $6.14 \mathrm{E}+03$ & $6.90 \mathrm{E}+03$ \\
\hline${ }^{1}$ Li.icp.a & $\mu \mathrm{g} / \mathrm{mL}$ & $5.97 \mathrm{E}+00$ & $1.45 \mathrm{E}-01$ & 1 & $4.12 \mathrm{E}+00$ & $7.81 \mathrm{E}+00$ \\
\hline Mo.icp.a & $\mu \mathrm{g} / \mathrm{mL}$ & $8.26 \mathrm{E}+01$ & $2.50 \mathrm{E}-01$ & 1 & $7.94 \mathrm{E}+01$ & $8.57 \mathrm{E}+01$ \\
\hline
\end{tabular}


Table B3-12. Summary Statistics - Supernatant Composite (Core 152, Segments 1-14). (2 sheets)

\begin{tabular}{|c|c|c|c|c|c|c|}
\hline f inglyte & Units. & 19 & $\delta_{2}$ & if & III & U1: \\
\hline Mo.icp.adl & $\mu \mathrm{g} / \mathrm{mL}$ & $1.01 \mathrm{E}+02$ & $1.00 \mathrm{E}+00$ & 1 & $8.83 \mathrm{E}+01$ & $1.14 \mathrm{E}+02$ \\
\hline $\mathrm{NO}_{2}^{-}$-ic & $\mu \mathrm{g} / \mathrm{mL}$ & $1.31 \mathrm{E}+05$ & $5.00 \mathrm{E}+02$ & 1 & $1.24 \mathrm{E}+05$ & $1.37 \mathrm{E}+05$ \\
\hline $\mathrm{NO}_{3}^{-}$.ic & $\mu \mathrm{g} / \mathrm{mL}$ & $1.68 \mathrm{E}+05$ & $5.00 \mathrm{E}+02$ & 1 & $1.61 \mathrm{E}+05$ & $1.74 \mathrm{E}+05$ \\
\hline Na.icp.a & $\mu \mathrm{g} / \mathrm{mL}$ & $2.11 \mathrm{E}+05$ & $0.00 \mathrm{E}+00$ & 1 & $2.11 \mathrm{E}+05$ & $2.11 \mathrm{E}+05$ \\
\hline Na.icp.adl & $\mu \mathrm{g} / \mathrm{mL}$ & $2.56 \mathrm{E}+05$ & $1.50 \mathrm{E}+03$ & 1 & $2.36 \mathrm{E}+05$ & $2.75 E+05$ \\
\hline$\overline{\mathrm{OH}^{-}}$ & $\mu \mathrm{g} / \mathrm{mL}$ & $5.86 \mathrm{E}+04$ & $1.95 \mathrm{E}+03$ & 1 & $3.38 \mathrm{E}+04$ & $8.33 \mathrm{E}+04$ \\
\hline P.icp.a & $\mu \mathrm{g} / \mathrm{mL}$ & $3.40 \mathrm{E}+02$ & $3.50 \mathrm{E}+00$ & 1 & $2.95 \mathrm{E}+02$ & $3.84 \mathrm{E}+02$ \\
\hline P.icp.adl & $\mu \mathrm{g} / \mathrm{mL}$ & $3.93 \mathrm{E}+02$ & $2.50 \mathrm{E}+00$ & 1 & $3.61 \mathrm{E}+02$ & $4.24 \mathrm{E}+02$ \\
\hline $\mathrm{PO}_{4}{ }^{3-}$. ic & $\mu \mathrm{g} / \mathrm{mL}$ & $6.17 \mathrm{E}+02$ & $1.85 \mathrm{E}+01$ & 1 & $3.81 \mathrm{E}+02$ & $8.52 \mathrm{E}+02$ \\
\hline S.icp.a & $\mu \mathrm{g} / \mathrm{mL}$ & $8.77 \mathrm{E}+02$ & $3.00 \mathrm{E}+00$ & 1 & $8.39 \mathrm{E}+02$ & $9.15 \mathrm{E}+02$ \\
\hline S.icp.adl & $\mu \mathrm{g} / \mathrm{mL}$ & $1.01 \mathrm{E}+03$ & $1.85 \mathrm{E}+01$ & 1 & $7.76 \mathrm{E}+02$ & $1.25 \mathrm{E}+03$ \\
\hline $\mathrm{SO}_{4}{ }^{2-} . \mathrm{ic}$ & $\mu \mathrm{g} / \mathrm{mL}$ & $9.70 \mathrm{E}+02$ & $4.05 \mathrm{E}+01$ & 1 & $4.55 \mathrm{E}+02$ & $1.48 \mathrm{E}+03$ \\
\hline Si.icp.a & $\mu \mathrm{g} / \mathrm{mL}$ & $2.20 \mathrm{E}+02$ & $1.15 \mathrm{E}+01$ & 1 & $7.34 \mathrm{E}+01$ & $3.66 \mathrm{E}+02$ \\
\hline Si.icp.adl & $\mu \mathrm{g} / \mathrm{mL}$ & $1.66 \mathrm{E}+02$ & $0.00 \mathrm{E}+00$ & 1 & $1.66 \mathrm{E}+02$ & $1.66 \mathrm{E}+02$ \\
\hline${ }^{89 / 90} \mathrm{Sr}$ & $\mu \mathrm{Ci} / \mathrm{mL}$ & $4.14 \mathrm{E}-02$ & $6.00 \mathrm{E}-04$ & 1 & $3.38 \mathrm{E}-02$ & $4.90 \mathrm{E}-02$ \\
\hline TIC & $\mu \mathrm{g} / \mathrm{mL}$ & $2.20 \mathrm{E}+03$ & $2.50 \mathrm{E}+01$ & 1 & $1.88 \mathrm{E}+03$ & $2.51 \mathrm{E}+03$ \\
\hline TOC.coul & $\mu \mathrm{g} / \mathrm{mL}$ & $6.10 \mathrm{E}+03$ & $3.00 \mathrm{E}+01$ & 1 & $5.72 \mathrm{E}+03$ & $6.48 \mathrm{E}+03$ \\
\hline TOC.pers & $\mu \mathrm{g} / \mathrm{mL}$ & $2.59 \mathrm{E}+03$ & $1.50 \mathrm{E}+02$ & 1 & $6.84 \mathrm{E}+02$ & $4.50 \mathrm{E}+03$ \\
\hline${ }^{99} \mathrm{Tc}$ & $\mu \mathrm{Ci} / \mathrm{mL}$ & $2.74 \mathrm{E}-01$ & $5.50 \mathrm{E}-03$ & 1 & $2.04 \mathrm{E}-01$ & $3.43 \mathrm{E}-01$ \\
\hline Total Beta & $\mu \mathrm{Ci} / \mathrm{mL}$ & $5.38 \mathrm{E}+02$ & $4.00 \mathrm{E}+00$ & 1 & $4.87 \mathrm{E}+02$ & $5.89 \mathrm{E}+02$ \\
\hline Zn.icp.a & $\mu \mathrm{g} / \mathrm{mL}$ & $7.61 \mathrm{E}+01$ & $1.60 \mathrm{E}+00$ & 1 & $5.58 \mathrm{E}+01$ & $9.64 \mathrm{E}+01$ \\
\hline Zn.icp.adl & $\mu \mathrm{g} / \mathrm{mL}$ & $1.76 \mathrm{E}+01$ & $5.00 \mathrm{E}-01$ & 1 & $1.12 \mathrm{E}+01$ & $2.40 \mathrm{E}+01$ \\
\hline SpG & -- & $1.42 \mathrm{E}+00$ & $7.00 \mathrm{E}-03$ & 1 & $1.33 \mathrm{E}+00$ & $1.50 \mathrm{E}+00$ \\
\hline U.phos & $\mu \mathrm{g} / \mathrm{mL}$ & $3.57 \mathrm{E}+00$ & \begin{tabular}{|l|}
$1.95 \mathrm{E}-01$ \\
\end{tabular} & 1 & $1.09 \mathrm{E}+00$ & $6.04 \mathrm{E}+00$ \\
\hline
\end{tabular}

Note:

${ }^{1} \mathrm{Li}$ is one of the tracers for hydrostatic head fluid. 
HNF-SD-WM-ER-678 Rev. 0

Table B3-13. Supernatant Composite (Core 152, Segments 1-14) Analytes with $>50$ Percent "Less Than" Values. ( 3 sheets)

\begin{tabular}{|c|c|c|}
\hline . & Orit? & Maximinn Resill \\
\hline${ }^{241} \mathrm{Am}$. aea & $\mu \mathrm{Ci} / \mathrm{mL}$ & $<3.91 \mathrm{E}-05$ \\
\hline${ }^{241} \mathrm{Am}$.gea & $\mu \mathrm{Ci} / \mathrm{mL}$ & $<8.57 \mathrm{E}-01$ \\
\hline As.icp.a & $\mu \mathrm{g} / \mathrm{mL}$ & $<5.24 \mathrm{E}+01$ \\
\hline As.icp.adl & $\mu \mathrm{g} / \mathrm{mL}$ & $<6.01 \mathrm{E}+01$ \\
\hline Ba.icp.a & $\mu \mathrm{g} / \mathrm{mL}$ & $<2.62 \mathrm{E}+01$ \\
\hline Ba.icp.adl & $\mu \mathrm{g} / \mathrm{mL}$ & $<3.01 \mathrm{E}+01$ \\
\hline Be.icp.a & $\mu \mathrm{g} / \mathrm{mL}$ & $<2.62 \mathrm{E}+00$ \\
\hline Be.icp.adl & $\mu \mathrm{g} / \mathrm{mL}$ & $<3.00 \mathrm{E}+00$ \\
\hline Bi.icp.a & $\mu \mathrm{g} / \mathrm{mL}$ & $<5.24 \mathrm{E}+01$ \\
\hline Bi.icp.adl & $\mu \mathrm{g} / \mathrm{mL}$ & $<6.01 \mathrm{E}+01$ \\
\hline${ }^{1} \mathrm{Br}$.ic & $\mu \mathrm{g} / \mathrm{mL}$ & $<5.22 \mathrm{E}+02$ \\
\hline Ca.icp.adl & $\mu \mathrm{g} / \mathrm{mL}$ & $<6.01 \mathrm{E}+01$ \\
\hline Cd.icp.a & $\mu \mathrm{g} / \mathrm{mL}$ & $<2.62 \mathrm{E}+00$ \\
\hline Cd.icp.adl & $\mu \mathrm{g} / \mathrm{mL}$ & $<3.00 \mathrm{E}+00$ \\
\hline Ce.icp.a & $\mu \mathrm{g} / \mathrm{mL}$ & $<5.24 \mathrm{E}+01$ \\
\hline Ce.icp.adl & $\mu \mathrm{g} / \mathrm{mL}$ & $<6.01 \mathrm{E}+01$ \\
\hline${ }^{243 / 244} \mathrm{Cm}$ & $\mu \mathrm{Ci} / \mathrm{mL}$ & $<3.91 \mathrm{E}-05$ \\
\hline Co.icp.a & $\mu \mathrm{g} / \mathrm{mL}$ & $<1.05 \mathrm{E}+01$ \\
\hline Co.icp.adl & $\mu \mathrm{g} / \mathrm{mL}$ & $<1.20 \mathrm{E}+01$ \\
\hline${ }^{60} \mathrm{Co}$.gea & $\mu \mathrm{Ci} / \mathrm{mL}$ & $<1.30 \mathrm{E}-02$ \\
\hline Cu.icp.a & $\mu \mathrm{g} / \mathrm{mL}$ & $<6.29 \mathrm{E}+00$ \\
\hline Cu.icp.adl & $\mu \mathrm{g} / \mathrm{mL}$ & $<6.01 E+00$ \\
\hline${ }^{154}$ Eu.gea & $\mu \mathrm{Ci} / \mathrm{mL}$ & $<9.74 \mathrm{E}-02$ \\
\hline${ }^{155}$ Eu.gea & $\mu \mathrm{Ci} / \mathrm{mL}$ & $<3.26 \mathrm{E}-01$ \\
\hline F'.ic & $\mu \mathrm{g} / \mathrm{mL}$ & $<5.38 \mathrm{E}+01$ \\
\hline Fe.icp.adl & $\mu \mathrm{g} / \mathrm{mL}$ & $<3.01 \mathrm{E}+01$ \\
\hline Hg.cvaa & $\mu \mathrm{g} / \mathrm{mL}$ & $<7.00 \mathrm{E}-02$ \\
\hline La.icp.a & $\mu \mathrm{g} / \mathrm{mL}$ & $<2.62 \mathrm{E}+01$ \\
\hline La.icp.adl & $\mu \mathrm{g} / \mathrm{mL}$ & $<3.01 \mathrm{E}+01$ \\
\hline${ }^{1}$ Li.icp.adl & $\mu \mathrm{g} / \mathrm{mL}$ & $<6.01 \mathrm{E}+00$ \\
\hline
\end{tabular}


Table B3-13. Supernatant Composite (Core 152, Segments 1-14) Analytes with $>50$ Percent "Less Than" Values. (3 sheets)

\begin{tabular}{|c|c|c|}
\hline \%: : . Intyte & 1.: $6 \mathrm{mi}$ & . Mrainam Reril \\
\hline Mg.icp.a & $\mu \mathrm{g} / \mathrm{mL}$ & $<5.24 \mathrm{E}+01$ \\
\hline Mg.icp.adl & $\mu \mathrm{g} / \mathrm{mL}$ & $<6.01 \mathrm{E}+01$ \\
\hline Mn.icp.a & $\mu \mathrm{g} / \mathrm{mL}$ & $<5.24 \mathrm{E}+00$ \\
\hline Mn.icp.adl & $\mu \mathrm{g} / \mathrm{mL}$ & $<6.01 \mathrm{E}+00$ \\
\hline Nd.icp.a & $\mu \mathrm{g} / \mathrm{mL}$ & $<5.24 \mathrm{E}+01$ \\
\hline Nd.icp.adl & $\mu \mathrm{g} / \mathrm{mL}$ & $<6.01 \mathrm{E}+01$ \\
\hline Ni.icp.a & $\mu \mathrm{g} / \mathrm{mL}$ & $<1.05 \mathrm{E}+01$ \\
\hline Ni.icp.adl & $\mu \mathrm{g} / \mathrm{mL}$ & $<1.20 \mathrm{E}+01$ \\
\hline${ }^{237} \mathrm{~Np}$ & $\mu \mathrm{Ci} / \mathrm{mL}$ & $<2.21 \mathrm{E}-05$ \\
\hline Oxalate.ic & $\mu \mathrm{g} / \mathrm{mL}$ & $<4.35 \mathrm{E}+02$ \\
\hline Pb.icp.a & $\mu \mathrm{g} / \mathrm{mL}$ & $<5.24 \mathrm{E}+01$ \\
\hline Pb.icp.adl & $\mu \mathrm{g} / \mathrm{mL}$ & $<6.01 \mathrm{E}+01$ \\
\hline${ }^{239 / 240} \mathrm{Pu}$ & $\mu \mathrm{Ci} / \mathrm{mL}$ & $<3.72 \mathrm{E}-05$ \\
\hline Sb.icp.a & $\mu \mathrm{g} / \mathrm{mL}$ & $<3.14 \mathrm{E}+01$ \\
\hline Sb.icp.adl & $\mu \mathrm{g} / \mathrm{mL}$ & $<3.61 \mathrm{E}+01$ \\
\hline Se.icp.a & $\mu \mathrm{g} / \mathrm{mL}$ & $<5.24 \mathrm{E}+01$ \\
\hline Se.icp.adl & $\mu \mathrm{g} / \mathrm{mL}$ & $<6.01 \mathrm{E}+01$ \\
\hline Sm.icp.a & $\mu \mathrm{g} / \mathrm{mL}$ & $<5.24 \mathrm{E}+01$ \\
\hline Sm.icp.adl & $\mu \mathrm{g} / \mathrm{mL}$ & $<6.01 \mathrm{E}+01$ \\
\hline Sr.icp.a & $\mu \mathrm{g} / \mathrm{mL}$ & $<5.24 \mathrm{E}+00$ \\
\hline Sr.icp.adl & $\mu \mathrm{g} / \mathrm{mL}$ & $<6.01 \mathrm{E}+00$ \\
\hline Ti.icp.a & $\mu \mathrm{g} / \mathrm{mL}$ & $<5.24 \mathrm{E}+00$ \\
\hline Ti.icp.adl & $\mu \mathrm{g} / \mathrm{mL}$ & $<6.01 \mathrm{E}+00$ \\
\hline T1.icp.a & $\mu \mathrm{g} / \mathrm{mL}$ & $<1.05 \mathrm{E}+02$ \\
\hline Tl.icp.adl & $\mu \mathrm{g} / \mathrm{mL}$ & $<1.20 \mathrm{E}+02$ \\
\hline Total alpha & $\mu \mathrm{Ci} / \mathrm{mL}$ & $<1.54 \mathrm{E}-02$ \\
\hline Tritium & $\mu \mathrm{Ci} / \mathrm{mL}$ & $<3.96 \mathrm{E}-05$ \\
\hline U.icp.a & $\mu \mathrm{g} / \mathrm{mL}$ & $<2.62 \mathrm{E}+02$ \\
\hline U.icp.adl & $\mu \mathrm{g} / \mathrm{mL}$ & $<3.00 \mathrm{E}+02$ \\
\hline V.icp.a & $\mu \mathrm{g} / \mathrm{mL}$ & $<2.62 \mathrm{E}+01$ \\
\hline
\end{tabular}


Table B3-13. Supernatant Composite (Core 152, Segments 1-14) Analytes with $>50$ Percent "Less Than" Values. (3 sheets)

\begin{tabular}{|c|c|c|}
\hline (1) & Unit & Maxinimin Resuir \\
\hline V.icp.adl & $\mu \mathrm{g} / \mathrm{mL}$ & $<3.01 \mathrm{E}+01$ \\
\hline Zr.icp.a & $\mu \mathrm{g} / \mathrm{mL}$ & $<5.24 \mathrm{E}+00$ \\
\hline Zr.icp.adl & $\mu \mathrm{g} / \mathrm{mL}$ & $<6.01 \mathrm{E}+00$ \\
\hline
\end{tabular}

Note:

${ }^{1} \mathrm{Li}$ and ${ }^{1} \mathrm{Br}$ are used for tracers in hydrostatic head fluid.

B3.4.1.2 Salt Slurry (Sludge). The mean concentration estimates for the sludge layer, along with the two-sided 95 percent confidence interval for the mean concentration, are given in Table B3-14 (nested ANOVA with the riser term) and Table B3-15 (nested ANOVA without the riser term) for those analytes with at least 50 percent of the reported data as quantitative values. For some of the analytes, the lower limit of the 95 percent confidence interval was a negative value due to the magnitude of the variability. Because the actual concentration of a tank sample cannot be less than zero, the lower limit is reported as zero. The analytes in Table B3-14 where $\hat{\sigma}_{\text {riser }}$ is significantly different from zero are marked with a "**". The riser variable is an indicator of horizontal homogeneity.

For those analytes where $\hat{\sigma}_{\text {riser }}$ is not significantly different from zero, the mean concentrations and the variances of the mean concentrations calculated using the two statistical models (nested ANOVA with the riser term and nested ANOVA without the riser term) are not significantly different. In these cases, the nested ANOVA model without the riser term is more appropriate. The analytes (sludge layer), which had less than 50 percent of the reported data as quantitative values, are listed in Table B3-16. Table B3-16 cites the largest value observed from the analytical results. 
Table B3-14. Summary Statistics (per g basis) - Sludge (Nested ANOVA with Riser Term). (2 sheets)

\begin{tabular}{|c|c|c|c|c|c|c|}
\hline Mrolute. & Whits & V. & 8 & 10 & 16 & W1. \\
\hline$\%$ water.tga & wt\% & $4.44 \mathrm{E}+01$ & $1.21 \mathrm{E}+00$ & 1 & $2.91 \mathrm{E}+01$ & $5.98 \mathrm{E}+01$ \\
\hline Acetate.ic.w & $\mu \mathrm{g} / \mathrm{g}$ & $5.93 E+02$ & $6.22 \mathrm{E}+01$ & 1 & $0.00 \mathrm{E}+00$ & $1.38 E+03$ \\
\hline Acetate.ic.wo & $\mu \mathrm{g} / \mathrm{g}$ & $6.74 \mathrm{E}+02$ & $2.71 \mathrm{E}+01$ & 1 & $3.29 \mathrm{E}+02$ & $1.02 \mathrm{E}+03$ \\
\hline Ag.icp.a & $\mu \mathrm{g} / \mathrm{g}$ & $1.94 \mathrm{E}+01$ & $5.00 \mathrm{E}+00$ & 1 & $0.00 \mathrm{E}+00$ & $8.30 \mathrm{E}+01$ \\
\hline Al.icp.a & $\mu \mathrm{g} / \mathrm{g}$ & $2.21 \mathrm{E}+04$ & $9.96 \mathrm{E}+02$ & 1 & $9.46 \mathrm{E}+03$ & $3.48 \mathrm{E}+04$ \\
\hline Al.icp.f & $\mu \mathrm{g} / \mathrm{g}$ & $2.31 \mathrm{E}+04$ & $8.27 \mathrm{E}+02$ & 1 & $1.26 \mathrm{E}+04$ & $3.36 \mathrm{E}+04$ \\
\hline B.icp.a * & $\mu \mathrm{g} / \mathrm{g}$ & $8.98 \mathrm{E}+01$ & $1.35 \mathrm{E}+01$ & 1 & $0.00 \mathrm{E}+00$ & $2.61 \mathrm{E}+02$ \\
\hline Ca.icp.a * & $\mu \mathrm{g} / \mathrm{g}$ & $6.42 \mathrm{E}+02$ & $3.32 \mathrm{E}+02$ & 1 & $0.00 \mathrm{E}+00$ & $4.86 \mathrm{E}+03$ \\
\hline Cd.icp.a & $\mu \mathrm{g} / \mathrm{g}$ & $1.16 \mathrm{E}+01$ & $2.02 \mathrm{E}+00$ & 1 & $0.00 \mathrm{E}+00$ & $3.73 \mathrm{E}+01$ \\
\hline $\mathrm{Cl}^{-}$.ic & $\mu \mathrm{g} / \mathrm{g}$ & $5.18 \mathrm{E}+03$ & $2.42 \mathrm{E}+02$ & 1 & $2.10 \mathrm{E}+03$ & $8.25 E+03$ \\
\hline Cr.icp.a & $\mu \mathrm{g} / \mathrm{g}$ & $2.34 \mathrm{E}+03$ & $3.32 E+02$ & 1 & $0.00 \mathrm{E}+00$ & $6.56 \mathrm{E}+03$ \\
\hline Cr.jep.f & $\mu \mathrm{g} / \mathrm{g}$ & $2.48 \mathrm{E}+03$ & $4.43 E+02$ & 1 & $0.00 \mathrm{E}+00$ & $8.11 \mathrm{E}+03$ \\
\hline Cu.icp.a.w & $\mu \mathrm{g} / \mathrm{g}$ & $7.05 \mathrm{E}+00$ & $5.85 \mathrm{E}-01$ & 1 & $0.00 \mathrm{E}+00$ & $1.45 \mathrm{E}+01$ \\
\hline Cu.icp.a.wo & $\mu \mathrm{g} / \mathrm{g}$ & $7.12 \mathrm{E}+00$ & $5.24 \mathrm{E}-01$ & 1 & $4.63 \mathrm{E}-01$ & $1.38 \mathrm{E}+01$ \\
\hline DSC.dry & $\mathrm{J} / \mathrm{g}$ dry & $6.38 \mathrm{E}+01$ & $1.29 \mathrm{E}+01$ & 1 & $0.00 \mathrm{E}+00$ & $2.27 \mathrm{E}+02$ \\
\hline DSC.wet & $\mathrm{J} / \mathrm{g}$ wet & $3.52 \mathrm{E}+01$ & $6.47 \mathrm{E}+00$ & 1 & $0.00 \mathrm{E}+00$ & $1.17 \mathrm{E}+02$ \\
\hline $\mathrm{F}^{-}$.ic & $\mu \mathrm{g} / \mathrm{g}$ & $1.85 \mathrm{E}+03$ & $5.09 \mathrm{E}+02$ & 1 & $0.00 \mathrm{E}+00$ & $8.32 \mathrm{E}+03$ \\
\hline Fe.icp.a & $\mu \mathrm{g} / \mathrm{g}$ & $1.19 \mathrm{E}+02$ & $2.62 \mathrm{E}+01$ & 1 & $0.00 \mathrm{E}+00$ & $4.52 \mathrm{E}+02$ \\
\hline Formate.ic & $\mu \mathrm{g} / \mathrm{g}$ & $1.06 \mathrm{E}+03$ & $3.88 \mathrm{E}+01$ & 1 & $5.67 E+02$ & $1.55 \mathrm{E}+03$ \\
\hline K.icp.a & $\mu \mathrm{g} / \mathrm{g}$ & $3.45 \mathrm{E}+03$ & $1.44 \mathrm{E}+02$ & 1 & $1.62 \mathrm{E}+03$ & $5.28 \mathrm{E}+03$ \\
\hline${ }^{1}$ Li.icp.a.w & $\mu \mathrm{g} / \mathrm{g}$ & $1.97 \mathrm{E}+01$ & $5.02 \mathrm{E}+00$ & 1 & $0.00 \mathrm{E}+00$ & $8.35 \mathrm{E}+01$ \\
\hline${ }^{1}$ Li.icp.a.wo & $\mu \mathrm{g} / \mathrm{g}$ & $2.05 \mathrm{E}+01$ & $4.84 \mathrm{E}+00$ & 1 & $0.00 \mathrm{E}+00$ & $8.20 \mathrm{E}+01$ \\
\hline Mn.icp.a.w & $\mu \mathrm{g} / \mathrm{g}$ & $2.08 \mathrm{E}+01$ & $9.48 \mathrm{E}+00$ & 1 & $0.00 \mathrm{E}+00$ & $1.41 \mathrm{E}+02$ \\
\hline Mn.icp.a.wo & $\mu \mathrm{g} / \mathrm{g}$ & $2.14 \mathrm{E}+01$ & $9.77 \mathrm{E}+00$ & 1 & $0.00 \mathrm{E}+00$ & $1.46 \mathrm{E}+02$ \\
\hline Mo.icp.a* & $\mu \mathrm{g} / \mathrm{g}$ & $5.15 \mathrm{E}+01$ & $3.07 \mathrm{E}+00$ & 1 & $1.25 \mathrm{E}+01$ & $9.04 \mathrm{E}+01$ \\
\hline $\mathrm{NO}_{2}^{-}$.ic & $\mu \mathrm{g} / \mathrm{g}$ & $6.39 E+04$ & $2.50 \mathrm{E}+03$ & 1 & $3.21 \mathrm{E}+04$ & $9.56 \mathrm{E}+04$ \\
\hline $\mathrm{NO}_{3}{ }^{-}$.ic & $\mu \mathrm{g} / \mathrm{g}$ & $1.11 E+05$ & $7.09 E+03$ & 1 & $2.11 \mathrm{E}+04$ & $2.01 \mathrm{E}+05$ \\
\hline Na.icp.a * & $\mu \mathrm{g} / \mathrm{g}$ & $1.83 \mathrm{E}+05$ & $8.73 E+03$ & 1 & $7.17 \mathrm{E}+04$ & $2.94 \mathrm{E}+05$ \\
\hline Na.icp.f* & $\mu \mathrm{g} / \mathrm{g}$ & $2.28 \mathrm{E}+05$ & $5.88 \mathrm{E}+03$ & 1 & $1.53 \mathrm{E}+05$ & $3.03 E+05$ \\
\hline Ni.icp.a & $\mu \mathrm{g} / \mathrm{g}$ & $4.07 E+01$ & $5.14 E+00$ & 1 & $0.00 \mathrm{E}+00$ & $1.06 \mathrm{E}+02$ \\
\hline
\end{tabular}


Table B3-14. Summary Statistics (per g basis) - Sludge (Nested ANOVA with Riser Term). ( 2 sheets)

\begin{tabular}{|c|c|c|c|c|c|c|}
\hline Analyte & Units. & f & \% & drf & W. & (6) \\
\hline Ni.icp.f.w * & $\mu \mathrm{g} / \mathrm{g}$ & $1.36 \mathrm{E}+03$ & $4.89 \mathrm{E}+02$ & 1 & $0.00 \mathrm{E}+00$ & $7.57 \mathrm{E}+03$ \\
\hline Ni.icp.f.wo & $\mu \mathrm{g} / \mathrm{g}$ & $1.74 \mathrm{E}+03$ & $3.64 \mathrm{E}+02$ & 1 & $0.00 \mathrm{E}+00$ & $6.37 E+03$ \\
\hline Oxalate.ic & $\mu \mathrm{g} / \mathrm{g}$ & $6.97 \mathrm{E}+03$ & $1.08 \mathrm{E}+03$ & 1 & $0.00 \mathrm{E}+00$ & $2.07 \mathrm{E}+04$ \\
\hline P.icp.a & $\mu \mathrm{g} / \mathrm{g}$ & $2.46 \mathrm{E}+03$ & $1.66 \mathrm{E}+03$ & 1 & $0.00 \mathrm{E}+00$ & $2.35 \mathrm{E}+04$ \\
\hline $\mathrm{PO}_{4}^{3-}$.ic.w & $\mu \mathrm{g} / \mathrm{g}$ & $8.01 \mathrm{E}+03$ & $5.89 \mathrm{E}+03$ & 1 & $0.00 \mathrm{E}+00$ & $8.29 \mathrm{E}+04$ \\
\hline $\mathrm{PO}_{4}{ }^{3-}$.ic.wo & $\mu \mathrm{g} / \mathrm{g}$ & $8.01 \mathrm{E}+03$ & $5.89 \mathrm{E}+03$ & 1 & $0.00 \mathrm{E}+00$ & $8.29 E+04$ \\
\hline Pb.icp.a.w & $\mu \mathrm{g} / \mathrm{g}$ & $4.75 E+01$ & $6.14 \mathrm{E}+00$ & 1 & $0.00 \mathrm{E}+00$ & $1.26 \mathrm{E}+02$ \\
\hline Pb.icp.a.wo & $\mu \mathrm{g} / \mathrm{g}$ & $5.03 \mathrm{E}+01$ & $8.16 \mathrm{E}+00$ & 1 & $0.00 \mathrm{E}+00$ & $1.54 \mathrm{E}+02$ \\
\hline S.icp.a * & $\mu \mathrm{g} / \mathrm{g}$ & $2.51 \mathrm{E}+03$ & $3.32 \mathrm{E}+02$ & 1 & $0.00 \mathrm{E}+00$ & $6.73 E+03$ \\
\hline S.icp.f.w & $\mu \mathrm{g} / \mathrm{g}$ & $2.77 \mathrm{E}+03$ & $1.59 \mathrm{E}+02$ & 1 & $7.51 \mathrm{E}+02$ & $4.80 \mathrm{E}+03$ \\
\hline S.icp.f.wo & $\mu \mathrm{g} / \mathrm{g}$ & $2.81 \mathrm{E}+03$ & $1.49 E+02$ & 1 & $9.22 \mathrm{E}+02$ & $4.71 E+03$ \\
\hline $\mathrm{SO}_{4}^{2-} . \mathrm{ic}$ & $\mu \mathrm{g} / \mathrm{g}$ & $6.27 E+03$ & $6.34 \mathrm{E}+02$ & 1 & $0.00 E+00$ & $1.43 \mathrm{E}+04$ \\
\hline Si.icp.a.w & $\mu \mathrm{g} / \mathrm{g}$ & $1.34 \mathrm{E}+02$ & $6.65 \mathrm{E}+00$ & 1 & $4.99 \mathrm{E}+01$ & $2.19 \mathrm{E}+02$ \\
\hline Si.icp.a.wo & $\mu \mathrm{g} / \mathrm{g}$ & $1.38 \mathrm{E}+02$ & $8.61 \mathrm{E}+00$ & 1 & $2.84 \mathrm{E}+01$ & $2.47 \mathrm{E}+02$ \\
\hline Zn.icp.a * & $\mu \mathrm{g} / \mathrm{g}$ & $4.34 \mathrm{E}+01$ & $6.12 \mathrm{E}+00$ & 1 & $0.00 \mathrm{E}+00$ & $1.21 \mathrm{E}+02$ \\
\hline Zr.icp.a.w & $\mu \mathrm{g} / \mathrm{g}$ & $1.13 \mathrm{E}+01$ & $3.93 \mathrm{E}+00$ & 1 & $0.00 \mathrm{E}+00$ & $6.13 E+01$ \\
\hline Zr.icp.a.wo & $\mu \mathrm{g} / \mathrm{g}$ & $1.19 \mathrm{E}+01$ & $4.21 \mathrm{E}+00$ & 1 & $0.00 \mathrm{E}+00$ & $6.54 \mathrm{E}+01$ \\
\hline Total Alpha.w & $\mu \mathrm{Ci} / \mathrm{g}$ & $2.28 \mathrm{E}-02$ & $1.04 \mathrm{E}-02$ & 1 & $0.00 \mathrm{E}+00$ & $1.55 \mathrm{E}-01$ \\
\hline Total Alpha.wo & $\mu \mathrm{Ci} / \mathrm{g}$ & $2.30 \mathrm{E}-02$ & $1.46 \mathrm{E}-02$ & 1 & $0.00 \mathrm{E}+00$ & $2.08 \mathrm{E}-01$ \\
\hline
\end{tabular}

Notes:

$*=\hat{\boldsymbol{o}}_{\text {ties }}$ is significantly different from zero (evidence of horizontal heterogeneity).

${ }^{\prime} \mathrm{Li}$ is one of the tracers for hydrostatic head fluid. 
Table B3-15. Summary Statistics (per g basis) - Sludge (Nested ANOVA without Riser). (2 sheets)

\begin{tabular}{|c|c|c|c|c|c|c|}
\hline Mnorito & Whits & 12 & $\%$ & Ifo & 1. & 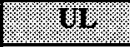 \\
\hline \%water.tga & wt\% & $4.44 \mathrm{E}+01$ & $7.87 \mathrm{E}-01$ & 10 & $4.27 \mathrm{E}+01$ & $4.62 \mathrm{E}+01$ \\
\hline Acetate.ic.w & $\mu \mathrm{g} / \mathrm{g}$ & $5.94 \mathrm{E}+02$ & $5.18 \mathrm{E}+01$ & 10 & $4.79 \mathrm{E}+02$ & $7.10 \mathrm{E}+02$ \\
\hline Acetate.ic.wo & $\mu \mathrm{g} / \mathrm{g}$ & $6.74 \mathrm{E}+02$ & $2.71 \mathrm{E}+01$ & 10 & $6.14 \mathrm{E}+02$ & $7.35 \mathrm{E}+02$ \\
\hline Ag.icp.a & $\mu \mathrm{g} / \mathrm{g}$ & $1.94 \mathrm{E}+01$ & $5.00 \mathrm{E}+00$ & 10 & $8.28 \mathrm{E}+00$ & $3.06 \mathrm{E}+01$ \\
\hline Al.icp.a & $\mu \mathrm{g} / \mathrm{g}$ & $2.21 \mathrm{E}+04$ & $7.10 \mathrm{E}+02$ & 10 & $2.05 \mathrm{E}+04$ & $2.36 \mathrm{E}+04$ \\
\hline Al.icp.f & $\mu \mathrm{g} / \mathrm{g}$ & $2.31 E+04$ & $8.27 E+02$ & 10 & $2.13 \mathrm{E}+04$ & $2.50 \mathrm{E}+04$ \\
\hline Cd.icp.a & $\mu \mathrm{g} / \mathrm{g}$ & $1.16 \mathrm{E}+01$ & $2.02 \mathrm{E}+00$ & 10 & $7.14 \mathrm{E}+00$ & $1.61 \mathrm{E}+01$ \\
\hline $\mathrm{Cl}^{-}$.ic & $\mu \mathrm{g} / \mathrm{g}$ & $5.18 \mathrm{E}+03$ & $2.02 E+02$ & 10 & $4.73 E+03$ & $5.63 E+03$ \\
\hline Cr.icp.a & $\mu \mathrm{g} / \mathrm{g}$ & $2.34 \mathrm{E}+03$ & $3.24 \mathrm{E}+02$ & 10 & $1.62 \mathrm{E}+03$ & $3.06 \mathrm{E}+03$ \\
\hline Cr.icp.f & $\mu \mathrm{g} / \mathrm{g}$ & $2.48 \mathrm{E}+03$ & $3.47 \mathrm{E}+02$ & 10 & $1.71 \mathrm{E}+03$ & $3.26 \mathrm{E}+03$ \\
\hline Cu.icp.a.w & $\mu \mathrm{g} / \mathrm{g}$ & $7.03 \mathrm{E}+00$ & $5.15 \mathrm{E}-01$ & 10 & $5.89 E+00$ & $8.18 \mathrm{E}+00$ \\
\hline Cu.icp.a.wo & $\mu \mathrm{g} / \mathrm{g}$ & $7.11 \mathrm{E}+00$ & $5.02 \mathrm{E}-01$ & 10 & $5.99 \mathrm{E}+00$ & $8.23 \mathrm{E}+00$ \\
\hline DSC.dry & $\mathrm{J} / \mathrm{g}$ dry & $6.38 \mathrm{E}+01$ & $8.31 \mathrm{E}+00$ & 10 & $4.53 \mathrm{E}+01$ & $8.23 \mathrm{E}+01$ \\
\hline DSC.wet & $\mathrm{J} / \mathrm{g}$ wet & $3.52 \mathrm{E}+01$ & $4.70 \mathrm{E}+00$ & 10 & $2.48 \mathrm{E}+01$ & $4.57 \mathrm{E}+01$ \\
\hline $\mathrm{F}^{-}$.ic & $\mu \mathrm{g} / \mathrm{g}$ & $1.85 E+03$ & $5.09 \mathrm{E}+02$ & 10 & $7.11 \mathrm{E}+02$ & $2.98 \mathrm{E}+03$ \\
\hline Fe.icp.a & $\mu \mathrm{g} / \mathrm{g}$ & $1.19 \mathrm{E}+02$ & $2.62 \mathrm{E}+01$ & 10 & $6.08 \mathrm{E}+01$ & $1.77 \mathrm{E}+02$ \\
\hline Formate.ic & $\mu \mathrm{g} / \mathrm{g}$ & $1.06 \mathrm{E}+03$ & $3.88 \mathrm{E}+01$ & 10 & $9.73 \mathrm{E}+02$ & $1.15 \mathrm{E}+03$ \\
\hline K.icp.a & $\mu \mathrm{g} / \mathrm{g}$ & $3.45 \mathrm{E}+03$ & $1.18 \mathrm{E}+02$ & 10 & $3.18 \mathrm{E}+03$ & $3.71 E+03$ \\
\hline${ }^{1}$ Li.icp.a.w & $\mu \mathrm{g} / \mathrm{g}$ & $1.97 \mathrm{E}+01$ & $5.02 \mathrm{E}+00$ & 10 & $8.54 \mathrm{E}+00$ & $3.09 \mathrm{E}+01$ \\
\hline${ }^{1}$ Li.icp.a.wo & $\mu \mathrm{g} / \mathrm{g}$ & $2.05 \mathrm{E}+01$ & $4.84 \mathrm{E}+00$ & 10 & $9.75 \mathrm{E}+00$ & $3.13 \mathrm{E}+01$ \\
\hline Mn.icp.a.w & $\mu \mathrm{g} / \mathrm{g}$ & $2.08 \mathrm{E}+01$ & $9.48 \mathrm{E}+00$ & 10 & $0.00 \mathrm{E}+00$ & $4.19 \mathrm{E}+01$ \\
\hline Mn.icp.a.wo & $\mu \mathrm{g} / \mathrm{g}$ & $2.14 \mathrm{E}+01$ & $9.77 \mathrm{E}+00$ & 10 & $0.00 \mathrm{E}+00$ & $4.32 \mathrm{E}+01$ \\
\hline $\mathrm{NO}_{2} . \mathrm{ic}$ & $\mu \mathrm{g} / \mathrm{g}$ & $6.39 \mathrm{E}+04$ & $2.50 \mathrm{E}+03$ & 10 & $5.83 E+04$ & $6.94 E+04$ \\
\hline $\mathrm{NO}_{3}^{-} . \mathrm{ic}$ & $\mu \mathrm{g} / \mathrm{g}$ & $1.11 \mathrm{E}+05$ & $7.09 \mathrm{E}+03$ & 10 & $9.54 \mathrm{E}+04$ & $1.27 \mathrm{E}+05$ \\
\hline Ni.icp.a & $\mu \mathrm{g} / \mathrm{g}$ & $4.07 \mathrm{E}+01$ & $5.14 \mathrm{E}+00$ & 10 & $2.93 \mathrm{E}+01$ & $5.21 \mathrm{E}+01$ \\
\hline Ni.icp.f.wo & $\mu \mathrm{g} / \mathrm{g}$ & $1.74 \mathrm{E}+03$ & $3.64 \mathrm{E}+02$ & 9 & $9.19 \mathrm{E}+02$ & $2.57 \mathrm{E}+03$ \\
\hline Oxalate.ic & $\mu \mathrm{g} / \mathrm{g}$ & $6.94 \mathrm{E}+03$ & $6.68 \mathrm{E}+02$ & 10 & $5.45 \mathrm{E}+03$ & $8.43 E+03$ \\
\hline P.icp.a & $\mu \mathrm{g} / \mathrm{g}$ & $2.46 \mathrm{E}+03$ & $1.66 \mathrm{E}+03$ & 10 & $0.00 \mathrm{E}+00$ & $6.14 \mathrm{E}+03$ \\
\hline $\mathrm{PO}_{4}{ }^{3-}$.ic.w & $\mu \mathrm{g} / \mathrm{g}$ & $8.01 \mathrm{E}+03$ & $5.89 E+03$ & 10 & $0.00 \mathrm{E}+00$ & $2.11 \mathrm{E}+04$ \\
\hline $\mathrm{PO}_{4}^{3-}$.ic.wo & $\mu \mathrm{g} / \mathrm{g}$ & $8.01 \mathrm{E}+03$ & $5.89 E+03$ & 10 & $0.00 \mathrm{E}+00$ & $2.11 \mathrm{E}+04$ \\
\hline
\end{tabular}


Table B3-15. Summary Statistics (per g basis) - Sludge (Nested ANOVA without Riser). ( 2 sheets)

\begin{tabular}{|c|c|c|c|c|c|c|}
\hline Aralyite & Wints: & 11 & \% & of & 1.1. & U1. \\
\hline Pb.icp.a.w & $\mu \mathrm{g} / \mathrm{g}$ & $4.74 \mathrm{E}+01$ & $4.40 \mathrm{E}+00$ & 10 & $3.77 \mathrm{E}+01$ & $5.72 \mathrm{E}+01$ \\
\hline Pb.icp.a.wo & $\mu \mathrm{g} / \mathrm{g}$ & $4.95 \mathrm{E}+01$ & $6.43 \mathrm{E}+00$ & 7 & $3.43 \mathrm{E}+01$ & $6.47 \mathrm{E}+01$ \\
\hline S.icp.f.w & $\mu \mathrm{g} / \mathrm{g}$ & $2.77 \mathrm{E}+03$ & $1.59 \mathrm{E}+02$ & 10 & $2.42 \mathrm{E}+03$ & $3.13 \mathrm{E}+03$ \\
\hline S.icp.f.wo & $\mu \mathrm{g} / \mathrm{g}$ & $2.81 \mathrm{E}+03$ & $1.49 \mathrm{E}+02$ & 10 & $2.48 \mathrm{E}+03$ & $3.15 \mathrm{E}+03$ \\
\hline $\mathrm{SO}_{4}{ }^{2}$.ic & $\mu \mathrm{g} / \mathrm{g}$ & $6.26 \mathrm{E}+03$ & $5.24 \mathrm{E}+02$ & 10 & $5.10 \mathrm{E}+03$ & $7.43 \mathrm{E}+03$ \\
\hline Si.icp.a.w & $\mu \mathrm{g} / \mathrm{g}$ & $1.34 \mathrm{E}+02$ & $6.65 \mathrm{E}+00$ & 10 & $1.20 \mathrm{E}+02$ & $1.49 \mathrm{E}+02$ \\
\hline Si.icp.a.wo & $\mu \mathrm{g} / \mathrm{g}$ & $1.38 \mathrm{E}+02$ & $5.88 \mathrm{E}+00$ & 10 & $1.25 \mathrm{E}+02$ & $1.51 \mathrm{E}+02$ \\
\hline Zr.icp.a.w & $\mu \mathrm{g} / \mathrm{g}$ & $1.13 \mathrm{E}+01$ & $3.93 \mathrm{E}+00$ & 10 & $2.58 \mathrm{E}+00$ & $2.01 \mathrm{E}+01$ \\
\hline Zr.icp.a.wo & $\mu \mathrm{g} / \mathrm{g}$ & $1.19 \mathrm{E}+01$ & $4.21 \mathrm{E}+00$ & 10 & $2.50 \mathrm{E}+00$ & $2.13 \mathrm{E}+01$ \\
\hline Total Alpha.w & $\mu \mathrm{Ci} / \mathrm{g}$ & $2.28 \mathrm{E}-02$ & $1.04 \mathrm{E}-02$ & 10 & $0.00 \mathrm{E}+00$ & 4.60 \\
\hline Total Alpha.wo & $\mu \mathrm{Ci} / \mathrm{g}$ & $2.30 \mathrm{E}-02$ & $1.46 \mathrm{E}-02$ & 7 & $0.00 \mathrm{E}+00$ & $5.75 \mathrm{E}-02$ \\
\hline
\end{tabular}

Note:

' $\mathrm{Li}$ is one of the tracers for hydrostatic head fluid. 
Table B3-16. Analytes (Sludge - per g basis) with $>50$ Percent "Less Than" Values. (2 sheets)

\begin{tabular}{|c|c|c|}
\hline $12:$ Analyte & 1. UMil & 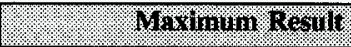 \\
\hline Ag.icp.f & $\mu \mathrm{g} / \mathrm{g}$ & $<212$ \\
\hline As.icp.a & $\mu \mathrm{g} / \mathrm{g}$ & $<48.9$ \\
\hline As.icp.f & $\mu \mathrm{g} / \mathrm{g}$ & $<2120$ \\
\hline B.icp.f & $\mu \mathrm{g} / \mathrm{g}$ & $<1060$ \\
\hline Ba.icp.a & $\mu \mathrm{g} / \mathrm{g}$ & $<24.4$ \\
\hline Ba.icp.f & $\mu \mathrm{g} / \mathrm{g}$ & $<1060$ \\
\hline Be.icp.a & $\mu \mathrm{g} / \mathrm{g}$ & 3.11 \\
\hline Be.icp.f & $\mu \mathrm{g} / \mathrm{g}$ & $<106$ \\
\hline Bi.icp.a & $\mu \mathrm{g} / \mathrm{g}$ & 71.8 \\
\hline Bi.icp.f & $\mu \mathrm{g} / \mathrm{g}$ & $<2120$ \\
\hline $\mathrm{Br}$.ic & $\mu \mathrm{g} / \mathrm{g}$ & $<1070$ \\
\hline Ca.icp.f & $\mu \mathrm{g} / \mathrm{g}$ & $<2120$ \\
\hline Cd.icp.f & $\mu \mathrm{g} / \mathrm{g}$ & $<106$ \\
\hline Ce.icp.a & $\mu \mathrm{g} / \mathrm{g}$ & $<48.9$ \\
\hline Ce.icp.f & $\mu \mathrm{g} / \mathrm{g}$ & $<2120$ \\
\hline Co.icp.a & $\mu \mathrm{g} / \mathrm{g}$ & $<9.78$ \\
\hline Co.icp.f & $\mu \mathrm{g} / \mathrm{g}$ & $<425$ \\
\hline Cu.icp.f & $\mu \mathrm{g} / \mathrm{g}$ & $<212$ \\
\hline Fe.icp.f & $\mu \mathrm{g} / \mathrm{g}$ & $<1060$ \\
\hline La.icp.a & $\mu \mathrm{g} / \mathrm{g}$ & $<24.4$ \\
\hline La.icp.f & $\mu \mathrm{g} / \mathrm{g}$ & $<1060$ \\
\hline 'Li.icp.f & $\mu \mathrm{g} / \mathrm{g}$ & $<212$ \\
\hline Mg.icp.a & $\mu \mathrm{g} / \mathrm{g}$ & 60.9 \\
\hline Mg.icp.f & $\mu \mathrm{g} / \mathrm{g}$ & $<2120$ \\
\hline Mn.icp.f & $\mu \mathrm{g} / \mathrm{g}$ & $<212$ \\
\hline Mo.icp.f & $\mu \mathrm{g} / \mathrm{g}$ & $<1060$ \\
\hline \begin{tabular}{|l|} 
Nd.icp.a \\
\end{tabular} & $\mu \mathrm{g} / \mathrm{g}$ & $<48.9$ \\
\hline Nd.icp.f & $\mu \mathrm{g} / \mathrm{g}$ & $<2120$ \\
\hline P.icp.f & $\mu \mathrm{g} / \mathrm{g}$ & 35700 \\
\hline Pb.icp.f & $\mu \mathrm{g} / \mathrm{g}$ & $<2120$ \\
\hline
\end{tabular}


Table B3-16. Analytes (Sludge - per $g$ basis) with $>50$ Percent "Less Than" Values. (2 sheets)

\begin{tabular}{|c|c|c|}
\hline 1. Anolyte & W. Whit & I. Marimun Resul \\
\hline Sb.icp.a & $\mu \mathrm{g} / \mathrm{g}$ & $<29.3$ \\
\hline Sb.icp.f & $\mu \mathrm{g} / \mathrm{g}$ & $<1270$ \\
\hline Se.icp.a & $\mu \mathrm{g} / \mathrm{g}$ & $<48.9$ \\
\hline Se.icp.f & $\mu \mathrm{g} / \mathrm{g}$ & $<2120$ \\
\hline Si.icp.f & $\mu \mathrm{g} / \mathrm{g}$ & 2450 \\
\hline Sm.icp.a & $\mu \mathrm{g} / \mathrm{g}$ & $<48.9$ \\
\hline Sm.icp.f & $\mu \mathrm{g} / \mathrm{g}$ & $<2120$ \\
\hline Sr.icp.a & $\mu \mathrm{g} / \mathrm{g}$ & 9.73 \\
\hline Sr.icp.f & $\mu \mathrm{g} / \mathrm{g}$ & $<212$ \\
\hline Ti.icp.a & $\mu \mathrm{g} / \mathrm{g}$ & $<4.89$ \\
\hline Ti.icp.f & $\mu \mathrm{g} / \mathrm{g}$ & $<212$ \\
\hline T1.icp.a & $\mu \mathrm{g} / \mathrm{g}$ & $<97.8$ \\
\hline Tl.icp.f & $\mu \mathrm{g} / \mathrm{g}$ & $<4250$ \\
\hline U.icp.a & $\mu \mathrm{g} / \mathrm{g}$ & $<244$ \\
\hline U.icp.f & $\mu \mathrm{g} / \mathrm{g}$ & $<10600$ \\
\hline V.icp.a & $\mu \mathrm{g} / \mathrm{g}$ & $<24.4$ \\
\hline V.icp.f & $\mu \mathrm{g} / \mathrm{g}$ & $<1060$ \\
\hline Zn.icp.f & $\mu \mathrm{g} / \mathrm{g}$ & 810 \\
\hline Zr.icp.f & $\mu \mathrm{g} / \mathrm{g}$ & 480 \\
\hline
\end{tabular}

Note:

${ }^{\mathrm{I}} \mathrm{Li}$ is one of the tracers for hydrostatic head fluid. 
Two sludge composite samples were formed from Core 152 segments. The first sludge composite consisted of material from segments 14 through 20 . The second composite consisted of material from segment 22 . The arithmetic mean, the associated variability, and a two-sided 95 percent confidence interval for the mean concentration were calculated for each analyte with at least 50 percent of the reported data as quantitative values. The confidence interval takes into account only the analytical uncertainty. The summary statistics are listed in Tables B3-17 for the first sludge composite and Tables B3-19 for the second sludge composite. Tables B-18 and B-20 contain the analytes which had less than 50 percent of the reported data as quantative values.

Table B3-17. Summary Statistics - Sludge Composite (Core 152, Segments 14-20). (2 sheets)

\begin{tabular}{|c|c|c|c|c|c|c|}
\hline Analyte & Units & 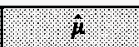 & 6. & df & II. & U1 \\
\hline \%water.tga & wt\% & $4.57 \mathrm{E}+01$ & $4.50 \mathrm{E}-01$ & 1 & $4.00 \mathrm{E}+01$ & $5.15 \mathrm{E}+01$ \\
\hline Acetate.ic & $\mu \mathrm{g} / \mathrm{g}$ & $4.72 \mathrm{E}+02$ & $2.00 \mathrm{E}+00$ & 1 & $4.47 \mathrm{E}+02$ & $4.97 \mathrm{E}+02$ \\
\hline Ag.icp.a & $\mu \mathrm{g} / \mathrm{g}$ & $1.19 \mathrm{E}+01$ & $4.00 \mathrm{E}-01$ & 1 & $6.82 \mathrm{E}+00$ & $1.70 \mathrm{E}+01$ \\
\hline Al.icp.a & $\mu \mathrm{g} / \mathrm{g}$ & $2.05 E+04$ & $2.50 \mathrm{E}+02$ & 1 & $1.73 \mathrm{E}+04$ & $2.36 \mathrm{E}+04$ \\
\hline${ }^{241}$ Am.aea & $\mu \mathrm{Ci} / \mathrm{g}$ & $4.10 \mathrm{E}-03$ & $8.55 \mathrm{E}-04$ & 1 & $0.00 \mathrm{E}+00$ & $1.50 \mathrm{E}-02$ \\
\hline B.icp.a & $\mu \mathrm{g} / \mathrm{g}$ & $1.17 \mathrm{E}+02$ & $1.15 \mathrm{E}+01$ & 1 & $0.00 \mathrm{E}+00$ & $2.63 E+02$ \\
\hline Ca.icp.a & $\mu \mathrm{g} / \mathrm{g}$ & $2.16 \mathrm{E}+02$ & $6.50 \mathrm{E}+00$ & 1 & $1.33 \mathrm{E}+02$ & $2.98 \mathrm{E}+02$ \\
\hline Cd.icp.a & $\mu \mathrm{g} / \mathrm{g}$ & $7.30 \mathrm{E}+00$ & $8.35 \mathrm{E}-01$ & 1 & $0.00 \mathrm{E}+00$ & $1.79 \mathrm{E}+01$ \\
\hline $\mathrm{Cl}^{-}$.ic & $\mu \mathrm{g} / \mathrm{g}$ & $5.88 \mathrm{E}+03$ & $3.00 \mathrm{E}+01$ & 1 & $5.50 \mathrm{E}+03$ & $6.26 \mathrm{E}+03$ \\
\hline Cr.icp.a & $\mu \mathrm{g} / \mathrm{g}$ & $1.49 \mathrm{E}+03$ & $1.15 \mathrm{E}+02$ & 1 & $2.38 \mathrm{E}+01$ & $2.95 \mathrm{E}+03$ \\
\hline $\mathrm{Cr}(\mathrm{VI})$. spec & $\mu \mathrm{g} / \mathrm{g}$ & $1.19 \mathrm{E}+02$ & $1.00 \mathrm{E}+00$ & 1 & $1.06 \mathrm{E}+02$ & $1.32 \mathrm{E}+02$ \\
\hline${ }^{137}$ Cs.gea & $\mu \mathrm{Ci} / \mathrm{g}$ & $3.04 \mathrm{E}+02$ & $1.00 \mathrm{E}+00$ & 1 & $2.91 \mathrm{E}+02$ & $3.17 \mathrm{E}+02$ \\
\hline Cu.icp.a & $\mu \mathrm{g} / \mathrm{g}$ & $5.11 \mathrm{E}+00$ & $2.30 \mathrm{E}-01$ & 1 & $2.19 \mathrm{E}+00$ & $8.03 \mathrm{E}+00$ \\
\hline DSC.dry & Joules/g dry & $6.95 \mathrm{E}+01$ & $1.11 \mathrm{E}+01$ & 1 & $0.00 \mathrm{E}+00$ & $2.10 \mathrm{E}+02$ \\
\hline DSC.wet & Joules/g wet & $3.77 \mathrm{E}+01$ & $6.00 \mathrm{E}+00$ & 1 & $0.00 \mathrm{E}+00$ & $1.14 \mathrm{E}+02$ \\
\hline F.ic & $\mu \mathrm{g} / \mathrm{g}$ & $1.14 \mathrm{E}+03$ & $2.11 \mathrm{E}+02$ & 1 & $0.00 \mathrm{E}+00$ & $3.81 \mathrm{E}+03$ \\
\hline Fe.icp.a & $\mu \mathrm{g} / \mathrm{g}$ & $6.12 \mathrm{E}+01$ & $1.06 \mathrm{E}+01$ & 1 & $0.00 \mathrm{E}+00$ & $1.95 \mathrm{E}+02$ \\
\hline Formate.ic & $\mu \mathrm{g} / \mathrm{g}$ & $1.23 \mathrm{E}+03$ & $5.00 \mathrm{E}+00$ & 1 & $1.16 \mathrm{E}+03$ & $1.29 \mathrm{E}+03$ \\
\hline K.icp.a & $\mu \mathrm{g} / \mathrm{g}$ & $3.09 \mathrm{E}+03$ & $2.00 \mathrm{E}+01$ & 1 & $2.84 \mathrm{E}+03$ & $3.34 \mathrm{E}+03$ \\
\hline${ }^{1}$ Li.icp.a & $\mu \mathrm{g} / \mathrm{g}$ & $8.70 \mathrm{E}+00$ & $1.00 \mathrm{E}-01$ & 1 & $7.43 \mathrm{E}+00$ & $9.97 \mathrm{E}+00$ \\
\hline Mn.icp.a & $\mu \mathrm{g} / \mathrm{g}$ & $7.82 \mathrm{E}+00$ & $6.40 \mathrm{E}-01$ & 1 & $0.00 \mathrm{E}+00$ & $1.60 \mathrm{E}+01$ \\
\hline Mo.icp.a & $\mu \mathrm{g} / \mathrm{g}$ & $4.59 \mathrm{E}+01$ & $5.00 \mathrm{E}-02$ & 1 & $4.52 \mathrm{E}+01$ & $4.65 \mathrm{E}+01$ \\
\hline
\end{tabular}


Table B3-17. Summary Statistics - Sludge Composite (Core 152, Segments 14-20).

(2 sheets)

\begin{tabular}{|c|c|c|c|c|c|c|}
\hline oraly/te. & Vrits: & 9 & 6. & af & 1.1. & U1. \\
\hline $\mathrm{NO}_{2}^{-}$. ic & $\mu \mathrm{g} / \mathrm{g}$ & $7.31 \mathrm{E}+04$ & $1.50 \mathrm{E}+02$ & 1 & $7.11 \mathrm{E}+04$ & $7.50 \mathrm{E}+04$ \\
\hline $\mathrm{NO}_{3}^{-}$.ic & $\mu \mathrm{g} / \mathrm{g}$ & $1.13 \mathrm{E}+05$ & $4.00 \mathrm{E}+03$ & 1 & $6.22 \mathrm{E}+04$ & $1.64 \mathrm{E}+05$ \\
\hline Na.icp.a & $\mu \mathrm{g} / \mathrm{g}$ & $1.55 \mathrm{E}+05$ & $2.00 \mathrm{E}+03$ & 1 & $1.30 \mathrm{E}+05$ & $1.80 \mathrm{E}+05$ \\
\hline Ni.icp.a & $\mu \mathrm{g} / \mathrm{g}$ & $3.18 \mathrm{E}+01$ & $3.15 \mathrm{E}+00$ & 1 & $0.00 \mathrm{E}+00$ & $7.18 \mathrm{E}+01$ \\
\hline $237 \mathrm{~Np}$ & $\mu \mathrm{Ci} / \mathrm{g}$ & $1.06 \mathrm{E}-02$ & $3.00 \mathrm{E}-04$ & 1 & $6.79 \mathrm{E}-03$ & $1.44 \mathrm{E}-02$ \\
\hline$\overline{\mathrm{OH}^{-}}$ & $\mu \mathrm{g} / \mathrm{g}$ & $2.41 \mathrm{E}+04$ & $4.50 \mathrm{E}+02$ & 1 & $1.83 \mathrm{E}+04$ & $2.98 \mathrm{E}+04$ \\
\hline Oxalate.ic & $\mu \mathrm{g} / \mathrm{g}$ & $6.49 \mathrm{E}+03$ & $2.00 \mathrm{E}+02$ & 1 & $3.95 \mathrm{E}+03$ & $9.03 E+03$ \\
\hline P.icp.a & $\mu \mathrm{g} / \mathrm{g}$ & $4.02 \mathrm{E}+02$ & $1.60 \mathrm{E}+01$ & 1 & $1.99 \mathrm{E}+02$ & $6.05 \mathrm{E}+02$ \\
\hline $\mathrm{PO}_{4}{ }^{3} \cdot$.ic & $\mu \mathrm{g} / \mathrm{g}$ & $1.21 \mathrm{E}+03$ & $7.50 \mathrm{E}+01$ & 1 & $2.52 \mathrm{E}+02$ & $2.16 \mathrm{E}+03$ \\
\hline S.icp.a & $\mu \mathrm{g} / \mathrm{g}$ & $2.18 \mathrm{E}+03$ & $1.45 \mathrm{E}+02$ & 1 & $3.33 \mathrm{E}+02$ & $4.02 \mathrm{E}+03$ \\
\hline $\mathrm{SO}_{4}^{2-}$.ic & $\mu \mathrm{g} / \mathrm{g}$ & $7.48 \mathrm{E}+03$ & $2.55 \mathrm{E}+02$ & 1 & $4.23 \mathrm{E}+03$ & $1.07 \mathrm{E}+04$ \\
\hline Si.icp.a & $\mu \mathrm{g} / \mathrm{g}$ & $1.79 \mathrm{E}+02$ & $3.95 \mathrm{E}+01$ & 1 & $0.00 \mathrm{E}+00$ & $6.80 \mathrm{E}+02$ \\
\hline${ }^{89190} \mathrm{Sr}$ & $\mu \mathrm{Ci} / \mathrm{g}$ & $9.16 \mathrm{E}+00$ & $2.50 \mathrm{E}-01$ & 1 & $5.98 \mathrm{E}+00$ & $1.23 \mathrm{E}+01$ \\
\hline TIC & $\mu \mathrm{g} / \mathrm{g}$ & $1.31 \mathrm{E}+04$ & $5.50 \mathrm{E}+02$ & 1 & $6.06 \mathrm{E}+03$ & $2.00 \mathrm{E}+04$ \\
\hline TOC.pers & $\mu \mathrm{g} / \mathrm{g}$ & $5.43 \mathrm{E}+03$ & $5.25 \mathrm{E}+02$ & 1 & $0.00 \mathrm{E}+00$ & $1.21 \mathrm{E}+04$ \\
\hline${ }^{999} \mathrm{Tc}$ & $\mu \mathrm{Ci} / \mathrm{g}$ & $1.81 \mathrm{E}-01$ & $5.00 \mathrm{E}-04$ & 1 & $1.74 \mathrm{E}-01$ & $1.87 \mathrm{E}-01$ \\
\hline Total Alpha & $\mu \mathrm{Ci} / \mathrm{g}$ & $1.36 \mathrm{E}-02$ & $1.60 \mathrm{E}-03$ & 1 & $0.00 \mathrm{E}+00$ & $3.39 \mathrm{E}-02$ \\
\hline Total Beta & $\mu \mathrm{Ci} / \mathrm{g}$ & $3.27 \mathrm{E}+02$ & $1.50 \mathrm{E}+00$ & 1 & $3.07 \mathrm{E}+02$ & $3.46 \mathrm{E}+02$ \\
\hline Tritium & $\mu \mathrm{Ci} / \mathrm{g}$ & $2.20 \mathrm{E}-03$ & $2.10 \mathrm{E}-04$ & 1 & $0.00 \mathrm{E}+00$ & $4.87 \mathrm{E}-03$ \\
\hline U.phos & $\mu \mathrm{g} / \mathrm{g}$ & $8.74 \mathrm{E}+01$ & $1.20 \mathrm{E}+00$ & 1 & $7.22 \mathrm{E}+01$ & $1.03 \mathrm{E}+02$ \\
\hline Zn.icp.a & $\mu \mathrm{g} / \mathrm{g}$ & $8.18 \mathrm{E}+00$ & $4.00 \mathrm{E}-01$ & 1 & $3.10 \mathrm{E}+00$ & $1.33 \mathrm{E}+01$ \\
\hline
\end{tabular}

Note:

' $\mathrm{Li}$ is one of the tracers for hydrostatic head fluid. 
Table B3-18. Sludge Composite (Core 152, Segments 14-20) Analytes with $>50$ Percent "Less Than" Values.

\begin{tabular}{|c|c|c|}
\hline 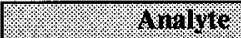 & VInt & Maxinim Resull \\
\hline${ }^{241}$ Am.gea & $\mu \mathrm{Ci} / \mathrm{g}$ & $<8.25 \mathrm{E}-01$ \\
\hline As.icp.a & $\mu \mathrm{Ci} / \mathrm{g}$ & $<4.03 \mathrm{E}+01$ \\
\hline Ba.icp.a & $\mu \mathrm{g} / \mathrm{g}$ & $<2.02 \mathrm{E}+01$ \\
\hline Be.icp.a & $\mu \mathrm{g} / \mathrm{g}$ & $<2.02 \mathrm{E}+00$ \\
\hline Bi.icp.a & $\mu \mathrm{g} / \mathrm{g}$ & $<4.03 \mathrm{E}+01$ \\
\hline${ }^{1} \mathrm{Br}^{-} . \mathrm{ic}$ & $\mu \mathrm{g} / \mathrm{g}$ & $<5.58 \mathrm{E}+02$ \\
\hline Ce.icp.a & $\mu \mathrm{g} / \mathrm{g}$ & $<4.03 \mathrm{E}+01$ \\
\hline${ }^{243 / 244} \mathrm{Cm}$ & $\mu \mathrm{Ci} / \mathrm{g}$ & $<9.67 \mathrm{E}-04$ \\
\hline Co.icp.a & $\mu \mathrm{g} / \mathrm{g}$ & $<8.06 \mathrm{E}+00$ \\
\hline${ }^{60}$ Co.gea & $\mu \mathrm{Ci} / \mathrm{g}$ & $<3.75 \mathrm{E}-02$ \\
\hline${ }^{154}$ Eu.gea & $\mu \mathrm{Ci} / \mathrm{g}$ & $<9.92 \mathrm{E}-02$ \\
\hline${ }^{155}$ Eu.gea & $\mu \mathrm{Ci} / \mathrm{g}$ & $<3.87 \mathrm{E}-01$ \\
\hline${ }^{129} \mathrm{I}$ & $\mu \mathrm{Ci} / \mathrm{g}$ & $<3.92 \mathrm{E}-03$ \\
\hline La.icp.a & $\mu \mathrm{g} / \mathrm{g}$ & $<2.02 \mathrm{E}+01$ \\
\hline Mg.icp.a & $\mu \mathrm{g} / \mathrm{g}$ & $<4.03 \mathrm{E}+01$ \\
\hline Nd.icp.a & $\mu \mathrm{g} / \mathrm{g}$ & $<4.03 \mathrm{E}+01$ \\
\hline Pb.icp.a & $\mu \mathrm{g} / \mathrm{g}$ & $<4.03 \mathrm{E}+01$ \\
\hline${ }^{239 / 240} \mathrm{Pu}$ & $\mu \mathrm{Ci} / \mathrm{g}$ & $<1.81 \mathrm{E}-03$ \\
\hline Sb.icp.a & $\mu \mathrm{g} / \mathrm{g}$ & $<2.42 \mathrm{E}+01$ \\
\hline Se.icp.a & $\mu \mathrm{g} / \mathrm{g}$ & $<4.03 \mathrm{E}+01$ \\
\hline Sm.icp.a & $\mu \mathrm{g} / \mathrm{g}$ & $<4.03 \mathrm{E}+01$ \\
\hline Sr.icp.a & $\mu \mathrm{g} / \mathrm{g}$ & $<4.03 \mathrm{E}+00$ \\
\hline Ti.icp.a & $\mu \mathrm{g} / \mathrm{g}$ & $<4.03 \mathrm{E}+00$ \\
\hline Tl.icp.a & $\mu \mathrm{g} / \mathrm{g}$ & $<8.06 \mathrm{E}+01$ \\
\hline U.icp.a & $\mu \mathrm{g} / \mathrm{g}$ & $<2.02 \mathrm{E}+02$ \\
\hline V.icp.a & $\mu \mathrm{g} / \mathrm{g}$ & $<2.02 \mathrm{E}+01$ \\
\hline Zr.icp.a & $\mu \mathrm{g} / \mathrm{g}$ & $<4.51 \mathrm{E}+00$ \\
\hline
\end{tabular}

Note:

${ }^{\prime} \mathrm{Br}$ is one of the tracers for hydrostatic head fluid. 
Table B3-19. Summary Statistics - Sludge Composite (Core 152, Segment 22). (2 sheets)

\begin{tabular}{|c|c|c|c|c|c|c|}
\hline Mnalue & Uniss. & \%. & $6 \%$ & ge & Wh & WI. \\
\hline$\%$ water.tga & wt\% & $4.27 \mathrm{E}+01$ & $1.30 \mathrm{E}-01$ & 1 & $4.11 \mathrm{E}+01$ & $4.44 \mathrm{E}+01$ \\
\hline Ag.icp.a & $\mu \mathrm{g} / \mathrm{g}$ & $4.25 \mathrm{E}+01$ & $2.35 E+00$ & 1 & $1.26 \mathrm{E}+01$ & $7.23 \mathrm{E}+01$ \\
\hline Al.icp.a & $\mu \mathrm{g} / \mathrm{g}$ & $1.45 E+04$ & $7.50 \mathrm{E}+02$ & 1 & $4.92 \mathrm{E}+03$ & $2.40 \mathrm{E}+04$ \\
\hline${ }^{241}$ Am.aea & $\mu \mathrm{Ci} / \mathrm{g}$ & $3.89 \mathrm{E}-02$ & $4.40 \mathrm{E}-03$ & 1 & $0.00 \mathrm{E}+00$ & $9.48 \mathrm{E}-02$ \\
\hline B.icp.a & $\mu \mathrm{g} / \mathrm{g}$ & $1.14 \mathrm{E}+02$ & $2.24 \mathrm{E}+01$ & 1 & $0.00 \mathrm{E}+00$ & $3.98 \mathrm{E}+02$ \\
\hline Ca.icp.a & $\mu \mathrm{g} / \mathrm{g}$ & $3.29 \mathrm{E}+02$ & $2.65 \mathrm{E}+01$ & 1 & $0.00 \mathrm{E}+00$ & $6.65 \mathrm{E}+02$ \\
\hline Cd.icp.a & $\mu \mathrm{g} / \mathrm{g}$ & $1.39 \mathrm{E}+01$ & $5.50 \mathrm{E}-01$ & 1 & $6.86 \mathrm{E}+00$ & $2.08 \mathrm{E}+01$ \\
\hline $\mathrm{Cl}^{-}$.ic & $\mu \mathrm{g} / \mathrm{g}$ & $4.07 \mathrm{E}+03$ & $2.70 \mathrm{E}+02$ & 1 & $6.39 E+02$ & $7.50 \mathrm{E}+03$ \\
\hline Cr.icp.a & $\mu \mathrm{g} / \mathrm{g}$ & $2.74 \mathrm{E}+03$ & $1.60 \mathrm{E}+02$ & 1 & $7.07 \mathrm{E}+02$ & $4.77 E+03$ \\
\hline Cr(VI).spec & $\mu \mathrm{g} / \mathrm{g}$ & $1.04 \mathrm{E}+02$ & $3.00 \mathrm{E}+00$ & 1 & $6.59 \mathrm{E}+01$ & $1.42 \mathrm{E}+02$ \\
\hline${ }^{137}$ Cs.gea & $\mu \mathrm{Ci} / \mathrm{g}$ & $2.06 \mathrm{E}+02$ & $1.00 \mathrm{E}+00$ & 1 & $1.93 \mathrm{E}+02$ & $2.19 \mathrm{E}+02$ \\
\hline Cu.icp.a & $\mu \mathrm{g} / \mathrm{g}$ & $6.03 E+00$ & $1.01 \mathrm{E}+00$ & 1 & $0.00 \mathrm{E}+00$ & $1.89 \mathrm{E}+01$ \\
\hline DSC.dry & $\mathrm{J} / \mathrm{g}$ dry & $1.00 \mathrm{E}+02$ & $5.75 \mathrm{E}+00$ & 1 & $2.72 \mathrm{E}+01$ & $1.73 \mathrm{E}+02$ \\
\hline DSC.wet & $\mathrm{J} / \mathrm{g}$ wet & $5.73 \mathrm{E}+01$ & $3.15 \mathrm{E}+00$ & 1 & $1.72 \mathrm{E}+01$ & $9.73 \mathrm{E}+01$ \\
\hline $\mathrm{F}^{-} . \mathrm{ic}$ & $\mu \mathrm{g} / \mathrm{g}$ & $5.03 \mathrm{E}+03$ & $1.55 \mathrm{E}+02$ & 1 & $3.06 \mathrm{E}+03$ & $6.99 E+03$ \\
\hline Fe.icp.a & $\mu \mathrm{g} / \mathrm{g}$ & $1.47 \mathrm{E}+02$ & $7.50 \mathrm{E}+00$ & 1 & $5.12 \mathrm{E}+01$ & $2.42 \mathrm{E}+02$ \\
\hline Formate.ic & $\mu \mathrm{g} / \mathrm{g}$ & $1.12 \mathrm{E}+03$ & $5.00 \mathrm{E}+00$ & 1 & $1.05 \mathrm{E}+03$ & $1.18 \mathrm{E}+03$ \\
\hline K.icp.a & $\mu \mathrm{g} / \mathrm{g}$ & $2.15 \mathrm{E}+03$ & $9.50 \mathrm{E}+01$ & 1 & $9.38 \mathrm{E}+02$ & $3.35 \mathrm{E}+03$ \\
\hline${ }^{1}$ Li.icp.a & $\mu \mathrm{g} / \mathrm{g}$ & $1.47 \mathrm{E}+01$ & $7.39 \mathrm{E}+00$ & 1 & $0.00 \mathrm{E}+00$ & $1.09 \mathrm{E}+02$ \\
\hline Mn.icp.a & $\mu \mathrm{g} / \mathrm{g}$ & $4.94 \mathrm{E}+01$ & $2.80 \mathrm{E}+00$ & 1 & $1.38 \mathrm{E}+01$ & $8.50 \mathrm{E}+01$ \\
\hline Mo.icp.a & $\mu \mathrm{g} / \mathrm{g}$ & $3.54 \mathrm{E}+01$ & $2.00 \mathrm{E}+00$ & 1 & $9.99 \mathrm{E}+00$ & $6.08 E+01$ \\
\hline $\mathrm{NO}_{2}^{-} \cdot \mathrm{ic}$ & $\mu \mathrm{g} / \mathrm{g}$ & $4.77 \mathrm{E}+04$ & $3.40 \mathrm{E}+03$ & 1 & $4.50 \mathrm{E}+03$ & $9.09 \mathrm{E}+04$ \\
\hline $\mathrm{NO}_{3}$.ic & $\mu \mathrm{g} / \mathrm{g}$ & $1.61 \mathrm{E}+05$ & $1.40 \mathrm{E}+04$ & 1 & $0.00 \mathrm{E}+00$ & $3.39 \mathrm{E}+05$ \\
\hline Na.icp.a & $\mu \mathrm{g} / \mathrm{g}$ & $1.65 \mathrm{E}+05$ & $1.05 \mathrm{E}+04$ & 1 & $3.11 \mathrm{E}+04$ & $2.98 \mathrm{E}+05$ \\
\hline Ni.icp.a & $\mu \mathrm{g} / \mathrm{g}$ & $3.37 \mathrm{E}+01$ & $2.85 \mathrm{E}+00$ & 1 & $0.00 \mathrm{E}+00$ & $6.99 \mathrm{E}+01$ \\
\hline $\mathrm{OH}^{-}$ & $\mu \mathrm{g} / \mathrm{g}$ & $2.20 \mathrm{E}+04$ & $4.00 \mathrm{E}+02$ & 1 & $1.69 \mathrm{E}+04$ & $2.71 \mathrm{E}+04$ \\
\hline Oxalate.ic & $\mu \mathrm{g} / \mathrm{g}$ & $5.88 \mathrm{E}+03$ & $4.20 \mathrm{E}+02$ & 1 & $5.43 \mathrm{E}+02$ & $1.12 \mathrm{E}+04$ \\
\hline P.icp.a & $\mu \mathrm{g} / \mathrm{g}$ & $1.41 \mathrm{E}+04$ & $9.00 \mathrm{E}+02$ & 1 & $2.66 \mathrm{E}+03$ & $2.55 \mathrm{E}+04$ \\
\hline $\mathrm{PO}_{4}^{3-}$.ic & $\mu \mathrm{g} / \mathrm{g}$ & $4.57 \mathrm{E}+04$ & $2.30 \mathrm{E}+03$ & 1 & $1.65 \mathrm{E}+04$ & $7.49 \mathrm{E}+04$ \\
\hline Pb.icp.a & $\mu \mathrm{g} / \mathrm{g}$ & $4.97 \mathrm{E}+01$ & $2.10 \mathrm{E}+00$ & 1 & $2.30 \mathrm{E}+01$ & $7.64 \mathrm{E}+01$ \\
\hline${ }^{239 / 240} \mathrm{Pu}$ & $\mu \mathrm{Ci} / \mathrm{g}$ & $7.55 \mathrm{E}-03$ & $2.50 \mathrm{E}-05$ & 1 & $7.23 \mathrm{E}-03$ & $7.86 \mathrm{E}-03$ \\
\hline
\end{tabular}


Table B3-19. Summary Statistics - Sludge Composite (Core 152, Segment 22). (2 sheets)

\begin{tabular}{|c|c|c|c|c|c|c|}
\hline Walyto & Whits & 9 & (1) & III & W & W \\
\hline S.icp.a & $\mu \mathrm{g} / \mathrm{g}$ & $1.45 \mathrm{E}+03$ & $8.50 \mathrm{E}+01$ & 1 & $3.65 \mathrm{E}+02$ & $2.53 \mathrm{E}+03$ \\
\hline $\mathrm{SO}_{4}^{2-}$.ic & $\mu \mathrm{g} / \mathrm{g}$ & $4.18 \mathrm{E}+03$ & $2.20 \mathrm{E}+02$ & 1 & $1.38 \mathrm{E}+03$ & $6.98 \mathrm{E}+03$ \\
\hline Si.icp.a & $\mu \mathrm{g} / \mathrm{g}$ & $4.75 \mathrm{E}+02$ & $1.47 \mathrm{E}+02$ & 1 & $0.00 \mathrm{E}+00$ & $2.34 E+03$ \\
\hline${ }^{89 / 90} \mathrm{Sr}$ & $\mu \mathrm{Ci} / \mathrm{g}$ & $3.66 \mathrm{E}+01$ & $7.50 \mathrm{E}-01$ & 1 & $2.70 \mathrm{E}+01$ & $4.61 \mathrm{E}+01$ \\
\hline TIC & $\mu \mathrm{g} / \mathrm{g}$ & $8.25 E+03$ & $2.60 \mathrm{E}+02$ & 1 & $4.95 \mathrm{E}+03$ & $1.16 \mathrm{E}+04$ \\
\hline TOC.pers & $\mu \mathrm{g} / \mathrm{g}$ & $4.48 E+03$ & $1.50 \mathrm{E}+02$ & 1 & $2.57 \mathrm{E}+03$ & $6.39 E+03$ \\
\hline${ }^{99} \mathrm{Tc}$ & $\mu \mathrm{Ci} / \mathrm{g}$ & $1.34 \mathrm{E}-01$ & $8.00 \mathrm{E}-03$ & 1 & $3.24 \mathrm{E}-02$ & $2.36 \mathrm{E}-01$ \\
\hline Total Alpha & $\mu \mathrm{Ci} / \mathrm{g}$ & $5.80 \mathrm{E}-02$ & $7.95 \mathrm{E}-03$ & 1 & $0.00 \mathrm{E}+00$ & $1.59 \mathrm{E}-01$ \\
\hline Total Beta & $\mu \mathrm{Ci} / \mathrm{g}$ & $2.75 E+02$ & $6.50 \mathrm{E}+00$ & 1 & $1.92 \mathrm{E}+02$ & $3.57 \mathrm{E}+02$ \\
\hline U.phos & $\mu \mathrm{g} / \mathrm{g}$ & $8.25 E+01$ & $1.85 \mathrm{E}+00$ & 1 & $5.89 \mathrm{E}+01$ & $1.06 \mathrm{E}+02$ \\
\hline Zn.icp.a & $\mu \mathrm{g} / \mathrm{g}$ & $1.16 \mathrm{E}+01$ & $1.85 E+00$ & 1 & $0.00 \mathrm{E}+00$ & $3.51 \mathrm{E}+01$ \\
\hline Zr.icp.a & $\mu \mathrm{g} / \mathrm{g}$ & $5.59 \mathrm{E}+01$ & $3.15 \mathrm{E}+00$ & 1 & $1.58 \mathrm{E}+01$ & $9.59 \mathrm{E}+01$ \\
\hline
\end{tabular}

Note:

${ }^{1} \mathrm{Li}$ is one of the tracers for hydrostatic head fluid. 
Table B3-20. Sludge Composite (Core 152, Segment 22) Analytes with $>50$ Percent "Less Than" Values.

\begin{tabular}{|c|c|c|}
\hline T:1: Amalye & U.t. & 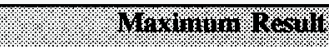 \\
\hline Acetate.ic & $\mu \mathrm{g} / \mathrm{g}$ & $<7.90 \mathrm{E}+01$ \\
\hline${ }^{241}$ Am.gea & $\mu \mathrm{Ci} / \mathrm{g}$ & $<4.95 \mathrm{E}-01$ \\
\hline As.icp.a & $\mu \mathrm{Ci} / \mathrm{g}$ & $<3.89 \mathrm{E}+01$ \\
\hline Ba.icp.a & $\mu \mathrm{g} / \mathrm{g}$ & $<1.95 \mathrm{E}+01$ \\
\hline Be.icp.a & $\mu \mathrm{g} / \mathrm{g}$ & $<1.95 \mathrm{E}+00$ \\
\hline Bi.icp.a & $\mu \mathrm{g} / \mathrm{g}$ & $<3.89 \mathrm{E}+01$ \\
\hline${ }^{1} \mathrm{Br} . i \mathrm{c}$ & $\mu \mathrm{g} / \mathrm{g}$ & $<4.94 \mathrm{E}+02$ \\
\hline Ce.icp.a & $\mu \mathrm{g} / \mathrm{g}$ & $<3.89 \mathrm{E}+01$ \\
\hline $243 / 244 \mathrm{Cm}$ & $\mu \mathrm{Ci} / \mathrm{g}$ & $<5.63 \mathrm{E}-03$ \\
\hline Co.icp.a & $\mu \mathrm{g} / \mathrm{g}$ & $<7.79 \mathrm{E}+00$ \\
\hline${ }^{60}$ Co.gea & $\mu \mathrm{Ci} / \mathrm{g}$ & $<1.80 \mathrm{E}-02$ \\
\hline${ }^{154}$ Eu.gea & $\mu \mathrm{Ci} / \mathrm{g}$ & $<7.58 \mathrm{E}-02$ \\
\hline${ }^{155}$ Eu.gea & $\mu \mathrm{Ci} / \mathrm{g}$ & $<2.30 \mathrm{E}-01$ \\
\hline${ }^{129} \mathrm{I}$ & $\mu \mathrm{Ci} / \mathrm{g}$ & $<3.11 \mathrm{E}-03$ \\
\hline La.icp.a & $\mu \mathrm{g} / \mathrm{g}$ & $<1.95 \mathrm{E}+01$ \\
\hline Mg.icp.a & $\mu \mathrm{g} / \mathrm{g}$ & $<3.89 \mathrm{E}+01$ \\
\hline Nd.icp.a & $\mu \mathrm{g} / \mathrm{g}$ & $<3.89 \mathrm{E}+01$ \\
\hline${ }^{237} \mathrm{~Np}$ & $\mu \mathrm{Ci} / \mathrm{g}$ & $<1.62 \mathrm{E}-02$ \\
\hline Sb.icp.a & $\mu \mathrm{g} / \mathrm{g}$ & $<2.34 \mathrm{E}+01$ \\
\hline Se.icp.a & $\mu \mathrm{g} / \mathrm{g}$ & $<3.89 \mathrm{E}+01$ \\
\hline Sm.icp.a & $\mu \mathrm{g} / \mathrm{g}$ & $<3.89 \mathrm{E}+01$ \\
\hline Sr.icp.a & $\mu \mathrm{g} / \mathrm{g}$ & $<3.89 \mathrm{E}+00$ \\
\hline Ti.icp.a & $\mu \mathrm{g} / \mathrm{g}$ & $<3.89 \mathrm{E}+00$ \\
\hline Tl.icp.a & $\mu \mathrm{g} / \mathrm{g}$ & $<7.79 \mathrm{E}+01$ \\
\hline Tritium & $\mu \mathrm{Ci} / \mathrm{g}$ & $<6.33 \mathrm{E}-04$ \\
\hline U.icp.a & $\mu \mathrm{g} / \mathrm{g}$ & $<1.95 \mathrm{E}+02$ \\
\hline V.icp.a & $\mu \mathrm{g} / \mathrm{g}$ & $<1.95 \mathrm{E}+01$ \\
\hline
\end{tabular}

Note:

'Br is one of the tracers for hydrostatic head fluid. 
In order to determine the tank sludge inventory, the sludge data were converted from $\mu \mathrm{g} / \mathrm{g}$ to $\mu \mathrm{g} / \mathrm{mL}$ using the following equation, where i represents the segment portion. The radionuclide sludge data were converted using the same equation substituting $\mathrm{Ci}$ for $\mathrm{g}$.

$$
\frac{\mu g}{m L}=\frac{\mu g}{g_{i}} \times \frac{g}{m L}{ }_{i}
$$

The densities are listed in Table B3-21. The converted sludge data were then analyzed using the ANOVA techniques explained above. The mean concentration estimates, along with the two-sided 95 percent confidence interval for the mean concentration, are given in Table B3-22 (nested ANOVA with the riser term), Table B3-23 (nested ANOVA without the riser term), and Table B3-24 ("less than" results).

Table B3-21. Tank 241-AN-105 Sludge Bulk Density Values.

\begin{tabular}{|c|c|c|c|c|c|c|c|}
\hline $\mathrm{RH}$ & Segment & Sortorl & Mensity & porer & Soprient & Poromin & Bsing \\
\hline 152 & 14 & Lower half & $1.53^{1}$ & 153 & 14 & Lower half & 1.56 \\
\hline 152 & 14 & Upper half & 1.53 & 153 & 15 & Lower half & 1.56 \\
\hline 152 & 16 & Lower half & 1.58 & 153 & 15 & Upper half & 1.52 \\
\hline 152 & 16 & Upper half & 1.59 & 153 & 17 & Lower half & 1.53 \\
\hline 152 & 18 & Lower half & 1.64 & 153 & 17 & Upper half & 1.52 \\
\hline 152 & 18 & Upper half & 1.66 & 153 & 19 & Lower half & 1.56 \\
\hline 152 & 20 & Lower half & 1.60 & 153 & 21 & Lower half & 1.57 \\
\hline 152 & 20 & Upper half & 1.58 & 153 & 22 & Lower half & 1.60 \\
\hline 152 & 22 & Upper half & 1.64 & 153 & 22 & Upper half & 1.58 \\
\hline
\end{tabular}

Note:

'No bulk density measurement; used the value from the upper half subsample. 
Table B3-22. Summary Statistics (per $\mathrm{mL}$ basis) - Sludge

(Nested ANOVA with Riser Term). (2 sheets)

\begin{tabular}{|c|c|c|c|c|c|c|}
\hline 6rallyte & Units: & 10 & $\sigma_{\%}$ & 18 & U1. & U1. \\
\hline Water.tga & $\mathrm{g} / \mathrm{mL}$ & $7.00 \mathrm{E}-01$ & 1.13E-02 & 1 & 5.57E-01 & $8.43 \mathrm{E}-01$ \\
\hline Acetate.ic.w & $\mu \mathrm{g} / \mathrm{mL}$ & $9.34 \mathrm{E}+02$ & $1.09 \mathrm{E}+02$ & 1 & $0.00 \mathrm{E}+00$ & $2.32 \mathrm{E}+03$ \\
\hline Acetate.ic.wo & $\mu \mathrm{g} / \mathrm{mL}$ & $1.06 \mathrm{E}+03$ & $4.29 \mathrm{E}+01$ & 1 & $5.15 \mathrm{E}+02$ & $1.61 \mathrm{E}+03$ \\
\hline Ag.icp.a & $\mu \mathrm{g} / \mathrm{mL}$ & $3.08 \mathrm{E}+01$ & $8.03 \mathrm{E}+00$ & 1 & $0.00 \mathrm{E}+00$ & $1.33 \mathrm{E}+02$ \\
\hline Al.icp.a & $\mu \mathrm{g} / \mathrm{mL}$ & $3.49 \mathrm{E}+04$ & $2.04 \mathrm{E}+03$ & 1 & $8.94 \mathrm{E}+03$ & $6.08 \mathrm{E}+04$ \\
\hline Al.icp.f & $\mu \mathrm{g} / \mathrm{mL}$ & $3.64 \mathrm{E}+04$ & $1.21 \mathrm{E}+03$ & 1 & $2.10 \mathrm{E}+04$ & $5.18 \mathrm{E}+04$ \\
\hline B.icp. ${ }^{*}$ & $\mu \mathrm{g} / \mathrm{mL}$ & $1.41 \mathrm{E}+02$ & $1.95 \mathrm{E}+01$ & 1 & $0.00 \mathrm{E}+00$ & $3.89 \mathrm{E}+02$ \\
\hline Ca.icp.a * & $\mu \mathrm{g} / \mathrm{mL}$ & $1.01 \mathrm{E}+03$ & $5.13 \mathrm{E}+02$ & 1 & $0.00 \mathrm{E}+00$ & $7.52 \mathrm{E}+03$ \\
\hline Cd.icp.a & $\mu \mathrm{g} / \mathrm{mL}$ & $1.84 \mathrm{E}+01$ & $3.25 \mathrm{E}+00$ & 1 & $0.00 \mathrm{E}+00$ & $5.98 \mathrm{E}+01$ \\
\hline $\mathrm{Cl}^{-}$.ic & $\mu \mathrm{g} / \mathrm{mL}$ & $8.14 \mathrm{E}+03$ & $3.03 \mathrm{E}+02$ & 1 & $4.29 \mathrm{E}+03$ & $1.20 \mathrm{E}+04$ \\
\hline Cr.icp.a & $\mu \mathrm{g} / \mathrm{mL}$ & $3.69 \mathrm{E}+03$ & $5.13 \mathrm{E}+02$ & 1 & $0.00 \mathrm{E}+00$ & $1.02 \mathrm{E}+04$ \\
\hline Cr.icp.f & $\mu \mathrm{g} / \mathrm{mL}$ & $3.91 \mathrm{E}+03$ & $6.47 \mathrm{E}+02$ & 1 & $0.00 \mathrm{E}+00$ & $1.21 \mathrm{E}+04$ \\
\hline Cu.icp.a.w & $\mu \mathrm{g} / \mathrm{mL}$ & $1.11 \mathrm{E}+01$ & 7.94E-01 & 1 & $1.01 \mathrm{E}+00$ & $2.12 \mathrm{E}+01$ \\
\hline Cu.icp.a.wo & $\mu \mathrm{g} / \mathrm{mL}$ & $1.12 \mathrm{E}+01$ & $7.72 \mathrm{E}-01$ & 1 & $1.40 \mathrm{E}+00$ & $2.10 \mathrm{E}+01$ \\
\hline \begin{tabular}{|l|} 
DSC.dry \\
\end{tabular} & Joules/g dry & $6.38 \mathrm{E}+01$ & $1.29 \mathrm{E}+01$ & 1 & $0.00 \mathrm{E}+00$ & $2.27 \mathrm{E}+02$ \\
\hline DSC.wet & Joules/g wet & $3.52 \mathrm{E}+01$ & $6.47 \mathrm{E}+00$ & 1 & $0.00 \mathrm{E}+00$ & $1.17 \mathrm{E}+02$ \\
\hline $\mathrm{F}^{-}$.ic & $\mu \mathrm{g} / \mathrm{mL}$ & $2.93 \mathrm{E}+03$ & $8.18 \mathrm{E}+02$ & 1 & $0.00 \mathrm{E}+00$ & $1.33 \mathrm{E}+04$ \\
\hline Fe.icp.a & $\mu \mathrm{g} / \mathrm{mL}$ & $1.89 \mathrm{E}+02$ & $4.22 \mathrm{E}+01$ & 1 & $0.00 \mathrm{E}+00$ & $7.25 \mathrm{E}+02$ \\
\hline Formate.ic & $\mu \mathrm{g} / \mathrm{mL}$ & $1.67 \mathrm{E}+03$ & $6.46 \mathrm{E}+01$ & 1 & $8.47 \mathrm{E}+02$ & $2.49 \mathrm{E}+03$ \\
\hline K.icp.a & $\mu \mathrm{g} / \mathrm{mL}$ & $5.44 \mathrm{E}+03$ & $3.01 \mathrm{E}+02$ & 1 & $1.62 \mathrm{E}+03$ & $9.26 \mathrm{E}+03$ \\
\hline${ }^{1}$ Li.icp.a.w & $\mu \mathrm{g} / \mathrm{mL}$ & $3.12 \mathrm{E}+01$ & $8.02 \mathrm{E}+00$ & 1 & $0.00 \mathrm{E}+00$ & $1.33 \mathrm{E}+02$ \\
\hline${ }^{1}$ Li.icp.a.wo & $\mu \mathrm{g} / \mathrm{mL}$ & $3.25 \mathrm{E}+01$ & $7.80 \mathrm{E}+00$ & 1 & $0.00 \mathrm{E}+00$ & $1.32 \mathrm{E}+02$ \\
\hline Mn.icp.a.w & $\mu \mathrm{g} / \mathrm{mL}$ & $3.32 \mathrm{E}+01$ & $1.52 \mathrm{E}+01$ & 1 & $0.00 \mathrm{E}+00$ & $2.26 \mathrm{E}+02$ \\
\hline Mn.icp.a.wo & $\mu \mathrm{g} / \mathrm{mL}$ & $3.42 \mathrm{E}+01$ & $1.57 \mathrm{E}+01$ & 1 & $0.00 \mathrm{E}+00$ & $2.33 \mathrm{E}+02$ \\
\hline Mo.icp.a * & $\mu \mathrm{g} / \mathrm{mL}$ & $8.12 \mathrm{E}+01$ & $5.92 \mathrm{E}+00$ & 1 & $5.96 \mathrm{E}+00$ & $1.56 \mathrm{E}+02$ \\
\hline $\mathrm{NO}_{2}^{-}$.ic & $\mu \mathrm{g} / \mathrm{mL}$ & $1.00 \mathrm{E}+05$ & $3.74 \mathrm{E}+03$ & 1 & $5.29 \mathrm{E}+04$ & $1.48 \mathrm{E}+05$ \\
\hline $\mathrm{NO}_{3}$. ic & $\mu \mathrm{g} / \mathrm{mL}$ & $1.76 \mathrm{E}+05$ & $1.17 \mathrm{E}+04$ & 1 & $2.65 \mathrm{E}+04$ & $3.25 \mathrm{E}+05$ \\
\hline Na.icp.a * & $\mu \mathrm{g} / \mathrm{mL}$ & $2.89 \mathrm{E}+05$ & $1.77 \mathrm{E}+04$ & 1 & $6.42 \mathrm{E}+04$ & $5.13 \mathrm{E}+05$ \\
\hline Na.icp.f* & $\mu \mathrm{g} / \mathrm{mL}$ & $3.60 \mathrm{E}+05$ & $1.41 \mathrm{E}+04$ & 1 & $1.81 \mathrm{E}+05$ & $5.38 \mathrm{E}+05$ \\
\hline Ni.icp.a & $\mu \mathrm{g} / \mathrm{mL}$ & $6.43 \mathrm{E}+01$ & $8.24 \mathrm{E}+00$ & 1 & $0.00 \mathrm{E}+00$ & $1.69 \mathrm{E}+02$ \\
\hline
\end{tabular}


Table B3-22. Summary Statistics (per mL basis) - Sludge

(Nested ANOVA with Riser Term). (2 sheets)

\begin{tabular}{|c|c|c|c|c|c|c|}
\hline Warigle & Orints & 1. & (1) & $d f$ & 11. & 61\% \\
\hline Ni.icp.f.w* & $\mu \mathrm{g} / \mathrm{mL}$ & $2.15 \mathrm{E}+03$ & $7.82 \mathrm{E}+02$ & 1 & $0.00 \mathrm{E}+00$ & $1.21 \mathrm{E}+04$ \\
\hline Ni.icp.f.wo & $\mu \mathrm{g} / \mathrm{mL}$ & $2.76 \mathrm{E}+03$ & $5.71 \mathrm{E}+02$ & 1 & $0.00 \mathrm{E}+00$ & $1.00 \mathrm{E}+04$ \\
\hline Oxalate.ic & $\mu \mathrm{g} / \mathrm{mL}$ & $1.10 \mathrm{E}+04$ & $1.60 \mathrm{E}+03$ & 1 & $0.00 \mathrm{E}+00$ & $3.13 \mathrm{E}+04$ \\
\hline P.icp.a & $\mu \mathrm{g} / \mathrm{mL}$ & $3.92 \mathrm{E}+03$ & $2.65 \mathrm{E}+03$ & 1 & $0.00 \mathrm{E}+00$ & $3.75 \mathrm{E}+04$ \\
\hline $\mathrm{PO}_{4}^{3-}$.ic. W & $\mu \mathrm{g} / \mathrm{mL}$ & $1.28 \mathrm{E}+04$ & $9.41 \mathrm{E}+03$ & 1 & $0.00 \mathrm{E}+00$ & $1.32 \mathrm{E}+05$ \\
\hline $\mathrm{PO}_{4}{ }^{3-}$.ic.wo & $\mu \mathrm{g} / \mathrm{mL}$ & $1.28 \mathrm{E}+04$ & $9.41 \mathrm{E}+03$ & 1 & $0.00 \mathrm{E}+00$ & $1.32 \mathrm{E}+05$ \\
\hline Pb.icp.a.w & $\mu \mathrm{g} / \mathrm{mL}$ & $7.49 \mathrm{E}+01$ & $8.77 \mathrm{E}+00$ & 1 & $0.00 \mathrm{E}+00$ & $1.86 \mathrm{E}+02$ \\
\hline Pb.icp.a.wo & $\mu \mathrm{g} / \mathrm{mL}$ & $7.96 \mathrm{E}+01$ & $1.17 \mathrm{E}+01$ & 1 & $0.00 \mathrm{E}+00$ & $2.28 \mathrm{E}+02$ \\
\hline S.icp.a * & $\mu \mathrm{g} / \mathrm{mL}$ & $3.96 \mathrm{E}+03$ & $5.80 E+02$ & 1 & $0.00 \mathrm{E}+00$ & $1.13 \mathrm{E}+04$ \\
\hline S.icp.f.w & $\mu \mathrm{g} / \mathrm{mL}$ & $4.39 E+03$ & $2.76 \mathrm{E}+02$ & 1 & $8.83 E+02$ & $7.89 \mathrm{E}+03$ \\
\hline S.icp.f.wo & $\mu \mathrm{g} / \mathrm{mL}$ & $4.45 \mathrm{E}+03$ & $2.63 \mathrm{E}+02$ & 1 & $1.10 \mathrm{E}+03$ & $7.79 \mathrm{E}+03$ \\
\hline $\mathrm{SO}_{4}{ }^{2-} \cdot \mathrm{ic}$ & $\mu \mathrm{g} / \mathrm{mL}$ & $9.92 E+03$ & $1.16 \mathrm{E}+03$ & 1 & $0.00 \mathrm{E}+00$ & $2.46 \mathrm{E}+04$ \\
\hline Si.icp.a.w & $\mu \mathrm{g} / \mathrm{mL}$ & $2.11 E+02$ & $9.39 \mathrm{E}+00$ & 1 & $9.20 \mathrm{E}+01$ & $3.31 \mathrm{E}+02$ \\
\hline Si.icp.a.wo & $\mu \mathrm{g} / \mathrm{mL}$ & $2.16 \mathrm{E}+02$ & $1.11 \mathrm{E}+01$ & 1 & $7.57 \mathrm{E}+01$ & $3.57 \mathrm{E}+02$ \\
\hline Zn.icp.a * & $\mu \mathrm{g} / \mathrm{mL}$ & $6.84 E+01$ & $8.84 \mathrm{E}+00$ & 1 & $0.00 \mathrm{E}+00$ & $1.81 E+02$ \\
\hline Zr.icp.a.w & $\mu \mathrm{g} / \mathrm{mL}$ & $1.81 \mathrm{E}+01$ & $6.33 \mathrm{E}+00$ & 1 & $0.00 \mathrm{E}+00$ & $9.84 \mathrm{E}+01$ \\
\hline Zr.icp.a.wo & $\mu \mathrm{g} / \mathrm{mL}$ & $1.89 \mathrm{E}+01$ & $6.77 \mathrm{E}+00$ & 1 & $0.00 \mathrm{E}+00$ & $1.05 \mathrm{E}+02$ \\
\hline Total Alpha.w & $\mu \mathrm{Ci} / \mathrm{mL}$ & $3.63 \mathrm{E}-02$ & $1.67 \mathrm{E}-02$ & 1 & $0.00 \mathrm{E}+00$ & $2.49 \mathrm{E}-01$ \\
\hline Total Alpha.wo & $\mu \mathrm{Ci} / \mathrm{mL}$ & $3.67 \mathrm{E}-02$ & $2.34 \mathrm{E}-02$ & 1 & $0.00 \mathrm{E}+00$ & $3.34 \mathrm{E}-01$ \\
\hline
\end{tabular}

Notes:

* $\hat{\sigma}_{\text {riser }}$ is significantly different from zero (evidence of horizontal heterogeneity).

' $\mathrm{Li}$ is used as tracer in hydrostatic head fluid. 
Table B3-23. Summary Statistics (per mL basis) - Sludge (Nested ANOVA without Riser). (2 sheets)

\begin{tabular}{|c|c|c|c|c|c|c|}
\hline Alpirto & Wuits & 16 & 6 & of. & 1. & M!. \\
\hline Water.tga & $\mathrm{g} / \mathrm{mL}$ & $7.00 \mathrm{E}-01$ & $1.13 \mathrm{E}-02$ & 10 & $6.75 \mathrm{E}-01$ & $7.25 \mathrm{E}-01$ \\
\hline Acetate.ic.w & $\mu \mathrm{g} / \mathrm{mL}$ & $9.37 \mathrm{E}+02$ & $8.20 \mathrm{E}+01$ & 10 & $7.54 \mathrm{E}+02$ & $1.12 \mathrm{E}+03$ \\
\hline Acetate.ic.wo & $\mu \mathrm{g} / \mathrm{mL}$ & $1.06 \mathrm{E}+03$ & $4.29 \mathrm{E}+01$ & 10 & $9.65 \mathrm{E}+02$ & $1.16 \mathrm{E}+03$ \\
\hline Ag.icp.a & $\mu \mathrm{g} / \mathrm{mL}$ & $3.08 \mathrm{E}+01$ & $8.03 E+00$ & 10 & $1.29 \mathrm{E}+01$ & $4.86 \mathrm{E}+01$ \\
\hline Al.icp.a & $\mu \mathrm{g} / \mathrm{mL}$ & $3.47 \mathrm{E}+04$ & $1.07 \mathrm{E}+03$ & 10 & $3.23 E+04$ & $3.71 \mathrm{E}+04$ \\
\hline Al.icp.f & $\mu \mathrm{g} / \mathrm{mL}$ & $3.64 \mathrm{E}+04$ & $1.21 \mathrm{E}+03$ & 10 & $3.37 \mathrm{E}+04$ & $3.91 \mathrm{E}+04$ \\
\hline Cd.icp.a & $\mu \mathrm{g} / \mathrm{mL}$ & $1.84 \mathrm{E}+01$ & $3.25 \mathrm{E}+00$ & 10 & $1.12 \mathrm{E}+01$ & $2.57 \mathrm{E}+01$ \\
\hline $\mathrm{Cl}^{-}$.ic & $\mu \mathrm{g} / \mathrm{mL}$ & $8.14 \mathrm{E}+03$ & $3.03 \mathrm{E}+02$ & 10 & $7.47 \mathrm{E}+03$ & $8.82 E+03$ \\
\hline Cr.icp.a & $\mu \mathrm{g} / \mathrm{mL}$ & $3.69 \mathrm{E}+03$ & $5.13 \mathrm{E}+02$ & 10 & $2.55 \mathrm{E}+03$ & $4.83 E+03$ \\
\hline Cr.icp.f & $\mu \mathrm{g} / \mathrm{mL}$ & $3.91 \mathrm{E}+03$ & $5.41 \mathrm{E}+02$ & 10 & $2.70 \mathrm{E}+03$ & $5.12 \mathrm{E}+03$ \\
\hline Cu.icp.a.w & $\mu \mathrm{g} / \mathrm{mL}$ & $1.11 \mathrm{E}+01$ & $7.86 \mathrm{E}-01$ & 10 & $9.35 \mathrm{E}+00$ & $1.28 \mathrm{E}+01$ \\
\hline Cu.icp.a.wo & $\mu \mathrm{g} / \mathrm{mL}$ & $1.12 \mathrm{E}+01$ & $7.72 \mathrm{E}-01$ & 10 & $9.49 \mathrm{E}+00$ & $1.29 E+01$ \\
\hline DSC.dry & $J / g$ dry & $6.38 \mathrm{E}+01$ & $8.31 \mathrm{E}+00$ & 10 & $4.53 \mathrm{E}+01$ & $8.23 E+01$ \\
\hline DSC.wet & $\mathrm{J} / \mathrm{g}$ wet & $3.52 \mathrm{E}+01$ & $4.70 \mathrm{E}+00$ & 10 & $2.48 \mathrm{E}+01$ & $4.57 \mathrm{E}+01$ \\
\hline $\mathrm{F}^{-}$.ic & $\mu \mathrm{g} / \mathrm{mL}$ & $2.93 \mathrm{E}+03$ & $8.18 \mathrm{E}+02$ & 10 & $1.11 \mathrm{E}+03$ & $4.75 \mathrm{E}+03$ \\
\hline Fe.icp.a & $\mu \mathrm{g} / \mathrm{mL}$ & $1.89 \mathrm{E}+02$ & $4.22 \mathrm{E}+01$ & 10 & $9.53 \mathrm{E}+01$ & $2.83 \mathrm{E}+02$ \\
\hline Formate.ic & $\mu \mathrm{g} / \mathrm{mL}$ & $1.67 \mathrm{E}+03$ & $6.02 E+01$ & 10 & $1.53 \mathrm{E}+03$ & $1.80 \mathrm{E}+03$ \\
\hline K.icp.a & $\mu \mathrm{g} / \mathrm{mL}$ & $5.42 \mathrm{E}+03$ & $1.77 \mathrm{E}+02$ & 10 & $5.03 \mathrm{E}+03$ & $5.82 E+03$ \\
\hline${ }^{1}$ Li.icp.a.w & $\mu \mathrm{g} / \mathrm{mL}$ & $3.12 \mathrm{E}+01$ & $8.02 \mathrm{E}+00$ & 10 & $1.33 \mathrm{E}+01$ & $4.91 \mathrm{E}+01$ \\
\hline${ }^{1}$ Li.icp.a.wo & $\mu \mathrm{g} / \mathrm{mL}$ & $3.25 \mathrm{E}+01$ & $7.80 \mathrm{E}+00$ & 10 & $1.51 \mathrm{E}+01$ & $4.99 \mathrm{E}+01$ \\
\hline Mn.icp.a.w & $\mu \mathrm{g} / \mathrm{mL}$ & $3.32 \mathrm{E}+01$ & $1.52 \mathrm{E}+01$ & 10 & $0.00 \mathrm{E}+00$ & $6.70 \mathrm{E}+01$ \\
\hline Mn.icp.a.wo & $\mu \mathrm{g} / \mathrm{mL}$ & $3.42 \mathrm{E}+01$ & $1.57 \mathrm{E}+01$ & 10 & $0.00 \mathrm{E}+00$ & $6.91 E+01$ \\
\hline $\mathrm{NO}_{2}^{-} \cdot \mathrm{ic}$ & $\mu \mathrm{g} / \mathrm{mL}$ & $1.00 \mathrm{E}+05$ & $3.74 \mathrm{E}+03$ & 10 & $9.21 \mathrm{E}+04$ & $1.09 \mathrm{E}+05$ \\
\hline $\mathrm{NO}_{3} \cdot \mathrm{ic}$ & $\mu \mathrm{g} / \mathrm{mL}$ & $1.76 \mathrm{E}+05$ & $1.17 \mathrm{E}+04$ & 10 & $1.50 \mathrm{E}+05$ & $2.02 \mathrm{E}+05$ \\
\hline Ni.icp.a & $\mu \mathrm{g} / \mathrm{mL}$ & $6.43 E+01$ & $8.24 \mathrm{E}+00$ & 10 & $4.59 \mathrm{E}+01$ & $8.26 \mathrm{E}+01$ \\
\hline Ni.icp.f.wo & $\mu \mathrm{g} / \mathrm{mL}$ & $2.76 \mathrm{E}+03$ & $5.71 \mathrm{E}+02$ & 9 & $1.47 \mathrm{E}+03$ & $4.05 \mathrm{E}+03$ \\
\hline Oxalate.ic & $\mu \mathrm{g} / \mathrm{mL}$ & $1.09 \mathrm{E}+04$ & $9.89 \mathrm{E}+02$ & 10 & $8.71 \mathrm{E}+03$ & $1.31 \mathrm{E}+04$ \\
\hline P.icp.a & $\mu \mathrm{g} / \mathrm{mL}$ & $3.92 E+03$ & $2.65 E+03$ & 10 & $0.00 \mathrm{E}+00$ & $9.82 \mathrm{E}+03$ \\
\hline $\mathrm{PO}_{4}^{3-}$.ic.w & $\mu \mathrm{g} / \mathrm{mL}$ & $1.28 \mathrm{E}+04$ & $9.41 \mathrm{E}+03$ & 10 & $0.00 \mathrm{E}+00$ & $3.37 \mathrm{E}+04$ \\
\hline $\mathrm{PO}_{4}^{3-}$.ic.wo & $\mu \mathrm{g} / \mathrm{mL}$ & $1.28 \mathrm{E}+04$ & $9.41 \mathrm{E}+03$ & 10 & $0.00 \mathrm{E}+00$ & $3.37 \mathrm{E}+04$ \\
\hline
\end{tabular}


Table B3-23. Summary Statistics (per mL basis) - Sludge (Nested ANOVA without Riser). (2 sheets)

\begin{tabular}{|c|c|c|c|c|c|c|}
\hline Anipilyte & Uinits & 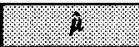 & 6 & dif & (1) & 161\% \\
\hline Pb.icp.a.w & $\mu \mathrm{g} / \mathrm{mL}$ & $7.48 \mathrm{E}+01$ & $7.08 \mathrm{E}+00$ & 10 & $5.90 \mathrm{E}+01$ & $9.06 \mathrm{E}+01$ \\
\hline Pb.icp.a.wo & $\mathrm{g} / \mathrm{mL}$ & $7.90 \mathrm{E}+01$ & $1.03 \mathrm{E}+01$ & 7 & $46 \mathrm{E}+01$ & $1.03 E+02$ \\
\hline S.icp.f.w & $\mu \mathrm{g} / \mathrm{mL}$ & $39 \mathrm{E}+03$ & $2.76 \mathrm{E}+02$ & 10 & -03 & $.00 \mathrm{E}+03$ \\
\hline S.icp.f.wo & $\mu \mathrm{g} / \mathrm{mL}$ & $4.45 \mathrm{E}+03$ & $2.62 \mathrm{E}+02$ & 10 & $3.86 \mathrm{E}+03$ & $5.03 E+03$ \\
\hline $\mathrm{SO}_{4}^{2-}$.ic & $\mu \mathrm{g} / \mathrm{mL}$ & $90 \mathrm{E}+03$ & $8.69 \mathrm{E}+02$ & 10 & $7.96 \mathrm{E}+03$ & $1.18 \mathrm{E}+04$ \\
\hline Si.icp.a.w & $\mu \mathrm{g} / \mathrm{mL}$ & $11 \mathrm{E}+02$ & $9.39 \mathrm{E}+00$ & 10 & $1.90 \mathrm{E}+02$ & $2.32 \mathrm{E}+02$ \\
\hline Si.icp.a.wo & $\mu \mathrm{g} / \mathrm{mL}$ & $6 \mathrm{E}+02$ & $8.24 \mathrm{E}+00$ & 10 & $98 \mathrm{E}+02$ & $2.35 \mathrm{E}+02$ \\
\hline Zr.icp.a.w & $\mu \mathrm{g} / \mathrm{mL}$ & $1.81 \mathrm{E}+01$ & $6.33 \mathrm{E}+00$ & 10 & $3.97 \mathrm{E}+00$ & $3.22 \mathrm{E}+01$ \\
\hline Zr.icp.a.wo & $\mu \mathrm{g} / \mathrm{mL}$ & $1.89 \mathrm{E}+01$ & $6.77 \mathrm{E}+00$ & 10 & $3.85 \mathrm{E}+00$ & $3.40 \mathrm{E}+01$ \\
\hline Total Alpha.w & $\mu \mathrm{Ci} / \mathrm{mL}$ & $3.63 \mathrm{E}-02$ & $1.67 \mathrm{E}-02$ & 10 & $0.00 \mathrm{E}+00$ & $7.35 \mathrm{E}-02$ \\
\hline Total Alpha.wo & $\mu \mathrm{Ci} / \mathrm{mL}$ & $3.66 \mathrm{E}-02$ & $2.34 \mathrm{E}-02$ & 7 & $0.00 \mathrm{E}+00$ & $9.19 \mathrm{E}-02$ \\
\hline
\end{tabular}

Note:

${ }^{1} \mathrm{Li}$ is used as tracer in hydrostatic head fluid. 
Table B3-24. Sludge (per L basis) - Analytes with > 50 Percent "Less Than" Values.

(2 sheets)

\begin{tabular}{|c|c|c|}
\hline Analyte & Unit & Maxlinum Resoll \\
\hline Ag.icp.f & $\mathrm{g} / \mathrm{L}$ & $<3.48 \mathrm{E}-01$ \\
\hline As.icp.a & $g / L$ & $<7.82 \mathrm{E}-02$ \\
\hline As.icp.f & $g / L$ & $<3.48 \mathrm{E}+00$ \\
\hline B.icp.f & $g / L$ & $<1.74 \mathrm{E}+00$ \\
\hline Ba.icp.a & $g / L$ & $<3.90 \mathrm{E}-02$ \\
\hline Ba.icp.f & $g / L$ & $<1.74 \mathrm{E}+00$ \\
\hline Be.icp.a & $g / L$ & $<4.76 \mathrm{E}-03$ \\
\hline Be.icp.f & $g / L$ & $<1.74 \mathrm{E}-01$ \\
\hline Bi.icp.a & $g / L$ & $<1.15 \mathrm{E}-01$ \\
\hline Bi.icp.f & $\mathrm{g} / \mathrm{L}$ & $<3.48 \mathrm{E}+00$ \\
\hline${ }^{1} \mathrm{Br}$.ic & $g / L$ & $<1.74 \mathrm{E}+00$ \\
\hline Ca.icp.f & $\mathrm{g} / \mathrm{L}$ & $<3.48 \mathrm{E}+00$ \\
\hline Cd.icp.f & $\mathrm{g} / \mathrm{L}$ & $<1.74 \mathrm{E}-01$ \\
\hline Ce.icp.a & $g / L$ & $<7.82 \mathrm{E}-02$ \\
\hline Ce.icp.f & $g / L$ & $<3.48 \mathrm{E}+00$ \\
\hline Co.icp.a & $g / L$ & $<1.56 \mathrm{E}-02$ \\
\hline Co.icp.f & $\mathrm{g} / \mathrm{L}$ & $<6.97 \mathrm{E}-01$ \\
\hline Cu.icp.f & $\mathrm{g} / \mathrm{L}$ & $<3.48 \mathrm{E}-01$ \\
\hline Fe.icp.f & $\mathrm{g} / \mathrm{L}$ & $<1.74 \mathrm{E}+00$ \\
\hline La.icp.a & $\mathrm{g} / \mathrm{L}$ & $<3.90 \mathrm{E}-02$ \\
\hline La.icp.f & $g / L$ & $<1.74 \mathrm{E}+00$ \\
\hline${ }^{1}$ Li.icp.f & $g / L$ & $<3.48 \mathrm{E}-01$ \\
\hline Mg.icp.a & $\mathrm{g} / \mathrm{L}$ & $<9.74 \mathrm{E}-02$ \\
\hline Mg.icp.f & $\mathrm{g} / \mathrm{L}$ & $<3.48 \mathrm{E}+00$ \\
\hline Mn.icp.f & $g / L$ & $<3.48 \mathrm{E}-01$ \\
\hline Mo.icp.f & $\mathrm{g} / \mathrm{L}$ & $<1.74 \mathrm{E}+00$ \\
\hline Nd.icp.a & $\mathrm{g} / \mathrm{L}$ & $<7.82 \mathrm{E}-02$ \\
\hline Nd.icp.f & $g / L$ & $<3.48 \mathrm{E}+00$ \\
\hline P.icp.f & $g / L$ & $<5.71 \mathrm{E}+01$ \\
\hline Pb.icp.f & $\mathrm{g} / \mathrm{L}$ & $<3.48 \mathrm{E}+00$ \\
\hline
\end{tabular}


Table B3-24. Sludge (per L basis) - Analytes with $>50$ Percent "Less Than" Values.

(2 sheets)

\begin{tabular}{|c|c|c|}
\hline Anavie & 1. VEit & Maximum Resill \\
\hline Sb.icp.a & $\mathrm{g} / \mathrm{L}$ & $<4.69 \mathrm{E}-02$ \\
\hline Sb.icp.f & $g / L$ & $<2.08 \mathrm{E}+00$ \\
\hline Se.icp.a & $\mathrm{g} / \mathrm{L}$ & $<7.82 \mathrm{E}-02$ \\
\hline Se.icp.f & $\mathrm{g} / \mathrm{L}$ & $<3.48 \mathrm{E}+00$ \\
\hline Si.icp.f & $g / L$ & $<3.82 \mathrm{E}+00$ \\
\hline Sm.icp.a & $\mathrm{g} / \mathrm{L}$ & $<7.82 \mathrm{E}-02$ \\
\hline Sm.icp.f & $g / L$ & $<3.48 \mathrm{E}+00$ \\
\hline Sr.icp.a & $\mathrm{g} / \mathrm{L}$ & $<1.56 \mathrm{E}-02$ \\
\hline Sr.icp.f & $\mathrm{g} / \mathrm{L}$ & $<3.48 \mathrm{E}-01$ \\
\hline Ti.icp.a & $g / L$ & $<7.82 \mathrm{E}-03$ \\
\hline Ti.icp.f & $g / L$ & $<3.48 \mathrm{E}-01$ \\
\hline Tl.icp.a & $\mathrm{g} / \mathrm{L}$ & $<1.56 \mathrm{E}-01$ \\
\hline Tl.icp.f & $g / L$ & $<6.97 \mathrm{E}+00$ \\
\hline U.icp.a & $g / L$ & $<3.90 \mathrm{E}-01$ \\
\hline U.icp.f & $g / L$ & $<1.74 \mathrm{E}+01$ \\
\hline V.icp.a & $\mathrm{g} / \mathrm{L}$ & $<3.90 \mathrm{E}-02$ \\
\hline V.icp.f & $g / L$ & $<1.74 \mathrm{E}+00$ \\
\hline Zn.icp.f & $\mathrm{g} / \mathrm{L}$ & $<1.26 \mathrm{E}+00$ \\
\hline Zr.icp.f & $g / L$ & $<7.68 \mathrm{E}-01$ \\
\hline
\end{tabular}

Note:

${ }^{1} \mathrm{Li}$ and ${ }^{1} \mathrm{Br}$ are the tracers for hydrostatic head fluid. 
B3.4.1.3 Crust. The mean concentration estimates for the crust (the mean of two analytical results for the one segment), along with the two-sided 95 percent confidence interval for the mean concentration, are given in Table B3-25 for those analytes with at least 50 percent of the reported data as quantitative values. The confidence interval takes into account only the analytical variability. For some of the analytes, the lower limit of the 95 percent confidence interval was a negative value due to the magnitude of the variability. Because the actual concentration of a tank sample cannot be less than zero, the lower limit is reported as zero. The analytes (crust), which had less than 50 percent of the reported data as quantitative values, are listed in Table B3-26. Table B3-26 cites the largest value observed from the analytical results.

Table B3-25. Summary Statistics (per g basis) - Crust. (2 sheets)

\begin{tabular}{|c|c|c|c|c|c|c|}
\hline Analyte & Units: & 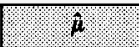 & $\sigma_{0}$ & dif & (1) & 11. \\
\hline$\%$ water.tga & wt\% & $4.37 \mathrm{E}+01$ & $2.94 \mathrm{E}+00$ & 1 & $6.45 E+00$ & $8.10 \mathrm{E}+01$ \\
\hline Ag.icp.a & $\mu \mathrm{g} / \mathrm{g}$ & $1.36 \mathrm{E}+01$ & $1.50 \mathrm{E}-01$ & 1 & $1.16 \mathrm{E}+01$ & $1.55 \mathrm{E}+01$ \\
\hline Al.icp.a & $\mu \mathrm{g} / \mathrm{g}$ & $2.50 \mathrm{E}+04$ & $1.50 \mathrm{E}+02$ & 1 & $2.30 \mathrm{E}+04$ & $2.69 \mathrm{E}+04$ \\
\hline \begin{tabular}{|l|} 
Al.icp.f \\
\end{tabular} & $\mu \mathrm{g} / \mathrm{g}$ & $2.50 \mathrm{E}+04$ & $6.00 \mathrm{E}+02$ & 1 & $1.74 \mathrm{E}+04$ & $3.26 \mathrm{E}+04$ \\
\hline B.icp.a & $\mu \mathrm{g} / \mathrm{g}$ & $1.04 \mathrm{E}+02$ & $6.80 \mathrm{E}+00$ & 1 & $1.78 \mathrm{E}+01$ & $1.91 \mathrm{E}+02$ \\
\hline Ca.icp.a & $\mu \mathrm{g} / \mathrm{g}$ & $1.45 \mathrm{E}+02$ & $3.70 \mathrm{E}+01$ & 1 & $0.00 \mathrm{E}+00$ & $6.15 \mathrm{E}+02$ \\
\hline Cd.icp.a & $\mu \mathrm{g} / \mathrm{g}$ & $3.54 \mathrm{E}+00$ & $2.25 \mathrm{E}-01$ & 1 & $6.76 \mathrm{E}-01$ & $6.39 \mathrm{E}+00$ \\
\hline $\mathrm{Cl}$.ic & $\mu \mathrm{g} / \mathrm{g}$ & $5.49 \mathrm{E}+03$ & $1.35 \mathrm{E}+02$ & 1 & $3.77 \mathrm{E}+03$ & $7.20 \mathrm{E}+03$ \\
\hline Cr.icp.a & $\mu \mathrm{g} / \mathrm{g}$ & $7.46 \mathrm{E}+02$ & $1.25 \mathrm{E}+01$ & 1 & $5.87 \mathrm{E}+02$ & $9.04 \mathrm{E}+02$ \\
\hline Cr.icp.f & $\mu \mathrm{g} / \mathrm{g}$ & $7.17 \mathrm{E}+02$ & $4.50 \mathrm{E}+00$ & 1 & $6.59 \mathrm{E}+02$ & $7.74 \mathrm{E}+02$ \\
\hline Cu.icp.a & $\mu \mathrm{g} / \mathrm{g}$ & $4.52 \mathrm{E}+00$ & $1.95 \mathrm{E}-01$ & 1 & $2.04 \mathrm{E}+00$ & $6.99 \mathrm{E}+00$ \\
\hline DSC.dry & Joules/g dry & $1.09 \mathrm{E}+01$ & $2.06 \mathrm{E}+00$ & 1 & $0.00 \mathrm{E}+00$ & $3.71 \mathrm{E}+01$ \\
\hline DSC.wet & Joules/g wet & $6.15 \mathrm{E}+00$ & $1.15 \mathrm{E}+00$ & 1 & $0.00 \mathrm{E}+00$ & $2.08 \mathrm{E}+01$ \\
\hline $\mathrm{F}^{-}$.ic & $\mu \mathrm{g} / \mathrm{g}$ & $3.98 \mathrm{E}+02$ & $1.29 \mathrm{E}+02$ & 1 & $0.00 \mathrm{E}+00$ & $2.04 \mathrm{E}+03$ \\
\hline Fe.icp.a & $\mu \mathrm{g} / \mathrm{g}$ & $3.88 \mathrm{E}+01$ & $6.00 \mathrm{E}+00$ & 1 & $0.00 \mathrm{E}+00$ & $1.15 \mathrm{E}+02$ \\
\hline Formate.ic & $\mu \mathrm{g} / \mathrm{g}$ & $1.30 \mathrm{E}+03$ & $6.00 \mathrm{E}+01$ & 1 & $5.38 \mathrm{E}+02$ & $2.06 \mathrm{E}+03$ \\
\hline K.icp.a & $\mu \mathrm{g} / \mathrm{g}$ & $3.88 \mathrm{E}+03$ & $8.50 \mathrm{E}+01$ & 1 & $2.79 \mathrm{E}+03$ & $4.96 \mathrm{E}+03$ \\
\hline Mo.icp.a & $\mu \mathrm{g} / \mathrm{g}$ & $5.72 \mathrm{E}+01$ & $6.00 \mathrm{E}-01$ & 1 & $4.96 \mathrm{E}+01$ & $6.48 \mathrm{E}+01$ \\
\hline $\mathrm{NO}_{2}$. ic & $\mu \mathrm{g} / \mathrm{g}$ & $7.29 \mathrm{E}+04$ & $1.60 \mathrm{E}+03$ & 1 & $5.26 \mathrm{E}+04$ & $9.32 \mathrm{E}+04$ \\
\hline $\mathrm{NO}_{3}{ }^{-}$.ic & $\mu \mathrm{g} / \mathrm{g}$ & $1.54 \mathrm{E}+05$ & $4.50 \mathrm{E}+03$ & 1 & $9.63 \mathrm{E}+04$ & $2.11 \mathrm{E}+05$ \\
\hline Na.icp.a & $\mu \mathrm{g} / \mathrm{g}$ & $1.83 \mathrm{E}+05$ & $4.50 \mathrm{E}+03$ & 1 & $1.25 \mathrm{E}+05$ & $2.40 \mathrm{E}+05$ \\
\hline Na.icp.f & $\mu \mathrm{g} / \mathrm{g}$ & $2.46 \mathrm{E}+05$ & $8.00 \mathrm{E}+03$ & 1 & $1.44 \mathrm{E}+05$ & $3.48 \mathrm{E}+05$ \\
\hline
\end{tabular}


Table B3-25. Summary Statistics (per $\mathrm{g}$ basis) - Crust. (2 sheets)

\begin{tabular}{|c|c|c|c|c|c|c|}
\hline Analyte & Virits. & in & 6 & if & II. & प1. \\
\hline Ni.icp.a & $\mu \mathrm{g} / \mathrm{g}$ & $1.45 \mathrm{E}+01$ & $1.85 \mathrm{E}+00$ & 1 & $0.00 \mathrm{E}+00$ & $3.80 \mathrm{E}+01$ \\
\hline Ni.icp.f & $\mu \mathrm{g} / \mathrm{g}$ & $1.85 \mathrm{E}+03$ & $1.07 \mathrm{E}+03$ & 1 & $0.00 \mathrm{E}+00$ & $1.54 \mathrm{E}+04$ \\
\hline Oxalate.ic & $\mu \mathrm{g} / \mathrm{g}$ & $4.48 \mathrm{E}+03$ & $8.50 \mathrm{E}+01$ & 1 & $3.39 \mathrm{E}+03$ & $5.56 \mathrm{E}+03$ \\
\hline P.icp.a & $\mu \mathrm{g} / \mathrm{g}$ & $1.13 \mathrm{E}+03$ & $1.96 \mathrm{E}+02$ & 1 & $0.00 \mathrm{E}+00$ & $3.62 \mathrm{E}+03$ \\
\hline $\mathrm{PO}_{4}^{3-}$. . & $\mu \mathrm{g} / \mathrm{g}$ & $3.59 \mathrm{E}+03$ & $3.50 \mathrm{E}+01$ & 1 & $3.14 \mathrm{E}+03$ & $4.03 \mathrm{E}+03$ \\
\hline S.icp.a & $\mu \mathrm{g} / \mathrm{g}$ & $1.54 \mathrm{E}+03$ & $4.00 \mathrm{E}+01$ & 1 & $1.03 \mathrm{E}+03$ & $2.05 E+03$ \\
\hline $\mathrm{SO}_{4}{ }^{2-}$.ic & $\mu \mathrm{g} / \mathrm{g}$ & $3.44 \mathrm{E}+03$ & $1.00 \mathrm{E}+01$ & 1 & $3.31 \mathrm{E}+03$ & $3.57 \mathrm{E}+03$ \\
\hline Si.icp.a & $\mu \mathrm{g} / \mathrm{g}$ & $2.10 \mathrm{E}+02$ & $9.00 \mathrm{E}+00$ & 1 & $9.56 \mathrm{E}+01$ & $3.24 \mathrm{E}+02$ \\
\hline Zn.icp.a & $\mu \mathrm{g} / \mathrm{g}$ & $3.17 \mathrm{E}+01$ & $5.00 \mathrm{E}-01$ & 1 & $2.53 \mathrm{E}+01$ & $3.81 \mathrm{E}+01$ \\
\hline Zn.icp.f & $\mu \mathrm{g} / \mathrm{g}$ & $6.45 \mathrm{E}+02$ & $5.90 \mathrm{E}+01$ & 1 & $0.00 \mathrm{E}+00$ & $1.39 \mathrm{E}+03$ \\
\hline
\end{tabular}


Table B3-26. Analytes (Crust - per $\mathrm{g}$ basis) with $>50$ Percent "Less Than" Values. (2 sheets)

\begin{tabular}{|c|c|c|}
\hline Analyte & I. Unt & Maximuin Resill \\
\hline Acetate.ic & $\mu \mathrm{g} / \mathrm{g}$ & $<7.57 \mathrm{E}+02$ \\
\hline Ag.icp.f & $\mu \mathrm{g} / \mathrm{g}$ & $<2.01 \mathrm{E}+02$ \\
\hline As.icp.a & $\mu \mathrm{g} / \mathrm{g}$ & $<4.03 \mathrm{E}+01$ \\
\hline As.icp.f & $\mu \mathrm{g} / \mathrm{g}$ & $<2.01 \mathrm{E}+03$ \\
\hline B.icp.f & $\mu \mathrm{g} / \mathrm{g}$ & $<1.01 \mathrm{E}+03$ \\
\hline Ba.icp.a & $\mu \mathrm{g} / \mathrm{g}$ & $<2.01 \mathrm{E}+01$ \\
\hline Ba.icp.f & $\mu \mathrm{g} / \mathrm{g}$ & $<1.01 \mathrm{E}+03$ \\
\hline Be.icp.a & $\mu \mathrm{g} / \mathrm{g}$ & $<2.01 \mathrm{E}+00$ \\
\hline Be.icp.f & $\mu \mathrm{g} / \mathrm{g}$ & $<1.01 \mathrm{E}+02$ \\
\hline Bi.icp.a & $\mu \mathrm{g} / \mathrm{g}$ & $<4.03 \mathrm{E}+01$ \\
\hline Bi.icp.f & $\mu \mathrm{g} / \mathrm{g}$ & $<2.01 \mathrm{E}+03$ \\
\hline${ }^{1} \mathrm{Br}$.ic & $\mu \mathrm{g} / \mathrm{g}$ & $<5.14 \mathrm{E}+02$ \\
\hline Ca.icp.f & $\mu \mathrm{g} / \mathrm{g}$ & $<2.01 \mathrm{E}+03$ \\
\hline Cd.icp.f & $\mu \mathrm{g} / \mathrm{g}$ & $<1.01 \mathrm{E}+02$ \\
\hline Ce.icp.a & $\mu \mathrm{g} / \mathrm{g}$ & $<4.03 \mathrm{E}+01$ \\
\hline Ce.icp.f & $\mu \mathrm{g} / \mathrm{g}$ & $<2.01 \mathrm{E}+03$ \\
\hline Co.icp.a & $\mu \mathrm{g} / \mathrm{g}$ & $<8.06 \mathrm{E}+00$ \\
\hline Co.icp.f & $\mu \mathrm{g} / \mathrm{g}$ & $<4.02 \mathrm{E}+02$ \\
\hline Cu.icp.f & $\mu \mathrm{g} / \mathrm{g}$ & $<2.01 \mathrm{E}+02$ \\
\hline Fe.icp.f & $\mu \mathrm{g} / \mathrm{g}$ & $<1.01 \mathrm{E}+03$ \\
\hline La.icp.a & $\mu \mathrm{g} / \mathrm{g}$ & $<2.01 \mathrm{E}+01$ \\
\hline La.icp.f & $\mu \mathrm{g} / \mathrm{g}$ & $<1.01 \mathrm{E}+03$ \\
\hline${ }^{1}$ Li.icp.a & $\mu \mathrm{g} / \mathrm{g}$ & $<4.03 \mathrm{E}+00$ \\
\hline${ }^{1}$ Li.icp.f & $\mu \mathrm{g} / \mathrm{g}$ & $<2.01 \mathrm{E}+02$ \\
\hline Mg.icp.a & $\mu \mathrm{g} / \mathrm{g}$ & $<4.03 \mathrm{E}+01$ \\
\hline Mg.icp.f & $\mu \mathrm{g} / \mathrm{g}$ & $<2.01 \mathrm{E}+03$ \\
\hline Mn.icp.a & $\mu \mathrm{g} / \mathrm{g}$ & $<4.03 \mathrm{E}+00$ \\
\hline Mn.icp.f & $\mu \mathrm{g} / \mathrm{g}$ & $<2.01 \mathrm{E}+02$ \\
\hline Mo.icp.f & $\mu \mathrm{g} / \mathrm{g}$ & $<1.01 \mathrm{E}+03$ \\
\hline Nd.icp.a & $\mu \mathrm{g} / \mathrm{g}$ & $<4.03 \mathrm{E}+01$ \\
\hline
\end{tabular}


Table B3-26. Analytes (Crust - per g basis) with > 50 Percent "Less Than" Values.

(2 sheets)

\begin{tabular}{|l|l|l|}
\hline \multicolumn{1}{|c|}{ Analye } & & Unit \\
\hline Nd.icp.f & $\mu \mathrm{g} / \mathrm{g}$ & $<2.01 \mathrm{E}+03$ \\
\hline P.icp.f & $\mu \mathrm{g} / \mathrm{g}$ & $<4.02 \mathrm{E}+03$ \\
\hline Pb.icp.a & $\mu \mathrm{g} / \mathrm{g}$ & $<4.14 \mathrm{E}+01$ \\
\hline Pb.icp.f & $\mu \mathrm{g} / \mathrm{g}$ & $<2.01 \mathrm{E}+03$ \\
\hline S.icp.f & $\mu \mathrm{g} / \mathrm{g}$ & $<2.01 \mathrm{E}+03$ \\
\hline Sb.icp.a & $\mu \mathrm{g} / \mathrm{g}$ & $<2.42 \mathrm{E}+01$ \\
\hline Sb.icp.f & $\mu \mathrm{g} / \mathrm{g}$ & $<1.21 \mathrm{E}+03$ \\
\hline Se.icp.a & $\mu \mathrm{g} / \mathrm{g}$ & $<4.03 \mathrm{E}+01$ \\
\hline Se.icp.f & $\mu \mathrm{g} / \mathrm{g}$ & $<2.01 \mathrm{E}+03$ \\
\hline Si.icp.f & $\mu \mathrm{g} / \mathrm{g}$ & $<1.01 \mathrm{E}+03$ \\
\hline Sm.icp.a & $\mu \mathrm{g} / \mathrm{g}$ & $<4.03 \mathrm{E}+01$ \\
\hline Sm.icp.f & $\mu \mathrm{g} / \mathrm{g}$ & $<2.01 \mathrm{E}+03$ \\
\hline Sr.icp.a & $\mu \mathrm{g} / \mathrm{g}$ & $<4.03 \mathrm{E}+00$ \\
\hline Sr.icp.f & $\mu \mathrm{g} / \mathrm{g}$ & $<2.01 \mathrm{E}+02$ \\
\hline Ti.icp.a & $\mu \mathrm{g} / \mathrm{g}$ & $<4.03 \mathrm{E}+00$ \\
\hline Ti.icp.f & $\mu \mathrm{g} / \mathrm{g}$ & $<2.01 \mathrm{E}+02$ \\
\hline Tl.icp.a & $\mu \mathrm{g} / \mathrm{g}$ & $<8.06 \mathrm{E}+01$ \\
\hline Tl.icp.f & $\mu \mathrm{g} / \mathrm{g}$ & $<4.02 \mathrm{E}+03$ \\
\hline Total Alpha & $\mu \mathrm{Ci} / \mathrm{g}$ & $<2.28 \mathrm{E}-02$ \\
\hline U.icp.a & $\mu \mathrm{g} / \mathrm{g}$ & $<2.01 \mathrm{E}+02$ \\
\hline U.icp.f & $\mu \mathrm{g} / \mathrm{g}$ & $<1.01 \mathrm{E}+04$ \\
\hline V.icp.a & $\mu \mathrm{g} / \mathrm{g}$ & $<2.01 \mathrm{E}+01$ \\
\hline V.icp.f & $\mu \mathrm{g} / \mathrm{g}$ & $<1.01 \mathrm{E}+03$ \\
\hline Zr.icp.a & $\mu \mathrm{g} / \mathrm{g}$ & $<4.03 \mathrm{E}+00$ \\
\hline Zr.icp.f & $\mu \mathrm{g} / \mathrm{g}$ & $<2.01 \mathrm{E}+02$ \\
\hline & & \\
\hline
\end{tabular}

Note:

${ }^{1} \mathrm{Li}$ and ${ }^{1} \mathrm{Br}$ are the tracers for hydrostatic head fluid. 


\section{B3.4.2 Analysis of Variance Model}

A statistical model is needed to account for the spatial and measurement variability in $\hat{\sigma}_{\hat{\mu}}$. This cannot be done using an ordinary standard deviation of the data (Snedecor 1980).

B3.4.2.1 Supernatant. The data were statistically evaluated using two different models. The first model used a nested analysis of variance. The nested analysis of variance statistical model used to describe the structure of the data is

where

$$
\begin{gathered}
Y_{i j k}=\mu+R_{i}+S_{i j}+A_{i j k}, \\
i=1,2, \ldots, a ; j=1,2, \ldots, b_{i} ; k=1,2, \ldots, n_{i j} ;
\end{gathered}
$$

$$
\begin{aligned}
& \mathrm{Y}_{\mathrm{ijk}}=\quad \text { concentration from the } \mathrm{k}^{\text {th }} \text { analytical result from the } \mathrm{j}^{\text {th }} \text { segment } \\
& \text { from the } i^{\text {th }} \text { riser } \\
& \mu \quad=\quad \text { the grand mean } \\
& \mathbf{R}_{\mathbf{i}}=\text { the effect of the } i^{\text {th }} \text { riser } \\
& S_{\mathrm{ij}}=\text { the effect of the } \mathrm{j}^{\text {th }} \text { segment from the } \mathrm{i}^{\text {th }} \text { riser } \\
& \mathrm{A}_{\mathrm{ijk}} \quad=\quad \text { the effect of the } \mathrm{k}^{\text {th }} \text { analytical result from the } \mathrm{j}^{\text {th }} \text { segment from } \\
& \text { the } i^{\text {th }} \text { riser } \\
& \text { a } \quad=\quad \text { the number of risers } \\
& b_{i} \quad=\quad \text { the number of segments from the } i^{\text {th }} \text { riser } \\
& \mathrm{n}_{\mathrm{ij}}=\quad \text { the number of analytical results from the } \mathrm{j}^{\text {th }} \text { segment from the } \\
& i^{\text {th }} \text { riser. }
\end{aligned}
$$

The variables $R_{i}$ and $S_{i j}$ are assumed to be a random effects. These variables, as well as $A_{i j k}$, are assumed to be uncorrelated and normally distributed with means zero and variances $\sigma^{2}(\mathrm{R}), \sigma^{2}(\mathrm{~S})$, and $\sigma^{2}(\mathrm{~A})$, respectively. Estimates of $\sigma^{2}(\mathrm{R}), \sigma^{2}(\mathrm{~S})$, and $\sigma^{2}(\mathrm{~A})$ were obtained using Restricted Maximum Likelihood Estimation (REML) method. This method applied to variance component estimation is described in Harville, 1977. The results using the REML techniques were obtained using the statistical analysis package S-PLUS ${ }^{1}$ (Statistical Sciences, 1993). The $d f$ associated with the standard deviation of the mean (a function of $\sigma^{2}(R), \sigma^{2}(S)$, and $\sigma^{2}(\mathrm{~A})$ ) is the number of risers minus one.

\footnotetext{
${ }^{1}$ S-PLUS is a trademark of Statistical Sciences, Incorporated, Seattle, Washington.
} 
The second model used one-way analysis of variance. The one-way analysis of variance statistical model used to describe the structure of the data is

$$
\begin{gathered}
\mathrm{Y}_{\mathrm{ij}}=\mu+\mathrm{S}_{\mathrm{i}}+\mathrm{A}_{\mathrm{ij}}, \\
\mathrm{i}=1,2, \ldots, \mathrm{a}, \mathrm{j}=1,2, \ldots, \mathrm{n}_{\mathrm{i}},
\end{gathered}
$$

where

$$
\begin{array}{ll}
Y_{i j} & =\text { concentration from the } j^{\text {th }} \text { analytical result from the } i^{\text {th }} \text { segment } \\
\mu & =\text { the grand mean } \\
S_{i} & =\text { the effect of the } i^{\text {th }} \text { segment } \\
\mathrm{A}_{\mathrm{ij}} & =\text { the effect of the } \mathrm{j}^{\text {th }} \text { analytical result from the } \mathrm{i}^{\text {th }} \text { segment } \\
\mathrm{a} & \quad \text { the number of segments } \\
\mathrm{n}_{\mathrm{i}} & \text { the number of analytical results from the } \mathrm{i}^{\text {th }} \text { segment. }
\end{array}
$$

The variable $S_{i}$ is assumed to be a random effect. This variable, as well as $A_{i j}$, are assumed to be uncorrelated and normally distributed with means zero and variances $\sigma^{2}(S)$ and $\sigma^{2}(A)$, respectively. Estimates of $\sigma^{2}(\mathrm{~S})$ and $\sigma^{2}(\mathrm{~A})$ were obtained using REML techniques. The results using the REML techniques were obtained using the statistical analysis package S-PLUS ${ }^{\text {TM }}$ (Statistical Sciences, 1993). The $d f$ associated with the standard deviation of the mean (a function of $\sigma^{2}(S)$ and $\sigma^{2}(A)$ ) is the number of segments minus one.

B3.4.2.2 Salt Slurry (Sludge). The data were statistically evaluated using two different models. The first model used a nested analysis of variance with a riser term. The nested analysis of variance statistical model used to describe the structure of the data is

$$
\begin{gathered}
Y_{i j k l}=\mu+R_{i}+S_{i j}+L_{i j k}+A_{i j k l}, \\
j=1,2, \ldots, a ; j=1,2, \ldots, b_{j} ; k=1,2, \ldots, c_{i j} ; l=1,2, \ldots, n_{i j k} ;
\end{gathered}
$$

where

$$
\begin{aligned}
& \mathrm{Y}_{\mathrm{ijk} \mathrm{k}}=\quad \text { concentration from the } \mathrm{l}^{\text {th }} \text { analytical result from the } \mathrm{k}^{\text {th }} \text { location } \\
& \text { from the } \mathrm{j}^{\text {th }} \text { segment from the } i^{\text {th }} \text { riser } \\
& \mu \quad=\quad \text { the grand mean } \\
& \mathrm{R}_{\mathrm{i}} \quad=\quad \text { the effect of the } \mathrm{i}^{\text {th }} \text { riser } \\
& \mathrm{S}_{\mathrm{ij}} \quad=\quad \text { the effect of the } \mathrm{j}^{\text {th }} \text { segment from the } \mathrm{i}^{\text {th }} \text { riser }
\end{aligned}
$$




$$
\begin{aligned}
& \mathrm{L}_{\mathrm{ijk}} \quad=\quad \text { the effect of the } \mathrm{k}^{\text {th }} \text { location from the } \mathrm{j}^{\text {th }} \text { segment from the } \\
& i^{\text {th }} \text { riser } \\
& \mathrm{A}_{\mathrm{ijk} 1} \quad=\quad \text { the effect of the } \mathrm{l}^{\text {th }} \text { analytical result from the } \mathrm{k}^{\text {th }} \text { location from } \\
& \text { the } j^{\text {th }} \text { segment from the } i^{\text {th }} \text { riser } \\
& \text { a } \quad=\quad \text { the number of risers } \\
& b_{i}=\text { the number of segments from the } i^{\text {th }} \text { riser } \\
& c_{i j} \quad=\quad \text { the number of locations from the } j^{\text {th }} \text { segment from the } i^{\text {th }} \text { riser } \\
& \mathrm{n}_{\mathrm{ijk}}=\quad \text { the number of analytical results from the } \mathrm{k}^{\text {th }} \text { location from the } \mathrm{j}^{\text {th }} \\
& \text { segment from the } \mathrm{i}^{\text {th }} \text { riser. }
\end{aligned}
$$

The variables $R_{i}, S_{i j}$, and $L_{i j k}$ are assumed to be random effects. These variables, as well as $\mathrm{A}_{\mathrm{ijk}}$, are assumed to be uncorrelated and normally distributed with means zero and variances $\sigma^{2}(\mathrm{R}), \sigma^{2}(\mathbf{S}), \sigma^{2}(\mathrm{~L})$, and $\sigma^{2}(\mathrm{~A})$, respectively. Estimates of $\sigma^{2}(\mathrm{R}), \sigma^{2}(\mathbf{S}), \sigma^{2}(\mathrm{~L})$, and $\sigma^{2}(\mathrm{~A})$ were obtained using REML techniques. This method applied to variance component estimation is described in Harville, 1977. The resuits using the REML techniques were obtained using the statistical analysis package S-PLUS ${ }^{\text {ru }}$ (Statistical Sciences, 1993). The $d f$ associated with the standard deviation of the mean (a function of $\sigma^{2}(\mathrm{R}), \sigma^{2}(\mathrm{~S}), \sigma^{2}(\mathrm{~L})$, and $\sigma^{2}(\mathrm{~A})$ ) is the number of risers minus one.

The second model used nested analysis of variance without the riser term. This nested ANOVA model is

where

$$
\begin{gathered}
Y_{i j k}=\mu+S_{i}+L_{i j}+A_{i j k}, \\
i=1,2, \ldots, a ; j=1,2, \ldots, b_{i} ; k=1,2, \ldots, n_{i j} ;
\end{gathered}
$$

$$
\begin{aligned}
& \mathrm{Y}_{\mathrm{ij \textrm {j }}}=\quad \text { concentration from the } \mathrm{k}^{\text {th }} \text { analytical result from the } \mathrm{j}^{\text {th }} \text { location } \\
& \text { from the } i^{\text {th }} \text { segment } \\
& \mu \quad=\quad \text { the grand mean } \\
& \mathrm{S}_{\mathrm{i}} \quad=\quad \text { the effect of the } \mathrm{i}^{\text {th }} \text { segment } \\
& L_{i j} \quad=\quad \text { the effect of the } j^{\text {th }} \text { location from the } i^{\text {th }} \text { segment } \\
& \mathrm{A}_{\mathrm{ijk}}=\quad \text { the effect of the } \mathrm{k}^{\text {th }} \text { analytical result from the } \mathrm{j}^{\mathrm{th}} \text { location from } \\
& \text { the } \mathrm{i}^{\text {th }} \text { segment } \\
& \text { a } \quad=\quad \text { the number of segments }
\end{aligned}
$$




$$
\begin{aligned}
& \mathrm{b}_{\mathrm{i}} \quad=\quad \text { the number of locations from the } \mathrm{i}^{\text {th }} \text { segment } \\
& \mathrm{n}_{\mathrm{ij}} \quad=\quad \text { the number of analytical results from the } \mathrm{j}^{\text {th }} \text { location from the } \\
& i^{\text {th }} \text { segment. }
\end{aligned}
$$

The variables $S_{i}$ and $L_{i j}$ are assumed to be a random effects. These variables, as well as $A_{i j k}$, are assumed to be uncorrelated and normally distributed with means zero and variances $\sigma^{2}(S)$, $\sigma^{2}(\mathrm{~L})$, and $\sigma^{2}(\mathrm{~A})$, respectively. Estimates of $\sigma^{2}(\mathrm{~S}), \sigma^{2}(\mathrm{~L})$, and $\sigma^{2}(\mathrm{~A})$ were obtained using REML techniques. This method applied to variance component estimation is described in Harville, 1977. The results using the REML techniques were obtained using the statistical analysis package S-PLUS ${ }^{\mathrm{TM}}$ (Statistical Sciences, 1993). The $d f$ associated with the standard deviation of the mean (a function of $\sigma^{2}(S), \sigma^{2}(L)$, and $\sigma^{2}(A)$ ) is the number of segments minus one.

\section{B3.4.3 Sampling Based Tank Inventory}

The sampling based tank inventory for each analyte is calculated by multiplying the tank volume $(4,270 \mathrm{~kL})$ by the mean concentration. The supernatant tank volume is estimated to be 60 percent of the tank volume or $2,562 \mathrm{~kL}$. The salt slurry tank volume is estimated to be 40 percent of the tank volume or $1,708 \mathrm{~kL}$. The lower and upper limits were calculated by multiplying the tank volume by the lower and upper limits from the two-sided 95 percent confidence interval for the mean concentration. The tank inventory for the supernatant for each analyte, along with the upper and lower limits, are presented in Table B3-27 (nested ANOVA), Table B3-28 (one-way ANOVA), Table B3-29 ("less than" results), and Table B3-30 (supernatant composite - Core 152, segments 1-14). The tank inventory for the sludge for each analyte, along with the upper and lower limits, are presented in Table B3-31 (nested ANOVA with the riser term), Table B3-32 (nested ANOVA without the riser term), and Table B3-33 ("less than" results). For those analytes with less than 50 percent of the data reported as quantitative values, the tank inventory was calculated by multiplying the tank volume by the maximum concentration listed in previous tables. Values for the lower limit and the upper limit are not possible.

The salt slurry tank inventory determined from the composite sample prepared from sludge segments 14-20 is presented in Table B3-34. The volume for sludge segments (14-21) was estimated to be 91 percent $\left(142^{\prime \prime} / 156^{\prime \prime}\right)$ of the total sludge inventory $(1,708 \mathrm{~kL})$, which is equal to $1,554 \mathrm{~kL}$. The salt slurry tank inventory determined from the composite sample prepared from sludge segment 22 is presented in Table B3-35. The volume for sludge segment 22 was estimated to be 9 percent $\left(14^{\prime \prime} / 156^{\prime \prime}\right)$ of the total sludge inventory $(1,708$ $\mathrm{kL}$ ), which is equal to $154 \mathrm{~kL}$.

The tank inventory based on the segment data is presented in Table B3-36. The tank inventory was determined by summing the supernatant inventory (Tables B3-27 and B3-29) and the salt slurry inventory values (Tables B3-31 and B3-33). The tank inventory based on the composite data is presented in Table B3-37. The tank inventory was determined by 
summing the supernatant inventory (Table B3-30) and the salt slurry inventory values (Tables B3-34 and B3-35). Since the analyte means were calculated using different data sets (.w, .wo, .ed, .adl and so forth), Tables B3-36 and B3-37 also list the analyte name for the supernatant mean (first column) and the analyte name for the salt slurry mean (second column).

Table B3-27. Analytical-Based Supernate Inventory for Tank 241-AN-105 (Nested ANOVA - Segment Data). (2 sheets)

\begin{tabular}{|c|c|c|c|}
\hline Arialyte & I) Tank Iryeritory & 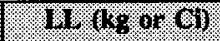 & $11 \%(180101$ \\
\hline Water.tga & $1.28 \mathrm{E}+06 \mathrm{~kg}$ & $1.16 \mathrm{E}+06$ & $1.40 \mathrm{E}+06$ \\
\hline Water.tga.ed & $1.29 \mathrm{E}+06 \mathrm{~kg}$ & $1.23 \mathrm{E}+06$ & $1.34 \mathrm{E}+06$ \\
\hline Acetate.ic.w & $1.54 \mathrm{E}+03 \mathrm{~kg}$ & $0.00 \mathrm{E}+00$ & $3.77 \mathrm{E}+03$ \\
\hline Acetate.ic.wo & $1.57 \mathrm{E}+03 \mathrm{~kg}$ & $0.00 \mathrm{E}+00$ & $3.46 \mathrm{E}+03$ \\
\hline Ag.icp.adl & $4.56 \mathrm{E}+01 \mathrm{~kg}$ & $3.43 \mathrm{E}+01$ & $5.69 \mathrm{E}+01$ \\
\hline Al.icp.adl & $1.07 \mathrm{E}+05 \mathrm{~kg}$ & $8.93 \mathrm{E}+04$ & $1.24 \mathrm{E}+05$ \\
\hline B.icp.adl & $1.91 \mathrm{E}+02 \mathrm{~kg}$ & $1.66 \mathrm{E}+02$ & $2.15 \mathrm{E}+02$ \\
\hline${ }^{1} \mathrm{Br}^{-}$.ic.w & $2.47 \mathrm{E}+03 \mathrm{~kg}$ & $0.00 \mathrm{E}+00$ & $5.20 \mathrm{E}+03$ \\
\hline${ }^{1} \mathrm{Br}^{-}$.ic.wo & $2.50 \mathrm{E}+03 \mathrm{~kg}$ & $3.46 \mathrm{E}+02$ & $4.66 \mathrm{E}+03$ \\
\hline $\mathrm{Cl}^{\prime}$.ic & $2.62 \mathrm{E}+04 \mathrm{~kg}$ & $5.07 \mathrm{E}+03$ & $4.74 \mathrm{E}+04$ \\
\hline $\mathrm{Cl}^{-}$.ic.ed & $2.54 \mathrm{E}+04 \mathrm{~kg}$ & $2.27 \mathrm{E}+04$ & $2.82 \mathrm{E}+04$ \\
\hline Cr.icp.adl & $5.76 \mathrm{E}+02 \mathrm{~kg}$ & $1.08 \mathrm{E}+02$ & $1.04 \mathrm{E}+03$ \\
\hline F-ic.w & $7.95 \mathrm{E}+02 \mathrm{~kg}$ & $0.00 \mathrm{E}+00$ & $1.99 \mathrm{E}+03$ \\
\hline F-ic.w.ed & $7.52 \mathrm{E}+02 \mathrm{~kg}$ & $0.00 \mathrm{E}+00$ & $2.39 \mathrm{E}+03$ \\
\hline \begin{tabular}{|l|} 
F-ic.wo \\
\end{tabular} & $1.25 \mathrm{E}+03 \mathrm{~kg}$ & $0.00 \mathrm{E}+00$ & $3.10 \mathrm{E}+03$ \\
\hline F'.ic.wo.ed & $1.16 \mathrm{E}+03 \mathrm{~kg}$ & $5.04 \mathrm{E}+02$ & $1.81 \mathrm{E}+03$ \\
\hline Formate.ic & $4.00 \mathrm{E}+03 \mathrm{~kg}$ & $1.30 \mathrm{E}+03$ & $6.69 \mathrm{E}+03$ \\
\hline K.icp.adl & $1.68 \mathrm{E}+04 \mathrm{~kg}$ & $1.45 \mathrm{E}+04$ & $1.91 \mathrm{E}+04$ \\
\hline Mo.icp.adl & $2.48 \mathrm{E}+02 \mathrm{~kg}$ & $2.15 \mathrm{E}+02$ & $2.80 \mathrm{E}+02$ \\
\hline $\mathrm{NO}_{2}^{-} \cdot \mathrm{ic}$ & $3.24 \mathrm{E}+05 \mathrm{~kg}$ & $4.61 \mathrm{E}+04$ & $6.02 \mathrm{E}+05$ \\
\hline $\mathrm{NO}_{2}$.ic.ed & $3.13 \mathrm{E}+05 \mathrm{~kg}$ & $2.77 \mathrm{E}+05$ & $3.49 \mathrm{E}+05$ \\
\hline $\mathrm{NO}_{3}^{-} \cdot \mathrm{ic}$ & $4.25 \mathrm{E}+05 \mathrm{~kg}$ & $7.10 \mathrm{E}+04$ & $7.79 \mathrm{E}+05$ \\
\hline $\mathrm{NO}_{3}{ }^{-}$.ic.ed & $4.12 \mathrm{E}+05 \mathrm{~kg}$ & $3.55 \mathrm{E}+05$ & $4.69 \mathrm{E}+05$ \\
\hline $\mathrm{Na}$.icp.adl & $6.33 \mathrm{E}+05 \mathrm{~kg}$ & $5.02 \mathrm{E}+05$ & $7.64 \mathrm{E}+05$ \\
\hline P.icp.adl & $1.02 \mathrm{E}+03 \mathrm{~kg}$ & $3.38 \mathrm{E}+02$ & $1.71 \mathrm{E}+03$ \\
\hline
\end{tabular}


Table B3-27. Analytical-Based Supernate Inventory for Tank 241-AN-105 -

(Nested ANOVA - Segment Data). (2 sheets)

\begin{tabular}{|c|c|c|c|}
\hline (1): Anlyte: & Tant Inventory & Ift of or of & 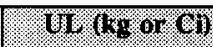 \\
\hline P.icp.adl.ed & $9.86 \mathrm{E}+02 \mathrm{~kg}$ & $3.66 \mathrm{E}+02$ & $1.61 \mathrm{E}+03$ \\
\hline $\mathrm{PO}_{4}^{3-}$.ic.w & $2.71 \mathrm{E}+03 \mathrm{~kg}$ & $0.00 \mathrm{E}+00$ & $1.50 \mathrm{E}+04$ \\
\hline $\mathrm{PO}_{4}{ }^{3-}$.ic.w.ed & $1.93 \mathrm{E}+03 \mathrm{~kg}$ & $0.00 \mathrm{E}+00$ & $4.57 \mathrm{E}+03$ \\
\hline$\overline{\mathrm{PO}_{4}{ }^{3-} \text {.ic.wo }}$ & $4.00 \mathrm{E}+03 \mathrm{~kg}$ & $0.00 \mathrm{E}+00$ & $3.35 \mathrm{E}+04$ \\
\hline $\mathrm{PO}_{4}{ }^{3-}$.ic.wo.ed & $2.15 \mathrm{E}+03 \mathrm{~kg}$ & $0.00 \mathrm{E}+00$ & $7.50 \mathrm{E}+03$ \\
\hline Pb.icp.adl.w & $1.38 \mathrm{E}+02 \mathrm{~kg}$ & $9.14 \mathrm{E}+01$ & $1.84 \mathrm{E}+02$ \\
\hline Pb.icp.adl.wo & $1.27 \mathrm{E}+02 \mathrm{~kg}$ & $7.49 \mathrm{E}+01$ & $1.79 \mathrm{E}+02$ \\
\hline S.icp.adl & $2.40 \mathrm{E}+03 \mathrm{~kg}$ & $1.77 \mathrm{E}+03$ & $3.03 \mathrm{E}+03$ \\
\hline $\mathrm{SO}_{4}{ }^{2-}$.ic.w & $3.03 \mathrm{E}+03 \mathrm{~kg}$ & $0.00 \mathrm{E}+00$ & $6.46 \mathrm{E}+03$ \\
\hline $\mathrm{SO}_{4}^{2-}$.ic.w.ed & $2.82 \mathrm{E}+03 \mathrm{~kg}$ & $1.95 \mathrm{E}+03$ & $3.69 \mathrm{E}+03$ \\
\hline $\mathrm{SO}_{4}{ }^{2-}$.ic.wo & $3.01 \mathrm{E}+03 \mathrm{~kg}$ & $0.00 \mathrm{E}+00$ & $6.31 \mathrm{E}+03$ \\
\hline $\mathrm{SO}_{4}{ }^{2}$.ic.wo.ed & $2.79 \mathrm{E}+03 \mathrm{~kg}$ & $2.04 \mathrm{E}+03$ & $3.54 \mathrm{E}+03$ \\
\hline Si.icp.adl & $5.59 \mathrm{E}+02 \mathrm{~kg}$ & $3.77 \mathrm{E}+02$ & $7.41 \mathrm{E}+02$ \\
\hline Zn.icp.adl & $8.00 \mathrm{E}+01 \mathrm{~kg}$ & $0.00 \mathrm{E}+00$ & $3.02 \mathrm{E}+02$ \\
\hline
\end{tabular}

Note:

'Br is one of the tracers for hydrostatic head fluid. 
Table B3-28. Analytical-Based Supernate Inventory for Tank 241-AN-105 (One-way ANOVA - Segment Data). (2 sheets)

\begin{tabular}{|c|c|c|c|}
\hline 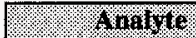 & . & 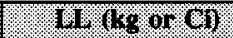 & 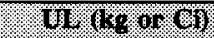 \\
\hline Water.tga & $1.28 \mathrm{E}+06 \mathrm{~kg}$ & $1.26 \mathrm{E}+06$ & $1.30 \mathrm{E}+06$ \\
\hline Water.tga.ed & $1.29 \mathrm{E}+06 \mathrm{~kg}$ & $1.28 \mathrm{E}+06$ & $1.29 \mathrm{E}+06$ \\
\hline Acetate.ic.w & $1.53 \mathrm{E}+03 \mathrm{~kg}$ & $1.32 \mathrm{E}+03$ & $1.74 \mathrm{E}+03$ \\
\hline Acetate.ic.wo & $1.57 \mathrm{E}+03 \mathrm{~kg}$ & $1.35 \mathrm{E}+03$ & $1.78 \mathrm{E}+03$ \\
\hline Ag.icp.adl & $4.56 \mathrm{E}+01 \mathrm{~kg}$ & $4.46 \mathrm{E}+01$ & $4.66 \mathrm{E}+01$ \\
\hline Al.icp.adl & $1.07 \mathrm{E}+05 \mathrm{~kg}$ & $1.04 \mathrm{E}+05$ & $1.09 \mathrm{E}+05$ \\
\hline B.icp.adl & $1.91 \mathrm{E}+02 \mathrm{~kg}$ & $1.87 \mathrm{E}+02$ & $1.94 \mathrm{E}+02$ \\
\hline${ }^{1} \mathrm{Br}^{-}$.ic.w & $2.48 \mathrm{E}+03 \mathrm{~kg}$ & $2.19 \mathrm{E}+03$ & $2.76 \mathrm{E}+03$ \\
\hline${ }^{1} \mathrm{Br}^{-}$.ic.wo & $2.51 \mathrm{E}+03 \mathrm{~kg}$ & $2.22 \mathrm{E}+03$ & $2.79 \mathrm{E}+03$ \\
\hline $\mathrm{Cl}^{-}$.ic & $2.62 \mathrm{E}+04 \mathrm{~kg}$ & $2.28 \mathrm{E}+04$ & $2.97 \mathrm{E}+04$ \\
\hline $\mathrm{Cl}^{-}$.ic.ed & $2.54 \mathrm{E}+04 \mathrm{~kg}$ & $2.50 \mathrm{E}+04$ & $2.59 \mathrm{E}+04$ \\
\hline Cr.icp.adl & $5.75 \mathrm{E}+02 \mathrm{~kg}$ & $5.33 E+02$ & $6.17 \mathrm{E}+02$ \\
\hline $\mathrm{F}^{-}$.ic.w & $7.95 \mathrm{E}+02 \mathrm{~kg}$ & $6.01 E+02$ & $9.88 \mathrm{E}+02$ \\
\hline$F^{-}$.ic.w.ed & $7.50 \mathrm{E}+02 \mathrm{~kg}$ & $5.56 \mathrm{E}+02$ & $9.45 \mathrm{E}+02$ \\
\hline Formate.ic & $3.99 \mathrm{E}+03 \mathrm{~kg}$ & $3.57 \mathrm{E}+03$ & $4.42 \mathrm{E}+03$ \\
\hline K.icp.adl & $1.68 \mathrm{E}+04 \mathrm{~kg}$ & $1.65 \mathrm{E}+04$ & $1.71 \mathrm{E}+04$ \\
\hline Mo.icp.adl & $2.48 \mathrm{E}+02 \mathrm{~kg}$ & $2.42 \mathrm{E}+02$ & $2.53 E+02$ \\
\hline $\mathrm{NO}_{2}^{-} \cdot \mathrm{ic}$ & $3.24 \mathrm{E}+05 \mathrm{~kg}$ & $2.79 \mathrm{E}+05$ & $3.69 \mathrm{E}+05$ \\
\hline $\mathrm{NO}_{2}^{-}$.ic.ed & $3.13 \mathrm{E}+05 \mathrm{~kg}$ & $3.07 \mathrm{E}+05$ & $3.19 E+05$ \\
\hline $\mathrm{NO}_{3}^{-} \cdot \mathrm{ic}$ & $4.25 \mathrm{E}+05 \mathrm{~kg}$ & $3.68 \mathrm{E}+05$ & $4.82 \mathrm{E}+05$ \\
\hline $\mathrm{NO}_{3}^{-}$.ic.ed & $4.12 \mathrm{E}+05 \mathrm{~kg}$ & $4.03 \mathrm{E}+05$ & $4.21 \mathrm{E}+05$ \\
\hline Na.icp.adl & $6.33 \mathrm{E}+05 \mathrm{~kg}$ & $6.20 \mathrm{E}+05$ & $6.45 \mathrm{E}+05$ \\
\hline P.icp.adl & $1.02 \mathrm{E}+03 \mathrm{~kg}$ & $9.14 \mathrm{E}+02$ & $1.14 \mathrm{E}+03$ \\
\hline P.icp.adl.ed & $9.85 \mathrm{E}+02 \mathrm{~kg}$ & $9.15 \mathrm{E}+02$ & $1.06 \mathrm{E}+03$ \\
\hline $\mathrm{PO}_{4}{ }^{3-}$.ic.w & $2.73 \mathrm{E}+03 \mathrm{~kg}$ & $1.10 \mathrm{E}+03$ & $4.35 \mathrm{E}+03$ \\
\hline $\mathrm{PO}_{4}{ }^{3-}$.ic.w.ed & $1.93 \mathrm{E}+03 \mathrm{~kg}$ & $1.66 \mathrm{E}+03$ & $2.21 \mathrm{E}+03$ \\
\hline $\mathrm{PO}_{4}{ }^{3-}$.ic.wo & $3.96 \mathrm{E}+03 \mathrm{~kg}$ & $1.39 \mathrm{E}+02$ & $7.77 \mathrm{E}+03$ \\
\hline Pb.icp.adl.w & $1.38 \mathrm{E}+02 \mathrm{~kg}$ & $1.31 E+02$ & $1.44 \mathrm{E}+02$ \\
\hline Pb.icp.adl.wo & $1.28 \mathrm{E}+02 \mathrm{~kg}$ & $1.22 \mathrm{E}+02$ & $1.34 \mathrm{E}+02$ \\
\hline S.icp.adl & $2.40 \mathrm{E}+03 \mathrm{~kg}$ & $2.30 \mathrm{E}+03$ & $2.50 \mathrm{E}+03$ \\
\hline
\end{tabular}


Table B3-28. Analytical-Based Supernate Inventory for Tank 241-AN-105 (One-way ANOVA - Segment Data). (2 sheets)

\begin{tabular}{|c|c|c|c|}
\hline Analy te & Tant Inventory & M. (kg or (9) & WU $(k$, or $(1)$ \\
\hline $\mathrm{SO}_{4}{ }^{2-}$.ic.w & $3.03 \mathrm{E}+03 \mathrm{~kg}$ & $2.58 \mathrm{E}+03$ & $3.48 \mathrm{E}+03$ \\
\hline $\mathrm{SO}_{4}{ }^{2-}$.ic.w.ed & $2.82 \mathrm{E}+03 \mathrm{~kg}$ & $2.69 \mathrm{E}+03$ & $2.95 \mathrm{E}+03$ \\
\hline $\mathrm{SO}_{4}{ }^{2-}$.ic.wo & $3.01 \mathrm{E}+03 \mathrm{~kg}$ & $2.54 \mathrm{E}+03$ & $3.48 \mathrm{E}+03$ \\
\hline $\mathrm{SO}_{4}{ }^{2-}$.ic.wo.ed & $2.79 \mathrm{E}+03 \mathrm{~kg}$ & $2.67 \mathrm{E}+03$ & $2.91 \mathrm{E}+03$ \\
\hline Si.icp.adl & $5.59 \mathrm{E}+02 \mathrm{~kg}$ & $5.30 \mathrm{E}+02$ & $5.89 \mathrm{E}+02$ \\
\hline
\end{tabular}

Note:

${ }^{1} \mathrm{Br}$ is one of the tracers for hydrostatic head fluid.

Table B3-29. Analytical-Based Supernatant Inventory for Tank 241-AN-105 ("Less Thans" - Segment Data). (2 sheets)

\begin{tabular}{|c|c|c|c|}
\hline Analye & Toni Truenlory & Let $1 \mathrm{gg}$ or 6 & (1) $(\mathrm{kg}, 0 . \mathrm{C})$ \\
\hline As.icp.adl & $<1.54 \mathrm{E}+02 \mathrm{~kg}$ & NA & NA \\
\hline Ba.icp.adl & $<7.71 \mathrm{E}+01 \mathrm{~kg}$ & NA & NA \\
\hline Be.icp.adl & $<7.69 \mathrm{E}+00 \mathrm{~kg}$ & NA & NA \\
\hline Bi.icp.adl & $<1.54 \mathrm{E}+02 \mathrm{~kg}$ & NA & $\mathrm{NA}$ \\
\hline Ca.icp.adl & $<1.54 \mathrm{E}+02 \mathrm{~kg}$ & NA & NA \\
\hline Cd.icp:adl & $<7.69 \mathrm{E}+00 \mathrm{~kg}$ & NA & $\overline{N A}$ \\
\hline Ce.icp.adl & $<1.54 \mathrm{E}+02 \mathrm{~kg}$ & NA & NA \\
\hline Co.icp.adl & $<3.07 \mathrm{E}+01 \mathrm{~kg}$ & NA & $\mathrm{NA}$ \\
\hline Cu.icp.adl & $<2.61 \mathrm{E}+01 \mathrm{~kg}$ & NA & $\mathrm{NA}$ \\
\hline Fe.icp.adl & $<7.71 \mathrm{E}+01 \mathrm{~kg}$ & NA & $\mathrm{NA}$ \\
\hline La.icp.adl & $<7.71 \mathrm{E}+01 \mathrm{~kg}$ & NA & NA \\
\hline${ }^{l}$ Li.icp.adl & $<6.99 \mathrm{E}+01 \mathrm{~kg}$ & NA & NA \\
\hline Mg.icp.adl & $<1.54 \mathrm{E}+02 \mathrm{~kg}$ & NA & NA \\
\hline Mn.icp.adl & $<1.54 \mathrm{E}+01 \mathrm{~kg}$ & NA & NA \\
\hline Nd.icp.adl & $<1.54 \mathrm{E}+02 \mathrm{~kg}$ & NA & NA \\
\hline Ni.icp.adl & $<3.07 \mathrm{E}+01 \mathrm{~kg}$ & NA & NA \\
\hline Oxalate.ic & $4.12 \mathrm{E}+04 \mathrm{~kg}$ & NA & NA \\
\hline Sb.icp.adl & $<9.25 \mathrm{E}+01 \mathrm{~kg}$ & NA & $\mathrm{NA}$ \\
\hline
\end{tabular}


Table B3-29. Analytical-Based Supernatant Inventory for Tank 241-AN-105 ("Less Thans" - Segment Data). (2 sheets)

\begin{tabular}{|c|c|c|c|}
\hline Arate & WHar GYoron & $1.6,0 \%$ & 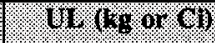 \\
\hline Se.icp.adl & $<1.54 \mathrm{E}+02 \mathrm{~kg}$ & NA & NA \\
\hline Sm.icp.adl & $<1.54 \mathrm{E}+02 \mathrm{~kg}$ & NA & NA \\
\hline Sr.icp.adl & $<1.54 \mathrm{E}+01 \mathrm{~kg}$ & NA & NA \\
\hline Ti.icp.adl & $<1.54 \mathrm{E}+01 \mathrm{~kg}$ & NA & NA \\
\hline T1.icp.adl & $<3.07 \mathrm{E}+02 \mathrm{~kg}$ & NA & NA \\
\hline Total Alpha & $<3.71 \mathrm{E}+02 \mathrm{Ci}$ & NA & NA \\
\hline U.icp.adl & $<7.69 \mathrm{E}+02 \mathrm{~kg}$ & NA & NA \\
\hline V.icp.adl & $<7.71 \mathrm{E}+01 \mathrm{~kg}$ & NA & NA \\
\hline Zr.icp.adl & $<1.56 \mathrm{E}+01 \mathrm{~kg}$ & NA & NA \\
\hline
\end{tabular}

Note:

${ }^{1} \mathrm{Li}$ is one of the tracers for hydrostatic head fluid. 
Table B3-30. Analytical-Based Supernatant Inventory for Tank 241-AN-105 - (Supernatant

Composite Sample - Core 152, Segments 1-14). (4 sheets)

\begin{tabular}{|c|c|c|c|}
\hline 141016 & That worlory & 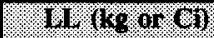 & 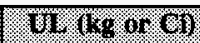 \\
\hline Water.tga & $1.82 \mathrm{E}+06 \mathrm{~kg}$ & $2.29 \mathrm{E}+05$ & $3.42 \mathrm{E}+06$ \\
\hline Acetate.ic & $1.64 \mathrm{E}+03 \mathrm{~kg}$ & $1.63 \mathrm{E}+03$ & $1.66 \mathrm{E}+03$ \\
\hline Ag.icp.a & $3.88 \mathrm{E}+01 \mathrm{~kg}$ & $3.07 \mathrm{E}+01$ & $4.70 \mathrm{E}+01$ \\
\hline Ag.icp.adl & $4.61 \mathrm{E}+01 \mathrm{~kg}$ & $2.98 \mathrm{E}+01$ & $6.24 \mathrm{E}+01$ \\
\hline Al.icp.a & $9.15 \mathrm{E}+04 \mathrm{~kg}$ & $8.82 \mathrm{E}+04$ & $9.47 \mathrm{E}+04$ \\
\hline Al.icp.adl & $1.12 \mathrm{E}+05 \mathrm{~kg}$ & $1.05 \mathrm{E}+05$ & $1.18 \mathrm{E}+05$ \\
\hline B.icp.a & $4.34 \mathrm{E}+02 \mathrm{~kg}$ & $0.00 \mathrm{E}+00$ & $8.74 \mathrm{E}+02$ \\
\hline B.icp.adl & $1.86 \mathrm{E}+02 \mathrm{~kg}$ & $1.39 \mathrm{E}+02$ & $2.34 \mathrm{E}+02$ \\
\hline Ca.icp.a & $2.25 \mathrm{E}+03 \mathrm{~kg}$ & $1.44 \mathrm{E}+03$ & $3.06 \mathrm{E}+03$ \\
\hline $\mathrm{Cl}^{-}$.ic & $2.61 \mathrm{E}+04 \mathrm{~kg}$ & $2.61 \mathrm{E}+04$ & $2.61 E+04$ \\
\hline Cr.icp.a & $4.79 \mathrm{E}+02 \mathrm{~kg}$ & $4.79 \mathrm{E}+02$ & $4.79 \mathrm{E}+02$ \\
\hline Cr.icp.adl & $5.70 \mathrm{E}+02 \mathrm{~kg}$ & $5.21 \mathrm{E}+02$ & $6.19 \mathrm{E}+02$ \\
\hline Cr(VI).spec & $3.93 \mathrm{E}+02 \mathrm{~kg}$ & $3.77 \mathrm{E}+02$ & $4.10 \mathrm{E}+02$ \\
\hline${ }^{137}$ Cs.gea & $1.22 \mathrm{E}+03 \mathrm{Ci}$ & $1.17 \mathrm{E}+03$ & $1.26 \mathrm{E}+03$ \\
\hline Fe.icp.a & $1.63 \mathrm{E}+02 \mathrm{~kg}$ & $0.00 \mathrm{E}+00$ & $4.47 \mathrm{E}+02$ \\
\hline Formate.ic & $4.15 \mathrm{E}+03 \mathrm{~kg}$ & $4.15 \mathrm{E}+03$ & $4.15 E+03$ \\
\hline${ }^{129} I$ & $6.85 \mathrm{E}-01 \mathrm{Ci}$ & $0.00 \mathrm{E}+00$ & $1.39 \mathrm{E}+00$ \\
\hline K.icp.a & $1.36 \mathrm{E}+04 \mathrm{~kg}$ & $1.25 \mathrm{E}+04$ & $1.47 \mathrm{E}+04$ \\
\hline K.icp.adl & $1.67 \mathrm{E}+04 \mathrm{~kg}$ & $1.57 \mathrm{E}+04$ & $1.77 \mathrm{E}+04$ \\
\hline${ }^{1}$ Li.icp.a & $1.53 \mathrm{E}+01 \mathrm{~kg}$ & $1.06 \mathrm{E}+01$ & $2.00 \mathrm{E}+01$ \\
\hline Mo.icp.a & $2.11 \mathrm{E}+02 \mathrm{~kg}$ & $2.03 E+02$ & $2.20 \mathrm{E}+02$ \\
\hline Mo.icp.adl & $2.59 \mathrm{E}+02 \mathrm{~kg}$ & $2.26 \mathrm{E}+02$ & $2.91 \mathrm{E}+02$ \\
\hline $\mathrm{NO}_{2}^{-} \cdot \mathrm{ic}$ & $3.34 \mathrm{E}+05 \mathrm{~kg}$ & $3.18 \mathrm{E}+05$ & $3.51 \mathrm{E}+05$ \\
\hline $\mathrm{NO}_{3}^{-} \cdot \mathrm{ic}$ & $4.29 \mathrm{E}+05 \mathrm{~kg}$ & $4.13 E+05$ & $4.45 \mathrm{E}+05$ \\
\hline Na.icp.a & $5.41 \mathrm{E}+05 \mathrm{~kg}$ & $5.41 \mathrm{E}+05$ & $5.41 \mathrm{E}+05$ \\
\hline Na.icp.adl & $6.55 \mathrm{E}+05 \mathrm{~kg}$ & $6.06 \mathrm{E}+05$ & $7.03 \mathrm{E}+05$ \\
\hline$\overline{\mathrm{OH}^{-}}$ & $1.50 \mathrm{E}+05 \mathrm{~kg}$ & $8.65 E+04$ & $2.13 \mathrm{E}+05$ \\
\hline P.icp.a & $8.70 \mathrm{E}+02 \mathrm{~kg}$ & $7.56 \mathrm{E}+02$ & $9.84 \mathrm{E}+02$ \\
\hline P.icp.adl & $1.01 \mathrm{E}+03 \mathrm{~kg}$ & $9.24 \mathrm{E}+02$ & $1.09 \mathrm{E}+03$ \\
\hline $\mathrm{PO}_{4}{ }^{3-} \cdot \mathrm{ic}$ & $1.58 \mathrm{E}+03 \mathrm{~kg}$ & $9.77 \mathrm{E}+02$ & $2.18 \mathrm{E}+03$ \\
\hline
\end{tabular}


Table B3-30. Analytical-Based Supernatant Inventory for Tank 241-AN-105 - (Supernatant

Composite Sample - Core 152, Segments 1-14). (4 sheets)

\begin{tabular}{|c|c|c|c|}
\hline A14lote & 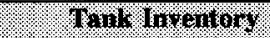 & Wl. ks or oly & 161. 48 . 0\% (6) \\
\hline S.icp.a & $2.25 \mathrm{E}+03 \mathrm{~kg}$ & $2.15 \mathrm{E}+03$ & $2.34 \mathrm{E}+03$ \\
\hline S.icp.adl & $2.59 \mathrm{E}+03 \mathrm{~kg}$ & $1.99 \mathrm{E}+03$ & $3.19 \mathrm{E}+03$ \\
\hline $\mathrm{SO}_{4}^{2-}$.ic & $2.48 \mathrm{E}+03 \mathrm{~kg}$ & $1.17 \mathrm{E}+03$ & $3.80 \mathrm{E}+03$ \\
\hline Si.icp.a & $5.62 \mathrm{E}+02 \mathrm{~kg}$ & $1.88 \mathrm{E}+02$ & $9.37 \mathrm{E}+02$ \\
\hline Si.icp.adl & $4.25 \mathrm{E}+02 \mathrm{~kg}$ & $4.25 \mathrm{E}+02$ & $4.25 \mathrm{E}+02$ \\
\hline${ }^{89 / 90} \mathrm{Sr}$ & $1.06 \mathrm{E}+02 \mathrm{Ci}$ & $8.65 \mathrm{E}+01$ & $1.26 \mathrm{E}+02$ \\
\hline TIC & $5.62 \mathrm{E}+03 \mathrm{~kg}$ & $4.81 E+03$ & $6.44 \mathrm{E}+03$ \\
\hline TOC.coul & $1.56 \mathrm{E}+04 \mathrm{~kg}$ & $1.47 \mathrm{E}+04$ & $1.66 \mathrm{E}+04$ \\
\hline TOC.pers & $6.64 \mathrm{E}+03 \mathrm{~kg}$ & $1.75 \mathrm{E}+03$ & $1.15 \mathrm{E}+04$ \\
\hline${ }^{99} \mathrm{Tc}$ & $7.01 \mathrm{E}+02 \mathrm{Ci}$ & $5.22 \mathrm{E}+02$ & $8.80 \mathrm{E}+02$ \\
\hline Total Beta & $1.38 \mathrm{E}+06 \mathrm{Ci}$ & $1.25 \mathrm{E}+06$ & $1.51 \mathrm{E}+06$ \\
\hline Zn.icp.a & $1.95 \mathrm{E}+02 \mathrm{~kg}$ & $1.43 \mathrm{E}+02$ & $2.47 \mathrm{E}+02$ \\
\hline Zn.icp.adl & $4.51 \mathrm{E}+01 \mathrm{~kg}$ & $2.88 \mathrm{E}+01$ & $6.14 \mathrm{E}+01$ \\
\hline U.phos & $9.13 \mathrm{E}+00 \mathrm{~kg}$ & $2.79 \mathrm{E}+00$ & $1.55 \mathrm{E}+01$ \\
\hline${ }^{241} \mathrm{Am}$.aea & $<1.00 \mathrm{E}-01 \mathrm{Ci}$ & NA & NA \\
\hline${ }^{241}$ Am.gea & $<2.20 \mathrm{E}+03 \mathrm{Ci}$ & NA & NA \\
\hline As.icp.a & $<1.34 \mathrm{E}+02 \mathrm{~kg}$ & NA & $\overline{N A}$ \\
\hline As.icp.adl & $<1.54 \mathrm{E}+02 \mathrm{~kg}$ & NA & NA \\
\hline Ba.icp.a & $<6.71 \mathrm{E}+01 \mathrm{~kg}$ & NA & NA \\
\hline Ba.icp.adl & $<7.71 \mathrm{E}+01 \mathrm{~kg}$ & NA & $\mathrm{NA}$ \\
\hline Be.icp.a & $<6.71 \mathrm{E}+00 \mathrm{~kg}$ & NA & NA \\
\hline Be.icp.adl & $<7.69 \mathrm{E}+00 \mathrm{~kg}$ & NA & NA \\
\hline Bi.icp.a & $<1.34 \mathrm{E}+02 \mathrm{~kg}$ & NA & NA \\
\hline Bi.icp.adl & $<1.54 \mathrm{E}+02 \mathrm{~kg}$ & $\mathrm{NA}$ & NA \\
\hline${ }^{1} \mathrm{Br}^{-}$.ic & $<1.34 \mathrm{E}+03 \mathrm{~kg}$ & NA & NA \\
\hline Ca.icp.adl & $<1.54 \mathrm{E}+02 \mathrm{~kg}$ & NA & NA \\
\hline Cd.icp.a & $<6.71 \mathrm{E}+00 \mathrm{~kg}$ & $\mathrm{NA}$ & NA \\
\hline Cd.icp.adl & $<7.69 \mathrm{E}+00 \mathrm{~kg}$ & NA & NA \\
\hline Ce.icp.a & $<1.34 \mathrm{E}+02 \mathrm{~kg}$ & NA & NA \\
\hline Ce.icp.adl & $<1.54 \mathrm{E}+02 \mathrm{~kg}$ & NA & NA \\
\hline
\end{tabular}


Table B3-30. Analytical-Based Supernatant Inventory for Tank 241-AN-105 - (Supernatant Composite Sample - Core 152, Segments 1-14). (4 sheets)

\begin{tabular}{|c|c|c|c|}
\hline $1+19410$ & Tonis inverion. & 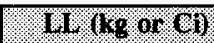 & ML (Ko or 81$)$ \\
\hline${ }^{243 / 244} \mathrm{Cm}$ & $<1.00 \mathrm{E}-01 \mathrm{Ci}$ & NA & NA \\
\hline Co.icp.a & $<2.69 \mathrm{E}+01 \mathrm{~kg}$ & NA & NA \\
\hline Co.icp.adl & $<3.07 \mathrm{E}+01 \mathrm{~kg}$ & $\mathrm{NA}$ & NA \\
\hline${ }^{60}$ Co.gea & $<3.33 \mathrm{E}-02 \mathrm{Ci}$ & NA & NA \\
\hline Cu.icp.a & $<1.61 \mathrm{E}+01 \mathrm{~kg}$ & NA & NA \\
\hline Cu.icp.adl & $<1.54 \mathrm{E}+01 \mathrm{~kg}$ & NA & NA \\
\hline${ }^{154}$ Eu.gea & $<2.50 \mathrm{E}+02 \mathrm{Ci}$ & NA & NA \\
\hline${ }^{155}$ Eu.gea & $<8.35 \mathrm{E}+02 \mathrm{Ci}$ & NA & NA \\
\hline $\mathrm{F}^{-}$.ic & $<1.38 \mathrm{E}+02 \mathrm{~kg}$ & NA & NA \\
\hline Fe.icp.adl & $<7.71 \mathrm{E}+01 \mathrm{~kg}$ & NA & NA \\
\hline Hg.cvaa & $<1.79 \mathrm{E}-01 \mathrm{~kg}$ & NA & NA \\
\hline La.icp.a & $<6.71 \mathrm{E}+01 \mathrm{~kg}$ & NA & NA \\
\hline La.icp.adl & $<7.71 \mathrm{E}+01 \mathrm{~kg}$ & NA & NA \\
\hline${ }^{1}$ Li.icp.adl & $<1.54 \mathrm{E}+01 \mathrm{~kg}$ & $\mathrm{NA}$ & NA \\
\hline Mg.icp.a & $<1.34 \mathrm{E}+02 \mathrm{~kg}$ & $\mathrm{NA}$ & NA \\
\hline Mg.icp.adl & $<1.54 \mathrm{E}+02 \mathrm{~kg}$ & NA & NA \\
\hline Mn.icp.a & $<1.34 \mathrm{E}+01 \mathrm{~kg}$ & NA & NA \\
\hline Mn.icp.adl & $<1.54 \mathrm{E}+01 \mathrm{~kg}$ & NA & NA \\
\hline Nd.icp.a & $<1.34 \mathrm{E}+02 \mathrm{~kg}$ & NA & NA \\
\hline Nd.icp.adl & $<1.54 \mathrm{E}+02 \mathrm{~kg}$ & $\mathrm{NA}$ & NA \\
\hline Ni.icp.a & $<2.69 \mathrm{E}+01 \mathrm{~kg}$ & NA & NA \\
\hline Ni.icp.adl & $<3.07 \mathrm{E}+01 \mathrm{~kg}$ & NA & NA \\
\hline${ }^{237} \mathrm{~Np}$ & $<5.66 \mathrm{E}-02 \mathrm{Ci}$ & NA & NA \\
\hline Oxalate.ic & $<1.11 \mathrm{E}+03 \mathrm{~kg}$ & NA & NA \\
\hline Pb.icp.a & $<1.34 \mathrm{E}+02 \mathrm{~kg}$ & $\mathrm{NA}$ & $\mathrm{NA}$ \\
\hline Pb.icp.adl & $<1.54 \mathrm{E}+02 \mathrm{~kg}$ & $\mathrm{NA}$ & $\mathrm{NA}$ \\
\hline${ }^{239 / 240} \mathrm{Pu}$ & $<9.53 \mathrm{E}-02 \mathrm{Ci}$ & NA & NA \\
\hline Sb.icp.a & $<8.04 \mathrm{E}+01 \mathrm{~kg}$ & NA & $\mathrm{NA}$ \\
\hline Sb.icp.adl & $<9.25 \mathrm{E}+01 \mathrm{~kg}$ & $\mathrm{NA}$ & NA \\
\hline Se.icp.a & $<1.34 \mathrm{E}+02 \mathrm{~kg}$ & $\mathrm{NA}$ & NA \\
\hline
\end{tabular}


Table B3-30. Analytical-Based Supernatant Inventory for Tank 241-AN-105 - (Supernatant Composite Sample - Core 152, Segments 1-14). (4 sheets)

\begin{tabular}{|c|c|c|c|}
\hline 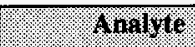 & ThML Bryontoy & 1. 1 (6) $01 \%$ & 112. (k) or 01$)$ \\
\hline Se.icp.adl & $<1.54 \mathrm{E}+02 \mathrm{~kg}$ & NA & NA \\
\hline Sm.icp.a & $<1.34 \mathrm{E}+02 \mathrm{~kg}$ & NA & NA \\
\hline Sm.icp.adl & $<1.54 \mathrm{E}+02 \mathrm{~kg}$ & NA & NA \\
\hline Sr.icp.a & $<1.34 \mathrm{E}+01 \mathrm{~kg}$ & NA & NA \\
\hline Sr.icp.adl & $<1.54 \mathrm{E}+01 \mathrm{~kg}$ & $\widehat{\mathrm{NA}}$ & NA \\
\hline Ti.icp.a & $<1.34 \mathrm{E}+01 \mathrm{~kg}$ & NA & NA \\
\hline Ti.icp.adl & $<1.54 \mathrm{E}+01 \mathrm{~kg}$ & NA & NA \\
\hline Tl.icp.a & $<2.69 \mathrm{E}+02 \mathrm{~kg}$ & NA & NA \\
\hline Tl.icp.adl & $<3.07 \mathrm{E}+02 \mathrm{~kg}$ & NA & NA \\
\hline Total Alpha & $<3.95 \mathrm{E}+01 \mathrm{Ci}$ & NA & NA \\
\hline Tritium & $<1.01 \mathrm{E}-04 \mathrm{Ci}$ & NA & NA \\
\hline U.icp.a & $<6.71 \mathrm{E}+02 \mathrm{~kg}$ & NA & NA \\
\hline U.icp.adl & $<7.69 \mathrm{E}+02 \mathrm{~kg}$ & NA & NA \\
\hline V.icp.a & $<6.71 \mathrm{E}+01 \mathrm{~kg}$ & NA & NA \\
\hline V.icp.adl & $<7.71 \mathrm{E}+01 \mathrm{~kg}$ & NA & NA \\
\hline Zr.icp.a & $<1.34 \mathrm{E}+01 \mathrm{~kg}$ & NA & NA \\
\hline Zr.icp.adl & $<1.54 \mathrm{E}+01 \mathrm{~kg}$ & NA & $\mathrm{NA}$ \\
\hline
\end{tabular}

Note:

${ }^{\mathrm{L}} \mathrm{Li}$ and ${ }^{~} \mathrm{Br}$ are the tracers for hydrostatic head fluid.

Table B3-31. Analytical-Based Salt Slurry Inventory for Tank 241-AN-105 - (nested ANOVA with the Riser Term - Segment Data). (3 sheets)

\begin{tabular}{|c|c|c|c|}
\hline Whalyte & Trat inventory & 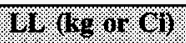 & W. $(k g .0101)$ \\
\hline Water.tga & $1.20 \mathrm{E}+06 \mathrm{~kg}$ & $9.51 \mathrm{E}+05$ & $1.44 \mathrm{E}+06$ \\
\hline Acetate.ic.w & $1.60 \mathrm{E}+03 \mathrm{~kg}$ & $0.00 \mathrm{E}+00$ & $3.97 \mathrm{E}+03$ \\
\hline Acetate.ic.wo & $1.81 \mathrm{E}+03 \mathrm{~kg}$ & $8.80 \mathrm{E}+02$ & $2.74 \mathrm{E}+03$ \\
\hline Ag.icp.a & $5.25 \mathrm{E}+01 \mathrm{~kg}$ & $0.00 \mathrm{E}+00$ & $2.27 \mathrm{E}+02$ \\
\hline Al.icp.a & $5.96 \mathrm{E}+04 \mathrm{~kg}$ & $1.53 \mathrm{E}+04$ & $1.04 \mathrm{E}+05$ \\
\hline
\end{tabular}


Table B3-31. Analytical-Based Salt Slurry Inventory for Tank 241-AN-105 - (nested ANOVA with the Riser Term - Segment Data). (3 sheets)

\begin{tabular}{|c|c|c|c|}
\hline Minjorto & 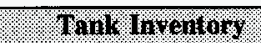 & 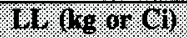 & (1) $/\left(\mathrm{A}_{6} / 01 \%\right)$ \\
\hline Al.icp.f & $6.22 \mathrm{E}+04 \mathrm{~kg}$ & $3.59 \mathrm{E}+04$ & $8.85 \mathrm{E}+04$ \\
\hline B.icp.a & $2.41 \mathrm{E}+02 \mathrm{~kg}$ & $0.00 \mathrm{E}+00$ & $6.64 \mathrm{E}+02$ \\
\hline Ca.icp.a & $1.73 \mathrm{E}+03 \mathrm{~kg}$ & $0.00 \mathrm{E}+00$ & $1.28 \mathrm{E}+04$ \\
\hline Cd.icp.a & $3.15 \mathrm{E}+01 \mathrm{~kg}$ & $0.00 \mathrm{E}+00$ & $1.02 \mathrm{E}+02$ \\
\hline $\mathrm{Cl}^{-}$.ic & $1.39 \mathrm{E}+04 \mathrm{~kg}$ & $7.32 \mathrm{E}+03$ & $2.05 \mathrm{E}+04$ \\
\hline Cr.icp.a & $6.30 \mathrm{E}+03 \mathrm{~kg}$ & $0.00 \mathrm{E}+00$ & $1.74 \mathrm{E}+04$ \\
\hline Cr.icp.f & $6.67 \mathrm{E}+03 \mathrm{~kg}$ & $0.00 \mathrm{E}+00$ & $2.07 \mathrm{E}+04$ \\
\hline Cu.icp.a.w & $1.90 \mathrm{E}+01 \mathrm{~kg}$ & $1.72 \mathrm{E}+00$ & $3.62 \mathrm{E}+01$ \\
\hline Cu.icp.a.wo & $1.91 \mathrm{E}+01 \mathrm{~kg}$ & $2.39 \mathrm{E}+00$ & $3.59 \mathrm{E}+01$ \\
\hline $\mathrm{F}^{-}$.ic & $5.01 \mathrm{E}+03 \mathrm{~kg}$ & $0.00 \mathrm{E}+00$ & $2.28 \mathrm{E}+04$ \\
\hline Fe.icp.a & $3.23 \mathrm{E}+02 \mathrm{~kg}$ & $0.00 \mathrm{E}+00$ & $1.24 \mathrm{E}+03$ \\
\hline Formate.ic & $2.85 \mathrm{E}+03 \mathrm{~kg}$ & $1.45 \mathrm{E}+03$ & $4.25 E+03$ \\
\hline K.icp.a & $9.30 \mathrm{E}+03 \mathrm{~kg}$ & $2.77 \mathrm{E}+03$ & $1.58 \mathrm{E}+04$ \\
\hline${ }^{1}$ Li.icp.a.w & $5.33 \mathrm{E}+01 \mathrm{~kg}$ & $0.00 \mathrm{E}+00$ & $2.27 \mathrm{E}+02$ \\
\hline${ }^{1}$ Li.icp.a.wo & $5.55 \mathrm{E}+01 \mathrm{~kg}$ & $0.00 \mathrm{E}+00$ & $2.25 \mathrm{E}+02$ \\
\hline Mn.icp.a.w & $5.67 \mathrm{E}+01 \mathrm{~kg}$ & $0.00 \mathrm{E}+00$ & $3.86 \mathrm{E}+02$ \\
\hline Mn.icp.a.wo & $5.85 \mathrm{E}+01 \mathrm{~kg}$ & $0.00 \mathrm{E}+00$ & $3.98 \mathrm{E}+02$ \\
\hline Mo.icp.a & $1.39 \mathrm{E}+02 \mathrm{~kg}$ & $1.02 \mathrm{E}+01$ & $2.67 \mathrm{E}+02$ \\
\hline $\mathrm{NO}_{2}^{-} \cdot \mathrm{ic}$ & $1.72 \mathrm{E}+05 \mathrm{~kg}$ & $9.03 \mathrm{E}+04$ & $2.53 \mathrm{E}+05$ \\
\hline $\mathrm{NO}_{3} \cdot \mathrm{ic}$ & $3.00 \mathrm{E}+05 \mathrm{~kg}$ & $4.53 \mathrm{E}+04$ & $5.55 \mathrm{E}+05$ \\
\hline Na.icp.a & $4.93 \mathrm{E}+05 \mathrm{~kg}$ & $1.10 \mathrm{E}+05$ & $8.76 \mathrm{E}+05$ \\
\hline Na.icp.f & $6.14 \mathrm{E}+05 \mathrm{~kg}$ & $3.09 \mathrm{E}+05$ & $9.20 \mathrm{E}+05$ \\
\hline Ni.icp.a & $1.10 \mathrm{E}+02 \mathrm{~kg}$ & $0.00 \mathrm{E}+00$ & $2.89 \mathrm{E}+02$ \\
\hline Ni.icp.f.w & $3.68 \mathrm{E}+03 \mathrm{~kg}$ & $0.00 \mathrm{E}+00$ & $2.07 \mathrm{E}+04$ \\
\hline Ni.icp.f.wo & $4.72 \mathrm{E}+03 \mathrm{~kg}$ & $0.00 \mathrm{E}+00$ & $1.71 E+04$ \\
\hline Oxalate.ic & $1.87 \mathrm{E}+04 \mathrm{~kg}$ & $0.00 \mathrm{E}+00$ & $5.34 \mathrm{E}+04$ \\
\hline P.icp.a & $6.70 \mathrm{E}+03 \mathrm{~kg}$ & $0.00 \mathrm{E}+00$ & $6.41 \mathrm{E}+04$ \\
\hline $\mathrm{PO}_{4}^{3-}$.ic.w & $2.18 \mathrm{E}+04 \mathrm{~kg}$ & $0.00 \mathrm{E}+00$ & $2.26 \mathrm{E}+05$ \\
\hline $\mathrm{PO}_{4}{ }^{3-}$.ic.wo & $2.18 \mathrm{E}+04 \mathrm{~kg}$ & $0.00 \mathrm{E}+00$ & $2.26 \mathrm{E}+05$ \\
\hline Pb.icp.a.w & $1.28 \mathrm{E}+02 \mathrm{~kg}$ & $0.00 \mathrm{E}+00$ & $3.18 \mathrm{E}+02$ \\
\hline
\end{tabular}


Table B3-31. Analytical-Based Salt Slurry Inventory for Tank 241-AN-105 - (nested ANOVA with the Riser Term - Segment Data). (3 sheets)

\begin{tabular}{|c|c|c|c|}
\hline 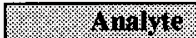 & Thol $110 \% 110 \%$ & $1.0 .0 .01)$ & Wul (kg or or) \\
\hline Pb.icp.a.wo & $1.36 \mathrm{E}+02 \mathrm{~kg}$ & $0.00 \mathrm{E}+00$ & $3.90 \mathrm{E}+02$ \\
\hline S.icp.a & $6.77 \mathrm{E}+03 \mathrm{~kg}$ & $0.00 \mathrm{E}+00$ & $1.94 \mathrm{E}+04$ \\
\hline S.icp.f.w & $7.49 \mathrm{E}+03 \mathrm{~kg}$ & $1.51 \mathrm{E}+03$ & $1.35 \mathrm{E}+04$ \\
\hline S.icp.f.wo & $7.59 \mathrm{E}+03 \mathrm{~kg}$ & $1.88 \mathrm{E}+03$ & $1.33 \mathrm{E}+04$ \\
\hline $\mathrm{SO}_{4}{ }^{2-}$.ic & $1.69 \mathrm{E}+04 \mathrm{~kg}$ & $0.00 \mathrm{E}+00$ & $4.21 \mathrm{E}+04$ \\
\hline Si.icp.a.w & $3.61 \mathrm{E}+02 \mathrm{~kg}$ & $1.57 \mathrm{E}+02$ & $5.65 \mathrm{E}+02$ \\
\hline Si.icp.a.wo & $3.70 \mathrm{E}+02 \mathrm{~kg}$ & $1.29 \mathrm{E}+02$ & $6.10 \mathrm{E}+02$ \\
\hline$\overline{Z n . i c p . a}$ & $1.17 \mathrm{E}+02 \mathrm{~kg}$ & $0.00 \mathrm{E}+00$ & $3.09 E+02$ \\
\hline Zr.icp.a.w & $3.08 \mathrm{E}+01 \mathrm{~kg}$ & $0.00 \mathrm{E}+00$ & $1.68 \mathrm{E}+02$ \\
\hline Zr.icp.a.wo & $3.23 \mathrm{E}+01 \mathrm{~kg}$ & $0.00 \mathrm{E}+00$ & $1.79 \mathrm{E}+02$ \\
\hline Total Alpha.w & $6.20 \mathrm{E}+01 \mathrm{Ci}$ & $0.00 \mathrm{E}+00$ & $4.25 \mathrm{E}+02$ \\
\hline Total Alpha.2o & $6.25 \mathrm{E}+01 \mathrm{Ci}$ & $0.00 \mathrm{E}+00$ & $5.70 \mathrm{E}+02$ \\
\hline
\end{tabular}

Note:

${ }^{1} \mathrm{Li}$ is one of the tracers for hydrostatic head fluid.

Tabie B3-32. Analytical-Based Salt Slurry Inventory for Tank 241-AN-105 - (nested ANOVA without the Riser Term - Segment Data). (2 sheets)

\begin{tabular}{|c|c|c|c|}
\hline 4 alde & Thil mentom, & $(4,(1001 / 4)$ & U. (1) or (6) \\
\hline Water.tga & $1.20 \mathrm{E}+06 \mathrm{~kg}$ & $1.15 \mathrm{E}+06$ & $1.24 \mathrm{E}+06$ \\
\hline Acetate.ic.w & $1.60 \mathrm{E}+03 \mathrm{~kg}$ & $1.29 \mathrm{E}+03$ & $1.91 \mathrm{E}+03$ \\
\hline Acetate.ic.wo & $1.81 \mathrm{E}+03 \mathrm{~kg}$ & $1.65 \mathrm{E}+03$ & $1.97 \mathrm{E}+03$ \\
\hline Ag.icp.a & $5.25 \mathrm{E}+01 \mathrm{~kg}$ & $2.20 \mathrm{E}+01$ & $8.31 \mathrm{E}+01$ \\
\hline Al.icp.a & $5.93 \mathrm{E}+04 \mathrm{~kg}$ & $5.52 \mathrm{E}+04$ & $6.34 \mathrm{E}+04$ \\
\hline Al.icp.f & $6.22 \mathrm{E}+04 \mathrm{~kg}$ & $5.75 E+04$ & $6.68 \mathrm{E}+04$ \\
\hline Cd.icp.a & $3.15 \mathrm{E}+01 \mathrm{~kg}$ & $1.91 \mathrm{E}+01$ & $4.39 \mathrm{E}+01$ \\
\hline $\mathrm{Cl}^{-}$.ic & $1.39 \mathrm{E}+04 \mathrm{~kg}$ & $1.28 \mathrm{E}+04$ & $1.51 \mathrm{E}+04$ \\
\hline Cr.icp.a & $6.30 \mathrm{E}+03 \mathrm{~kg}$ & $4.35 \mathrm{E}+03$ & $8.26 \mathrm{E}+03$ \\
\hline Cr.icp.f & $6.68 \mathrm{E}+03 \mathrm{~kg}$ & $4.62 \mathrm{E}+03$ & $8.74 E+03$ \\
\hline Cu.icp.a.w & $1.90 \mathrm{E}+01 \mathrm{~kg}$ & $1.60 \mathrm{E}+01$ & $2.19 \mathrm{E}+01$ \\
\hline
\end{tabular}


Table B3-32. Analytical-Based Salt Slurry Inventory for Tank 241-AN-105 - (nested ANOVA without the Riser Term - Segment Data). (2 sheets)

\begin{tabular}{|c|c|c|c|}
\hline Whalyte & 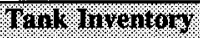 & 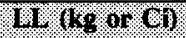 & 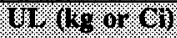 \\
\hline Cu.icp.a.wo & $1.91 \mathrm{E}+01 \mathrm{~kg}$ & $1.62 \mathrm{E}+01$ & $2.21 \mathrm{E}+01$ \\
\hline$F^{-}$.ic & $5.01 \mathrm{E}+03 \mathrm{~kg}$ & $1.89 \mathrm{E}+03$ & $8.12 \mathrm{E}+03$ \\
\hline Fe.icp.a & $3.23 \mathrm{E}+02 \mathrm{~kg}$ & $1.63 \mathrm{E}+02$ & $4.84 \mathrm{E}+02$ \\
\hline Formate.ic & $2.85 \mathrm{E}+03 \mathrm{~kg}$ & $2.62 \mathrm{E}+03$ & $3.08 \mathrm{E}+03$ \\
\hline K.icp.a & $9.26 \mathrm{E}+03 \mathrm{~kg}$ & $8.59 \mathrm{E}+03$ & $9.94 \mathrm{E}+03$ \\
\hline${ }^{1}$ Li.icp.a.w & $5.33 \mathrm{E}+01 \mathrm{~kg}$ & $2.27 \mathrm{E}+01$ & $8.38 \mathrm{E}+01$ \\
\hline${ }^{1}$ Li.icp.a.wo & $5.55 \mathrm{E}+01 \mathrm{~kg}$ & $2.58 \mathrm{E}+01$ & $8.52 \mathrm{E}+01$ \\
\hline Mn.icp.a.w & $5.67 \mathrm{E}+01 \mathrm{~kg}$ & $0.00 \mathrm{E}+00$ & $1.14 \mathrm{E}+02$ \\
\hline Mn.icp.a.wo & $5.85 \mathrm{E}+01 \mathrm{~kg}$ & $0.00 \mathrm{E}+00$ & $1.18 \mathrm{E}+02$ \\
\hline $\mathrm{NO}_{2}^{-} \cdot \mathrm{ic}$ & $1.72 \mathrm{E}+05 \mathrm{~kg}$ & $1.57 \mathrm{E}+05$ & $1.86 \mathrm{E}+05$ \\
\hline $\mathrm{NO}_{3}^{-} \cdot \mathrm{ic}$ & $3.00 \mathrm{E}+05 \mathrm{~kg}$ & $2.55 \mathrm{E}+05$ & $3.45 \mathrm{E}+05$ \\
\hline Ni.icp.a & $1.10 \mathrm{E}+02 \mathrm{~kg}$ & $7.84 \mathrm{E}+01$ & $1.41 \mathrm{E}+02$ \\
\hline Ni.icp.f.wo & $4.72 \mathrm{E}+03 \mathrm{~kg}$ & $2.51 \mathrm{E}+03$ & $6.93 E+03$ \\
\hline Oxalate.ic & $1.86 \mathrm{E}+04 \mathrm{~kg}$ & $1.49 \mathrm{E}+04$ & $2.24 \mathrm{E}+04$ \\
\hline P.icp.a & $6.70 \mathrm{E}+03 \mathrm{~kg}$ & $0.00 \mathrm{E}+00$ & $1.68 \mathrm{E}+04$ \\
\hline $\mathrm{PO}_{4}^{3-}$.ic.w & $2.18 \mathrm{E}+04 \mathrm{~kg}$ & $0.00 \mathrm{E}+00$ & $5.76 \mathrm{E}+04$ \\
\hline $\mathrm{PO}_{4}^{3-}$.ic.wo & $2.18 \mathrm{E}+04 \mathrm{~kg}$ & $0.00 \mathrm{E}+00$ & $5.76 \mathrm{E}+04$ \\
\hline Pb.icp.a.w & $1.28 \mathrm{E}+02 \mathrm{~kg}$ & $1.01 \mathrm{E}+02$ & $1.55 \mathrm{E}+02$ \\
\hline Pb.icp.a.wo & $1.35 \mathrm{E}+02 \mathrm{~kg}$ & $9.33 \mathrm{E}+01$ & $1.76 \mathrm{E}+02$ \\
\hline S.icp.f.w & $7.49 \mathrm{E}+03 \mathrm{~kg}$ & $6.44 \mathrm{E}+03$ & $8.54 \mathrm{E}+03$ \\
\hline S.icp.f.wo & $7.59 \mathrm{E}+03 \mathrm{~kg}$ & $6.59 \mathrm{E}+03$ & $8.59 \mathrm{E}+03$ \\
\hline $\mathrm{SO}_{4}^{2-} . \mathrm{ic}$ & $1.69 \mathrm{E}+04 \mathrm{~kg}$ & $1.36 \mathrm{E}+04$ & $2.02 \mathrm{E}+04$ \\
\hline Si.icp.a.w & $3.61 \mathrm{E}+02 \mathrm{~kg}$ & $3.25 \mathrm{E}+02$ & $3.97 \mathrm{E}+02$ \\
\hline Si.icp.a.wo & $3.69 \mathrm{E}+02 \mathrm{~kg}$ & $3.38 \mathrm{E}+02$ & $4.01 \mathrm{E}+02$ \\
\hline Zr.icp.a.w & $3.08 \mathrm{E}+01 \mathrm{~kg}$ & $6.78 \mathrm{E}+00$ & $5.49 \mathrm{E}+01$ \\
\hline Zr.icp.a.wo & $3.23 \mathrm{E}+01 \mathrm{~kg}$ & $6.58 \mathrm{E}+00$ & $5.81 \mathrm{E}+01$ \\
\hline Total Alpha.w & $6.20 \mathrm{E}+01 \mathrm{Ci}$ & $0.00 \mathrm{E}+00$ & $1.26 \mathrm{E}+02$ \\
\hline Total Alpha.wo & $6.25 \mathrm{E}+01 \mathrm{Ci}$ & $0.00 \mathrm{E}+00$ & $1.57 \mathrm{E}+02$ \\
\hline
\end{tabular}

Note:

' $\mathrm{Li}$ is one of the tracers for hydrostatic head fluid. 
Table B3-33. Analytes (Sludge - Segment Data) with >50 Percent "Less Than" Values.

(2 sheets)

\begin{tabular}{|c|c|c|c|}
\hline Ansilyte & That Thventory & WI (Ko or ol) & U1 (k8. or (61) \\
\hline Ag.icp.f & $<5.94 \mathrm{E}+02 \mathrm{~kg}$ & NA & NA \\
\hline As.icp.a & $<1.34 \mathrm{E}+02 \mathrm{~kg}$ & NA & NA \\
\hline As.icp.f & $<5.94 \mathrm{E}+03 \mathrm{~kg}$ & NA & NA \\
\hline B.icp.f & $<2.97 \mathrm{E}+03 \mathrm{~kg}$ & $\mathrm{NA}$ & $\widehat{\mathrm{NA}}$ \\
\hline Ba.icp.a & $<6.67 \mathrm{E}+01 \mathrm{~kg}$ & NA & NA \\
\hline Ba.icp.f & $<2.97 \mathrm{E}+03 \mathrm{~kg}$ & NA & NA \\
\hline Be.icp.a & $<8.13 \mathrm{E}+00 \mathrm{~kg}$ & NA & NA \\
\hline Be.icp.f & $<2.97 \mathrm{E}+02 \mathrm{~kg}$ & $\mathrm{NA}$ & NA \\
\hline Bi.icp.a & $<1.96 \mathrm{E}+02 \mathrm{~kg}$ & $\mathrm{NA}$ & NA \\
\hline Bi.icp.f & $<5.94 \mathrm{E}+03 \mathrm{~kg}$ & $\mathrm{NA}$ & NA \\
\hline${ }^{1} \mathrm{Br}$.ic & $<2.97 \mathrm{E}+03 \mathrm{~kg}$ & NA & NA \\
\hline Ca.icp.f & $<5.94 \mathrm{E}+03 \mathrm{~kg}$ & NA & NA \\
\hline Cd.icp.f & $<2.97 \mathrm{E}+02 \mathrm{~kg}$ & NA & NA \\
\hline Ce.icp.a & $<1.34 \mathrm{E}+02 \mathrm{~kg}$ & NA & NA \\
\hline Ce.icp.f & $<5.94 \mathrm{E}+03 \mathrm{~kg}$ & NA & NA \\
\hline Co.icp.a & $<2.67 \mathrm{E}+01 \mathrm{~kg}$ & NA & NA \\
\hline Co.icp.f & $<1.19 \mathrm{E}+03 \mathrm{~kg}$ & NA & NA \\
\hline Cu.icp.f & $<5.94 \mathrm{E}+02 \mathrm{~kg}$ & NA & NA \\
\hline Fe.icp.f & $<2.97 \mathrm{E}+03 \mathrm{~kg}$ & NA & $\mathrm{NA}$ \\
\hline La.icp.a & $<6.67 \mathrm{E}+01 \mathrm{~kg}$ & NA & NA \\
\hline La.icp.f & $<2.97 \mathrm{E}+03 \mathrm{~kg}$ & NA & NA \\
\hline${ }^{1}$ Li.icp.f & $<5.94 \mathrm{E}+02 \mathrm{~kg}$ & NA & NA \\
\hline Mg.icp.a & $<1.66 \mathrm{E}+02 \mathrm{~kg}$ & NA & NA \\
\hline Mg.icp.f & $<5.94 \mathrm{E}+03 \mathrm{~kg}$ & NA & NA \\
\hline Mn.icp.f & $<5.94 \mathrm{E}+02 \mathrm{~kg}$ & NA & $\mathrm{NA}$ \\
\hline Mo.icp.f & $<2.97 \mathrm{E}+03 \mathrm{~kg}$ & NA & NA \\
\hline Nd.icp.a & $<1.34 \mathrm{E}+02 \mathrm{~kg}$ & NA & NA \\
\hline Nd.icp.f & $<5.94 \mathrm{E}+03 \mathrm{~kg}$ & NA & NA \\
\hline P.icp.f & $<9.76 \mathrm{E}+04 \mathrm{~kg}$ & NA & NA \\
\hline Pb.icp.f & $<5.94 \mathrm{E}+03 \mathrm{~kg}$ & NA & NA \\
\hline
\end{tabular}


Table B3-33. Analytes (Sludge - Segment Data) with > 50 Percent "Less Than" Values. ( 2 sheets)

\begin{tabular}{|c|c|c|c|}
\hline inave & Irinl Inyentory & 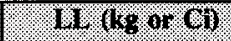 & 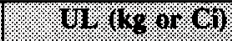 \\
\hline Sb.icp.a & $<8.01 \mathrm{E}+01 \mathrm{~kg}$ & NA & NA \\
\hline Sb.icp.f & $<3.56 \mathrm{E}+03 \mathrm{~kg}$ & NA & NA \\
\hline Se.icp.a & $<1.34 \mathrm{E}+02 \mathrm{~kg}$ & NA & NA \\
\hline Se.icp.f & $<5.94 \mathrm{E}+03 \mathrm{~kg}$ & $\mathrm{NA}$ & NA \\
\hline Si.icp.f & $<6.53 \mathrm{E}+03 \mathrm{~kg}$ & NA & NA \\
\hline Sm.icp.a & $<1.34 \mathrm{E}+02 \mathrm{~kg}$ & NA & NA \\
\hline Sm.icp.f & $<5.94 \mathrm{E}+03 \mathrm{~kg}$ & NA & NA \\
\hline Sr.icp.a & $<2.66 \mathrm{E}+01 \mathrm{~kg}$ & $\mathrm{NA}$ & NA \\
\hline$\overline{\text { Sr.icp.f }}$ & $<5.94 \mathrm{E}+02 \mathrm{~kg}$ & NA & NA \\
\hline Ti.icp.a & $<1.34 \mathrm{E}+01 \mathrm{~kg}$ & NA & NA \\
\hline Ti.icp.f & $<5.94 \mathrm{E}+02 \mathrm{~kg}$ & NA & NA \\
\hline Tl.icp.a & $<2.67 \mathrm{E}+02 \mathrm{~kg}$ & NA & NA \\
\hline Tl.icp.f & $<1.19 \mathrm{E}+04 \mathrm{~kg}$ & $\mathrm{NA}$ & NA \\
\hline U.icp.a & $<6.67 \mathrm{E}+02 \mathrm{~kg}$ & NA & NA \\
\hline U.icp.f & $<2.97 \mathrm{E}+04 \mathrm{~kg}$ & NA & NA \\
\hline V.icp.a & $<6.67 \mathrm{E}+01 \mathrm{~kg}$ & NA & NA \\
\hline V.icp.f & $<2.97 \mathrm{E}+03 \mathrm{~kg}$ & NA & NA \\
\hline Zn.icp.f & $<2.16 \mathrm{E}+03 \mathrm{~kg}$ & NA & $\mathrm{NA}$ \\
\hline Zr.icp.f & $<1.31 \mathrm{E}+03 \mathrm{~kg}$ & $\widehat{N A}$ & NA \\
\hline
\end{tabular}

Note:

${ }^{\mathrm{t}} \mathrm{Li}$ and ${ }^{\mathrm{B}} \mathrm{Br}$ are the tracers for hydrostatic head fluid. 
Table B3-34. Analytical-Based Salt Slurry Tank Inventory - Composite (Core 152, Segments 14-20). (3 sheets)

\begin{tabular}{|c|c|c|c|}
\hline Anivite & T: Tank Inventory & $41.08 .6 r(9)$ & WI $16 \%$ or CI \\
\hline Water.tga & $1.11 \mathrm{E}+06 \mathrm{~kg}$ & $9.70 \mathrm{E}+05$ & $1.25 \mathrm{E}+06$ \\
\hline Acetate.ic & $1.14 \mathrm{E}+03 \mathrm{~kg}$ & $1.08 \mathrm{E}+03$ & $1.21 \mathrm{E}+03$ \\
\hline Ag.icp.a & $2.88 \mathrm{E}+01 \mathrm{~kg}$ & $1.65 \mathrm{E}+01$ & $4.12 \mathrm{E}+01$ \\
\hline \begin{tabular}{|l} 
Al.icp.a \\
\end{tabular} & $4.96 \mathrm{E}+04 \mathrm{~kg}$ & $4.19 \mathrm{E}+04$ & $5.73 \mathrm{E}+04$ \\
\hline${ }^{241}$ Am.aea & $9.93 \mathrm{E}+00 \mathrm{Ci}$ & $0.00 \mathrm{E}+00$ & $3.63 \mathrm{E}+01$ \\
\hline B.icp.a & $2.82 \mathrm{E}+02 \mathrm{~kg}$ & $0.00 \mathrm{E}+00$ & $6.37 \mathrm{E}+02$ \\
\hline Ca.icp.a & $5.22 \mathrm{E}+02 \mathrm{~kg}$ & $3.22 \mathrm{E}+02$ & $7.23 \mathrm{E}+02$ \\
\hline Cd.icp.a & $1.77 \mathrm{E}+01 \mathrm{~kg}$ & $0.00 \mathrm{E}+00$ & $4.34 \mathrm{E}+01$ \\
\hline Cl.ic & $1.43 \mathrm{E}+04 \mathrm{~kg}$ & $1.33 \mathrm{E}+04$ & $1.52 \mathrm{E}+04$ \\
\hline Cr.icp.a & $3.60 \mathrm{E}+03 \mathrm{~kg}$ & $5.77 \mathrm{E}+01$ & $7.14 \mathrm{E}+03$ \\
\hline $\mathrm{Cr}(\mathrm{VI})$.spec & $2.88 \mathrm{E}+02 \mathrm{~kg}$ & $2.58 \mathrm{E}+02$ & $3.19 \mathrm{E}+02$ \\
\hline${ }^{137} \mathrm{Cs}$.gea & $7.37 \mathrm{E}+05 \mathrm{Ci}$ & $7.06 \mathrm{E}+05$ & $7.68 \mathrm{E}+05$ \\
\hline Cu.icp.a & $1.24 \mathrm{E}+01 \mathrm{~kg}$ & $5.30 \mathrm{E}+00$ & $1.95 \mathrm{E}+01$ \\
\hline$\overline{F^{-} . \text {ic }}$ & $2.76 \mathrm{E}+03 \mathrm{~kg}$ & $0.00 \mathrm{E}+00$ & $9.25 \mathrm{E}+03$ \\
\hline Fe.icp.a & $1.48 \mathrm{E}+02 \mathrm{~kg}$ & $0.00 \mathrm{E}+00$ & $4.73 \mathrm{E}+02$ \\
\hline Formate.ic & $2.97 \mathrm{E}+03 \mathrm{~kg}$ & $2.82 \mathrm{E}+03$ & $3.12 \mathrm{E}+03$ \\
\hline K.icp.a & $7.49 \mathrm{E}+03 \mathrm{~kg}$ & $6.87 \mathrm{E}+03$ & $8.11 \mathrm{E}+03$ \\
\hline${ }^{1}$ Li.icp.a & $2.11 \mathrm{E}+01 \mathrm{~kg}$ & $1.80 \mathrm{E}+01$ & $2.42 \mathrm{E}+01$ \\
\hline Mn.icp.a & $1.90 \mathrm{E}+01 \mathrm{~kg}$ & $0.00 \mathrm{E}+00$ & $3.87 \mathrm{E}+01$ \\
\hline Mo.icp.a & $1.11 \mathrm{E}+02 \mathrm{~kg}$ & $1.10 \mathrm{E}+02$ & $1.13 \mathrm{E}+02$ \\
\hline $\mathrm{NO}_{2}^{-} \cdot \mathrm{ic}$ & $1.77 \mathrm{E}+05 \mathrm{~kg}$ & $1.72 \mathrm{E}+05$ & $1.82 \mathrm{E}+05$ \\
\hline $\mathrm{NO}_{3}^{-}$.ic & $2.74 \mathrm{E}+05 \mathrm{~kg}$ & $1.51 \mathrm{E}+05$ & $3.97 \mathrm{E}+05$ \\
\hline Na.icp.a & $3.76 \mathrm{E}+05 \mathrm{~kg}$ & $3.14 \mathrm{E}+05$ & $4.37 \mathrm{E}+05$ \\
\hline Ni.icp.a & $7.70 \mathrm{E}+01 \mathrm{~kg}$ & $0.00 \mathrm{E}+00$ & $1.74 \mathrm{E}+02$ \\
\hline${ }^{237} \mathrm{~Np}$ & $2.57 \mathrm{E}+01 \mathrm{Ci}$ & $1.65 \mathrm{E}+01$ & $3.49 \mathrm{E}+01$ \\
\hline $\mathrm{OH}^{-}$ & $5.83 \mathrm{E}+04 \mathrm{~kg}$ & $4.44 \mathrm{E}+04$ & $7.22 \mathrm{E}+04$ \\
\hline Oxalate.ic & $1.57 \mathrm{E}+04 \mathrm{~kg}$ & $9.57 \mathrm{E}+03$ & $2.19 \mathrm{E}+04$ \\
\hline P.icp.a & $9.75 \mathrm{E}+02 \mathrm{~kg}$ & $4.82 \mathrm{E}+02$ & $1.47 \mathrm{E}+03$ \\
\hline $\mathrm{PO}_{4}{ }^{3-}$-ic & $2.92 \mathrm{E}+03 \mathrm{~kg}$ & $6.11 \mathrm{E}+02$ & $5.23 \mathrm{E}+03$ \\
\hline S.icp.a & $5.27 \mathrm{E}+03 \mathrm{~kg}$ & $8.06 \mathrm{E}+02$ & $9.74 \mathrm{E}+03$ \\
\hline
\end{tabular}


Table B3-34. Analytical-Based Salt Slurry Tank Inventory - Composite (Core 152, Segments 14-20). (3 sheets)

\begin{tabular}{|c|c|c|c|}
\hline Tralite & Tand Irvenitory & fy (kg or ( 91$)$ & (1) (kg or G \\
\hline $\mathrm{SO}_{4}^{2-}$.ic & $1.81 \mathrm{E}+04 \mathrm{~kg}$ & $1.03 \mathrm{E}+04$ & $2.60 \mathrm{E}+04$ \\
\hline Si.icp.a & $4.33 \mathrm{E}+02 \mathrm{~kg}$ & $0.00 \mathrm{E}+00$ & $1.65 \mathrm{E}+03$ \\
\hline${ }^{89 / 90} \mathrm{Sr}$ & $2.22 \mathrm{E}+04 \mathrm{Ci}$ & $1.45 \mathrm{E}+04$ & $2.99 \mathrm{E}+04$ \\
\hline TIC & $3.16 \mathrm{E}+04 \mathrm{~kg}$ & $1.47 \mathrm{E}+04$ & $4.86 \mathrm{E}+04$ \\
\hline TOC.pers & $1.32 \mathrm{E}+04 \mathrm{~kg}$ & $0.00 \mathrm{E}+00$ & $2.93 \mathrm{E}+04$ \\
\hline${ }^{99} \mathrm{Tc}$ & $4.38 \mathrm{E}+02 \mathrm{Ci}$ & $4.22 \mathrm{E}+02$ & $4.53 \mathrm{E}+02$ \\
\hline Total Alpha & $3.30 \mathrm{E}+01 \mathrm{Ci}$ & $0.00 \mathrm{E}+00$ & $8.23 \mathrm{E}+01$ \\
\hline Total Beta & $7.92 \mathrm{E}+05 \mathrm{Ci}$ & $7.45 \mathrm{E}+05$ & $8.38 \mathrm{E}+05$ \\
\hline Tritium & $5.33 \mathrm{E}+00 \mathrm{Ci}$ & $0.00 \mathrm{E}+00$ & $1.18 \mathrm{E}+01$ \\
\hline U.phos & $2.12 \mathrm{E}+02 \mathrm{~kg}$ & $1.75 \mathrm{E}+02$ & $2.49 \mathrm{E}+02$ \\
\hline Zn.icp.a & $1.98 \mathrm{E}+01 \mathrm{~kg}$ & $7.51 \mathrm{E}+00$ & $3.22 \mathrm{E}+01$ \\
\hline & & & \\
\hline${ }^{241} \mathrm{Am}$.gea & $<2.00 \mathrm{E}+03 \mathrm{Ci}$ & $\mathrm{NA}$ & NA \\
\hline As.icp.a & $<9.77 \mathrm{E}+01 \mathrm{~kg}$ & NA & NA \\
\hline Ba.icp.a & $<4.90 \mathrm{E}+01 \mathrm{~kg}$ & NA & NA \\
\hline Be.icp.a & $<4.90 \mathrm{E}+00 \mathrm{~kg}$ & NA & NA \\
\hline Bi.icp.a & $<9.77 \mathrm{E}+01 \mathrm{~kg}$ & $\mathrm{NA}$ & NA \\
\hline${ }^{3} \mathrm{Br}^{-}$.ic & $<1.35 \mathrm{E}+03 \mathrm{~kg}$ & NA & NA \\
\hline Ce.icp.a & $<9.77 \mathrm{E}+01 \mathrm{~kg}$ & NA & NA \\
\hline${ }^{243 / 244} \mathrm{Cm}$ & $<2.34 \mathrm{E}+00 \mathrm{Ci}$ & NA & NA \\
\hline Co.icp.a & $<1.95 \mathrm{E}+01 \mathrm{~kg}$ & NA & NA \\
\hline${ }^{60}$ Co.gea & $<9.09 \mathrm{E}+01 \mathrm{Ci}$ & NA & NA \\
\hline${ }^{154}$ Eu.gea & $<2.40 \mathrm{E}+02 \mathrm{Cj}$ & NA & NA \\
\hline${ }^{155}$ Eu.gea & $<9.38 \mathrm{E}+02 \mathrm{Ci}$ & NA & NA \\
\hline${ }^{129} \mathrm{I}$ & $<9.50 \mathrm{E}+00 \mathrm{Ci}$ & NA & $\mathrm{NA}$ \\
\hline La.icp.a & $<4.90 \mathrm{E}+01 \mathrm{~kg}$ & NA & $\mathrm{NA}$ \\
\hline Mg.icp.a & $<9.77 \mathrm{E}+01 \mathrm{~kg}$ & NA & $\mathrm{NA}$ \\
\hline Nd.icp.a & $<9.77 \mathrm{E}+01 \mathrm{~kg}$ & $\mathrm{NA}$ & NA \\
\hline Pb.icp.a & $<9.77 \mathrm{E}+01 \mathrm{~kg}$ & NA & NA \\
\hline${ }^{239 / 240} \mathrm{Pu}$ & $<4.39 \mathrm{E}+00 \mathrm{Ci}$ & NA & NA \\
\hline
\end{tabular}


Table B3-34. Analytical-Based Salt Slurry Tank Inventory - Composite (Core 152, Segments 14-20). (3 sheets)

\begin{tabular}{|c|c|c|c|}
\hline Analyte & Thank laveraory & W. ( w or or $)$ & 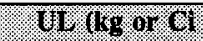 \\
\hline Sb.icp.a & $<5.87 \mathrm{E}+01 \mathrm{~kg}$ & NA & NA \\
\hline Se.icp.a & $<9.77 \mathrm{E}+01 \mathrm{~kg}$ & NA & NA \\
\hline Sm.icp.a & $<9.77 \mathrm{E}+01 \mathrm{~kg}$ & NA & NA \\
\hline Sr.icp.a & $<9.77 \mathrm{E}+00 \mathrm{~kg}$ & NA & NA \\
\hline Ti.icp.a & $<9.77 \mathrm{E}+00 \mathrm{~kg}$ & NA & NA \\
\hline Tl.icp.a & $<1.95 \mathrm{E}+02 \mathrm{~kg}$ & NA & NA \\
\hline U.icp.a & $<4.90 \mathrm{E}+02 \mathrm{~kg}$ & NA & NA \\
\hline V.icp.a & $<4.90 \mathrm{E}+01 \mathrm{~kg}$ & NA & NA \\
\hline Zr.icp.a & $<1.09 \mathrm{E}+01 \mathrm{~kg}$ & NA & NA \\
\hline
\end{tabular}

Note:

${ }^{1} \mathrm{Li}$ and ${ }^{\mathrm{B}} \mathrm{Br}$ are the tracers for hydrostatic head fluid. 
Table B3-35. Analytical-Based Salt Slurry Tank Inventory - Composite (Core 152, Segment 22). (3 sheets)

\begin{tabular}{|c|c|c|c|}
\hline Nanule & 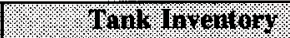 & (1) $(1 \times 8.0 \%$ & $101,16,00 \%$ \\
\hline Water.tga & $1.10 \mathrm{E}+05 \mathrm{~kg}$ & $1.06 \mathrm{E}+05$ & $1.14 \mathrm{E}+05$ \\
\hline Ag.icp.a & $1.09 \mathrm{E}+01 \mathrm{~kg}$ & $3.24 \mathrm{E}+00$ & $1.86 \mathrm{E}+01$ \\
\hline Al.icp.a & $3.72 \mathrm{E}+03 \mathrm{~kg}$ & $1.27 \mathrm{E}+03$ & $6.17 \mathrm{E}+03$ \\
\hline${ }^{241}$ Am.aea & $1.00 \mathrm{E}+01 \mathrm{Ci}$ & $0.00 \mathrm{E}+00$ & $2.44 \mathrm{E}+01$ \\
\hline B.icp.a & $2.92 \mathrm{E}+01 \mathrm{~kg}$ & $0.00 \mathrm{E}+00$ & $1.02 \mathrm{E}+02$ \\
\hline Ca.icp.a & $8.45 \mathrm{E}+01 \mathrm{~kg}$ & $0.00 \mathrm{E}+00$ & $1.71 \mathrm{E}+02$ \\
\hline Cd.icp.a & $3.56 \mathrm{E}+00 \mathrm{~kg}$ & $1.76 \mathrm{E}+00$ & $5.36 \mathrm{E}+00$ \\
\hline $\mathrm{Cl}^{-}$.ic & $1.05 \mathrm{E}+03 \mathrm{~kg}$ & $1.64 \mathrm{E}+02$ & $1.93 \mathrm{E}+03$ \\
\hline Cr.icp.a & $7.05 \mathrm{E}+02 \mathrm{~kg}$ & $1.82 \mathrm{E}+02$ & $1.23 \mathrm{E}+03$ \\
\hline Cr(VI).spec & $2.67 \mathrm{E}+01 \mathrm{~kg}$ & $1.69 \mathrm{E}+01$ & $3.66 \mathrm{E}+01$ \\
\hline${ }^{137}$ Cs.gea & $5.30 \mathrm{E}+04 \mathrm{Ci}$ & $4.97 E+04$ & $5.62 \mathrm{E}+04$ \\
\hline Cu.icp.a & $1.55 \mathrm{E}+00 \mathrm{~kg}$ & $0.00 \mathrm{E}+00$ & $4.85 \mathrm{E}+00$ \\
\hline $\mathrm{F}^{-}$.ic & $1.29 \mathrm{E}+03 \mathrm{~kg}$ & $7.86 \mathrm{E}+02$ & $1.80 \mathrm{E}+03$ \\
\hline Fe.icp.a & $3.77 \mathrm{E}+01 \mathrm{~kg}$ & $1.32 \mathrm{E}+01$ & $6.22 \mathrm{E}+01$ \\
\hline Formate.ic & $2.87 \mathrm{E}+02 \mathrm{~kg}$ & $2.70 \mathrm{E}+02$ & $3.03 E+02$ \\
\hline K.icp.a & $5.52 \mathrm{E}+02 \mathrm{~kg}$ & $2.41 \mathrm{E}+02$ & $8.62 E+02$ \\
\hline${ }^{1}$ Li.icp.a & $3.78 \mathrm{E}+00 \mathrm{~kg}$ & $0.00 \mathrm{E}+00$ & $2.79 \mathrm{E}+01$ \\
\hline Mn.icp.a & $1.27 \mathrm{E}+01 \mathrm{~kg}$ & $3.55 \mathrm{E}+00$ & $2.19 \mathrm{E}+01$ \\
\hline Mo.icp.a & $9.10 \mathrm{E}+00 \mathrm{~kg}$ & $2.57 \mathrm{E}+00$ & $1.56 \mathrm{E}+01$ \\
\hline $\mathrm{NO}_{2}^{-}$. ic & $1.23 \mathrm{E}+04 \mathrm{~kg}$ & $1.16 \mathrm{E}+03$ & $2.34 \mathrm{E}+04$ \\
\hline $\mathrm{NO}_{3}^{-} \cdot \mathrm{ic}$ & $4.14 \mathrm{E}+04 \mathrm{~kg}$ & $0.00 \mathrm{E}+00$ & $8.72 \mathrm{E}+04$ \\
\hline Na.icp.a & $4.23 \mathrm{E}+04 \mathrm{~kg}$ & $7.99 \mathrm{E}+03$ & $7.66 \mathrm{E}+04$ \\
\hline Ni.icp.a & $8.65 \mathrm{E}+00 \mathrm{~kg}$ & $0.00 \mathrm{E}+00$ & $1.80 \mathrm{E}+01$ \\
\hline $\mathrm{OH}^{-}$ & $5.66 \mathrm{E}+03 \mathrm{~kg}$ & $4.35 \mathrm{E}+03$ & $6.97 \mathrm{E}+03$ \\
\hline Oxalate.ic & $1.51 \mathrm{E}+03 \mathrm{~kg}$ & $1.40 \mathrm{E}+02$ & $2.88 \mathrm{E}+03$ \\
\hline P.icp.a & $3.63 \mathrm{E}+03 \mathrm{~kg}$ & $6.85 \mathrm{E}+02$ & $6.57 \mathrm{E}+03$ \\
\hline $\mathrm{PO}_{4}{ }^{3-} . \mathrm{ic}$ & $1.18 \mathrm{E}+04 \mathrm{~kg}$ & $4.24 \mathrm{E}+03$ & $1.93 E+04$ \\
\hline Pb.icp.a & $1.28 \mathrm{E}+01 \mathrm{~kg}$ & $5.92 \mathrm{E}+00$ & $1.96 \mathrm{E}+01$ \\
\hline${ }^{239 / 240} \mathrm{Pu}$ & $1.94 \mathrm{E}+00 \mathrm{Ci}$ & $1.86 \mathrm{E}+00$ & $2.02 \mathrm{E}+00$ \\
\hline S.icp.a & $3.72 \mathrm{E}+02 \mathrm{~kg}$ & $9.39 \mathrm{E}+01$ & $6.49 \mathrm{E}+02$ \\
\hline
\end{tabular}


Table B3-35. Analytical-Based Salt Slurry Tank Inventory - Composite (Core 152, Segment 22). (3 sheets)

\begin{tabular}{|c|c|c|c|}
\hline Analyte & Trinl Inveritory & H. (k: or Ci) & 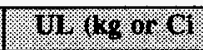 \\
\hline $\mathrm{SO}_{4}{ }^{2} \cdot \mathrm{ic}$ & $1.08 \mathrm{E}+03 \mathrm{~kg}$ & $3.56 \mathrm{E}+02$ & $1.79 \mathrm{E}+03$ \\
\hline Si.icp.a & $1.22 \mathrm{E}+02 \mathrm{~kg}$ & $0.00 \mathrm{E}+00$ & $6.01 \mathrm{E}+02$ \\
\hline${ }^{89 / 90} \mathrm{Sr}$ & $9.40 \mathrm{E}+03 \mathrm{Ci}$ & $6.95 \mathrm{E}+03$ & $1.19 \mathrm{E}+04$ \\
\hline TIC & $2.12 \mathrm{E}+03 \mathrm{~kg}$ & $1.27 \mathrm{E}+03$ & $2.97 \mathrm{E}+03$ \\
\hline TOC.pers & $1.15 \mathrm{E}+03 \mathrm{~kg}$ & $6.62 \mathrm{E}+02$ & $1.64 \mathrm{E}+03$ \\
\hline${ }^{99} \mathrm{Tc}$ & $3.45 \mathrm{E}+01 \mathrm{Ci}$ & $8.32 \mathrm{E}+00$ & $6.06 \mathrm{E}+01$ \\
\hline Total Alpha & $1.49 \mathrm{E}+01 \mathrm{Ci}$ & $0.00 \mathrm{E}+00$ & $4.09 \mathrm{E}+01$ \\
\hline Total Beta & $7.06 \mathrm{E}+04 \mathrm{Ci}$ & $4.94 \mathrm{E}+04$ & $9.18 \mathrm{E}+04$ \\
\hline U.phos & $2.12 \mathrm{E}+01 \mathrm{~kg}$ & $1.52 \mathrm{E}+01$ & $2.72 \mathrm{E}+01$ \\
\hline Zn.icp.a & $2.97 \mathrm{E}+00 \mathrm{~kg}$ & $0.00 \mathrm{E}+00$ & $9.02 \mathrm{E}+00$ \\
\hline Zr.icp.a & $1.44 \mathrm{E}+01 \mathrm{~kg}$ & $4.07 \mathrm{E}+00$ & $2.47 \mathrm{E}+01$ \\
\hline \multicolumn{4}{|c|}{ 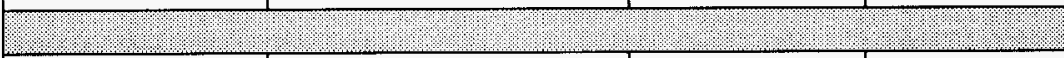 } \\
\hline Acetate.ic & $<2.03 \mathrm{E}+01 \mathrm{~kg}$ & NA & NA \\
\hline${ }^{241}$ Am.gea & $<1.27 \mathrm{E}+02 \mathrm{Ci}$ & NA & NA \\
\hline As.icp.a & $<1.00 \mathrm{E}+01 \mathrm{~kg}$ & NA & NA \\
\hline Ba.icp.a & $<5.02 \mathrm{E}+00 \mathrm{~kg}$ & NA & NA \\
\hline Be.icp.a & $<5.02 \mathrm{E}-01 \mathrm{~kg}$ & NA & NA \\
\hline Bi.icp.a & $<1.00 \mathrm{E}+01 \mathrm{~kg}$ & NA & NA \\
\hline${ }^{1} \mathrm{Br}$.ic & $<1.27 \mathrm{E}+02 \mathrm{~kg}$ & NA & NA \\
\hline Ce.icp.a & $<1.00 \mathrm{E}+01 \mathrm{~kg}$ & $\mathrm{NA}$ & NA \\
\hline${ }^{243 / 244} \mathrm{Cm}$ & $<1.45 \mathrm{E}+00 \mathrm{Ci}$ & NA & NA \\
\hline Co.icp.a & $<2.00 \mathrm{E}+00 \mathrm{~kg}$ & NA & NA \\
\hline${ }^{60} \mathrm{Co}$.gea & $<4.63 \mathrm{E}+00 \mathrm{Ci}$ & NA & NA \\
\hline${ }^{154} \mathrm{Eu}$. gea & $<1.95 \mathrm{E}+01 \mathrm{Ci}$ & NA & NA \\
\hline${ }^{155}$ Eu.gea & $<5.92 \mathrm{E}+01 \mathrm{Ci}$ & NA & NA \\
\hline${ }^{129} \mathrm{I}$ & $<8.00 \mathrm{E}-01 \mathrm{Ci}$ & NA & NA \\
\hline La.icp.a & $<5.02 \mathrm{E}+00 \mathrm{~kg}$ & NA & NA \\
\hline Mg.icp.a & $<1.00 \mathrm{E}+01 \mathrm{~kg}$ & NA & NA \\
\hline Nd.icp.a & $<1.00 \mathrm{E}+01 \mathrm{~kg}$ & NA & NA \\
\hline${ }^{237} \mathrm{~Np}$ & $<4.17 \mathrm{E}+00 \mathrm{Ci}$ & NA & NA \\
\hline
\end{tabular}


Table B3-35. Analytical-Based Salt Slurry Tank Inventory - Composite (Core 152, Segment 22). (3 sheets)

\begin{tabular}{|c|c|c|c|}
\hline Analve & Iant Irvenitory & 11) (k. or (c) & 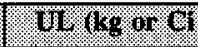 \\
\hline Sb.icp.a & $<6.02 \mathrm{E}+00 \mathrm{~kg}$ & NA & NA \\
\hline Se.icp.a & $<1.00 \mathrm{E}+01 \mathrm{~kg}$ & NA & NA \\
\hline Sm.icp.a & $<1.00 \mathrm{E}+01 \mathrm{~kg}$ & NA & $\mathrm{NA}$ \\
\hline Sr.icp.a & $<1.00 \mathrm{E}+00 \mathrm{~kg}$ & NA & NA \\
\hline Ti.icp.a & $<1.00 \mathrm{E}+00 \mathrm{~kg}$ & NA & NA \\
\hline Tl.icp.a & $<2.00 \mathrm{E}+01 \mathrm{~kg}$ & NA & $\mathrm{NA}$ \\
\hline Tritium & $<1.63 \mathrm{E}-01 \mathrm{Ci}$ & NA & NA \\
\hline U.icp.a & $<5.02 \mathrm{E}+01 \mathrm{~kg}$ & NA & NA \\
\hline V.icp.a & $<5.02 \mathrm{E}+00 \mathrm{~kg}$ & NA & $\mathrm{NA}$ \\
\hline
\end{tabular}

Note:

${ }^{1} \mathrm{Li}$ and ${ }^{\mathrm{I}} \mathrm{Br}$ are the tracers for hydrostatic head fluid. 
Table B3-36. Tank Inventory - Based on Segment Data. (4 sheets)

\begin{tabular}{|c|c|c|c|c|}
\hline Sup rmatan. & Swpennataint & 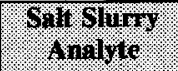 & $610 \%$ & I \\
\hline Water.tga & $1.28 \mathrm{E}+06 \mathrm{~kg}$ & Water.tga & $1.20 \mathrm{E}+06 \mathrm{~kg}$ & $2.48 \mathrm{E}+06 \mathrm{~kg}$ \\
\hline Water.tga.ed & $1.29 \mathrm{E}+06 \mathrm{~kg}$ & Water.tga & $1.20 \mathrm{E}+06 \mathrm{~kg}$ & $2.49 \mathrm{E}+06 \mathrm{~kg}$ \\
\hline Acetate.ic.w & $1.54 \mathrm{E}+03 \mathrm{~kg}$ & Acetate.ic.w & $1.60 \mathrm{E}+03 \mathrm{~kg}$ & $3.14 \mathrm{E}+03 \mathrm{~kg}$ \\
\hline Acetate.ic.wo & $1.57 \mathrm{E}+03 \mathrm{~kg}$ & Acetate.ic.wo & $1.81 \mathrm{E}+03 \mathrm{~kg}$ & $3.38 \mathrm{E}+03 \mathrm{~kg}$ \\
\hline Ag.icp.adl & $4.56 \mathrm{E}+01 \mathrm{~kg}$ & Ag.icp.a & $5.25 \mathrm{E}+01 \mathrm{~kg}$ & $9.81 \mathrm{E}+01 \mathrm{~kg}$ \\
\hline Ag.icp.adl & $4.56 \mathrm{E}+01 \mathrm{~kg}$ & Ag.icp.f & $<5.94 \mathrm{E}+02 \mathrm{~kg}$ & $<6.40 \mathrm{E}+02 \mathrm{~kg}$ \\
\hline Al.icp.adl & $1.07 \mathrm{E}+05 \mathrm{~kg}$ & Al.icp.a & $5.96 \mathrm{E}+04 \mathrm{~kg}$ & $1.67 \mathrm{E}+05 \mathrm{~kg}$ \\
\hline Al.icp.adl & $1.07 \mathrm{E}+05 \mathrm{~kg}$ & Al.icp.f & $6.22 \mathrm{E}+04 \mathrm{~kg}$ & $1.69 \mathrm{E}+05 \mathrm{~kg}$ \\
\hline As.icp.adl & $<1.54 \mathrm{E}+02 \mathrm{~kg}$ & As.icp.a & $<1.34 \mathrm{E}+02 \mathrm{~kg}$ & $<2.88 \mathrm{E}+02 \mathrm{~kg}$ \\
\hline As.icp.adl & $<1.54 \mathrm{E}+02 \mathrm{~kg}$ & As.icp.f & $<5.94 \mathrm{E}+03 \mathrm{~kg}$ & $<6.09 \mathrm{E}+03 \mathrm{~kg}$ \\
\hline B.icp.adl & $1.91 \mathrm{E}+02 \mathrm{~kg}$ & B.icp.a & $2.41 \mathrm{E}+02 \mathrm{~kg}$ & $4.32 \mathrm{E}+02 \mathrm{~kg}$ \\
\hline B.icp.adl & $1.91 \mathrm{E}+02 \mathrm{~kg}$ & B.icp.f & $<2.97 \mathrm{E}+03 \mathrm{~kg}$ & $<3.16 \mathrm{E}+03 \mathrm{~kg}$ \\
\hline Ba.icp.adl & $<7.71 \mathrm{E}+01 \mathrm{~kg}$ & Ba.icp.a & $<6.67 \mathrm{E}+01 \mathrm{~kg}$ & $<1.44 \mathrm{E}+02 \mathrm{~kg}$ \\
\hline Ba.icp.adl & $<7.71 \mathrm{E}+01 \mathrm{~kg}$ & Ba.icp.f & $<2.97 \mathrm{E}+03 \mathrm{~kg}$ & $<3.05 \mathrm{E}+03 \mathrm{~kg}$ \\
\hline Be.icp.adl & $<7.69 \mathrm{E}+00 \mathrm{~kg}$ & Be.icp.a & $<8.13 \mathrm{E}+00 \mathrm{~kg}$ & $<1.58 \mathrm{E}+01 \mathrm{~kg}$ \\
\hline Be.icp.adl & $<7.69 \mathrm{E}+00 \mathrm{~kg}$ & Be.icp.f & $<2.97 \mathrm{E}+02 \mathrm{~kg}$ & $<3.05 \mathrm{E}+02 \mathrm{~kg}$ \\
\hline Bi.icp.adl & $<1.54 \mathrm{E}+02 \mathrm{~kg}$ & Bi.icp.a & $1.96 \mathrm{E}+02 \mathrm{~kg}$ & $<3.50 \mathrm{E}+02 \mathrm{~kg}$ \\
\hline Bi.icp.adl & $<1.54 \mathrm{E}+02 \mathrm{~kg}$ & Bi.icp.f & $<5.94 \mathrm{E}+03 \mathrm{~kg}$ & $<6.09 \mathrm{E}+03 \mathrm{~kg}$ \\
\hline${ }^{1} \mathrm{Br}^{-}$.ic.w & $2.47 \mathrm{E}+03 \mathrm{~kg}$ & $\mathrm{Br} . \mathrm{ic}$ & $<2.97 \mathrm{E}+03 \mathrm{~kg}$ & $<5.44 \mathrm{E}+03 \mathrm{~kg}$ \\
\hline${ }^{1} \mathrm{Br}$.ic.wo & $2.50 \mathrm{E}+03 \mathrm{~kg}$ & $\mathrm{Br} . \mathrm{ic}$ & $<2.97 \mathrm{E}+03 \mathrm{~kg}$ & $<5.47 \mathrm{E}+03 \mathrm{~kg}$ \\
\hline Ca.icp.adl & $<1.54 \mathrm{E}+02 \mathrm{~kg}$ & Ca.icp.a & $1.73 \mathrm{E}+03 \mathrm{~kg}$ & $<1.88 \mathrm{E}+03 \mathrm{~kg}$ \\
\hline Ca.icp.adl & $<1.54 \mathrm{E}+02 \mathrm{~kg}$ & Ca.icp.f & $<5.94 \mathrm{E}+03 \mathrm{~kg}$ & $<6.09 \mathrm{E}+03 \mathrm{~kg}$ \\
\hline Cd.icp.adl & $<7.69 \mathrm{E}+00 \mathrm{~kg}$ & Cd.icp.a & $3.15 \mathrm{E}+01 \mathrm{~kg}$ & $<3.92 \mathrm{E}+01 \mathrm{~kg}$ \\
\hline Cd.icp.adl & $<7.69 \mathrm{E}+00 \mathrm{~kg}$ & Cd.icp.f & $<2.97 \mathrm{E}+02 \mathrm{~kg}$ & $<3.05 \mathrm{E}+02 \mathrm{~kg}$ \\
\hline Ce.icp.ad1 & $<1.54 \mathrm{E}+02 \mathrm{~kg}$ & Ce.icp.a & $<1.34 \mathrm{E}+02 \mathrm{~kg}$ & $<2.88 \mathrm{E}+02 \mathrm{~kg}$ \\
\hline Ce.icp.ad1 & $<1.54 \mathrm{E}+02 \mathrm{~kg}$ & Ce.icp.f & $<5.94 \mathrm{E}+03 \mathrm{~kg}$ & $<6.09 \mathrm{E}+03 \mathrm{~kg}$ \\
\hline $\mathrm{Cl}^{-}$ic & $2.62 \mathrm{E}+04 \mathrm{~kg}$ & $\mathrm{Cl}^{-}$.ic & $1.39 \mathrm{E}+04 \mathrm{~kg}$ & $4.01 \mathrm{E}+04 \mathrm{~kg}$ \\
\hline $\mathrm{Cl}^{-}$.ic.ed & $2.54 \mathrm{E}+04 \mathrm{~kg}$ & $\mathrm{Cl}^{-}$.ic & $1.39 \mathrm{E}+04 \mathrm{~kg}$ & $3.93 \mathrm{E}+04 \mathrm{~kg}$ \\
\hline Co.icp.adl & $<3.07 \mathrm{E}+01 \mathrm{~kg}$ & Co.icp.a & $<2.67 \mathrm{E}+01 \mathrm{~kg}$ & $<5.74 \mathrm{E}+01 \mathrm{~kg}$ \\
\hline Co.icp.adl & $<3.07 \mathrm{E}+01 \mathrm{~kg}$ & Co.icp.f & $<1.19 \mathrm{E}+03 \mathrm{~kg}$ & $<1.22 \mathrm{E}+03 \mathrm{~kg}$ \\
\hline Cr.icp.adl & $5.76 \mathrm{E}+02 \mathrm{~kg}$ & Cr.icp.a & $6.30 \mathrm{E}+03 \mathrm{~kg}$ & $6.88 \mathrm{E}+03 \mathrm{~kg}$ \\
\hline Cr.icp.adl & $5.76 \mathrm{E}+02 \mathrm{~kg}$ & Cr.icp.f & $6.67 \mathrm{E}+03 \mathrm{~kg}$ & $7.25 \mathrm{E}+03 \mathrm{~kg}$ \\
\hline
\end{tabular}


Table B3-36. Tank Inventory - Based on Segment Data. (4 sheets)

\begin{tabular}{|c|c|c|c|c|}
\hline Sipermant & Supringtar & Sall alury & Soll onimy & 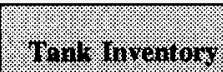 \\
\hline Cu.icp.adl & $2.61 \mathrm{E}+01 \mathrm{~kg}$ & Cu.icp.a.w & $1.90 \mathrm{E}+01 \mathrm{~kg}$ & $4.51 \mathrm{E}+01 \mathrm{~kg}$ \\
\hline Cu.icp.adl & $2.61 \mathrm{E}+01 \mathrm{~kg}$ & Cu.icp.a.wo & $1.91 \mathrm{E}+01 \mathrm{~kg}$ & $4.52 \mathrm{E}+01 \mathrm{~kg}$ \\
\hline Cu.icp.adl & $2.61 \mathrm{E}+01 \mathrm{~kg}$ & Cu.icp.f & $<5.94 \mathrm{E}+02 \mathrm{~kg}$ & $<6.20 \mathrm{E}+02 \mathrm{~kg}$ \\
\hline F.ic.w & $7.95 \mathrm{E}+02 \mathrm{~kg}$ & F-ic & $5.01 \mathrm{E}+03 \mathrm{~kg}$ & $5.81 \mathrm{E}+03 \mathrm{~kg}$ \\
\hline F-ic.w.ed & $7.52 \mathrm{E}+02 \mathrm{~kg}$ & $\mathrm{~F}^{-}$.ic & $5.01 \mathrm{E}+03 \mathrm{~kg}$ & $5.76 \mathrm{E}+03 \mathrm{~kg}$ \\
\hline $\mathrm{F}^{-}$.ic.wo & $1.25 \mathrm{E}+03 \mathrm{~kg}$ & F.ic & $5.01 \mathrm{E}+03 \mathrm{~kg}$ & $6.26 \mathrm{E}+03 \mathrm{~kg}$ \\
\hline $\mathrm{F}^{-}$.ic.wo.ed & $1.16 \mathrm{E}+03 \mathrm{~kg}$ & F.ic & $5.01 \mathrm{E}+03 \mathrm{~kg}$ & $6.17 \mathrm{E}+03 \mathrm{~kg}$ \\
\hline Fe.icp.adl & $<7.71 \mathrm{E}+01 \mathrm{~kg}$ & Fe.icp.a & $3.23 \mathrm{E}+02 \mathrm{~kg}$ & $<4.00 \mathrm{E}+02 \mathrm{~kg}$ \\
\hline Fe.icp.adl & $<7.71 \mathrm{E}+01 \mathrm{~kg}$ & Fe.icp.f & $<2.97 \mathrm{E}+03 \mathrm{~kg}$ & $<3.05 \mathrm{E}+03 \mathrm{~kg}$ \\
\hline Formate.ic & $4.00 \mathrm{E}+03 \mathrm{~kg}$ & Formate.ic & $2.85 \mathrm{E}+03 \mathrm{~kg}$ & $6.85 \mathrm{E}+03 \mathrm{~kg}$ \\
\hline K.icp.adl & $1.68 \mathrm{E}+04 \mathrm{~kg}$ & K.icp.a & $9.30 \mathrm{E}+03 \mathrm{~kg}$ & $2.61 \mathrm{E}+04 \mathrm{~kg}$ \\
\hline La.icp.adl & $<7.71 \mathrm{E}+01 \mathrm{~kg}$ & La.icp.a & $<6.67 \mathrm{E}+01 \mathrm{~kg}$ & $<1.44 \mathrm{E}+02 \mathrm{~kg}$ \\
\hline La.icp.adl & $<7.71 \mathrm{E}+01 \mathrm{~kg}$ & La.icp.f & $<2.97 \mathrm{E}+03 \mathrm{~kg}$ & $<3.05 \mathrm{E}+03 \mathrm{~kg}$ \\
\hline \begin{tabular}{|l}
${ }^{1}$ Li.icp.adl \\
\end{tabular} & $6.99 \mathrm{E}+01 \mathrm{~kg}$ & Li.icp.a.w & $5.33 \mathrm{E}+01 \mathrm{~kg}$ & $1.23 \mathrm{E}+02 \mathrm{~kg}$ \\
\hline${ }^{1}$ Li.icp.adl & $6.99 \mathrm{E}+01 \mathrm{~kg}$ & Li.icp.a.wo & $5.55 \mathrm{E}+01 \mathrm{~kg}$ & $1.25 \mathrm{E}+02 \mathrm{~kg}$ \\
\hline${ }^{1}$ Li.icp.adl & $6.99 \mathrm{E}+01 \mathrm{~kg}$ & Li.icp.f & $<5.94 \mathrm{E}+02 \mathrm{~kg}$ & $<6.64 \mathrm{E}+02 \mathrm{~kg}$ \\
\hline Mg.icp.adl & $<1.54 \mathrm{E}+02 \mathrm{~kg}$ & Mg.icp.a & $<1.66 \mathrm{E}+02 \mathrm{~kg}$ & $<3.20 \mathrm{E}+02 \mathrm{~kg}$ \\
\hline Mg.icp.adl & $<1.54 \mathrm{E}+02 \mathrm{~kg}$ & Mg.icp.f & $<5.94 \mathrm{E}+03 \mathrm{~kg}$ & $<6.09 \mathrm{E}+03 \mathrm{~kg}$ \\
\hline Mn.icp.adl & $<1.54 \mathrm{E}+01 \mathrm{~kg}$ & Mn.icp.a.w & $5.67 \mathrm{E}+01 \mathrm{~kg}$ & $<7.21 \mathrm{E}+01 \mathrm{~kg}$ \\
\hline Mn.icp.adl & $<1.54 \mathrm{E}+01 \mathrm{~kg}$ & Mn.icp.a.wo & $5.85 \mathrm{E}+01 \mathrm{~kg}$ & $<7.39 \mathrm{E}+01 \mathrm{~kg}$ \\
\hline Mn.icp.adl & $<1.54 \mathrm{E}+01 \mathrm{~kg}$ & Mn.icp.f & $<5.94 \mathrm{E}+02 \mathrm{~kg}$ & $<6.09 \mathrm{E}+02 \mathrm{~kg}$ \\
\hline Mo.icp.adl & $2.48 \mathrm{E}+02 \mathrm{~kg}$ & Mo.icp.a & $1.39 \mathrm{E}+02 \mathrm{~kg}$ & $3.87 \mathrm{E}+02 \mathrm{~kg}$ \\
\hline Mo.icp.adl & $2.48 \mathrm{E}+02 \mathrm{~kg}$ & Mo.icp.f & $<2.97 \mathrm{E}+03 \mathrm{~kg}$ & $<3.22 \mathrm{E}+03 \mathrm{~kg}$ \\
\hline $\mathrm{NO}_{2}$.ic & $3.24 \mathrm{E}+05 \mathrm{~kg}$ & $\mathrm{NO}_{2}^{-}$. ic & $1.72 \mathrm{E}+05 \mathrm{~kg}$ & $4.96 \mathrm{E}+05 \mathrm{~kg}$ \\
\hline $\mathrm{NO}_{2}^{-}$.ic.ed & $3.13 \mathrm{E}+05 \mathrm{~kg}$ & $\mathrm{NO}_{2}^{-}$.ic & $1.72 \mathrm{E}+05 \mathrm{~kg}$ & $4.85 \mathrm{E}+05 \mathrm{~kg}$ \\
\hline $\mathrm{NO}_{3}^{-} \cdot \mathrm{ic}$ & $4.25 \mathrm{E}+05 \mathrm{~kg}$ & $\mathrm{NO}_{3}^{-}$.ic & $3.00 \mathrm{E}+05 \mathrm{~kg}$ & $7.25 \mathrm{E}+05 \mathrm{~kg}$ \\
\hline $\mathrm{NO}_{3}{ }^{-}$.ic.ed & $4.12 \mathrm{E}+05 \mathrm{~kg}$ & $\mathrm{NO}_{3}^{-} \cdot \mathrm{ic}$ & $3.00 \mathrm{E}+05 \mathrm{~kg}$ & $7.12 \mathrm{E}+05 \mathrm{~kg}$ \\
\hline Na.icp.adl & $6.33 \mathrm{E}+05 \mathrm{~kg}$ & Na.icp.a & $4.93 \mathrm{E}+05 \mathrm{~kg}$ & $1.13 \mathrm{E}+06 \mathrm{~kg}$ \\
\hline Na.icp.adl & $6.33 \mathrm{E}+05 \mathrm{~kg}$ & Na.icp.f & $6.14 \mathrm{E}+05 \mathrm{~kg}$ & $1.25 \mathrm{E}+06 \mathrm{~kg}$ \\
\hline Nd.icp.adl & $<1.54 \mathrm{E}+02 \mathrm{~kg}$ & Nd.icp.a & $<1.34 \mathrm{E}+02 \mathrm{~kg}$ & $<2.88 \mathrm{E}+02 \mathrm{~kg}$ \\
\hline Nd.icp.adl & $<1.54 \mathrm{E}+02 \mathrm{~kg}$ & Nd.icp.f & $<5.94 \mathrm{E}+03 \mathrm{~kg}$ & $<6.09 \mathrm{E}+03 \mathrm{~kg}$ \\
\hline Ni.icp.adl & $<3.07 \mathrm{E}+01 \mathrm{~kg}$ & Ni.icp.a & $1.10 \mathrm{E}+02 \mathrm{~kg}$ & $<1.41 \mathrm{E}+02 \mathrm{~kg}$ \\
\hline
\end{tabular}


Table B3-36. Tank Inventory - Based on Segment Data. (4 sheets)

\begin{tabular}{|c|c|c|c|c|}
\hline suparmarint & Supquntort & Shlughry & 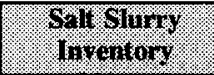 & Mrat \\
\hline Ni.icp.adl & $<3.07 \mathrm{E}+01 \mathrm{~kg}$ & Ni.icp.f.w & $3.68 \mathrm{E}+03 \mathrm{~kg}$ & $<3.71 \mathrm{E}+03 \mathrm{~kg}$ \\
\hline Ni.icp.adl & $<3.07 \mathrm{E}+01 \mathrm{~kg}$ & Ni.icp.f.wo & $4.72 \mathrm{E}+03 \mathrm{~kg}$ & $<4.75 \mathrm{E}+03 \mathrm{~kg}$ \\
\hline Oxalate.ic & $4.12 \mathrm{E}+04 \mathrm{~kg}$ & Oxalate.ic & $1.87 \mathrm{E}+04 \mathrm{~kg}$ & $5.99 \mathrm{E}+04 \mathrm{~kg}$ \\
\hline P.icp.adl & $1.02 \mathrm{E}+03 \mathrm{~kg}$ & P.icp.a & $6.70 \mathrm{E}+03 \mathrm{~kg}$ & $7.72 \mathrm{E}+03 \mathrm{~kg}$ \\
\hline P.icp.adl & $1.02 \mathrm{E}+03 \mathrm{~kg}$ & P.icp.f & $9.76 \mathrm{E}+04 \mathrm{~kg}$ & $9.86 \mathrm{E}+04 \mathrm{~kg}$ \\
\hline P.icp.adl.ed & $9.86 \mathrm{E}+02 \mathrm{~kg}$ & P.icp.a & $6.70 \mathrm{E}+03 \mathrm{~kg}$ & $7.69 \mathrm{E}+03 \mathrm{~kg}$ \\
\hline P.icp.adl.ed & $9.86 \mathrm{E}+02 \mathrm{~kg}$ & P.icp.f & $9.76 \mathrm{E}+04 \mathrm{~kg}$ & $9.86 \mathrm{E}+04 \mathrm{~kg}$ \\
\hline $\mathrm{PO}_{4}^{3+}$.ic.w & $2.71 \mathrm{E}+03 \mathrm{~kg}$ & $\mathrm{PO}_{4}^{3-}$.ic. $\mathrm{W}$ & $2.18 \mathrm{E}+04 \mathrm{~kg}$ & $2.45 \mathrm{E}+04 \mathrm{~kg}$ \\
\hline $\mathrm{PO}_{4}^{3-}$.ic.w & $2.71 \mathrm{E}+03 \mathrm{~kg}$ & $\mathrm{PO}_{4}{ }^{3-}$.ic.wo & $2.18 \mathrm{E}+04 \mathrm{~kg}$ & $2.45 \mathrm{E}+04 \mathrm{~kg}$ \\
\hline $\mathrm{PO}_{4}{ }^{3-}$.ic.w.ed & $1.93 \mathrm{E}+03 \mathrm{~kg}$ & $\mathrm{PO}_{4}{ }^{3-}$.ic.w & $2.18 \mathrm{E}+04 \mathrm{~kg}$ & $2.37 \mathrm{E}+04 \mathrm{~kg}$ \\
\hline $\mathrm{PO}_{4}{ }^{3}$.ic.w.ed & $1.93 \mathrm{E}+03 \mathrm{~kg}$ & $\mathrm{PO}_{4}{ }^{3-}$.ic.wo & $2.18 \mathrm{E}+04 \mathrm{~kg}$ & $2.37 \mathrm{E}+\mathrm{C}$ \\
\hline $\mathrm{PO}_{4}{ }^{3-}$.ic.wo & $4.00 \mathrm{E}+03 \mathrm{~kg}$ & $\mathrm{PO}_{4}^{3-}$.ic.w & $2.18 \mathrm{E}+04 \mathrm{~kg}$ & $2.58 \mathrm{E}+04 \mathrm{~kg}$ \\
\hline $\mathrm{PO}_{4}{ }^{3+}$.ic.wo & $4.00 \mathrm{E}+03 \mathrm{~kg}$ & $\mathrm{PO}_{4}{ }^{3-}$.ic.wo & $2.18 \mathrm{E}+04 \mathrm{~kg}$ & $2.58 \mathrm{E}+04 \mathrm{~kg}$ \\
\hline $\mathrm{PO}_{4}{ }^{3}$.ic.wo.ed & $2.15 \mathrm{E}+03 \mathrm{~kg}$ & $\mathrm{PO}_{4}^{3-}$.ic. $\mathrm{W}$ & $2.18 \mathrm{E}+04 \mathrm{~kg}$ & $2.40 \mathrm{E}+04 \mathrm{~kg}$ \\
\hline $\mathrm{PO}_{4}{ }^{3-}$.ic.wo.ed & $2.15 \mathrm{E}+03 \mathrm{~kg}$ & $\mathrm{PO}_{4}{ }^{3-}$.ic.wo & $2.18 \mathrm{E}+04 \mathrm{~kg}$ & $2.40 \mathrm{E}+04 \mathrm{~kg}$ \\
\hline Pb.icp.adl.w & $1.38 \mathrm{E}+02 \mathrm{~kg}$ & Pb.icp.a.w & $1.28 \mathrm{E}+02 \mathrm{~kg}$ & $2.66 \mathrm{E}+02 \mathrm{~kg}$ \\
\hline Pb.icp.adl.w & $1.38 \mathrm{E}+02 \mathrm{~kg}$ & Pb.icp.a.wo & $1.36 \mathrm{E}+02 \mathrm{~kg}$ & $2.74 \mathrm{E}+02 \mathrm{~kg}$ \\
\hline Pb.icp.adl.w & $1.38 \mathrm{E}+02 \mathrm{~kg}$ & Pb.icp.f & $<5.94 \mathrm{E}+03 \mathrm{~kg}$ & $<6.08 \mathrm{E}+03 \mathrm{~kg}$ \\
\hline Pb.icp.adl.wo & $1.27 \mathrm{E}+02 \mathrm{~kg}$ & Pb.icp.a.w & $1.28 \mathrm{E}+02 \mathrm{~kg}$ & $2.55 \mathrm{E}+02 \mathrm{~kg}$ \\
\hline Pb.icp.adl.wo & $1.27 \mathrm{E}+02 \mathrm{~kg}$ & Pb.icp.a.wo & $1.36 \mathrm{E}+02 \mathrm{~kg}$ & $2.63 \mathrm{E}+02 \mathrm{~kg}$ \\
\hline Pb.icp.adl.wo & $1.27 \mathrm{E}+02 \mathrm{~kg}$ & Pb.icp.f & $<5.94 \mathrm{E}+03 \mathrm{~kg}$ & $<6.07 \mathrm{E}+03 \mathrm{~kg}$ \\
\hline S.icp.adl & $2.40 \mathrm{E}+03 \mathrm{~kg}$ & S.icp.a & $6.77 \mathrm{E}+03 \mathrm{~kg}$ & $9.17 \mathrm{E}+03 \mathrm{~kg}$ \\
\hline S.icp.adl & $2.40 \mathrm{E}+03 \mathrm{~kg}$ & S.icp.f.w & $7.49 \mathrm{E}+03 \mathrm{~kg}$ & $9.89 \mathrm{E}+03 \mathrm{~kg}$ \\
\hline S.icp.adl & $2.40 \mathrm{E}+03 \mathrm{~kg}$ & S.icp.f.wo & $7.59 \mathrm{E}+03 \mathrm{~kg}$ & $9.99 \mathrm{E}+03 \mathrm{~kg}$ \\
\hline $\mathrm{SO}_{4}^{2-}$.ic.W & $3.03 \mathrm{E}+03 \mathrm{~kg}$ & $\mathrm{SO}_{4}{ }^{2-} \cdot \mathrm{ic}$ & $1.69 \mathrm{E}+04 \mathrm{~kg}$ & $1.99 \mathrm{E}+04 \mathrm{~kg}$ \\
\hline $\mathrm{SO}_{4}^{2-}$.ic.w.ed & $2.82 \mathrm{E}+03 \mathrm{~kg}$ & $\mathrm{SO}_{4}{ }^{2-} \cdot \mathrm{ic}$ & $1.69 \mathrm{E}+04 \mathrm{~kg}$ & $1.97 \mathrm{E}+04 \mathrm{~kg}$ \\
\hline $\mathrm{SO}_{4}^{2-}$.ic.wo & $3.01 \mathrm{E}+03 \mathrm{~kg}$ & $\mathrm{SO}_{4}{ }^{2-} . \mathrm{ic}$ & $1.69 \mathrm{E}+04 \mathrm{~kg}$ & $1.99 \mathrm{E}+04 \mathrm{~kg}$ \\
\hline $\mathrm{SO}_{4}{ }^{2-}$.ic.wo.ed & $2.79 \mathrm{E}+03 \mathrm{~kg}$ & $\mathrm{SO}_{4}{ }^{2-} . \mathrm{ic}$ & $1.69 \mathrm{E}+04 \mathrm{~kg}$ & $1.97 \mathrm{E}+04 \mathrm{~kg}$ \\
\hline Sb.icp.adl & $<9.25 \mathrm{E}+01 \mathrm{~kg}$ & Sb.icp.a & $<8.01 \mathrm{E}+01 \mathrm{~kg}$ & $<1.73 \mathrm{E}+02 \mathrm{~kg}$ \\
\hline Sb.icp.adl & $<9.25 \mathrm{E}+01 \mathrm{~kg}$ & Sb.icp.f & $<3.56 \mathrm{E}+03 \mathrm{~kg}$ & $<3.65 \mathrm{E}+03 \mathrm{~kg}$ \\
\hline Se.icp.adl & $<1.54 \mathrm{E}+02 \mathrm{~kg}$ & Se.icp.a & $<1.34 \mathrm{E}+02 \mathrm{~kg}$ & $<2.88 \mathrm{E}+02 \mathrm{~kg}$ \\
\hline Se.icp.adl & $<1.54 \mathrm{E}+02 \mathrm{~kg}$ & Se.icp.f & $<5.94 \mathrm{E}+03 \mathrm{~kg}$ & $<6.09 \mathrm{E}+03 \mathrm{~kg}$ \\
\hline
\end{tabular}


Table B3-36. Tank Inventory - Based on Segment Data. (4 sheets)

\begin{tabular}{|c|c|c|c|c|}
\hline Shpormarit & Whowntrint & WuThrol & 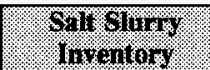 & 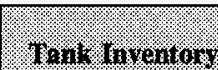 \\
\hline Si.icp.adl & $5.59 \mathrm{E}+02 \mathrm{~kg}$ & Si.icp.a.w & $3.61 \mathrm{E}+02 \mathrm{~kg}$ & $9.20 \mathrm{E}+02 \mathrm{~kg}$ \\
\hline Si.icp.adl & $5.59 \mathrm{E}+02 \mathrm{~kg}$ & Si.icp.a.wo & $3.70 \mathrm{E}+02 \mathrm{~kg}$ & $9.29 \mathrm{E}+02 \mathrm{~kg}$ \\
\hline Si.icp.adl & $5.59 \mathrm{E}+02 \mathrm{~kg}$ & Si.icp.f & $6.53 \mathrm{E}+03 \mathrm{~kg}$ & $7.09 \mathrm{E}+03 \mathrm{~kg}$ \\
\hline Sm.icp.adl & $<1.54 \mathrm{E}+02 \mathrm{~kg}$ & Sm.icp.a & $<1.34 \mathrm{E}+02 \mathrm{~kg}$ & $<2.88 \mathrm{E}+02 \mathrm{~kg}$ \\
\hline Sm.icp.adl & $<1.54 \mathrm{E}+02 \mathrm{~kg}$ & Sm.icp.f & $<5.94 \mathrm{E}+03 \mathrm{~kg}$ & $<6.09 \mathrm{E}+03 \mathrm{~kg}$ \\
\hline Sr.icp.adl & $<1.54 \mathrm{E}+01 \mathrm{~kg}$ & Sr.icp.a & $2.66 \mathrm{E}+01 \mathrm{~kg}$ & $<4.20 \mathrm{E}+01 \mathrm{~kg}$ \\
\hline Sr.icp.adl & $<1.54 \mathrm{E}+01 \mathrm{~kg}$ & Sr.icp.f & $<5.94 \mathrm{E}+02 \mathrm{~kg}$ & $<6.09 \mathrm{E}+02 \mathrm{~kg}$ \\
\hline Ti.icp.adl & $<1.54 \mathrm{E}+01 \mathrm{~kg}$ & Ti.icp.a & $<1.34 \mathrm{E}+01 \mathrm{~kg}$ & $<2.88 \mathrm{E}+01 \mathrm{~kg}$ \\
\hline Ti.icp.adl & $<1.54 \mathrm{E}+01 \mathrm{~kg}$ & Ti.icp.f & $<5.94 \mathrm{E}+02 \mathrm{~kg}$ & $<6.09 \mathrm{E}+02 \mathrm{~kg}$ \\
\hline Tl.icp.adl & $<3.07 \mathrm{E}+02 \mathrm{~kg}$ & Tl.icp.a & $<2.67 \mathrm{E}+02 \mathrm{~kg}$ & $<5.74 \mathrm{E}+02 \mathrm{~kg}$ \\
\hline Tl.icp.adl & $<3.07 \mathrm{E}+02 \mathrm{~kg}$ & Tl.icp.f & $<1.19 \mathrm{E}+04 \mathrm{~kg}$ & $<1.22 \mathrm{E}+04 \mathrm{~kg}$ \\
\hline $\mathrm{AT}$ & $<3.71 \mathrm{E}+02 \mathrm{Ci}$ & AT.w & $6.20 \mathrm{E}+01 \mathrm{Ci}$ & $<4.33 \mathrm{E}+02 \mathrm{Ci}$ \\
\hline AT & $<3.71 \mathrm{E}+02 \mathrm{Ci}$ & AT.wo & $6.26 \mathrm{E}+01 \mathrm{Ci}$ & $<4.34 \mathrm{E}+02 \mathrm{Ci}$ \\
\hline U.icp.adl & $<7.69 \mathrm{E}+02 \mathrm{~kg}$ & U.icp.a & $<6.67 \mathrm{E}+02 \mathrm{~kg}$ & $<1.44 \mathrm{E}+03 \mathrm{~kg}$ \\
\hline U.icp.adl & $<7.69 \mathrm{E}+02 \mathrm{~kg}$ & U.icp.f & $<2.97 \mathrm{E}+04 \mathrm{~kg}$ & $<3.05 \mathrm{E}+04 \mathrm{~kg}$ \\
\hline V.icp.adl & $<7.71 \mathrm{E}+01 \mathrm{~kg}$ & V.icp.a & $<6.67 \mathrm{E}+01 \mathrm{~kg}$ & $<1.44 \mathrm{E}+02 \mathrm{~kg}$ \\
\hline V.icp.adl & $<7.71 \mathrm{E}+01 \mathrm{~kg}$ & V.icp.f & $<2.97 \mathrm{E}+03 \mathrm{~kg}$ & $<3.05 \mathrm{E}+03 \mathrm{~kg}$ \\
\hline Zn.icp.adl & $8.00 \mathrm{E}+01 \mathrm{~kg}$ & Zn.icp.a & $1.17 \mathrm{E}+02 \mathrm{~kg}$ & $1.97 \mathrm{E}+02 \mathrm{~kg}$ \\
\hline Zn.icp.adl & $8.00 \mathrm{E}+01 \mathrm{~kg}$ & Zn.icp.f & $2.16 \mathrm{E}+03 \mathrm{~kg}$ & $2.24 \mathrm{E}+03 \mathrm{~kg}$ \\
\hline Zr.icp.adl & $1.56 \mathrm{E}+01 \mathrm{~kg}$ & Zr.icp.a.w & $3.08 \mathrm{E}+01 \mathrm{~kg}$ & $4.64 \mathrm{E}+01 \mathrm{~kg}$ \\
\hline Zr.icp.adl & $1.56 \mathrm{E}+01 \mathrm{~kg}$ & Zr.icp.a.wo & $3.23 \mathrm{E}+01 \mathrm{~kg}$ & $4.79 \mathrm{E}+01 \mathrm{~kg}$ \\
\hline Zr.icp.adl & $1.56 \mathrm{E}+01 \mathrm{~kg}$ & Zr.icp.f & $1.31 \mathrm{E}+03 \mathrm{~kg}$ & $1.33 \mathrm{E}+03 \mathrm{~kg}$ \\
\hline
\end{tabular}

Note:

${ }^{1} \mathrm{Li}$ and ${ }^{1} \mathrm{Br}$ are the tracers for hydrostatic head fluid. 
Table B3-37. Tank Inventory - Based on Composite Data. (6 sheets)

\begin{tabular}{|c|c|c|c|c|c|}
\hline SHinglanil & Sumprintaril & Slinge Analyte & Sril sinyy & 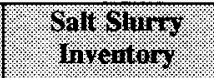 & Hail Larion \\
\hline${ }^{129} \mathrm{I}$ & $6.85 \mathrm{E}-01 \mathrm{Ci}$ & ${ }^{129} \mathbf{I}$ & $<9.50 \mathrm{E}+00 \mathrm{Ci}$ & $<8.00 \mathrm{E}-01 \mathrm{Ci}$ & $<.10 \mathrm{E}+01 \mathrm{i}$ \\
\hline${ }^{137}$ Cs.gea & $1.22 \mathrm{E}+03 \mathrm{Ci}$ & ${ }^{137} \mathrm{Cs}$.gea & $7.37 \mathrm{E}+05 \mathrm{Ci}$ & $5.30 \mathrm{E}+04 \mathrm{Ci}$ & $7.91 \mathrm{E}+05 \mathrm{Ci}$ \\
\hline${ }^{154}$ Eu.gea & $<2.50 \mathrm{E}+02 \mathrm{Ci}$ & ${ }^{154}$ Eu.gea & $<2.40 \mathrm{E}+02 \mathrm{Ci}$ & $<1.95 \mathrm{E}+01 \mathrm{Ci}$ & $<5.10 \mathrm{E}+02 \mathrm{Ci}$ \\
\hline${ }^{155}$ Eu.gea & $<8.35 \mathrm{E}+02 \mathrm{Ci}$ & ${ }^{155}$ Eu.gea & $<9.38 \mathrm{E}+02 \mathrm{Ci}$ & $<5.92 \mathrm{E}+01 \mathrm{Ci}$ & $<1.83 \mathrm{E}+03 \mathrm{Ci}$ \\
\hline${ }^{237} \mathrm{~Np}$ & $<5.66 \mathrm{E}-02 \mathrm{Ci}$ & ${ }^{237} \mathrm{~Np}$ & $2.57 \mathrm{E}+01 \mathrm{Ci}$ & $<4.17 \mathrm{E}+00 \mathrm{Ci}$ & $<2.99 \mathrm{E}+01 \mathrm{Ci}$ \\
\hline${ }^{239 / 240} \mathrm{Pu}$ & $<9.53 \mathrm{E}-02 \mathrm{Ci}$ & ${ }^{239 / 240} \mathrm{Pu}$ & $<4.39 \mathrm{E}+00 \mathrm{Ci}$ & $1.94 \mathrm{E}+00 \mathrm{Ci}$ & $<6.43 \mathrm{E}+00 \mathrm{Ci}$ \\
\hline${ }^{241} \mathrm{Am}$.aea & $<1.00 \mathrm{E}-01 \mathrm{Ci}$ & ${ }^{241}$ Am.aea & $9.93 \mathrm{E}+00 \mathrm{Ci}$ & $1.00 \mathrm{E}+01 \mathrm{Ci}$ & $<2.00 \mathrm{E}+01 \mathrm{Ci}$ \\
\hline${ }^{241}$ Am.gea & $<2.20 \mathrm{E}+03 \mathrm{Ci}$ & ${ }^{241} \mathrm{Am}$.gea & $<2.00 \mathrm{E}+03 \mathrm{Ci}$ & $<1.27 \mathrm{E}+02 \mathrm{Ci}$ & $<4.33 \mathrm{E}+03 \mathrm{Ci}$ \\
\hline${ }^{243 / 244} \mathrm{Cm}$ & $<1.00 \mathrm{E}-01 \mathrm{Ci}$ & ${ }^{243 / 244} \mathrm{Cm}$ & $<2.34 \mathrm{E}+00 \mathrm{Ci}$ & $<1.45 \mathrm{E}+00 \mathrm{Ci}$ & $<3.89 \mathrm{E}+00 \mathrm{Ci}$ \\
\hline${ }^{60} \mathrm{Co}$ gea & $<3.33 \mathrm{E}-02 \mathrm{Ci}$ & ${ }^{60} \mathrm{Co}$. gea & $<9.09 \mathrm{E}+01 \mathrm{Ci}$ & $<4.63 \mathrm{E}+00 \mathrm{Ci}$ & $<9.56 \mathrm{E}+01 \mathrm{Ci}$ \\
\hline${ }^{89 / 90} \mathrm{Sr}$ & $1.06 \mathrm{E}+02 \mathrm{Ci}$ & ${ }^{89 / 90} \mathrm{Sr}$ & $2.22 \mathrm{E}+04 \mathrm{Ci}$ & $9.40 \mathrm{E}+03 \mathrm{Ci}$ & $3.17 \mathrm{E}+04 \mathrm{Ci}$ \\
\hline${ }^{99} \mathrm{Tc}$ & $7.01 \mathrm{E}+02 \mathrm{Ci}$ & ${ }^{956} \mathrm{Tc}$ & $4.38 \mathrm{E}+02 \mathrm{Ci}$ & $3.45 \mathrm{E}+01 \mathrm{Ci}$ & $1.17 \mathrm{E}+03 \mathrm{Ci}$ \\
\hline Acetate.ic & $1.64 \mathrm{E}+03 \mathrm{~kg}$ & Acetate.ic & $1.14 \mathrm{E}+03 \mathrm{~kg}$ & $<2.03 \mathrm{E}+01 \mathrm{~kg}$ & $<2.80 \mathrm{E}+03 \mathrm{~kg}$ \\
\hline Ag.icp.a & $3.88 \mathrm{E}+01 \mathrm{~kg}$ & Ag.icp.a & $2.88 \mathrm{E}+01 \mathrm{~kg}$ & $1.09 \mathrm{E}+01 \mathrm{~kg}$ & $7.85 \mathrm{E}+01 \mathrm{~kg}$ \\
\hline Ag.icp.adl & $4.61 \mathrm{E}+01 \mathrm{~kg}$ & Ag.icp.a & $2.88 \mathrm{E}+01 \mathrm{~kg}$ & $1.09 \mathrm{E}+01 \mathrm{~kg}$ & $8.58 \mathrm{E}+01 \mathrm{~kg}$ \\
\hline Al.icp.a & $9.15 \mathrm{E}+04 \mathrm{~kg}$ & Al.icp.a & $4.96 \mathrm{E}+04 \mathrm{~kg}$ & $3.72 \mathrm{E}+03 \mathrm{~kg}$ & $1.45 \mathrm{E}+05 \mathrm{~kg}$ \\
\hline Al.icp.adl & $1.12 \mathrm{E}+05 \mathrm{~kg}$ & Al.icp.a & $4.96 \mathrm{E}+04 \mathrm{~kg}$ & $3.72 \mathrm{E}+03 \mathrm{~kg}$ & $1.65 \mathrm{E}+05 \mathrm{~kg}$ \\
\hline As.icp.a & $<1.34 \mathrm{E}+02 \mathrm{~kg}$ & As.icp.a & $<9.77 \mathrm{E}+01 \mathrm{~kg}$ & $<1.00 \mathrm{E}+01 \mathrm{~kg}$ & $<2.42 \mathrm{E}+02 \mathrm{~kg}$ \\
\hline As.icp.adl & $<1.54 \mathrm{E}+02 \mathrm{~kg}$ & As.icp.a & $<9.77 \mathrm{E}+01 \mathrm{~kg}$ & $<1.00 \mathrm{E}+01 \mathrm{~kg}$ & $<2.62 \mathrm{E}+02 \mathrm{~kg}$ \\
\hline B.icp.a & $4.34 \mathrm{E}+02 \mathrm{~kg}$ & B.icp.a & $2.82 \mathrm{E}+02 \mathrm{~kg}$ & $2.92 \mathrm{E}+01 \mathrm{~kg}$ & $7.45 \mathrm{E}+02 \mathrm{~kg}$ \\
\hline
\end{tabular}


Table B3-37. Tank Inventory - Based on Composite Data. (6 sheets)

\begin{tabular}{|c|c|c|c|c|c|}
\hline Superinglan & Superiagar & gluge 4 malyte & 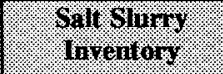 & 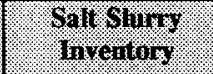 & 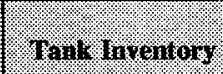 \\
\hline B.icp.adl & $1.86 \mathrm{E}+02 \mathrm{~kg}$ & B.icp.a & $2.82 \mathrm{E}+02 \mathrm{~kg}$ & $2.92 \mathrm{E}+01 \mathrm{~kg}$ & $4.97 \mathrm{E}+02 \mathrm{~kg}$ \\
\hline Ba.icp.a & $<6.71 \mathrm{E}+01 \mathrm{~kg}$ & Ba.icp.a & $<4.90 \mathrm{E}+01 \mathrm{~kg}$ & $<5.02 \mathrm{E}+00 \mathrm{~kg}$ & $<1.21 \mathrm{E}+02 \mathrm{~kg}$ \\
\hline Ba.icp.adl & $<7.71 \mathrm{E}+01 \mathrm{~kg}$ & Ba.icp.a & $<4.90 \mathrm{E}+01 \mathrm{~kg}$ & $<5.02 \mathrm{E}+00 \mathrm{~kg}$ & $<1.31 \mathrm{E}+02 \mathrm{~kg}$ \\
\hline Be.icp.a & $<6.71 \mathrm{E}+00 \mathrm{~kg}$ & Be.icp.a & $<4.90 \mathrm{E}+00 \mathrm{~kg}$ & $<5.02 \mathrm{E}-01 \mathrm{~kg}$ & $<1.21 \mathrm{E}+01 \mathrm{~kg}$ \\
\hline Be.icp.adl & $<7.69 \mathrm{E}+00 \mathrm{~kg}$ & Be.icp.a & $<4.90 \mathrm{E}+00 \mathrm{~kg}$ & $<5.02 \mathrm{E}-01 \mathrm{~kg}$ & $<1.31 \mathrm{E}+01 \mathrm{~kg}$ \\
\hline Bi.icp.a & $<1.34 \mathrm{E}+02 \mathrm{~kg}$ & Bi.icp.a & $<9.77 \mathrm{E}+01 \mathrm{~kg}$ & $<1.00 \mathrm{E}+01 \mathrm{~kg}$ & $<2.42 \mathrm{E}+02 \mathrm{~kg}$ \\
\hline Bi.icp.adl & $<1.54 \mathrm{E}+02 \mathrm{~kg}$ & Bi.icp.a & $<9.77 \mathrm{E}+01 \mathrm{~kg}$ & $<1.00 \mathrm{E}+01 \mathrm{~kg}$ & $<2.62 \mathrm{E}+02 \mathrm{~kg}$ \\
\hline${ }^{1} \mathrm{Br}^{-}$.ic & $<1.34 \mathrm{E}+03 \mathrm{~kg}$ & $\mathrm{Br} . \mathrm{ic}$ & $<1.35 \mathrm{E}+03 \mathrm{~kg}$ & $<1.27 \mathrm{E}+02 \mathrm{~kg}$ & $<2.82 \mathrm{E}+03 \mathrm{~kg}$ \\
\hline Ca.icp.a & $2.25 \mathrm{E}+03 \mathrm{~kg}$ & Ca.icp.a & $5.22 \mathrm{E}+02 \mathrm{~kg}$ & $8.45 \mathrm{E}+01 \mathrm{~kg}$ & $2.86 \mathrm{E}+03 \mathrm{~kg}$ \\
\hline Ca.icp.adl & $<1.54 \mathrm{E}+02 \mathrm{~kg}$ & Ca.icp.a & $5.22 \mathrm{E}+02 \mathrm{~kg}$ & $8.45 \mathrm{E}+01 \mathrm{~kg}$ & $<7.61 \mathrm{E}+02 \mathrm{~kg}$ \\
\hline Cd.icp.a & $<6.71 \mathrm{E}+00 \mathrm{~kg}$ & Cd.icp.a & $1.77 \mathrm{E}+01 \mathrm{~kg}$ & $3.56 \mathrm{E}+00 \mathrm{~kg}$ & $<2.80 \mathrm{E}+01 \mathrm{~kg}$ \\
\hline Cd.icp.adl & $<7.69 \mathrm{E}+00 \mathrm{~kg}$ & Cd.icp.a & $1.77 \mathrm{E}+01 \mathrm{~kg}$ & $3.56 \mathrm{E}+00 \mathrm{~kg}$ & $<2.90 \mathrm{E}+01 \mathrm{~kg}$ \\
\hline Ce.icp.a & $<1.34 \mathrm{E}+02 \mathrm{~kg}$ & Ce.icp.a & $<9.77 \mathrm{E}+01 \mathrm{~kg}$ & $<1.00 \mathrm{E}+01 \mathrm{~kg}$ & $<2.42 \mathrm{E}+02 \mathrm{~kg}$ \\
\hline Ce.icp.adl & $<1.54 \mathrm{E}+02 \mathrm{~kg}$ & Ce.icp.a & $<9.77 \mathrm{E}+01 \mathrm{~kg}$ & $<1.00 \mathrm{E}+01 \mathrm{~kg}$ & $<2.62 \mathrm{E}+02 \mathrm{~kg}$ \\
\hline $\mathrm{Cl}^{-} . \mathrm{ic}$ & $2.61 \mathrm{E}+04 \mathrm{~kg}$ & $\mathrm{Cl}^{-} . \mathrm{ic}$ & $1.43 \mathrm{E}+04 \mathrm{~kg}$ & $1.05 \mathrm{E}+03 \mathrm{~kg}$ & $4.15 \mathrm{E}+04 \mathrm{~kg}$ \\
\hline Co.icp.a & $<2.69 \mathrm{E}+01 \mathrm{~kg}$ & Co.icp.a & $<1.95 \mathrm{E}+01 \mathrm{~kg}$ & $<2.00 \mathrm{E}+00 \mathrm{~kg}$ & $<4.84 \mathrm{E}+01 \mathrm{~kg}$ \\
\hline Co.icp.adl & $<3.07 \mathrm{E}+01 \mathrm{~kg}$ & Co.icp.a & $<1.95 \mathrm{E}+01 \mathrm{~kg}$ & $<2.00 \mathrm{E}+00 \mathrm{~kg}$ & $<5.22 \mathrm{E}+01 \mathrm{~kg}$ \\
\hline Cr(VI).spec & $3.93 \mathrm{E}+02 \mathrm{~kg}$ & $\mathrm{Cr}(\mathrm{VI}) \cdot \mathrm{spec}$ & $2.88 \mathrm{E}+02 \mathrm{~kg}$ & $2.67 \mathrm{E}+01 \mathrm{~kg}$ & $7.08 \mathrm{E}+02 \mathrm{~kg}$ \\
\hline Cr.icp.a & $4.79 \mathrm{E}+02 \mathrm{~kg}$ & Cr.icp.a & $3.60 \mathrm{E}+03 \mathrm{~kg}$ & $7.05 \mathrm{E}+02 \mathrm{~kg}$ & $4.78 \mathrm{E}+03 \mathrm{~kg}$ \\
\hline Cr.icp.adl & $5.70 \mathrm{E}+02 \mathrm{~kg}$ & Cr.icp.a & $3.60 \mathrm{E}+03 \mathrm{~kg}$ & $7.05 \mathrm{E}+02 \mathrm{~kg}$ & $4.88 \mathrm{E}+03 \mathrm{~kg}$ \\
\hline
\end{tabular}


Table B3-37. Tank Inventory - Based on Composite Data. (6 sheets)

\begin{tabular}{|c|c|c|c|c|c|}
\hline Suporingan & Supronarit & 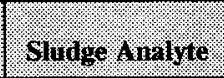 & Gals surry & Sall 5 hroy & Ganit loweritoy \\
\hline Cu.icp.a & $<1.61 \mathrm{E}+01 \mathrm{~kg}$ & Cu.icp.a & $1.24 \mathrm{E}+01 \mathrm{~kg}$ & $1.55 \mathrm{E}+00 \mathrm{~kg}$ & $<3.01 \mathrm{E}+01 \mathrm{~kg}$ \\
\hline Cu.icp.adl & $<1.54 \mathrm{E}+01 \mathrm{~kg}$ & Cu.icp.a & $1.24 \mathrm{E}+01 \mathrm{~kg}$ & $1.55 \mathrm{E}+00 \mathrm{~kg}$ & $<2.94 \mathrm{E}+01 \mathrm{~kg}$ \\
\hline $\mathrm{F}^{-}$ic & $<1.38 \mathrm{E}+02 \mathrm{~kg}$ & F.ic & $2.76 \mathrm{E}+03 \mathrm{~kg}$ & $1.29 \mathrm{E}+03 \mathrm{~kg}$ & $<4.19 \mathrm{E}+03 \mathrm{~kg}$ \\
\hline Fe.icp.a & $1.63 \mathrm{E}+02 \mathrm{~kg}$ & Fe.icp.a & $1.48 \mathrm{E}+02 \mathrm{~kg}$ & $3.77 \mathrm{E}+01 \mathrm{~kg}$ & $3.49 \mathrm{E}+02 \mathrm{~kg}$ \\
\hline Fe.icp.adl & $<7.71 \mathrm{E}+01 \mathrm{~kg}$ & Fe.icp.a & $1.48 \mathrm{E}+02 \mathrm{~kg}$ & $3.77 \mathrm{E}+01 \mathrm{~kg}$ & $<2.63 \mathrm{E}+02 \mathrm{~kg}$ \\
\hline Formate.ic & $4.15 \mathrm{E}+03 \mathrm{~kg}$ & Formate.ic & $2.97 \mathrm{E}+03 \mathrm{~kg}$ & $2.87 \mathrm{E}+02 \mathrm{~kg}$ & $7.41 \mathrm{E}+03 \mathrm{~kg}$ \\
\hline K.icp.a & $1.36 \mathrm{E}+04 \mathrm{~kg}$ & K.icp.a & $7.49 \mathrm{E}+03 \mathrm{~kg}$ & $5.52 \mathrm{E}+02 \mathrm{~kg}$ & $2.16 \mathrm{E}+04 \mathrm{~kg}$ \\
\hline K.icp.adl & $1.67 \mathrm{E}+04 \mathrm{~kg}$ & K.icp.a & $7.49 \mathrm{E}+03 \mathrm{~kg}$ & $5.52 \mathrm{E}+02 \mathrm{~kg}$ & $2.47 \mathrm{E}+04 \mathrm{~kg}$ \\
\hline La.icp.a & $<6.71 \mathrm{E}+01 \mathrm{~kg}$ & La.icp.a & $<4.90 \mathrm{E}+01 \mathrm{~kg}$ & $<5.02 \mathrm{E}+00 \mathrm{~kg}$ & $<1.21 \mathrm{E}+02 \mathrm{~kg}$ \\
\hline La.icp.adl & $<7.71 \mathrm{E}+01 \mathrm{~kg}$ & La.icp.a & $<4.90 \mathrm{E}+01 \mathrm{~kg}$ & $<5.02 \mathrm{E}+00 \mathrm{~kg}$ & $<1.31 \mathrm{E}+02 \mathrm{~kg}$ \\
\hline${ }^{1}$ Li.icp.a & $1.53 \mathrm{E}+01 \mathrm{~kg}$ & Li.icp.a & $2.11 \mathrm{E}+01 \mathrm{~kg}$ & $3.78 \mathrm{E}+00 \mathrm{~kg}$ & $4.02 \mathrm{E}+01 \mathrm{~kg}$ \\
\hline${ }^{1}$ Li.icp.adl & $<1.54 \mathrm{E}+01 \mathrm{~kg}$ & Li.icp.a & $2.11 \mathrm{E}+01 \mathrm{~kg}$ & $3.78 \mathrm{E}+00 \mathrm{~kg}$ & $<4.03 \mathrm{E}+01 \mathrm{~kg}$ \\
\hline Mg.icp.a & $<1.34 \mathrm{E}+02 \mathrm{~kg}$ & Mg.icp.a & $<9.77 \mathrm{E}+01 \mathrm{~kg}$ & $<1.00 \mathrm{E}+01 \mathrm{~kg}$ & $<2.42 \mathrm{E}+02 \mathrm{~kg}$ \\
\hline Mg.icp.adl & $<1.54 \mathrm{E}+02 \mathrm{~kg}$ & Mg.icp.a & $<9.77 \mathrm{E}+01 \mathrm{~kg}$ & $<1.00 \mathrm{E}+01 \mathrm{~kg}$ & $<2.62 \mathrm{E}+02 \mathrm{~kg}$ \\
\hline Mn.icp.a & $<1.34 \mathrm{E}+01 \mathrm{~kg}$ & Mn.icp.a & $1.90 \mathrm{E}+01 \mathrm{~kg}$ & $1.27 \mathrm{E}+01 \mathrm{~kg}$ & $<4.51 \mathrm{E}+01 \mathrm{~kg}$ \\
\hline Mn.icp.adl & $<1.54 \mathrm{E}+01 \mathrm{~kg}$ & Mn.icp.a & $1.90 \mathrm{E}+01 \mathrm{~kg}$ & $1.27 \mathrm{E}+01 \mathrm{~kg}$ & $<4.71 \mathrm{E}+01 \mathrm{~kg}$ \\
\hline Mo.icp.a & $2.11 \mathrm{E}+02 \mathrm{~kg}$ & Mo.icp.a & $1.11 \mathrm{E}+02 \mathrm{~kg}$ & $9.10 \mathrm{E}+00 \mathrm{~kg}$ & $3.31 \mathrm{E}+02 \mathrm{~kg}$ \\
\hline Mo.icp.adl & $2.59 \mathrm{E}+02 \mathrm{~kg}$ & Mo.icp.a & $1.11 \mathrm{E}+02 \mathrm{~kg}$ & $9.10 \mathrm{E}+00 \mathrm{~kg}$ & $3.79 \mathrm{E}+02 \mathrm{~kg}$ \\
\hline $\mathrm{NO}^{2-}$.ic & $3.34 \mathrm{E}+05 \mathrm{~kg}$ & $\mathrm{NO}_{2}^{-}$.ic & $1.77 \mathrm{E}+05 \mathrm{~kg}$ & $1.23 \mathrm{E}+04 \mathrm{~kg}$ & $5.23 \mathrm{E}+05 \mathrm{~kg}$ \\
\hline $\mathrm{NO}_{3}^{-}$.ic & $4.29 \mathrm{E}+05 \mathrm{~kg}$ & $\mathrm{NO}_{3}^{-} \cdot \mathrm{ic}$ & $2.74 \mathrm{E}+05 \mathrm{~kg}$ & $4.14 \mathrm{E}+04 \mathrm{~kg}$ & $7.44 \mathrm{E}+05 \mathrm{~kg}$ \\
\hline
\end{tabular}


Table B3-37. Tank Inventory - Based on Composite Data. (6 sheets)

\begin{tabular}{|c|c|c|c|c|c|}
\hline Supernatant & Gupriatant & olioge twalyte & 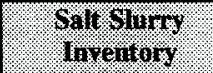 & Sall shmy & Tarit. inceritory \\
\hline Na.icp.a & $5.41 \mathrm{E}+05 \mathrm{~kg}$ & Na.icp.a & $3.76 \mathrm{E}+05 \mathrm{~kg}$ & $4.23 \mathrm{E}+04 \mathrm{~kg}$ & $9.59 \mathrm{E}+05 \mathrm{~kg}$ \\
\hline Na.icp.adl & $6.55 \mathrm{E}+05 \mathrm{~kg}$ & Na.icp.a & $3.76 \mathrm{E}+05 \mathrm{~kg}$ & $4.23 \mathrm{E}+04 \mathrm{~kg}$ & $1.07 \mathrm{E}+06 \mathrm{~kg}$ \\
\hline Nd.icp.a & $<1.34 \mathrm{E}+02 \mathrm{~kg}$ & Nd.icp.a & $<9.77 \mathrm{E}+01 \mathrm{~kg}$ & $<1.00 \mathrm{E}+01 \mathrm{~kg}$ & $<2.42 \mathrm{E}+02 \mathrm{~kg}$ \\
\hline Nd.icp.adl & $<1.54 \mathrm{E}+02 \mathrm{~kg}$ & Nd.icp.a & $<9.77 \mathrm{E}+01 \mathrm{~kg}$ & $<1.00 \mathrm{E}+01 \mathrm{~kg}$ & $<2.62 \mathrm{E}+02 \mathrm{~kg}$ \\
\hline Ni.icp.a & $<2.69 \mathrm{E}+01 \mathrm{~kg}$ & Ni.icp.a & $7.70 \mathrm{E}+01 \mathrm{~kg}$ & $8.65 \mathrm{E}+00 \mathrm{~kg}$ & $<1.13 \mathrm{E}+02 \mathrm{~kg}$ \\
\hline$\widehat{\text { Ni.icp.adl }}$ & $<3.07 \mathrm{E}+01 \mathrm{~kg}$ & Ni.icp.a & $7.70 \mathrm{E}+01 \mathrm{~kg}$ & $8.65 \mathrm{E}+00 \mathrm{~kg}$ & $<1.16 \mathrm{E}+02 \mathrm{~kg}$ \\
\hline$\overline{\mathrm{OH}^{-}}$ & $1.50 \mathrm{E}+05 \mathrm{~kg}$ & $\mathrm{OH}^{-}$ & $5.83 \mathrm{E}+04 \mathrm{~kg}$ & $5.66 \mathrm{E}+03 \mathrm{~kg}$ & $2.14 \mathrm{E}+05 \mathrm{~kg}$ \\
\hline Oxalate.ic & $<1.11 \mathrm{E}+03 \mathrm{~kg}$ & Oxalate.ic & $1.57 \mathrm{E}+04 \mathrm{~kg}$ & $1.51 \mathrm{E}+03 \mathrm{~kg}$ & $<1.83 \mathrm{E}+04 \mathrm{~kg}$ \\
\hline P.icp.a & $8.70 \mathrm{E}+02 \mathrm{~kg}$ & P.icp.a & $9.75 \mathrm{E}+02 \mathrm{~kg}$ & $3.63 \mathrm{E}+03 \mathrm{~kg}$ & $5.48 \mathrm{E}+03 \mathrm{~kg}$ \\
\hline P.icp.adl & $1.01 \mathrm{E}+03 \mathrm{~kg}$ & P.icp.a & $9.75 \mathrm{E}+02 \mathrm{~kg}$ & $3.63 \mathrm{E}+03 \mathrm{~kg}$ & $5.62 \mathrm{E}+03 \mathrm{~kg}$ \\
\hline $\mathrm{PO}_{4}{ }^{3-} . \mathrm{ic}$ & $1.58 \mathrm{E}+03 \mathrm{~kg}$ & $\mathrm{PO}_{4}{ }^{3-} \cdot \mathrm{ic}$ & $2.92 \mathrm{E}+03 \mathrm{~kg}$ & $1.18 \mathrm{E}+04 \mathrm{~kg}$ & $1.63 \mathrm{E}+04 \mathrm{~kg}$ \\
\hline Pb.icp.a & $<1.34 \mathrm{E}+02 \mathrm{~kg}$ & Pb.icp.a & $<9.77 \mathrm{E}+01 \mathrm{~kg}$ & $1.28 \mathrm{E}+01 \mathrm{~kg}$ & $<2.45 \mathrm{E}+02 \mathrm{~kg}$ \\
\hline Pb.icp.adl & $<1.54 \mathrm{E}+02 \mathrm{~kg}$ & Pb.icp.a & $<9.77 \mathrm{E}+01 \mathrm{~kg}$ & $1.28 \mathrm{E}+01 \mathrm{~kg}$ & $<2.65 \mathrm{E}+02 \mathrm{~kg}$ \\
\hline S.icp.a & $2.25 \mathrm{E}+03 \mathrm{~kg}$ & S.icp.a & $5.27 \mathrm{E}+03 \mathrm{~kg}$ & $3.72 \mathrm{E}+02 \mathrm{~kg}$ & $7.89 \mathrm{E}+03 \mathrm{~kg}$ \\
\hline S.icp.adl & $2.59 \mathrm{E}+03 \mathrm{~kg}$ & S.icp.a & $5.27 \mathrm{E}+03 \mathrm{~kg}$ & $3.72 \mathrm{E}+02 \mathrm{~kg}$ & $8.23 \mathrm{E}+03 \mathrm{~kg}$ \\
\hline $\mathrm{SO}_{4}^{2-}, \mathrm{ic}$ & $2.48 \mathrm{E}+03 \mathrm{~kg}$ & $\mathrm{SO}_{4}{ }^{2-} . \mathrm{ic}$ & $1.81 \mathrm{E}+04 \mathrm{~kg}$ & $1.08 \mathrm{E}+03 \mathrm{~kg}$ & $2.17 \mathrm{E}+04 \mathrm{~kg}$ \\
\hline Sb.icp.a & $<8.04 \mathrm{E}+01 \mathrm{~kg}$ & Sb.icp.a & $<5.87 \mathrm{E}+01 \mathrm{~kg}$ & $<6.02 \mathrm{E}+00 \mathrm{~kg}$ & $<1.45 \mathrm{E}+02 \mathrm{~kg}$ \\
\hline Sb.icp.adl & $<9.25 \mathrm{E}+01 \mathrm{~kg}$ & Sb.icp.a & $<5.87 \mathrm{E}+01 \mathrm{~kg}$ & $<6.02 \mathrm{E}+00 \mathrm{~kg}$ & $<1.57 \mathrm{E}+02 \mathrm{~kg}$ \\
\hline Se.icp.a & $<1.34 \mathrm{E}+02 \mathrm{~kg}$ & Se.icp.a & $<9.77 \mathrm{E}+01 \mathrm{~kg}$ & $<1.00 \mathrm{E}+01 \mathrm{~kg}$ & $<2.42 \mathrm{E}+02 \mathrm{~kg}$ \\
\hline Se.icp.adl & $<1.54 \mathrm{E}+02 \mathrm{~kg}$ & Se.icp.a & $<9.77 \mathrm{E}+01 \mathrm{~kg}$ & $<1.00 \mathrm{E}+01 \mathrm{~kg}$ & $<2.62 \mathrm{E}+02 \mathrm{~kg}$ \\
\hline
\end{tabular}


Table B3-37. Tank Inventory - Based on Composite Data. (6 sheets)

\begin{tabular}{|c|c|c|c|c|c|}
\hline Superiatant & Wuperinatant & Sludge 1 malute & Wollowing & hall shoms & Tani lingairoy \\
\hline Si.icp.a & $5.62 \mathrm{E}+02 \mathrm{~kg}$ & Si.icp.a & $4.33 \mathrm{E}+02 \mathrm{~kg}$ & $1.22 \mathrm{E}+02 \mathrm{~kg}$ & $1.12 \mathrm{E}+03 \mathrm{~kg}$ \\
\hline Si.icp.adl & $4.25 \mathrm{E}+02 \mathrm{~kg}$ & Si.icp.a & $4.33 \mathrm{E}+02 \mathrm{~kg}$ & $1.22 \mathrm{E}+02 \mathrm{~kg}$ & $9.80 \mathrm{E}+02 \mathrm{~kg}$ \\
\hline Sm.icp.a & $<1.34 \mathrm{E}+02 \mathrm{~kg}$ & Sm.icp.a & $<9.77 \mathrm{E}+01 \mathrm{~kg}$ & $<1.00 \mathrm{E}+01 \mathrm{~kg}$ & $<2.42 \mathrm{E}+02 \mathrm{~kg}$ \\
\hline Sm.icp.adl & $<1.54 \mathrm{E}+02 \mathrm{~kg}$ & Sm.icp.a & $<9.77 \mathrm{E}+01 \mathrm{~kg}$ & $<1.00 \mathrm{E}+01 \mathrm{~kg}$ & $<2.62 \mathrm{E}+02 \mathrm{~kg}$ \\
\hline Sr.icp.a & $<1.34 \mathrm{E}+01 \mathrm{~kg}$ & Sr.icp.a & $<9.77 \mathrm{E}+00 \mathrm{~kg}$ & $<1.00 \mathrm{E}+00 \mathrm{~kg}$ & $<2.42 \mathrm{E}+01 \mathrm{~kg}$ \\
\hline Sr.icp.adl & $<1.54 \mathrm{E}+01 \mathrm{~kg}$ & Sr.icp.a & $<9.77 \mathrm{E}+00 \mathrm{~kg}$ & $<1.00 \mathrm{E}+00 \mathrm{~kg}$ & $<2.62 \mathrm{E}+01 \mathrm{~kg}$ \\
\hline TIC & $5.62 \mathrm{E}+03 \mathrm{~kg}$ & TIC & $3.16 \mathrm{E}+04 \mathrm{~kg}$ & $2.12 \mathrm{E}+03 \mathrm{~kg}$ & $3.93 \mathrm{E}+04 \mathrm{~kg}$ \\
\hline TOC.coul & $1.56 \mathrm{E}+04 \mathrm{~kg}$ & TOC.pers & $1.32 \mathrm{E}+04 \mathrm{~kg}$ & $1.15 \mathrm{E}+03 \mathrm{~kg}$ & $3.00 \mathrm{E}+04 \mathrm{~kg}$ \\
\hline TOC.pers & $6.64 \mathrm{E}+03 \mathrm{~kg}$ & TOC.pers & $1.32 \mathrm{E}+04 \mathrm{~kg}$ & $1.15 \mathrm{E}+03 \mathrm{~kg}$ & $2.10 \mathrm{E}+04 \mathrm{~kg}$ \\
\hline Ti.icp.a & $<1.34 \mathrm{E}+01 \mathrm{~kg}$ & Ti.icp.a & $<9.77 \mathrm{E}+00 \mathrm{~kg}$ & $<1.00 \mathrm{E}+00 \mathrm{~kg}$ & $<2.42 \mathrm{E}+01 \mathrm{~kg}$ \\
\hline Ti.icp.adl & $<1.54 \mathrm{E}+01 \mathrm{~kg}$ & Ti.icp.a & $<9.77 \mathrm{E}+00 \mathrm{~kg}$ & $<1.00 \mathrm{E}+00 \mathrm{~kg}$ & $<2.62 \mathrm{E}+01 \mathrm{~kg}$ \\
\hline Tl.icp.a & $<2.69 \mathrm{E}+02 \mathrm{~kg}$ & Tl.icp.a & $<1.95 \mathrm{E}+02 \mathrm{~kg}$ & $<2.00 \mathrm{E}+01 \mathrm{~kg}$ & $<4.84 \mathrm{E}+02 \mathrm{~kg}$ \\
\hline Tl.icp.adl & $<3.07 \mathrm{E}+02 \mathrm{~kg}$ & Tl.icp.a & $<1.95 \mathrm{E}+02 \mathrm{~kg}$ & $<2.00 \mathrm{E}+01 \mathrm{~kg}$ & $<5.22 \mathrm{E}+02 \mathrm{~kg}$ \\
\hline AT & $<3.95 \mathrm{E}+01 \mathrm{Ci}$ & AT & $3.30 \mathrm{E}+01 \mathrm{Ci}$ & $1.49 \mathrm{E}+01 \mathrm{Ci}$ & $<8.74 \mathrm{E}+01 \mathrm{Ci}$ \\
\hline Total Beta & $1.38 \mathrm{E}+06 \mathrm{Ci}$ & Total Beta & $7.92 \mathrm{E}+05 \mathrm{Ci}$ & $7.06 \mathrm{E}+04 \mathrm{Ci}$ & $2.24 \mathrm{E}+06 \mathrm{Ci}$ \\
\hline Tritium & $<1.01 \mathrm{E}-04 \mathrm{Ci}$ & Tritium & $5.33 \mathrm{E}+00 \mathrm{Ci}$ & $<1.63 \mathrm{E}-01 \mathrm{Ci}$ & $<5.49 \mathrm{E}+00 \mathrm{Ci}$ \\
\hline U.icp.a & $<6.71 \mathrm{E}+02 \mathrm{~kg}$ & U.icp.a & $<4.90 \mathrm{E}+02 \mathrm{~kg}$ & $<5.02 \mathrm{E}+01 \mathrm{~kg}$ & $<1.21 \mathrm{E}+03 \mathrm{~kg}$ \\
\hline U.icp.adl & $<7.69 \mathrm{E}+02 \mathrm{~kg}$ & U.icp.a & $<4.90 \mathrm{E}+02 \mathrm{~kg}$ & $<5.02 \mathrm{E}+01 \mathrm{~kg}$ & $<1.31 \mathrm{E}+03 \mathrm{~kg}$ \\
\hline U.phos & $9.13 \mathrm{E}+00 \mathrm{~kg}$ & U.phos & $2.12 \mathrm{E}+02 \mathrm{~kg}$ & $2.12 \mathrm{E}+01 \mathrm{~kg}$ & $2.42 \mathrm{E}+02 \mathrm{~kg}$ \\
\hline V.icp.a & $<6.71 \mathrm{E}+01 \mathrm{~kg}$ & V.icp.a & $<4.90 \mathrm{E}+01 \mathrm{~kg}$ & $<5.02 \mathrm{E}+00 \mathrm{~kg}$ & $<1.21 \mathrm{E}+02 \mathrm{~kg}$ \\
\hline
\end{tabular}


Table B3-37. Tank Inventory - Based on Composite Data. (6 sheets)

\begin{tabular}{|c|c|c|c|c|c|}
\hline Sopromatan & Siperantail & Slunge analye & Wullow & Sall Swang? & 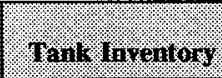 \\
\hline V.icp.adl & $<7.71 \mathrm{E}+01 \mathrm{~kg}$ & V.icp.a & $<4.90 \mathrm{E}+01 \mathrm{~kg}$ & $<5.02 \mathrm{E}+00 \mathrm{~kg}$ & $<1.31 \mathrm{E}+02 \mathrm{~kg}$ \\
\hline Water.tga & $1.82 \mathrm{E}+06 \mathrm{~kg}$ & Water.tga & $1.11 \mathrm{E}+06 \mathrm{~kg}$ & $1.10 \mathrm{E}+05 \mathrm{~kg}$ & $3.04 \mathrm{E}+06 \mathrm{~kg}$ \\
\hline Zn.icp.a & $1.95 \mathrm{E}+02 \mathrm{~kg}$ & Zn.icp.a & $1.98 \mathrm{E}+01 \mathrm{~kg}$ & $2.97 \mathrm{E}+00 \mathrm{~kg}$ & $2.18 \mathrm{E}+02 \mathrm{~kg}$ \\
\hline Zn.icp.adl & $4.51 \mathrm{E}+01 \mathrm{~kg}$ & Zn.icp.a & $1.98 \mathrm{E}+01 \mathrm{~kg}$ & $2.97 \mathrm{E}+00 \mathrm{~kg}$ & $6.79 \mathrm{E}+01 \mathrm{~kg}$ \\
\hline Zr.icp.a & $<1.34 \mathrm{E}+01 \mathrm{~kg}$ & Zr.icp.a & $1.09 \mathrm{E}+01 \mathrm{~kg}$ & $1.44 \mathrm{E}+01 \mathrm{~kg}$ & $<3.87 \mathrm{E}+01 \mathrm{~kg}$ \\
\hline Zr.icp.adl & $<1.54 \mathrm{E}+01 \mathrm{~kg}$ & Zr.icp.a & $1.09 \mathrm{E}+01 \mathrm{~kg}$ & $1.44 \mathrm{E}+01 \mathrm{~kg}$ & $<4.07 \mathrm{E}+01 \mathrm{~kg}$ \\
\hline
\end{tabular}

Note:

' $\mathrm{Li}$ and ${ }^{\mathrm{B}} \mathrm{Br}$ are the tracers for hydrostatic head fluid. 
An overall mean tank concentration was computed using (1) the segment data (per volume basis), (2) the segment data (per weight basis), (3) the composite data (per volume basis), and (4) the composite data (per weight basis). The equations for the mean tank concentration for each analyte based on the segment data are

$$
\begin{aligned}
& \hat{\mu}_{\text {tank }}=\hat{\mu}_{\text {supermatant }} \times 0.60+\hat{\mu}_{\text {salt slurry }} \times 0.40 \text { (per volume basis) } \\
& \hat{\mu}_{\text {tank }}=\hat{\mu}_{\text {supermatant }} \times 0.574+\hat{\mu}_{\text {salt slurry }} \times 0.426 \text { (per weight basis) }
\end{aligned}
$$

where $\hat{\mu}_{\text {supenatant }}$ is from the nested ANOVA results and $\hat{\mu}_{\text {salt slurry }}$ is from the nested ANOVA results with the riser term included in the model. For those analytes where $\hat{\sigma}_{\text {riser }}$ is not significantly different from zero, the mean concentrations calculated using the two statistical models (with the riser term and without the riser term) are not significantly different. For those analytes where $\hat{\sigma}_{\text {riser }}$ is significantly different from zero, the mean concentrations are calculated using the statistical model with the riser term. The mean tank concentrations based on the segment data are listed in Table B3-38 (per volume basis) and Table B3-39 (per weight basis). Because the analyte means were calculated using different data sets (.w, .wo, .ed, and so forth), Tables B3-38 and B3-39 also list the analyte name for the supernatant mean (first column) and the analyte name for the salt slurry mean (second column). The tank mean concentration is marked with a "*" if either the supernatant means or the salt slurry means were actually "less than" values. If the "less than" value was insignificant compared to the quantitative value, the analyte is marked with a $" * * "$. 
The equations for the mean tank concentration for each analyte based on the composite data are

$$
\begin{aligned}
& \hat{\mu}_{\text {tankc }}=\hat{\mu}_{\text {sup comp }} \times 0.60+\hat{\mu}_{\mathrm{SSCl}} \times 0.364+\hat{\mu}_{\mathrm{SSC} 2} \times 0.036 \text { (per volume basis) } \\
& \hat{\mu}_{\text {tankc }}=\hat{\mu}_{\text {sup comp }} \times 0.576+\hat{\mu}_{\mathrm{SSC} 1} \times 0.391+\hat{\mu}_{\mathrm{SsC} 2} \times 0.039 \text { (per weight basis) }
\end{aligned}
$$

where $\hat{\mu}_{\text {tankc }}$ is the mean tank concentration based on composite data, $\hat{\mu}_{\text {sup comp }}$ is the mean supernatant concentration based on composite data, $\hat{\mu}_{\mathrm{Ssc} 1}$ is the salt slurry mean concentration based on the composite sample from segments $14-20$, and $\hat{\mu}_{\mathrm{ssc} 2}$ is the salt slurry mean concentration based on the composite sample from segment 22 . The mean tank concentrations based on the composite data are listed in Table B3-40 (per volume basis) and Table B3-41 (per weight basis). Because the analyte means were calculated using different data sets (.w, .wo, .ed, and so forth), Tables B3-40 and B3-41 also list the analyte name for the supernatant mean (first column) and the analyte name for the salt slurry means (second column). The tank mean concentration is marked with a "*" if either the supernatant means or the salt slurry means were actually "less than" values. If the "less than" value was insignificant compared to the quantitative value, the analyte is marked with a "**".

Table B3-38. Tank Mean Concentration - Based on Segment Data (Per Volume Basis).

\begin{tabular}{|c|c|c|c|}
\hline Supeminunt Aingte & Salt Slumy analne & $1+1.1$. & Trits \\
\hline Water.tga & Water.tga & $7.05 \mathrm{E}-01$ & $\mathrm{~g} / \mathrm{mL}$ \\
\hline Water.tga.ed & Water.tga & $7.08 \mathrm{E}-01$ & $\mathrm{~g} / \mathrm{mL}$ \\
\hline Acetate.ic.w & Acetate.ic.w & $7.33 \mathrm{E}+02$ & $\mu \mathrm{g} / \mathrm{mL}$ \\
\hline Acetate.ic.wo & Acetate.ic.wo & $7.91 \mathrm{E}+02$ & $\mu \mathrm{g} / \mathrm{mL}$ \\
\hline Ag.icp.adl & Ag.icp.a & $2.30 \mathrm{E}+01$ & $\mu \mathrm{g} / \mathrm{mL}$ \\
\hline Ag.icp.adl & Ag.icp.f & $1.50 \mathrm{E}+02^{*}$ & $\mu \mathrm{g} / \mathrm{mL}$ \\
\hline Al.icp.adl & Al.icp.a & $3.89 \mathrm{E}+04$ & $\mu \mathrm{g} / \mathrm{mL}$ \\
\hline Al.icp.adl & Al.icp.f & $3.96 \mathrm{E}+04$ & $\mu \mathrm{g} / \mathrm{mL}$ \\
\hline As.icp.adl & As.icp.a & $6.74 \mathrm{E}+01 *$ & $\mu \mathrm{g} / \mathrm{mL}$ \\
\hline As.icp.adl & As.icp.f & $1.43 \mathrm{E}+03^{*}$ & $\mu \mathrm{g} / \mathrm{mL}$ \\
\hline B.icp.adl & B.icp.a & $1.01 \mathrm{E}+02$ & $\mu \mathrm{g} / \mathrm{mL}$ \\
\hline B.icp.adl & B.icp.f & $7.40 \mathrm{E}+02 *$ & $\mu \mathrm{g} / \mathrm{mL}$ \\
\hline Ba.icp.adl & Ba.icp.a & $3.37 \mathrm{E}+01 *$ & $\mu \mathrm{g} / \mathrm{mL}$ \\
\hline Ba.icp.adl & Ba.icp.f & $7.13 \mathrm{E}+02^{*}$ & $\mu \mathrm{g} / \mathrm{mL}$ \\
\hline Be.icp.adl & Be.icp.a & $3.70 \mathrm{E}+00^{*}$ & $\mu \mathrm{g} / \mathrm{mL}$ \\
\hline
\end{tabular}
(5 sheets) 
Table B3-38. Tank Mean Concentration - Based on Segment Data (Per Volume Basis). (5 sheets)

\begin{tabular}{|c|c|c|c|}
\hline 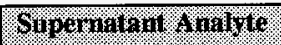 & 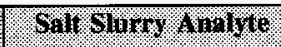 & H.1. & Uim \\
\hline Be.icp.adl & Be.icp.f & $7.13 \mathrm{E}+01^{*}$ & $\mu \mathrm{g} / \mathrm{mL}$ \\
\hline Bi.icp.adl & Bi.icp.a & $8.20 \mathrm{E}+01^{*}$ & $\mu \mathrm{g} / \mathrm{mL}$ \\
\hline Bi.icp.adl & Bi.icp.f & $1.43 \mathrm{E}+03^{*}$ & $\mu \mathrm{g} / \mathrm{mL}$ \\
\hline${ }^{1}$ Br-.ic.w & $\mathrm{Br}^{-}$.ic & $1.27 \mathrm{E}+03^{*}$ & $\mu \mathrm{g} / \mathrm{mL}$ \\
\hline${ }^{1} \mathrm{Br}$.ic.wo & $\mathrm{Br}$.ic & $1.28 \mathrm{E}+03^{*}$ & $\mu \mathrm{g} / \mathrm{mL}$ \\
\hline Ca.icp.adl & Ca.icp.a & $4.40 \mathrm{E}+02 * *$ & $\mu \mathrm{g} / \mathrm{mL}$ \\
\hline Ca.icp.adl & Ca.icp.f & $1.43 \mathrm{E}+03^{*}$ & $\mu \mathrm{g} / \mathrm{mL}$ \\
\hline Cd.icp.adl & Cd.icp.a & $9.17 \mathrm{E}+00^{*}$ & $\mu \mathrm{g} / \mathrm{mL}$ \\
\hline Cd.icp.adl & Cd.icp.f & $7.13 \mathrm{E}+01^{*}$ & $\mu \mathrm{g} / \mathrm{mL}$ \\
\hline Ce.icp.adl & Ce.icp.a & $6.74 \mathrm{E}+01^{*}$ & $\mu \mathrm{g} / \mathrm{mL}$ \\
\hline Ce.icp.adl & Ce.icp.f & $1.43 \mathrm{E}+03^{*}$ & $\mu \mathrm{g} / \mathrm{mL}$ \\
\hline $\mathrm{Cl}^{-}$.ic & $\mathrm{Cl}^{-} . \mathrm{ic}$ & $9.40 \mathrm{E}+03$ & $\mu \mathrm{g} / \mathrm{mL}$ \\
\hline Cl.ic.ed & $\mathrm{Cl}^{-}$ic & $9.21 \mathrm{E}+03$ & $\mu \mathrm{g} / \mathrm{mL}$ \\
\hline Co.icp.adl & Co.icp.a & $1.35 \mathrm{E}+01^{*}$ & $\mu \mathrm{g} / \mathrm{mL}$ \\
\hline Co.icp.adl & Co.icp.f & $2.86 \mathrm{E}+02^{*}$ & $\mu \mathrm{g} / \mathrm{mL}$ \\
\hline Cr.icp.adl & Cr.icp.a & $1.61 \mathrm{E}+03$ & $\mu \mathrm{g} / \mathrm{mL}$ \\
\hline Cr.icp.adl & Cr.icp.f & $1.70 \mathrm{E}+03$ & $\mu \mathrm{g} / \mathrm{mL}$ \\
\hline Cu.icp.adl & Cu.icp.a.w & $1.06 \mathrm{E}+01$ & $\mu \mathrm{g} / \mathrm{mL}$ \\
\hline Cu.icp.adl & Cu.icp.a.wo & $1.06 \mathrm{E}+01$ & $\mu \mathrm{g} / \mathrm{mL}$ \\
\hline Cu.icp.adl & Cu.icp.f & $1.45 \mathrm{E}+02^{*}$ & $\mu \mathrm{g} / \mathrm{mL}$ \\
\hline F-.ic.w & $\mathrm{F}^{-}$.ic & $1.36 \mathrm{E}+03$ & $\mu \mathrm{g} / \mathrm{mL}$ \\
\hline F-ic.w.ed & $\mathrm{F}^{-}$.ic & $1.35 \mathrm{E}+03$ & $\mu \mathrm{g} / \mathrm{mL}$ \\
\hline F.ic.wo & $\mathrm{F}$. ic & $1.46 \mathrm{E}+03$ & $\mu \mathrm{g} / \mathrm{mL}$ \\
\hline$F^{*}$.ic.wo.ed & F.ic & $1.44 \mathrm{E}+03$ & $\mu \mathrm{g} / \mathrm{mL}$ \\
\hline Fe.icp.adl & Fe.icp.a & $9.37 \mathrm{E}+01^{*}$ & $\mu \mathrm{g} / \mathrm{mL}$ \\
\hline Fe.icp.adl & Fe.icp.f & $7.13 \mathrm{E}+02^{*}$ & $\mu \mathrm{g} / \mathrm{mL}$ \\
\hline Formate.ic & Formate.ic & $1.60 \mathrm{E}+03$ & $\mu \mathrm{g} / \mathrm{mL}$ \\
\hline K.icp.adl & K.icp.a & $6.11 \mathrm{E}+03$ & $\mu \mathrm{g} / \mathrm{mL}$ \\
\hline La.icp.adl & La.icp.a & $3.37 \mathrm{E}+01^{*}$ & $\mu \mathrm{g} / \mathrm{mL}$ \\
\hline La.icp.adl & La.icp.f & $7.13 \mathrm{E}+02 *$ & $\mu \mathrm{g} / \mathrm{mL}$ \\
\hline
\end{tabular}


Table B3-38. Tank Mean Concentration - Based on Segment Data (Per Volume Basis). (5 sheets)

\begin{tabular}{|c|c|c|c|}
\hline 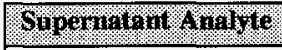 & 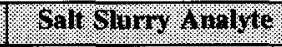 & 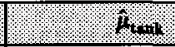 & Writus \\
\hline${ }^{1}$ Li.icp.adl & Li.icp.a.w & $2.89 \mathrm{E}+01$ & $\mu \mathrm{g} / \mathrm{mL}$ \\
\hline${ }^{1}$ Li.icp.adl & Li.icp.a.woo & $2.94 \mathrm{E}+01$ & $\mu \mathrm{g} / \mathrm{mL}$ \\
\hline${ }^{1}$ Li.icp.adl & Li.icp.f & $1.55 \mathrm{E}+02^{*}$ & $\mu \mathrm{g} / \mathrm{mL}$ \\
\hline Mg.icp.adl & Mg.icp.a & $7.50 \mathrm{E}+01^{*}$ & $\mu \mathrm{g} / \mathrm{mL}$ \\
\hline Mg.icp.adl & Mg.icp.f & $1.43 E+03^{*}$ & $\mu \mathrm{g} / \mathrm{mL}$ \\
\hline Mn.icp.adl & Mn.icp.a.w & $1.69 \mathrm{E}+01^{*}$ & $\mu \mathrm{g} / \mathrm{mL}$ \\
\hline Mn.icp.adl & Mn.icp.a.wo & $1.73 \mathrm{E}+01^{*}$ & $\mu \mathrm{g} / \mathrm{mL}$ \\
\hline Mn.icp.adl & Mn.icp.f & $1.43 \mathrm{E}+02 *$ & $\mu \mathrm{g} / \mathrm{mL}$ \\
\hline Mo.icp.adl & Mo.icp.a & $9.05 \mathrm{E}+01$ & $\mu \mathrm{g} / \mathrm{mL}$ \\
\hline Mo.icp.adl & Mo.icp.f & $7.53 \mathrm{E}+02^{*}$ & $\mu \mathrm{g} / \mathrm{mL}$ \\
\hline $\mathrm{NO}_{2}^{-}$.ic & $\mathrm{NO}_{2}^{-} \cdot \mathrm{ic}$ & $1.16 \mathrm{E}+05$ & $\mu \mathrm{g} / \mathrm{mL}$ \\
\hline $\mathrm{NO}_{2}$.ic.ed & $\mathrm{NO}_{2}^{-}, \mathrm{ic}$ & $1.14 \mathrm{E}+05$ & $\mu \mathrm{g} / \mathrm{mL}$ \\
\hline $\mathrm{NO}_{3}^{-} \cdot \mathrm{ic}$ & $\mathrm{NO}_{3}{ }^{-} . \mathrm{ic}$ & $1.70 \mathrm{E}+05$ & $\mu \mathrm{g} / \mathrm{mL}$ \\
\hline $\mathrm{NO}_{3}^{-}$.ic.ed & $\mathrm{NO}_{3}^{-}$.ic & $1.67 \mathrm{E}+05$ & $\mu \mathrm{g} / \mathrm{mL}$ \\
\hline Na.icp.adl & Na.icp.a & $2.64 E+05$ & $\mu \mathrm{g} / \mathrm{mL}$ \\
\hline Na.icp.adl & Na.icp.f & $2.92 \mathrm{E}+05$ & $\mu \mathrm{g} / \mathrm{mL}$ \\
\hline Nd.icp.adl & Nd.icp.a & $6.74 \mathrm{E}+01^{*}$ & $\mu \mathrm{g} / \mathrm{mL}$ \\
\hline Nd.icp.adl & Nd.icp.f & $1.43 \mathrm{E}+03^{*}$ & $\mu \mathrm{g} / \mathrm{mL}$ \\
\hline Ni.icp.adl & Ni.icp.a & $3.29 \mathrm{E}+01^{*}$ & $\mu \mathrm{g} / \mathrm{mL}$ \\
\hline Ni.icp.adl & Ni.icp.f.w & $8.69 \mathrm{E}+02 * *$ & $\mu \mathrm{g} / \mathrm{mL}$ \\
\hline Ni.icp.adl & Ni.icp.f.wo & $1.11 \mathrm{E}+03^{* *}$ & $\mu \mathrm{g} / \mathrm{mL}$ \\
\hline Oxalate.ic & Oxalate.ic & $1.40 \mathrm{E}+04$ & $\mu \mathrm{g} / \mathrm{mL}$ \\
\hline P.icp.adl & P.icp.a & $1.81 \mathrm{E}+03$ & $\mu \mathrm{g} / \mathrm{mL}$ \\
\hline P.icp.adl.ed & P.icp.a & $1.80 \mathrm{E}+03$ & $\mu \mathrm{g} / \mathrm{mL}$ \\
\hline P.icp.adl & P.jcp.f & $2.31 \mathrm{E}+04$ & $\mu \mathrm{g} / \mathrm{mL}$ \\
\hline P.icp.adl.ed & P.icp.f & $2.31 \mathrm{E}+04$ & $\mu \mathrm{g} / \mathrm{mL}$ \\
\hline $\mathrm{PO}_{4}^{3-}$.ic.w & $\mathrm{PO}_{4}{ }^{3-}$.ic. W & $5.74 \mathrm{E}+03$ & $\mu \mathrm{g} / \mathrm{mL}$ \\
\hline $\mathrm{PO}_{4}{ }^{3-}$.ic. $\mathrm{W}$ & $\mathrm{PO}_{4}{ }^{3}$.ic.wo & $5.75 E+03$ & $\mu \mathrm{g} / \mathrm{mL}$ \\
\hline $\mathrm{PO}_{4}{ }^{3-}$.ic.w.ed & $\mathrm{PO}_{4}{ }^{3-}$.ic.w & $5.56 \mathrm{E}+03$ & $\mu \mathrm{g} / \mathrm{mL}$ \\
\hline $\mathrm{PO}_{4}{ }^{3-}$.ic.w.ed & $\mathrm{PO}_{4}{ }^{3-}$.ic.wo & $5.56 \mathrm{E}+03$ & $\mu \mathrm{g} / \mathrm{mL}$ \\
\hline
\end{tabular}


Table B3-38. Tank Mean Concentration - Based on Segment Data (Per Volume Basis). (5 sheets)

\begin{tabular}{|c|c|c|c|}
\hline Superiatant d nalyte. & 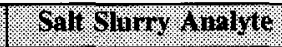 & $i_{1+1}$ & $1.6 \%$ WHito \\
\hline $\mathrm{PO}_{4}{ }^{3-}$.ic.wo & $\mathrm{PO}_{4}{ }^{3-}$.ic.w & $6.04 \mathrm{E}+03$ & $\mu \mathrm{g} / \mathrm{mL}$ \\
\hline $\mathrm{PO}_{4}^{3 \cdot}$.ic.wo & $\mathrm{PO}_{4}{ }^{3-}$.ic.wo & $6.05 \mathrm{E}+03$ & $\mu \mathrm{g} / \mathrm{mL}$ \\
\hline $\mathrm{PO}_{4}^{3-}$.ic.wo.ed & $\mathrm{PO}_{4}{ }^{3-}$.ic.w & $5.61 \mathrm{E}+03$ & $\mu \mathrm{g} / \mathrm{mL}$ \\
\hline $\mathrm{PO}_{4}{ }^{3-}$.ic.wo.ed & $\mathrm{PO}_{4}{ }^{3-}$.ic.wo & $5.61 \mathrm{E}+03$ & $\mu \mathrm{g} / \mathrm{mL}$ \\
\hline Pb.icp.adl.w & Pb.icp.a.w & $6.22 \mathrm{E}+01$ & $\mu \mathrm{g} / \mathrm{mL}$ \\
\hline Pb.icp.adl.w & Pb.icp.a.wo & $6.41 \mathrm{E}+01$ & $\mu \mathrm{g} / \mathrm{mL}$ \\
\hline Pb.icp.adl.w & Pb.icp.f & $1.42 \mathrm{E}+03^{*}$ & $\mu \mathrm{g} / \mathrm{mL}$ \\
\hline Pb.icp.adl.wo & Pb.icp.a.w & $5.97 \mathrm{E}+01$ & $\mu \mathrm{g} / \mathrm{mL}$ \\
\hline Pb.icp.adl.wo & Pb.icp.a.wo & $6.16 \mathrm{E}+01$ & $\mu \mathrm{g} / \mathrm{mL}$ \\
\hline Pb.icp.adl.wo & Pb.icp.f & $1.42 \mathrm{E}+03^{*}$ & $\mu \mathrm{g} / \mathrm{mL}$ \\
\hline S.icp.adl & S.icp.a & $2.15 \mathrm{E}+03$ & $\mu \mathrm{g} / \mathrm{mL}$ \\
\hline S.icp.adl & S.icp.f.w & $2.32 \mathrm{E}+03$ & $\mu \mathrm{g} / \mathrm{mL}$ \\
\hline S.icp.adl & S.icp.f.wo & $2.34 \mathrm{E}+03$ & $\mu \mathrm{g} / \mathrm{mL}$ \\
\hline $\mathrm{SO}_{4}^{2-} . \mathrm{ic} . \mathrm{W}$ & $\mathrm{SO}_{4}{ }^{2-} \cdot \mathrm{ic}$ & $4.68 \mathrm{E}+03$ & $\mu \mathrm{g} / \mathrm{mL}$ \\
\hline $\mathrm{SO}_{4}^{2-}$.ic.w.ed & $\mathrm{SO}_{4}{ }^{2-} \cdot \mathrm{ic}$ & $4.63 \mathrm{E}+03$ & $\mu \mathrm{g} / \mathrm{mL}$ \\
\hline $\mathrm{SO}_{4}^{2-}$.ic.wo & $\mathrm{SO}_{4}{ }^{2 \cdot} \cdot \mathrm{ic}$ & $4.67 \mathrm{E}+03$ & $\mu \mathrm{g} / \mathrm{mL}$ \\
\hline $\mathrm{SO}_{4}{ }^{2-}$.ic.wo.ed & $\mathrm{SO}_{4}{ }^{2-} . \mathrm{ic}$ & $4.62 E+03$ & $\mu \mathrm{g} / \mathrm{mL}$ \\
\hline Sb.icp.adl & Sb.icp.a & $4.04 \mathrm{E}+01^{*}$ & $\mu \mathrm{g} / \mathrm{mL}$ \\
\hline Sb.icp.adl & Sb.icp.f & $8.55 \mathrm{E}+02^{*}$ & $\mu \mathrm{g} / \mathrm{mL}$ \\
\hline Se.icp.adl & Se.icp.a & $6.74 \mathrm{E}+01^{*}$ & $\mu \mathrm{g} / \mathrm{mL}$ \\
\hline Se.icp.adl & Se.icp.f & $1.43 \mathrm{E}+03^{*}$ & $\mu \mathrm{g} / \mathrm{mL}$ \\
\hline Si.icp.adl & Si.icp.a.w & $2.15 \mathrm{E}+02$ & $\mu \mathrm{g} / \mathrm{mL}$ \\
\hline Si.icp.adl & Si.icp.a.wo & $2.18 \mathrm{E}+02$ & $\mu \mathrm{g} / \mathrm{mL}$ \\
\hline Si.icp.adl & Si.icp.f & $1.66 \mathrm{E}+03$ & $\mu \mathrm{g} / \mathrm{mL}$ \\
\hline Sm.icp.adl & Sm.icp.a & $6.74 E+01^{*}$ & $\mu \mathrm{g} / \mathrm{mL}$ \\
\hline Sm.icp.adl & Sm.icp.f & $1.43 \mathrm{E}+03^{*}$ & $\mu \mathrm{g} / \mathrm{mL}$ \\
\hline Sr.icp.adl & Sr.icp.a & $9.83 \mathrm{E}+00^{*}$ & $\mu \mathrm{g} / \mathrm{mL}$ \\
\hline Sr.icp.adl & Sr.icp.f & $1.43 \mathrm{E}+02^{*}$ & $\mu \mathrm{g} / \mathrm{mL}$ \\
\hline Ti.icp.adl & Ti.icp.a & $6.74 \mathrm{E}+00^{*}$ & $\mu \mathrm{g} / \mathrm{mL}$ \\
\hline Ti.icp.adl & Ti.icp.f & $1.43 \mathrm{E}+02 *$ & $\mu \mathrm{g} / \mathrm{mL}$ \\
\hline
\end{tabular}


Table B3-38. Tank Mean Concentration - Based on Segment Data (Per Volume Basis). (5 sheets)

\begin{tabular}{|c|c|c|c|}
\hline 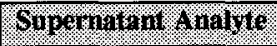 & 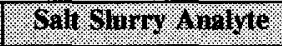 & $4 .+1$ & Whits \\
\hline Tl.icp.adl & Tl.icp.a & $1.35 \mathrm{E}+02^{*}$ & $\mu \mathrm{g} / \mathrm{mL}$ \\
\hline Tl.icp.adl & Tl.icp.f & $2.86 \mathrm{E}+03^{*}$ & $\mu \mathrm{g} / \mathrm{mL}$ \\
\hline Total Alpha & Total Alpha.w & $1.02 \mathrm{E}-01 *$ & $\mu \mathrm{Ci} / \mathrm{mL}$ \\
\hline Total Alpha & Total Alpha.wo & $1.02 \mathrm{E}-01 *$ & $\mu \mathrm{Ci} / \mathrm{mL}$ \\
\hline U.icp.adl & U.icp.a & $3 . \overline{36 \mathrm{E}+02^{*}}$ & $\mu \mathrm{g} / \mathrm{mL}$ \\
\hline U.icp.adl & U.icp.f & $7.13 \mathrm{E}+03^{*}$ & $\mu \mathrm{g} / \mathrm{mL}$ \\
\hline V.icp.adl & V.icp.a & 3.37E+01* & $\mu \mathrm{g} / \mathrm{mL}$ \\
\hline V.icp.adl & V.icp.f & $7.13 \mathrm{E}+02^{*}$ & $\mu \mathrm{g} / \mathrm{mL}$ \\
\hline Zn.icp.adl & Zn.icp.a & $4.61 \mathrm{E}+01$ & $\mu \mathrm{g} / \mathrm{mL}$ \\
\hline Zn.icp.adl & Zn.icp.f & $5.24 \mathrm{E}+02$ & $\mu \mathrm{g} / \mathrm{mL}$ \\
\hline Zr.icp.adl & Zr.icp.a.w & $1.09 \mathrm{E}+01$ & $\mu \mathrm{g} / \mathrm{mL}$ \\
\hline Zr.icp.adl & Zr.icp.a.wo & $1.12 \mathrm{E}+01$ & $\mu \mathrm{g} / \mathrm{mL}$ \\
\hline
\end{tabular}

Note:

${ }^{\mathrm{L}} \mathrm{Li}$ and ${ }^{1} \mathrm{Br}$ used for tracer in hydrostatic head fluid. 
Table B3-39. Tank Mean Concentration - Based on Segment Data (Per Weight Basis).

(4 sheets)

\begin{tabular}{|c|c|c|c|}
\hline 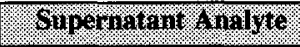 & Soll olury & . & Biniu \\
\hline Water.tga & Water.tga & $4.76 \mathrm{E}-01$ & $g / g$ \\
\hline Water.tga.ed & Water.tga & 4.78E-01 & $g / g$ \\
\hline Acetate.ic.w & Acetate.ic.w & $4.95 \mathrm{E}+02$ & $\mu \mathrm{g} / \mathrm{g}$ \\
\hline Acetate.ic.wo & Acetate.ic.wo & $5.34 \mathrm{E}+02$ & $\mu \mathrm{g} / \mathrm{g}$ \\
\hline Ag.icp.adl & Ag.icp.a & $1.55 \mathrm{E}+01$ & $\mu \mathrm{g} / \mathrm{g}$ \\
\hline Ag.icp.adl & Ag.icp.f & $9.75 \mathrm{E}+01 *$ & $\mu \mathrm{g} / \mathrm{g}$ \\
\hline Al.icp.adl & Al.icp.a & $2.63 \mathrm{E}+04$ & $\mu \mathrm{g} / \mathrm{g}$ \\
\hline Al.icp.adl & Al.icp.f & $2.67 \mathrm{E}+04$ & $\mu \mathrm{g} / \mathrm{g}$ \\
\hline As.icp.adl & As.icp.a & $4.51 \mathrm{E}+01^{*}$ & $\mu \mathrm{g} / \mathrm{g}$ \\
\hline As.icp.adl & As.icp.f & $9.27 \mathrm{E}+02^{*}$ & $\mu \mathrm{g} / \mathrm{g}$ \\
\hline B.icp.adl & B.icp.a & $6.83 \mathrm{E}+01$ & $\mu \mathrm{g} / \mathrm{g}$ \\
\hline B.icp.adl & B.icp.f & $4.82 \mathrm{E}+02 *$ & $\mu \mathrm{g} / \mathrm{g}$ \\
\hline Ba.icp.adl & Ba.icp.a & $2.26 \mathrm{E}+01^{*}$ & $\mu \mathrm{g} / \mathrm{g}$ \\
\hline Ba.icp.adl & Ba.icp.f & $4.64 \mathrm{E}+02^{*}$ & $\mu \mathrm{g} / \mathrm{g}$ \\
\hline Be.icp.adl & Be.icp.a & $2.54 \mathrm{E}+00^{*}$ & $\mu \mathrm{g} / \mathrm{g}$ \\
\hline Be.icp.adl & Be.icp.f & $4.64 \mathrm{E}+01^{*}$ & $\mu \mathrm{g} / \mathrm{g}$ \\
\hline Bi.icp.adl & Bi.icp.a & $5.49 \mathrm{E}+01^{*}$ & $\mu \mathrm{g} / \mathrm{g}$ \\
\hline Bi.icp.adl & Bi.icp.f & $9.27 \mathrm{E}+02^{*}$ & $\mu \mathrm{g} / \mathrm{g}$ \\
\hline${ }^{1} \mathrm{Br}$.ic.w & $\mathrm{Br}$. ic & $8.46 \mathrm{E}+02^{*}$ & $\mu \mathrm{g} / \mathrm{g}$ \\
\hline${ }^{1} \mathrm{Br}$.ic.wo & $\mathrm{Br}^{-}$.ic & $8.51 \mathrm{E}+02^{*}$ & $\mu \mathrm{g} / \mathrm{g}$ \\
\hline Ca.icp.adl & Ca.icp.a & $2.98 \mathrm{E}+02 * *$ & $\mu \mathrm{g} / \mathrm{g}$ \\
\hline Ca.icp.adl & Ca.icp.f & $9.27 \mathrm{E}+02^{*}$ & $\mu \mathrm{g} / \mathrm{g}$ \\
\hline Cd.icp.adl & Cd.icp.a & $6.17 \mathrm{E}+00^{*}$ & $\mu \mathrm{g} / \mathrm{g}$ \\
\hline Cd.icp.adl & Cd.icp.f & $4.64 \mathrm{E}+01^{*}$ & $\mu \mathrm{g} / \mathrm{g}$ \\
\hline Ce.icp.adl & Ce.icp.a & $4.51 \mathrm{E}+01 *$ & $\mu \mathrm{g} / \mathrm{g}$ \\
\hline Ce.icp.adl & Ce.icp.f & $9.27 \mathrm{E}+02^{*}$ & $\mu \mathrm{g} / \mathrm{g}$ \\
\hline $\mathrm{Cl}$.ic & $\mathrm{Cl}^{-} . \mathrm{ic}$ & $6.35 \mathrm{E}+03$ & $\mu \mathrm{g} / \mathrm{g}$ \\
\hline $\mathrm{Cl}^{-}$. ic.ed & $\mathrm{Cl}^{-}$.ic & $6.22 E+03$ & $\mu \mathrm{g} / \mathrm{g}$ \\
\hline Co.icp.adl & Co.icp.a & $9.02 \mathbf{E}+00^{*}$ & $\mu \mathrm{g} / \mathrm{g}$ \\
\hline Co.icp.adl & Co.icp.f & $1.86 \mathrm{E}+02^{*}$ & $\mu \mathrm{g} / \mathrm{g}$ \\
\hline
\end{tabular}


Table B3-39. Tank Mean Concentration - Based on Segment Data (Per Weight Basis). (4 sheets)

\begin{tabular}{|c|c|c|c|}
\hline Superinatant inngle & 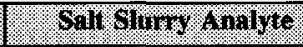 & $i_{2+4}$ & Unitios \\
\hline Cr.icp.adl & Cr.icp.a & $1.09 \mathrm{E}+03$ & $\mu \mathrm{g} / \mathrm{g}$ \\
\hline Cr.icp.adl & Cr.icp.f & $1.15 \mathrm{E}+03$ & $\mu \mathrm{g} / \mathrm{g}$ \\
\hline Cu.icp.adl & Cu.icp.a.w & $7.12 \mathrm{E}+00$ & $\mu \mathrm{g} / \mathrm{g}$ \\
\hline Cu.icp.adl & Cu.icp.a.wo & $7.15 \mathrm{E}+00$ & $\mu \mathrm{g} / \mathrm{g}$ \\
\hline Cu.icp.adl & Cu.icp.f & $1.52 \mathrm{E}+02 *$ & $\mu \mathrm{g} / \mathrm{g}$ \\
\hline F-ic.w & F.ic & $9.12 \mathrm{E}+02$ & $\mu \mathrm{g} / \mathrm{g}$ \\
\hline$F^{-}$.ic.w.ed & F.ic & $9.05 \mathrm{E}+02$ & $\mu \mathrm{g} / \mathrm{g}$ \\
\hline F-ic.wo & F-.ic & $9.83 \mathrm{E}+02$ & $\mu \mathrm{g} / \mathrm{g}$ \\
\hline F-ic.wo.ed & F-ic & $9.69 \mathrm{E}+02$ & $\mu \mathrm{g} / \mathrm{g}$ \\
\hline Fe.icp.adl & Fe.icp.a & $6.29 \mathrm{E}+01 *$ & $\mu \mathrm{g} / \mathrm{g}$ \\
\hline Fe.icp.adl & Fe.icp.f & $4.64 \mathrm{E}+02 *$ & $\mu \mathrm{g} / \mathrm{g}$ \\
\hline Formate.ic & Formate.ic & $1.08 \mathrm{E}+03$ & $\mu \mathrm{g} / \mathrm{g}$ \\
\hline K.icp.adl & K.icp.a & $4.12 \mathrm{E}+03$ & $\mu \mathrm{g} / \mathrm{g}$ \\
\hline La.icp.adl & La.icp.a & $2.26 \mathrm{E}+01^{*}$ & $\mu \mathrm{g} / \mathrm{g}$ \\
\hline La.icp.adl & La.icp.f & $4.64 \mathrm{E}+02 *$ & $\mu \mathrm{g} / \mathrm{g}$ \\
\hline${ }^{1}$ Li.icp.adl & Li.icp.a.w & $1.94 \mathrm{E}+01$ & $\mu \mathrm{g} / \mathrm{g}$ \\
\hline${ }^{1}$ Li.icp.adl & Li.icp.a.wo & $1.98 \mathrm{E}+01$ & $\mu \mathrm{g} / \mathrm{g}$ \\
\hline${ }^{1}$ Li.icp.adl & Li.icp.f & $1.01 \mathrm{E}+02^{*}$ & $\mu \mathrm{g} / \mathrm{g}$ \\
\hline Mg.icp.adl & Mg.icp.a & $5.02 \mathrm{E}+01 *$ & $\mu \mathrm{g} / \mathrm{g}$ \\
\hline Mg.icp.adl & Mg.icp.f & $9.27 \mathrm{E}+02 *$ & $\mu \mathrm{g} / \mathrm{g}$ \\
\hline Mn.icp.adl & Mn.icp.a.w & $1.13 \mathrm{E}+01^{*}$ & $\mu \mathrm{g} / \mathrm{g}$ \\
\hline Mn.icp.adl & Mn.icp.a.wo & $1.16 \mathrm{E}+01^{*}$ & $\mu \mathrm{g} / \mathrm{g}$ \\
\hline Mn.icp.adl & Mn.icp.f & $9.27 \mathrm{E}+01^{*}$ & $\mu \mathrm{g} / \mathrm{g}$ \\
\hline Mo.icp.adl & Mo.icp.a & $6.10 \mathrm{E}+01$ & $\mu \mathrm{g} / \mathrm{g}$ \\
\hline Mo.icp.adl & Mo.icp.f & $4.91 \mathrm{E}+02 *$ & $\mu \mathrm{g} / \mathrm{g}$ \\
\hline $\mathrm{NO}_{2}^{-}$.ic & $\mathrm{NO}_{2}^{-}$.ic & $7.84 \mathrm{E}+04$ & $\mu \mathrm{g} / \mathrm{g}$ \\
\hline $\mathrm{NO}_{2}$.ic.ed & $\mathrm{NO}_{2}^{-} \cdot \mathrm{ic}$ & $7.76 \mathrm{E}+04$ & $\mu \mathrm{g} / \mathrm{g}$ \\
\hline $\mathrm{NO}_{3}{ }^{\circ}$.ic & $\mathrm{NO}_{3}$. ic & $1.14 \mathrm{E}+05$ & $\mu \mathrm{g} / \mathrm{g}$ \\
\hline $\mathrm{NO}_{3}$.ic.ed & $\mathrm{NO}_{3}$. ic & $1.12 \mathrm{E}+05$ & $\mu \mathrm{g} / \mathrm{g}$ \\
\hline Na.icp.adl & Na.icp.a & $1.78 \mathrm{E}+05$ & $\mu \mathrm{g} / \mathrm{g}$ \\
\hline
\end{tabular}


Table B3-39. Tank Mean Concentration - Based on Segment Data (Per Weight Basis). (4 sheets)

\begin{tabular}{|c|c|c|c|}
\hline SHpernatant 1 nulve & Gart oluy $/$ nalyte & 2.1.1. & Winis \\
\hline Na.icp.adl & Na.icp.f & $1.97 E+05$ & $\mu \mathrm{g} / \mathrm{g}$ \\
\hline Nd.icp.adl & Nd.icp.a & $4.51 \mathrm{E}+01 *$ & $\mu \mathrm{g} / \mathrm{g}$ \\
\hline Nd.icp.adl & Nd.icp.f & $9.27 \mathrm{E}+02^{*}$ & $\mu \mathrm{g} / \mathrm{g}$ \\
\hline Ni.icp.adl & Ni.icp.a & $2.22 \mathrm{E}+01^{*}$ & $\mu \mathrm{g} / \mathrm{g}$ \\
\hline Ni.icp.adl & Ni.icp.f.w & $5.84 \mathrm{E}+02 * *$ & $\mu \mathrm{g} / \mathrm{g}$ \\
\hline Ni.icp.adl & Ni.icp.f.wo & $7.47 \mathrm{E}+02 * *$ & $\mu \mathrm{g} / \mathrm{g}$ \\
\hline Oxalate.ic & Oxalate.ic & $9.48 \mathrm{E}+03$ & $\mu \mathrm{g} / \mathrm{g}$ \\
\hline P.icp.adl & P.icp.a & $1.21 \mathrm{E}+03$ & $\mu \mathrm{g} / \mathrm{g}$ \\
\hline P.icp.adl.ed & P.icp.a & $1.20 \mathrm{E}+03$ & $\mu \mathrm{g} / \mathrm{g}$ \\
\hline P.icp.adl & P.icp.f & $1.54 \mathrm{E}+04$ & $\mu \mathrm{g} / \mathrm{g}$ \\
\hline P.icp.adl.ed & P.icp.f & $1.54 \mathrm{E}+04$ & $\mu \mathrm{g} / \mathrm{g}$ \\
\hline $\mathrm{PO}_{4}^{3+}$.ic.w & $\mathrm{PO}_{4}{ }^{3-}$. ic. $\mathrm{W}$ & $3.84 \mathrm{E}+03$ & $\mu \mathrm{g} / \mathrm{g}$ \\
\hline $\mathrm{PO}_{4}^{3-}$.ic.w & $\mathrm{PO}_{4}{ }^{3-}$.ic.wo & $3.84 E+03$ & $\mu \mathrm{g} / \mathrm{g}$ \\
\hline $\mathrm{PO}_{4}{ }^{3-}$.ic.w.ed & $\mathrm{PO}_{4}^{3-}$.ic. $\mathrm{w}$ & $3.72 \mathrm{E}+03$ & $\mu \mathrm{g} / \mathrm{g}$ \\
\hline $\mathrm{PO}_{4}{ }^{3-}$.ic.w.ed & $\mathrm{PO}_{4}{ }^{3-}$.ic.wo & $3.72 \mathrm{E}+03$ & $\mu \mathrm{g} / \mathrm{g}$ \\
\hline $\mathrm{PO}_{4}{ }^{3-}$.ic.wo & $\mathrm{PO}_{4}^{3-}$.ic. W & $4.04 \mathrm{E}+03$ & $\mu \mathrm{g} / \mathrm{g}$ \\
\hline $\mathrm{PO}_{4}^{3-}$.ic.wo & $\mathrm{PO}_{4}{ }^{3-}$.ic.wo & $4.04 \mathrm{E}+03$ & $\mu \mathrm{g} / \mathrm{g}$ \\
\hline $\mathrm{PO}_{4}{ }^{3-}$.ic.wo.ed & $\mathrm{PO}_{4}{ }^{3-}$.ic.w & $3.75 \mathrm{E}+03$ & $\mu \mathrm{g} / \mathrm{g}$ \\
\hline $\mathrm{PO}_{4}{ }^{3-}$.ic.wo.ed & $\mathrm{PO}_{4}{ }^{3-}$.ic.wo & $3.75 \mathrm{E}+03$ & $\mu \mathrm{g} / \mathrm{g}$ \\
\hline Pb.icp.adl.w & Pb.icp.a.w & $4.20 \mathrm{E}+01$ & $\mu \mathrm{g} / \mathrm{g}$ \\
\hline Pb.icp.adl.w & Pb.icp.a.wo & $4.32 \mathrm{E}+01$ & $\mu \mathrm{g} / \mathrm{g}$ \\
\hline Pb.icp.adl.w & Pb.icp.f & $9.25 \mathrm{E}+02 *$ & $\mu \mathrm{g} / \mathrm{g}$ \\
\hline Pb.icp.adl.wo & Pb.icp.a.w & $4.03 \mathrm{E}+01$ & $\mu \mathrm{g} / \mathrm{g}$ \\
\hline Pb.icp.adl.wo & Pb.icp.a.wo & $4.15 \mathrm{E}+01$ & $\mu \mathrm{g} / \mathrm{g}$ \\
\hline Pb.icp.adl.wo & Pb.icp.f & $9.23 \mathrm{E}+02^{*}$ & $\mu \mathrm{g} / \mathrm{g}$ \\
\hline S.icp.adl & S.icp.a & $1.45 \mathrm{E}+03$ & $\mu \mathrm{g} / \mathrm{g}$ \\
\hline S.icp.adl & S.icp.f.w & $1.56 \mathrm{E}+03$ & $\mu \mathrm{g} / \mathrm{g}$ \\
\hline S.icp.adl & S.icp.f.wo & $1.58 \mathrm{E}+03$ & $\mu \mathrm{g} / \mathrm{g}$ \\
\hline $\mathrm{SO}_{4}{ }^{2-}$.ic.w & $\mathrm{SO}_{4}{ }^{2-} . \mathrm{ic}$ & $3.15 \mathrm{E}+03$ & $\mu \mathrm{g} / \mathrm{g}$ \\
\hline $\mathrm{SO}_{4}{ }^{2-}$,ic.w.ed & $\mathrm{SO}_{4}{ }^{2-} . \mathrm{ic}$ & $3.12 \mathrm{E}+03$ & $\mu \mathrm{g} / \mathrm{g}$ \\
\hline
\end{tabular}


Table B3-39. Tank Mean Concentration - Based on Segment Data (Per Weight Basis). (4 sheets)

\begin{tabular}{|c|c|c|c|}
\hline 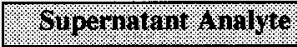 & 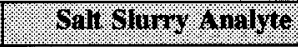 & 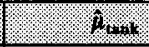 & (6) \\
\hline $\mathrm{SO}_{4}{ }^{2-}$.ic.wo & $\mathrm{SO}_{4}{ }^{2-} . \mathrm{ic}$ & $3.15 \mathrm{E}+03$ & $\mu \mathrm{g} / \mathrm{g}$ \\
\hline $\mathrm{SO}_{4}^{2-}$.ic.wo.ed & $\mathrm{SO}_{4}{ }^{2-} \cdot \mathrm{ic}$ & $3.11 \mathrm{E}+03$ & $\mu \mathrm{g} / \mathrm{g}$ \\
\hline Sb.icp.adl & Sb.icp.a & $2.71 \mathrm{E}+01^{*}$ & $\mu \mathrm{g} / \mathrm{g}$ \\
\hline Sb.icp.adl & Sb.icp.f & $5.56 \mathrm{E}+02 *$ & $\mu \mathrm{g} / \mathrm{g}$ \\
\hline Se.icp.adl & Se.icp.a & $4.51 \mathrm{E}+01^{*}$ & $\mu \mathrm{g} / \mathrm{g}$ \\
\hline Se.icp.adl & Se.icp.f & $9.27 \mathrm{E}+02 *$ & $\mu \mathrm{g} / \mathrm{g}$ \\
\hline Si.icp.adl & Si.icp.a.w & $1.45 \mathrm{E}+02$ & $\mu \mathrm{g} / \mathrm{g}$ \\
\hline Si.icp.adl & Si.icp.a.wo & $1.47 \mathrm{E}+02$ & $\mu \mathrm{g} / \mathrm{g}$ \\
\hline Si.icp.adl & Si.icp.f & $1.13 E+03$ & $\mu \mathrm{g} / \mathrm{g}$ \\
\hline Sm.icp.adl & Sm.icp.a & $4.51 \mathrm{E}+01 *$ & $\mu \mathrm{g} / \mathrm{g}$ \\
\hline Sm.icp.adl & Sm.icp.f & $9.27 \mathrm{E}+02 *$ & $\mu \mathrm{g} / \mathrm{g}$ \\
\hline Sr.icp.adl & Sr.icp.a & $6.57 \mathrm{E}+00^{*}$ & $\mu \mathrm{g} / \mathrm{g}$ \\
\hline Sr.icp.adl & Sr.icp.f & $9.27 \mathrm{E}+01^{*}$ & $\mu \mathrm{g} / \mathrm{g}$ \\
\hline Ti.icp.adl & Ti.icp.a & $4.51 \mathrm{E}+00^{*}$ & $\mu \mathrm{g} / \mathrm{g}$ \\
\hline Ti.icp.adl & Ti.icp.f & $9.27 \mathrm{E}+01 *$ & $\mu \mathrm{g} / \mathrm{g}$ \\
\hline Tl.icp.adl & T1.icp.a & $9.02 \mathrm{E}+01 *$ & $\mu \mathrm{g} / \mathrm{g}$ \\
\hline Tl.icp.adl & Tl.icp.f & $1.86 \mathrm{E}+03^{*}$ & $\mu \mathrm{g} / \mathrm{g}$ \\
\hline Total Alpha & Total Alpha.w & $6.83 \mathrm{E}-02 *$ & $\mu \mathrm{Ci} / \mathrm{g}$ \\
\hline Total Alpha & Total Alpha.wo & $6.84 \mathrm{E}-02^{*}$ & $\mu \mathrm{Ci} / \mathrm{g}$ \\
\hline U.icp.adl & U.icp.a & $2.25 \mathrm{E}+02 *$ & $\mu \mathrm{g} / \mathrm{g}$ \\
\hline U.icp.adl & U.icp.f & $4.64 \mathrm{E}+03^{*}$ & $\mu \mathrm{g} / \mathrm{g}$ \\
\hline V.icp.adl & V.icp.a & $2.26 \mathrm{E}+01^{*}$ & $\mu \mathrm{g} / \mathrm{g}$ \\
\hline V.icp.adl & V.icp.f & $4.64 \mathrm{E}+02 *$ & $\mu \mathrm{g} / \mathrm{g}$ \\
\hline Zn.icp.adl & Zn.icp.a & $3.11 \mathrm{E}+01$ & $\mu \mathrm{g} / \mathrm{g}$ \\
\hline Zn.icp.adl & Zn.icp.f & $3.58 \mathrm{E}+02$ & $\mu \mathrm{g} / \mathrm{g}$ \\
\hline Zr.icp.adl & Zr.icp.a.w & $7.29 \mathrm{E}+00$ & $\mu \mathrm{g} / \mathrm{g}$ \\
\hline Zr.icp.adl & Zr.icp.a.wo & $7.52 \mathrm{E}+00$ & $\mu \mathrm{g} / \mathrm{g}$ \\
\hline
\end{tabular}

Note:

${ }^{i} \mathrm{Li}$ and ${ }^{1} \mathrm{Br}$ are the tracers for hydrostatic head fluid. 
Table B3-40. Tank Mean Concentration - Based on Composite Sample Data (Per Volume Basis). (4 sheets)

\begin{tabular}{|c|c|c|c|}
\hline Supertiatant Amilyte & Sall Gling Inolyte & $1.1 \%$ & 1: : Uins \\
\hline Water.tga & Water.tga & $7.12 \mathrm{E}-01$ & $\mathrm{~g} / \mathrm{mL}$ \\
\hline Acetate.ic & Acetate.ic & $6.58 \mathrm{E}+02^{*}$ & $\mu \mathrm{g} / \mathrm{mL}$ \\
\hline Ag.icp.a & Ag.icp.a & $1.84 \mathrm{E}+01$ & $\mu \mathrm{g} / \mathrm{mL}$ \\
\hline Ag.icp.adl & Ag.icp.a & $2.01 \mathrm{E}+01$ & $\mu \mathrm{g} / \mathrm{mL}$ \\
\hline Al.icp.a & Al.icp.a & $3.39 \mathrm{E}+04$ & $\mu \mathrm{g} / \mathrm{mL}$ \\
\hline Al.icp.adl & Al.icp.a & $3.86 \mathrm{E}+04$ & $\mu \mathrm{g} / \mathrm{mL}$ \\
\hline${ }^{241} \mathrm{Am}$.aea & $\mathrm{Am}^{241}$.aea & $4.69 \mathrm{E}-03 * *$ & $\mu \mathrm{Ci} / \mathrm{mL}$ \\
\hline${ }^{241} \mathrm{Am}$.gea & ${ }^{241} \mathrm{Am}$.gea & $1.01 \mathrm{E}+00^{*}$ & $\mu \mathrm{Ci} / \mathrm{mL}$ \\
\hline As.icp.a & As.icp.a & $5.67 \mathrm{E}+01^{*}$ & $\mu \mathrm{g} / \mathrm{mL}$ \\
\hline As.icp.adl & As.icp.a & $6.13 \mathrm{E}+01^{*}$ & $\mu \mathrm{g} / \mathrm{mL}$ \\
\hline B.icp.a & B.icp.a & $1.75 \mathrm{E}+02$ & $\mu \mathrm{g} / \mathrm{mL}$ \\
\hline B.icp.adl & B.icp.a & $1.17 \mathrm{E}+02$ & $\mu \mathrm{g} / \mathrm{mL}$ \\
\hline Ba.icp.a & Ba.icp.a & $2.84 \mathrm{E}+01^{*}$ & $\mu \mathrm{g} / \mathrm{mL}$ \\
\hline Ba.icp.adl & Ba.icp.a & $3.07 \mathrm{E}+01^{*}$ & $\mu \mathrm{g} / \mathrm{mL}$ \\
\hline Be.icp.a & Be.icp.a & $2.84 \mathrm{E}+00^{*}$ & $\mu \mathrm{g} / \mathrm{mL}$ \\
\hline Be.icp.adl & Be.icp.a & $3.06 \mathrm{E}+00^{*}$ & $\mu \mathrm{g} / \mathrm{mL}$ \\
\hline Bi.icp.a & Bi.icp.a & $5.67 \mathrm{E}+01^{*}$ & $\mu \mathrm{g} / \mathrm{mL}$ \\
\hline Bi.icp.adl & Bi.icp.a & $6.13 \mathrm{E}+01^{*}$ & $\mu \mathrm{g} / \mathrm{mL}$ \\
\hline${ }^{1} \mathrm{Br}$.ic & $\mathrm{Br}$.ic & $6.60 \mathrm{E}+02^{*}$ & $\mu \mathrm{g} / \mathrm{mL}$ \\
\hline Ca.icp.a & Ca.icp.a & $6.69 \mathrm{E}+02 *$ & $\mu \mathrm{g} / \mathrm{mL}$ \\
\hline Ca.icp.adl & Ca.icp.a & $1.78 \mathrm{E}+02$ & $\mu \mathrm{g} / \mathrm{mL}$ \\
\hline Cd.icp.a & Cd.icp.a & $6.55 \mathrm{E}+00^{*}$ & $\mu \mathrm{g} / \mathrm{mL}$ \\
\hline Cd.icp.adl & Cd.icp.a & $6.78 \mathrm{E}+00^{*}$ & $\mu \mathrm{g} / \mathrm{mL}$ \\
\hline Ce.icp.a & Ce.icp.a & $5.67 \mathrm{E}+01 *$ & $\mu \mathrm{g} / \mathrm{mL}$ \\
\hline Ce.icp.adl & Ce.icp.a & $6.13 \mathrm{E}+01^{*}$ & $\mu \mathrm{g} / \mathrm{mL}$ \\
\hline $\mathrm{Cl}^{-}$.ic & $\mathrm{Cl}$.ic & $9.70 \mathrm{E}+03$ & $\mu \mathrm{g} / \mathrm{mL}$ \\
\hline${ }^{2434 / 244} \mathrm{Cm}$ & ${ }^{243 / 244} \mathrm{Cm}$ & $9.11 \mathrm{E}-04^{*}$ & $\mu \mathrm{Ci} / \mathrm{mL}$ \\
\hline Co.icp.a & Co.icp.a & $1.13 \mathrm{E}+01^{*}$ & $\mu \mathrm{g} / \mathrm{mL}$ \\
\hline Co.icp.adl & Co.icp.a & $1.22 \mathrm{E}+01^{*}$ & $\mu \mathrm{g} / \mathrm{mL}$ \\
\hline${ }^{60} \mathrm{Co}$.gea & ${ }^{60} \mathrm{Co}$.gea & $3.02 \mathrm{E}-02 *$ & $\mu \mathrm{Ci} / \mathrm{mL}$ \\
\hline
\end{tabular}


Table B3-40. Tank Mean Concentration - Based on Composite Sample Data (Per Volume Basis). (4 sheets)

\begin{tabular}{|c|c|c|c|}
\hline Supernatant Aralyte & 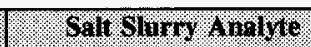 & I.1.1.1. & Uring \\
\hline Cr.icp.a & Cr.icp.a & $1.12 \mathrm{E}+03$ & $\mu \mathrm{g} / \mathrm{mL}$ \\
\hline Cr.icp.adl & Cr.icp.a & $1.14 \mathrm{E}+03$ & $\mu \mathrm{g} / \mathrm{mL}$ \\
\hline Cr(VI).spec & Cr(VI).spec & $1.66 \mathrm{E}+02$ & $\mu \mathrm{g} / \mathrm{mL}$ \\
\hline${ }^{137}$ Cs.gea & ${ }^{137}$ Cs.gea & $4.70 \mathrm{E}+02$ & $\mu \mathrm{Ci} / \mathrm{mL}$ \\
\hline Cu.icp.a & Cu.icp.a & $7.04 \mathrm{E}+00^{*}$ & $\mu \mathrm{g} / \mathrm{mL}$ \\
\hline Cu.icp.adl & Cu.icp.a & $6.87 \mathrm{E}+00^{*}$ & $\mu \mathrm{g} / \mathrm{mL}$ \\
\hline${ }^{154}$ Eu.gea & ${ }^{154}$ Eu.gea & $1.19 \mathrm{E}-01^{*}$ & $\mu \mathrm{Ci} / \mathrm{mL}$ \\
\hline${ }^{155}$ Eu.gea & ${ }^{155}$ Eu.gea & $4.29 \mathrm{E}-01^{*}$ & $\mu \mathrm{Ci} / \mathrm{mL}$ \\
\hline $\mathrm{F}^{-}$.ic & $F^{-}$.ic & $9.81 \mathrm{E}+02 * *$ & $\mu \mathrm{g} / \mathrm{mL}$ \\
\hline Fe.icp.a & Fe.icp.a & $8.18 \mathrm{E}+01$ & $\mu \mathrm{g} / \mathrm{mL}$ \\
\hline Fe.icp.adl & Fe.icp.a & $6.16 \mathrm{E}+01^{*}$ & $\mu \mathrm{g} / \mathrm{mL}$ \\
\hline Formate.ic & Formate.ic & $1.73 \mathrm{E}+03$ & $\mu \mathrm{g} / \mathrm{mL}$ \\
\hline${ }^{129} \mathbf{I}$ & ${ }^{129} \mathrm{I}$ & $2.57 \mathrm{E}-03^{*}$ & $\mu \mathrm{Ci} / \mathrm{mL}$ \\
\hline K.icp.a & K.icp.a & $5.07 \mathrm{E}+03$ & $\mu \mathrm{g} / \mathrm{mL}$ \\
\hline K.icp.adl & K.icp.a & $5.80 \mathrm{E}+03$ & $\mu \mathrm{g} / \mathrm{mL}$ \\
\hline La.icp.a & La.icp.a & $2.84 \mathrm{E}+01^{*}$ & $\mu \mathrm{g} / \mathrm{mL}$ \\
\hline La.icp.adl & La.icp.a & $3.07 \mathrm{E}+01^{*}$ & $\mu \mathrm{g} / \mathrm{mL}$ \\
\hline${ }^{\prime}$ Li.icp.a & Li.icp.a & $9.40 \mathrm{E}+00$ & $\mu \mathrm{g} / \mathrm{mL}$ \\
\hline 'Li.icp.adl & Li.icp.a & $9.43 \mathrm{E}+00^{*}$ & $\mu \mathrm{g} / \mathrm{mL}$ \\
\hline Mg.icp.a & Mg.icp.a & $5.67 \mathrm{E}+01 *$ & $\mu \mathrm{g} / \mathrm{mL}$ \\
\hline Mg.icp.adl & Mg.icp.a & $6.13 \mathrm{E}+01^{*}$ & $\mu \mathrm{g} / \mathrm{mL}$ \\
\hline Mn.icp.a & Mn.icp.a & $1.06 \mathrm{E}+01^{*}$ & $\mu \mathrm{g} / \mathrm{mL}$ \\
\hline Mn.icp.adl & Mn.icp.a & $1.10 \mathrm{E}+01^{*}$ & $\mu \mathrm{g} / \mathrm{mL}$ \\
\hline Mo.icp.a & Mo.icp.a & $7.77 \mathrm{E}+01$ & $\mu \mathrm{g} / \mathrm{mL}$ \\
\hline Mo.icp.adl & Mo.icp.a & $8.88 \mathrm{E}+01$ & $\mu \mathrm{g} / \mathrm{mL}$ \\
\hline $\mathrm{NO}_{2}^{-}$.ic & $\mathrm{NO}_{2}^{-}$.ic & $1.23 \mathrm{E}+05$ & $\mu \mathrm{g} / \mathrm{mL}$ \\
\hline $\mathrm{NO}_{3} \cdot \mathrm{ic}$ & $\mathrm{NO}_{3}{ }^{-}$.ic & $1.74 \mathrm{E}+05$ & $\mu \mathrm{g} / \mathrm{mL}$ \\
\hline Na.icp.a & Na.icp.a & $2.25 \mathrm{E}+05$ & $\mu \mathrm{g} / \mathrm{mL}$ \\
\hline Na.icp.adl & Na.icp.a & $2.51 \mathrm{E}+05$ & $\mu \mathrm{g} / \mathrm{mL}$ \\
\hline Nd.icp.a & Nd.icp.a & $5.67 \mathrm{E}+01^{*}$ & $\mu \mathrm{g} / \mathrm{mL}$ \\
\hline
\end{tabular}


Table B3-40. Tank Mean Concentration - Based on Composite Sample Data (Per Volume Basis). (4 sheets)

\begin{tabular}{|c|c|c|c|}
\hline Superantant Analyte & Sait silury Aralyte: & in..... & Units \\
\hline Nd.icp.adl & Nd.icp.a & $6.13 \mathrm{E}+01^{*}$ & $\mu \mathrm{g} / \mathrm{mL}$ \\
\hline Ni.icp.a & Ni.icp.a & $2.64 \mathrm{E}+01^{*}$ & $\mu \mathrm{g} / \mathrm{mL}$ \\
\hline Ni.icp.adl & Ni.icp.a & $2.73 \mathrm{E}+01^{*}$ & $\mu \mathrm{g} / \mathrm{mL}$ \\
\hline${ }^{237} \mathrm{~Np} \cdot \mathrm{tta}$ & ${ }^{237} \mathrm{~Np} . \mathrm{tta}$ & $7.01 \mathrm{E}-03^{*}$ & $\mu \mathrm{Ci} / \mathrm{mL}$ \\
\hline $\mathrm{OH}^{-}$ & $\overline{\mathrm{OH}^{-}}$ & $5.01 \mathrm{E}+04$ & $\mu \mathrm{g} / \mathrm{mL}$ \\
\hline Oxalate.ic & Oxalate.ic & $4.30 \mathrm{E}+03 * *$ & $\mu \mathrm{g} / \mathrm{mL}$ \\
\hline P.icp.a & P.icp.a & $1.28 \mathrm{E}+03$ & $\mu \mathrm{g} / \mathrm{mL}$ \\
\hline P.icp.adl & P.icp.a & $1.31 \mathrm{E}+03$ & $\mu \mathrm{g} / \mathrm{mL}$ \\
\hline $\mathrm{PO}_{4}^{3} \cdot \mathrm{ic}$ & $\mathrm{PO}_{4}^{3-} . \mathrm{ic}$ & $3.80 \mathrm{E}+03$ & $\mu \mathrm{g} / \mathrm{mL}$ \\
\hline Pb.icp.a & Pb.icp.a & $5.73 \mathrm{E}+01^{*}$ & $\mu \mathrm{g} / \mathrm{mL}$ \\
\hline Pb.icp.adl & Pb.icp.a & $6.19 \mathrm{E}+01^{*}$ & $\mu \mathrm{g} / \mathrm{mL}$ \\
\hline${ }^{239 / 240} \mathrm{Pu}$ & ${ }^{239 / 240} \mathrm{Pu}$ & $1.50 \mathrm{E}-03^{*}$ & $\mu \mathrm{Ci} / \mathrm{mL}$ \\
\hline S.icp.a & S.icp.a & $1.85 \mathrm{E}+03$ & $\mu \mathrm{g} / \mathrm{mL}$ \\
\hline S.icp.adl & S.icp.a & $1.93 \mathrm{E}+03$ & $\mu \mathrm{g} / \mathrm{mL}$ \\
\hline $\mathrm{SO}_{4}^{2-}$.ic & $\mathrm{SO}_{4}{ }^{2-}$.ic & $5.08 \mathrm{E}+03$ & $\mu \mathrm{g} / \mathrm{mL}$ \\
\hline Sb.icp.a & Sb.icp.a & $3.40 \mathrm{E}+01^{*}$ & $\mu \mathrm{g} / \mathrm{mL}$ \\
\hline Sb.icp.adl & Sb.icp.a & $3.68 \mathrm{E}+01^{*}$ & $\mu \mathrm{g} / \mathrm{mL}$ \\
\hline Se.icp.a & Se.icp.a & $5.67 \mathrm{E}+01^{*}$ & $\mu \mathrm{g} / \mathrm{mL}$ \\
\hline $\begin{array}{l}\text { Se.icp.adl } \\
\end{array}$ & Se.icp.a & $6.13 \mathrm{E}+01^{*}$ & $\mu \mathrm{g} / \mathrm{mL}$ \\
\hline Si.icp.a & Si.icp.a & $2.62 E+02$ & $\mu \mathrm{g} / \mathrm{mL}$ \\
\hline Si.icp.adl & Si.icp.a & $2.29 \mathrm{E}+02$ & $\mu \mathrm{g} / \mathrm{mL}$ \\
\hline Sm.icp.a & Sm.icp.a & $5.67 \mathrm{E}+01^{*}$ & $\mu \mathrm{g} / \mathrm{mL}$ \\
\hline Sm.icp.adl & Sm.icp.a & $6.13 \mathrm{E}+01^{*}$ & $\mu \mathrm{g} / \mathrm{mL}$ \\
\hline Sr.icp.a & Sr.icp.a & $5.67 \mathrm{E}+00^{*}$ & $\mu \mathrm{g} / \mathrm{mL}$ \\
\hline Sr.icp.adl & Sr.icp.a & $6.13 \mathrm{E}+00^{*}$ & $\mu \mathrm{g} / \mathrm{mL}$ \\
\hline${ }^{89 / 190} \mathrm{Sr}$ & ${ }^{89 / 90} \mathrm{Sr}$ & $7.42 \mathrm{E}+00$ & $\mu \mathrm{Ci} / \mathrm{mL}$ \\
\hline TIC & TIC & $9.22 \mathrm{E}+03$ & $\mu \mathrm{g} / \mathrm{mL}$ \\
\hline TOC.coul & TOC.pers & $7.01 \mathrm{E}+03$ & $\mu \mathrm{g} / \mathrm{mL}$ \\
\hline TOC.pers & TOC.pers & $4.90 \mathrm{E}+03$ & $\mu \mathrm{g} / \mathrm{mL}$ \\
\hline${ }^{99} \mathrm{Tc}$ & ${ }^{99} \mathrm{Tc}$ & $2.75 \mathrm{E}-01$ & $\mu \mathrm{Ci} / \mathrm{mL}$ \\
\hline
\end{tabular}


Table B3-40. Tank Mean Concentration - Based on Composite Sample Data

(Per Volume Basis). (4 sheets)

\begin{tabular}{|c|c|c|c|}
\hline Supernatint Arualye & Sall Slingry Awalyte & i. & Vaits \\
\hline Ti.icp.a & Ti.icp.a & $5.67 \mathrm{E}+00^{*}$ & $\mu \mathrm{g} / \mathrm{mL}$ \\
\hline Ti.icp.adl & Ti.icp.a & $6.13 \mathrm{E}+00^{*}$ & $\mu \mathrm{g} / \mathrm{mL}$ \\
\hline Tl.icp.a & Tl.icp.a & $1.13 \mathrm{E}+02^{*}$ & $\mu \mathrm{g} / \mathrm{mL}$ \\
\hline Tl.icp.adl & Tl.icp.a & $1.22 \mathrm{E}+02^{*}$ & $\mu \mathrm{g} / \mathrm{mL}$ \\
\hline Total Alpha & Total Alpha & $2.04 \mathrm{E}-02 *$ & $\mu \mathrm{Ci} / \mathrm{mL}$ \\
\hline Total Beta & Total Beta & $5.25 \mathrm{E}+02$ & $\mu \mathrm{Ci} / \mathrm{mL}$ \\
\hline \begin{tabular}{|l|} 
Tritium. 1 \\
\end{tabular} & \begin{tabular}{|l|} 
Tritium.1 \\
\end{tabular} & $1.31 \mathrm{E}-03^{*}$ & $\mu \mathrm{Ci} / \mathrm{mL}$ \\
\hline U.icp.a & U.icp.a & $2.84 \mathrm{E}+02 *$ & $\mu \mathrm{g} / \mathrm{mL}$ \\
\hline U.icp.adl & U.icp.a & $3.06 \mathrm{E}+02 *$ & $\mu \mathrm{g} / \mathrm{mL}$ \\
\hline U.phos & U.phos & $5.67 \mathrm{E}+01$ & $\mu \mathrm{g} / \mathrm{mL}$ \\
\hline V.icp.a & V.icp.a & $2.84 \mathrm{E}+01^{*}$ & $\mu \mathrm{g} / \mathrm{mL}$ \\
\hline V.icp.adl & V.icp.a & $3.07 \mathrm{E}+01^{*}$ & $\mu \mathrm{g} / \mathrm{mL}$ \\
\hline Zn.icp.a & Zn.icp.a & $5.10 \mathrm{E}+01$ & $\mu \mathrm{g} / \mathrm{mL}$ \\
\hline Zn.jep.adl & Zn.icp.a & $1.59 \mathrm{E}+01$ & $\mu \mathrm{g} / \mathrm{mL}$ \\
\hline Zr.icp.a & Zr.icp.a & $9.06 \mathrm{E}+00^{*}$ & $\mu \mathrm{g} / \mathrm{mL}$ \\
\hline Zr.icp.adl & Zr.icp.a & $9.52 \mathrm{E}+00^{*}$ & $\mu \mathrm{g} / \mathrm{mL}$ \\
\hline SpG & SpG & $1.48 \mathrm{E}+00$ & "g/mL" \\
\hline
\end{tabular}

Note:

${ }^{1} \mathrm{Li}$ and ${ }^{1} \mathrm{Br}$ are the tracers for hydrostatic head fluid. 
Table B3-41. Tank Mean Concentration - Based on Composite Sample Data (Per Weight Basis). (4 sheets)

\begin{tabular}{|c|c|c|c|}
\hline Superinatant Aralyte & Sall slurty inglyte. & $1.7 \%$ & 1. Inils \\
\hline Water.tga & Water.tga & $6.03 \mathrm{E}-01$ & $g / g$ \\
\hline Acetate.ic & Acetate.ic & $5.54 \mathrm{E}+02^{*}$ & $\mu \mathrm{g} / \mathrm{g}$ \\
\hline Ag.icp.a & Ag.icp.a & $1.50 \mathrm{E}+01$ & $\mu \mathrm{g} / \mathrm{g}$ \\
\hline Ag.icp.adl & Ag.icp.a & $1.66 \mathrm{E}+01$ & $\mu \mathrm{g} / \mathrm{g}$ \\
\hline Al.icp.a & Al.icp.a & $2.90 \mathrm{E}+04$ & $\mu \mathrm{g} / \mathrm{g}$ \\
\hline Al.icp.adl & Al.icp.a & $3.35 \mathrm{E}+04$ & $\mu \mathrm{g} / \mathrm{g}$ \\
\hline${ }^{241} \mathrm{Am}$.aea & $\mathrm{Am}^{241}$.aea & $3.15 \mathrm{E}-03 * *$ & $\mu \mathrm{Ci} / \mathrm{g}$ \\
\hline${ }^{241} \mathrm{Am}$. gea & ${ }^{241}$ Am.gea & $8.31 \mathrm{E}-01^{*}$ & $\mu \mathrm{Ci} / \mathrm{g}$ \\
\hline As.icp.a & As.icp.a & $4.72 \mathrm{E}+01^{*}$ & $\mu \mathrm{g} / \mathrm{g}$ \\
\hline As.icp.adl & As.icp.a & $5.16 \mathrm{E}+01^{*}$ & $\mu \mathrm{g} / \mathrm{g}$ \\
\hline B.icp.a & B.icp.a & $1.47 \mathrm{E}+02$ & $\mu \mathrm{g} / \mathrm{g}$ \\
\hline B.icp.adl & B.icp.a & $9.12 \mathrm{E}+01$ & $\mu \mathrm{g} / \mathrm{g}$ \\
\hline Ba.icp.a & Ba.icp.a & $2.36 \mathrm{E}+01 *$ & $\mu \mathrm{g} / \mathrm{g}$ \\
\hline Ba.icp.adl & Ba.icp.a & $2.59 \mathrm{E}+01^{*}$ & $\mu \mathrm{g} / \mathrm{g}$ \\
\hline Be.icp.a & Be.icp.a & $2.36 \mathrm{E}+00^{*}$ & $\mu \mathrm{g} / \mathrm{g}$ \\
\hline Be.icp.adl & Be.icp.a & $2.58 \mathrm{E}+00^{*}$ & $\mu \mathrm{g} / \mathrm{g}$ \\
\hline Bi.icp.a & Bi.icp.a & $4.72 \mathrm{E}+01^{*}$ & $\mu \mathrm{g} / \mathrm{g}$ \\
\hline Bi.icp.adl & Bi.icp.a & $5.16 \mathrm{E}+01^{*}$ & $\mu \mathrm{g} / \mathrm{g}$ \\
\hline${ }^{1} \mathrm{Br}$.ic & $\mathrm{Br}$.ic & $5.35 \mathrm{E}+02 *$ & $\mu \mathrm{g} / \mathrm{g}$ \\
\hline Ca.icp.a & Ca.icp.a & $6.02 \mathrm{E}+02^{*}$ & $\mu \mathrm{g} / \mathrm{g}$ \\
\hline Ca.icp.adl & Ca.icp.a & $1.31 \mathrm{E}+02$ & $\mu \mathrm{g} / \mathrm{g}$ \\
\hline Cd.icp.a & Cd.icp.a & $4.86 \mathrm{E}+00^{*}$ & $\mu \mathrm{g} / \mathrm{g}$ \\
\hline Cd.icp.adl & Cd.icp.a & $5.08 \mathrm{E}+00^{*}$ & $\mu \mathrm{g} / \mathrm{g}$ \\
\hline Ce.icp.a & Ce.icp.a & $4.72 \mathrm{E}+01^{*}$ & $\mu \mathrm{g} / \mathrm{g}$ \\
\hline Ce.icp.adl & Ce.icp.a & $5.16 \mathrm{E}+01^{*}$ & $\mu \mathrm{g} / \mathrm{g}$ \\
\hline $\mathrm{Cl}^{-}$.ic & $\mathrm{Cl}$.ic & $8.30 \mathrm{E}+03$ & $\mu \mathrm{g} / \mathrm{g}$ \\
\hline${ }^{2434 / 244} \mathrm{Cm}$ & ${ }^{243 / 244} \mathrm{Cm}$ & $6.19 \mathrm{E}-04^{*}$ & $\mu \mathrm{Ci} / \mathrm{g}$ \\
\hline Co.icp.a & Co.icp.a & $9.45 \mathrm{E}+00^{*}$ & $\mu \mathrm{g} / \mathrm{g}$ \\
\hline Co.icp.adl & Co.icp.a & $1.03 \mathrm{E}+01^{*}$ & $\mu \mathrm{g} / \mathrm{g}$ \\
\hline${ }^{60} \mathrm{Co}$. gea & ${ }^{60} \mathrm{Co}$ gea & $2.26 \mathrm{E}-02^{*}$ & $\mu \mathrm{Ci} / \mathrm{g}$ \\
\hline
\end{tabular}


Table B3-41. Tank Mean Concentration - Based on Composite Sample Data (Per Weight Basis). (4 sheets)

\begin{tabular}{|c|c|c|c|}
\hline Sinpernatant 6 nalyte & Salt Slumy Analye & $1 . a_{\text {matr }}$ & 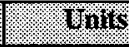 \\
\hline Cr.icp.a & Cr.icp.a & $7.88 \mathrm{E}+02$ & $\mu \mathrm{g} / \mathrm{g}$ \\
\hline Cr.icp.adl & Cr.icp.a & $8.08 \mathrm{E}+02$ & $\mu \mathrm{g} / \mathrm{g}$ \\
\hline $\mathrm{Cr}(\mathrm{VI})$. spec & $\mathrm{Cr}(\mathrm{VI}) . \mathrm{spec}$ & $1.38 \mathrm{E}+02$ & $\mu \mathrm{g} / \mathrm{g}$ \\
\hline${ }^{137} \mathrm{Cs}$.gea & ${ }^{137} \mathrm{Cs}$.gea & $3.98 \mathrm{E}+02$ & $\mu \mathrm{Ci} / \mathrm{g}$ \\
\hline Cu.icp.a & Cu.icp.a & $5.83 \mathrm{E}+00^{*}$ & $\mu \mathrm{g} / \mathrm{g}$ \\
\hline Cu.icp.adl & Cu.icp.a & $5.67 \mathrm{E}+00^{*}$ & $\mu \mathrm{g} / \mathrm{g}$ \\
\hline${ }^{154} \mathrm{Eu} . g e a$ & ${ }^{154}$ Eu.gea & $9.72 \mathrm{E}-02^{*}$ & $\mu \mathrm{Ci} / \mathrm{g}$ \\
\hline${ }^{155}$ Eu.gea & ${ }^{155}$ Eu.gea & $3.46 \mathrm{E}-01^{*}$ & $\mu \mathrm{Ci} / \mathrm{g}$ \\
\hline F-ic & $F^{-}$.ic & $6.70 \mathrm{E}+02 * *$ & $\mu \mathrm{g} / \mathrm{g}$ \\
\hline Fe.icp.a & Fe.icp.a & $6.61 \mathrm{E}+01$ & $\mu \mathrm{g} / \mathrm{g}$ \\
\hline Fe.icp.adl & Fe.icp.a & $4.67 \mathrm{E}+01^{*}$ & $\mu \mathrm{g} / \mathrm{g}$ \\
\hline Formate.ic & Formate.ic & $1.45 \bar{E}+03$ & $\mu \mathrm{g} / \mathrm{g}$ \\
\hline${ }^{129} \mathrm{I}$ & ${ }^{129} \mathrm{I}$ & $1.78 \mathrm{E}-03^{*}$ & $\mu \mathrm{Ci} / \mathrm{g}$ \\
\hline K.icp.a & K.icp.a & $4.33 \mathrm{E}+03$ & $\mu \mathrm{g} / \mathrm{g}$ \\
\hline K.icp.adl & K.icp.a & $5.03 \mathrm{E}+03$ & $\mu \mathrm{g} / \mathrm{g}$ \\
\hline La.icp.a & La.icp.a & $2.36 \mathrm{E}+01^{*}$ & $\mu \mathrm{g} / \mathrm{g}$ \\
\hline La.icp.ad1 & La.icp.a & $2.59 \mathrm{E}+01^{*}$ & $\mu \mathrm{g} / \mathrm{g}$ \\
\hline 'Li.icp.a & Li.icp.a & $7.37 \mathrm{E}+00$ & $\mu \mathrm{g} / \mathrm{g}$ \\
\hline${ }^{1}$ Li.icp.adl & Li.icp.a & $7.39 \mathrm{E}+00^{*}$ & $\mu \mathrm{g} / \mathrm{g}$ \\
\hline Mg.icp.a & Mg.icp.a & $4.72 \mathrm{E}+01 *$ & $\mu \mathrm{g} / \mathrm{g}$ \\
\hline Mg.icp.adl & Mg.icp.a & $5.16 \mathrm{E}+01^{*}$ & $\mu \mathrm{g} / \mathrm{g}$ \\
\hline Mn.icp.a & Mn.icp.a & $8.00 \mathrm{E}+00^{*}$ & $\mu \mathrm{g} / \mathrm{g}$ \\
\hline Mn.icp.adl & Mn.icp.a & $8.44 \mathrm{E}+00^{*}$ & $\mu \mathrm{g} / \mathrm{g}$ \\
\hline Mo.icp.a & Mo.icp.a & $6.66 \mathrm{E}+01$ & $\mu \mathrm{g} / \mathrm{g}$ \\
\hline Mo.icp.adl & Mo.icp.a & $7.72 \mathrm{E}+01$ & $\mu \mathrm{g} / \mathrm{g}$ \\
\hline $\mathrm{NO}_{2}^{-}$.ic & $\mathrm{NO}_{2}$. ic & $1.05 \bar{E}+05$ & $\mu \mathrm{g} / \mathrm{g}$ \\
\hline $\mathrm{NO}_{3}^{-}$.ic & $\mathrm{NO}_{3}$. $\mathrm{ic}$ & $1.46 \mathrm{E}+05$ & $\mu \mathrm{g} / \mathrm{g}$ \\
\hline Na.icp.a & Na.icp.a & $1.88 \mathrm{E}+05$ & $\mu \mathrm{g} / \mathrm{g}$ \\
\hline Na.icp.adl & Na.icp.a & $2.13 \mathrm{E}+05$ & $\mu \mathrm{g} / \mathrm{g}$ \\
\hline Nd.icp.a & Nd.icp.a & $4.72 \mathrm{E}+01^{*}$ & $\mu \mathrm{g} / \mathrm{g}$ \\
\hline
\end{tabular}


Table B3-41. Tank Mean Concentration - Based on Composite Sample Data (Per Weight Basis). (4 sheets)

\begin{tabular}{|c|c|c|c|}
\hline Supornatur f nule & 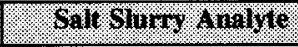 & 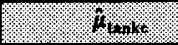 & Wous \\
\hline Nd.icp.adl & Nd.icp.a & $5.16 \mathrm{E}+01^{*}$ & $\mu \mathrm{g} / \mathrm{g}$ \\
\hline Ni.icp.a & Ni.icp.a & $1.96 \mathrm{E}+01 *$ & $\mu \mathrm{g} / \mathrm{g}$ \\
\hline Ni.icp.adl & Ni.icp.a & $2.05 \mathrm{E}+01 *$ & $\mu \mathrm{g} / \mathrm{g}$ \\
\hline${ }^{237} \mathrm{~Np} . \mathrm{tta}$ & ${ }^{237} \mathrm{~Np} \cdot \mathrm{tta}$ & $4.73 \mathrm{E}-03^{*}$ & $\mu \mathrm{Ci} / \mathrm{g}$ \\
\hline $\mathrm{OH}^{-}$ & $\mathrm{OH}^{-}$ & $4.38 \mathrm{E}+04$ & $\mu \mathrm{g} / \mathrm{g}$ \\
\hline Oxalate.ic & Oxalate.ic & $2.98 \mathrm{E}+03 * *$ & $\mu \mathrm{g} / \mathrm{g}$ \\
\hline P.icp.a & P.icp.a & $9.14 \mathrm{E}+02$ & $\mu \mathrm{g} / \mathrm{g}$ \\
\hline P.icp.adl & P.icp.a & $9.44 \mathrm{E}+02$ & $\mu \mathrm{g} / \mathrm{g}$ \\
\hline $\mathrm{PO}_{4}^{32} . \mathrm{ic}$ & $\mathrm{PO}_{4}{ }^{3-}$.ic & $2.65 \mathrm{E}+03$ & $\mu \mathrm{g} / \mathrm{g}$ \\
\hline Pb.icp.a & Pb.icp.a & $4.76 \mathrm{E}+01 *$ & $\mu \mathrm{g} / \mathrm{g}$ \\
\hline Pb.icp.adl & Pb.icp.a & $5.21 \mathrm{E}+01 *$ & $\mu \mathrm{g} / \mathrm{g}$ \\
\hline${ }^{239 / 240} \mathrm{Pu}$ & ${ }^{239 / 240} \mathrm{Pu}$ & $1.02 \mathrm{E}-03^{*}$ & $\mu \mathrm{Ci} / \mathrm{g}$ \\
\hline S.icp.a & S.icp.a & $1.40 \mathrm{E}+03$ & $\mu \mathrm{g} / \mathrm{g}$ \\
\hline S.icp.adl & S.icp.a & $1.48 \mathrm{E}+03$ & $\mu \mathrm{g} / \mathrm{g}$ \\
\hline $\mathrm{SO}_{4}^{2-} . \mathrm{ic}$ & $\mathrm{SO}_{4}^{2-} \cdot \mathrm{ic}$ & $3.60 \mathrm{E}+03$ & $\mu \mathrm{g} / \mathrm{g}$ \\
\hline Sb.icp.a & Sb.icp.a & $2.83 \mathrm{E}+01^{*}$ & $\mu \mathrm{g} / \mathrm{g}$ \\
\hline Sb.icp.adl & Sb.icp.a & $3.10 \mathrm{E}+01^{*}$ & $\mu \mathrm{g} / \mathrm{g}$ \\
\hline Se.icp.a & Se.icp.a & $4.72 \mathrm{E}+01^{*}$ & $\mu \mathrm{g} / \mathrm{g}$ \\
\hline Se.icp.adl & Se.icp.a & $5.16 \mathrm{E}+01^{*}$ & $\mu \mathrm{g} / \mathrm{g}$ \\
\hline Si.icp.a & Si.icp.a & $2.14 \mathrm{E}+02$ & $\mu \mathrm{g} / \mathrm{g}$ \\
\hline Si.icp.adl & Si.icp.a & $1.83 \mathrm{E}+02$ & $\mu \mathrm{g} / \mathrm{g}$ \\
\hline Sm.icp.a & Sm.icp.a & $4.72 \mathrm{E}+01^{*}$ & $\mu \mathrm{g} / \mathrm{g}$ \\
\hline Sm.icp.adl & Sm.icp.a & $5.16 \mathrm{E}+01^{*}$ & $\mu \mathrm{g} / \mathrm{g}$ \\
\hline Sr.icp.a & Sr.icp.a & $4.72 \mathrm{E}+00^{*}$ & $\mu \mathrm{g} / \mathrm{g}$ \\
\hline Sr.icp.adl & Sr.icp.a & $5.16 \mathrm{E}+00^{*}$ & $\mu \mathrm{g} / \mathrm{g}$ \\
\hline${ }^{89 / 90} \mathrm{Sr}$ & ${ }^{89 / 90} \mathrm{Sr}$ & $5.00 \mathrm{E}+00$ & $\mu \mathrm{Ci} / \mathrm{g}$ \\
\hline TIC & TIC & $6.61 E+03$ & $\mu \mathrm{g} / \mathrm{g}$ \\
\hline TOC.coul & TOC.pers & $5.78 \mathrm{E}+03$ & $\mu \mathrm{g} / \mathrm{g}$ \\
\hline TOC.pers & TOC.pers & $3.75 \mathrm{E}+03$ & $\mu \mathrm{g} / \mathrm{g}$ \\
\hline${ }^{99} \mathrm{Tc}$ & ${ }^{99} \mathrm{Tc}$ & $2.32 \mathrm{E}-01$ & $\mu \mathrm{Ci} / \mathrm{g}$ \\
\hline
\end{tabular}


Table B3-41. Tank Mean Concentration - Based on Composite Sample Data (Per Weight Basis). (4 sheets)

\begin{tabular}{|c|c|c|c|}
\hline Supenuatant knable & Sall Slumy Analyte & 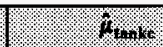 & . Uilis \\
\hline Ti.icp.a & Ti.icp.a & $4.72 \mathrm{E}+00^{*}$ & $\mu \mathrm{g} / \mathrm{g}$ \\
\hline Ti.icp.adl & Ti.icp.a & $5.16 \mathrm{E}+00^{*}$ & $\mu \mathrm{g} / \mathrm{g}$ \\
\hline T1.icp.a & Tl.icp.a & $9.45 \mathrm{E}+01^{*}$ & $\mu \mathrm{g} / \mathrm{g}$ \\
\hline Tl.icp.adl & Tl.icp.a & $1.03 \mathrm{E}+02^{*}$ & $\mu \mathrm{g} / \mathrm{g}$ \\
\hline Total Alpha & Total Alpha & $1.64 \mathrm{E}-02^{*}$ & $\mu \mathrm{Ci} / \mathrm{g}$ \\
\hline Total Beta & Total Beta & $4.46 \mathrm{E}+02$ & $\mu \mathrm{Ci} / \mathrm{g}$ \\
\hline Tritium.1 & Tritium.1 & $8.93 \mathrm{E}-04^{*}$ & $\mu \mathrm{Ci} / \mathrm{g}$ \\
\hline U.icp.a & U.icp.a & $2.36 \mathrm{E}+02^{*}$ & $\mu \mathrm{g} / \mathrm{g}$ \\
\hline U.icp.adl & U.icp.a & $2.58 \mathrm{E}+02^{*}$ & $\mu \mathrm{g} / \mathrm{g}$ \\
\hline U.phos & U.phos & $3.89 \mathrm{E}+01$ & $\mu \mathrm{g} / \mathrm{g}$ \\
\hline V.icp.a & V.icp.a & $2.36 \mathrm{E}+01^{*}$ & $\mu \mathrm{g} / \mathrm{g}$ \\
\hline V.icp.adl & V.icp.a & $2.59 \mathrm{E}+01^{*}$ & $\mu \mathrm{g} / \mathrm{g}$ \\
\hline Zn.icp.a & Zn.icp.a & $4.74 \mathrm{E}+01$ & $\mu \mathrm{g} / \mathrm{g}$ \\
\hline Zn.icp.adl & Zn.icp.a & $1.37 \mathrm{E}+01$ & $\mu \mathrm{g} / \mathrm{g}$ \\
\hline Zr.icp.a & Zr.icp.a & $6.98 \mathrm{E}+00^{*}$ & $\mu \mathrm{g} / \mathrm{g}$ \\
\hline Zr.icp.adl & Zr.icp.a & $7.43 \mathrm{E}+00^{*}$ & $\mu \mathrm{g} / \mathrm{g}$ \\
\hline
\end{tabular}

Note:

${ }^{\prime} \mathrm{Li}$ and ${ }^{~} \mathrm{Br}$ are the tracers for hydrostatic head fluid. 


\section{B4.0 APPENDIX B REFERENCES}

Dukelow, G. T., J. W. Hunt, H. Babad, and J. E. Meacham, 1995, Tank Safety Screening Data Quality Objective, WHC-SD-WM-SP-004, Rev. 2, Westinghouse Hanford Company, Richland, Washington.

Eggers, R. F., 1996, Tank 241-AN-105 Push Mode Core Sampling and Analysis Plan, WHC-SD-WM-TSAP-027, Rev. 1, Westinghouse Hanford Company, Richland, Washington.

Harville, D. A., 1977, "Maximum Likelihood Approaches to Variance Component Estimation and to Related Problems," Journal of the American Statistical Association, pp. 320-340.

Jansky, M. D., 1984, Laboratory Support for Upcoming 242-A Evaporator Campaign, (internal memorandum \#65453-84-134 to E. G. Gratny, May 10, 1984), Rockwell Hanford Operation, Richland, Washington.

Mauss, B. M., 1984, Chemical Compositions of 102-AY, 101-AW, 105-AN and 104-AW, (internal memorandum \#65453-84-348 to E. G. Gratny, November 9, 1984), Rockwell Hanford Operation, Richland, Washington.

McDuffie, N. G. and G. D Johnson, 1995, Flammable Gas Tank Safety Program: Data Requirements for Core Sample Analysis Developed Through the Data Quality Objectives (DQO) Process, WHC-SD-WM DQO-004, Rev. 2, Westinghouse Hanford Company, Richland, Washington.

Snedecor, G. W., and W. G. Cochran, 1980, Statistical Methods, 7th Edition, Iowa State University Press, Ames, Iowa.

Statistical Sciences, Inc. S-PLUS Reference Manual, Version 3.2, Seattle: StatSci, a division of MathSoft, Inc., 1993.

Steen, F. H., 1997, Final Report for Tank 241-AN-105, Cores 152 and 153, HNF-SD-WM-DP-199, Rev. 1, Rust Federal Services of Hanford, Inc., Richland, Washington. 
HNF-SD-WM-ER-678 Rev. 0

This page intentionally left blank. 
HNF-SD-WM-ER-678 Rev. 0

\section{APPENDIX C}

STATISTICAL ANALYSIS FOR ISSUE RESOLUTION

C-1 
HNF-SD-WM-ER-678 Rev. 0

This page intentionally left blank.

C-2 


\section{APPENDIX C}

\section{STATISTICAL ANALYSIS FOR ISSUE RESOLUTION}

In Appendix C, the results of analyses required for the applicable data quality objective (DQO) reports for tank 241-AN-105 are reported. Specifically, statistical and other numerical manipulations required in the DQO reports are documented in this appendix. The analysis required for tank 241-AN-105 are documented in the following sections:

- Section C1: Statistical analysis supporting the Safety Screening DQO (Dukelow et al. 1995). Specifically, confidence intervals were needed to support the DSC and plutonium (criticality) threshold limit.

\section{C1.0 STATISTICS FOR SAFETY SCREENING DATA QUALITY OBJECTIVE}

The safety screening DQO (Dukelow et al. 1995) defines acceptable decision confidence limits in terms of one-sided 95 percent confidence intervals. In this appendix, one-sided confidence limits supporting the safety screening DQO are calculated for tank 241-AN-105. The data in this section are from the 1996 sampling event for tank 241-AN-105.

Confidence intervals were computed for each sample number from tank 241-AN-105 analytical data (Steen 1997). The upper limit (UL) of a one-sided 95 percent confidence interval for the mean is

$$
\hat{\mu}+\mathrm{t}_{(\mathrm{dr}, 0.05)} * \hat{\sigma}_{\hat{\mu}} \text {. }
$$

In this equation, $\hat{\mu}$ is the arithmetic mean of the data, $\hat{\sigma}_{\hat{\mu}}$ is the estimate of the standard deviation of the mean, and $\mathrm{t}_{(\mathrm{df}, 0.05)}$ is the quantile from Student's $\mathrm{t}$ distribution with $d f$ degrees of freedom for a one-sided 95 percent confidence interval. For the tank 241-AN-105 data (per sample number), $d f$ equals the number of observations minus one.

\section{C1.1 DIFFERENTIAL SCANNING CALORIMETRY}

The upper limit of the 95 percent confidence interval was calculated for each subsample which had DSC measurements. The upper limit for each sample number is listed in Table C1-1. Each confidence interval can be used to make the following statement. If the upper limit is less than $480 \mathrm{~J} / \mathrm{g}$ dry, then one would reject the null hypothesis that DSC is 
greater than or equal to $480 \mathrm{~J} / \mathrm{g}$ dry at the 0.05 level of significance. All of the DSC dry upper limits were less than $480 \mathrm{~J} / \mathrm{g}$ dry. The hypothesis that the DSC results are greater than $480 \mathrm{~J} / \mathrm{g}$ dry is rejected for all the subsamples.

\section{C1.2 TOTAL ALPHA}

The upper limit of the 95 percent confidence interval was calculated for each subsample which had at least 50 percent of the reported total alpha data as quantitative values. The sample numbers and the upper limit of the 95 percent confidence intervals are listed in Table C1-2. Each confidence interval can be used to make the following statement. If the upper limit is less than $61.5 \mu \mathrm{Ci} / \mathrm{mL}$ or $37.0 \mu \mathrm{Ci} / \mathrm{g}$ (conversion used the maximum density of $1.66 \mathrm{~g} / \mathrm{mL}$ observed for the sludge subsamples), then one would reject the null hypothesis that total alpha is greater than or equal to $61.5 \mu \mathrm{Ci} / \mathrm{mL}$ (or $37.0 \mu \mathrm{Ci} / \mathrm{g}$ ) at the 0.05 level of significance. For all the subsamples with at least 50 percent of the reported total alpha data as quantitative values, the upper limits were less than $61.5 \mu \mathrm{Ci} / \mathrm{mL}$ or $37.0 \mu \mathrm{Ci} / \mathrm{g}$. For the subsamples where both analytical results were "less than" values, the maximum "less than" value is orders of magnitude smaller than either $61.5 \mu \mathrm{Ci} / \mathrm{mL}$ or $37.0 \mu \mathrm{Ci} / \mathrm{g}$. The hypothesis that the Total Alpha results are greater than $61.5 \mu \mathrm{Ci} / \mathrm{mL}$ or $37.0 \mu \mathrm{Ci} / \mathrm{g}$ is rejected for all subsamples. 
Table C1-1. Tank 241-AN-105 Differential Scanning Calorimetry Results (J/g dry). (2 sheets)

\begin{tabular}{|c|c|c|c|c|c|c|}
\hline Samuple Nurnber & Core & Segment & Wocrilon & 19. & 6 & (6) \\
\hline S96T003731 & 152 & 1 & Crust & $1.09 \mathrm{E}+01$ & $2.06 \mathrm{E}+00$ & $2.39 \mathrm{E}+01$ \\
\hline S96T003732 & 152 & 1 & $\mathrm{DL}$ & $0.00 \mathrm{E}+00$ & $0.00 \mathrm{E}+00$ & $0.00 \mathrm{E}+00$ \\
\hline S96T003739 & 152 & 2 & $\mathrm{DL}$ & $0.00 \mathrm{E}+00$ & $0.00 \mathrm{E}+00$ & $0.00 \mathrm{E}+00$ \\
\hline S96T003740 & 152 & 3 & $\overline{\mathrm{DL}}$ & $0.00 \mathrm{E}+00$ & $0.00 \mathrm{E}+00$ & $0.00 \mathrm{E}+00$ \\
\hline S96T003741 & 152 & 4 & $\mathrm{DL}$ & $0.00 \mathrm{E}+00$ & $0.00 \mathrm{E}+00$ & $0.00 \mathrm{E}+00$ \\
\hline S96T003742 & 152 & 5 & $\mathrm{DL}$ & $0.00 \mathrm{E}+00$ & $0.00 \mathrm{E}+00$ & $0.00 \mathrm{E}+00$ \\
\hline S96T003743 & 152 & 6 & $\mathrm{DL}$ & $0.00 \mathrm{E}+00$ & $0.00 \mathrm{E}+00$ & $0.00 \mathrm{E}+00$ \\
\hline S96T003744 & 152 & 7 & $\mathrm{DL}$ & $0.00 \mathrm{E}+00$ & $0.00 \mathrm{E}+00$ & $0.00 \mathrm{E}+00$ \\
\hline S96T003745 & 152 & 8 & $\mathrm{DL}$ & $0.00 \mathrm{E}+00$ & $0.00 \mathrm{E}+00$ & $0.00 \mathrm{E}+00$ \\
\hline S96T003746 & 152 & 9 & DL & $0.00 \mathrm{E}+00$ & $0.00 \mathrm{E}+00$ & $0.00 \mathrm{E}+00$ \\
\hline S96T003747 & 152 & 10 & $\overline{\mathrm{DL}}$ & $0.00 \mathrm{E}+00$ & $0.00 \mathrm{E}+00$ & $0.00 \mathrm{E}+00$ \\
\hline S96T003748 & 152 & 11 & $\overline{\mathrm{DL}}$ & $0.00 \mathrm{E}+00$ & $0.00 \mathrm{E}+00$ & $0.00 \mathrm{E}+00$ \\
\hline S96T003749 1 & 152 & 12 & $\mathrm{DL}$ & $1.08 \mathrm{E}+02$ & $2.27 \mathrm{E}+01$ & $2.51 \mathrm{E}+02$ \\
\hline S96T003750 & 152 & 13 & $\overline{\mathrm{DL}}$ & $0.00 \mathrm{E}+00$ & $0.00 \mathrm{E}+00$ & $0.00 \mathrm{E}+00$ \\
\hline S96T003751 & 152 & 14 & $\mathrm{DL}$ & $0.00 \mathrm{E}+00$ & $0.00 \mathrm{E}+00$ & $0.00 \mathrm{E}+00$ \\
\hline S96T004433 & 153 & 1 & DL & $2.84 \mathrm{E}+01$ & $3.15 \mathrm{E}+00$ & $4.82 \mathrm{E}+01$ \\
\hline S96T004308 & 153 & 2 & $\mathrm{DL}$ & $0.00 \mathrm{E}+00$ & $0.00 \mathrm{E}+00$ & $0.00 \mathrm{E}+00$ \\
\hline S96T004434 & 153 & 3 & $\mathrm{DL}$ & $3.77 \mathrm{E}+01$ & $3.15 \mathrm{E}+00$ & $5.75 \mathrm{E}+01$ \\
\hline S96T004419 & 153 & 5 & $\mathrm{DL}$ & $1.20 \mathrm{E}+02$ & $9.83 \mathrm{E}+01$ & $3.51 \mathrm{E}+02$ \\
\hline S96T004420 & 153 & 6 & DL & $0.00 \mathrm{E}+00$ & $0.00 \mathrm{E}+00$ & $0.00 \mathrm{E}+00$ \\
\hline S96T004421 & 153 & 7 & DL & $3.45 \mathrm{E}+01$ & $3.45 \mathrm{E}+01$ & $2.52 \mathrm{E}+02$ \\
\hline S96T003975 & 153 & 8 & DL & $0.00 \mathrm{E}+00$ & $0.00 \mathrm{E}+00$ & $0.00 \mathrm{E}+00$ \\
\hline S96T003976 & 153 & 9 & $\mathrm{DL}$ & $2.22 \mathrm{E}+00$ & $2.22 \mathrm{E}+00$ & $1.62 \mathrm{E}+01$ \\
\hline S96T004309 & 153 & 10 & $\mathrm{DL}$ & $3.24 \mathrm{E}+01$ & $5.85 \mathrm{E}+00$ & $6.93 \mathrm{E}+01$ \\
\hline S96T003979 & 153 & 11 & $\mathrm{DL}$ & $0.00 \mathrm{E}+00$ & $0.00 \mathrm{E}+00$ & $0.00 \mathrm{E}+00$ \\
\hline S96T003977 & 153 & 12 & DL & $5.25 \mathrm{E}+01$ & $5.25 \mathrm{E}+01$ & $3.84 \mathrm{E}+02$ \\
\hline S96T003978 & 153 & 13 & DL & $0.00 \mathrm{E}+00$ & $0.00 \mathrm{E}+00$ & $0.00 \mathrm{E}+00$ \\
\hline S96T004333 & 153 & 14 & $\mathrm{DL}$ & $9.21 \mathrm{E}+01$ & $2.30 \mathrm{E}+01$ & $2.37 \mathrm{E}+02$ \\
\hline S96T003980 & 153 & 21 & $\mathrm{DL}$ & $0.00 \mathrm{E}+00$ & $0.00 \mathrm{E}+00$ & $0.00 \mathrm{E}+00$ \\
\hline S96T003817 & 152 & 14 & $\mathrm{LH}$ & $2.13 \mathrm{E}+01$ & $7.60 \mathrm{E}+00$ & $6.93 \mathrm{E}+01$ \\
\hline
\end{tabular}


Table C1-1. Tank 241-AN-105 Differential Scanning Calorimetry

Results (J/g dry). (2 sheets)

\begin{tabular}{|c|c|c|c|c|c|c|}
\hline Sample Nworner & Core & Segrinen & Trocalion. & f. & 6. & OU. \\
\hline S96T003816 & 152 & 14 & UH & $4.96 \mathrm{E}+01$ & $3.95 \mathrm{E}+00$ & $7.45 \mathrm{E}+01$ \\
\hline S96T003793 & 152 & 16 & $\mathrm{LH}$ & $4.87 \mathrm{E}+01$ & $7.00 \mathrm{E}-01$ & $5.31 \mathrm{E}+01$ \\
\hline S96T003792 & 152 & 16 & $\mathrm{UH}$ & $5.54 \mathrm{E}+01$ & $1.20 \mathrm{E}+01$ & $1.31 \mathrm{E}+02$ \\
\hline S96T003795 & 152 & 18 & $\mathrm{LH}$ & $5.72 \mathrm{E}+01$ & $1.80 \mathrm{E}+00$ & $6.86 \mathrm{E}+01$ \\
\hline S96T003794 & 152 & 18 & $\mathrm{UH}$ & $4.50 \mathrm{E}+01$ & $1.95 \mathrm{E}+00$ & $5.73 \mathrm{E}+01$ \\
\hline S96T003797 & 152 & 20 & LH & $0.00 \mathrm{E}+00$ & $0.00 \mathrm{E}+00$ & $0.00 \mathrm{E}+00$ \\
\hline S96T003796 & 152 & 20 & UH & $7.19 \mathrm{E}+01$ & $1.10 \mathrm{E}+00$ & $7.88 \mathrm{E}+01$ \\
\hline S96T003798 & 152 & 22 & UH & $1.10 \mathrm{E}+02$ & $1.15 \mathrm{E}+01$ & $1.82 \mathrm{E}+02$ \\
\hline S96T004335 & 153 & 14 & LH & $6.53 \mathrm{E}+01$ & $7.45 \mathrm{E}+00$ & $1.12 \mathrm{E}+02$ \\
\hline S96T004311 & 153 & 15 & LH & $9.48 \mathrm{E}+01$ & $2.33 \mathrm{E}+01$ & $2.42 \mathrm{E}+02$ \\
\hline S96T004310 & 153 & 15 & $\mathrm{UH}$ & $6.16 \mathrm{E}+01$ & $1.58 \mathrm{E}+01$ & $1.61 \mathrm{E}+02$ \\
\hline S96T004436 & 153 & 17 & LH & $3.14 \mathrm{E}+01$ & $3.35 \mathrm{E}+00$ & $5.25 \mathrm{E}+01$ \\
\hline S96T004435 & 153 & 17 & UH & $1.22 \mathrm{E}+02$ & $3.89 \mathrm{E}+01$ & $3.68 \mathrm{E}+02$ \\
\hline S96T004437 & 153 & 19 & LH & $6.16 \mathrm{E}+01$ & $1.97 \mathrm{E}+01$ & $1.86 \mathrm{E}+02$ \\
\hline S96T003981 & 153 & 21 & LH & $3.59 \mathrm{E}+01$ & $2.55 \mathrm{E}+00$ & $5.20 \mathrm{E}+01$ \\
\hline S96T003982 & 153 & 22 & LH & $1.40 \mathrm{E}+02$ & $3.40 \mathrm{E}+01$ & $3.55 \mathrm{E}+02$ \\
\hline S96T003983 & 153 & 22 & UH & $7.79 \mathrm{E}+01$ & $8.45 \mathrm{E}+00$ & $1.31 \mathrm{E}+02$ \\
\hline S96T004039 & 152 & $1-14$ & Comp. & $0.00 \mathrm{E}+00$ & $0.00 \mathrm{E}+00$ & $0.00 \mathrm{E}+00$ \\
\hline S96T004254 & 152 & $14-20$ & Comp. & $6.95 \mathrm{E}+01$ & $1.11 \mathrm{E}+01$ & $1.39 \mathrm{E}+02$ \\
\hline S96T004259 & 152 & 22 & Comp. & $1.00 \mathrm{E}+02$ & $5.75 \mathrm{E}+00$ & $1.37 \mathrm{E}+02$ \\
\hline
\end{tabular}

Notes:

$\begin{array}{lll}\mathrm{DL} & = & \text { Drainable liquid } \\ \mathrm{LH} & = & \text { Lower half } \\ \mathrm{UH} & = & \text { Upper half } \\ \text { Comp. } & = & \text { Composite }\end{array}$


Table C1-2. Tank 241-AN-105 Total Alpha Results. (2 sheets)

\begin{tabular}{|c|c|c|c|c|c|c|}
\hline $\begin{array}{l}\text { Sample: } \\
\text { Namber. }\end{array}$ & Coro & Segmont & Ilacallion & Maginam & d. & 14. \\
\hline \multicolumn{7}{|l|}{ Liquid Samples } \\
\hline S96T003732* & 152 & 1 & $\overline{\mathrm{DL}}$ & $2.26 \mathrm{E}-02$ & $2.50 \mathrm{E}-04$ & $2.41 \mathrm{E}-02$ \\
\hline S96T003739 & 152 & 2 & $\overline{\mathrm{DL}}$ & $<2.72 \mathrm{E}-02$ & NA & NA \\
\hline S96T003740* & 152 & 3 & $\overline{\mathrm{DL}}$ & $2.99 \mathrm{E}-02$ & $1.34 \mathrm{E}-02$ & $1.15 \mathrm{E}-01$ \\
\hline S96T003741 & 152 & 4 & $\mathrm{DL}$ & $<2.47 \mathrm{E}-02$ & NA & $\mathrm{NA}$ \\
\hline S96T003742 & 152 & 5 & $\mathrm{DL}$ & $<2.28 \mathrm{E}-02$ & NA & NA \\
\hline S96T003743 & 152 & 6 & $\mathrm{DL}$ & $<4.23 \mathrm{E}-02$ & NA & NA \\
\hline S96T003744* & 152 & 7 & $\mathrm{DL}$ & $1.96 \mathrm{E}-02$ & $1.95 \mathrm{E}-03$ & $3.19 \mathrm{E}-02$ \\
\hline S96T003745 & 152 & 8 & $\mathrm{DL}$ & $<2.74 \mathrm{E}-02$ & NA & NA \\
\hline S96T003746 & 152 & 9 & $\overline{\mathrm{DL}}$ & $<2.74 \mathrm{E}-02$ & NA & NA \\
\hline S96T003747 & 152 & 10 & $\mathrm{DL}$ & $<3.33 \mathrm{E}-02$ & $\mathrm{NA}$ & NA \\
\hline S96T003748 & 152 & 11 & $\mathrm{DL}$ & $<8.27 \mathrm{E}-02$ & NA & NA \\
\hline S96T003749 & 152 & 12 & $\mathrm{DL}$ & $<5.59 \mathrm{E}-02$ & $\overline{\mathrm{NA}}$ & NA \\
\hline S96T003750 & 152 & 13 & $\mathrm{DL}$ & $<4.68 \mathrm{E}-02$ & NA & NA \\
\hline S96T003751 & 152 & 14 & $\mathrm{DL}$ & $<7.97 \mathrm{E}-02$ & NA & NA \\
\hline S96T004433 & 153 & 1 & $\overline{\mathrm{DL}}$ & $<2.86 \mathrm{E}-02$ & NA & NA \\
\hline S96T004308 & 153 & 2 & $\mathrm{DL}$ & $<1.36 \mathrm{E}-02$ & $\mathrm{NA}$ & NA \\
\hline S96T004434 & 153 & 3 & $\mathrm{DL}$ & $<1.87 \mathrm{E}-02$ & NA & NA \\
\hline S96T004419 & 153 & 5 & $\mathrm{DL}$ & $<1.87 \mathrm{E}-02$ & NA & $\mathrm{NA}$ \\
\hline S96T004420 & 153 & 6 & $\mathrm{DL}$ & $<1.54 \mathrm{E}-02$ & NA & $\mathrm{NA}$ \\
\hline S96T004421 & 153 & 7 & $\mathrm{DL}$ & $<1.42 \mathrm{E}-02$ & NA & NA \\
\hline S96T003975 & 153 & 8 & $\mathrm{DL}$ & $<1.45 \mathrm{E}-01$ & $\mathrm{NA}$ & NA \\
\hline S96T003976 * & 153 & 9 & $\mathrm{DL}$ & $3.71 \mathrm{E}-02$ & $7.70 \mathrm{E}-03$ & $8.57 \mathrm{E}-02$ \\
\hline S96T004309 & 153 & 10 & $\overline{\mathrm{DL}}$ & $<7.10 \mathrm{E}-03$ & NA & NA \\
\hline S96T003979 & 153 & 11 & $\mathrm{DL}$ & $<3.29 \mathrm{E}-02$ & NA & NA \\
\hline S96T003977 & 153 & 12 & $\mathrm{DL}$ & $<3.29 \mathrm{E}-02$ & $\mathrm{NA}$ & NA \\
\hline S96T003978 & 153 & 13 & $\mathrm{DL}$ & $<2.58 \mathrm{E}-02$ & NA & NA \\
\hline S96T004333 * & 153 & 14 & $\mathrm{DL}$ & $1.06 \mathrm{E}-02$ & $3.45 \mathrm{E}-03$ & $3.23 \mathrm{E}-02$ \\
\hline S96T003980 & 153 & 21 & $\mathrm{DL}$ & $<2.22 \mathrm{E}-02$ & NA & NA \\
\hline S96T004039 & 152 & $1-14$ & Comp. & $<1.54 \mathrm{E}-02$ & NA & NA \\
\hline
\end{tabular}


Table C1-2. Tank 241-AN-105 Total Alpha Results. (2 sheets)

\begin{tabular}{|c|c|c|c|c|c|c|}
\hline $\begin{array}{l}\text { Sample } \\
\text { Number. }\end{array}$ & Coro & segnent & 19eralion & $\begin{array}{l}\text { i or } \\
\text { Marimun }\end{array}$ & q. & w. \\
\hline \multicolumn{7}{|c|}{ Sludge samples $(\mu \mathrm{Ci} / \mathrm{g})$} \\
\hline S96T003893 & 152 & 1 & $\overline{\mathrm{UH}}$ & $<2.28 \mathrm{E}-02$ & NA & NA \\
\hline S96T003827 & 152 & 14 & $\mathrm{LH}$ & $<2.67 \mathrm{E}-02$ & NA & NA \\
\hline S96T003889 & 152 & 16 & $\overline{\mathrm{LH}}$ & $<3.16 \mathrm{E}-02$ & NA & NA \\
\hline S96T003890 & 152 & 18 & $\mathrm{LH}$ & $<1.92 \mathrm{E}-02$ & NA & NA \\
\hline S96T003891 & 152 & 20 & LH & $7.49 \mathrm{E}-03$ & $2.65 \mathrm{E}-04$ & $9.16 \mathrm{E}-03$ \\
\hline S96T003892 & 152 & 22 & $\overline{\mathrm{UH}}$ & $1.27 \mathrm{E}-02$ & $1.30 \mathrm{E}-03$ & $2.09 \mathrm{E}-02$ \\
\hline S96T004337* & 153 & 14 & LH & $3.97 \mathrm{E}-03$ & $1.25 \mathrm{E}-04$ & $4.75 \mathrm{E}-03$ \\
\hline S96T004317* & 153 & 15 & $\mathrm{LH}$ & $4.56 \mathrm{E}-03$ & $7.90 \mathrm{E}-04$ & $9.55 \mathrm{E}-03$ \\
\hline S96T004442 & 153 & 17 & $\overline{\mathrm{LH}}$ & $7.04 \mathrm{E}-03$ & $1.10 \mathrm{E}-04$ & $7.73 \mathrm{E}-03$ \\
\hline S96T004443 & 153 & 19 & $\mathrm{LH}$ & $1.45 \mathrm{E}-02$ & $3.15 \mathrm{E}-03$ & $3.43 \mathrm{E}-02$ \\
\hline S96T003984 & 153 & 21 & $\mathrm{LH}$ & $7.95 \mathrm{E}-03$ & $5.30 \mathrm{E}-04$ & $1.13 \mathrm{E}-02$ \\
\hline S96T003985 & 153 & 22 & $\overline{L H}$ & $1.25 \mathrm{E}-01$ & $8.50 \mathrm{E}-03$ & $1.78 \mathrm{E}-01$ \\
\hline S96T004256 & 152 & $14-20$ & Comp. & $1.36 \mathrm{E}-01$ & $1.60 \mathrm{E}-03$ & $2.37 \mathrm{E}-02$ \\
\hline S96T004261 & 152 & 22 & Comp. & $5.80 \mathrm{E}-02$ & $7.95 \mathrm{E}-03$ & $1.08 \mathrm{E}-01$ \\
\hline
\end{tabular}

Notes:

$\begin{array}{lll}\mathrm{DL} & = & \text { Drainable liquid } \\ \mathrm{LH} & = & \text { Lower half } \\ \mathrm{UH} & = & \text { Upper half } \\ * & = & \text { One of the two results was a less than value; it was treated as a real number. } \\ \text { Comp. } & = & \text { Composite }\end{array}$




\section{C2.0 APPENDIX C REFERENCES}

Dukelow, G. T., J. W. Hunt, H. Babad, and J. E. Meacham, 1995, Tank Safety Screening Data Quality Objective, WHC-SD-WM-SP-004, Rev. 2, Westinghouse Hanford Company, Richland, Washington.

Steen, F. H., 1997, Final Report for Tank 241-AN-105, Cores 152 and 153, HNF-SD-WM-DP-199, Rev. 1, Fluor Daniel Hanford, Inc., Richland, Washington. 
HNF-SD-WM-ER-678 Rev. 0

This page intentionally left blank.

C-10 
APPENDIX D

EVALUATION TO ESTABLISH BEST-BASIS

INVENTORY FOR TANK 241-AN-105

D-1 
HNF-SD-WM-ER-678 Rev. 0

This page intentionally left blank. 


\section{APPENDIX D}

\section{EVALUATION TO ESTABLISH BEST-BASIS INVENTORY FOR TANK 241-AN-105}

An effort is underway to provide waste inventory estimates that will serve as standard characterization source terms for the various waste management activities (Hodgson and LeClair 1996). As part of this effort, an evaluation of available information for tank 241-AN-105 was performed, and a best-basis inventory was established. This work, detailed in the following sections, follows the methodology that was established by the standard inventory task.

\section{D1.O CHEMICAL INFORMATION SOURCES}

Available composition information for the waste in tank $241-\mathrm{AN}-105$ is as follows:

- Steen (1997) and Appendix B of this report provides characterization results from the June 1996 core sampling event.

- A feed projection document that supported grout treatment facility studies provides estimates of the waste in tank $241-\mathrm{AN}-105$ based on 242-A Evaporator slurry product data (Hendrickson 1994).

- The HDW model document (Agnew et al. 1997) provides tank content estimates in terms of component concentrations and inventories. A complete list of data sources used in this evaluation is provided at the end of this section.

A complete list of data sources used in this evaluation is provided at the end of this section.

\section{D2.0 COMPARISON OF COMPONENT INVENTORY VALUES}

There have been no transfers (with exception of a small amount of flush water) into or out of tank 241-AN-105 since 1985. The HDW model provides composition estimates for the waste in tank 241-AN-105 as of January 1, 1994. Sample-based inventories derived from the June 1996 core samples and inventories generated by the HDW model (Agnew et al. 1997), are compared in Tables D2-1 and D2-2. A tank volume of $4,270 \mathrm{~kL}(1,129 \mathrm{kgal})$ was used to 
generate the sample-based and HDW inventories. The sample based densities are $1.42 \mathrm{~g} / \mathrm{mL}$ for the liquid and $1.59 \mathrm{~g} / \mathrm{mL}$ for the salt slurry. The sample based bulk density is 1.56 $\mathrm{g} / \mathrm{mL}$. The HDW model bulk density is $1.88 \mathrm{~g} / \mathrm{mL}$. The sample-based inventories were taken from the inventory tables in Section B3.4.3. The liquid comprises 60 percent of the total volume; the salt slurry occupies 40 percent. The inventories that included the less than values (those having a "w" extension) but excluded the outliers ("ed") were used in this assessment.

Table D2-1. Sampling and Hanford Defined Waste Model Inventory Estimates for Nonradioactive Components in Double-Shell

Tank 241-AN 105 (2 sheets)

\begin{tabular}{|c|c|c|c|c|}
\hline Analyte & $\begin{array}{l}\text { June } 1996 \\
\text { Inventory based } \\
\text { on segment data } \\
\text { from both core } \\
\text { samples }(\mathrm{kg})\end{array}$ & $\begin{array}{c}\text { June } 1996 \\
\text { Inventory } \\
\text { based on } \\
\text { composite of } \\
\text { core } 152(\mathrm{~kg})\end{array}$ & $\begin{array}{l}\text { HDW Model } \\
\text { Inventory }(\mathrm{kg})\end{array}$ & $\begin{array}{l}\text { Inventory based } \\
\text { on Evaporator } \\
\text { post-run data } \\
\text { (kg) }\end{array}$ \\
\hline $\mathrm{Al}$ & 168,000 & 145,000 & 271,000 & 201,000 \\
\hline $\mathrm{Bi}$ & 196 & $<232$ & 1,670 & NR \\
\hline $\mathrm{Ca}$ & $<1,880$ & 2,860 & 9,560 & NR \\
\hline $\mathrm{Cl}^{-}$ & 40,100 & 41,500 & 54,800 & 36,400 \\
\hline $\mathrm{CO}_{3}^{2-}$ & NR & 197,000 & 234,000 & 93,000 \\
\hline $\mathrm{Cr}$ & 4,890 & 5,200 & 35,600 & 2,890 \\
\hline $\mathrm{F}^{-}$ & 4,800 & 2,900 & 8,720 & NR \\
\hline $\mathrm{Fe}$ & $<263$ & 311 & 3,730 & 47.1 \\
\hline $\mathrm{Hg}$ & NR & NR & 11.8 & NR \\
\hline $\mathrm{K}$ & 26,100 & 24,700 & 17,800 & 26,200 \\
\hline $\mathrm{La}$ & $<144$ & $<131$ & 11.8 & NR \\
\hline $\mathrm{Mn}$ & $<71.8$ & $<47.1$ & 2,200 & NR \\
\hline $\mathrm{Na}$ & $1.13 \mathrm{E}+06$ & $1.07 \mathrm{E}+06$ & $2.02 \mathrm{E}+06$ & $1.18 \mathrm{E}+06$ \\
\hline $\mathrm{Ni}$ & $<141$ & 116.35 & 2,660 & NR \\
\hline $\mathrm{NO}_{2}^{-}$ & 485,000 & 523,000 & 609,000 & 513,000 \\
\hline $\mathrm{NO}_{3}$ & 712,000 & 744,000 & $1.88 \mathrm{E}+06$ & 826,000 \\
\hline $\mathrm{OH}^{-}$ & NR & 214,000 & 997,000 & 264,000 \\
\hline
\end{tabular}


Table D2-1. Sampling and Hanford Defined Waste Model Inventory Estimates for Nonradioactive Components in Double-Shell

Tank 241-AN 105 (2 sheets)

\begin{tabular}{|l|l|l|l|l|}
\hline Analyte & $\begin{array}{c}\text { June } 1996 \\
\text { Inventory based } \\
\text { on segment data } \\
\text { from both core } \\
\text { samples (kg) }\end{array}$ & $\begin{array}{c}\text { June } 1996 \\
\text { Inventory } \\
\text { based on } \\
\text { composite of } \\
\text { core } 152 \text { (kg) }\end{array}$ & $\begin{array}{l}\text { HDW Model } \\
\text { Inventory (kg) }\end{array}$ & $\begin{array}{l}\text { Inventory based } \\
\text { on Evaporator } \\
\text { post-run data } \\
\text { (kg) }\end{array}$ \\
\hline $\mathrm{Pb}$ & 266 & $<264$ & 1,540 & $\mathrm{NR}$ \\
\hline $\mathrm{PO}_{4}^{3-}$ & 23,600 & 16,800 & 74,700 & 8,110 \\
\hline $\mathrm{Si}$ & 920 & 980 & 13,600 & $\mathrm{NR}$ \\
\hline $\mathrm{SO}_{4}^{2-}$ & 24,100 & 21,700 & 159,000 & 28,000 \\
\hline $\mathrm{Sr}$ & 40 & $<24.2$ & 0 & $\mathrm{NR}$ \\
\hline $\mathrm{TOC}$ & $\mathrm{NR}$ & 30,000 & 109,000 & 14,400 \\
\hline $\mathrm{U}$ & $\mathrm{NR}$ & 242 & 13,800 & $\mathrm{NR}$ \\
\hline $\mathrm{Zr}$ & $<46.4$ & $<40.7$ & 149 & $\mathrm{NR}$ \\
\hline$\%$ water & 46.6 & 46.6 & 17.2 & $\mathrm{NR}$ \\
\hline density, g/mL & 1.56 & 1.56 & 1.88 & 1.50 \\
\hline
\end{tabular}

Notes:

$$
\begin{aligned}
& \text { NR = not reported } \\
& { }^{1} \text { Hendrickson } 1994 .
\end{aligned}
$$


Table D2-2. Sampling and Hanford Defined Waste Model Inventory Estimates for Radioactive Components in Double-Shell Tank 241-AN-105.

(Decayed to January 1, 1994)

\begin{tabular}{|c|c|c|c|c|}
\hline Analyte & $\begin{array}{l}\text { June } 1996 \\
\text { inventory based } \\
\text { on segment data } \\
\text { from both core } \\
\text { samples (Ci) }\end{array}$ & $\begin{array}{l}\text { June } 1996 \\
\text { inventory based } \\
\text { on composite of } \\
\text { core } 152 \text { (Ci) }\end{array}$ & $\begin{array}{l}\text { HDW model } \\
\text { inventory }(\mathrm{Ci})\end{array}$ & $\begin{array}{l}\text { Inventory based } \\
\text { on evaporator } \\
\text { post-run data }\end{array}$ \\
\hline${ }^{60} \mathrm{Co}$ & NR & $<6.46$ & 253 & NR \\
\hline${ }^{90} \mathrm{Sr}$ & NR & 33,600 & 665,000 & 13,800 \\
\hline${ }^{90} \mathrm{Y}$ & NR & 33,600 & 665,000 & NR \\
\hline${ }^{99} \mathrm{Tc}$ & NR & 1,174 & 1,500 & NR \\
\hline${ }^{129} \mathrm{~T}$ & NR & 11.0 & 2.89 & NR \\
\hline${ }^{137} \mathrm{Cs}$ & $\mathrm{NR}$ & 84,800 & $1.48 \mathrm{E}+06$ & 215,000 \\
\hline${ }^{137} \mathrm{mBa}$ & NR & 80,500 & $1.41 \mathrm{E}+06$ & NR \\
\hline${ }^{154} \mathrm{Eu}$ & NR & $<565$ & 4,020 & NR \\
\hline${ }^{155} \mathrm{Eu}$ & NR & $<2,250$ & 1,660 & NR \\
\hline${ }^{237} \mathrm{~Np}$ & NR & $<25.8$ & 5.28 & NR \\
\hline${ }^{239 / 240} \mathrm{Pu}$ & $\mathrm{NR}$ & 6.42 & 359 & 44.1 \\
\hline${ }^{241} \mathrm{Am}$ & NR & 10.1 & 429 & NR \\
\hline${ }^{244} \mathrm{Cm}$ & NR & $<4.27$ & 0.92 & NR \\
\hline
\end{tabular}

Note:

${ }^{1}$ Hendrickson 1994. 
The HDW model estimates for tank 241-AN-105 were derived by combining the supernatant streams that were believed to have been concentrated in the 242-A evaporator and then sent to tank 241-AN-105. This part of the HDW model is called the supernatant mixing model (SMM). The compositions of the supernatants are derived largely from bulk compositions of process waste streams that left the various processing facilities. To obtain the supernatant fractions, these bulk compositions are multiplied by solubility factors determined from the highest concentrations found among supernatant sample analyses. The higher inventories HDW model inventories for tank 241-AN-105 and other DST supernatants and slurries, when compared to their respective laboratory sample results, appear to be due to overestimation of the solubility of waste constituents in the process waste streams.

The periods in which tank 241-AN-105 received DSSF from the 242-A Evaporator and other waste tanks are accurately known (See Table A3-1, Appendix A). Slurry product concentration data from 242-A Evaporator post run documents can be obtained for each batch of feed processed. This data combined with the fill history of tank 241-AN-105 can be used to estimate the composition of tank 241-AN-105. This can serve as a check against the sample-based and HDW model inventories.

In 1994, an analysis of the DSSF in tanks 241-AN-104 and 241-AN-105 was performed (Hendrickson 1994). The results for tank 241-AN-105 are shown in column 5 of Tables D2-1 and D2-2. This table demonstrates close agreement between 242-A evaporator slurry data and the June 1996 core samples for all major components including $\mathrm{Na}, \mathrm{OH}^{-}, \mathrm{NO}_{2}^{-}$, and $\mathrm{NO}_{3}$. For $\mathrm{K}$, and $\mathrm{SO}_{4}^{2-}$, components present in lower concentrations, the June 1996 sample inventories and the inventories calculated from evaporator data are essentially the same. Al, $\mathrm{Cl}^{-}, \mathrm{Cr}, \mathrm{Fe}, \mathrm{PO}_{4}^{3-}, \mathrm{TOC},{ }^{90} \mathrm{Sr}$, and ${ }^{239 / 240} \mathrm{Pu}$ inventories determined from the evaporator post-run data are also in closer agreement to the June 1996 data than the HDW model.

\section{D3.0 COMPONENT INVENTORY EVALUATION}

The evaluation of the core sample data in Appendix B noted that the relative percent differences (RPD) between the samples and duplicates, and spike recoveries for a number of analytes during individual segment analyses were outside the specified ranges (See Section B2.0). These discrepancies were attributed to the heterogeneity of the waste. Another factor for analytes such as ${ }^{241} \mathrm{Am}, \mathrm{Ca}, \mathrm{Li}, \mathrm{Fe}, \mathrm{Zr}, \mathrm{Si}, \mathrm{Mn}$, and $\mathrm{Ni}$, whose RPDs were outside the required range is that their segment concentrations were at or near the detection limits. In Table D3-1, inventories for the supernatant and salt slurry portions based on the means of the segment data for both cores and the Core 152 composite are shown. Core 152 was taken from riser $12 \mathrm{~A}$ near the center of the tank and Core 153 was taken from riser $7 \mathrm{~B}$ twenty feet southwest of the center. 
Table D3-1. Comparison of June 1996 Core Sample-based Inventories.

\begin{tabular}{|c|c|c|c|c|c|c|}
\hline Wratros & 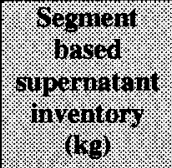 & 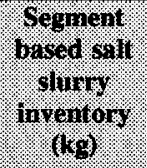 & 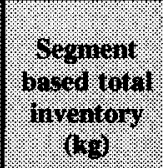 & 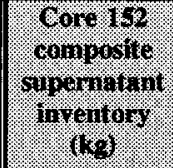 & 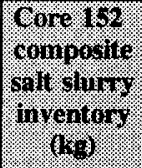 & 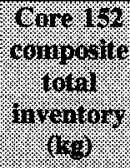 \\
\hline $\mathrm{Al}$ & 107,000 & 59,600 & 168,000 & 91,500 & 53,300 & 144,500 \\
\hline $\mathrm{Bi}$ & NR & 196 & 196 & $<134$ & $<97.7$ & $<232$ \\
\hline $\mathrm{Ca}$ & $<154$ & 1,730 & 1,880 & 2,250 & 606 & 2,860 \\
\hline $\mathrm{Cl}$ & 26,200 & 13,900 & 40,100 & 26,100 & 15,350 & 41,500 \\
\hline $\mathrm{CO}_{3}$ & NR & NR & NR & 28,100 & 169,000 & 197,000 \\
\hline $\mathrm{Cr}$ & 576 & 4,310 & 4,890 & 570 & 4,620 & 5,200 \\
\hline F & 752 & 4,050 & 4,800 & $<138$ & 2,760 & 2,900 \\
\hline $\mathrm{Fe}$ & $<77.1$ & 186 & 263 & 163 & 148 & 311 \\
\hline $\mathrm{K}$ & 16,800 & 9,300 & 26,100 & 16,700 & 8,040 & 24,700 \\
\hline $\mathrm{La}$ & $<77.1$ & $<66.7$ & $<144$ & $<77.1$ & $<54.02$ & $<131$ \\
\hline $\mathrm{Mn}$ & $<15.1$ & 56.7 & $<71.8$ & $<15.4$ & 31.7 & $<47.1$ \\
\hline $\mathrm{Na}$ & 633,000 & 493,000 & $1.13 E+06$ & 655,000 & 418,000 & $1.07 \mathrm{E}+06$ \\
\hline $\mathrm{Ni}$ & $<30.7$ & $110^{n}$ & 141 & $<30.7$ & 85.6 & 116 \\
\hline $\mathrm{NO}_{2}$ & 313,000 & 172,000 & 485,000 & 334,000 & 189,000 & 523,000 \\
\hline $\mathrm{NO}_{3}$ & 412,000 & 300,000 & 712,000 & 429,000 & 315,000 & 744,000 \\
\hline $\mathrm{OH}$ & NR & NR & NR & 150,000 & 64,000 & 214,000 \\
\hline $\mathrm{Pb}$ & 138 & 128 & 266 & $<154$ & $<110$ & $<264$ \\
\hline $\mathrm{PO}_{4}$ & 3,020 & 20,500 & 23,600 & 2,670 & 14,100 & 16,800 \\
\hline $\mathrm{Si}$ & 559 & 361 & 920 & 425 & 555 & 980 \\
\hline $\mathrm{SO}_{4}$ & 7,190 & 16,900 & 24,100 & 2,480 & 19,200 & 21,700 \\
\hline $\mathrm{Sr}$ & NR & 26.6 & 40 & $<13.4$ & $<10.1$ & $<24.2$ \\
\hline TOC & NR & NR & NR & 15,600 & 14,400 & 30,000 \\
\hline U & NR & NR & NR & 9.13 & 233 & 242 \\
\hline $\mathrm{Zr}$ & $<15.6$ & 30.8 & 46.4 & $<15.4$ & 25.3 & $<40.7$ \\
\hline
\end{tabular}

Note:

"Based on acid digest. $\mathrm{Ni}$ concentrations from fusion analyses are biased from $\mathrm{Ni}$ in the fusion crucible. 
For major components ( $\mathrm{Al}, \mathrm{Na}, \mathrm{NO}_{2}^{-}, \mathrm{NO}_{3}{ }^{-}$), the RPDs between the total inventories are less than 15 percent. For analytes with RPDs $<20$ percent, an average of the results in columns 4 and 7 are assumed to be appropriate for the best basis inventory. Generally, the mean concentrations from the segment data should be more accurate than the single core composite data because these means are based on concentrations taken from both cores. Reconciliation for analytes with RPDs $>20$ percent are provided in the list below.

\section{Calcium (Ca)}

The RPD for the two total inventories is 41 percent. From Table B2-38, Ca liquid concentrations in all of the segments from both cores are below the detection limit $(<42.3 \mu \mathrm{g} / \mathrm{g})$. The Ca liquid concentration in the Core 152 composite is $618 \mu \mathrm{g} / \mathrm{g}$. This number may be in error and may be a result of solids being present in the sample and included in the acid digestion. The $<154 \mathrm{~kg}$ value from the segment-based inventory is used for the best basis.

The salt slurry inventory for $\mathrm{Ca}$ in the segment-based sample is 2.85 times higher than the composite-based inventory. Table B2-31 for the solids acid digest results suggests horizontal heterogeneity between Core 152 and Core 153. An average of the salt slurry inventories in Table D3-1 is used in the best-basis inventory.

\section{Fluoride (F)}

The RPD for the two total inventories is 49 percent. Concentrations for $F$ from the segment data vary considerably from segment to segment for both cores. An average of the segment-based data and the core 152 composite is used to determine the best-basis inventory.

\section{Manganese (Mn)}

The RPD for the two total inventories is 42 percent. The calculated inventories are < $71.8 \mathrm{~kg}$ and $<47.1 \mathrm{~kg}$. Both cores show wide variation in $\mathrm{Mn}$ concentrations in the salt slurry segment concentrations. An average of the segment-based data and the core 152 composite is used to determine the best-basis inventory.

\section{Phosphate $\left(\mathrm{PO}_{4}{ }^{3}\right)$}

The RPD for $\mathrm{PO}_{4}{ }^{3-}$ for the two total inventories is 34 percent. The segment-based inventories for both the supernatant and the salt slurry are higher than the composite for Core 152. Table B2-59 shows that Core 153 contained higher $\mathrm{PO}_{4}$ concentrations than Core 152 , implying horizontal heterogeneity. An average of the segment-based data and the core 152 composite is used to determine the best-basis inventory. 


\section{D4.0 DEFINE THE BEST-BASIS AND ESTABLISH COMPONENT INVENTORIES}

Inventories based on the June 1996 sampling event should serve as the basis for the best estimate inventory to tank $241-\mathrm{AN}-105$ for the following reasons:

1. The June 1996 sampling event provides the most recent data for the waste.

2. Estimates based on 242-A Evaporator product sample data agrees with the June 1996 data.

3. The HDW model estimate overestimates slurry and supernatant inventories due to solubility assumptions that are biased high.

Best-basis inventory estimates for tank 241-AN-105 are presented in Tables D4-1 and D4-2. Radionuclide values are decayed to January 1, 1994.

Table D4-1. Best-Basis Inventory Estimates for Nonradioactive Components in Tank 241-AN-105 Supernatant as of May 31, 1996. (2 sheets)

\begin{tabular}{|c|c|c|c|}
\hline Analyte & $\begin{array}{l}\text { Superuatiant } \\
\text { inventory }(\mathrm{kg})\end{array}$ & (8, Misis or bi) & 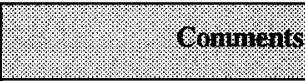 \\
\hline $\mathrm{Al}$ & 99,200 & $\mathrm{~S}$ & \\
\hline $\mathrm{Bi}$ & $<134$ & $S$ & \\
\hline $\mathrm{Ca}$ & $<154$ & $S$ & \\
\hline $\mathrm{Cl}$ & 26,100 & $S$ & \\
\hline TIC as $\mathrm{CO}_{3}$ & 26,200 & $S$ & Core composite data. \\
\hline $\mathrm{Cr}$ & 573 & $S$ & \\
\hline$F$ & 445 & $\mathrm{~S}$ & RPD $>20$ percent \\
\hline $\mathrm{Fe}$ & 120 & $S$ & Segment-based $<77.1$ \\
\hline $\mathrm{K}$ & 16,700 & $S$ & \\
\hline $\mathrm{La}$ & $<77.1$ & $S$ & \\
\hline $\mathrm{Mn}$ & $<15.2$ & S & \\
\hline $\mathrm{Na}$ & 644,000 & S & \\
\hline $\mathrm{Ni}$ & $<30.7$ & $S$ & \\
\hline $\mathrm{NO}_{2}$ & 323,000 & $S$ & \\
\hline $\mathrm{NO}_{3}$ & 420,500 & S & \\
\hline $\mathrm{OH}$ & 150,000 & $S$ & Core composite data. \\
\hline
\end{tabular}


Table D4-1. Best-Basis Inventory Estimates for Nonradioactive Components in Tank 241-AN-105 Supernatant as of May 31, 1996. (2 sheets)

\begin{tabular}{|c|c|c|c|}
\hline Analyte & $\begin{array}{l}\text { Sivperinatian: } \\
\text { inventory (ke) }\end{array}$ & (S. M. M. or E) & (1.5. \\
\hline $\mathrm{Pb}$ & 146 & $S$ & Composite $<154$ \\
\hline$\overline{\mathrm{PO}_{4}}$ & 2,850 & $S$ & \\
\hline $\mathrm{Si}$ & 492 & $S$ & \\
\hline $\mathrm{SO}_{4}$ & 4,840 & $S$ & \\
\hline $\mathrm{Sr}$ & $<13.4$ & $S$ & \\
\hline TOC & 15,600 & $S$ & Core composite data \\
\hline$\widetilde{\mathrm{U}_{\text {TOTAL }}}$ & 9.13 & $\mathrm{~S}$ & Core composite data \\
\hline $\mathrm{Zr}$ & $<15.5$ & $S$ & \\
\hline Percent water & 51.9 & $\mathrm{~S}$ & \\
\hline Density, $\mathrm{g} / \mathrm{mL}$ & 1.42 & S & \\
\hline
\end{tabular}

Notes:

${ }^{1} \mathrm{~S}=$ Sample-based, $\mathrm{M}=$ Hanford defined waste model-based, $\mathrm{E}=$ Engineering assessment-based.

Table D4-2. Best-Basis Inventory Estimates for Radioactive Components in Tank 241-AN-105 Supernatant as of May 31, 1996.

(Decayed to January 1, 1994) (2 sheets)

\begin{tabular}{|c|c|c|}
\hline f. & Supematant intentory (C)) & 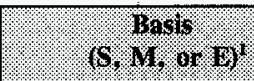 \\
\hline${ }^{60} \mathrm{Co}$ & $<0.050$ & $S$ \\
\hline${ }^{90} \mathrm{Sr}$ & 112 & $S$ \\
\hline${ }^{90} \mathrm{Y}$ & 112 & $S$ \\
\hline${ }^{99} \mathrm{Tc}$ & 701 & $S$ \\
\hline${ }^{129} I$ & 0.685 & $S$ \\
\hline${ }^{137} \mathrm{Cs}$ & 1,290 & $S$ \\
\hline${ }^{137 \mathrm{~m}} \mathrm{Ba}$ & 1,230 & $S$ \\
\hline${ }^{154} \mathrm{Eu}$ & $<250$ & $S$ \\
\hline${ }^{155} \mathrm{Eu}$ & $<835$ & $S$ \\
\hline${ }^{237} \mathrm{~Np}$ & $<0.0566$ & $S$ \\
\hline${ }^{239 / 240} \mathrm{Pu}$ & 0.0953 & $S$ \\
\hline
\end{tabular}


Table D4-2. Best-Basis Inventory Estimates for Radioactive Components in Tank 241-AN-105 Supernatant as of May 31, 1996.

(Decayed to January 1, 1994) (2 sheets)

\begin{tabular}{|c|c|c|}
\hline 1:1:1:0: Amalte & Superiatant inuentor (C) & (5. Masts: \\
\hline${ }^{241} \mathrm{Am}$ & 0.100 & $S$ \\
\hline $243 / 244 \mathrm{Cm}$ & 0.110 & $S$ \\
\hline
\end{tabular}

Notes:

$$
{ }^{1} \mathrm{~S}=\text { Sample-based, } \mathrm{M}=\text { Hanford defined waste model-based, } \mathrm{E}=\text { Engineering assessment-based }
$$


Table D4-3. Best-Basis Inventory Estimates for Nonradioactive Components in Tank 241-AN-105 Salt Slurry as of May 31, 1996.

\begin{tabular}{|c|c|c|c|}
\hline : Inalyte: & 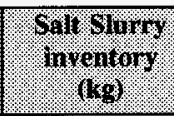 & $(6,19,0,0, E)$ & - Gomment: \\
\hline$\overline{\mathrm{Al}}$ & 56,500 & $S$ & \\
\hline$\overline{\mathrm{Bi}}$ & 147 & $S$ & \\
\hline $\mathrm{Ca}$ & 942 & $S$ & \\
\hline $\mathrm{Cl}$ & 14,620 & S & \\
\hline $\mathrm{TIC}$ as $\mathrm{CO}_{3}$ & 169,000 & $S$ & Core composite data. \\
\hline $\mathrm{Cr}$ & 4,470 & $S$ & \\
\hline $\mathrm{F}$ & 3,400 & $S$ & \\
\hline $\mathrm{Fe}$ & 167 & $S$ & \\
\hline $\bar{K}$ & 8,670 & $\mathrm{~S}$ & \\
\hline $\mathrm{La}$ & $<504$ & $S$ & \\
\hline $\mathrm{Mn}$ & 44.2 & S & \\
\hline $\mathrm{Na}$ & 456,000 & $S$ & \\
\hline $\mathrm{Ni}$ & 97.8 & S & \\
\hline $\mathrm{NO}_{2}$ & 181,000 & $S$ & \\
\hline $\mathrm{NO}_{3}$ & 308,000 & $S$ & \\
\hline$\overline{\mathrm{OH}}$ & 64,000 & $S$ & Core composite data. \\
\hline $\mathrm{Pb}$ & 119 & $S$ & \\
\hline $\mathrm{PO}_{4}$ & 17,300 & $S$ & \\
\hline$\widehat{\mathrm{Si}}$ & 458 & $S$ & \\
\hline $\mathrm{SO}_{4}$ & 18,000 & $S$ & \\
\hline $\mathrm{Sr}$ & $<18.7$ & $S$ & Core composite data. \\
\hline TOC & 14,300 & $S$ & Core composite data. \\
\hline $\mathrm{U}_{\text {TOTAL }}$ & 233 & $S$ & Core composite data. \\
\hline Percent water & 44.7 & $S$ & \\
\hline Density, $\mathrm{g} / \mathrm{mL}$ & 1.59 & $S$ & \\
\hline $\mathrm{Zr}$ & 20.0 & $S$ & \\
\hline
\end{tabular}

Notes:

${ }^{1} \mathrm{~S}=$ Sample-based, $\mathrm{M}=$ Hanford defined waste model-based, $\mathrm{E}=$ Engineering assessment-based. 
Table D4-4. Best-Basis Inventory Estimates for Radioactive Components in Tank 241-AN-105 Salt Slurry as of May 31, 1996.

(Decayed to January 1, 1994)

\begin{tabular}{|c|c|c|}
\hline 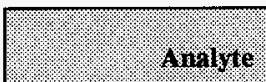 & Sall simy inventory (on) & 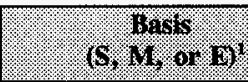 \\
\hline${ }^{60} \mathrm{Co}$ & 6.41 & $S$ \\
\hline${ }^{90} \mathrm{Sr}$ & 33,500 & $\mathrm{~s}$ \\
\hline${ }^{90} \mathrm{Y}$ & 33,500 & $\mathrm{~S}$ \\
\hline${ }^{994} \mathrm{TC}$ & 473 & $\bar{S}$ \\
\hline${ }^{129} I$ & 10.3 & S \\
\hline${ }^{137} \mathrm{Cs}$ & 83,500 & $S$ \\
\hline$\overline{137 \mathrm{~m}} \mathrm{Ba}$ & 79,300 & $S$ \\
\hline${ }^{154} \mathrm{Eu}$ & $<315$ & S \\
\hline${ }^{155} \mathrm{Eu}$ & $<1,420$ & $S$ \\
\hline${ }^{237} \mathrm{~Np}$ & $<25.7$ & $S$ \\
\hline${ }^{239 / 240} \mathrm{Pu}$ & 6.33 & $S$ \\
\hline $2{ }^{241} \mathrm{Am}$ & 9.97 & $S$ \\
\hline $243 / 244 \mathrm{Cm}$ & $<4.16$ & 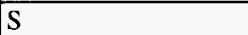 \\
\hline
\end{tabular}

Notes:

'S = Sample-based, $M=$ Hanford defined waste model-based, $E=$ Engineering assessment-based.

Table D4-5. Best-Basis Total Inventory Estimates for Nonradioactive Components in

Tank 241-AN-105 as of May 31, 1996. (2 sheets)

\begin{tabular}{|c|c|c|}
\hline (.). & Total inverion? & (S. Mr. or $\mathrm{g})$ \\
\hline $\mathrm{Al}$ & 156,000 & $S$ \\
\hline $\mathrm{Bi}$ & 214 & $S$ \\
\hline $\mathrm{Ca}$ & 2,140 & $S$ \\
\hline $\mathrm{Cl}$ & 40,800 & $S$ \\
\hline TIC as $\mathrm{CO}_{3}$ & 197,000 & $S$ \\
\hline $\mathrm{Cr}$ & 5,040 & $S$ \\
\hline $\mathrm{F}$ & 3,850 & $S$ \\
\hline $\mathrm{Fe}$ & 287 & $S$ \\
\hline
\end{tabular}


Table D4-5. Best-Basis Total Inventory Estimates for Nonradioactive Components in Tank 241-AN-105 as of May 31, 1996. (2 sheets)

\begin{tabular}{|c|c|c|}
\hline 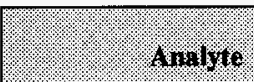 & 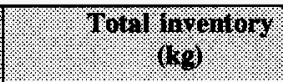 & 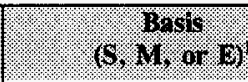 \\
\hline K & 25,400 & $S$ \\
\hline$\overline{\mathrm{La}}$ & $<581$ & $S$ \\
\hline $\mathrm{Mn}$ & $<59.4$ & $\mathrm{~S}$ \\
\hline$\overline{\mathrm{Na}}$ & $1.10 \mathrm{E}+06$ & S \\
\hline $\mathrm{Ni}$ & $<129$ & $S$ \\
\hline$\overline{\mathrm{NO}_{2}}$ & 504,000 & $S$ \\
\hline $\mathrm{NO}_{3}$ & 728,000 & $\mathrm{~S}$ \\
\hline$\overline{\mathrm{OH}}$ & 214,000 & $S$ \\
\hline$\overline{\mathrm{Pb}}$ & 265 & $S$ \\
\hline $\mathrm{PO}_{4}$ & 20,200 & $S$ \\
\hline $\mathrm{Si}$ & 950 & $S$ \\
\hline$\overline{\mathrm{SO}_{4}}$ & 22,900 & $S$ \\
\hline $\mathrm{Sr}$ & $<32.1$ & $S$ \\
\hline TOC & 29,900 & $S$ \\
\hline $\mathrm{U}_{\text {TOTAL }}$ & 242 & $S$ \\
\hline $\mathrm{Zr}$ & $<43.5$ & $S$ \\
\hline Percent water & 46.6 & $\mathrm{~S}$ \\
\hline Density, $\mathrm{g} / \mathrm{mL}$ & 1.56 & $S$ \\
\hline
\end{tabular}

Notes: ${ }^{1} \mathrm{~S}=$ Sample-based, $\mathrm{M}=$ Hanford Defined Waste model-based, $\mathrm{E}=$ Engineering assessment-based. 
Table D4-6. Best-Basis Total Inventory Estimates for Radioactive Components in Tank 241-AN-105 as of May 31, 1996. (Decayed to January 1, 1994)

\begin{tabular}{|c|c|c|}
\hline : Analve & 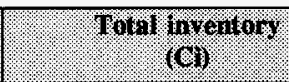 & 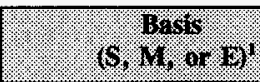 \\
\hline${ }^{60} \mathrm{Co}$ & $<6.46$ & $\mathrm{~S}$ \\
\hline${ }^{90} \mathrm{Sr}$ & 33,600 & $\mathrm{~S}$ \\
\hline${ }^{90} \mathrm{Y}$ & 33,600 & $S$ \\
\hline${ }^{99} \mathrm{Tc}$ & 1,170 & $S$ \\
\hline${ }^{129} \mathrm{I}$ & 11.0 & $S$ \\
\hline${ }^{137} \mathrm{Cs}$ & 84,800 & $S$ \\
\hline${ }^{137 \mathrm{~m}} \mathrm{Ba}$ & 80,600 & S \\
\hline${ }^{154} \mathrm{Eu}$ & $<565$ & $S$ \\
\hline${ }^{155} \mathrm{Eu}$ & $<2,260$ & $S$ \\
\hline${ }^{237} \mathrm{~Np}$ & 25.8 & $S$ \\
\hline${ }^{239 / 240} \mathrm{Pu}$ & 6.43 & S \\
\hline${ }^{241} \mathrm{Am}$ & 10.1 & $S$ \\
\hline${ }^{243 / 244} \mathrm{Cm}$ & $<4.27$ & $S$ \\
\hline
\end{tabular}

Notes:

${ }^{1} \mathrm{~S}=$ Sample-based, $\mathrm{M}=$ Hanford defined waste model-based, $\mathrm{E}=$ Engineering assessment-based. 


\section{D5.0 APPENDIX D REFERENCES}

Agnew, S. F., 1997, Hanford Tank Chemical and Radionuclide Inventories: HDW Model Rev. 4, Los Alamos National Laboratory, Los Alamos, New Mexico.

Hendrickson, D. W., 1994, Grout Treatment Facility Waste Feed Projections, WHC-SD-SMTI-528, Westinghouse Hanford Company, Richland, Washington.

Hodgson, K. M., and M. D. LeClair, 1996, Work Plan for Defining a Standard Inventory Estimate for Wastes Stored in Hanford Site Underground Tanks, WHC-SD-WM-WP-311, Rev. 1, Lockheed Martin Hanford Corporation, Richland, Washington.

Steen, F. H., 1997, Final Report for Tank 241-AN-105, Cores 152 and 153, HNF-SD-WM-DP-199, Rev. 1, Fluor Daniel Hanford, Inc., Richland, Washington. 
HNF-SD-WM-ER-678 Rev. 0

This page intentionally left blank. 
HNF-SD-WM-ER-678 Rev. 0

\section{APPENDIX E}

BIBLIOGRAPHY FOR TANK 241-AN-105

E-1 
HNF-SD-WM-ER-678 Rev. 0

This page intentionally left blank. 


\section{APPENDIX E}

\section{BIBLIOGRAPHY FOR TANK 241-AN-105}

Appendix E provides a bibliography of information that supports the characterization of tank 241-AN-105. This bibliography represents an in-depth literature search of all known information sources that provide sampling, analysis, surveillance, and modeling information, as well as processing occurrences associated with tank $241-\mathrm{AN}-105$ and its respective waste types.

The references in this bibliography are separated into three broad categories containing references broken down into subgroups. These categories and their subgroups are listed below.

\section{NON-ANALYTICAL DATA}

Ia. Models/Waste Type Inventories/Campaign Information

Ib. Fill History/Waste Transfer Records

Ic. Surveillance/Tank Configuration

Id. Sample Planning/Tank Prioritization

Ie. Data Quality Objectives/Customers of Characterization Data

\section{ANALYTICAL DATA - SAMPLING OF TANK WASTE AND WASTE TYPES}

Ila. Sampling of tank 241-AN-105

IIb. Sampling of 242-A Evaporator Streams

\section{COMBINED ANALYTICAL/NON-ANALYTICAL DATA}

IIIa. Inventories using both Campaign and Analytical Information (Best Estimate)

IIIb. Compendium of Existing Physical and Chemical Documented Data Sources

This bibliography is broken down into the appropriate sections of material to use, with an annotation at the end of each reference describing the information source. Where possible, a reference is provided for information sources. A majority of the information listed below may be found in the Westinghouse Hanford Company Tank Characterization Resource Center. 


\section{NON-ANALYTICAL DATA}

Ia. Models/Waste Type Inventories/Campaign Information

Agnew, S. F., J. Boyer, R. A. Corbin, T. B. Duran, J. R. Fitzpatrick, K. A. Jurgensen, T. P. Ortiz, and B. L. Young, 1997, Hanford Tank Chemical and Radionuclide Inventories: HDW Model Rev. 4, LA-UR-96-3680, Rev. 0, Los Alamos National Laboratory, Los Alamos, New Mexico.

- Document contains tank layer and supernatant models and the Historical Tank Content Estimate for Hanford Site underground waste storage tanks, as well as a list of Hanford Site waste types.

Ib. Fill History/Waste Transfer Records

Agnew, S. F., R. A. Corbin, T. B. Duran, K. A. Jurgensen, T. P. Ortiz, and B. L. Young, 1994, Waste Status and Transaction Record Summary, WSTRS Rev. 4, LA-UR-97-311, Rev. 0, Los Alamos National Laboratory, Los Alamos, New Mexico.

- Document contains spreadsheets depicting all available data on tank additions/transfers.

Koreski, G. M., and J. N. Strode, 1994, Operational Waste Volume Projections, WHC-SD-WM-ER-029, Rev. 20, Westinghouse Hanford Company, Richland, Washington.

- Document contains account of waste transfers for double-shell tanks, including waste type and volume, source, and destination.

Ic. Surveillance/Tank Configuration

Lipnizki, J., 1996, Waste Tank Risers Available for Sampling, WHC-SD-WM-TI-710, Rev. 3, Westinghouse Hanford Company, Richland, Washington.

- Document describing riser information.

Salazar, B. E. 1994, Double-Shell Underground Waste Storage Tank Riser Survey, WHC-SD-RE-TI-093, Rev. 4, Westinghouse Hanford Company, Richland, Washington.

- Document describing double-shell underground waste tank riser survey. 
Tran, T. T., 1993, Thermocouple Status Single Shell and Double Shell Waste Tanks, WHC-SD-WM-TI-553, Rev. 0, Westinghouse Hanford Company, Richland, Washington.

- Document contains information pertaining to thermocouple trees installed in the Hanford Site underground waste tanks, such as installation date, material condition, riser number, height of individual thermocouples above the bottom of the tank, high temperature reading, frequency of surveillance, and type of thermocouple.

Id. Sample Planning/Tank Prioritization

Baldwin, J. H., 1997, Push Mode Core Sampling and Analysis Plan for Tank 241-AN-105 in Support of the Privatization Request Tank for Proposal, WHC-SD-WM-TSAP-111, Rev. 0A, Westinghouse Hanford Company, Richland, Washington.

- Document contains detailed sampling and analysis procedure information for tank 241-AN-105 based on TWRS Privatization's request for proposal.

Bell, K. E., 1993, Tank Waste Remediation System Tank Waste Characterization Plan, WHC-SD-WM-PLN-047, Rev. 1, Westinghouse Hanford Company, Richland, Washington.

- Document coordinates the activities of the tank farms and the laboratories by establishing standard sample procurement and analysis procedures, standard quality control procedures and criteria, and prioritizing tank samples.

Brown, T. M., S. J. Eberlein, J. W. Hunt and T. J. Kunthara, 1996, Tank Waste Characterization Basis, WHC-SD-WM-TA-164, Rev. 2, Westinghouse Hanford Company, Richland, Washington.

- Document that summarizes the technical basis for characterizing the waste in the tanks and assigns a priority number to each tank.

Eggers, R. F., 1996, Tank 241-AN-105 Push Mode Core Sampling and Analysis Plan, WHC-SD-WM-TSAP-027, Rev. 1, Westinghouse Hanford Company, Richland, Washington.

- Document contains detailed sampling and analysis procedure information for tank 241-AN-105 based on applicable DQOs. 
Grimes, G. W., 1977, Hanford Long-Term Defense High-Level Waste Management Program Waste Sampling and Characterization Plan, RHO-CD-137, Rockwell Hanford Operations, Richland, Washington.

- Early characterization planning document.

Phillips, J. R., R. Shekarriz, J. M. Bates, 1996, Sampling Plan for Tank 241-AN-105 Retained Gas Samples Deployment, PNNLMIT:030796, Pacific Northwest National Laboratory, Richland, Washington.

- Letter report (and May 30, 1996 Addendum) provide RGS requirements input for the 241-AN-105 TSAP.

Winkelman, W. D., 1996, Tank 241-AN-105 Tank Characterization Plan, WHC-SD-WM-SD-TP-385, Rev. 3, Westinghouse Hanford Company, Richland, Washington.

- Document discusses any and all relevant DQOs and how they will be met for tank 241-AN-105.

Winkelman, W. D., J. W. Hunt and L. J. Fergstrom, 1996, FY 1997 Tank Waste Analysis Plan, WHC-SD-WM-PLN-120, Rev. 1, Lockheed Martin Hanford Corporation, Richland, Washington.

- Document contains Tri-Party Agreement (see Ecology et al. 1994 listing in Section 5.0) requirement-driven TWRS Characterization Program information and a list of tanks addressed in fiscal year 1997.

Winters, W. I., 1996, Privatization Samples for Envelopes " $A$ ", " $B$ " and " $C$ ", WHC-SD-WM-TP-495, Rev. 2, Westinghouse Hanford Company, Richland, Washington.

- Document containing test plan to describe the method for sampling and analysis for privatization. 
Ie. Data Quality Objectives (DQO) and Customers of Characterization Data

Carothers, K. G., 1994, Data Quality Objectives for the Waste Compatibility Program, WHC-SD-WM-DQO-001, Rev. 0, Westinghouse Hanford Company, Richland, Washington.

- Document provides requirements for safe and efficient combination of waste from different tanks and tank systems..

Dukelow, G. T., J. W. Hunt, H. Babad, and J. E. Meacham, 1995, Tank Safety Screening Data Quality Objective, WHC-SD-WM-SP-004, Rev. 2, Westinghouse Hanford Company, Richland, Washington.

- DQO used to determine if tanks are under safe operating conditions.

Jones, T. E. and K. D. Wiemers, 1996, Data Requirements for TWRS Privitization Characterization of Potential Low Activity Waste Feed, WHC-SD-WM-DQO-023, Rev. 0, Westinghouse Hanford Company, Richland, Washington.

- Documents the need of the privitization function within TWRS.

McDuffie, N. G. and G. D. Johnson, 1995, Flammable Gas Tank Safety Program: Data Requirements for Core Sample Analysis Developed Through the Data Quality Objectives (DQO) Process, WHC-SD-WM-DQO-004, Rev.2, Westinghouse Hanford Company, Richland Washington.

- Document provides data needs for evaluating the flammability issues in the tank.

Minteer, D. J., 1996, Waste Tank 214-AN-105 Gas Release Response Recommendations, WHC-SD-WM-ER-524, Rev. 0, Westinghouse Hanford Company, Richland, Washington.

- Document describing gas release response recommendations.

Slankas, T. J., M. J. Kupfer, and W. W. Schultz, 1995, Data Needs and Attendant Data Quality Objectives for Tank Waste Pretreatment and Disposal, WHC-SD-WM-DQO-022, Rev. 0, Westinghouse Hanford Company, Richland, Washington.

- Documents the needs of the pretreatment function within TWRS. 


\section{ANALYTICAL DATA - SAMPLING OF TANK WASTE AND WASTE TYPES}

IIa. Sampling of tank 241-AN-105

Harmon, H. D., 1991, Watch List for Tanks Which May Have Hydrogen Build up, (letter 9001478B R1 to R. E. Gerton, February 8), Westinghouse Hanford Company, Richland, Washington.

- Letter reports the results of the slurry growth study of actual waste.

Jansky, M. T., 1984, Laboratory Batch Boil Down on Actual Double Shell Slurry Feed: $A N-104$ \& AN-105 Blend, (letter 65453-84-090 to L. M. Sasaki, April 2), Rockwell Hanford Operation, Richland, Washington.

- Memo reports the results of the slurry growth study of actual waste.

Jansky, M. T., 1984, Boil Down \& Slurry Growth Experiment with Tank AN-104 \& AN-105 Double-Shell Slurry Feed (DSSF), (letter \# 65611-84-008 to L. M. Sasaki, January 19, 1984), Rockwell Hanford Operation, Richland, Washington.

- Memo to provide information in support of DSS flowsheet and for determination of scale-up effects on slurry growth.

King, A. G., 1996, TWRS Privatization Private Contractor Samples for Low Activity Waste Envelope B Tank 241-AN-105 Preliminary Analytical Report, (letter 9655753 to C. G. Mattsson, November 20), Rust Federal Services of Hanford, Inc., Richland, Washington.

- Letter provides analyses of TWRS privatization contract samples, low-activity waste feed envelope B. Tank.

Mauss, B. M., 1984, Chemical Compositions of 102-AY, 101-AW, 105-AN, and 104-AN, (internal memorandum to E. G. Gratny from B. M Mauss, 65453-84-348, November 9), Rockwell International, Richland, Washington.

- Memorandum of chemical compositions of 105-AN tank. 
Payne, M. A., 1992, Addition of Water to Watch List Tanks, (letter 9251519 to R. E. Gerton, March 12), Westinghouse Hanford Company, Richland, Washington.

- Letter reports the results of the slurry growth study of actual waste.

Sasaki L. M., 1984, Results of Organic Analysis of Double Shell Slurry, (letter 65611-84-053 to D. J. Fisher, April 5), Rockwell Hanford Operation, Richland, Washington.

- Memo reports the results of the slurry growth study of actual waste. Also supports complexant removal studies.

Stewart, C. W., J. M. Alzheimer, C. L. Shepard, G. Terrones, G. Chen, N. E. Wilkins, 1996, In Situ Determination of Rheological Properties and Void Fraction: Hanford Waste Tank 241-AN-105, PNNLMIT:021696, Pacific Northwest National Laboratory, Richland, Washington.

- Document presents the results from operating the ball rheometer and void fraction instrument in Hanford Site waste tank 241-AN-105.

Steen, F. H., 1997, Final Report for Tank 241-AN-105, Cores 152 and 153, HNF-SD-WM-DP-199, Rev. 1, Rust Federal Services of Hanford, Inc., Richland, Washington.

- Document contains sample analyses from 1996 tank 241-AN-105 core sampling event.

Steen, F. H., 1996, Tank 241-AN-105, Cores 152 and 153, Analytical Results for The 45 Day Report, WHC-SD-WM-DP-199, Rev. 0, Westinghouse Hanford Company, Richland, Washington.

- Document contains safety screening sample analyses from 1996 tank 241-AN-105 core sampling event.

Shekarriz, A., R. E. Bauer, D. R. Rector, N. S. Cannon, L. A. Mahoney, B. E. Hey, M. A. Chieda, C. G. Linshooten, J. M. Bates, F. J. Reitz, E. R. Siciliano., 1997, Retained Gas Sampler Measurement Results for Hanford Waste Tanks 241-AW-101, 241-A-101, 241-AN-105, 241-AN-104 241-AN-103, PNNL-11450, Rev. 1, Pacific Northwest National Laboratory, Richland, Washington.

- Document contains the measurements resulting from retained gas sampling tanks. 
Wilkins, N. E., 1995, Results of Gas Monitoring of Double-Shell Flammable Gas Watch List Tanks, WHC-SD-WM-TI-682, Rev. OA, Westinghouse Hanford Company, Richland, Washington.

- Document describing gas monitoring of double-shell tanks.

Wilkins, N. E., R. E. Bauer, D. M. Ogden, 1996, Results of Vapor Space Monitoring of Flammable Gas Watch List Tanks, HNF-SD-WM-TI-797, Lockheed Martin Hanford Corporation, Richland, Washington.

- Document contains results of SHMS, vapor grab sampling, and gas character system monitoring of tank $241-\mathrm{AN}-105$ as well as other watch list tanks.

IIb. Sampling of 242-A Evaporator Streams

Cash, R. J. and J. W. Lentch, 1996, Flammable Gas Safety Program report on May 30, 1996 Gas Release Event in Tank AN-105, 1996, (letter 9652960 to J. K. McClusky, July 3), Westinghouse Hanford Company, Richland, Washington.

- Letter written to report assessment and actions in response to gas release event of May 30, 1996.

Jansky, M. T., 1984, Laboratory Support for Upcoming 242-Evaporator Campaign, Run 84-5, (letter 65453-84-134 to E. F. Gratny, May 10), Rockwell Hanford Operation, Richland, Washington.

- Letter reports the analytical data to support Evaporator Campaign, Run 84-5.

Johnson, G. D. and M. A. Payne, 1994, Tank AN-105 Gas Release Event of May 1994, (letter 9455249 to distribution, July 29), Westinghouse Hanford Company, Richland, Washington.

- Letter written to review data and evaluate the safety implications of Gas Release Event of May 17, 1994.

Pontious, N. L., 1986, 242-A Evaporator/Crystal; FY-1985 Campaign 85-3 Post-Run Document, WHC-SD-WM-PE-023, Westinghouse Hanford Company, Richland, Washington.

- Document describing evaporator run campaign 85-3. 
Von Bergen, B. H., 1987, 242-A Evaporator Run Schedule for Room 88-1 (Internal memorandum 13331-87-975 to G. L. Dunford, December 18), Westinghouse Hanford Company, Richland, Washington.

- Memorandum contains 1988 evaporator run planning information.

\section{COMBINED ANALYTICAL/NON-ANALYTICAL DATA}

IIIa. Inventories from Campaign and Analytical Information

Agnew, S. F., J. Boyer, R. A. Corbin, T. B. Duran, J. R. Fitzpatrick, K. A. Jurgensen, T. P. Ortiz, and B. L. Young, 1997, Hanford Tank Chemical and Radionuclide Inventories: HDW Rev. 4, LA-UR-96-3680, Rev. 0, Los Alamos National Laboratory, Los Alamos, New Mexico.

- Document contains waste type summaries as well as primary chemical compound/analyte and Radionuclide estimates for sludge, supernatant, and solids.

IIIb. Compendium of data from other sources physical and chemical

Agnew, S. F., and J. G. Watkin, 1994, Estimation of Limiting Solubilities for Ionic Species in Hanford Waste Tank Supernates, LAUR-94-3590, Los Alamos National Laboratory, Los Alamos, New Mexico.

- Document gives solubility ranges used for key chemical and radionuclide components based on supernatant sample analyses.

Brevick, C. H., L. A. Gaddis, and E. D. Johnson, 1995, Tank Waste Source Term Inventory Validation, Vol I \& II., WHC-SD-WM-ER-400, Rev. 0, Westinghouse Hanford Company, Richland, Washington.

- Document contains a quick reference to sampling information in spreadsheet or graphical form for 23 chemicals and 11 radionuclides for all the tanks.

Brevick, C. H., L. A. Gaddis, and S. D. Consort, 1995, Supporting Document for the Southeast Quadrant Historical Tank Content Estimate for AN Tank Farm (Vol I and II), WHC-SD-WM-ER-314, Rev. 0, Westinghouse Hanford Company, Richland, Washington.

- Document describing the AN Tank Farm. 
Hanlon, B. M., 1996, Waste Tank Summary Repeat for Month Ending May 31, 1996, HNF-EP-0182-98, Westinghouse Hanford Company, Richland, Washington.

- Document describing waste tank summary report.

Husa, E. I., 1993, Hanford Site Waste Storage Tank Information Notebook, WHC-EP-0625, Westinghouse Hanford Company, Richland, Washington.

- Document contains in-tank photos as well as summaries on the tank description, leak detection system, and tank status.

Husa, E. I., 1995, Hanford Waste Tank Preliminary Dryness Evaluation, WHC-SD-WM-TI-703, Rev. 0, Westinghouse Hanford Company, Richland, Washington.

- Document gives assessment of relative dryness between tanks.

Olson N. J., 1989, Electrochemical Testing A537 Carbon Steel Purex Scrub Solutions For Corrosion Behavior-Preliminary Report, (letter LET-011689 to Reynolds, D. A., January 16), Pacific Northwest National Laboratory, Richland, Washington.

- Letter presents the results from corrosion testing with scrub solutions for corrosion behavior.

Van Vlect, R. J., 1993, Radionuclide and Chemical Inventories for DoubleShell Tanks, WHC-SD-WM-TI-543, Rev. 1, Westinghouse Hanford Company, Richland, Washington.

- Report containing radionuclide chemical inventories for double-shell tanks. 


\section{DISTRIBUTION SHEET}

\begin{tabular}{|c|c|c|c|c|c|}
\hline \multirow{2}{*}{$\begin{array}{l}\text { To } \\
\text { Distribution }\end{array}$} & \multirow{2}{*}{\multicolumn{3}{|c|}{ From }} & \multicolumn{2}{|l|}{ Page 1 of 2} \\
\hline & & & & \multicolumn{2}{|c|}{$04 / 30 / 97$} \\
\hline \multicolumn{4}{|l|}{ Project Title/Work Order } & \multicolumn{2}{|c|}{ EDT No. EDT-617654 } \\
\hline \multicolumn{4}{|c|}{$\begin{array}{l}\text { Tank Characterization Report for Double-She17 Tank 241-AN-105, } \\
\text { HNF-SD-WM-ER-678. Rev. } 0\end{array}$} & \multicolumn{2}{|c|}{ ECN No. $\quad N / A$} \\
\hline Name & MSIN & $\begin{array}{c}\text { Text } \\
\text { With All } \\
\text { Attach. }\end{array}$ & Text Only & $\begin{array}{l}\text { Attach./ } \\
\text { Appendix } \\
\text { Oniy }\end{array}$ & $\begin{array}{l}\text { EDT/ECN } \\
\text { Only }\end{array}$ \\
\hline
\end{tabular}

OFFSITE

Sandia National Laboratory

P.O. Box 5800

MS-0744, Dept. 6404

Albuquerque, NM 87815

D. Powers

Nuclear Consulting Services Inc.

P. 0. Box 29151

Columbus, OH 43229-01051

J. L. Kovach

$X$

Chemical Reaction Sub-TAP

P.0. Box 271

Lindsborg. KS 67456

B. C. Hudson

$X$

SAIC

20300 Century Boulevard. Suite 200-B

Germantown, MD 20874

H. Sutter

$x$

Los Alamos Laboratory

CST-14 MS-J586

P. 0. Box 1663

Los Alamos, NM 87545

S. F. Agnew

$x$

Los Alamos Technical Associates

T. T. Tran

B1-44 $\quad X$

Tank Advisory Panel

102 Windham Road

Oak Ridge, TN 37830

D. 0. Campbe11 


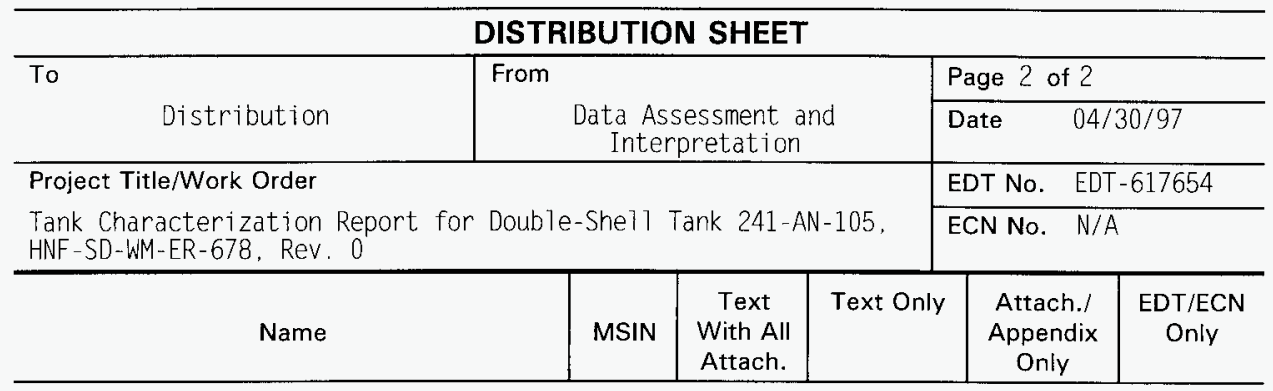

\section{ONSITE}

Department of Energy - Richland Operations

J. F. Thompson

W. S. Liou

J. A. Poppiti

N. W. Withis

DE\&S Hanford, Inc.

R. J. Cash

W. L. Cowley

G. L. Dunford

G. D. Johnson

J. E. Meacham

Fluor Daniel Northwest

J. L. Stroup

Lockheed Martin Hanford, Corp.

K. M. Hodgson

J. Jo

T. J. Kelley

L. M. Sasaki

B. C. Simpson

L. R. Webb

ERC (Environmental Resource Center)

T.C.S.R.C.

Lockheed Martin Services, Inc.

B. G. Lauzon

Central Files

EDMC

Numatec Hanford Corporation

J. S. Garfield

J. S. Hertzel

D. L. Lamberd

Pacific Northwest National Laboratory

A. F. Noonan

Rust Federal Services of Hanford, Inc.

C. T. Narquis

$\begin{array}{ll}57.54 & x \\ 57-54 & x \\ 57-54 & x \\ 57-54 & x\end{array}$

S7- $14 \quad x$

R2-54 $\quad x$

A2-34 $\quad X$

$57-14 \quad x$

$57-14 \quad x$

S3-09 $\quad x$

$\mathrm{H} 0-34 \quad X$

R2-12 $\quad X$

S7-21 $\quad X$

R2 $-12 \quad x$

R2 $-12 \quad X$

$R 2-12 \quad x$

R1-51 X

R1-08 5

R1-08 $\quad X$

A3-88 $\quad x$

H6-08 $X$

H5-49 $\quad x$

$H 5-61 \quad X$

H5-61 $x$

$K 9-91 \quad X$

T6-16 $\quad x$ 